\title{
Population Balance Models: A useful complementary modelling framework for future WWTP modelling
}

Nopens, Ingmar; Torfs, Elena ; Ducoste, Joel ; Vanrolleghem, Peter; Gernaey, Krist

Published in:

Proceedings. 4th IWA/WEF Wastewater treatment modelling seminar

Publication date:

2014

Document Version

Publisher's PDF, also known as Version of record

Link back to DTU Orbit

Citation (APA):

Nopens, I., Torfs, E., Ducoste, J., Vanrolleghem, P., \& Gernaey, K. (2014). Population Balance Models: A useful complementary modelling framework for future WWTP modelling. In Proceedings. 4th IWA/WEF Wastewater treatment modelling seminar (pp. 129-138). Water Environment Federation.

\section{General rights}

Copyright and moral rights for the publications made accessible in the public portal are retained by the authors and/or other copyright owners and it is a condition of accessing publications that users recognise and abide by the legal requirements associated with these rights.

- Users may download and print one copy of any publication from the public portal for the purpose of private study or research.

- You may not further distribute the material or use it for any profit-making activity or commercial gain

- You may freely distribute the URL identifying the publication in the public portal 


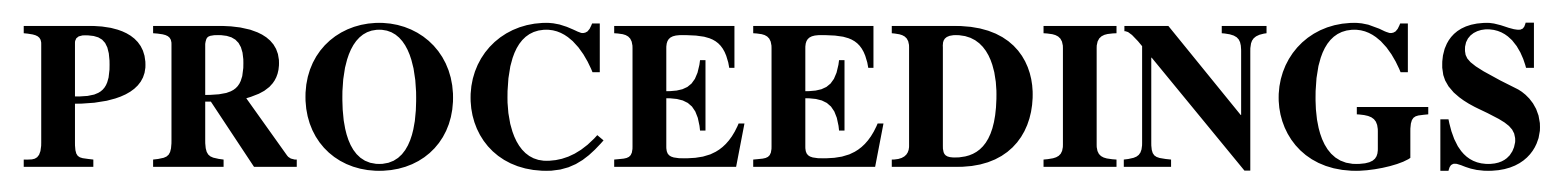

4th IWA/WEF Wastewater treatment modelling seminar

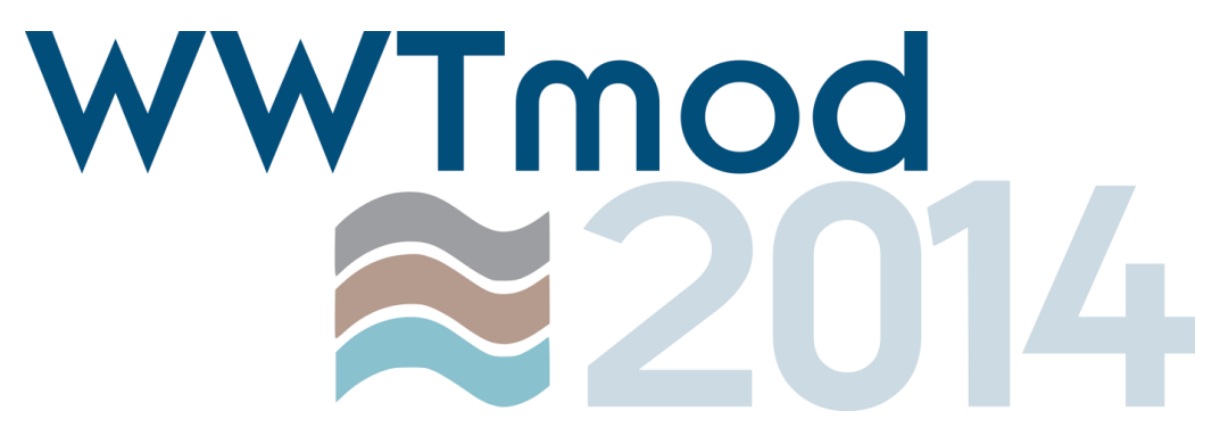

Spa, Belgium, March 30th - April 2nd, 2014

DHI)

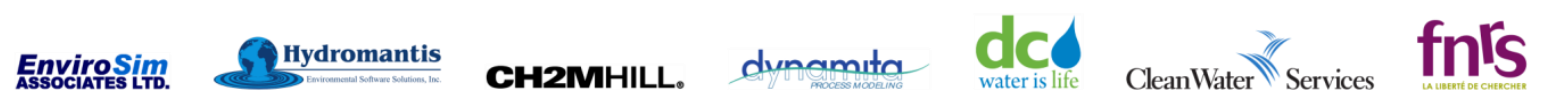



WWTmod2014 is the fourth international seminar on wastewater treatment (WWT) modelling after successful events in 2008, 2010 and 2012. WWTmod provides a platform upon which any relevant aspect of WWT modeling may be scrutinized. The main objective of WWTmod is consensus building. The process of consensus building is supported by obtaining insights from a diverse group of leading professionals: researchers, consultants, utilities, regulators, manufacturers, and software developers.

Stricter effluent limits for nutrients and other contaminants, and concerns about plant efficiency, climate change, and emerging contaminants are driving new model development efforts and more and more sophisticated application of modelling.

The widespread use of wastewater treatment models depends on the development of widely accepted standards and procedures. The organizers hope this seminar will contribute to the further development of "Good Modelling Practice" in this field.
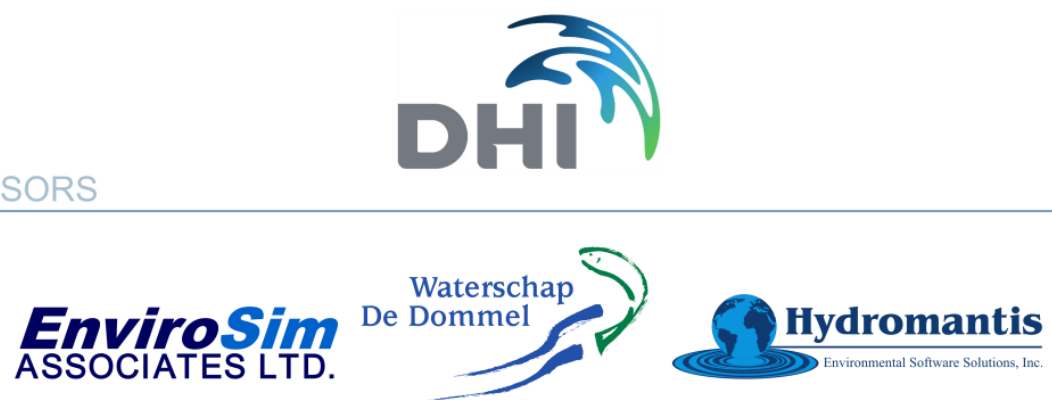

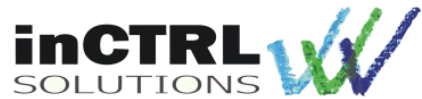

SILVER SPONSORS
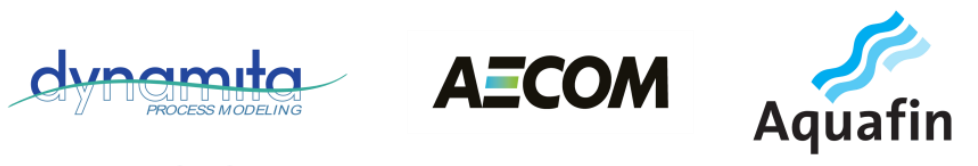

CH2MHILL。

UTILITY ENDORSEMENT
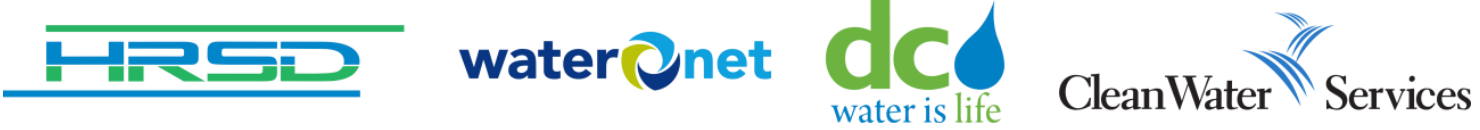

SUPPORTING ORGANIZATIONS

$$
\text { fwo: }
$$




\section{WWTmod}

\section{FINAL PROGRAMME}

\section{Saturday, March $29^{\text {th }} 2014$}

\section{9:00 - 17:00 YWP Workshop}

09:00-09:15 Overview

Part I: Data Issues in Process Modeling

09:15-10:15 Presentations

10:15-10:30 Coffee Break

10:30-11:45 Presentations

11:45-13:00 Lunch

Part II: Identifying Barriers to Innovation in Process Modelling

13:00-15:00 Presentations and Small Group Discussions

15:00-15:30 Coffee Break

Part III: Finding Solutions

15:30-16:00 Summary of small group discussions and of proposed solutions

16:00-16:45 Panel Discussion: Overcoming barriers to innovation

16:45- 17:00 Wrap-up, closing summary

YWP Workshop Organizing Committee:

Magnus Arnell, Lund University, Sweden

Hélène Hauduc, LISBP - INSA de Toulouse, France

Thomas Maere, Ghent University, Belgium

Adrienne Menniti, Clean Water Services, USA 


\section{WWTmod}

Final Programme

\section{Sunday, March $30^{\text {th }} 2014$}

08:30-08:45 Welcome by Ingmar Nopens (Chair Scientific Committee, Chair B-IWA)

08:45-09:45 Opening Lecture by Daniel A. Nolasco, Nolasco \& Associates:

Waste Water Treatment Plant (WWTP) Modeling from a consultant point of view.

09:45-10:45 Presentations and Discussion

09:45 - 17:15 Parallel full-day workshops

10:45-11:15 Coffee break

11:15-12:45 Presentations and Discussion

12:45-13:45 Lunch break

13:45-15:15 Presentations and Discussion

15:15-15:45 Coffee break

15:45-16:45 Presentations and Discussion

16:45-17:15 Wrap-up

Themes:

1. Where are we and where should we go with MBR modelling?

Thomas Maere and Joaquim Comas

2. What do we need for "Total" Nitrogen Modelling?

Peter Dold and José C. Porro

3. How can modelling be effectively used for energy balance optimization?

Adrienne Menniti, Tom Johnson and Leon Downing

4. Revisiting phosphorus removal: do the models give the answers we want?

Youri Amerlinck, Albert Guisasola and David Ikumi

5. Linking WWTP modelling with Life Cycle Assessment (LCA) and other Holistic models

Andrew Shaw and Lluis Corominas

6. Wet-weather modelling: Why and how should we tame the beast?

Lorenzo Benedetti and Peter Vanrolleghem

17:15-18:00 Report preparation (workshop chairs)

18:30 Welcome reception and dinner at Casino of Spa 


\section{WWTmod}

Final Programme

\section{Monday, March $31^{\text {st }} 2014$}

08:30-08:45 Opening by IWA president Glen Daigger

08:45-09:15 Report from Saturday YWP workshop and Discussion

09:15-10:15 Reports and discussion from Sunday workshops 1\&2

Moderator: Peter Vanrolleghem, Co-moderator: Kimberly Solon

10:15-10:45 Coffee break

10:45-12:15 Session 1: Influent characterisation for full scale modelling Moderator: Bruce Johnson, Co-moderator: Hélène Hauduc

Characterization and separation of unbiodegradable matter in WRRFs

Majdala Mansour-Geoffrion, Peter L. Dold, Alain Gadbois, Stéphane Déléris and Yves Comeau

Influent generator for probabilistic design of nutrient removal wastewater treatment plants

Mansour Talebizadeh, Evangelia Belia and Peter A. Vanrolleghem

12:15-13:30 Lunch

13:30-15:00 Session 2: Impact of diffusion

Moderator: Damien Batstone, Co-moderator: Marina Arnaldos

Increasing complexity in biofilm reactor models: How far do we need to go?

George Wells, Thomas Vannecke, Nathalie Hubaux, Eberhard Morgenroth and

Eveline Volcke (Fusion paper)

\section{The Case of the Ks: Diffusion versus Strategy}

Andrew Shaw, Imre Takacs, Krishna Pagilla, Rumana Riffat, Haydee

De Clippeleir, Christopher Wilson and Sudhir Murthy

15:00-15:30 Coffee break

15:30-17:00 Session 3: Nitrogen modeling extended/revisited

Moderator: Sylvie Gillot, Co-moderator: Magnus Arnell

Evaluating two concepts for the modelling of biological denitrification

Pan, Bing-Jie Ni, Huijie Lu, Kartik Chandran, David Richardson and Zhiguo Yuan

Calibration of nitrous oxide production models with continuous long-term process data

Mathieu Spérandio, Mathieu Pocquet, Lisha Guo, Peter A. Vanrolleghem, Bing-Jie $\mathrm{Ni}$ and Zhiguo Yuan

19:00 Dinner at venue 


\section{WWTmod}

Final Programme

\section{Tuesday, April $1^{\text {st }} 2014$}

08:30-09:15 Keynote lecture

Robert Schröder, Policy officer DG Environment

Water policy from a European perspective

09:15-10:15 Reports from Sunday workshops 3\&4

Moderator: Andy Shaw, Co-moderator: Laura Snip

10:15-10:45 Coffee break

10:45-12:15 Session 4: WWTP modeling: back to the future

Moderator: Eberhard Morgenroth, Co-moderator: Sherri Cook

Towards BSM2-GPS-X: A plant-wide benchmark simulation model not only for carbon and nitrogen, but also for greenhouse gases (G), phosphorus (P), sulphur (S) and micropollutants (X), all within the fence of WWTPs/WRRFs Peter Vanrolleghem, Xavier Flores-Alsina, Lisha Guo, Kimberly Solon, David Ikumi, Damien Batstone, Chris Brouckaert, Imre Takács, Paloma Grau, George Ekama, Ulf Jeppsson and Krist V. Gernaey

Population Balance Models: A useful complementary modelling framework for future WWTP modelling

Ingmar Nopens, Elena Torfs, Joel Ducoste, Peter A. Vanrolleghem and Krist V. Gernaey

12:15-13:30 Lunch

13:30-15:00 Session 5: Primary/secondary sedimentation

Moderator: Imre Takacs, Co-moderator: Adrienne Menniti

Modelling and characterisation of primary settlers in view of whole plant and resource recovery modelling

Giulia Bachis, Thibaud Maruéjouls, Sovanna Tik, Youri Amerlinck, Henryk Melcer Ingmar Nopens, Paul Lessard and Peter A. Vanrolleghem

Modelling the impact of filamentous bacteria abundance in a secondary settling tank: CFD sub-models optimization using long-term experimental data

Elham Ramin, Dorottya S. Wágner, Lars Yde, Peter Szabo, Michael R. Rasmussen, Arnaud Dechesne, Barth F. Smets, Peter Steen Mikkelsen, Benedek Gy. Plósz

15:00-15:30 Coffee break

15:30-16:30 Reports from Sunday workshops 5\&6

Moderator: Yves Comeau, Co-moderator: Elena Torfs

19:00

Gala dinner at Abbey of Stavelot 


\section{WWTmod}

Final Programme

\section{Wednesday, April $2^{\text {nd }} 2014$}

08:30-08:45 Welcome address

WEF represenative

08:45-10:15 Session 6: Models for new processes

Moderator: Eveline Volcke, Co-moderator: Bing-Jie Ni

Model based evaluation of mechanisms and benefits of nitrogen shortcut processes

Ahmed Al-Omari, Bernhard Wett, Ingmar Nopens, Haydee De Clippeleir, Mofei Han, Pusker Regmi, Charles Bott and Sudhir Murthy

Modeling of Organic Substrate Transformation in the High-Rate Activated Sludge Process: Why Current Models Don't Work and a Recommended Unified Model Approach

Thomas Nogaj, Andrew Randall, Jose Jimenez, Imre Takacs, Charles Bott, Mark Miller, Sudhir Murthy and Bernhard Wett

10:15-10:45 Coffee break

10:45-11:45 Closing session

11:45-12:00 Summary of WWTmod2014 by Josh Boltz (WWTmod2014 Chair)

12:00-12:15 Incoming Chair: Outlook on WWTmod2016

12:15-13:30 Lunch 


\section{WWTmod}

Final Programme

\section{Poster sessions}

Modelling of phosphorous removal

Towards Calibration of Phosphorus (P) Removal Plant-Wide Models

David S. Ikumi, Peter A. Vanrolleghem, Christopher J. Brouckaert, Marc B.

Neumann and George A. Ekama

Modelling the behaviour of Polyphosphate Accumulating Organisms (PAOs) In biological nutrient removal processes in the presence of external carbon Xiang Hu, Dominika Sobotka, Krzysztof Czerwionka, Qi Zhou, Li Xie, Giulio Munz, Jan A Oleszkiewicz and Jacek Makinia

A dynamic model for physicochemical phosphorus removal: validation and integration in ASM2d

Hélène Hauduc, Imre Takacs, Scott Smith, Anita Szabo, Sudhir Murthy, Glen T. Daigger, and Mathieu Sperandio

A control-based approach to achieve efficient biological phosphorus removal In WWTPs: design, simulation, optimisation and experimental validation Javier Guerrero, Albert Guisasola and Juan A. Baeza

Advanced physical-chemical WWTP modeling

Validation of spontaneous mineral precipitation models

Christian Kazadi Mbamba, Damien Batstone, Stephan Tait

Incorporating aquatic chemistry into wastewater treatment process models: a critical review of different approaches

Izaro Lizarralde, Christopher J Brouckaert, Peter A. Vanrolleghem, David S. Ikumi, George A. Ekama, Eduardo Ayesa and Paloma Grau

Computational Fluid Dynamics as a supportive tool for Wastewater Treatment Plant modelling

Julien Laurent, Randal. W. Samstag, Joel M. Ducoste, Alonso Griborio, Ingmar Nopens, Damien J. Batstone, Jim D. Wicks, Stephen Saunders, , Olivier Potier

Dynamic Modelling of Diffused Aeration Systems Lu-Man Jiang, Riccardo Gori, Thomas Gocke, Diego Rosso 


\section{WWTmod}

Final Programme

Advanced biological WWTP modelling

The application of removal coefficients for viruses in different wastewater treatment processes calculated using stochastic modelling

Edgard Dias, James Ebdon and Huw Taylor

Early-stage design of municipal wastewater treatment plants - presentation and discussion of an optimisation based concept

Hande Bozkurt, Alberto Quaglia, Krist V. Gernaey and Gürkan Sin

Empirical vs. knowledge-based modelling of filtration in submerged anaerobic MBRs (SAnMBRs)

Ángel Robles, Gergö Zajzon, Laura Jurecska, María Victoria Ruano, Josep Ribes, Aurora Seco and José Ferrer

Removal of pharmaceuticals in biological wastewater treatment systems: model generalisation and implications for environmental risk predictions Fabio Polesel, Katherine H. Langford, Stefan Trapp, Kevin V. Thomas, Benedek Gy. Plósz

Nutrient removal and recovery modelling

Modelling aerobic methane oxidation in a municipal WWTP

Matthijs R.J. Daelman, Tamara Van Eynde, Mark C.M. van Loosdrecht and Eveline I.P. Volcke

Innovative Modelling in the Design of the Sacramento Regional Wastewater Treatment Plant for Biological Nutrient Removal

Patrick Dunlap, Andrew Shaw, James Barnard, Heather Phillips, Daniel Wilson and Ken Abraham

Modeling bioaugmentation with nitrifiers in membrane bioreactors

Alberto Mannucci, Giulio Munz, Gualtiero Mori, Jacek Makinia, Claudio Lubello and Jan A Oleszkiewicz

A Green Micro-Algal Growth Model developed in the Activated Sludge Modelling Framework (ASM-A)

Borja Valverde-Pérez, Dorottya Sarolta Wágner, Mariann Sæbø, Jonathan Van

Wagenen, Irini Angelidaki, Barth F. Smets, Benedek Gy. Plósz 


\section{WWTmod}

Final Programme

Anaerobic digestion modelling

ADM1 implementation with an innovative organic matter characterization methodology based on chemical sequential extractions and 3D fluorescence spectroscopy: extension to organic micropollutants fate.

Julie Jimenez, Quentin Aemig, Jean-Philippe Steyer, Dominique Patureau

Establishing design quidelines for anaerobic co-digestion using a novel design-oriented analysis and ADM1

Sherri Cook, Steven Skerlos, Nancy Love

Model based approach to maximize gas production for high-loaded digestion Process

Ryu Suzuki, Sudhir Murthy, Bernhard Wett and Imre Takács

Modeling mixing and mixing models

A Protocol for Optimization of Activated Sludge Mixing

Randal W. Samstag and Edward Wicklein

A general three-dimensional extension to ADM1: the importance of an integrated fluid flow model

David L. F. Gaden and Eric L. Bibeau

Modelling bacterial selection during the plug-flow feeding phase of aerobic granular sludge biofilm reactors

David G. Weissbrodt, Christof Holliger, Eberhard Morgenroth

ASM input/output analysis

Should activated sludge models consider influent seeding of nitrifiers? Field characterization of nitrifying bacteria

Shameem Jauffur, Siavash Isazadeh and Dominic Frigon

Generation of (synthetic) influent data for performing wastewater treatment modelling studies

Xavier Flores-Alsina, Christoph Ort, Cristina Martin, Lorenzo Benedetti, Evangelina Belia, Laura Snip, Ramesh Saagi, Mansour Talebizadeh, Peter A. Vanrolleghem, Ulf Jeppsson, Krist V. Gernaey

A simple yet efficient colour-based system analysis tool for ASM Amerlinck, Y., Cierkens, K., Nopens, I. 


\section{WWTmod}

Final Programme

\section{Greenhouse gas modelling}

Modelling N2O dynamics in the engineered N cycle: Evaluation of alternate model structures

Barth F. Smets, Carles Pellicer-Nàcher, Carlos Domingo-Félez, Marlene Mark Jensen, Elham Ramin, Benedek Gy. Plósz, Gürkan Sin, Krist V. Gernaey.

Modelling simultaneous anaerobic methane and ammonium removal in a granular sludge reactor

Mari- Karoliina. H. Winkler, Thomas P.W. Vannecke, Andrew Bogdan and Eveline I. P. Volcke

Risk assessment modelling of $\mathrm{N} 2 \mathrm{O}$ production in activated sludge systems; a knowledge-based approach

Jose Porro, Costanza Milleri, Joaquim Comas, Ignasi Rodriguez-Roda, Maite Pijuan, Lluís Corominas, Lisha Guo, Matthijs Daelman, Eveline Volcke, Mark van Loosdrecht, Peter A. Vanrolleghem and Ingmar Nopens

Estimation of dynamic apparent nitrification kinetics as the key for reliable greenhouse gas emission prediction

Haydee De Clippeleir, Imre Takacs, Bernhard Wett, Kartik Chandran and Sudhir Murthy 
WWTmod

WORKSHOPS 


\section{Young Water Professional Workshop}

The 2014 YWP workshop will be divided into two parts. We will explore data issues in process modelling for the morning session. The afternoon session will be a highly interactive look at barriers to innovation in process modelling and how we can overcome them. Our goal is to create a dialog on important topics relevant to young water professionals and encourage networking between young and senior water professionals. YWPs are invited to submit this questions or experiences on either topic to the LindedIn forum: WWTmod 2014 YWP workshop. The contributions will be used as discussion starters in the workshop.

\section{Data Issues in Process Modelling}

Any simulation exercise has to rely on data to build, calibrate and validate the model. However, collecting data from wastewater treatment processes, lab or full scale, is always tedious and delicate. YWP, whether from academia, consultancy or utilities, are confronted with many questions, often without having enough experience to address them:

- Which kind of results are of interest to practitioners? Scientists? Are these results compatible with the data collected by utilities?

- Depending on the usage (knowledge build-up, scenario analysis, control), which data need to be collected (sensors, logbooks, dedicated measurement campaign) and at which frequency?

- How to deal with data storage? How to insure data quality (reconciliation, truncation, time stamps)?

Collecting and preparing a full-plant dataset that is reliable enough for a modelling exercise is a huge effort that may have additional value for other purposes. The question of sharing datasets, making them available for the whole community, will also be discussed in this morning session.

The workshop will contain 3 presentations from junior and senior experts on data issues and will be followed by a group discussion. The expert panel will also elaborate on data issues from the audience, which can be proposed through the LinkedIn forum: WWTmod 2014 YWP workshop.

\section{Overcoming Barriers to Innovation in Process Modelling}

Innovations in process modelling, as in any field, are encouraged by interdisciplinary collaboration, open exchange of ideas at conferences and in peer-reviewed publications, and freedom to explore without fear of failure. The process of transferring new ideas from academia to application relies on innovation by academics, consultants and utilities. As funding pressures in all three areas become more intense, the activities that foster innovation are reduced in favor of activities that increase the chances of winning research funding, improve profitability, or reduce "extraneous" operating expenses.

The three presentations will summarize the barriers to innovation in academia, consulting and utilities. Each presentation will be developed by a team of contributors to generate a consensus view that includes junior and senior viewpoints as well as male and female and European and north American. Each presentation will address the following questions:

- What are the contributions of each field to innovations in process modeling?

- What are the barriers to innovation?

- How do those barriers hinder YWP career development and vice versa?

- What solutions could address the issues identified?

The remainder of the afternoon will be spent in interactive discussion, exploring the topic further and brainstorming solutions. 
YWP Workshop

\section{Workshop Agenda:}

\begin{tabular}{|c|c|c|c|}
\hline \multicolumn{2}{|c|}{ Time } & \multirow{2}{*}{$\begin{array}{l}\text { Topic } \\
\text { Overview }\end{array}$} & \multirow[t]{2}{*}{ Potential Speaker } \\
\hline 9:00 & $9: 15$ & & \\
\hline \multicolumn{4}{|c|}{ Part I: Data Issues in Process Modeling } \\
\hline $9: 15$ & $9: 45$ & $\begin{array}{l}\text { Presentation ( } 20 \text { minutes): } \\
\text { Data issues in process modelling: It all starts } \\
\text { with sampling }\end{array}$ & Lina Belia, Primodal, Canada \\
\hline $9: 45$ & $10: 15$ & $\begin{array}{l}\text { Presentation (20 minutes): } \\
\text { Mass balancing and sensor placement for data } \\
\text { reconciliation and fault detection - Basic } \\
\text { concepts }\end{array}$ & Kris Villez, Eawag, Switzerland \\
\hline $10: 15$ & $10: 30$ & Break & \\
\hline $10: 30$ & 11:00 & $\begin{array}{l}\text { Presentation ( } 20 \text { minutes): } \\
\text { Data validation and model calibration: which } \\
\text { one needs to be perfect? }\end{array}$ & $\begin{array}{l}\text { Jeroen Langeveld, TU Delft, the } \\
\text { Netherlands }\end{array}$ \\
\hline 11:00 & $11: 45$ & Group discussion on issues from the audience & \\
\hline $11: 45$ & $13: 00$ & LUNCH & \\
\hline \multicolumn{4}{|c|}{ Part II: Identifying Barriers to Innovation in Process Modelling } \\
\hline 13:00 & 13:25 & $\begin{array}{l}\text { Presentation (20 minutes): } \\
\text { Barriers to Innovation in Academia }\end{array}$ & Christoph Ort, Eawag, Switzerland \\
\hline $13: 25$ & $13: 50$ & $\begin{array}{l}\text { Presentation (20 minutes): } \\
\text { Barriers to Innovation in Consultancy }\end{array}$ & $\begin{array}{l}\text { Leon Downing, Donohue \& } \\
\text { Associated, USA }\end{array}$ \\
\hline $13: 50$ & 14:15 & $\begin{array}{l}\text { Presentation ( } 20 \text { minutes): } \\
\text { Barriers to Innovation in Utilities }\end{array}$ & $\begin{array}{l}\text { Magnus Arnell, Lund University, } \\
\text { Sweden }\end{array}$ \\
\hline $14: 15$ & $15: 00$ & $\begin{array}{l}\text { Small Group Discussions: } \\
\text { Issues and Solutions }\end{array}$ & \\
\hline $15: 00$ & $15: 30$ & Break & \\
\hline \multicolumn{4}{|c|}{ Part III: Finding Solutions } \\
\hline $15: 30$ & 16:00 & $\begin{array}{l}\text { Discussion Primer: } \\
\text { Summary of small group discussions } \\
\text { Summary of solutions proposed by speakers }\end{array}$ & \\
\hline $16: 00$ & $16: 45$ & $\begin{array}{l}\text { Panel Discussion: } \\
\text { Overcoming barriers to innovation }\end{array}$ & \\
\hline $16: 45$ & 17:00 & Wrap-up, closing summary & \\
\hline
\end{tabular}

YWP Workshop Organizing Committee:

$\begin{array}{ll}\text { Magnus Arnell } & \text { Lund Unversity, Sweden } \\ \text { Hélène Hauduc } & \text { LISBP - INSA de Toulouse, France } \\ \text { Thomas Maere } & \text { Ghent University, Belgium } \\ \text { Adrienne Menniti } & \text { Clean Water Services, USA }\end{array}$


Maere \& Comas et al.

\section{Workshop: Where are we and where should we go with MBR modelling?}

he application of membrane bioreactors (MBR) for wastewater treatment has significantly increased over the last decades, despite the inevitable occurrence of membrane fouling which is one of the main drawbacks of MBR technology and is associated with high energy expenditures compared to conventional activated sludge technologies. Most of the MBR plants are working in a conservative way, with quite significant room for optimisation. The use of models for MBRs can be a really useful approach to optimise their operation. In this respect, the proposed workshop focuses on the current state of the art and the future needs in MBR modelling to move towards a) widely accepted MBR models and submodels and b) practically relevant models to describe the biological (aerobic and anaerobic) and filtration processes.

\section{Expected discussions and results}

The workshop consists of a series of invited presentations followed by moderated panel discussions in the morning and breakout sessions followed by a group discussion in the afternoon. The series of invited presentations focuses on the currently available models applied in MBR technology (where are we?). Specifically, the following main topics will be covered: (1) the modelling of the biological process, (2) the modelling of the filtration process, (3) current practices in optimisation and control of MBR systems. The breakout sessions will focus on future aspects of MBR modelling (where should we go?), with mainly 1) new modelling topics and frameworks and 2) full-scale applications.

The specialist presentation on biological process modelling will address the possibility of adapting the current plant-wide models or classical activated sludge models (ASM) and the anaerobic digestion model (ADM) for modelling the biological process in both aerobic and anaerobic MBRs, respectively. Therefore, this topic will focus on the necessity (or not) of adapting existing models based on membrane specificities (e.g. including soluble microbial products) in order to move towards an integrated MBR model. The importance to practice will be discussed.

The specialist presentation on filtration process modelling will focus on the necessity of developing feasible filtration process models which facilitate the design, operation and control of membrane technology. In particular, the applicability of the currently available deterministic and empirical models to different operating conditions, environments (aerobic and anaerobic), and membrane types will be discussed. The presentation will address the advantages and disadvantages of using integrated models or standalone filtration models as well. The link to practice will also be included.

The specialist presentation on optimisation and control will give an overview of current practices and tools used to optimise the operation and design of MBRs. A clear distinction will be made between tools that have been proposed in literature which have not yet been applied or validated, lab- and pilot-scale investigations and finally control systems that have been used in full-scale practice.

The breakout sessions are targeting the current needs and promising future paths in MBR modelling. Three presentations are planned: (1) new modelling topics (e.g. particle size distributions, viscosity, mixing, aeration, energy) and modelling approaches for these topics (e.g. computational fluid dynamics, population balance modelling); (2) full-scale applications (in response to a lack of full-scale studies in literature); (3) a topic from the audience which will be announced and decided upon through Linked-in and mail. 
Each presentation will be prepared by $2-3$ experts. The aim of having multiple people for a talk is to make sure that the various aspects of a topic are covered (academic, consultancy, utilities, companies). The presentations will be short, refreshing and thought-provoking to stimulate a lively discussion afterwards. The workshop is meant to be constructive and consensus-aiming.

We will sum up the main ideas highlighted by each speaker together with the key points from the specific discussions. Then, we will finish with a reflection on the main issues we wanted to cover within this workshop: "where we are in MBR modelling and where we need to go", taking into account the practitioners' point of view and demand from the field. Finally, we want to discuss if a review paper on certain topics is needed, or maybe even the formation of a task group focussed on crucial MBR modelling issues.

\section{Workshop set-up}

In the morning, the workshop is structured along a number of keynote presentations on the state of the art in biological and filtration modelling and control. The presentations are followed by moderated round table discussions to reach consensus. To assure an objective, critical and holistic view on the addressed topics, the presentations will be prepared by multiple people.

In the afternoon, outbreak sessions are planned on a couple of crucial topics, aiming to identify the key issues that have to be addressed and possible solutions. The day ends with an overview of the topics on which consensus is reached (or not) and future plans (e.g. formation of a task group, review paper, etc.).

In order to promote discussion, a discussion forum will be launched on Linked-in before the start of the conference. Important remarks and suggestions for topics will be taken into account in the actual workshop. Speakers and attendees will be invited to the forum.

\section{Chairs / co-chairs}

Chairs: Thomas Maere (Biomath, Ghent University, Belgium), Joaquim Comas (Lequia, University of Girona, Spain)

Co-Chairs: Vicky Ruano (Aqualia, Spain), Ángel Robles (University of València, Spain), Ignasi Rodriguez-Roda (Catalan Institute for Water Research, Spain), Ingmar Nopens (Biomath, Ghent University, Belgium)

\section{Speakers / collaborators}

From practice:

- Christoph Brepols (Erftverband, Germany)

- Murat Sarioglu (MWH global, Turkey)

- André Lerch (Samsung Cheil Industries, Germany)

- Marina Arnaldos Orts (Acciona Agua, Spain)

- Vicky Ruano (aqualia, Spain)

From academia:

- Ángel Robles (University of Valencia, Spain)

- Ignasi Rodriguez-Roda (ICRA, Spain)

- Joaquim Comas (University of Girona, Spain)

- Wouter Naessens (Ghent University, Belgium)

- Thomas Maere (Ghent University, Belgium) 
- Ingmar Nopens (Ghent University, Belgium)

\section{Target Participants}

This workshop should draw the attention of experts in membrane bioreactor technology both from practice and academia, with or without specific modelling expertise, as well as general WWTP modelling experts (e.g. ASM, ADM, CFD, control, energy) who want to learn about the specificities of MBR modelling.

\section{Programme}

\begin{tabular}{|c|c|c|}
\hline Time & Topic & Presenter/Moderator \\
\hline 09:45 - 09:55 & $\begin{array}{l}\text { Introduction: Motivation, scope, and objectives. Present } \\
\text { workshop structure, participants, etc. }\end{array}$ & Chairs \& co-chairs \\
\hline 09:55 - 10:20 & $\begin{array}{l}\text { Presentation \#1: Biological process modelling for aerobic } \\
\text { and anaerobic MBR }\end{array}$ & $\begin{array}{l}\text { Team \#1: } \\
\text { - T. Maere } \\
\text { - Á. Robles } \\
\text { - M. Sarioglu }\end{array}$ \\
\hline $10: 20-10: 45$ & Discussion Period & Chairs \& co-chairs \\
\hline $10: 45-11: 15$ & \multicolumn{2}{|l|}{ Coffee break } \\
\hline $11: 15-11: 45$ & $\begin{array}{l}\text { Presentation \#2: Filtration process modelling for aerobic } \\
\text { and anaerobic MBR }\end{array}$ & $\begin{array}{l}\text { Team \#2: } \\
\text { - Á. Robles } \\
\text { - W. Naessens }\end{array}$ \\
\hline $11: 45-12: 15$ & Presentation \#3: Control \& optimisation: current practices & $\begin{array}{l}\text { Team \#3: } \\
\text { - Q. Comas } \\
\text { - M. Arnaldos Orts }\end{array}$ \\
\hline $12: 15-12: 45$ & Discussion Period & Chairs \& co-chairs \\
\hline $12: 45-13: 45$ & \multicolumn{2}{|l|}{ Lunch break } \\
\hline $13: 45-14: 25$ & Outbreak session 1: New modelling topics and frameworks & $\begin{array}{l}\text { Team \#4: } \\
\text { - I. Rodriguez-Roda } \\
\text { - A. Lerch } \\
\text { - I. Nopens }\end{array}$ \\
\hline $14: 25-15: 05$ & Outbreak session 2: Full-scale applications & $\begin{array}{l}\text { Team \#5: } \\
\text { - V. Ruano } \\
\text { - C. Brepols }\end{array}$ \\
\hline $15: 05-15: 15$ & Group discussion & Chairs \& co-chairs \\
\hline $15: 15-15: 45$ & \multicolumn{2}{|l|}{ Coffee break } \\
\hline $15: 45-16: 15$ & Public outbreak session: Topics from the audience & Chairs \& co-chairs \\
\hline $16: 15-16: 45$ & $\begin{array}{l}\text { Plenum discussion } \\
\text { - } \quad \text { Future needs } \\
\text { - }\end{array}$ & Chairs \& co-chairs \\
\hline $16: 45-17: 15$ & Wrap-up, composing summary, report and presentation & Chairs \& co-chairs \\
\hline
\end{tabular}




\section{Workshop: What do we need for "Total" Nitrogen Modelling?}

Our community strives to provide models that reflect the behaviour in the many situations in wastewater treatment systems. Simulating nitrogen $(\mathrm{N})$ transformations is challenging because there are many $\mathrm{N}$ states of concern (ammonia, nitrite, nitrate, $\mathrm{NO}, \mathrm{N}_{2} \mathrm{O}, \mathrm{N}_{2}$, organic $\mathrm{N}$ ) with several organism groups involved, and components may be involved in multiple processes simultaneously.

Nitrogen transformations are implicated in so many issues: GHG emissions, energy reduction (e.g. nitrite shunt), alternative external carbon sources, attaining low TN limits, many sidestream treatment options, etc.

The proposed workshop will provide a forum to review, discuss and present ideas on the structure of models for nitrification and denitrification (and associated $\mathrm{N}_{2} \mathrm{O}$ production). For each main topic discussed, a discussion on the following will be included:

- Model structure: How well do existing models reflect the mechanisms?

- Interactions: How well do models capture the interactions between competing processes?

- Mechanisms: What mechanisms may be at play that we are overlooking? How do we uncover them?

- Data needs: In what areas, if any, do we need more data for model development and testing?

- Can we unify models to achieve multiple objectives for a more holistic Total Nitrogen Modelling?

\section{Expected discussions and results}

The topic area of Nitrogen Modelling has been viewed as five subtopics (with interest areas in parentheses):

1. Nitrification (SND and shortcut nitrite shunt)

2. Anammox processes (model structure)

3. Heterotrophic denitrification (Electron competition; model structure; pathways)

4. N modelling in practice (Achieving Low TN limits)

5. Nitrous oxide production (Mechanisms; model structure/performance; measurements; risk assessment modelling)

The day, from 9:30 a.m. to 5 p.m., will be mainly divided into series of presentation/discussion periods (see Programme) with morning and afternoon 30 minute coffee breaks and a 1 hour lunch break. The final slots in the morning and the afternoon are set aside for longer discussions on the topics. 
Presenters will include a mix of academics and practitioners to ensure well-rounded discussions, linking research to practice.

A workshop report will be compiled upon the completion of the workshop summarizing the key points discussed, consensus gained on topics, and action items for the $\mathrm{N}$ modelling community to fill gaps in knowledge and practice.

Plans for the workshop subject include a white paper or journal article such as WS\&T or WP\&T.

\section{Workshop set-up}

The workshop set up will consist of a series of presentations followed by a discussion period. A larger discussion period will follow the presentation/discussion slots in each the morning and afternoon sessions. This format will generally lead to significantly more discussion time than presentation time. This will be critical for reach consensus on topics and identifying research and practice needs for advancing $\mathrm{N}$ modelling.

\section{Chair and Co-chairs}

Chair: Peter Dold (EnviroSim Associates Ltd., Hamilton, Ontario, Canada)

Co-Chair: José C. Porro (LEQUiA, University of Girona, Spain)

\section{Speakers / Discussion Leaders}

Dwight Houweling (CH2M Hill, Canada)

Weiwei Du (EnviroSim, Canada)

Zhiguo Yuan (University of Queensland, Australia)

Eveline Volcke (Ghent University, Belgium)

Ed Becker (ARCADIS, USA)

Barth Smets (DTU, Denmark)

Marlies Kampschreur (Waterboard Aa en Maas, The Netherlands)

Mathieu Spérandio (University of Toulouse, France)

Haydee De Clippeleir (Columbia University, USA)

Maite Pijuan (ICRA, Spain)

\section{Target Participants}

Target participants include researchers active in $\mathrm{N}$ modelling, seasoned professionals who can lend invaluable insight on approaches, and practitioners interested in applying various $\mathrm{N}$ models for wastewater treatment plant design and operations. Target participants will be interested in attending as the workshop will create opportunities for research and practice collaboration, building consensus on ongoing efforts, and understanding how different data collection/modelling approaches can be applied to best meet different objectives. 


\section{Programme}

\begin{tabular}{|c|c|c|}
\hline Time & Topic & Presenter/Moderator \\
\hline $9: 45-10: 05$ & $\begin{array}{l}\text { Introduction: Motivation, scope, and objectives Present } \\
\text { workshop structure, participants, etc. }\end{array}$ & P. Dold \\
\hline $10: 05-10: 25$ & $\begin{array}{l}\text { Presentation/Discussion \#1: Nitrite Shunt, SND in FS } \\
\text { plants }\end{array}$ & D. Houweling \\
\hline $10: 25-10: 45$ & $\begin{array}{l}\text { Presentation/Discussion \#2: Experimental data and } \\
\text { denitrification model structure }\end{array}$ & W. Du \\
\hline $10: 45-11: 15$ & \multicolumn{2}{|l|}{ Coffee break } \\
\hline $11: 15 \quad 11: 35$ & $\begin{array}{l}\text { Presentation/Discussion \#3: Electron Competition in } \\
\text { denitrification }\end{array}$ & Z. Yuan \\
\hline $11: 35 \quad 12: 00$ & $\begin{array}{l}\text { Presentation/Discussion \#4: Anammox Processes and } \\
\text { Models }\end{array}$ & E. Volcke \\
\hline $12: 00-12: 45$ & Nitrification / Denitrification / Anammox Discussion & P. Dold \\
\hline $12: 45-13: 45$ & \multicolumn{2}{|l|}{ Lunch break } \\
\hline $13: 45-14: 05$ & $\begin{array}{l}\text { Presentation/Discussion \#5: The state of } \mathrm{N} \text { models for } \\
\text { achieving Low TN limits in practice }\end{array}$ & E. Becker \\
\hline $14: 05-14: 45$ & $\begin{array}{l}\text { Presentation/Discussion \#6: } \mathrm{N}_{2} \mathrm{O} \text { emission from } \\
\text { wastewater treatment: formation mechanisms and } \\
\text { models }\end{array}$ & $\begin{array}{l}\text { B. Smets, M. } \\
\text { Kampschreur, E. } \\
\text { Volcke }\end{array}$ \\
\hline $14: 45-15: 15$ & $\begin{array}{l}\text { Presentation/Discussion \#7: } \mathrm{N}_{2} \mathrm{O} \text { Model Calibration and } \\
\text { parameters }\end{array}$ & $\begin{array}{l}\text { M. Spérandio, } \\
\text { H. De Clippeleir }\end{array}$ \\
\hline $15: 15-15: 45$ & \multicolumn{2}{|l|}{ Coffee break } \\
\hline $15: 45-16: 10$ & $\begin{array}{l}\text { Presentation/Discussion \#8: } \mathrm{N}_{2} \mathrm{O} \text { measurements - new } \\
\text { methods and guidance }\end{array}$ & E. Volcke, M. Pijuan \\
\hline $16: 10-16: 25$ & \begin{tabular}{|llll}
$\begin{array}{l}\text { Presentation/Discussion } \\
\text { approach and modelling }\end{array}$ & $\# 9: \quad \mathrm{N}_{2} \mathrm{O} \quad$ knowledge-based \\
\end{tabular} & J. Porro \\
\hline $16: 25-17: 00$ & Total Nitrogen Modelling Checklist Discussion & P. Dold, J. Porro \\
\hline $17: 00-17: 15$ & Wrap-up, concluding remarks & P. Dold \\
\hline
\end{tabular}




\section{Workshop: How can modelling be effectively used for energy balance optimization?}

The wastewater industry is moving rapidly toward the goal of energy neutrality. As energy costs rise, improving the energy footprint for wastewater treatment is not just a sustainability goal but an essential step toward reducing operating costs. The ability to accurately predict energy consumption and production is critical to identifying and evaluating opportunities for energy balance improvement. The purpose of this workshop is to critically evaluate how modeling (both steady state and dynamic) can be effectivley used for estimating a plant energy balance and its associated cost implications.

\section{Expected discussions and results}

Achieving the goal of energy neutrality requires a balanced approach of decreasing energy demand, increasing energy production, and justifying the capital expense. The workshop will emulate this by being organized into three main sections. We will first examine energy demand and energy production, concentrating on the two largest contributors to the plant energy balance - energy demand due to aeration and energy production from biogas. Next, the translation of energy predictions to costs is explored. Each section will involve brief presentations highlighting the approaches and challenges in a given area, followed by a focused discussion in both large and small group formats.

The end of the workshop will "bring it all together", discussing the overall challenges with energy modelling in wastewater systems and identifying the key future prospects. The chairs will develop a summary of the first three sections during the afternoon break. An inspirational presentation followed by summary points from the earlier sessions will be used to stimulate an end of the day discussion focused on the workshop's overriding question: How can modelling be effectively used for energy balance optimization?

The sections will be organized into the following categories:

\section{Part I: Modeling Energy Reduction in Aeration Design and Operation}

- Main question: How is modelling used for effective design of aeration systems and in aeration system control?

- Discussion format: guided group discussion focused on the Part I main question

\section{Part II: Modeling Energy Production}

- Main question: Where are the gaps in energy production modeling?

- Discussion format: guided group discussion focused on the Part II main question

Part III: Modeling the Cost Implications of Energy Reduction and Production

- Main question: Can we effectively model the energy balance and energy cost components in a wastewater system?

- Discussion format: small group discussions to address the question - which smaller energy uses make sense to model? 


\section{Part IV: Discussion of the overall challenges and opportunities in wastewater energy modeling}

- Main question: What is the role of energy modeling, and where are the gaps?

- Presentation: Speaker will give a "motivational" speech on the importance of energy in wastewater modeling, with the aim to re-stimulate the workshop attendees

- Discussion format: the chairs will provide bulleted list of main takeaways from Part I, II, and III, and the a group discussion to identify the largest gaps/needs and key future prospects in energy modelling

\section{How will you summarize results for larger WWTmod group?}

The workshop results summary will focus on a major takeaway from Parts I, II, and III, and a list of the largest gaps/needs and key future prospects in energy modelling.

\section{What are plans for workshop subject after WWTmod? (i.e. white paper, publications, other?)}

The workshop outcomes will be summarized into a position paper: Where are the Knowledge Gaps and Future Prospects in Wastewater Energy Modelling? The paper will be submitted for consideration for publication in WS\&T. One possible workshop outcome could be the generation of a working group to establish best practices for energy modelling.

\section{Workshop set-up}

The workshop will rely on large group discussions and breakout discussions. The discussions will be preceded by a collaborative presentation where the speakers highlight key considerations in each area of energy modelling. The group discussions will focus on how modelling is used in energy related wastewater applications, and where the gaps in current model structure and/or modelling practice exist.

\section{Chairs}

Adrienne Menniti, Clean Water Services (Portland, Oregon, USA)

Tom Johnson, CH2MHill (Charlotte, North Carolina, USA)

Leon Downing, Donohue \& Associates (Sheboygan, Wisconsin, USA)

\section{Speakers / Moderators}

The speakers are all WWTMod veterans, and represent a cross section of the fields involved in wastewater modelling (academic, practitioners, utilities), as well as representatives from the Americas and Europe.

Speaker \#1 Tom Johnson (CH2MHill, Charlotte, North Carolina, USA)

Speaker \#2 Leon Downing (Donohue \& Associates, Sheboygan, Wisconsin, USA)

Speaker \#3 Sylvie Gillot (Irstea, France)

Speaker \#4 Bernhard Wett (ARA Consult, Innsbruck, Austria)

Speaker \#5 Sherri Cook (University of Michigan, Ann Arbor, Michigan, USA)

Speaker \#6 Adrienne Menniti (Clean Water Services, Portland, Oregon, USA)

Speaker \#7 Leiv Rieger (inCTRL Solutions, Oakville, Canada)

Speaker \#8 Lluís Corominas (ICRA, Girona, Spain)

Speaker \#9 Diego Rosso (UC Irvine, Irvine California, USA) 


\section{WWTmod}

Menniti, Johnson, and Downing

Programme

\begin{tabular}{|c|c|c|c|}
\hline \multicolumn{2}{|c|}{ Time } & Topic & Speaker \\
\hline 9:45 & 9:50 & Overview: What are the challenges? & Workshop Co-Chairs \\
\hline \multicolumn{4}{|c|}{ Part I: Modeling Energy Reduction in Aeration Design and Operation } \\
\hline 9:50 & $10: 20$ & 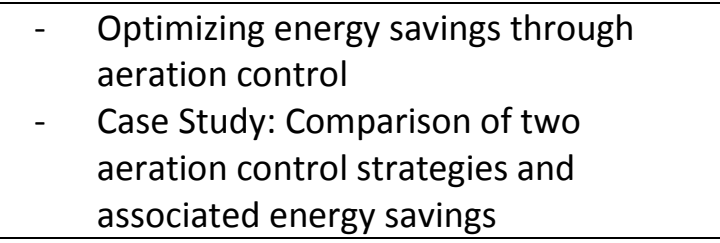 & $\begin{array}{l}\text { Leiv Rieger, InCTRL } \\
\text { Sylvie Gillot, Irstea } \\
\end{array}$ \\
\hline $10: 20$ & $10: 45$ & $\begin{array}{l}\text { Large Group Discussion: What are the Best } \\
\text { Practices for Aeration System Modeling for } \\
\text { Design and Operation? }\end{array}$ & \\
\hline $10: 45$ & $11: 15$ & Break & \\
\hline \multicolumn{4}{|c|}{ Part II: Modeling Energy Production } \\
\hline $11: 15$ & $12: 00$ & $\begin{array}{ll}\text { - } & \text { Predicting CAMBI performance } \\
\text { - } & \text { The benefits and limitations of } \\
\text { modeling co-digestion using ADM1 } \\
\text { - Case Study: Modeling Co-digestion in } \\
\text { Practice }\end{array}$ & $\begin{array}{l}\text { Bernhard Wett, ARA Consult } \\
\text { Sherri Cook, University of } \\
\text { Michigan } \\
\text { Adrienne Menniti, Clean } \\
\text { Water Services }\end{array}$ \\
\hline $12: 00$ & $12: 45$ & $\begin{array}{l}\text { Large Group Discussion: Where are the } \\
\text { knowledge gaps and opportunities in } A D \\
\text { Modeling? }\end{array}$ & \\
\hline $12: 45$ & $13: 45$ & LUNCH & \\
\hline \multicolumn{4}{|c|}{ Part III: Modeling the Cost Implications of Energy Reduction and Production } \\
\hline 13:45 & $14: 15$ & $\begin{array}{l}\text { - Modeling vs. Operation: How Closely } \\
\text { Can We Predict a Plant Energy } \\
\text { Balance? } \\
\text { - The "Hidden" Cost: Modeling Energy } \\
\text { Tariff/Demand Charges }\end{array}$ & $\begin{array}{l}\text { Tom Johnson, } \mathrm{CH} 2 \mathrm{MHill} \\
\text { Leon Downing, Donohue \& } \\
\text { Associates } \\
\text { Lluis Corominas, ICRA } \\
\end{array}$ \\
\hline $14: 15$ & $15: 15$ & $\begin{array}{l}\text { Break Out Group Discussion: Which smaller } \\
\text { energy uses make sense to model? }\end{array}$ & \\
\hline $15: 15$ & $15: 45$ & Break & \\
\hline \multicolumn{4}{|c|}{ Part IV: Discussion of the overall challenges with wastewater energy modeling } \\
\hline $15: 45$ & $16: 00$ & $\begin{array}{l}\text { The Resource Recovery Facility: What is the } \\
\text { Role of Energy Modeling, and Where are the } \\
\text { Gaps? }\end{array}$ & Diego Rosso, UC Irvine \\
\hline 16:00 & $17: 00$ & $\begin{array}{l}\text { Large Group Discussion: Where are the gaps } \\
\text { and where are the key future prospects? }\end{array}$ & \\
\hline $17: 00$ & $17: 15$ & Wrap-up, closing summary & Workshop Co-Chairs \\
\hline
\end{tabular}




\section{Revisiting phosphorus removal: do the models give the answers we want?}

Looking from a whole plant perspective, including biological and chemical processes.

Whole plant modelling gets increasing attention (Grau et al., 2007) and deals with linking the different models available for activated sludge, anaerobic digestion and anoxic-aerobic digestion. Due to the complexities in modelling P removal, the whole plant models also will have to deal with the effect of combining the biological and chemical models and their effect on each other (Barat et al., 2008; de Haas et al., 2000; Schonborn et al., 2001). Describing and predicting phosphorus (P) removal means looking at both biological and chemical processes. Modelling these processes has evolved over the years and result in a different status.

Modelling of biological phosphorus removal and in particular enhanced biological phosphorus removal (EBPR) has gotten a lot of attention during the 1990s resulting in the publication of the ASM2d (Henze et al., 1999). In the same period metabolic models (Lopez-Vazquez et al., 2009; Schuler and Jenkins, 2003; Smolders et al., 1995) also have shown to be promising for modelling the EBPR. More recently, a lot of criticism arose about ASM2d concerning the inability to account for several processes and many extensions have been published (García-Usach et al., 2010; Larrea et al., 2002; Makinia et al., 2006; Manga et al., 2001).

Driven by problems of struvite precipitation, phosphate recovery and the need to predict $\mathrm{pH}$, modelling chemical $\mathrm{P}$ removal has been getting a lot of attention in recent years, which led to the start of an IWA task group on a Generalized Physicochemical Framework (Batstone et al., 2012).

Today, it is clear that for modelling phosphorus removal at wastewater treatment plants a whole plant context is required, including both biological and chemical processes. But there are a lot of models and extensions of models available. The choice of model leads to a large uncertainty in the model outcome and reduces the confidence in its predictions.

\section{Expected discussions and results}

\section{Questions}

What are the prerequisites of the models to deal with practical questions (in regard to design and operation of wastewater treatment plants)?

- What unit processes (AD, primary sedimentation, secondary sedimentation...) are insufficiently modelled to describe the fate of phosphorus in a plant wide modelling context?

- What different populations do we need to consider in the model without including unnecessary detail and complexity?

- What are the missing links/components to truly model physical-chemical processes?

- What is the impact of EBPR on other biological processes and vice versa?

- Are the currently known models sufficient?

Is there a need for a consensus model to make the modelling of phosphorus removal a mature methodology? 


\section{How}

The workshop will be organized using four different session styles.

The first session style is based on presentations to set the stage for further discussion. Several wellknown researchers will give an overview of the state-of-art on different aspects of whole plant modelling for phosphorus removal.

The second session style is based on repeated breakout sessions. The four topics, guided by a moderator, will be discussed twice for half an hour with different groups. Participants can choose 2 out of the 4 topics: (i) From practical questions to whole plant model prerequisites, (ii) impact of the sulfate cycle on P removal, (iii) the impact of biological reactions on chemical conditions and viceversa) and (iv) the effect of high temperature on EBPR. At the end of the two sessions, the moderators will present the results to the whole group.

The third session style is based on the 'Open Space Technology'. This is a method for convening groups around a specific question or task of importance and giving them responsibility for creating both their own agenda and experience. In first instance the participants are invited to propose topics. In a second step the remaining participants choose the topic of their interest and discuss it. One fundamental in this process is 'the law of two feet'. I.e. if at any time during the time together you find yourself in any situation where you are neither learning nor contributing, use your two feet, go to another discussion.

The fourth session style is a typical round table discussion around the questions the workshop wants to answer. The moderator will be in charge of challenging the audience in the round table and wrapping up the main conclusions in the final session

\section{After}

After the workshop we will work towards a position paper. As a first step we suggest to submit a a short report (2-3 pages max.) with the final conclusions of the workshop. The report will be distributed to the IWA task group on a Generalized Physicochemical Framework, to the IWA task group on Benchmarking of Control Strategies for Wastewater Treatment Plants and it will be uploaded into the MIA web (or send to the MIA group). As a second step we will invite the participants to contribute to the position paper.

If the group sees an interest in a consensus model, the position paper can be the starting point for setting up an IWA task group with the goal of formulating a successor of ASM2d.

\section{Chair/Co-chair}

Chair Youri Amerlinck (BIOMATH, Ghent University, Ghent, Belgium)

Co-Chair\#1 Albert Guisasola (Universitat Autònoma de Barcelona, Barcelona, Spain)

Co-Chair\#2 Hélène Hauduc (Institut National des Sciences Appliquées de Toulouse, France)

Co-Chair\#3 David Ikumi (University of Cape Town, Cape Town, South Africa)

\section{Speakers / Moderators}

Moderator \#1 Marjoleine Weemaes (Aquafin, Aartselaar, Belgium)

Moderator \#2 Chris Brouckaert (University of KwaZulu-Natal, Durban, South Africa)

Moderator \#3 Hélène Hauduc (Institut National des Sciences Appliquées de Toulouse, France) 
Moderator \#4 Guclu Insel (Istanbul Technical University, Istanbul, Turkey)

Moderator \#5 Albert Guisasola (Universitat Autònoma de Barcelona, Barcelona, Spain)

Moderator \#6 David Ikumi (University of Cape Town, Cape Town, South Africa)

Moderator \#7 Youri Amerlinck (BIOMATH, Ghent University, Ghent, Belgium)

Speaker \#1 Yves Comeau (Ecole Polytechnique de Montréal, Montréal, Canada)

Speaker \#2 Damien Batstone (University of Queensland, Brisbane, Australia)

Speaker \#3 Imre Takacs (Dynamita, Nyons, France)

Speaker \#4 George Ekama (University of Cape Town, Cape Town, South Africa)

Speaker \#5 Juan Antonio Baeza (Universitat Autònoma de Barcelona, Barcelona, Spain)

\section{Target Participants}

Everyone who is dealing with phosphorus removal (either chemical, biological or both) at wastewater treatment plants.

- Plant staff

- Consultants

- Researchers

\section{Programme}

\begin{tabular}{|c|c|c|}
\hline Time & Topic & Presenter/Moderator \\
\hline $09: 45-10: 00$ & Introduction: Motivation, scope, and objectives. & Youri Amerlinck \\
\hline $10: 00-10: 20$ & Presentation: Microbiological aspects of EBPR. & Yves Comeau \\
\hline $10: 20-10: 40$ & Presentation: Chemical phosphorus removal. & Damien Batstone \\
\hline $10: 40-11: 00$ & Presentation: Overview of the existing models. & $\begin{array}{l}\text { Imre Takacs } \\
\text { George Ekama }\end{array}$ \\
\hline $11: 00-11: 30$ & Coffee break & \\
\hline $11: 30-12: 45$ & 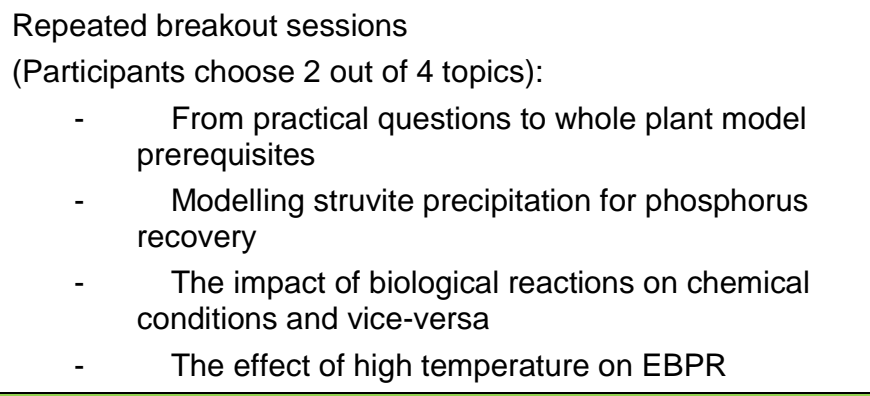 & $\begin{array}{l}\text { Marjoleine Weemaes } \\
\text { Chris Brouckaert } \\
\text { Hélène Hauduc } \\
\text { Guclu Insel }\end{array}$ \\
\hline $12: 45-13: 45$ & Lunch break & \\
\hline $13: 45-14: 15$ & Presentation: Control of phosphorus removal processes. & Juan Antonio Baeza \\
\hline $14: 15-15: 15$ & 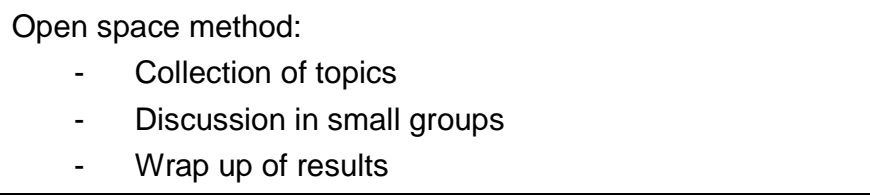 & \\
\hline $15: 15-15: 45$ & Coffee break & \\
\hline $15: 45-16: 15$ & $\begin{array}{l}\text { Round table discussion: the need for an consensus model for } \\
\text { EBPR }\end{array}$ & Albert Guisasola \\
\hline
\end{tabular}


Amerlinck et al.

\begin{tabular}{|c|l|l|}
\hline $16: 15-16: 45$ & $\begin{array}{l}\text { Round table discussion: modelling phosphorus removal in a whole } \\
\text { plant context: SWOT }\end{array}$ & $\begin{array}{l}\text { Hélène Hauduc } \\
\text { David lkumi }\end{array}$ \\
\hline $16: 45-17: 15$ & Wrap-up, composing summary, report and presentation. & Youri Amerlinck \\
\hline
\end{tabular}

\section{References}

Barat, R., T. Montoya, L. Borrás, J. Ferrer, and A. Seco, 2008, Interactions between calcium precipitation and the polyphosphate-accumulating bacteria metabolism: Water Research, v. 42, p. 3415-3424.

Batstone, D. J., Y. Amerlinck, G. Ekama, R. Goel, P. Grau, B. Johnson, I. Kaya, J. P. Steyer, S. Tait, I. Takacs, P. A. Vanrolleghem, C. J. Brouckaert, and E. Volcke, 2012, Towards a generalized physicochemical framework: Water Science and Technology, v. 66, p. 11471161.

de Haas, D. W., M. C. Wentzel, and G. A. Ekama, 2000, The use of simultaneous chemical precipitation in modified activated sludge systems exhibiting biological excess phosphate removal Part 1: Literature review: Water Sa, v. 26, p. 439-452.

García-Usach, F., J. Ribes, J. Ferrer, and A. Seco, 2010, Calibration of denitrifying activity of polyphosphate accumulating organisms in an extended ASM2d model: Water Research, v. 44, p. 5284-5297.

Grau, P., M. de Gracia, P. A. Vanrolleghem, and E. Ayesa, 2007, A new plant-wide modelling methodology for WWTPs: Water Research, v. 41, p. 4357-4372.

Henze, M., W. Gujer, T. Mino, T. Matsuo, M. C. Wentzel, G. V. R. Marais, and M. C. M. Van Loosdrecht, 1999, Activated Sludge Model No.2d, ASM2d: Water Science and Technology, v. 39, p. 165-182.

Larrea, L., I. Irizar, and M. E. Hidalgo, 2002, Improving the predictions of ASM2d through modelling in practice: Water Science and Technology, v. 45, p. 199-208.

Lopez-Vazquez, C. M., A. Oehmen, C. M. Hooijmans, D. Brdjanovic, H. J. Gijzen, Z. G. Yuan, and M. C. M. van Loosdrecht, 2009, Modeling the PAO-GAO competition: Effects of carbon source, $\mathrm{pH}$ and temperature: Water Research, v. 43, p. 450-462.

Makinia, J., K. H. Rosenwinkel, M. Swinarski, and E. Dobiegala, 2006, Experimental and modelbased evaluation of the role of denitrifying polyphosphate accumulating organisms at two large scale WWTPs in northern Poland: Water Science and Technology, v. 54, p. 73-81.

Manga, J., J. Ferrer, F. Garcia-Usach, and A. Seco, 2001, A modification to the Activated Sludge Model No. 2 based on the competition between phosphorus-accumulating organisms and glycogen-accumulating organisms: Water Science and Technology, v. 43, p. 161-171.

Schonborn, C., H. D. Bauer, and I. Roske, 2001, Stability of enhanced biological phosphorus removal and composition of polyphosphate granules: Water Research, v. 35, p. 3190-3196.

Schuler, A. J., and D. Jenkins, 2003, Enhanced biological phosphorus removal from wastewater by biomass with different phosphorus contents, part I: Experimental results and comparison with metabolic models: Water Environment Research, v. 75, p. 485-498.

Smolders, G. J. F., J. Vandermeij, M. C. M. Vanloosdrecht, and J. J. Heijnen, 1995, A structured metabolic model for anaerobic and aerobic stoichiometry and kinetics of the biological phosphorus removal process: Biotechnology and Bioengineering, v. 47, p. 277-287. 
Shaw et al.

\section{Workshop: Linking WWTP modelling with Life Cycle Assessment (LCA) and other Holistic models.}

The main goal of this workshop is to provide a forum for experts in wastewater treatment plant modelling to think about how current process modelling fits with LCA and how current models might be expanded to create more synergies with LCA and other holistic models.

\section{Expected discussions and results}

Decisions about wastewater treatment have traditionally been driven by considerations of technical aspects and cost-benefit analyses. In order to assess sustainability it is essential also to incorporate environmental and social aspects. In this line of thinking, several methods for sustainability assessment of wastewater treatment technologies have been proposed and evaluated in literature. Focusing on environmental performance, Life Cycle Assessment (LCA) (ISO 14040, 2006) is an accepted tool that has also been used to evaluate potential environmental impacts from environmental processes (Finnveden et al., 2009) including wastewater treatment processes throughout their whole life cycle (Guest et al., 2009). The success of this method is demonstrated by the large number of published studies which have applied LCA to the wastewater treatment field (Corominas et al., 2013). The published studies so far have been applied to estimate the impact of different wastewater treatment plants (WWTPs) and to compare conventional and new wastewater treatment technologies.

The organization of this workshop links to current activities of the IWA Working Group for Life Cycle Assessment of Water and Wastewater Treatment (LCA-Water WG) which was established in September 2012 to facilitate the exchange of ideas, and develop consensual methodologies, to promote better use of LCA in urban water systems. One of the main activities is to organize workshops to disseminate the use of LCA and to create synergies with other organizations (e.g. Task groups or working groups within IWA). WWTmod2014 is a great opportunity to define the synergies between that working group and other task forces that the modelling community on wastewater systems is running. The outcomes from the workshop might be bidirectional: from one side the LCA community can provide the wastewater process modelling community with complementary tools that can be coupled to existing models to expand how they are used to include environmental impacts and at the same time the LCA community involved in the field of WWT may benefit from close collaboration with the wastewater process engineers and managers who have extensive firsthand experience, resulting in a clearer modelling of the environmental impacts (and associated processes' variability particularly important for, e.g. GHG air emissions on the Global Warming category). On the other side, the wastewater process modelling community can learn from the LCA community which are the key issues from an environmental assessment point of view that should be addressed in the future (e.g. answering the question on where to put more effort: in modelling greenhouse gases emissions or microcontaminants, for example? What is more relevant for the environment?). Hence, the key questions from the workshop will be:

- How can the wastewater process modelling community benefit from environmental assessment tools? 
- Which are the key issues that should be addressed in the future research by the wastewater process modelling community to help filling the gaps in the environmental assessment field?

- What would be key issues in LCA models to address with future research in order to offer accurate models to be of use to the WWT process community?

\section{How will the workshop go about answering the posed question?}

In order to answer the questions above posed, some fundamentals on LCA will be introduced first and then, several group discussions will be organized where the following points will be addressed that cover the different steps of the LCA ISO standard:

- Goal and scope definition. To identify different types of studies where LCA coupled to wastewater treatment models might play an important role.

- Inventory analysis. To identify which data required in the LCA studies can be obtained from wastewater treatment models.

- Impact assessment. To establish the link between LCA impact assessment methods with current simulation platforms.

- Interpretation. To discuss which wastewater treatment modelling challenges are more relevant from an environmental assessment point of view.

In order to have dynamic presentations promoting discussion we will address these issues by presenting successful examples of LCA studies and practices.

Who will be presenting in the workshop, and how will they contribute to the discussions?

Members of the LCA-Water WG will be introducing general concepts and moderate the discussions and other invited speakers covering different areas of expertise within the wastewater treatment modelling community will be involved to ensure bilateral discussions. (LCA vs wastewater treatment modelling).

How will you summarize results for larger WWTmod group?

A wrap-up session is planned by the end of the meeting where the key points will be identified and a PowerPoint presentation will be prepared with the key messages of the workshop.

What are plans for workshop subject after WWTmod? i.e. white paper, publications, other?

A white paper on linking current wastewater treatment modelling practices with LCA.

\section{References.}

Finnveden, G., Hauschild, M.Z., Ekvall, T., Guinée, J., Heijungs, R., Hellweg, S., Koehler, A., Pennington, D., Suh, S., 2009. Recent developments in Life Cycle Assessment. J. Environ. Manage. 91(1), 1-21. 
Corominas, L., Foley, J., Guest, J. S., Hospido, a, Larsen, H. F., Morera, S., \& Shaw, a. (2013). Life cycle assessment applied to wastewater treatment: State of the art. Water research, 47(15), 5480-92.

\section{Workshop set-up}

The workshop will be organized as a round table discussion after some keynote presentations.

The workshop will briefly define some fundamentals on LCA supported by wastewater treatment examples and will present the state-of-the-art of LCA and wastewater treatment. Then, the workshop will move to a more interactive stage with brief presentations and examples followed by discussion.

\section{Chair/Co-chair}

Andrew Shaw (Black \& Veatch and Illinois Institute of Technology, USA)

Lluís Corominas (ICRA, Spain)

\section{Speakers / Moderators}

Gonzalo Rodriguez-García (University of Santiago de Compostela, Spain and Helmholtz-Institute Ulm, Germany)

Eva Risch (ELSA, France)

Haydee De Clippelier (DC Water, USA)

Jens Alex (IFAK, Germany)

\section{Target Participants}

- Process modellers wishing to know more about LCA

- LCA practitioners wishing to understand how process models might fit with LCA

- Anyone wishing to increase their understanding of LCA for wastewater treatment 


\section{Programme}

\begin{tabular}{|c|c|c|c|}
\hline Time & & Topic & Presenter/Moderator \\
\hline 09:00 & - 09:10 & $\begin{array}{l}\text { Introduction: Motivation, scope, and objectives Present } \\
\text { workshop structure, participants, etc. }\end{array}$ & Lluis Corominas \\
\hline 09:10 & $-09: 30$ & $\begin{array}{l}\text { Presentation \#1: Beyond Carbon Footprinting (Intro to } \\
\text { LCA) }\end{array}$ & Andrew Shaw \\
\hline 09:30 & $-10: 00$ & $\begin{array}{l}\text { Case study \#1: LCA for WWT } \\
\text { Case study \#2: LCA in the Water-Energy Nexus }\end{array}$ & $\begin{array}{l}\text { Gonzalo Rodriguez- } \\
\text { García } \\
\text { Eva Risch }\end{array}$ \\
\hline $10: 00$ & $-10: 30$ & Coffee break & \\
\hline $10: 30$ & $-10: 50$ & Presentation \#2: Current state of the art in LCA & Lluis Corominas \\
\hline $10: 50$ & $-11: 20$ & $\begin{array}{l}\text { Case study \#3: Strass LCA } \\
\text { Case study \#4: Blue Plains LCA }\end{array}$ & $\begin{array}{l}\text { Haydee De Clippelier } \\
\text { Andrew Shaw }\end{array}$ \\
\hline $11: 20$ & $-12: 00$ & Discussion Period: Experiences and perceptions of LCA & $\begin{array}{l}\text { Gonzalo Rodriguez- } \\
\text { García } \\
\text { (facilitator) }\end{array}$ \\
\hline $12: 00$ & $-13: 30$ & Lunch break & \\
\hline 13:30 & $-13: 50$ & Presentation \#3: Incorporating LCA into Decision Making & Andrew Shaw \\
\hline 13:50 & $-15: 00$ & $\begin{array}{l}\text { Group discussion: Brainstorming on how LCA is/should } \\
\text { be used for WWT }\end{array}$ & $\begin{array}{l}\text { Gonzalo Rodriguez- } \\
\text { García (facilitator) }\end{array}$ \\
\hline $15: 00$ & $-15: 30$ & Coffee break & \\
\hline 15:30 & $-15: 50$ & $\begin{array}{l}\text { Presentation \#4: Incorporating LCA into Process } \\
\text { Simulators }\end{array}$ & Jens Alex \\
\hline $15: 50$ & $-16: 30$ & $\begin{array}{l}\text { Panel discussion: Should WWTmod step out of its box to } \\
\text { include LCA, integrated modelling etc? }\end{array}$ & $\begin{array}{l}\text { Andrew Shaw } \\
\text { (facilitator) }\end{array}$ \\
\hline $16: 30$ & - 17:00 & Wrap up and next steps & Lluis Corominas \\
\hline
\end{tabular}


Benedetti et al.

\section{Workshop: Wet-weather modelling: Why and how should we tame the beast?}

Wet-weather (WW) events and periods have a large impact on the Water Resource Recovery Facility (WRRF) behaviour and on its capability to comply with regulations. There has been a significant increase in interest in this topic in recent years. Publications like "Guide for Municipal Wet Weather Strategies" published this year by the Water Environment Federation (WEF) as well as "Design and Operational Considerations for the Management of Wet Weather Flows at Water Resource Recovery Facilities" (to be published in early 2014 by WEF as well), are meant to provide guidance to those involved in solving the unique challenges associated with the management of WW events. Both of these publications emphasize the importance dynamic modelling plays in providing adequate support in understanding WW phenomena and in evaluating design and operation options with regard to their performance during WW events.

The workshop will tackle the issue by promoting discussion around three main questions:

- Why is WW modelling important?

- What is happening in a WRRF during WW and how can we model it?

- How can we model measures that mitigate the effects of WW?

\section{Expected discussions and results}

In recent years municipal utilities have been facing the need to provide significant improvements on how they manage WW-related flows in their WRRFs as the result of increased regulatory and public pressures. These flows and loads, which result from combined sewer systems or even separate systems that have significant amounts of infiltration and inflow, can in many cases exceed the treatment capacity of existing facilities. However, the overall approach necessary to identify, evaluate, and eventually select the "best" WW flow management scenario for a particular WRRF in terms of process units and operational requirements is one that contrasts significantly from that normally used by the same utilities in dealing with dry weather (DW) treatment needs.

It can be argued that one of the most appropriate fields of application of dynamic WRRF modelling is indeed the study of WW events and periods, given the extremely time-varying nature of such phenomena, with typical time scales ranging from minutes to days. The behaviour of the facility in WW is very different from DW, especially with regard to influent flows and loads, primary and secondary settling efficiency, mixing, biological treatment performance, oxygen transfer and solids inventory transfer between the biological reactors and secondary settling tanks. This requires additional modelling efforts compared to DW, to enable decision support regarding design and operation alternatives devised to achieve permit compliance of the facility.

The topics covered by the workshop comprise an introduction to regulatory requirements and benefits of WW dynamic modelling, an overview of the main phenomena occurring at WRRFs during WW and of modelling of selected design and operation WW strategies. The presenters are based in both Europe and North America, and from both academia and consultancy. Plenary open discussion ( $60 \%$ of the total time) is allocated after each of the three presentation blocks and at the 
end of the workshop, with the participation of a panel of invited experts. "On the fly" model runs will be performed during the discussion periods to interactively illustrate/test specific aspects.

A summary of the presentations and of the discussion will be shared with the WWTmod group.

Expected outcomes:

- dissemination of current status in WW management and its modelling, with focus on implications for WWRF design and operation, leading the way to a deeper understanding of the aspects affecting the WRRF's performance under WW;

- identification of main challenges, gaps and opportunities, both for model development and application;

- a white paper on the state of the art and challenges related to WW modelling, with contributions by the presenters and by interested workshop participants.

\section{Workshop set-up}

The workshop is organized as follows:

- one presentation provides an introduction to regulatory requirements related to $\mathrm{WW}$, and a summary of dynamic WW modelling benefits; discussion follows

- two presentations illustrate the modelling (limitations) of the phenomena associated to WW, causing the facility's disturbance by WW (influent flows and loads) and occurring at the plant (mixing, settling, oxygen transfer, etc.); discussion follows

- two presentations introduce design and operation options aimed at dealing with WW, and in particular their specific modelling (limitations) issues; discussion follows

- a last discussion period is dedicated to more clearly identifying and finding consensus on the current status, directions, opportunities and gaps in WW knowledge and modelling.

\section{Chair/Co-chair}

Lorenzo Benedetti (Waterways, Italy)

Peter Vanrolleghem (Université Laval, Canada)

\section{Speakers / Moderators}

Lorenzo Benedetti (Waterways, Italy)

Charles Bott (Chief of Research and Development, HRSD, USA)

Jose Jimenez (Brown \& Caldwell, USA)

Dave Kinnear (HDR, USA)

Paul Krauth (Utah Division of Water Quality, USA)

Cristina Martin Andonegui (DEUSTOTECH, Spain)

Julian Sandino (CH2M HILL, USA)

Oliver Schraa (Hydromantis, Canada)

Peter Vanrolleghem (Université Laval, Canada)

Stefan Weijers (Waterschap De Dommel, Netherlands)

\section{Invited panel (confirmed)}

Gerda Hald (Director, Planning \& Investments, VandCenter Syd, Denmark)

Jeroen Langeveld (RHDHV / Delft University, Netherlands) 


\section{Target Participants}

- Utilities/operators: stimulate ideas on how to improve the performance of their systems.

- Consultants: find and show ways to better serve their clients.

- Academics: opportunity to show current activities and to identify research needs expressed by utilities and consultants.

\section{Programme}

\begin{tabular}{|c|c|c|}
\hline Time & Topic & Speaker/Moderator \\
\hline 09:45 - 09:55 & $\begin{array}{l}\text { Introduction: Motivation, scope, and objectives. Present } \\
\text { workshop structure, participants, etc. }\end{array}$ & Lorenzo Benedetti \\
\hline 09:55 - 10:15 & $\begin{array}{l}\text { Presentation \#1: "Why do we have to tame the beast?" } \\
\text { Regulatory, design and operational aspects of WW; support } \\
\text { provided by modelling in design and operation. }\end{array}$ & $\begin{array}{l}\text { Julian Sandino } \\
\text { Stefan Weijers }\end{array}$ \\
\hline $10: 15-10: 45$ & Discussion Period: regulation & Paul Krauth \\
\hline $10: 45-11: 15$ & Coffee break & \\
\hline $11: 15-11: 35$ & $\begin{array}{l}\text { Presentation \#2: "What is making the beast angry?" } \\
\text { Modelling WW influent aspects: flows, loads, and variability. }\end{array}$ & $\begin{array}{l}\text { Cristina Martin } \\
\text { Andonegui }\end{array}$ \\
\hline $10: 35-11: 55$ & $\begin{array}{l}\text { Presentation \#3: "What are the aspects of the beast's anger?" } \\
\text { Modelling WW impact on plant behaviour: mixing, settling, } \\
\text { aeration, etc. }\end{array}$ & Peter Vanrolleghem \\
\hline $11: 55-12: 45$ & Discussion Period: modelling & Dave Kinnear \\
\hline $12: 45-13: 45$ & Lunch break & \\
\hline $13: 45-14: 05$ & $\begin{array}{l}\text { Presentation \#4: "How do we tame the beast? The hard way." } \\
\text { Modelling design options for WW mitigation. }\end{array}$ & Jose Jimenez \\
\hline $14: 05-14: 25$ & $\begin{array}{l}\text { Presentation \#5: "How do we tame the beast? The soft way." } \\
\text { Modelling control options for WW mitigation. }\end{array}$ & Oliver Schraa \\
\hline $14: 25-15: 15$ & Discussion Period: mitigation & Charles Bott \\
\hline $15: 15-15: 45$ & Coffee break & \\
\hline $15: 45-16: 45$ & $\begin{array}{ll}\text { Discussion Period: general and conclusions } \\
\text { - } & \text { Summary } \\
\text { - } & \text { State-of-the-art } \\
\text { - } & \text { Future } \\
\text { - } & \text { Next steps } \\
\end{array}$ & Julian Sandino \\
\hline $16: 45-17: 15$ & Wrap-up, composing summary, report and presentation & \\
\hline
\end{tabular}


WWTmod

ORAL

CONTRIBUTIONS 


\title{
Characterization and separation of unbiodegradable matter in WRRFs
}

\author{
Majdala Mansour-Geoffrion ${ }^{1}$, Peter L. Dold ${ }^{2}$, Alain Gadbois ${ }^{3}$, Stéphane Déléris ${ }^{4}$ and Yves Comeau ${ }^{5}$ \\ ${ }^{1}$ BPR - Tetra Tech, 1205, Ampère Street, Boucherville, Quebec J4B 7M6, Canada (Email: \\ majdala.geoffrion@tetratech.com) \\ ${ }^{2}$ EnviroSim Associates Ltd., McMaster Innovation Park, 175 Longwood Rd South, Suite 114A, Hamilton, Ontario \\ L8P 0A1, Canada (dold@envirosim.com) \\ ${ }^{3}$ John Meunier Inc., 4105, Sartelon Street, Saint-Laurent, Quebec H4S 2B3, Canada (Email: \\ alain.gadbois@veoliawater.com) \\ ${ }^{4}$ Veolia Water, Technical Direction, Building Giovanni Battista B, 1, rue Giovanni Battista Pirelli, 94417 Saint- \\ Maurice, Cedex, France (Email: stephane.deleris@ veoliawater.com) \\ ${ }^{5}$ Department of Civil, Geological and Mining Engineering, Polytechnique Montreal, P.O. Box 6079, Station \\ Centre-Ville, Montreal, Quebec H3C 3A7, Canada (Email: majdala.mansour-geoffrion@ polymtl.ca; \\ yves.comeau@polymtl.ca)
}

\begin{abstract}
The purpose of this paper is to present a critical review on the characterization of influent unbiodegradable organic (trash) and inorganic (grit) particulate matter, to propose a fractionation of particulate unbiodegradable matter and to how the fractionation can be used to evaluate trash and grit removal efficiencies of physical separation units.
\end{abstract}

Keywords

Trash, grit, unbiodegradable organic, inorganic, characterization, fractionation, activated sludge

\section{INTRODUCTION}

The modelling of systems with long sludge retention times (membrane bioreactors, extended aeration and Cannibal-type systems) is particularly sensitive to the fractionation of wastewater unbiodegradable matter. Indeed, unbiodegradable influent particulate organic (trash; $\mathrm{X}_{\mathrm{U}, \mathrm{NNF}}$ ) and inorganic (grit; $\mathrm{X}_{\mathrm{Ig}, \mathrm{INF}}$ ) matter directly affect predictions of sludge production in water resource recovery facilities (WRRFs). The purpose of this paper is to present a critical review of the characterization of trash and grit, to propose a fractionation of unbiodegradable organic and inorganic material in activated sludge and to show how the fractionation can be used to evaluate trash and grit removal efficiencies of physical separation units.

\section{CHARACTERIZATION OF $\mathrm{X}_{\mathrm{U}, \mathrm{INF}}$}

Trash $\left(\mathrm{X}_{\mathrm{U}, \mathrm{Inf}}\right)$ can be defined as unbiodegradable particulate organic material originating from the influent. During model calibration, the $\mathrm{X}_{\mathrm{U}, \mathrm{In} f}$ fraction is sometimes modified so that the simulated and observed sludge productions correspond. It has been suggested that the development of methods to better represent $X_{U, \text { Inf }}$ would help avoid this fine-tuning (Choubert et al., 2013).

Trash is removed in the screenings at the headworks of WRRFs. Materials composing trash include toilet paper, hair, leaves, rags, sticks, food particles, plastics, bottle caps, tree roots, wood chips, etc. (WEF, 2010; Metcalf \& Eddy, 2014). The lack of data on trash and screenings is often mentioned in the literature and may be attributed to analytical challenges (Clay et al., 1996; Le Hyaric et al., 2009). Several studies have focused on characterizing the quantity, specific gravity, organic matter (volatile fraction), nutrients and biodegradability of these materials (UKWIR, 2000; Le Hyaric et al., 2009; Mansour-Geoffrion, 2012). Screenings characteristics can be influenced by the type and 
length of collection system, pumping stations (and whether screening is present at pumping station), rainfall, influent screen bar spacing, organic matter content, storage conditions, dewatering, solids content (Le Hyaric et al., 2009; WEF, 2010). Short, gently sloping collection systems with low turbulence generally produce more screenings than long steep interceptor systems with pumping stations because of differing degrees of organic solids disintegration (WEF, 2010). Typically, the shorter the sewer network, the greater the amount of screenings removed at the WRRF. This can be explained by less dilacerations of suspended solids and the decreased likelihood of pumping stations (can favor settling during dry weather and disintegration of solids (Canler \& Perret, 2004). Combined sewer systems produce several times the coarse screenings compared to separate systems (WEF, 2010). Peak wet-weather removal from combined systems may vary by as much as 20:1 on an hourly basis from average dry weather conditions (WEF, 2010).

\section{Quantities and composition of $\mathbf{X}_{\mathrm{U}, \mathrm{Inf}}$}

Typical reported quantities and characteristics of screenings collected at WRRFs are presented in Table 1. The average consumption of toilet paper in the United States is $23 \mathrm{~kg}_{\text {capita }}{ }^{-1} \mathrm{y}^{-1}$ (Haase, 2010). Assuming most of the toilet paper is flushed in the toilet and ends up in the sewer system, almost all of it should end up at the WRRF with the exception of combined sewer overflows. The average yearly screenings production at the influent of wastewater treatment plants in the United States was $4.5 \mathrm{~kg} /$ capita (Table 1), representing $20 \%$ of the toilet paper consumed. It can therefore be deduced that much of the toilet paper and most likely other materials considered to be trash are not entirely intercepted by influent screens and probably end up either in primary sludge, secondary sludge or effluents of WRRFs.

Reported solids content of screenings vary between 10 and 50\%, while bulk densities, which depend (among other factors) on solids content, are $510-1100 \mathrm{~kg} / \mathrm{m}^{3}$. As expected, screenings are mainly organic as evidenced by their high volatile fraction $\left(0.77-0.90 \mathrm{~g} \mathrm{X}_{\mathrm{VSS}} / \mathrm{g} \mathrm{X}_{\mathrm{TSS}}\right)$.

Table 1: Summary of quantities and characteristics of screenings collected at WRRFs

\begin{tabular}{|c|c|c|c|c|c|c|c|}
\hline & & UKWIR (2000) & Le Hyaric (2009) & Le Hyaric (2009) & $\begin{array}{c}\text { MOP } 8 \text { (WEF, } \\
2010)\end{array}$ & $\begin{array}{c}\text { Canler \& Perret } \\
(2004)\end{array}$ & $\begin{array}{c}\text { Metcalf \& } \\
\text { Eddy (2014) }\end{array}$ \\
\hline Parameter & Units & $\begin{array}{l}\text { Literature review } \\
\text { and field study }\end{array}$ & Literature review & Field study & $\begin{array}{l}\text { Survey of } 328 \\
\text { U.S. WRRFs }\end{array}$ & Literature review & (Handbook) \\
\hline \multirow{4}{*}{ Quantity } & L/capita·year & $3.7-11.0$ & $1.3-18.8$ & -- & 5.6 & $1.1-16.5$ & -- \\
\hline & $\mathrm{kg} /$ capita·year & -- & $1-15$ & $1-2.5$ & 4.5 & -- & -- \\
\hline & $\mathrm{L} / \mathrm{m}^{3}$ wastewater & -- & -- & -- & $0.74-148$ & -- & $4-100$ \\
\hline & $\mathrm{kg} / \mathrm{m}^{3}$ wastewater & -- & -- & -- & $0.01-0.3$ & -- & -- \\
\hline \multirow{2}{*}{$\%$ Dry solids } & uncompacted & $10-20 \%$ & $10-30 \%$ & $15 \%$ & $10-20 \%$ & $8 \%$ & \\
\hline & compacted & -- & $20-45 \%$ & $30 \%$ & -- & -- & $10-50 \%$ \\
\hline Bulk density & $\mathrm{kg} / \mathrm{m}^{3}$ & $600-900$ & $600-1000$ & $510-800$ & $600-1100$ & -- & $600-1100$ \\
\hline Volatile fraction $\left(f_{\mathrm{VT}}\right)$ & $\mathrm{g} \mathrm{X}_{\mathrm{VSS}} / \mathrm{g} X_{\mathrm{TSS}}$ & $0.80-0.90$ & $>0.80$ & $0.77-0.88$ & -- & 0.86 & -- \\
\hline Calorific value & $\mathrm{kJ} / \mathrm{kg}$ & $15 \times 10^{3}$ & $6-25 \times 10^{3}$ & -- & -- & -- & -- \\
\hline
\end{tabular}

Typical components of trash were characterized and compared to characteristics of microscreenings obtained from experiments conducted on activated sludge from nine full-scale WRRFs in Quebec (Canada) and Morongo (California, USA; Table 2)). The Morongo WRRF was the only facility tested where a full-scale microscreen was installed on the return activated sludge stream for the purpose of trash removal from sludge. The $\mathrm{f}_{\mathrm{VT}}$ (volatile to total suspended solids ratio), $\mathrm{f}_{\mathrm{CV}}$ (chemical oxygen demand to volatile suspended solids ratio), phosphorus content $\left(\mathrm{T}_{\mathrm{P}}\right)$ and total Kjeldahl nitrogen $\left(\mathrm{T}_{\mathrm{N}}\right)$ content of toilet paper, brown paper (for drying hands), Kleenex, live plant leaves and dead leaves were measured. Samples were dried $\left(103^{\circ} \mathrm{C}\right.$ for $24 \mathrm{~h}$ ), weighed, diluted in reverse osmosis water and blended (Ultra-Turrax ${ }^{\circledR} \mathrm{T} 25$ digital, IKA) prior to $\mathrm{X}_{\mathrm{VSS}}, \mathrm{X}_{\mathrm{TSS}}, \mathrm{T}_{\mathrm{COD}}, \mathrm{T}_{\mathrm{P}}$ 
and $\mathrm{T}_{\mathrm{N}}$ analyses. $\mathrm{T}_{\mathrm{P}}$ and $\mathrm{T}_{\mathrm{N}}$ were measured after digestion using an automated ion analyzer (Quickchem ${ }^{\circledR}$ AE model, Lachat Instruments, Inc., Loveland, CO) according to Standard Methods (APHA et al., 2005).

Results showed that paper products were almost entirely volatile $\left(f_{\mathrm{VT}}=98-100 \%\right)$ while dead leaves contained more inorganic matter $\left(f_{\mathrm{VT}}=90 \%\right)$ than live plant leaves $\left(f_{\mathrm{VT}}=96 \%\right)$. The $\mathrm{f}_{\mathrm{CV}}$ values obtained for microscreenings from Morongo and Quebec WRRFs were in the same range as the $\mathrm{f}_{\mathrm{CV}}$ values measured on typical components of microscreenings.

Low $f_{P V}$ and $f_{N V}$ values measured on Morongo microscreenings were characteristic of values measured on typical components of microscreenings, with the exception of live plant leaves. Live plant leaves contained much more $\mathrm{P}$ and $\mathrm{N}$ than paper products and even dead leaves, but they are probably a minor component of microscreenings as indicated by the low $f_{\mathrm{PV}}$ and $\mathrm{f}_{\mathrm{NV}}$ of microscreenings as well as observations.

Table 2. $\mathrm{f}_{\mathrm{VT}}, \mathrm{f}_{\mathrm{CV}}, \mathrm{f}_{\mathrm{NV}}$ and $\mathrm{f}_{\mathrm{PV}}$ of typical $\mathrm{X}_{\mathrm{U} \text {,Inf }}$ components, Morongo and Quebec sludge and microscreenings samples

\begin{tabular}{|c|c|c|c|c|}
\hline Samples & $\begin{array}{c}\mathbf{f}_{\mathrm{VT}} \\
\mathbf{g} X_{\mathrm{VSS}} / \mathbf{g} X_{\mathrm{TSS}} \\
\end{array}$ & $\mathbf{g} X_{\mathrm{coD}} \mathbf{f}_{\mathrm{cv}} / \mathbf{g} X_{\mathrm{vss}} \mathbf{g}$ & $\begin{array}{c}\mathbf{f}_{\mathrm{NV}} \\
X_{\mathrm{TKN}} / \mathbf{g} X_{\mathrm{VSS}} \mathbf{g}\end{array}$ & $\begin{array}{c}f_{P V} \\
X_{P} / g X_{v S S}\end{array}$ \\
\hline \multicolumn{5}{|l|}{ Slowly biodegradable WW components } \\
\hline Toilet paper & $100 \pm 1 \%$ & 1.39 & $0.04 \%$ & $0.006 \%$ \\
\hline Brown paper & $98 \pm 0 \%$ & 1.55 & $0.16 \%$ & $0.006 \%$ \\
\hline Kleenex & $100 \pm 0 \%$ & 1.51 & $0.05 \%$ & $0.003 \%$ \\
\hline Plant leaves (alive) & $96 \pm 1 \%$ & 2.77 & $3.34 \%$ & $0.535 \%$ \\
\hline Dead leaves & $90 \pm 1 \%$ & 2.18 & $1.54 \%$ & $0.074 \%$ \\
\hline \multicolumn{5}{|l|}{ Morongo WRRF (lab-scale tests) } \\
\hline Mixed liquor & $74 \%$ & 1.46 & $7.46 \%$ & $1.8 \%$ \\
\hline Mixed liquor microscreenings $(250 \mu \mathrm{m})$ & $87 \%$ & 1.49 & $0.06 \%$ & $0.013 \%$ \\
\hline \multicolumn{5}{|l|}{ Quebec WRRFs (lab-scale tests) } \\
\hline Activated sludge & $73 \pm 8 \%$ & $1.46 \pm 0.12$ & na & na \\
\hline $\begin{array}{l}\text { Microscreenings }(200-500 \mu \mathrm{m}) \\
\text { na: not available }\end{array}$ & $88 \pm 4 \%$ & $1.59 \pm 0.72$ & na & na \\
\hline
\end{tabular}

\section{Biodegradability of $\mathbf{X}_{\mathbf{U}, \text { Inf }}$}

Materials composing $X_{U, \text { Inf }}$ are often assumed to be unbiodegradable (Lei et al., 2010), however there is evidence in the literature that cellulose (toilet paper) is biodegradable in activated sludge systems (Verachtert et al., 1982) and that activated sludge microscreenings, which have similar characteristics to influent screenings, exhibit a 20-day carbonaceous biochemical oxygen demand ( $\mathrm{X}_{\mathrm{cBOD} 20}$; Mansour-Geoffrion, 2012). There is no standard procedure for the evaluation of screenings biodegradability. Several approaches for evaluating screenings biodegradability, such as manual sorting and identification of materials, biochemical oxygen demand and biomethane potential (BMP) have been proposed. The purpose of manually sorting screenings materials is to evaluate the variability of screenings composition and to identify biodegradable components of screenings. Screenings may be sorted into several categories (Table 3) after draining for thirty minutes (UKWIR, 2000) or after being partially dried at $80^{\circ} \mathrm{C}$ for 10 days (Le Hyaric, 2009). 
Mansour-Geoffrion et al.

Table 3: Categories for manual sorting of screenings materials (biodegradability evaluation)

\begin{tabular}{cc}
\hline UKWIR (2000) & Le Hyaric (2009) \\
\hline Faeces & Fines (less than 20 mm) \\
Sanitary products & Sanitary textiles \\
Fine Paper & Vegetation \\
Leaves & Paper-cardboard \\
Other Material & Plastics \\
& Textiles \\
& Metals, Aluminum \\
& Composites \\
& Combustibles \\
& Incombustibles \\
\hline
\end{tabular}

BOD tests have also been used to evaluate screenings and microscreenings biodegradability, but procedures in the literature present some major differences. Microscreenings biodegradability was evaluated using the $f_{B C}$ index $\left(X_{C B O D 20}\right.$ to $X_{C O D}$ ratio) as an indicator of organic matter biodegradability: the higher the ratio, the more biodegradable the organic matter. Ten microscreening experiments were conducted on sludge from Quebec WRRFs and $\mathrm{f}_{\mathrm{BC}}$ was measured on sludge fed to the microscreen (MSF) and on microscreenings (SCR). Results indicated that microscreenings were composed of biodegradable matter as they exhibited an $\mathrm{X}_{\mathrm{CBOD} 20}$ and were therefore not unbiodegradable as previously assumed (data not shown; Lei, 2010). Whether microscreenings are more or less biodegradable than the sludge they came from was unclear: in six runs microscreenings were more biodegradable than microscreen feed sludge $\left(f_{B C} S C R>f_{B C} M S F\right)$ and in five runs they were less biodegradable ( $f_{B C} S C R<f_{B C}$ MSF). Difficulties evaluating the biodegradability, or rather "unbiodegradability", of microscreenings were also encountered during the characterization of the Micronair ${ }^{\mathrm{TM}}$ system at the Winter Haven (Florida, USA) WRRF which included microscreening and hydrocycloning the return activated sludge stream for trash and grit removal and claimed "zero-biosolids disposal". Oxygen uptake rate of activated sludge microscreenings was $0.81 \mathrm{mg} \mathrm{kg}^{-1} \mathrm{~h}^{-1}$ at the Winter Haven plant (Bizier, 1999), which would also correspond to an $\mathrm{f}_{\mathrm{BC}}$ in the range of 0.2 to $0.3 \mathrm{~g} \mathrm{X}_{\mathrm{cBOD} 20} / \mathrm{g} \mathrm{X}_{\mathrm{COD}}$. Another approach to using the BOD test to evaluate screenings biodegradability is to rinse a wet, $200 \mathrm{~g}$ sample of food-processed screenings ten times, to filter the sample $(100 \mu \mathrm{m})$ while compressing it and to measure the $\mathrm{BOD}_{5}$ on the filtrate (UKWIR, 2000). This approach assumes that the fraction of the sample greater than $100 \mu \mathrm{m}$ does not contribute to BOD, which, considering the evidence in the literature of the biodegradability of screenings materials, seems to be unreasonable.

The slow biodegradability of at least some fraction of screenings and microscreenings is expected as cellulose forms one of the major structural components of all plant material such as wood and cotton (and hence paper products) and active cellulolysis has been shown to occur in activated sludge by enumeration of cellulolytic microorganisms, determination of cellulase activity, by the degradation of cellulose contained in Nylon bags suspended in mixed liquor at a WWTP and by the determination of cellulose and lignin in activated sludge and anaerobic digestion sludge (Verachtert et al., 1982; Ramasamy \& Verachtert, 1980; Cheung \& Anderson, 1997).

\section{CHARACTERIZATION OF $\mathbf{X}_{\text {GRIT }}$}

Typical reported quantities and characteristics of grit $\left(\mathrm{X}_{\mathrm{GRIT}}\right)$ collected at WRRFs are presented in Table 4. $\mathrm{X}_{\text {GRIT }}$ definitions vary but they typically include inorganic particles between 50 and 1000 $\mu \mathrm{m}$ and a range of densities between 1.1 - 2.65 (Reddy \& Pagilla, 2009; WEF, 2010). Sand, gravel, 
pebbles, ashes and eggshells are considered to be a part of grit. Certain references surprisingly claim there is a volatile fraction to "grit" - this is most likely due to $\mathrm{X}_{\mathrm{GRIT}}$ being wrongly defined as suspended solids removed by the grit removal system. Suspended solids removed by the grit removal system are not exclusively composed of $\mathrm{X}_{\mathrm{GRIT}}$ and contain $\mathrm{X}_{\mathrm{U}, \mathrm{Inf}}$ and other organics. $\mathrm{X}_{\mathrm{GRIT}}$ is widely accepted as inorganic suspended solids originating from the influent by definition. The quantity, characteristics and particle size distribution of influent grit depend on several factors: type of sewer network, sewer condition, season, rainfall, industrial loading and nature of industries, maintenance work, construction work, gravel and salt spreading on roads, nature of soils in the area and the height of the water table (Reddy \& Pagilla, 2009; Rippon et al., 2010). Sampling location and methods can also influence the interpretation of grit characteristics due to spatial stratification inside pipes and channels (Reddy \& Pagilla, 2009). Similarly to $X_{U, \text { Inf }}, X_{G R I T}$ is typically removed in the headworks and primary clarifiers of WRRFs, but still accumulates in downstream processes. Inadequate $\mathrm{X}_{\mathrm{GRIT}}$ removal at the headworks of WRRFs can damage process equipment (pumps, air diffusers) by abrasion. Insufficient grit removal can also lead to decreased capacity of bioreactors if $\mathrm{X}_{\mathrm{GRIT}}$ is allowed to settle and accumulate in bioreactors (reduction of effective volume and treatment capacity). Much smaller quantities of $\mathrm{X}_{\mathrm{GRIT}}$ are removed from WRRFS than $\mathrm{X}_{\mathrm{U}, \mathrm{Inf}}$ as can be seen by comparing Tables 1 and 4 . $\mathrm{X}_{\mathrm{GRIT}}$ is more easily dewatered than $\mathrm{X}_{\mathrm{U}, \mathrm{Inf}}$ and its density is typically higher than that of $\mathrm{X}_{\mathrm{U}, \text { Inf }}$.

Table 4: Summary of quantities and characteristics of grit collected at WRRFs

\begin{tabular}{|c|c|c|c|c|c|}
\hline & & $\begin{array}{l}\text { Metcalf \& Eddy } \\
(2014)\end{array}$ & $\begin{array}{l}\text { MOP } 8 \text { (WEF, } \\
2010)\end{array}$ & $\begin{array}{l}\text { Borneman \& } \\
\text { Gress (2011) }\end{array}$ & $\begin{array}{c}\text { Canler \& Perret } \\
(2004)\end{array}$ \\
\hline Parameter & Units & (Handbook) & $\begin{array}{l}\text { Survey of } 328 \\
\text { U.S. WRRFs }\end{array}$ & $\begin{array}{l}\text { WEFTEC } 2011 \text { - } \\
\text { Grit Workshop }\end{array}$ & Literature review \\
\hline $\begin{array}{l}\text { Quantity (separate sewers) } \\
\text { Quantity (combined sewers) }\end{array}$ & $\begin{array}{l}\mathrm{L} / \mathrm{m}^{3} \text { wastewater } \\
\mathrm{L} / \mathrm{m}^{3} \text { wastewater }\end{array}$ & $\begin{array}{c}0.004-0.037 \\
0.004-0.2\end{array}$ & $0.0037-0.148$ & $\begin{array}{l}0.004-0.037 \\
0.004-0.018\end{array}$ & $0.1-0.3$ \\
\hline$\%$ Dry solids & dewatered & $35-87 \%$ & $35-80 \%$ & -- & $80 \%$ \\
\hline Density & $\mathrm{kg} / \mathrm{m}^{3}$ & -- & $1100-2200$ & -- & 2650 \\
\hline Volatile fraction $\left(f_{\mathrm{VT}}\right)$ & $g X_{V S S} / g X_{T S S}$ & $0.01-0.56$ & $0.01-0.55$ & -- & $0.3-0.5$ \\
\hline Particle size & $\mu \mathrm{m}$ & $>150^{*}$ & -- & $50-1000$ & $>200$ \\
\hline
\end{tabular}

${ }^{*}$ in some cases, such as in the southeastern U.S., less than $60 \%$ of grit retained on 150 um screen due to presence of fine sand ("sugar sand")

\section{PROPOSED FRACTIONATION OF ACTIVATED SLUDGE}

Activated sludge matter can be fractionated according to size, volatility and biodegradability (Figure 1).Total solids (TS) can be fractionated into volatile (VS) and inorganic (IS) solids depending on whether they are volatilized at $550^{\circ} \mathrm{C}$. VS are assumed to be organic. VS and IS can each be fractionated according to size into filtered (FVS and FIS; $<0.45 \mu \mathrm{m}$ ) and suspended (VSS and ISS; > $1.2 \mu \mathrm{m}$ ) solids. Particles sized between 0.45 and $1.2 \mu \mathrm{m}$ - too large to be considered "filtered" and too small to be considered "suspended" - are in a no man's land of the solids tests and are thus not considered with regards to fractionation. Filtered matter is commonly termed "soluble" but since colloidal material $(0.08-1 \mu \mathrm{m}$; Odegaard, 2000) is present in both filtered and unfiltered samples, the term filtered rather than soluble is preferred. Total activated sludge organic matter (volatile solids, VS) can be classified as biodegradable $\left(\mathrm{T}_{\mathrm{B}}\right)$ and unbiodegradable $\left(\mathrm{T}_{\mathrm{U}}\right)$ organic matter. Each of these can be further fractionated according to size into filtered $(0.45 \mu \mathrm{m})$ and particulate $(1.2 \mu \mathrm{m})$ biodegradable organic matter $\left(\mathrm{XS}_{\mathrm{B}}\right)$, filtered unbiodegradable matter $\left(\mathrm{S}_{\mathrm{U}}\right)$ and particulate unbiodegradable matter $\left(\mathrm{X}_{\mathrm{U}}\right) . \mathrm{XS}_{\mathrm{B}}$ includes filtered $\left(\mathrm{S}_{\mathrm{B}}\right)$ and particulate $\left(\mathrm{X}_{\mathrm{B}}\right)$ 
biodegradable matter that can be used as substrate for biomass growth and heterotrophic biomass $\left(\mathrm{X}_{\mathrm{H}}\right)$. Filtered and particulate unbiodegradable organic matter $\left(\mathrm{S}_{\mathrm{U}}\right.$ and $\left.\mathrm{Xu}\right)$ can originate in the influent $\left(S_{U, \text { Inf }}\right.$ and $\left.X_{U, \text { Inf }}\right)$ or from endogenous respiration $\left(S_{E}\right.$ and $\left.X_{E}\right) . X_{U, \text { Inf }}(\operatorname{trash})$ and $X_{\mathrm{GRIT}}$ are the main focus of this paper. Salts are an example of filtered inorganic matter $\left(\mathrm{S}_{\mathrm{Ig}}\right)$. Particulate inorganic matter can be classified as associated to biomass cellular material $\mathrm{X}_{\mathrm{Ig}, \mathrm{Cel}}$ such as cell membranes or intracellular inorganic compounds of $\mathrm{X}_{\mathrm{H}}\left(\mathrm{X}_{\mathrm{Ig}, \mathrm{H}}\right)$ and $\mathrm{X}_{\mathrm{E}}\left(\mathrm{X}_{\mathrm{Ig}, \mathrm{E}}\right)$. Inorganic compounds associated to $\mathrm{X}_{\mathrm{U}, \mathrm{Inf}}\left(\mathrm{X}_{\mathrm{Ig}, \mathrm{U}, \mathrm{Inf}}\right)$ are also included in $\mathrm{X}_{\mathrm{Ig}, \mathrm{Cel}}$. Grit $\left(\mathrm{X}_{\mathrm{GRIT}}\right)$ and precipitates $\left(\mathrm{X}_{\mathrm{PPT}}\right)$ make up the extracellular particulate inorganic compounds $\left(\mathrm{X}_{\mathrm{Ig}, \mathrm{EC}}\right)$ of activated sludge. Important issues with fractionation include recognizing the type of filter used to define "filtered" components $(0.45$ vs $1.2 \mu \mathrm{m})$ and the categorization of colloidal material.

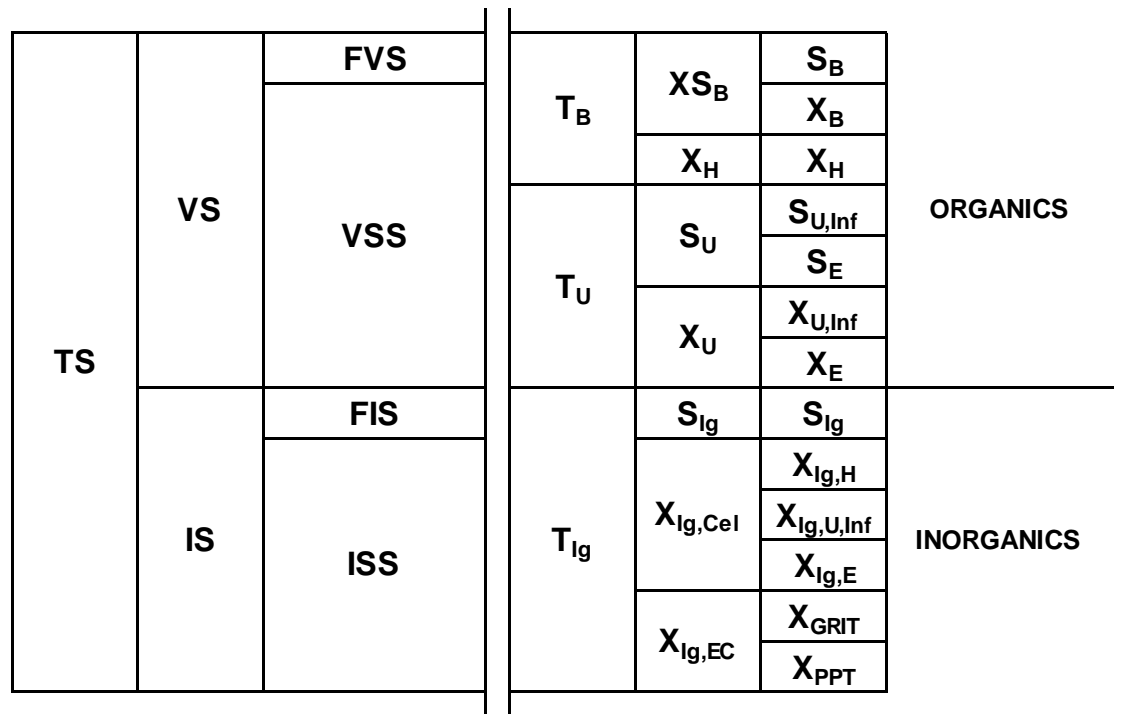

Figure 1: Activated sludge fractionation.

\section{EVALUATION OF $\mathbf{X}_{\mathrm{U} \text {,Inf }}$ and $\mathrm{X}_{\mathrm{GRIT}}$ REMOVALS USING PROPOSED FRACTIONATION}

Lab, pilot and full-scale tests were conducted on microscreens for trash removal and hydrocyclones for grit removal on activated sludge from nine WRRFs in Quebec (Canada) and Morongo (CA, USA). Below are presented the approaches used to

\section{$\mathrm{X}_{\mathrm{U}, \mathrm{Inf}}$ removal by microscreen}

The maximum removal of $\mathrm{X}_{\mathrm{U}, \mathrm{Inf}}$ from microscreen feed sludge (MSF) by microscreening was calculated according to equations 1 and 2 .

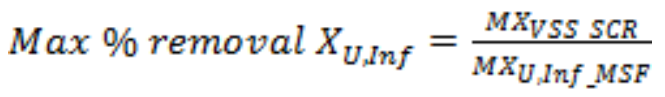

$$
\begin{aligned}
& M X_{U_{\Perp} I n f_{-} M S F}=f_{X U_{\Perp} I n f_{\_} S R T} \cdot M X_{V S S_{-} M S F}
\end{aligned}
$$

where:

$\mathrm{MX}_{\mathrm{VSS} \_\mathrm{SCR}}=$ mass of $\mathrm{X}_{\mathrm{VSS}}$ in microscreenings $\left(\mathrm{g} \mathrm{X}_{\mathrm{VSS}}\right)$;

$\mathrm{MX}_{\mathrm{U}, \mathrm{Inf} \_\mathrm{MSF}}=$ mass of trash in $\mathrm{MSF}\left(\mathrm{g} \mathrm{X}_{\mathrm{VSS}}\right)$; 
$\mathrm{fX}_{\mathrm{U}, \text { Inf_SRT }}=$ mass fraction of trash in MSF at sampling day solids retention time (SRT; $\mathrm{g}$ VSS/g VSS); and

$\mathrm{MX}_{\mathrm{VSS} \_\mathrm{MSF}}=$ mass of $\mathrm{X}_{\mathrm{VSS}}$ in MSF (g VSS).

The fraction of $\mathrm{X}_{\mathrm{U}, \text { Inf }}\left(\mathrm{fX}_{\mathrm{U}, \text { Inf_SRT }}\right)$ in the activated sludge samples from each WRRF was determined knowing the solids retention time (SRT) at the facility on the sampling day. Three underlying assumptions were made:

- influent wastewater fractionation at the WRRFs was typical for raw influent and primary effluent (characterization from Dold, 2007);

- SRTs calculated by WRRFs were correct; and

- all $\mathrm{X}_{\mathrm{VSS}}$ retained by microscreen is trash (hence "maximum").

Using this approach to fractionate the activated sludge solids, it was determined that $14 \%$ of trash contained in activated sludge from WRRFs with no primary clarification could be removed by microscreening, while only $3 \%$ of trash in activated sludge from WRRFs equipped with primary clarifiers could be removed (Figure 2).

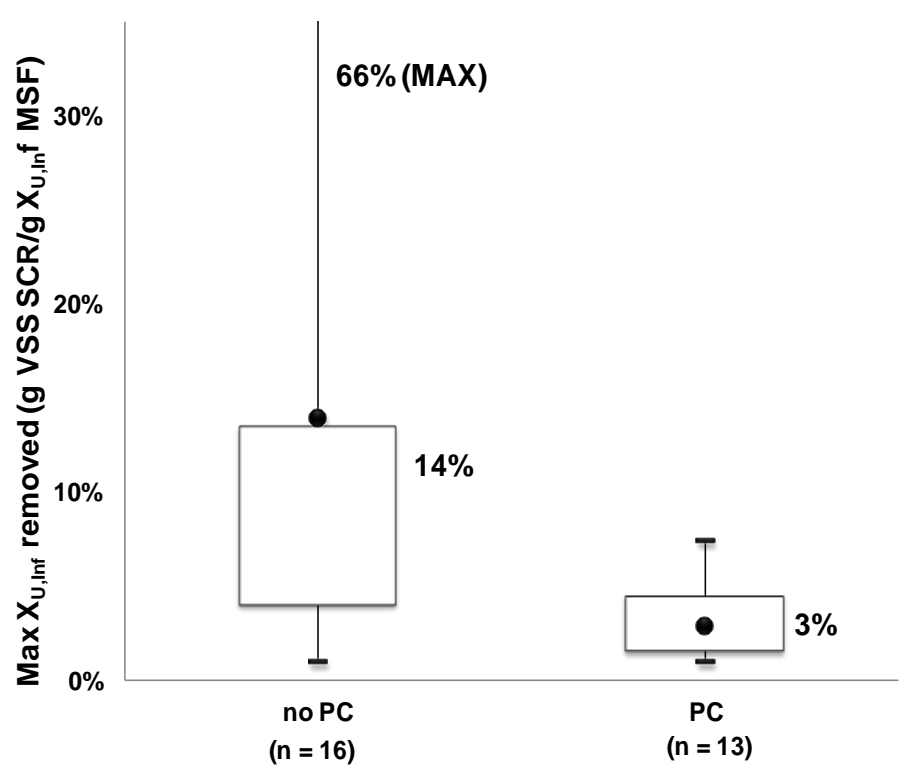

Figure 2: Maximum amount of $\mathrm{X}_{\mathrm{U}, \mathrm{Inf}}$ removed by the lab-scale microscreen (data from Quebec WRRFs). Data grouped according to presence/absence of primary clarifiers (PC).

\section{$\mathrm{X}_{\text {GRIT }}$ removal by hydrocyclone}

Concentration factors $(\mathrm{CF})$ for $\mathrm{X}_{\mathrm{VSS}}$ and $\mathrm{X}_{\mathrm{Ig}}\left(\mathrm{X}_{\mathrm{VSS} \text {, underflow }} / \mathrm{X}_{\mathrm{VSS} \text {,feed }} ; \mathrm{X}_{\mathrm{Ig}, \text { underflow }} / \mathrm{X}_{\mathrm{Ig}, \text { feed }}\right)$ were used to describe hydrocyclone performance. CFs were also calculated for $\mathrm{X}_{\mathrm{Ig}, \mathrm{Cel}}$ (intracellular inorganic particulate matter) and $\mathrm{X}_{\mathrm{Ig}, \mathrm{EC}}$ (extracellular inorganic particulate matter) by assuming $\mathrm{X}_{\mathrm{Ig}, \mathrm{Cel}}$ had the same $\mathrm{CF}$ as $\mathrm{X}_{\mathrm{VSS}}$ since $\mathrm{X}_{\mathrm{Ig}, \mathrm{Cel}}$ are associated to the organic matter. Another assumption made was that the inorganic content of the volatile matter was $0.08 \mathrm{~g} \mathrm{X}_{\mathrm{Ig}, \mathrm{Cel}} / \mathrm{g} \mathrm{X}_{\mathrm{TSS}}$ (Ramdani et al., 2010). The $\mathrm{CF}_{\mathrm{XIg}, \mathrm{EC}}\left(\approx \mathrm{CF}_{\mathrm{GRIT}}\right.$ if no precipitates) could then be calculated by mass balance. Results for lab-scale hydrocyclone experiments on activated sludge are presented in Figure 3 (data grouped according to presence/absence of primary clarifier). Results indicated that the hydrocyclone was more efficient at 
separating grit $\left(\mathrm{X}_{\mathrm{Ig}, \mathrm{EC}}\right)$ from activated sludge in WRRFs with no primary clarifiers than from sludge from WRRFs with primary clarifiers.

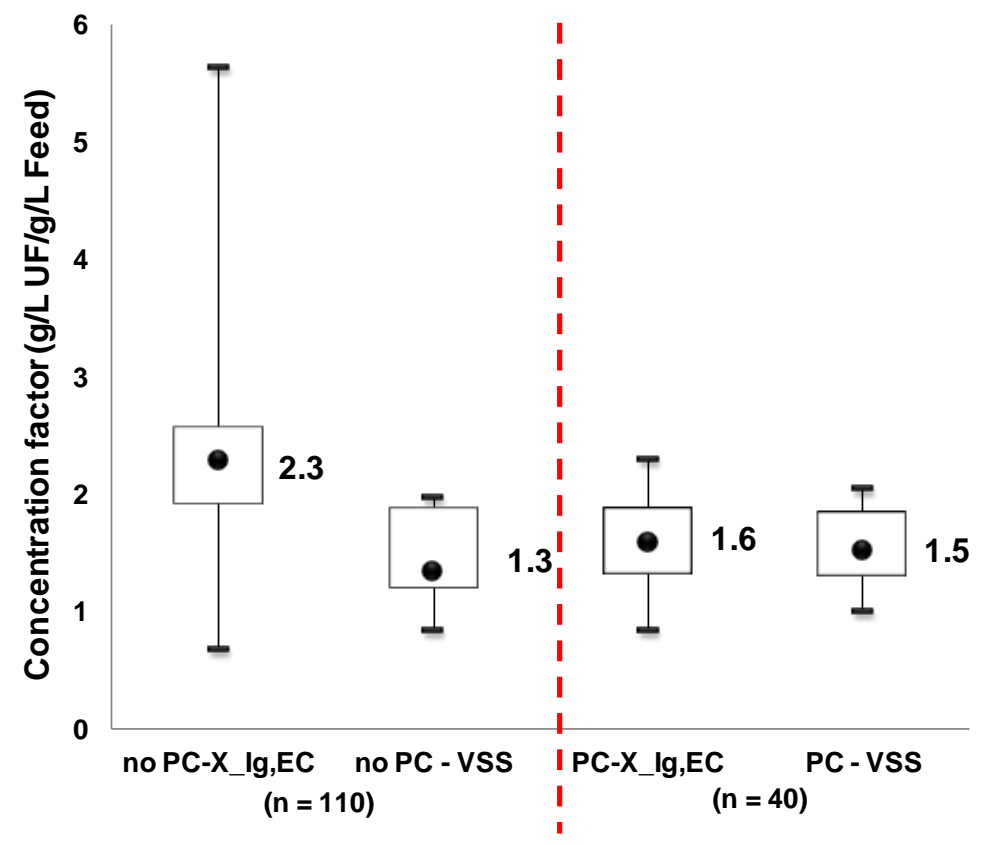

Figure 3: Influence of primary treatment on hydrocyclone concentration factors for a $13 \mathrm{~mm}$ labscale hydrocyclone and activated sludge samples from Quebec and Morongo WWTPs. UF: underflow, PC: primary clarifier, $\mathrm{X}_{\mathrm{Ig}, \mathrm{EC}}$ : extracellular inorganic suspended solids, VSS: volatile suspended solids.

\section{REFERENCES}

APHA, AWWA, \& WEF. (2005). Standard Methods for the Examination of Water and Wastewater (21 ${ }^{\mathrm{st}}$ ed.). Washington: American Public Health Association, American Water Works Association, Water Environment Federation.

Bizier, P.A. (1999). Evaluation of a biosolids minimization system. Environmental Engineering 1999, ASCE-CSCE National Conference on Environmental Engineering, July 25-28, Norfolk, USA (397-406). Reston: American Society of Civil Engineers.

Borneman, R. \& Gress, S.P. (2011). Fundamentals of Grit Removal and Grit System Evaluation. Workshop 102, WEFTEC 2011, 84 ${ }^{\text {th }}$ Annual Conference and Exposition of the Water Environment Federation, October 15-19, Los Angeles, CA, U.S.A.

Canler, J.P. \& Perret J.M. (2004). Études des prétraitements compacts basés uniquement sur le tamisage fin (FNDAE no 28). Lyon : Cemagref Editions.

Clay, S., Hodgkinson, A., Upton, J., \& Green, M. (1996). Developing acceptable sewage screening practices. Wat. Sci. Tech., 33(12), 229-234.

Cheung, S.W. \& Anderson B.C. (1997). Laboratory investigation of ethanol production from municipal primary wastewater. Bioresource Technology, 59 (1), 81-96.

Choubert, J. M., Rieger, L., Shaw, A., Copp, J., Spérandio, M., Sørensen, K., Ronner-Holm, S., Morgenroth, E., Melcer, H. \& Gillot, S. (2013). Rethinking wastewater characterisation methods for activated sludge systems-a position paper. Wat. Sci. Tech., 67(11), 2363-2373.

Dold, P. L. (2007). Quantifying sludge production in municipal treatment plants. Proceedings of WEFTEC 2007, 80 ${ }^{\text {th }}$ Annual Conference and Exposition of the Water Environment Federation, October 13-17, San Diego, CA, U.S.A. $(17,1522-1549)$.

Haase, S. (2010). Meeting passionate advocates of toilet paper. International Paper World, 6(2010), 32-33. 
Mansour-Geoffrion, M. (2012). Trash and grit removal from activated sludge: evaluating potential for sludge reduction. Thesis, Polytechnique Montreal, Montreal, QC, Canada.

Metcalf \& Eddy. (2014). Wastewater Engineering: Treatment and Resource Recovery (5 ${ }^{\text {th }}$ ed.). New York: McGrawHill.

Le Hyaric, R. (2009). Caractérisation, traitabilité et valorisation des refus de dégrillage des stations d'épuration. Thesis, INSA de Lyon, Lyon, France.

Le Hyaric, R., Canler, J. P., Barillon, B., Naquin, P., \& Gourdon, R. (2009). Characterization of screenings from three municipal wastewater treatment plants in the Region Rhône-Alpes. Wat. Sci. Tech., 60(2), 525-531.

Lei, L., Johnson, B., Sandino, J., \& Whitlock, D. (2010). A Proposed Approach for Modeling Sludge Reduction Technologies. Proceedings of WEFTEC 2010, 83rd Annual Conference and Exposition of the Water Environment Federation, October 2-6, New Orleans, LA, U.S.A. (12, 4850-4860).

Ramasamy, K. \& Verachtert, H. (1980). Localization of cellulase components in Pseudomonas sp. isolated from activated sludge. Journal of General Microbiology, 117(1), 181-191.

Ramdani, A., Dold, P., Déléris, S., Lamarre, D., Gadbois, A., \& Comeau, Y. (2010). Biodegradation of the endogenous residue of activated sludge. Water Research, 44(7), 2179-2188.

Reddy, M.P. and K. Pagilla. (2009). Integrated Methods for Wastewater Treatment Plant Upgrading and Optimization, Final Report. Alexandria: Water Environment Research Foundation.

Rippon, D., Higgins, R., Mrkvicka, R.S. (2010). Grit Characterization and the Impact on Grit Removal Systems. Proceedings of WEFTEC 2010, $83^{\text {rd }}$ Annual Conference and Exposition of the Water Environment Federation, October 2-6, New Orleans, LA, U.S.A. (10, 5963-5989).

UK Water Industry Research (UKWIR). (2000). Screenings: Quality and quantity, Report Ref. No. 00/WW/06/3.

WEF. (2010). Chapter 11: Preliminary Treatment. In Design of Municipal Wastewater Treatment Plants: WEF Manual of Practice No. 8 ( $5^{\text {th }}$ ed., 2, 1-90). Alexandria: Reston: Water Environment Federation; American Society of Civil Engineers/Environmental and Water Resources Institute. 


\title{
Influent generator for probabilistic modeling of nutrient removal wastewater treatment plants
}

\author{
Mansour Talebizadeh ${ }^{1}$, Evangelia Belia ${ }^{2}$, and Peter A. Vanrolleghem ${ }^{1}$ \\ ${ }^{1}$ modelEAU, Département de génie civil et de génie des eaux, Université Laval, 1065 av. de la Médecine, Québec \\ (QC) G1V 0A6, Canada (Email : mansour.talebizadehsardari.1@ulaval.ca;Peter.Vanrolleghem@gci.ulaval.ca) \\ ${ }^{2}$ Primodal Inc., 145 Aberdeen, Québec, QC G1R 2C9, Canada (Email: belia@primodal.com)
}

\begin{abstract}
The availability of influent wastewater time series is crucial when using models to assess the performance of a wastewater treatment plant (WWTP) under dynamic flow and loading conditions. Given the difficulty of collecting sufficient data, synthetic generation could be the only option. In this paper a hybrid of statistical and conceptual modeling techniques is proposed for synthetic generation of influent time series. The time series of rainfall and influent in DWF conditions were generated using two types of statistical models (a periodic-multivariate time series model for influent in DWF conditions and a two-state Markov chain-gamma model for rainfall). These two time series serve as inputs to a conceptual sewer model for generation of influent time series during WWF conditions. The effect of total model uncertainty on the generated outputs is taken into account through a Bayesian calibration and is communicated to the user by constructing uncertainty bands with a desired level of confidence. The proposed influent generator is a powerful tool for realistic generation of the influent time series and is well-suited for risk-based design of WWTPs as it considers both the effect of input variability (i.e. variability in rainfall and influent during DWF) and total model uncertainty in the generation of the influent. Considering the fact that the proposed influent generator only requires readily-available or easy-to-obtain information and data on climate and the general characteristics of sewershed, it is an attractive tool for practical applications.
\end{abstract}

Keywords

Bayesian estimation; uncertainty analysis; urban hydrology; wastewater composition; probabilistic design

\section{INTRODUCTION}

One of the major sources of uncertainty/variability that both plant designers and operators must deal with is the dynamics of the influent. The recent advances in mathematical modeling and improved computational power have enabled researchers to better understand the performance of different WWTP design alternatives (Hao et al., 2001; Salem et al., 2002) and/or evaluate control strategies under dynamic flow and loading conditions. However, the application of mathematical models used for simulating the performance of a WWTP could be misleading unless, among others, models are provided with representative influent time series. One of the problems that arise in this regard is the scarcity or even lack of long-term influent data. To remedy this problem, some researchers have proposed models for synthetic dynamic influent time series scenarios (Bechmann et al., 1999; Gernaey et al., 2011).

One of the simplest approaches in synthetic generation of influent time series is the application of empirical stochastic models (Capodaglio et al., 1990; Martin et al., 2007). However, these models may have a poor performance especially during wet weather flow conditions as different complex processes affect the dynamics of the influent. Indeed, such statistical models do not consider the underlying elements and processes that govern the generation and the dynamics of the influent. To 
consider the underlying phenomena that are involved, some researchers have advocated the use of detailed and physically-based models (Hernebring et al., 2002; Temprano et al., 2007). The application of these complex models might be useful for certain purposes, (e.g. evaluating the performance of different operating strategies in a sewer system). However, in cases in which the overall behavior of the influent time series is of interest, they might not be very useful as they require detailed information on the sewage system and running them for a large number of times could be computationally expensive.

Some researchers have proposed parsimonious conceptual models as an alternative to the complex mathematical models that require detailed information and data (Achleitner et al., 2007; Gernaey et al., 2011). In these models a conceptual view of the main phenomena and interactive processes contributing to the influent are formulated in terms of mathematical equations. Despite successful application of these models (at least in giving an overall view of the system), the performance of these models to a great extent depends on the proper choice of model parameters. Since some of the parameters may not have a clear physical meaning they are usually estimated through model calibration. In cases in which there is no measured data available for model calibration, only a rough estimate or a range of values could be inferred from the values reported in literature. Besides, it is almost impossible to have a complete similarity between the model output(s) and the observed values owing to the inextricable uncertainties (e.g. input data uncertainty and/or model structure uncertainty) in any modeling exercise (Belia et al., 2009; Freni and Mannina, 2010).

Given the importance of the issue of uncertainty, several studies have been conducted to consider its effect on both water quality and quantity in urban drainage modeling (Freni et al., 2009; Dotto et al., 2012). However, in these studies, only the effect of model uncertainty under a set of historical rain events (WWF conditions) has been considered (i.e. the time series of rainfall and also the contribution of wastewater in DWF conditions were known a priori). In this study on the contrary not only are we interested in considering the effect of model uncertainty, but also in the variability of rainfall and influent time series in DWF conditions which significantly affect both the amount and the dynamics of the influent.

\section{PROPOSED INFLUENT GENERATOR}

In this paper, a hybrid of statistical and conceptual modeling tools is proposed for synthetic generation of influent time series considering both the effect of model uncertainty and input variability. Given the importance of rainfall time series in the generation of the influent, a two-state Markov chain-gamma model (Richardson, 1981) in conjunction with two time series disaggregation methods were used for stochastic generation of rainfall time series with a high temporal resolution (i.e. 15-minute). To generate the influent time series in DWF conditions taking into account the daily periodic variation, auto-correlation, and cross-correlation in time, a multivariate time series models was developed and its parameters were estimated using the methodology proposed by Neumaier and Schneider (2001). The proposed stochastic model is superior to previous attempts in the generation of influent, as in previous studies the diurnal variation of the influent in DWF conditions was modeled using univariate time series models (Martin et al., 2007), or by multiplying the daily average influent values to a set of coefficients representing the ratio of influent at different times of a day to its average value with or without addition of a noise term to the generated time series (Achleitner et al., 2007; Langergraber et al., 2008; Gernaey et al., 2011). The outputs of the two statistical models used for the generation of rainfall and influent time series in DWF conditions are then input to a conceptual model for modeling the influent time series in WWF conditions. In this study the CITYDRAIN model (Achleitner et al., 2007) was selected as the conceptual model 
Talebizadeh et al.

owing to its flexibility and parsimony. The CITYDRAIN model of the sewershed is calibrated using the measured influent data through a Bayesian calibration procedure to account for the total model uncertainty. Finally, different realizations of the influent time series can be generated by running the calibrated CITYDRAIN model using an instance of a generated time series of rainfall and an instance of influent under DWF conditions (i.e. the two stochastic input time series). Figure shows the schematic of the proposed influent generator.

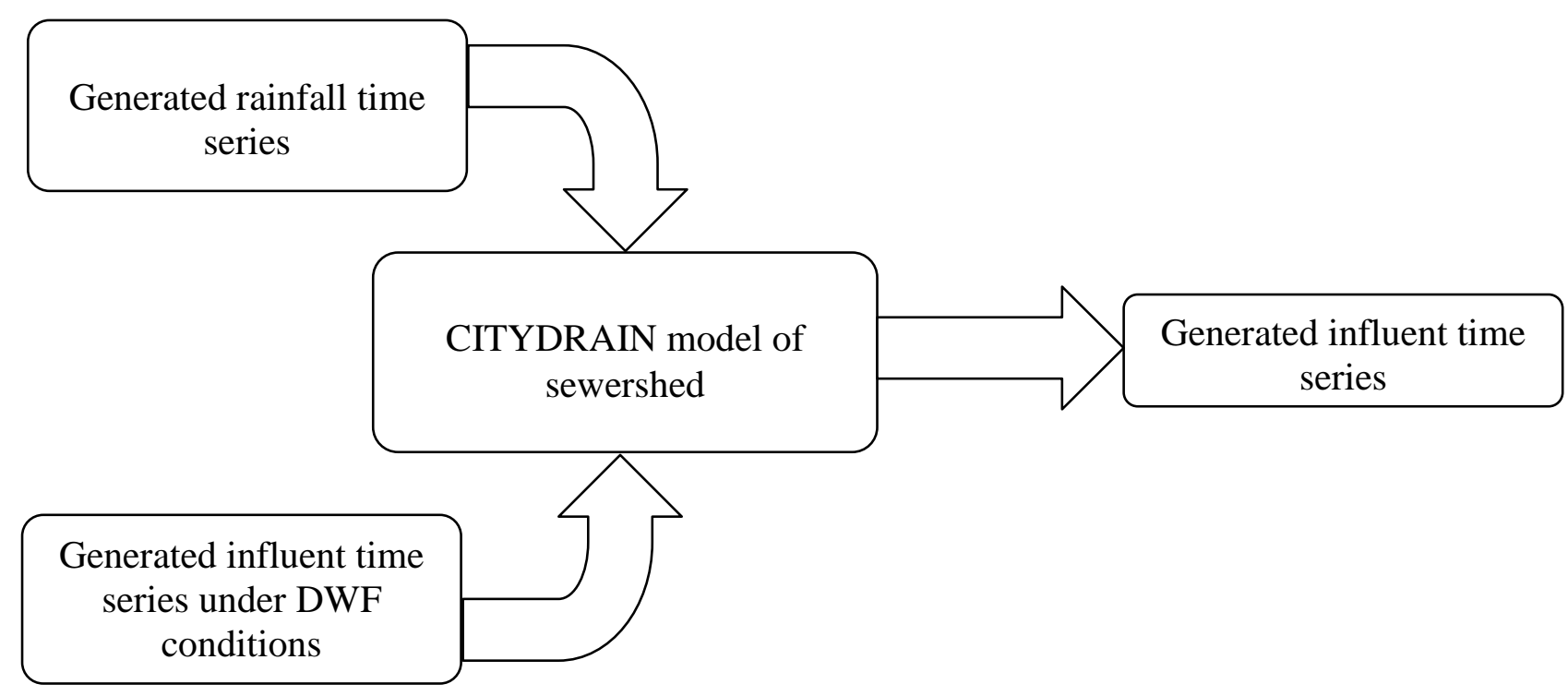

Figure 1 Schematic of the proposed influent generator

The main objective of the proposed influent generator is to produce a dynamic influent time series of flow and traditional wastewater component concentrations (TSS, COD, TN, TP, $\mathrm{NH}_{4}$ ) with 15min temporal resolution in order to capture the sub-daily time variations of the influent which could affect the operating parameters and the performance of WWTPs. One of the constraints was that the generator should only be using limited information on climate and the general characteristics of combined sewer systems. Depending on the biological model that would be used for modelling the biological processes inside a WWTP system, an influent fractionation block must be added to convert the generated traditional wastewater composition into state variables of the adopted biological models, e.g. the ASM models. The generated influent time series using the proposed tools can be used among others for the design of WWTPs under uncertainty (Martin et al., 2012).

\section{Data and case study}

The Eindhoven WWTP with a design capacity of 750000 population equivalent (PE) is the third largest WWTP in the Netherlands. The sewershed served by the Eindhoven WWTP has a total area of approximately $600 \mathrm{~km}^{2}$ and comprises of three main sub-sewersheds called Nuenen/Son, Eindhoven Stad, and Riool-Zuid. The influent data used in this study are related to sensor data of flow, ammonia (measured using an ion-selective sensor) soluble COD, total COD, and TSS (the latter 3 measured using an UV/VIS-based sensor) in the period of September 2011 to September 2012 at the outlet of the Nuenen/Son, Eindhoven Stad, and Riool-Zuid sub-sewersheds. It should be noted that the raw sensor data were cleaned up using visual inspection and a wavelet-based denoising strategy (details are not included in this paper). 
Talebizadeh et al.

The long-term daily rainfall data and also rainfall data with finer temporal resolution provided by KNMI (Royal Netherlands Meteorological Institute) and Waterschap De Dommel were used for estimating the parameters of the weather generator proposed in this paper.

\section{Weather generator}

Realistic generation of rainfall time series is crucial as it is one of the most important factors that affect the dynamics of the influent during WWF conditions. In this study a stochastic model proposed by Richardson (1981) was used for the synthetic generation of daily rainfall and air temperature time series. According to this method the sequence of dry and wet days is generated using a two-state Markov chain model with parameters $P(W \mid W)$ and $P(W \mid D)$ which represent the probability of having a wet day at day $t$ given a wet day at day $t-1$ and the probability of having a wet day at time $t$ given a dry day at time $t-1$ respectively (Figure ).

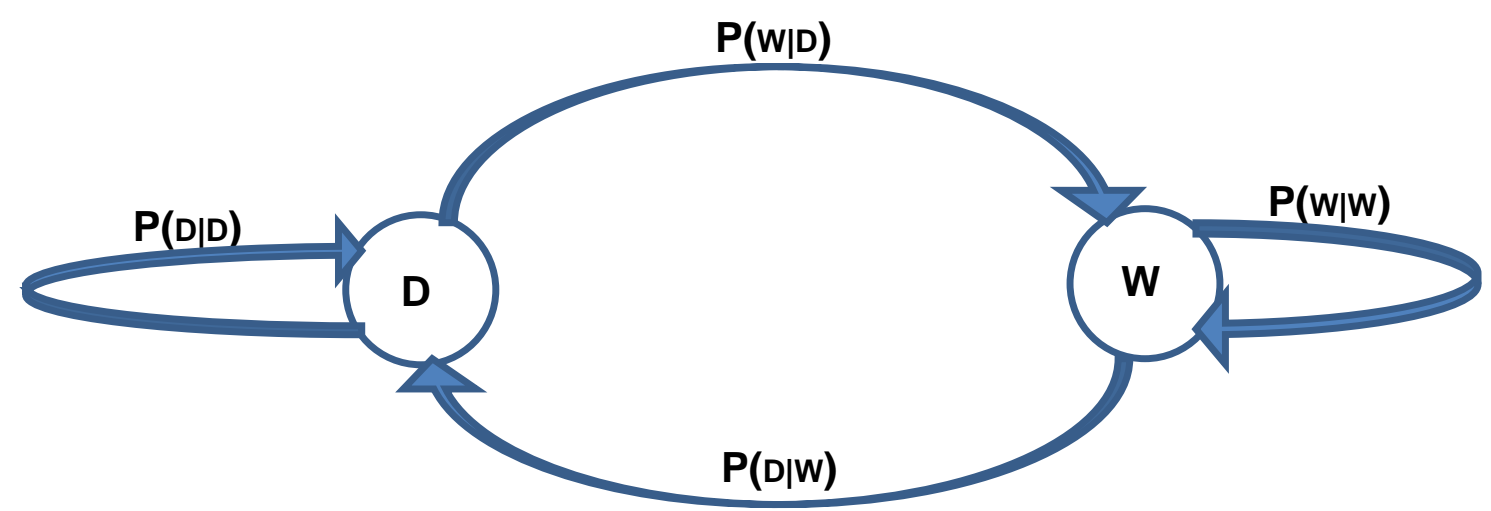

Figure 2 Schematic of a two-state Markov chain, i.e. wet (W) or dry (D)

The other two parameters of the transition matrix needed for generation of dry and wet days (i.e. $P(D \mid D)$ the probability of having a dry day at day $t$ given a dry day at day $t-1$ and $P(D \mid W)$ the probability of having a dry day at day $t$ given a wet day at day $t-1)$ can be calculated using Equation 1 and Equation 2.

$$
\begin{array}{ll}
P(D \mid D)=1-P(W \mid D) & \text { Equation 1 } \\
P(D \mid W)=1-P(W \mid W) & \text { Equation 2 }
\end{array}
$$

Once the sequence of wet and dry days is generated, the amount of rainfall in a wet day is generated by sampling from a gamma probability distribution (Equation 3)

$$
f(x)=\frac{(x / \beta)^{\alpha-1} \exp (-x / \beta)}{\beta \Gamma(\alpha)}
$$

Equation 3

where $x$ is the depth of daily rainfall, $\alpha$ and $\beta$ are the two parameters of the distribution, and $\Gamma(\alpha)$ represents the gamma function evaluated at $\alpha$. The time series of minimum and maximum air temperature are generated conditioned on the state of the day (i.e. wet or dry) using a multivariate linear first-order time series model (Matalas, 1967). The above weather generator is suited for random generation of daily rainfall and temperature. However, in this study we need to generate rainfall time series with a finer temporal resolution than daily resolution (15-min temporal resolution, comparable to the temporal resolution of rainfall in the BSM influent model (Gernaey et al., 2011)). Some methodologies have been proposed for random generation of hourly rainfall time 
Talebizadeh et al.

series based on historical hourly rainfall data. However, long-term hourly rainfall data may not be available in every region and using a limited hourly rainfall record for random generation of longterm hourly rainfall time series may result in misrepresentation of the inter-annual variability in rainfall.

That being said, in this study it is proposed to combine the Richardson-based weather generator (i.e. which is used for daily rainfall generation) with two time series disaggregation techniques. In other words, daily rainfall time series is first generated using the Richardson (1981) method and then two time series disaggregation models, including a daily-to-hourly model (Koutsoyiannis and Onof, 2001) and an hourly-to-15-minutes model (Ormsbee, 1989) are applied for generation of long-term rainfall time series with 15-minute temporal resolution. Moreover, the original Richardson-based weather generator is also suited for the generation of daily air temperature. However, in this study not the air temperature but the wastewater temperature is of interest as it affects the rate of many biological processes taking place in the bioreactors. To estimate the wastewater temperature a simple linear regression model was fitted between the daily air temperatures and the corresponding wastewater temperature measured during the period of September 2011 to September 2012. The fitted regression model was used to calculate the daily wastewater temperature as a function of daily air temperature generated using the Richardson-based weather generator.

\section{Influent generation in DWF conditions}

The influent time series in DWF conditions usually shows specific periodic patterns which can be mainly attributed to the socio-economic fabric of society and also to the physical characteristics of the wastewater collection system. To mimic these variations in time, it is common practice to estimate representative values (e.g. multiplying flow per person to the total population for estimating flow) for flow and loads and then multiplying them to a set of normalized coefficients reflecting diurnal, weekly and seasonal time variation of the influent time series (Jeppsson et al., 2007; Gernaey et al., 2011; Flores-Alsina et al., 2014). Moreover, Gernaey et al. (2011) proposed to add a noise term to the deterministic influent profile in order to avoid generating the same influent time series in subsequent days. In this study the application of a multivariate auto-regressive model (Neumaier and Schneider, 2001) with periodic components is proposed.

To estimate the parameters of the proposed time series model, the influent time series during DWF conditions were extracted and analyzed for estimating the parameters of the multivariate autoregressive model. First, the seasonal (e.g. associated to groundwater infiltration) and diurnal periodic components of flow and other wastewater constituents were estimated using different Fourier series approximations and removed from the original influent time series to calculate the residual time series. The zero-mean residual time series of influent flow and composition were furthered standardized to have an influent time series with a zero mean and unit standard deviation. The parameters of the multivariate autoregressive model in Equation 4 (i.e. $p, A_{l}, C$ ) were then estimated through a stepwise least square algorithm proposed by Neumaier and Schneider (2001).

$$
v_{t}=\sum_{l=1}^{p} A_{l} \times v_{t-l}+\varepsilon_{t}
$$

Equation 4

In Equation 4, $v_{t}$ is an m-dimensional vector (i.e. for our application $\mathrm{m}=5$ which corresponds to the flow and the four wastewater compositions) containing the generated influent component at time $t$, $p$ is the order of the auto-regressive model, $A_{1}, \ldots, A_{p}$ are the coefficient matrices of the autoregressive model, and $\varepsilon_{t}$ is a noise term generated from an uncorrelated zero-mean multivariate normal distribution with the covariance matrix $C$ (i.e. $\varepsilon_{t} \sim N(0, C)$ ). Different realizations of the 


\section{WWTmod}

Talebizadeh et al.

residual influent time series can be generated using this time series model and converted to the original scale depending on the mean and standard deviation of the original influent time series.

\section{Influent generation in WWF conditions}

Synthetic generation of the influent time series during WWF conditions is relatively more complicated than the generation of the influent time series during DWF conditions. Difficulties arise as various phenomena are occurring during WWF conditions and as the availability of measured data is usually scarce for these periods. Hence, using a purely statistical model may result in significant discrepancies between simulated and observed time series. Therefore, we used a combination of statistical modeling techniques and a conceptual model to generate the time series of the influent during WWF conditions. The CITYDRAIN model (Achleitner et al., 2007) was selected as the conceptual model as it takes into account the basic phenomena that govern the amount and dynamics of the influent and also requires only a small number of parameters whose values or ranges of values can be inferred from the basic information of a sewershed.

\section{Flow}

CITYDRAIN calculates the amount of effective rainfall by adopting the concept of virtual basins in which effective rainfall is calculated by subtracting the initial loss from rainfall and then multiplying it with the runoff coefficient. The height of the effective rainfall is then multiplied by the fraction of sewershed area which contributes to the generation of runoff to calculate flow. A simplified routing method based on the well-known Muskingum method is then used for routing flow and pollutants inside the sewer system.

\section{Composition}

For the generation of pollutant time series in WWF conditions, CITYDRAIN uses a rather simplistic approach in which a fixed pollutant concentration is imposed to the system:

$$
\left\{\begin{array}{l}
C(t)=C \text { if } h_{e}>0 \\
C(t)=0 \text { if } h_{e}=0
\end{array}\right.
$$

Equation 5

where, $C(t)$ is the generated pollutant concentration in time, $C$ is a model parameter representing the concentration in WWF conditions, and $h_{e}$ is the effective rainfall. Given the importance of the influent time series in WWF conditions, a more appropriate conceptual model was used for simulating the accumulation-wash off processes corresponding to the particulate concentrations. To this aim, a new block was developed and implemented in CITYDRAIN to generate the pollutant concentration time series in WWF conditions. Equation 6 shows the mathematical formulation of the selected accumulation-wash off model (Kanso et al., 2005).

$$
\left\{\begin{array}{l}
\text { Accumulation model: } \frac{d M_{(t)}}{d t}=K_{a}\left(m_{\lim } \times S_{i m p}-M_{(t)}\right) \\
\text { Wash off model }: \frac{d M_{(t)}}{d t}=-W_{e} \times I_{(t)}{ }^{w} \times M_{(t)}
\end{array}\right.
$$

Equation 6

where, $M_{(t)}$ is the vailable pollutant mass on the sewershed at time $t(\mathrm{~kg}), K_{a}$ is the accumulation coefficinet (1/day), $m_{\lim }$ is the maximum accumulated mass $(\mathrm{kg} / \mathrm{ha}), S_{i m p}$ is the impervious area (ha), $I_{(t)}$ is the rainfall intensity $(\mathrm{mm} / \mathrm{hr}), W_{e}$, and $w$ are calibration parameters. 
Talebizadeh et al.

\section{Bayesian model calibration and long-term influent generation}

As explained in the previous section, the dynamics of the influent time series in WWF conditions is modeled using the CITYDRAIN model. However, one should be aware of the fact that modeling the influent time series in WWF conditions using a conceptual model may not lead to reliable results unless the model is calibrated and the effect of different sources of uncertainties on the outputs (e.g. flow and other pollutants) are taken into account. To this aim, a Bayesian framework was used to update the ranges of values that were initially assigned to the parameters of the CITYDRAIN model (i.e. estimating the posterior distribution of parameters using their prior distribution and the measured data on flow and pollutant concentrations). In general, the posterior distribution of parameters using Bayes' theorem can be formulated by Equation 7 .

$$
h(\theta \mid \text { Data })=\frac{f(\text { Data } \mid \theta) p(\theta)}{f(\text { Data })}
$$

Equation 7

where $h(\theta \mid$ Data $)$ is the posterior distribution, $p(\theta)$ is the prior distribution, $f($ Data $)$ is merely a proportionality constant so that $\int h(\theta \mid$ Data $)=1$, and $f($ Data $\mid \theta)$ constitutes the likelihood function which measures the likelihood that the data correspond to the model outputs with parameter set $\boldsymbol{\theta}$. Assuming homoscedastic uncorrelated Gaussian error terms the likelihood function function can be formulated according Equation 8 (Bates and Campbell, 2001; Marshall et al., 2004).

$$
f(\text { Data } \mid \boldsymbol{\theta})=\left(2 \pi \sigma^{2}\right)^{-n / 2} \prod_{t}^{n} \exp \left\{-\frac{\left[\text { Data }_{t}-R\left(x_{t} ; \boldsymbol{\theta}\right)\right]^{2}}{2 \sigma^{2}}\right\}
$$

Equation 8

where $n$ is the number of observations, $\sigma^{2}$ is the variance of the residual error (i.e. the difference between model predictions and observed values), Data ${ }_{t}$ is the observed variable at time $t, x_{t}$ is the set of inputs at time $t, \boldsymbol{\theta}$ is the set of model parameters and $R\left(x_{t} ; \boldsymbol{\theta}\right)$ represents the model output as a function of $x_{t}$ and $\theta$.

A specific form of Markov chain Monte Carlo (MCMC) sampler known as differential evolution adaptive Metropolis or DREAM (Vrugt et al., 2008) was used to efficiently estimate the posterior distribution of the CITYDRAIN model parameters given the time series of flow and influent composition of the Eindhoven WWTP. It should be noted that the proposed Bayesian approach is not only capable of capturing the effect of model parameter uncertainty, but also of capturing the effect of other sources of uncertainties that could result in some discrepancies between the simulated influent time series and the observed series.

Once the uncertainty ranges of the CITYDRAIN model parameters are updated, synthetic influent time series for a desired number of years considering the variability in the inputs of the CITYDRAIN model (i.e. rainfall and influent time series in DWF conditions) and also the total uncertainty can be obtained as follows:

1. Synthetic generation of the 15-minute time series of rainfall for one year

2. Synthetic generation of the 15-minute time series of the influent in DWF conditions for one year

3. Sampling a point from the posterior distribution of the CITYDRAIN model parameters 


\section{WWTmod}

Talebizadeh et al.

4. Inputting the generated time series 1) and 2) and the parameters sampled in 3) and running the CITYDRAIN model for one year

5. Repeating 1) to 4) for a desired number of years

\section{RESULTS AND DISCUSSION}

This section presents the outputs and some discussion on the results of different components of the proposed influent generator. The performance of the weather generator and the influent generator under DWF conditions are evaluated by comparing the statistical properties of the generated time series with those of the historical time series. The results corresponding to the Bayesian calibration of CITYDRAIN model are explained and at the end a 7-day snapshot of generated one year influent time series is presented and discussed.

\section{Synthetic generation of rainfall}

The parameters of the statistical Markov-gamma model were estimated using the recorded rainfall data in the studied Eindhoven catchment. The results indicate that not only are the basic yearly statistics (i.e. average and variance) of the generated rainfall time series consistent with the recorded rainfall time series, but also the seasonal variations in rainfall intensity and frequency of wet days are respected (Figure and Table 1).
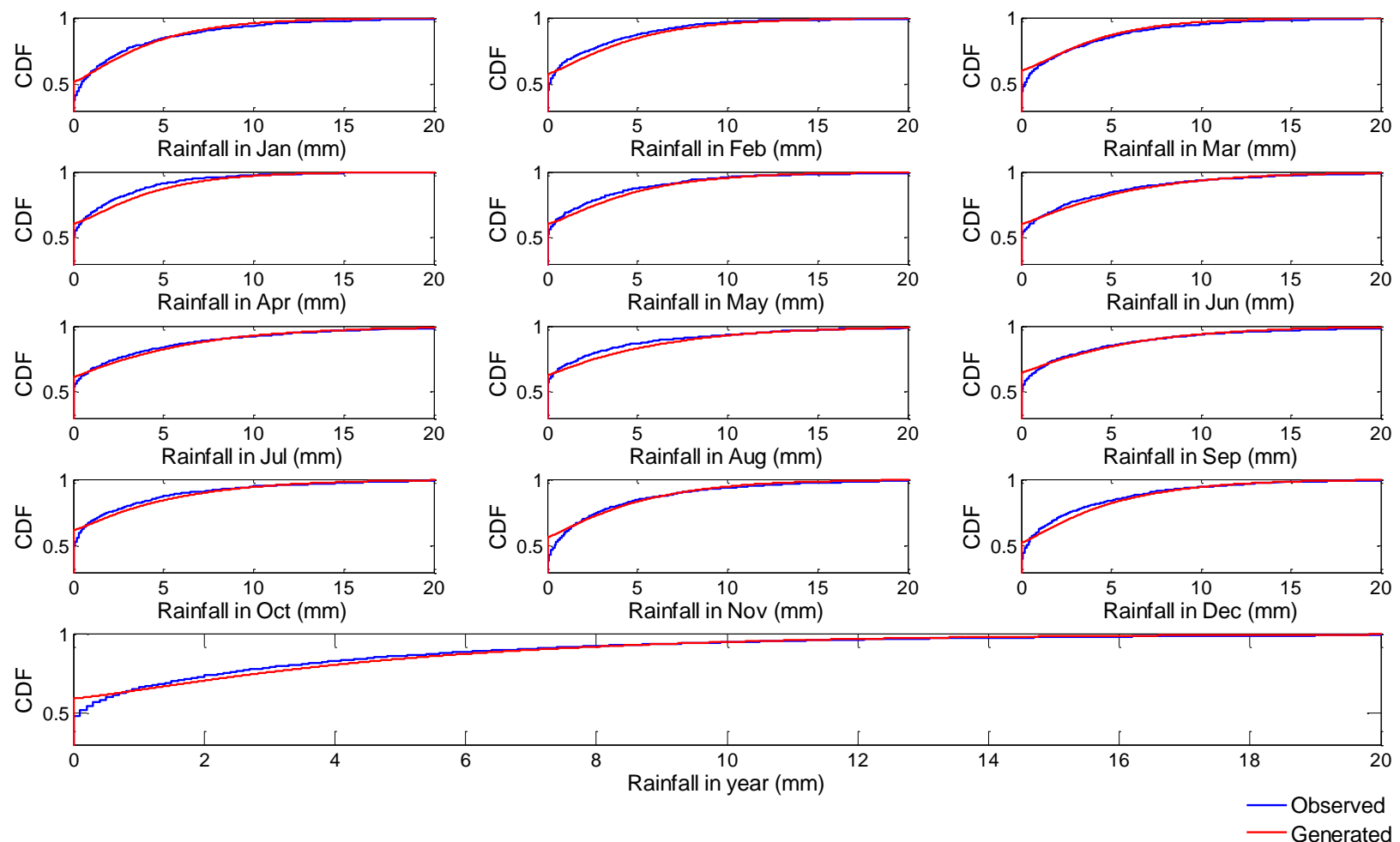

Figure 3 Cumulative distribution function of daily rainfall in the studied Eindhoven catchment

Moreover, Table shows that the hourly time series of rainfall which was generated using the time disaggregation method (i.e. disaggregation of daily to hourly time series) has the same statistical characteristics as the observed one. Overall, the synthetic generation of rainfall in which the statistical properties of the time series is respected across different time scales is a significant 
Talebizadeh et al.

improvement compared to the rainfall generation in for instance the BSM influent generator in which there is no clear way for extracting and incorporating the statistical properties of available recorded rainfall data into synthetic rainfall time series generation. Besides, the flexibility of the proposed rainfall generator allows users to define different scenarios reflecting future changes in precipitation regime (e.g. due to climate change (Chen et al., 2010)) and its effect on the influent time series (e.g. what would happen if the amount of precipitation increases by $20 \%$ ).

Table 1 Average rainfall amount and number of wet days for Eindhoven catchment

\begin{tabular}{|l|c|c|c|c|}
\hline \multicolumn{1}{|c|}{ Month } & \multicolumn{2}{|c|}{ Amount of Rainfall (mm) } & \multicolumn{2}{c|}{ Expected number of Wet Days } \\
\hline & Observed & Generated & 16 & 14 \\
\hline Jan & 72.3 & 67.0 & 12 & 11 \\
\hline Feb & 52.0 & 57.0 & 13 & 12 \\
\hline Mar & 63.4 & 54.4 & 12 & 12 \\
\hline April & 44.1 & 51.9 & 12 & 11 \\
\hline May & 58.3 & 60.9 & 12 & 11 \\
\hline Jun & 68.0 & 68.4 & 12 & 11 \\
\hline Jul & 74.7 & 73.5 & 11 & 11 \\
\hline Aug & 64.6 & 71.0 & 12 & 12 \\
\hline Sep & 67.9 & 62.1 & 12 & 14 \\
\hline Oct & 62.0 & 65.0 & 14 & 141 \\
\hline Nov & 71.1 & 66.4 & 152 & 11 \\
\hline Dec & 70.0 & 74.0 & & 17 \\
\hline Annual & 768 & 772 & & 12 \\
\hline
\end{tabular}

Table 2 Basic statistics of hourly rainfall data for Eindhoven catchment

\begin{tabular}{|c|c|c|c|}
\hline Statistics & Unit & Observed Value & Simulated Value \\
\hline Mean & $\mathrm{mm}$ & 0.08 & 0.08 \\
\hline Standard deviation & $\mathrm{mm}$ & 0.60 & 0.60 \\
\hline Lag 1 auto-correlation & --- & 0.33 & 0.36 \\
\hline Proportion of dry hours & --- & 0.92 & 0.94 \\
\hline
\end{tabular}

\section{Synthetic generation of influent temperature}

As mentioned in the methodology section, the daily temperature of wastewater is estimated through a linear regression model which relates the daily average wastewater temperature to the daily average air temperature. Figure illustrates a random generation of air and wastewater temperature time series for one year. The linear model in Figure shows that the average wastewater temperature can be estimated reasonably $\left(R^{2}=0.70\right)$ as a linear function of air temperature. To further disaggregate the daily average wastewater temperature into a time series with 15-minute temporal resolution, the average diurnal variation of wastewater temperature which was extracted and smoothed using a first order Fourier series estimate (Figure c) was multiplied to the daily average wastewater temperature. Despite the fact that the diurnal variation pattern in Figure c clearly shows a periodic behavior in time (which corresponds to the diurnal variation of wastewater temperature), there is no significant difference between the highest and lowest temperature throughout a day (i.e. the highest temperature is only around 1.001 times the daily average wastewater temperature and 
Talebizadeh et al.

the lowest temperature is around 0.9985 times the daily average wastewater temperature). Therefore, in practical applications (at least for the case study in this research), the diurnal temperature variation can be ignored.
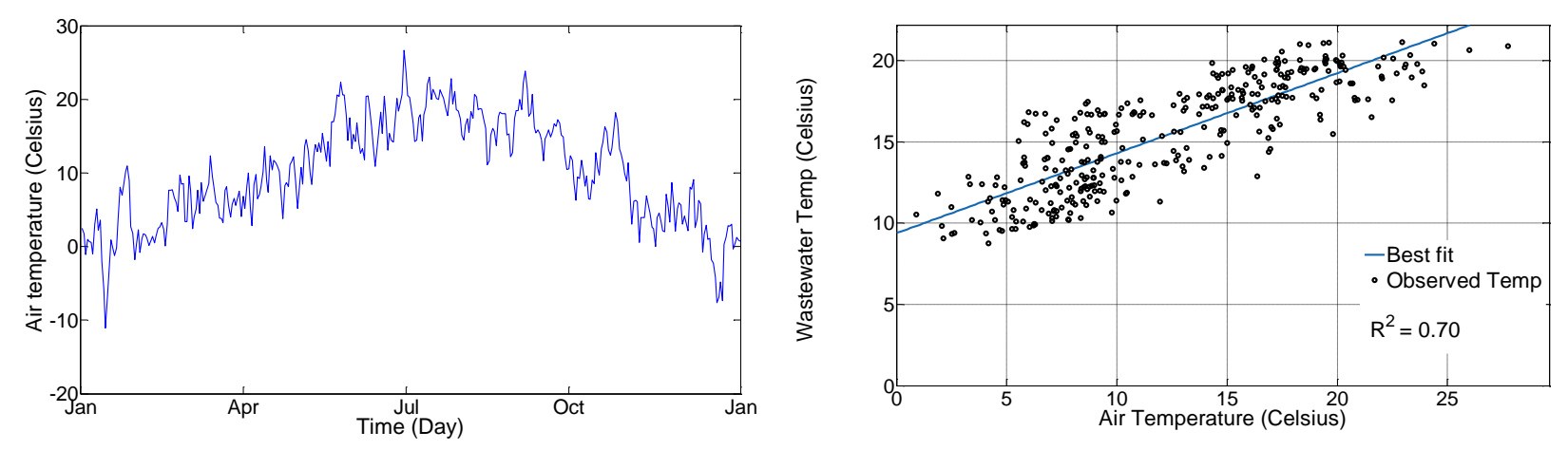

a)

b)
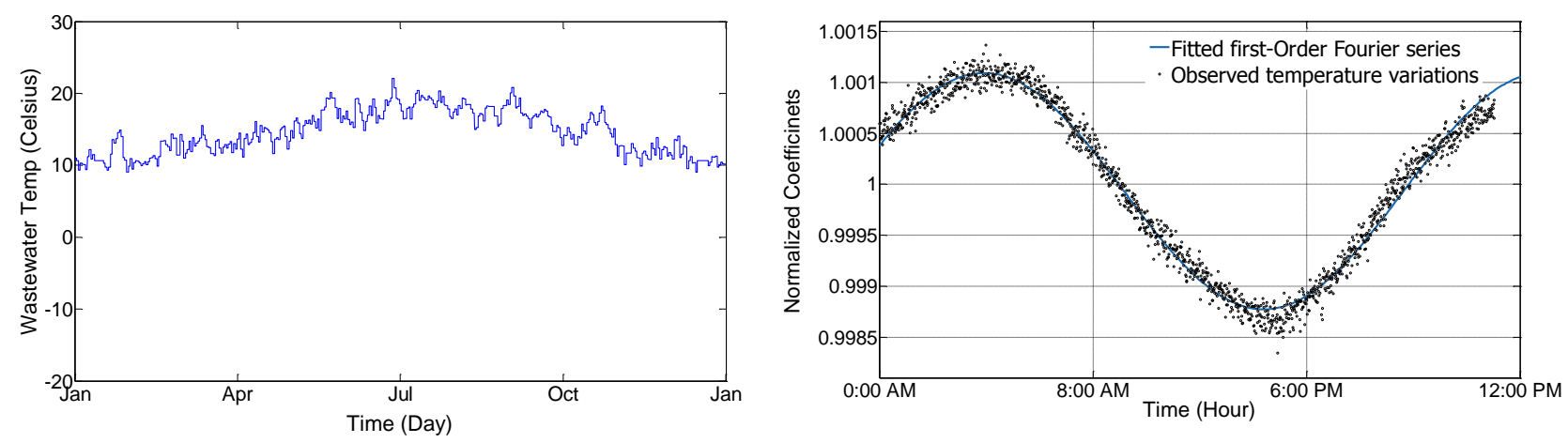

d)

c)

Figure 4 Random generation of air and wastewater temperature for one year for the Eindhoven WWTP:

a) Randomly generated average daily air temperature for a year, b) linear regression model for calculating the average daily wastewater temperature as a function of average daily air temperature, c) the average and fitted normalized coefficients (the normalized coefficients for each day were calculated by dividing the influent temperature at different moments of a day by the daily average influent temperature in the same day) for calculating the diurnal wastewater temperature variations, and d) randomly generated wastewater temperature time series with 15-minute temporal resolution.

\section{Multivariate auto-regressive model for DWF generation}

As explained, the parameters of the multivariate auto-regressive model were estimated using a specific least square algorithm (Neumaier and Schneider, 2001). Figure shows a continuous 3-day DWF influent time series with the results corresponding to the fitted multivariate auto-regressive model. The uncertainty band was generated through random generation of the noise term (i.e. $p, A_{l}$ in Equation 4 were fixed and the noise term was generated from $\varepsilon_{t} \sim N(0, C)$ ).

One of the main advantages of the proposed multivariate time series model over univariate time series models (Martin et al., 2007) or the DWF generator in the BSM influent generator (Gernaey et al., 2005) is that not only are the auto-correlation structures in time respected but also the crosscorrelation structures. Table shows the correlation matrix for the randomly generated and observed influent time series in DWF conditions. 


\section{WWTmod}

Talebizadeh et al.
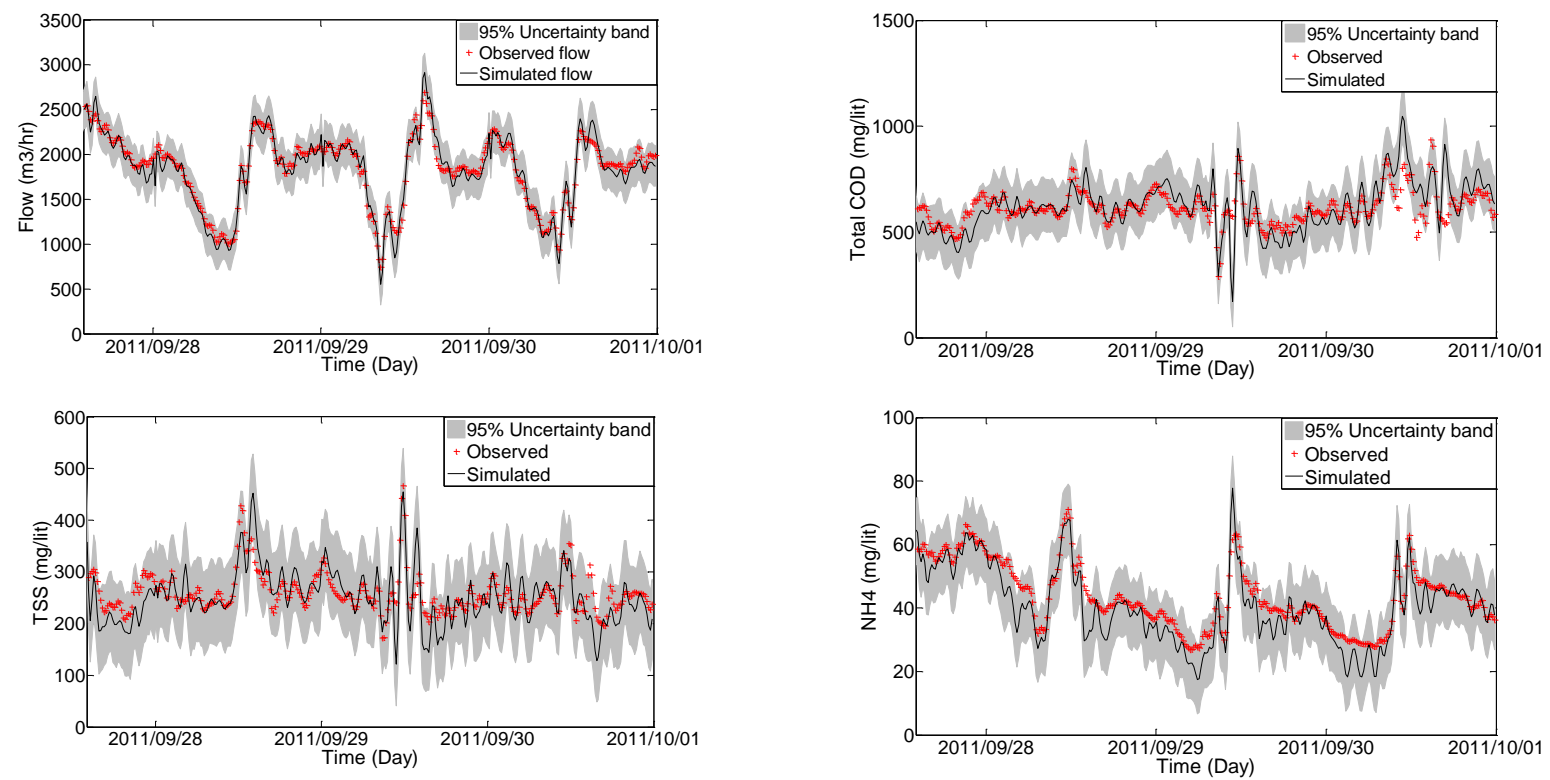

Figure 5 Observed and simulated influent time series under DWF conditions

Table 3 Correlation matrix for the generated and observed influent time series in DWF

\begin{tabular}{|c|c|c|c|c|c|c|c|c|c|c|c|}
\hline \multicolumn{6}{|c|}{ Generated influent time series } & \multicolumn{6}{|c|}{ Observed influent time series } \\
\hline & Flow & $\begin{array}{c}\text { Soluble } \\
\text { COD }\end{array}$ & $\begin{array}{l}\text { Total } \\
\text { COD }\end{array}$ & TSS & NH4 & & Flow & $\begin{array}{c}\text { Soluble } \\
\text { COD }\end{array}$ & $\begin{array}{l}\text { Total } \\
\text { COD }\end{array}$ & TSS & NH4 \\
\hline Flow & 1.00 & & & & & Flow & 1.00 & & & & \\
\hline Soluble COD & -0.11 & 1.00 & & & & Soluble COD & -0.12 & 1.00 & & & \\
\hline Total COD & -0.04 & 0.77 & 1.00 & & & Total COD & -0.06 & 0.77 & 1.00 & & \\
\hline TSS & 0.06 & 0.32 & 0.80 & 1.00 & & TSS & 0.05 & 0.33 & 0.81 & 1.00 & \\
\hline $\mathrm{NH} 4$ & -0.43 & -0.04 & -0.06 & -0.04 & 1.00 & $\mathrm{NH} 4$ & -0.46 & 0.00 & -0.02 & -0.03 & 1.00 \\
\hline
\end{tabular}

\section{CITYDRAIN model calibration and synthetic influent generation}

As explained in the methodology section, the CITYDRAIN model was used for modeling the dynamics of the influent time series during WWF conditions. Uniform distributions representing the initial knowledge on parameters were selected as prior distributions and their corresponding posterior distributions were estimated by sampling from Equation 7 using the DREAM sampler. Figure and Figure show the posterior distributions of the CITYDRAIN model after calibrating the model for flow and TSS time series in WWF conditions (three days of simulations were used as the warm-up period to set the initial conditions of the system).

As indicated in Figure and Figure, there exists some correlation among the parameters of the CITYDRAIN model. For example in Figure, the parameters that affect the generation of effective rainfall (i.e. runoff coefficient, initial loss, and permanent loss) are correlated meaning that different combinations of these parameters could result in the same amount of effective rainfall given the same inputs and values for other parameters. However, given the narrow ranges associated to the parameters that affect the amount of rainfall, the uncertainty band for flow relating to the total model uncertainty is mainly affected by the standard deviation of the residual error (i.e. Sigma in Figure ) and not by the uncertainty of the CITYDRAIN model parameters.

The parameters that affect the accumulation of pollutant (i.e. $\mathrm{m}$-lim, and Ka) and those that affect the wash-off of pollutants are also correlated. Given the different correlation structures that exist among some parameters it is very important to sample from the joint distribution of parameters to propagate the effect of parameter uncertainties to the outputs. 


\section{WWTmod}

Talebizadeh et al.

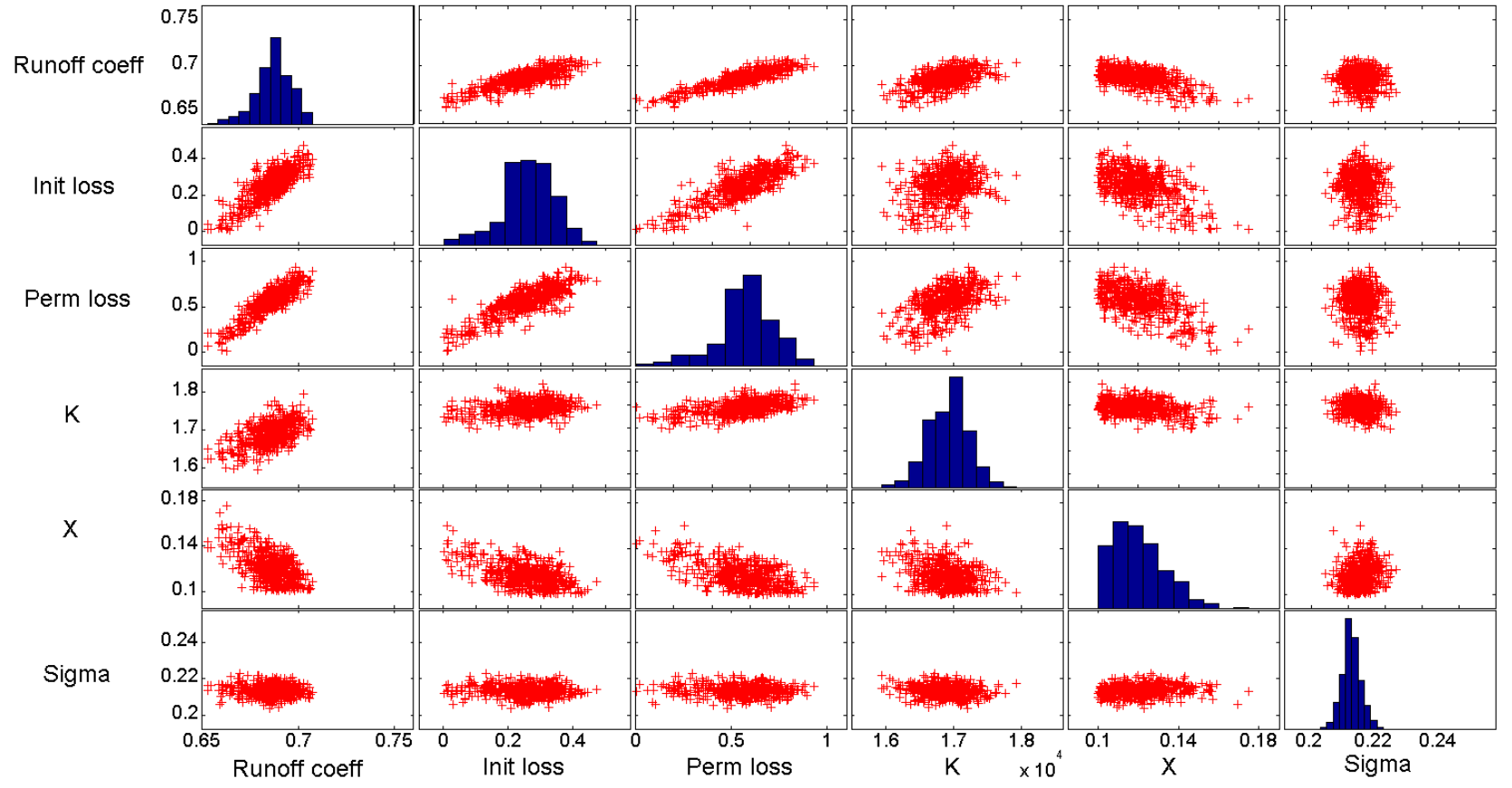

Figure 6 Posterior distribution of parameters for flow calibration where, runoff coeff, init loss, and perm loss are respectively the runoff coefficient, initial loss $(\mathrm{mm})$, permanent loss $(\mathrm{mm} /$ day) parmeters in the virtual basins model that is used in the CITYDRAIN model, $\mathrm{K}(\mathrm{sec})$ and $\mathrm{X}$ are the routing parameters used in the Muskingum method, and Sigma is the standard deviation of the residual error.

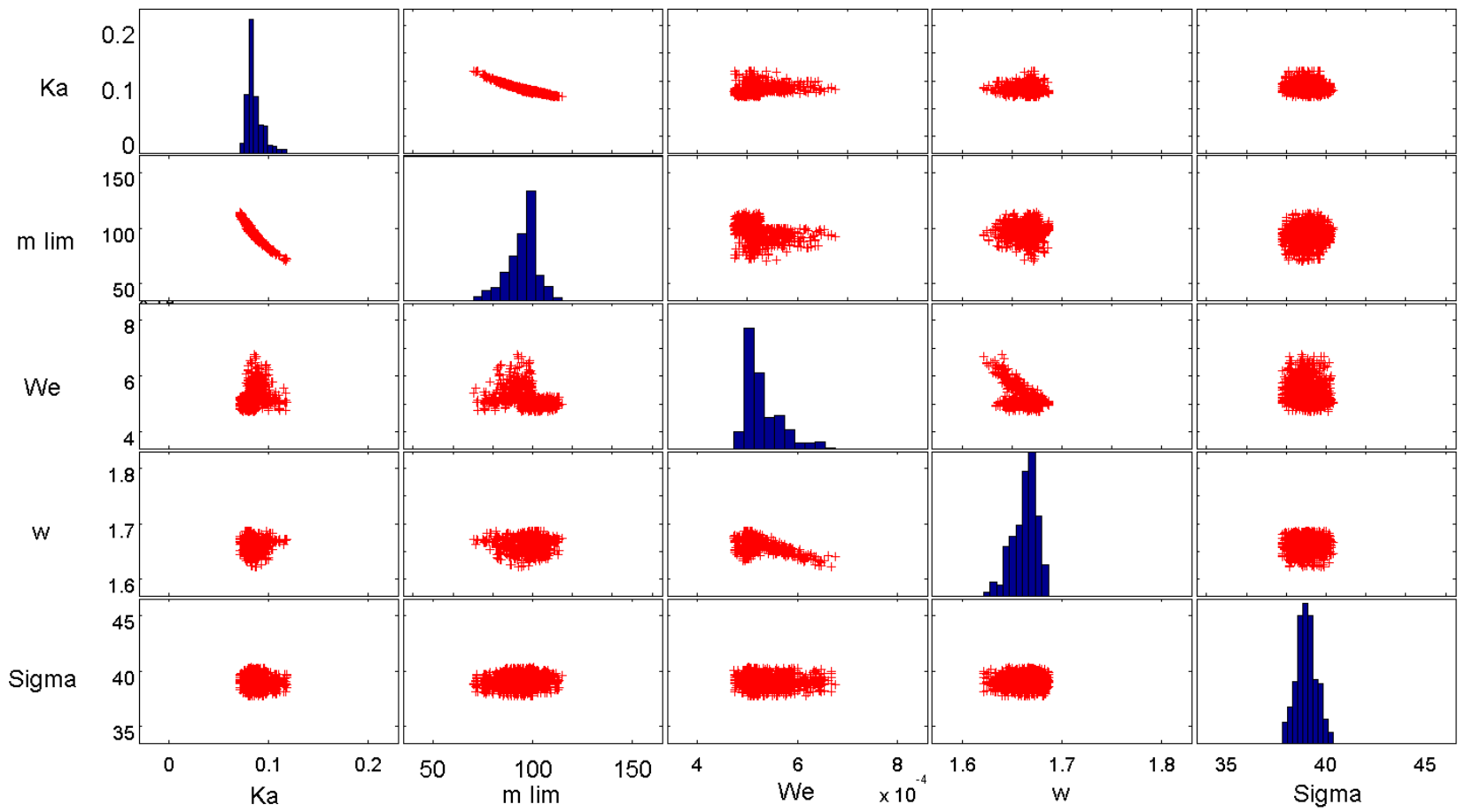

Figure 7 Posterior distribution of parameters for TSS calibration where Ka is the accumulation coefficient (1/day), $\mathrm{m} \_$lim is the maximum accumulated mass $(\mathrm{kg} / \mathrm{ha}), \mathrm{We}$, and $\mathrm{w}$ are the calibration parameters (Equation 6). 
Talebizadeh et al.

To consider the effect of total model uncertainty on the outputs of CITYDRAIN model, a Monte Carlo simulation was performed by sampling from the joint posterior distribution of parameters and running the model for 1000 times for a particular rainfall time series. Figure illustrates the $95 \%$ uncertainty band for flow and TSS which was constructed by selecting the 2.5 and 97.5 percentiles of the cumulative distribution of flow and TSS as the lower and upper limits of uncertainty of simulation with the rainfall time series shown in the figure. The figure also presents the observed and the best simulated time series. The latter corresponds to the set of parameters that has the highest likelihood function value.
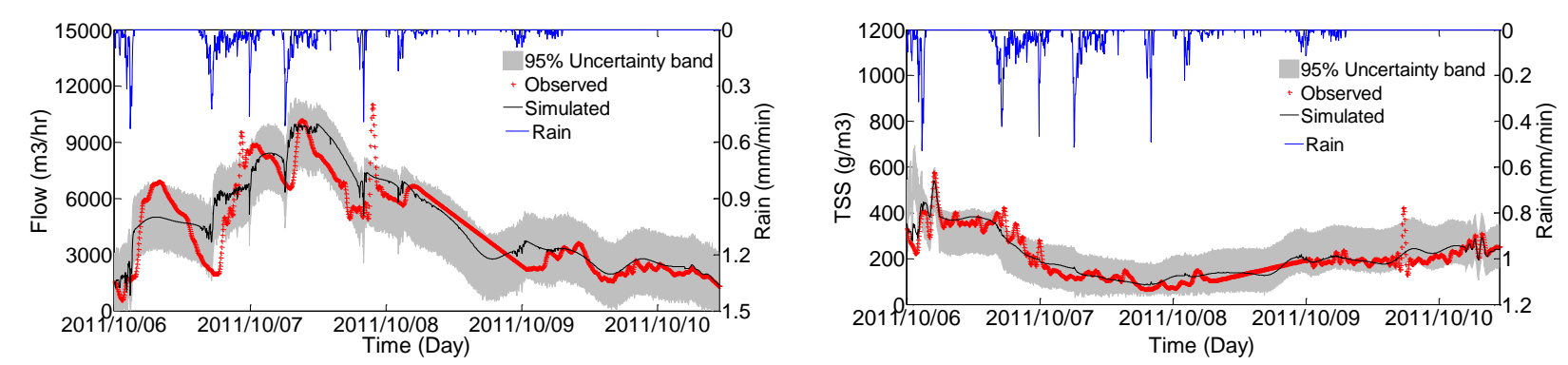

Figure 8 Uncertainty bands for flow (left) and TSS concentration (right) in a 4-day wet weather period

To further analyze the statistical properties of the simulated influent time series during both the DWF and WWF conditions, the cumulative distribution function (CDF) of the simulated and observed influent flow and pollutant load were compared in Figure and Figure 4. The simulated and observed influent time series with 15-minute temporal resolution were aggregated to construct the corresponding daily and hourly influent series. Figure and Figure 4 show that the influent generator has excellent performance when it comes to predicting the daily and hourly influent flow and pollutant load values.
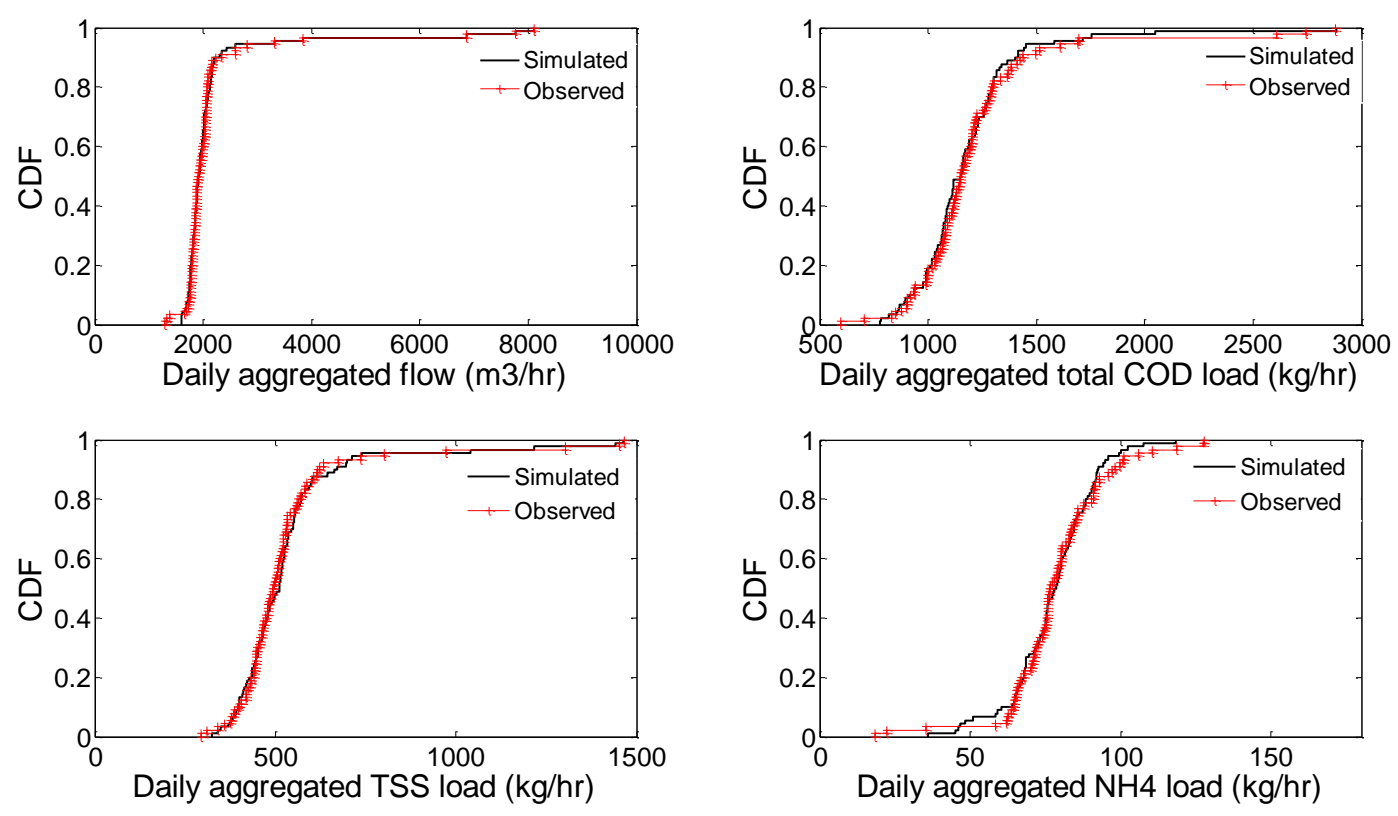

Figure 9 CDFs of daily-aggregated influent flow and load of influent pollutants 

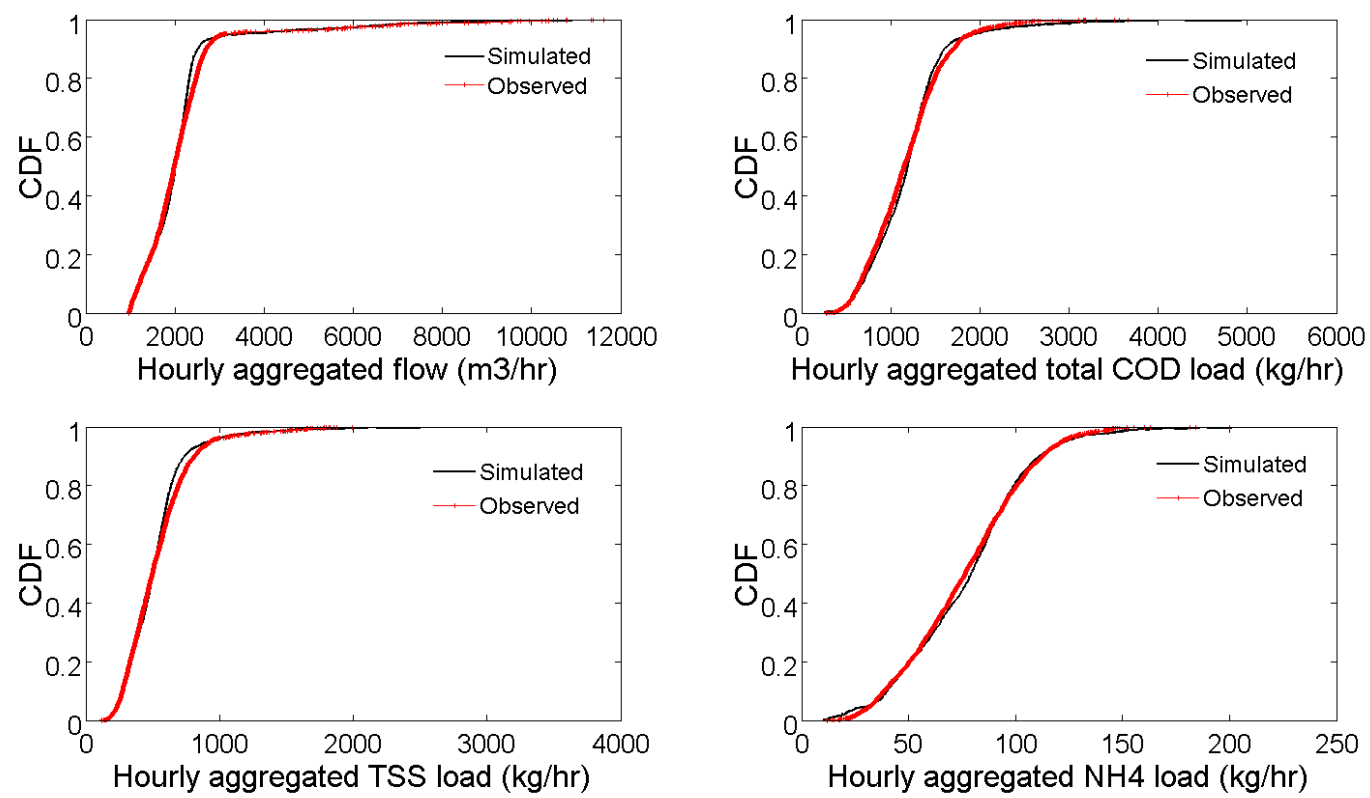

Figure 4 CDFs of hourly-aggregated influent flow and load of influent pollutants

It can be concluded from Figure and Figure 4 that the statistical properties of the simulated time series are similar to the properties of the observed series once the model is fed with the observed rainfall time series. As explained in the methodology section, synthetic generation of a one year influent time series with 15-minute temporal resolution is thus possible by sampling from the posterior distribution of the CITYDRAIN model parameters and inputting the model with synthetically-generated rainfall and influent time series for DWF conditions (both with 15-minute temporal resolution). The latter two series are to be generated using the proposed rainfall and DWF generators respectively.
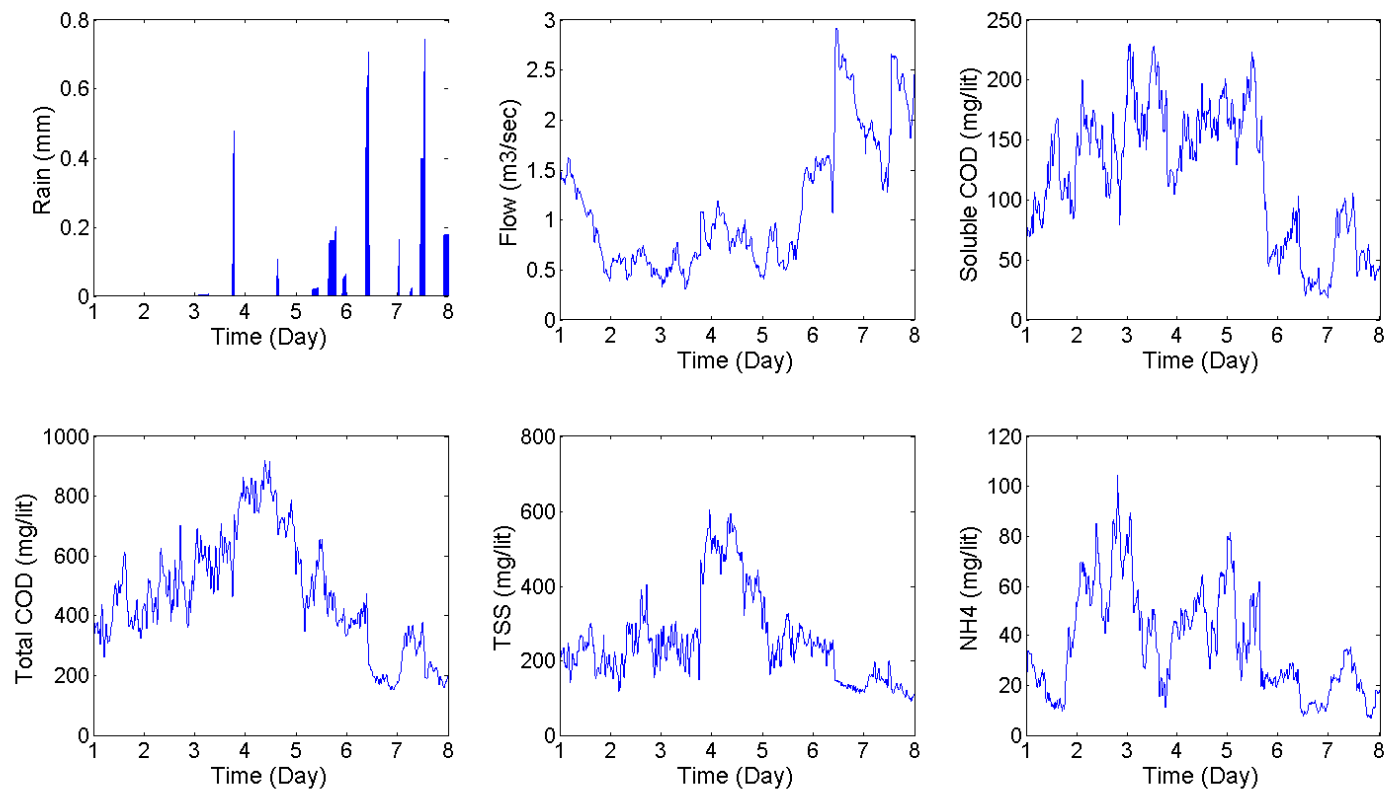

Figure 5 A 7-day realization of rainfall and influent time series 
Talebizadeh et al.

Figure 5 shows a 7-day snapshot of a generated one year influent time series. During the hours of the first day the time series of flow has a descending trend as the runoff produced by rainfall event just before the first day (not depicted in Figure 5) exits the sewer system and the flow time series reaches its DWF conditions with a typical periodic pattern (the second day in Figure 5). During the last hours of the third day another rainfall event occurs and the flow time series increases while the time series of soluble COD and ammonia drop due to dilution of wastewater by runoff. However, during the same period of time there is a sudden increase in the total COD and TSS concentrations due to the wash-off of particulate material. After the wash-off of the particulates during the last hours of the fourth day, the dilution effect starts to dominate again and the time series of total COD and TSS drop due to the dilution of the wastewater by runoff.

\section{CONCLUSION}

In this paper a combination of statistical and conceptual modeling tools was proposed for synthetic generation of dynamic influent time series of flows and pollutant concentrations with 15-miniute temporal resolution. The rainfall generator is capable of considering the annual and inter-annual rainfall regimes and keeping the consistency of the generated rainfall time series across different temporal resolutions. Comparison between observed and simulated influent time series for the Eindhoven case study proved the capability of the proposed multivariate auto-regressive model in generating realistic influent time series in DWF conditions. Moreover, long-term generation of influent time series under dry and wet weather conditions could be achieved by running the CITYDRAIN model of the sewershed using the generated stochastic inputs (i.e. rainfall and influent time series in DWF condition). Uncertainty could be captured by sampling different vectors of the model parameters from the posterior distribution obtained after Bayesian parameter estimation on the basis of the case study data.

Overall, the proposed influent generator provides a clear and coherent method to incorporate the general and easy-to-obtain information on the physical characteristics of the sewershed as well as climate conditions of the region into the synthetic generation of the influent of a treatment plant. The flexibility of the presented influent generator allows the users to define different scenarios reflecting the projected change in climate and the characteristics of the sewershed (e.g. population growth, change in pervious area) and evaluate their effect on the generated influent time series.

\section{ACKNOWLEDGEMENT}

The authors would like to thank Stefan Weijers, and Petra van Daal-Rombouts from Waterschap De Dommel (Eindhoven, The Netherlands) and Youri Amerlinck from BIOMATH-Ghent University (Belgium) for providing the influent and rainfall data for this study. This research work was funded by the Natural Sciences and Engineering Research Council of Canada (NSERC) and Primodal Inc. as well as the financial support of Hampton Roads Sanitation District, Virginia. Peter Vanrolleghem holds the Canada Research Chair in Water Quality Modeling.

\section{REFERENCES}

Achleitner S., Möderl M. and Rauch W. (2007). CITY DRAIN @ - An open source approach for simulation of integrated urban drainage systems. Environmental Modelling \& Software 22(8), 1184-95.

Bates B. C. and Campbell E. P. (2001). A Markov Chain Monte Carlo Scheme for parameter estimation and inference in conceptual rainfall-runoff modeling. Water Resources Research 37(4), 937-947.

Bechmann H., Nielsen M. K., Madsen H. and Kjølstad Poulsen N. (1999). Grey-box modelling of pollutant loads from a sewer system. Urban Water 1(1), 71-8.

Belia E., Amerlinck Y., Benedetti L., Johnson B., Sin G., Vanrolleghem P.A., Gernaey K.V., Gillot S., Neumann M.B., Rieger L., Shaw A. and Villez K. (2009). Wastewater treatment modelling: Dealing with uncertainties. Water Science and Technology 60(8), 1929-1941. 
Capodaglio A., Zheng S., Novotny V. and Feng X. (1990). Stochastic system identification of sewer-flow models. Journal of Environmental Engineering 116(2), 284-98.

Chen J., Brissette F. P. and Leconte R. (2010). A daily stochastic weather generator for preserving low-frequency of climate variability. Journal of Hydrology 388(3-4), 480-90.

Dotto C. B. S., Mannina G., Kleidorfer M., Vezzaro L., Henrichs M., McCarthy D. T., Freni G., Rauch W. and Deletic A. (2012). Comparison of different uncertainty techniques in urban stormwater quantity and quality modelling. Water Research 46(8), 2545-58.

Flores-Alsina X., Saagi R., Lindblom E., Thirsing C., Thornberg D., Gernaey K. V. and Jeppsson U. (2014). Calibration and validation of a phenomenological influent pollutant disturbance scenario generator using full-scale data. Water Research 51(0), 172-85.

Freni G. and Mannina G. (2010). Bayesian approach for uncertainty quantification in water quality modelling: The influence of prior distribution. Journal of Hydrology 392(1), 31-9.

Freni G., Mannina G. and Viviani G. (2009). Uncertainty assessment of an integrated urban drainage model. Journal of Hydrology 373(3), 392-404.

Gernaey, K., Rosen, C. and Jeppsson, U. (2005). BSM2: A model for dynamic influent data generation. Technical report. Department of Industrial Electrical Engineering and Automation. Lund University, Lund, Sweden.

Gernaey K. V., Flores-Alsina X., Rosen C., Benedetti L. and Jeppsson U. (2011). Dynamic influent pollutant disturbance scenario generation using a phenomenological modelling approach. Environmental Modelling \& Software 26(11), 1255-67.

Hao X., Loosdrecht M. C. M. V., Meijer S. C. F. and Qian Y. (2001). Model-based evaluation of two BNR processesUCT and A2N. Water Research 35(12), 2851-60.

Hernebring C., Jönsson L., Thorn U. and Møller A. (2002). Dynamic online sewer modelling in Helsingborg. Water Science and Technology 45(4-5), 429-36.

Jeppsson U., Pons M., Nopens I., Alex J., Copp J., Gernaey K., Rosen C., Steyer J. and Vanrolleghem P. A. (2007). Benchmark Simulation Model no 2: General protocol and exploratory case studies. Water Science and technology 56(8), 67-78.

Kanso A., Tassin B. and Chebbo G. (2005). A benchmark methodology for managing uncertainties in urban runoff quality models. Water Science and Technology 51(02), 163-70.

Koutsoyiannis D. and Onof C. (2001). Rainfall disaggregation using adjusting procedures on a Poisson cluster model. Journal of Hydrology 246(1-4), 109-22.

Langergraber G., Alex J., Weissenbacher N., Woerner D., Ahnert M., Frehmann T., Halft N., Hobus I., Plattes M. and Spering V. (2008). Generation of diurnal variation for influent data for dynamic simulation. Water Science and Technology 57(9), 1483.

Marshall L., Nott D. and Sharma A. (2004). A comparative study of Markov chain Monte Carlo methods for conceptual rainfall-runoff modeling. Water Resources Research 40(2), W02501.

Martin C., Eguinoa I., McIntyre N. R., García-Sanz M. and Ayesa E. (2007). ARMA models for uncertainty assessment of time series data: application to Galindo-Bilbao WWTP. In: Proceedings of the Seventh International IWA Symposium on Systems Analysis and Integrated Assessment in Water Management (WATERMATEX 2007). Washington DC, USA, 7th-9th May 2007.

Martin C., Neumann M. B., Altimir J. and Vanrolleghem P. A. (2012) A tool for optimum design of WWTPs under uncertainty: Estimating the probability of compliance In: Proceedings International Congress on Environmental Modelling and Software (iEMSs2012). Leipzig, Germany, 1th-5th July 2012.

Matalas N. C. (1967). Mathematical assessment of synthetic hydrology. Water Resources Research 3(4), 937-45.

Neumaier A. and Schneider T. (2001). Estimation of parameters and eigenmodes of multivariate autoregressive models. ACM Transactions on Mathematical Software (TOMS) 27(1), 27-57.

Ormsbee L. (1989). Rainfall disaggregation model for continuous hydrologic modeling. Journal of Hydraulic Engineering 115(4), 507-25.

Richardson C. W. (1981). Stochastic simulation of daily precipitation, temperature, and solar radiation. Water Resources Research 17(1), 182-90.

Salem S., Berends D., Heijnen J. and Van Loosdrecht M. (2002). Model-based evaluation of a new upgrading concept for N-removal. Water Science and Technology 45(6), 169-76.

Temprano J., Arango Ó., Cagiao J., Suárez J. and Tejero I. (2007). Stormwater quality calibration by SWMM: A case study in Northern Spain. Water SA 32(1), 55-63.

Vrugt J. A., ter Braak C. J. F., Clark M. P., Hyman J. M. and Robinson B. A. (2008). Treatment of input uncertainty in hydrologic modeling: Doing hydrology backward with Markov chain Monte Carlo simulation. Water Resources Research 44(12), W00B9. 


\title{
Increasing complexity in biofilm reactor models: How far do we need to go?
}

\author{
George Wells ${ }^{1,2}$, Thomas P.W. Vannecke ${ }^{3}$, Nathalie Hubaux ${ }^{1}$, Eberhard Morgenroth ${ }^{1,4}$, Eveline I.P. Volcke $^{3}$ \\ ${ }^{1}$ Department of Process Engineering, Eawag-Swiss Federal Institute of Aquatic Science and Technology, \\ Dübendorf, Switzerland (Emails: nathalie.hubaux@gmail.com; Eberhard.Morgenroth@eawag.ch) \\ ${ }^{2}$ Department of Civil and Environmental Engineering, Northwestern University, Evanston, USA (Email: \\ george.wells@northwestern.edu) \\ ${ }^{3}$ Department of Biosystems Engineering, Ghent University, Belgium (Emails: Thomas.Vannecke@UGent.be, \\ Eveline.Volcke@UGent.be) \\ ${ }^{4}$ Institute of Environmental Engineering, ETH Zürich, Zürich, Switzerland \\ *Corresponding author: george.wells@northwestern.edu
}

\begin{abstract}
A model should be as simple as possible - but not simpler. The appropriate level complexity depends both on the type of system and on the intended use of the model. This paper addresses the critical question of which purposes justify an increased complexity of biofilm (reactor) models. Additional model features considered are (1) of the distinction between flocs and granules in putatively granular sludge reactors and (2) the inclusion of microbial diversity, distinguishing between different species performing the same function. The impact of these features are assessed for a partial nitritation-anammox process and a conventional nitrification process, respectively. It was shown that the addition of a small level of flocs (5\% of total biomass) can have a significant impact on macroscale process performance and on microbial population and activity distributions in putatively granular sludge reactors. With a multispecies model considering interspecies diversity (10 species of ammonium-oxidizers and 10 species of nitrite-oxidizers), it was demonstrated that a constant macroscopic reactor performance not necessary reflects steady state conditions on the microscale. The biomass distribution in time and in space could be explained through the underlying microbial characteristics. Based on these case studies, we argue that increased complexity in biofilm (reactor) models will be likely more useful when the focus is on understanding fundamental microscale outputs, particularly under dynamic conditions or in cases of microbial cross-feeding and/or balanced aerobic/anoxic conditions. When the focus is on macroscale outputs (e.g. substrate removal rates, optimal bulk conditions), this complexity is likely not always necessary. However, under specific conditions, additional model features can be critically informative for bulk reactor behavior, prediction, or understanding.
\end{abstract}

Keywords

Biofilm reactors; population dynamics; anammox; nitrification; microbial coexistence; granules and flocs 


\section{INTRODUCTION}

Mathematical biofilm (reactor) models are excellent tools for both predicting patterns of behavior in overall process performance (macroscale outputs) and for understanding fundamental phenomena such as microbial interactions, segregation, or competition (microscale outputs). Deciding which features to include in biofilm or biofilm reactor models is a critical component of model structure selection. Wanner et al. (2006) emphasize the value of identifying model features that can be omitted without decreasing the utility of the model for its intended purpose, as summarized in their "golden rule" of modeling: "a model should be as simple as possible, and only as complex as needed." In essence, decreasing model complexity via simplifying assumptions can greatly ease computational requirements and interpretation of model outputs. The level of complexity to include in a model depends in large part on its intended use, but determining this level is not always straightforward.

One example of the utility of increasing biofilm model complexity in certain circumstances is the use of multidimensional $(2 \mathrm{D}, 3 \mathrm{D})$ simulations instead of the simpler, and more common, 1D models. Multidimensional models have been shown to be highly useful when the intent of the model is to understand the impact of microscale physical heterogeneity in biofilm solid matrix - for example, shape or local density variations that lead to microscale multidirectional concentration gradients - or when transport processes outside of molecular diffusion (e.g. advection or turbulent dispersion) of dissolved components in the biofilm liquid phase are of interest (Morgenroth et al. 2000a; Picioreanu et al. 2004; Eberl et al. 2006). If average microscale concentration profiles or macroscale outputs alone are of interest to the model user and in case vertical gradients are orders of magnitude higher than those in the directions parallel to the carrier surface, conventional 1D models that are much less computationally intensive can often perform well (Wanner \& Gujer 1986; Morgenroth et al. 2000a).

A range of complexity exists even within numerical 1D biofilm models. We focus our efforts here on numerical 1D biofilm models with stratification of biomass, multiple substrates, and multiple functional guilds. Two common simplifying assumptions in such biofilm models are: 1) to ignore mesoscale heterogeneity in aggregate structure (e.g. size distribution of granules, or variation in transport properties of a hybrid mixture of aggregate types, such as flocs and granules), and 2) to neglect microbial diversity and resulting internal microbial competition within function guilds. However, experimental observations have highlighted routine coexistence of multiple types of biomass aggregate types in a single biofilm reactor. Specifically, for granular nitritation-anammox reactors, flocs are commonly observed to be present in putative granular sludge reactors and reciprocally granules in suspended sludge reactors, suggesting that these two types of biomass aggregates coexist more often than a priori supposed (Innerebner et al. 2007; Vlaeminck et al. 2009; Vlaeminck et al. 2010; Winkler et al. 2012). In addition, experimental observations have demonstrated diverse assemblages of microbial populations within individual functional guilds in, for example, nitrifying biofilm reactors, where several genetically different populations of ammonium-oxidizers (Schramm et al. 2000; Bernet et al. 2004; Lydmark et al. 2006; Volcke et al. 2008; Terada et al. 2010; Almstrand et al. 2013) or nitrite-oxidizers (Schramm et al. 1998; Schramm et al. 2000; Downing \& Nerenberg 2008) were observed to coexist in the biofilm.

Moreover, and of critical importance to this paper, both diversity within functional guilds and heterogeneous aggregate characteristics have been proposed to influence macroscale reactor performance and process stability (Wittebolle et al. 2005; Siripong \& Rittmann 2007; Wett 2007). Indeed, the possible role of suspended biomass in influencing performance in biofilm reactors, and the general lack of consideration of this fraction in biofilm models, was noted as a possible oversimplification by Morgenroth et al. (2000b). Also, mathematical models including microbial 
community information have proven useful in investigating the link between observed microbial community shifts and the macroscopic reactor behavior (Downing \& Nerenberg 2008; Volcke et al. 2008; Ramirez et al. 2009; Nielsen et al. 2010; Wett et al. 2011; Brockmann et al. 2013; Vannecke et al. 2014). Taken together, these previous experimental studies suggests that, in at least some cases, increasing model complexity by incorporation of these often neglected features of biofilm systems (mesoscale heterogeneity in aggregate structure, and microbial diversity) may illuminate important aspects of both macroscale and microscale outputs.

We focus in this paper on the critical question of which purposes increases in complexity in standard biofilm or biofilm reactor models may be justified by providing two case studies of increases in complexity beyond standard biofilm model formulations that provide new insights at both the macroscale and microscale. The two case studies deal with

1) the influence of small levels of floccular biomass in a granular sludge combined nitritationanammox reactor on macroscale $\mathrm{N}$ removal efficiency, optimal dissolved oxygen (DO) conditions, and microscale microbial population and activity distribution; and

2) the influence of microbial diversity on biofilm development and microscale microbial population dynamics in a nitrifying biofilm, considering competition between 10 ammonium-oxidizing species and 10 nitrite-oxidizing species.

\section{BIOFILM MODELS}

\section{General model features}

Two 1-dimensional multispecies biofilm models were developed in Aquasim (Reichert et al. 1995). The modelled biofilm reactors were operated under similar conditions (Table ). Both case studies concern biological nitrogen removal processes. The first one considers completely autotrophic nitrogen removal through partial nitritation and anammox in a granular sludge reactor. The second case study concerns conventional ammonium oxidation to nitrate in a flat biofilm. The general stoichiometric matrix and kinetics for both case studies are based on Ni et al. (2009), Volcke et al. (2010) and on Vannecke et al. (2014), respectively.

Table 1. Influent characteristics and reactor operating conditions.

\begin{tabular}{lcc} 
& Case study 1 & Case study 2 \\
\hline Temperature $\left({ }^{\circ} \mathrm{C}\right)$ & 30 & 30 \\
$\mathrm{pH}$ & 7.5 & 7.5 \\
Nitrogen loading rate $\left(\mathrm{kg} \mathrm{N} \cdot \mathrm{m}^{-3} \cdot \mathrm{d}^{-1}\right)$ & 0.5 & 0.9 \\
Initial biofilm thickness $(\mu \mathrm{m})$ & 10 & 1 \\
Steady state biofilm thickness $(\mu \mathrm{m})$ & 750 & 1000 \\
Biofilm porosity & $80 \%$ & $80 \%$ \\
Influent NH$_{4}{ }^{+}\left(\mathrm{g} \mathrm{N} \cdot \mathrm{m}^{-3}\right)$ & 300 & 250 \\
Influent sCOD $\left(\mathrm{gCOD} \cdot \mathrm{m}^{-3}\right)$ & 10 & 0 \\
Reactor volume $\left(\mathrm{m}^{3}\right)$ & 400 & $2 \mathrm{e}-003$ \\
\hline
\end{tabular}

Case study 1: Modeling heterogeneity in aggregate structure

To assess the importance of small levels of flocs in putatively granular sludge combined nitritation-anammox reactors, two multispecies biofilm models were developed in Aquasim. The first model included only granular biomass in a continuous flow bioreactor, while the second model structure included both granular and floccular biomass. Two primary properties differentiated these two aggregate fractions: 1) biomass distribution was assumed to be heterogeneous in granules, as is 


\section{WWTmod}

Wells et al.

standard in biofilm models, and homogeneous in flocs due to flocculation and deflocculation; and 2) granules were mass transfer (diffusion) limited, while flocs did not exhibit mass transfer limitations. Both models included growth and decay (death-regeneration) of ammonium-oxidizing organisms (AOO), nitrite-oxidizing organisms (NOO), ordinary heterotrophic organisms (OHO) under both aerobic and denitrifying conditions, and anammox (AMO). OHO grew on organics arising from biomass decay or from exogenous organic carbon. In both model structures, granules were considered to be symmetrical spherical biofilms with a rigid biofilm matrix and negligible external mass transfer limitations. Solids detached from biofilm (granule) surfaces into the bulk phase were homogenously distributed and potentially active before being removed proportionally to the effluent flow. In the model structure considering both floccular and granular biomass, flocs were modeled by implementing a bifurcation from the effluent to the inlet, thus recycling part of the biomass detached from the granule surface:

$$
\operatorname{Load}_{X_{\imath} R \theta C}=Q_{\text {in }} *\left(1+\alpha-\frac{\theta_{H}}{S R T}\right) * X
$$

where $\operatorname{Load}_{X, R e c}$ is the loading of particulate (floccular) material in the recycle, $Q_{\text {in }}$ the influent flow rate, $\alpha$ the recycle ratio (here $=0.1), \theta_{H}$ is the hydraulic retention time $(0.5 \mathrm{~d}), S R T$ the sludge residence time (40d) and $X$ is the floccular biomass concentration in the reactor. The SRT was held constant by wasting a portion of the floccular biomass from the recycle.

\section{Case study 2: Modeling multispecies competition}

To model microbial competition between nitrifying species performing the same function in the biofilm, and to verify the importance of various microbial parameters in determining the competition outcome, a two-step nitrification biofilm model including the growth and endogenous respiration of 10 ammonium-oxidizing species (AOO) and 10 nitrite-oxidizing species (NOO) was used. Possible ranges of values for maximum growth rate $\left(\mu_{\max }\right)$, yield $(Y)$, affinity for the nitrogen substrate $\left(\mathrm{K}_{\mathrm{NH}}^{\mathrm{AOO}}\right.$ and $\left.\mathrm{K}_{\mathrm{NO2}}^{\mathrm{NOO}}\right)$ and the affinity for oxygen $\left(\mathrm{K}_{\mathrm{O} 2}^{\mathrm{AOO}}\right.$ and $\left.\mathrm{K}_{\mathrm{O} 2}^{\mathrm{NOO}}\right)$ were determined based on an extensive literature study. For each considered microbial parameter, a normal bimodal distribution was constructed as in Ramirez et al. (2009). The eight bimodal distributions were each typified by two means $\left(\mu_{1}=0.6 \times \mathrm{k} ; \mu_{2}=1.4 \times \mathrm{k}\right.$ ) and standard deviations of $\sigma_{1,2}=0.125 \times \mathrm{k}$, with $\mathrm{k}$ the average value of the range of values found in literature for the corresponding parameter. Ten species per type were then constructed by picking 10 random numbers from each bimodal distribution. Parameters employed in the final model are given in Table 2. The endogenous respiration rate for each species was assumed to be $5 \%$ of its corresponding maximum growth rate. The initial concentration of each AOO and NOO species was equal for all species of the same type (AOO: 7000 g COD. $\mathrm{m}^{-3}$ and NOO: 2333 g COD.m ${ }^{-3}$ ). As heterotrophic growth on biomass decay products can be neglected (Mozumder et al. 2013), and the influent did not contain an organic carbon source, heterotrophic growth was not considered in this model. The initial concentration of ammonium in the bulk liquid was set equal to the influent ammonium concentration $\left(250 \mathrm{~g} \mathrm{~N}^{-3} \mathrm{~m}^{-3}\right)$ while the initial concentrations of nitrite and nitrate were negligible $\left(1 \mathrm{~g} \mathrm{~N} . \mathrm{m}^{-3}\right)$. The bulk liquid oxygen concentration was kept constant at $3 \mathrm{~g} \mathrm{O}_{2} \cdot \mathrm{m}^{-3}$ during the simulations. The simulations were run during a sufficient amount of time to assure steady state reactor conditions, both at micro- and macroscale. 
Wells et al.

Table 2. Microbial parameters characterizing the AOO and NOO species included in Case study 2.

\begin{tabular}{|c|c|c|c|c|c|c|c|c|c|}
\hline & $\begin{array}{c}\mu_{\max }^{\mathrm{AOO}} \\
{\left[\mathrm{d}^{-1}\right]} \\
\end{array}$ & $\begin{array}{c}\mathbf{K}_{\mathrm{NH}}^{\mathrm{AOO}} \\
{\left[\mathrm{g} \mathrm{N}^{-3} \mathrm{~m}^{-3}\right]} \\
\end{array}$ & $\begin{array}{c}\mathbf{K}_{02}^{\mathrm{AOO}} \\
{\left[\mathrm{g} \mathrm{O}_{2} \cdot \mathrm{m}^{-3}\right]}\end{array}$ & $\begin{array}{c}\mathbf{Y}^{A O O} \\
{\left[\frac{g C O D}{g N}\right]}\end{array}$ & & $\begin{array}{c}\mu_{\max }^{\mathrm{NOO}} \\
{\left[\mathrm{d}^{-1}\right]}\end{array}$ & $\begin{array}{c}\mathbf{K}_{\mathrm{NO}}^{\mathrm{NoO}} \\
{\left[\mathrm{g} \text { N.m }{ }^{-3}\right]}\end{array}$ & $\begin{array}{c}\mathbf{K}_{\mathrm{O} 2}^{\mathrm{NOO}} \\
{\left[\mathrm{g} \mathrm{O}_{2} \cdot \mathrm{m}^{-3}\right]}\end{array}$ & $\begin{array}{c}\mathbf{Y}^{\mathrm{NOO}} \\
{\left[\frac{g \operatorname{COD}}{g N}\right]}\end{array}$ \\
\hline AOO1 & 1.10 & 2.84 & 0.95 & 0.23 & NOO1 & 1.77 & 4.31 & 0.99 & 0.10 \\
\hline AOO2 & 2.41 & 6.51 & 0.37 & 0.11 & $\mathrm{NOO2}$ & 0.74 & 1.91 & 1.69 & 0.11 \\
\hline AOO3 & 1.91 & 12.97 & 0.35 & 0.07 & NOO3 & 0.74 & 4.45 & 0.84 & 0.10 \\
\hline AOO4 & 0.79 & 4.82 & 0.47 & 0.08 & NOO4 & 0.87 & 3.84 & 0.66 & 0.09 \\
\hline AOO5 & 2.08 & 10.54 & 0.33 & 0.24 & NOO5 & 0.66 & 1.98 & 1.75 & 0.04 \\
\hline AOO6 & 2.22 & 5.96 & 0.36 & 0.10 & NOO6 & 1.67 & 2.73 & 1.58 & 0.09 \\
\hline AOO7 & 0.71 & 4.62 & 0.82 & 0.25 & NOO7 & 0.71 & 5.07 & 0.67 & 0.04 \\
\hline AOO8 & 1.77 & 4.71 & 0.83 & 0.21 & NOO8 & 0.50 & 5.16 & 0.99 & 0.08 \\
\hline AOO9 & 0.59 & 12.10 & 0.91 & 0.08 & NOO9 & 1.54 & 4.45 & 2.05 & 0.06 \\
\hline AOO10 & 0.68 & 12.27 & 0.27 & 0.13 & NOO10 & 0.63 & 4.26 & 0.73 & 0.10 \\
\hline
\end{tabular}

\section{RESULTS AND DISCUSSION}

\section{Case study 1: Impact of coexistence of flocs and granules: considering multiple aggregate} fractions

For both granular sludge combined-nitritation anammox model structures (granules alone, and combined granular and floccular biomass), the maximum $\mathrm{N}$ removal efficiency achieved was about $95 \%$ (Fig 1A). In both reactors at DO $<0.1 \mathrm{mg} / \mathrm{l}$, the low oxygen concentration limited AOO activity and thus hampered the $\mathrm{N}$ removal process, and for $\mathrm{DO}>0.5 \mathrm{mg} / \mathrm{l}$, the increasing bulk DO concentration gradually inhibited AMO and improved the conditions for the NOO growth. However, the addition of small levels ( $\sim 5 \%$ of total biomass) of flocs changes the predicted overall reactor performance in two critical ways. Firstly, the maximum $\mathrm{N}$ removal efficiency was achieved at lower DO with flocs. Secondly, the N removal peak was narrower, suggesting that small levels of floccular material may decrease process robustness to bulk oxygen changes in granular sludge reactors.

In addition to impacting overall $\mathrm{N}$ removal performance, the addition of flocs also exerted a substantial influence on microscale segregation of microbial processes and activities, as illustrated by $\mathrm{NH}_{4}{ }^{+}, \mathrm{NO}_{2}{ }^{-}$, and $\mathrm{NO}_{3}{ }^{-}$fluxes into granules under a variety of bulk DO conditions in the presence and absence of flocs (Fig. 1B-D). The addition of 5\% floccular material reduced $\mathrm{NH}_{4}{ }^{+}$flux into granules by half with no impact on overall $\mathrm{NH}_{4}{ }^{+}$removal, indicating partial conversion of $\mathrm{NH}_{4}{ }^{+}$to $\mathrm{NO}_{2}{ }^{-}$in the bulk liquid (Fig. 1B). $\mathrm{NO}_{2}{ }^{-}$flux to the granules was correspondingly increased (Fig. 1C). Above $\mathrm{DO}=0.5 \mathrm{mg} / \mathrm{L}, \mathrm{AMO}$ activity is minimal in the presence and absence of flocs, and all $\mathrm{NH}_{4}{ }^{+}$is converted to $\mathrm{NO}_{3}{ }^{-}$(Fig. 1D). These patterns in $\mathrm{NH}_{4}{ }^{+}, \mathrm{NO}_{2}{ }^{-}$, and $\mathrm{NO}_{3}{ }^{-}$fluxes into granules indicate that even small levels of flocs can lead to significant task segregation between biomass fractions, with AOO activity concentrated in floccular material and Ax activity concentrated in granular biofilms. 

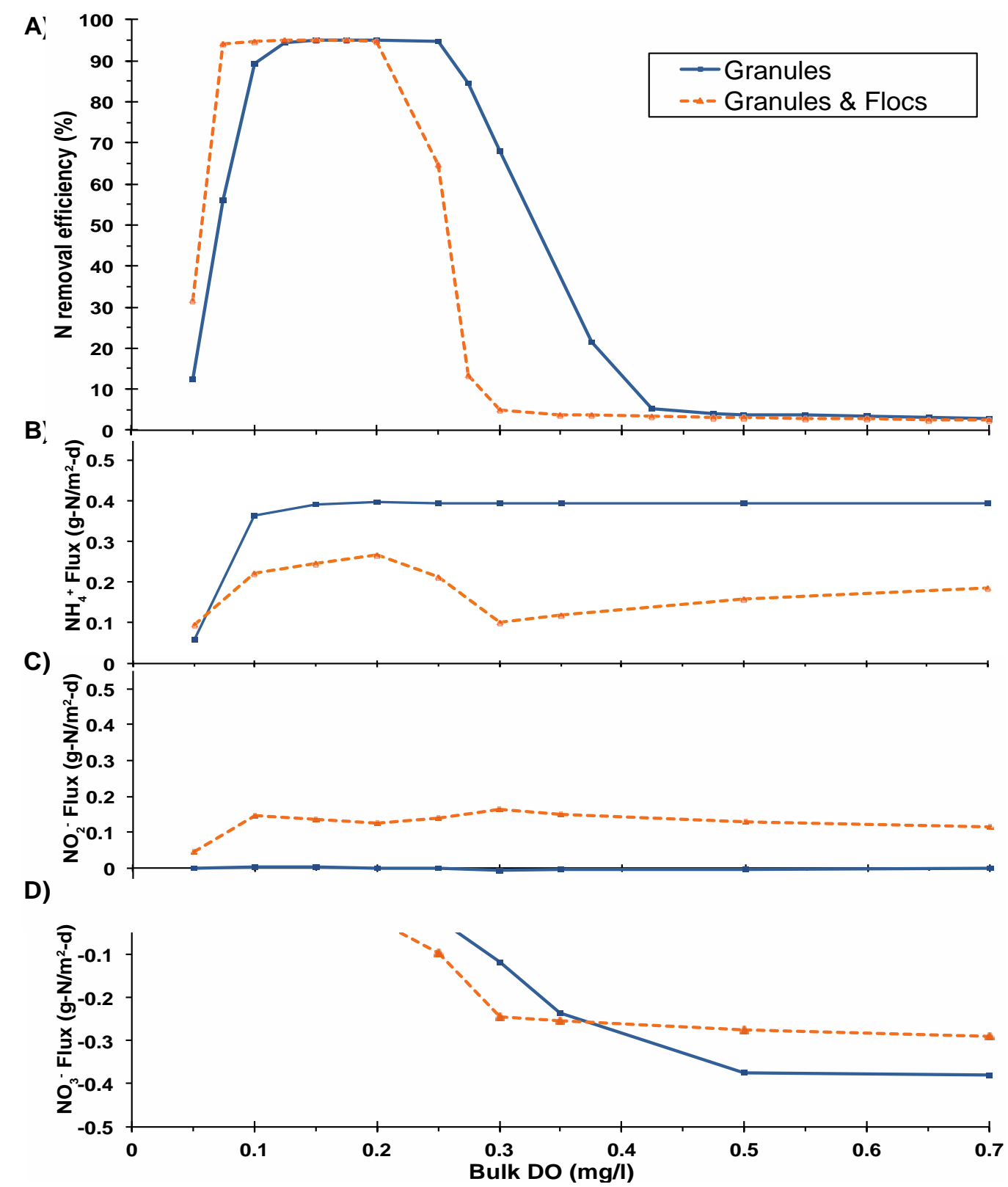

Figure 6. A: Nitrogen removal efficiency at steady state in granular sludge reactors with and without flocs with respect to bulk oxygen concentration. B-D: Ammonia, nitrite and nitrate fluxes into granules in a granular sludge reactor with and without flocs. $\mathrm{N}$ surface load: $0.45 \mathrm{gN} / \mathrm{m}^{2} / \mathrm{d}$, COD surface load: $0.015 \mathrm{gCOD} / \mathrm{m}^{2} / \mathrm{d}$.

Flux patterns were paralleled by segregation in microbial population distributions (Fig. 2). AOO predominate in flocs, resulting in relatively AMO-rich granules. Similarly, OHO populations are concentrated in the floccular fraction under low DO conditions when both biomass fractions are included. In the combined granular and floccular sludge reactor, NOO started to grow at $\mathrm{DO}=0.25 \mathrm{mg} / \mathrm{l}$ in the bulk and accounted up to about $30 \%$ of the total biomass in the granules at $\mathrm{DO}=0.3 \mathrm{mg} / \mathrm{l}$ (Figure 2, right). In the exclusively granular sludge reactor, NOO reached a similar fraction only at $\mathrm{DO}=0.5 \mathrm{mg} / \mathrm{l}$ (Figure 2 , left). These patterns in population and activity segregation can be explained in part by the lack of diffusive mass transfer limitation in floccular biomass, such that organisms in this fraction were directly exposed to oxygen. 


\section{WWTmod}

A study focused on organotrophic anammox activity in MBBR and granular sludge combined nitritation-anammox reactors by Winkler et al. (2012) offers an initial experimental assessment of such activity and population differences between floccular and biofilm aggregate fractions. Winkler documented substantially higher aerobic activity (AOO, NOO, and heterotrophs) in the floccular compared to biofilm fraction in the MBBR via oxygen uptake rate measurements (the granular sludge biomass was not subjected this analysis), in qualitative agreement with our model. Furthermore, the Winkler et al. remarked on the dominance in the floccular fraction of both reactors of AOO via FISH analyses.

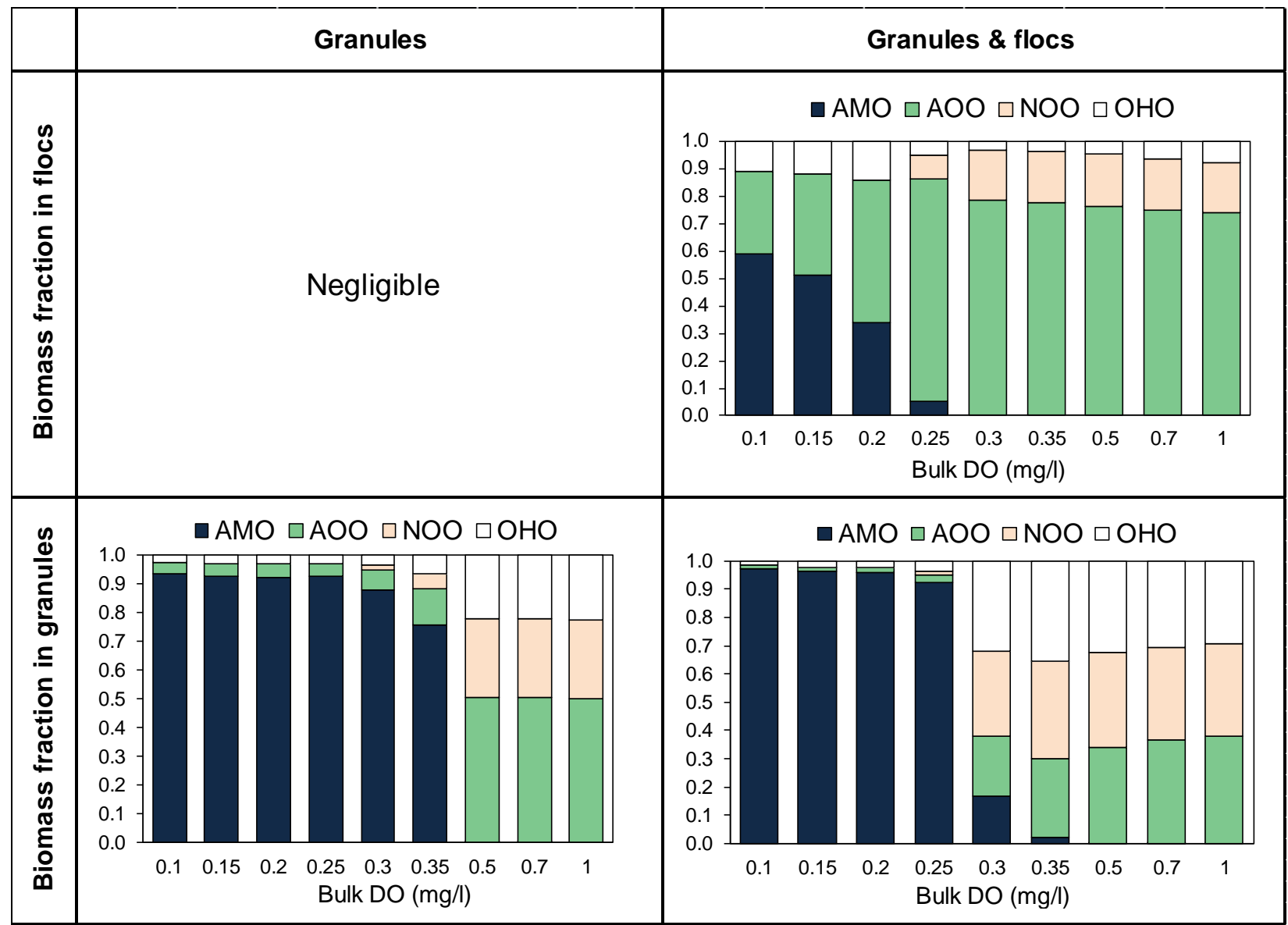

Figure 27. Steady state active biomass partitioning between flocs (5\% total biomass, above) and granules (below) in a granular sludge reactor (left) and in a mixed granules-flocs reactor (right) for different bulk oxygen concentrations. $\mathrm{N}$ surface load: $0.45 \mathrm{gN} / \mathrm{m}^{2} / \mathrm{d}$, COD surface load: $0.015 \mathrm{gCOD} / \mathrm{m}^{2} / \mathrm{d}$ (COD: $\left.\mathrm{N}=0.1: 3\right)$.

Volcke et al. (2012) assessed the influence of a non-uniform granule size distribution on granular nitritation-anammox reactor macroscale and microscale characteristics, and concluded that size distribution influences microscale solute transport due to increased relative abundance of AOO in smaller granules and AMO in larger granules. Interestingly, our model indicated a similar segregation of microbial populations and activities due to the inclusion of a second biomass fraction without mass transport limitations (flocs), with AMO concentrated in (uniform size distribution) granules and AOO predominating in floccular biomass.

Taken together, our results indicate that even small levels of floccular biomass in biofilm reactors can have important implications for reactor performance and optimization under certain operating conditions and for segregation of linked microbial processes. This suggests that the 
common practice of neglecting small levels of heterogeneity in aggregate structure (e.g. small levels of flocs in granular sludge reactors) in biofilm models may lead to erroneous patterns or results at the microscale, and also for macroscale performance in some cases.

\section{Case study 2: Modeling microbial competition in biofilm reactors: considering multiple taxa in a single functional group}

Using the two-step nitrification biofilm model implementing the growth and endogenous respiration of $10 \mathrm{AOO}$ and $10 \mathrm{NOO}$ species, it was observed that the macroscopic reactor behavior, in terms of nitrifying performance, was already at steady state within 10 days after start-up (Fig. 3A). At first, nitrite accumulated to a maximum concentration of $185 \mathrm{~g} \mathrm{NO}_{2}^{-}-\mathrm{N} . \mathrm{m}^{-3}$ on day 1 , but was completely converted after four days. At steady state, ammonium was almost completely converted to nitrate, resulting in a nitrate effluent concentration of $241 \mathrm{~g} \mathrm{NO}_{3}{ }^{-}-\mathrm{N} \cdot \mathrm{m}^{-3}$.
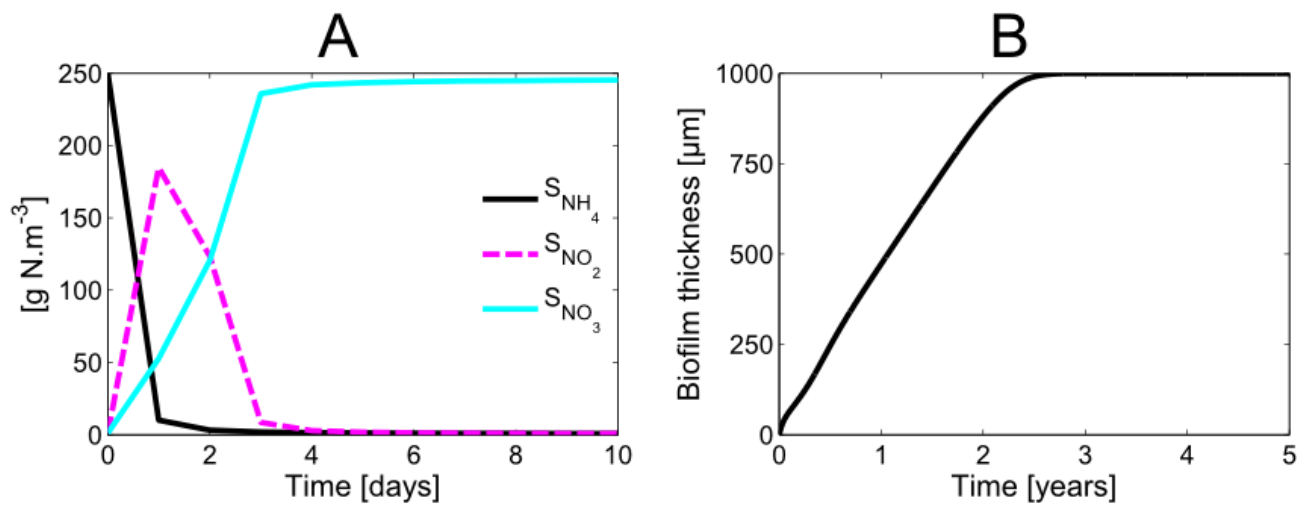

Figure 3. Bulk liquid concentration of nitrogen components (A) and the biofilm thickness (B) in function of time. Mind the different scale and units (days versus years) of the $x$-axis in both figures.

In contrast to the macroscopic reactor behavior, the steady state biofilm thickness of $1 \mathrm{~mm}$ was only reached after about 2.5 years (Fig. 3B), indicating that constant reactor performance not necessarily implies that the steady state biofilm thickness is already reached. The biofilm thickness increased linearly due to the formation of active biomass by microbial growth and the formation of inert particulate components by endogenous respiration. Inert particulate components made up more than $90 \%$ of the total particulate mass in the biofilm at steady state.

The steady state conditions of the microbial community were only reached after about 12 years (Table 2). A major microbial community shift was even observed after 5 years of operation. Initially, all AOO species made up $7.5 \%$ and all NOO species $2.5 \%$ of the total particulate matter mass (100 g COD) in the biofilm. Due to microbial competition, the initial fraction of each species evolved in time to its steady state value. In the AOO community, species AOO1 became dominant. In the NOO community, NOO6 remained dominant for about 7 years. However, after 5 years, species NOO2, which was virtually absent in the biofilm for 3 years, reappeared in the biofilm. This species became dominant after 8 years and remained the dominant NOO species at steady state. At steady state, 3 dominant species coexisted in the biofilm: AOO1, NOO6 and NOO2. All the others species could be considered absent and not contributing to the microbial conversions. However, it is assumed that when the operation conditions change, these species could re-emerge when the new conditions are favorable for them, as their concentrations were negligible, but nonzero. 
Wells et al.

Table 3. Evolution of the percentage of the total particulate matter ( $100 \mathrm{~g}$ COD) made up by each species in the biofilm through time. Percentages of individual $\mathrm{AOO}$ and NOO species are visualized by color codes from $0 \%$ (white) to $25 \%$ (black).

\begin{tabular}{|c|c|c|c|c|c|c|c|c|c|c|c|c|c|c|c|}
\hline $\begin{array}{l}\text { Time (months) } \rightarrow \\
\text { Fraction (\%) } \downarrow\end{array}$ & $\mathbf{0}$ & 10 & 20 & 30 & 40 & 50 & 60 & 70 & 80 & 90 & 100 & 110 & 120 & 130 & 140 \\
\hline AOO1 & 7.5 & 24.63 & 13.98 & 7.83 & 5.55 & 5.52 & 5.51 & 5.51 & 5.50 & 5.48 & 5.40 & 5.31 & 5.26 & 5.25 & 5.25 \\
\hline AOO2 & 7.5 & 0.002 & 0 & 0 & 0 & 0 & 0 & 0 & 0 & 0 & 0 & 0 & 0 & 0 & 0 \\
\hline AOO3 & 7.5 & 0 & 0 & 0 & 0 & 0 & 0 & 0 & 0 & 0 & 0 & 0 & 0 & 0 & 0 \\
\hline AOO4 & 7.5 & 0.002 & 0 & 0 & 0 & 0 & 0 & 0 & 0 & 0 & 0 & 0 & 0 & 0 & 0 \\
\hline AOO5 & 7.5 & 0 & 0 & 0 & 0 & 0 & 0 & 0 & 0 & 0 & 0 & 0 & 0 & 0 & 0 \\
\hline AOO6 & 7.5 & 0.029 & 0 & 0 & 0 & 0 & 0 & 0 & 0 & 0 & 0 & 0 & 0 & 0 & 0 \\
\hline AOO7 & 7.5 & 0.002 & 0 & 0 & 0 & 0 & 0 & 0 & 0 & 0 & 0 & 0 & 0 & 0 & 0 \\
\hline AOO8 & 7.5 & 0.069 & 0 & 0 & 0 & 0 & 0 & 0 & 0 & 0 & 0 & 0 & 0 & 0 & 0 \\
\hline AOO9 & 7.5 & 0 & 0 & 0 & 0 & 0 & 0 & 0 & 0 & 0 & 0 & 0 & 0 & 0 & 0 \\
\hline AOO10 & 7.5 & 0 & 0 & 0 & 0 & 0 & 0 & 0 & 0 & 0 & 0 & 0 & 0 & 0 & 0 \\
\hline Total AOO & 75 & 24.73 & 13.98 & 7.82 & 5.55 & 5.52 & 5.51 & 5.51 & 5.50 & 5.48 & 5.40 & 5.31 & 5.26 & 5.25 & 5.25 \\
\hline NOO1 & 2.5 & 0.003 & 0 & 0 & 0 & 0 & 0 & 0 & 0 & 0 & 0 & 0 & 0 & 0 & 0 \\
\hline $\mathrm{NOO2}$ & 2.5 & 0.10 & 0.002 & 0 & 0 & 0 & 0.007 & 0.042 & 0.17 & 0.63 & 1.60 & 2.69 & 3.25 & 3.44 & 3.49 \\
\hline NOO3 & 2.5 & 0.001 & 0 & 0 & 0 & 0 & 0 & 0 & 0 & 0 & 0 & 0 & 0 & 0 & 0 \\
\hline $\mathrm{NOO4}$ & 2.5 & 0.005 & 0 & 0 & 0 & 0 & 0 & 0 & 0 & 0 & 0 & 0 & 0 & 0 & 0 \\
\hline NOO5 & 2.5 & 0.037 & 0 & 0 & 0 & 0 & 0 & 0.001 & 0.001 & 0 & 0 & 0 & 0 & 0 & 0 \\
\hline NOO6 & 2.5 & 7.06 & 3.90 & 2.35 & 1.85 & 1.84 & 1.83 & 1.82 & 1.77 & 1.59 & 1.19 & 0.70 & 0.43 & 0.34 & 0.31 \\
\hline NOO7 & 2.5 & 0.001 & 0 & 0 & 0 & 0 & 0 & 0 & 0 & 0 & 0 & 0 & 0 & 0 & 0 \\
\hline NOO8 & 2.5 & 0.001 & 0 & 0 & 0 & 0 & 0 & 0 & 0 & 0 & 0 & 0 & 0 & 0 & 0 \\
\hline NOO9 & 2.5 & 0 & 0 & 0 & 0 & 0 & 0 & 0 & 0 & 0 & 0 & 0 & 0 & 0 & 0 \\
\hline NOO10 & 2.5 & 0.002 & 0 & 0 & 0 & 0 & 0 & 0 & 0 & 0 & 0 & 0 & 0 & 0 & 0 \\
\hline Total NOO & 25 & 7.22 & 3.90 & 2.35 & 1.85 & 1.84 & 1.84 & 1.86 & 1.94 & 2.22 & 2.79 & 3.38 & 3.68 & 3.77 & 3.80 \\
\hline Total XI & $\mathbf{0}$ & 68.05 & 82.12 & 89.82 & 92.61 & 92.64 & 92.64 & 92.63 & 92.55 & 92.30 & 91.80 & 91.31 & 91.06 & 90.98 & 90.96 \\
\hline
\end{tabular}

The steady state substrate gradients are displayed in Fig. 4. In this study, ammonium and nitrite were especially limiting, as the concentrations prevailing in the biofilm of these substrates were much lower than the affinity constants considered. Indeed, species with a rather high affinity for ammonium (AOO1) and nitrite (NOO2 and NOO6) were selected.

From the biomass concentration profile (Fig. 4D), it is observed that at steady state, NOO6 remained present in a small concentration at the surface of the biofilm while NOO2 had the highest concentration $83 \mu \mathrm{m}$ below the surface of the biofilm. The coexistence of two genetically and morphologically different populations of NOO with different distribution patterns in a biofilm was already observed experimentally by Schramm et al. (1998). When coexistence of species performing the same function is observed, a distinction is typically made between slow growing species with a high substrate affinity (K-strategists) and fast growing species with a low substrate affinity (r-strategists). The r- and K-selection strategy (Andrews \& Harris 1986) was already used previously to explain experimentally observed population shifts and microbial coexistence in nitrifying biofilms (Schramm et al. 2000; Volcke et al. 2008; Terada et al. 2010; Almstrand et al. 
2013). In the NOO community considered in this study, NOO6 was an r-strategist with a relatively high growth rate (high $\mu_{\max }^{\mathrm{NOO6}}$ ) and $\mathrm{NOO} 2$ was a K-strategist with a relatively high affinity for nitrite (low $\mathrm{K}_{\mathrm{NO2}}^{\mathrm{NOO2}}$ ). The r-strategist NOO6 was able to survive close to the surface due to the higher substrate concentrations prevailing there, in combination with its high maximum growth rate. As a K-strategist, NOO2 was able to cope with the limiting substrate concentrations deeper in the biofilm. Considering the evolution of the NOO community in time, it was observed that the rstrategist NOO6 was able to cope rapidly with the prevailing conditions and grew at a high rate due to its relatively high maximum growth rate. After 8 years, the slow growing K-strategist NOO2 became dominant over NOO6 due to its higher affinity for nitrite. It can thus be concluded that the $r$ - and K-selection strategy not only can be used here to explain the steady state microbial distribution profile but also the development of the microbial community composition over time.
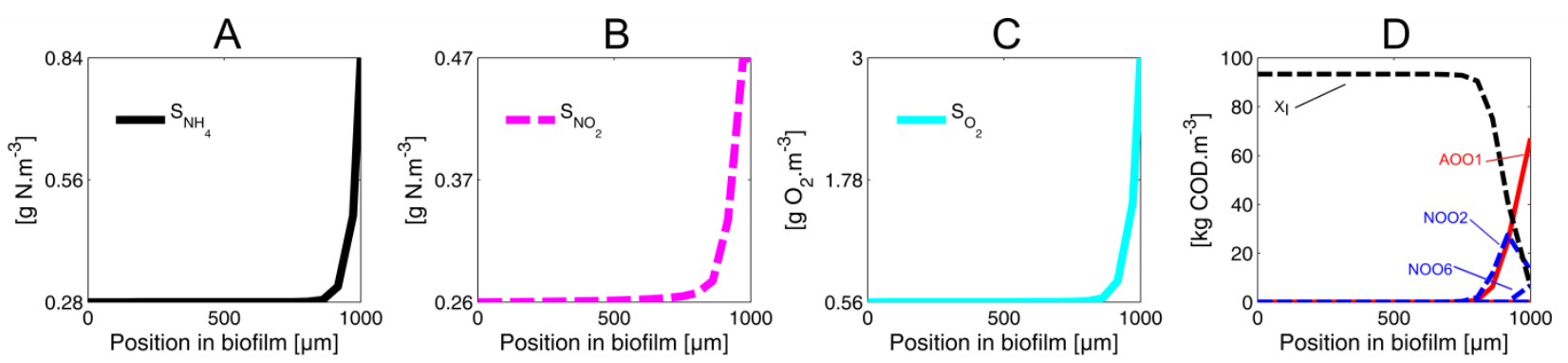

Figure 4. Steady state concentration profiles for ammonium (A), nitrite (B), oxygen (C) and particulate matter (D) in function of the position of the biofilm $(0 \mu \mathrm{m}=$ bottom, $1000 \mu \mathrm{m}=$ surface of the biofilm). Mind the different scale of the $y$-axis of the substrate concentration profiles.

By using a multispecies nitrification biofilm model, the individual role of various microbial characteristics on the microbial population dynamics was demonstrated. The coexistence of several species of the same type, in this case several types of nitrite-oxidizers, may be explained based on their difference in maximum growth rate and affinity for the limiting substrate. It was shown that the biomass distribution profiles at steady state of the coexisting NOO reflected the ecological niches created by the substrate gradients. Furthermore, it was concluded that constant macroscopic reactor performance not necessary reflects steady state conditions on the microscale. Significantly more time is needed for the biofilm and the microbial community to reach steady state compared to the macroscopic reactor behavior.

\section{CONCLUSIONS \& OUTLOOK}

We highlight above two instances in which additional model complexity is included beyond the conventional formulation for numerical 1-dimensional biofilm models. In Case study 1, our model suggests that even low levels of flocs can have a significant impact on process performance, optimal operating ranges, and microbial population and activity distributions in combined nitritation-anammox granular sludge reactors. The implication is that a better characterization of size distribution, mass transfer properties, and microbial population segregation of microbial aggregates - including flocs and granules- could improve operation of these reactors and contribute to better understanding of unexpected reactor behaviors. In Case study 2, it was shown that multispecies models are a useful tool to investigate the individual influence of various microbial characteristics on microbial population dynamics, and that coexistence of several species performing the same function is linked to the ecological niches created by the substrate concentration gradients in the biofilm. Nitrifying biofilm models including the growth of several species performing the same function not only demonstrate that a constant macroscopic reactor 
behavior may be hiding major microbial community shifts, but can also be used to investigate major microbial population shifts resulting in a different nitrifying performance (Vannecke et al. 2014).

Additional model complexity considered here had a substantial impact on macroscale outputs in some specific conditions, and on microscale outputs (namely, spatial distribution of dissolved and particulate components) under all conditions. It is likely a general rule that increased complexity will be more useful when the focus is on understanding fundamental microscale outputs. When the focus is on macroscale outputs (e.g. substrate removal rates, optimal bulk conditions), this complexity is clearly not always necessary. However, under specific conditions, additional model features can be critically informative for bulk reactor behavior prediction or understanding. Based on the results presented here, we suggest that two instances where additional biofilm model complexity may be warranted are 1) assessment of performance under dynamic process conditions; and 2) characterization of processes with a balance between aerobic and anoxic metabolisms.

Ample future work is warranted to delineate implications of both heterogeneity in mesoscale aggregate structure and of microbial diversity to biofilm models. Regarding the former, evaluating the combined influence of granule size distribution with small levels of floccular material in both granular nitritation-anammox reactors and in other biofilm systems, particularly those that involve cross-feeding between multiple functional groups, is a logical next step. In addition, the model presented here evaluated only two aggregate fractions (flocs and granules), whereas in reality there appears to be a continuum of structures between the two. For example, granules in some systems have been observed embedded in floccular sludge (Innerebner et al. 2007), thus potentially increasing locally the connections between a couple of aggregates and leading to more or less active flocs. In addition, Arrojo et al. (2006) demonstrated experimentally that aggregate size distribution varied over time when the mixing changed in the reactor. This suggests that the relative abundance of granules and flocs is probably not constant, and that flocs might become granules at some point and vice versa, which supports the presence of different types of flocs in reactors. Future modeling efforts are warranted to test the implicit assumption here that we can safely neglect these additional structural complexities under all relevant conditions when evaluating macroscale outputs, and to assess their influence on microscale microbial distribution and solute exchange.

From the standpoint of microbial diversity, further simulation studies based on multispecies nitrification biofilm models are required to investigate the individual role of various microbial characteristics and operation conditions on microbial competition. In the near term, a worthwhile future goal would be to assess the influence of both multiple taxa within the same functional group and heterogeneity in mesoscale aggregate architecture. In addition, there is increasing interest in explicitly incorporating our rapidly expanding understanding of microbial community structure and dynamics via molecular tools into predictive process models. Seshan et al. (2014) present an excellent example of this via a support vector regression model using microbial community diversity indices derived from DNA fingerprinting (T-RFLP) to predict reactor removal performance for COD, ammonia, nitrate, and 3-chloroanaline. Wastewater treatment modelers would also be well served by adapting emerging techniques in this direction in biogeochemical modeling. For example, Reed et al. (2014) provide a gene-based framework for incorporating environmental genomics data into a model of nitrogen cycling in the Arabian Sea oxygen minimum zone. A similar approach may be possible in bioprocess modeling to refine our understanding of the role of microbial diversity and community dynamics on both microscale and macroscale outputs in biofilm reactors. 


\section{ACKNOWLEDGEMENTS}

G.F. Wells was supported by an Eawag Postdoctoral Fellowship and by the US National Science Foundation under IRFP Grant No. 1064615. T.P.W. Vannecke is supported by the Research Foundation-Flanders (FWO) through a Ph.D. fellowship.

\section{REFERENCES}

Almstrand R., Lydmark P., Lindgren P. E., Sörensson F. and Hermansson M. (2013). Dynamics of specific ammonia-oxidizing bacterial populations and nitrification in response to controlled shifts of ammonium concentrations in wastewater. Applied Microbiology and Biotechnology 97(5), 2183-91.

Andrews J. H. and Harris R. F. (1986). R-selection and K-selection and microbial ecology. Advances in Microbial Ecology 9, 99-147.

Arrojo B., Mosquera-Corral A., Campos J. L. and Méndez R. (2006). Effects of mechanical stress on Anammox granules in a sequencing batch reactor (SBR). Journal of Biotechnology 123(4), 453-63.

Bernet N., Sanchez O., Dabert P., Olaizola A., Godon J. J. and Delgenes J. P. (2004). Effect of solid hold-up on nitrite accumulation in a biofilm reactor - molecular characterization of nitrifying communities. Water Science and Technology 49(11-12), 123-30.

Brockmann D., Caylet A., Escudié R., Steyer J. P. and Bernet N. (2013). Biofilm model calibration and microbial diversity study using Monte Carlo simulations. Biotechnology and Bioengineering 110(5), 1323-32.

Downing L. S. and Nerenberg R. (2008). Effect of Oxygen Gradients on the Activity and Microbial Community Structure of a Nitrifying, Membrane-Aerated Biofilm. Biotechnology and Bioengineering 101(6), 1193-204.

Eberl H., Morgenroth E., Noguera D. R., Picioreanu C., Rittmann B. E., van Loosdrecht M. and Wanner O. (2006). Mathematical Modeling of Biofilms. IWA Publishing, London, UK.

Innerebner G., Insam H., Franke-Whittle I. H. and Wett B. (2007). Identification of anammox bacteria in a full-scale deammonification plant making use of anaerobic ammonia oxidation. Systematic and Applied Microbiology 30(5), 408-12.

Lydmark P., Lind M., Sorensson F. and Hermansson M. (2006). Vertical distribution of nitrifying populations in bacterial biofilms from a full-scale nitrifying trickling filter. Environmental Microbiology 8(11), 2036-49.

Morgenroth E., Eberl H. and van Loosdrecht M. C. M. (2000a). Evaluating 3-D and 1-D mathematical models for mass transport in heterogeneous biofilms. Water Science and Technology 41(4-5), 347-56.

Morgenroth E., van Loosdrecht M. and Wanner O. (2000b). Biofilm models for the practitioner Water Science \& Technology 41(4-5), 509-12.

Mozumder M. S. I., Picioreanu C., van Loosdrecht M. C. M. and Volcke E. I. P. (2013). Effect of heterotrophic growth on autotrophic nitrogen removal in a granular sludge reactor. Environmental Technology, 1-11.

Ni B. J., Chen Y. P., Liu S. Y., Fang F., Xie W. M. and Yu H. Q. (2009). Modeling a GranuleBased Anaerobic Ammonium Oxidizing (ANAMMOX) Process. Biotechnology and Bioengineering 103(3), 490-9.

Nielsen P. H., Mielczarek A. T., Kragelund C., Nielsen J. L., Saunders A. M., Kong Y. H., Hansen A. A. and Vollertsen J. (2010). A conceptual ecosystem model of microbial communities in enhanced biological phosphorus removal plants. Water Research 44(17), 5070-88.

Picioreanu C., Kreft J.-U. and van Loosdrecht M. C. (2004). Particle-based multidimensional multispecies biofilm model. Applied and Environmental Microbiology 70(5), 3024-40. 
Ramirez I., Volcke E. I. P., Rajinikanth R. and Steyer J. P. (2009). Modeling microbial diversity in anaerobic digestion through an extended ADM1 model. Water Research 43(11), 2787-800.

Reed D. C., Algar C. K., Huber J. A. and Dick G. J. (2014). Gene-centric approach to integrating environmental genomics and biogeochemical models. Proceedings of the National Academy of Sciences.

Reichert P., Vonschulthess R. and Wild D. (1995). Use of AQUASIM for estimating parameters of activated sludge models. Water Science and Technology 31(2), 135.

Schramm A., De Beer D., Gieseke A. and Amann R. (2000). Microenvironments and distribution of nitrifying bacteria in a membrane-bound biofilm. Environmental Microbiology 2(6), 680-6.

Schramm A., de Beer D., Wagner M. and Amann R. (1998). Identification and activities in situ of Nitrosospira and Nitrospira spp. as dominant populations in a nitrifying fluidized bed reactor. Applied and Environmental Microbiology 64(9), 3480-5.

Seshan H., Goyal M. K., Falk M. W. and Wuertz S. Support Vector Regression Model of Wastewater Bioreactor Performance Using Microbial Community Diversity Indices: Effect of Stress and Bioaugmentation. Water Research $(0)$.

Seshan H., Goyal M. K., Falk M. W. and Wuertz S. (2014). Support vector regression model of wastewater bioreactor performance using microbial community diversity indices: Effect of stress and bioaugmentation. Water Research 53(0), 282-96.

Siripong S. and Rittmann B. E. (2007). Diversity study of nitrifying bacteria in full-scale municipal wastewater treatment plants. Water Research 41(5), 1110-20.

Terada A., Lackner S., Kristensen K. and Smets B. F. (2010). Inoculum effects on community composition and nitritation performance of autotrophic nitrifying biofilm reactors with counter-diffusion geometry. Environmental Microbiology 12(10), 2858-72.

Vannecke T. P., Bernet N., Steyer J. P. and Volcke E. I. (2014). Modelling ammonium-oxidizing population shifts in a biofilm reactor. Water Science and Technology 69(1), 208-16.

Vlaeminck S. E., Cloetens L. F. F., Carballa M., Boon N. and Verstraete W. (2009). Granular biomass capable of partial nitritation and anammox. Water Science and Technology 59(3), 609-17.

Vlaeminck S. E., Terada A., Smets B. F., De Clippeleir H., Schaubroeck T., Bolca S., Demeestere L., Mast J., Boon N., Carballa M. and Verstraete W. (2010). Aggregate Size and Architecture Determine Microbial Activity Balance for One-Stage Partial Nitritation and Anammox. Applied and Environmental Microbiology 76(3), 900-9.

Volcke E. I. P., Picioreanu C., De Baets B. and van Loosdrecht M. C. M. (2010). Effect of granule size on autotrophic nitrogen removal in a granular sludge reactor. Environmental Technology 31(11), 1271-80.

Volcke E. I. P., Picioreanu C., De Baets B. and van Loosdrecht M. C. M. (2012). The granule size distribution in an anammox-based granular sludge reactor affects the conversionImplications for modeling. Biotechnology and Bioengineering 109(7), 1629-36.

Volcke E. I. P., Sanchez O., Steyer J. P., Dabert P. and Bernet N. (2008). Microbial population dynamics in nitrifying reactors: Experimental evidence explained by a simple model including interspecies competition. Process Biochemistry 43(12), 1398-406.

Wanner O. and Gujer W. (1986). A Multispecies Biofilm Model. Biotechnology and Bioengineering 28(3), 314-28.

Wett B. (2007). Development and Implementation of a Robust Deammonification Process. Water Science \& Technology 56(7), 81-8. 
Wells et al.

Wett B., Jimenez J. A., Takacs I., Murthy S., Bratby J. R., Holm N. C. and Ronner-Holm S. G. E. (2011). Models for nitrification process design: one or two AOB populations. Water Science \& Technology 64(3), 568-78.

Winkler M. K. H., Yang J. J., Kleerebezem R., Plaza E., Trela J., Hultman B. and van Loosdrecht M. C. M. (2012). Nitrate reduction by organotrophic Anammox bacteria in a nitritation/anammox granular sludge and a moving bed biofilm reactor. Bioresource Technology 114, 217-23.

Wittebolle L., Boon N., Vanparys B., Heylen K., De Vos P. and Verstraete W. (2005). Failure of the ammonia oxidation process in two pharmaceutical wastewater treatment plants is linked to shifts in the bacterial communities. Journal of Applied Microbiology 99(5), 997-1006. 


\title{
The Case of the Ks: Diffusion versus Strategy
}

\author{
Andrew Shaw ${ }^{1,3}$, Imre Takacs ${ }^{2}$, Krishna Pagilla ${ }^{3}$, Rumana Riffat ${ }^{4}$, Haydee DeClippeleir ${ }^{5}$, Christopher \\ Wilson $^{6}$, and Sudhir Murthy ${ }^{7}$ \\ ${ }^{1}$ Global Practice \& Technology Leader, Black \& Veatch, 8400 Ward Parkway, Kansas City, Missouri, USA \\ (Email: shawar@bv.com ) \\ ${ }^{2}$ Dynamita (Email: imre@dynamita.com ) \\ ${ }^{3}$ Illinois Institute of Technology, Chicago, IL, USA (Email: pagilla@iit.edu) \\ ${ }^{4}$ George Washington University, Washington DC, USA (Email: riffat@gwu.edu ) \\ ${ }^{5}$ Columbia University, New York City, NY, USA (Email: $\underline{h d 2296 @ \text { columbia.edu) }}$ \\ ${ }^{6}$ Greeley \& Hansen, Richmond, VA, USA (Email: cwilson@greeley-hansen.com ) \\ ${ }^{7}$ DC Water, Washington DC, USA (Email: Sudhir.Murthy@dcwater.com )
}

\begin{abstract}
Observed (extant) $K_{S}$ is strongly influenced by diffusion. This paper argues that diffusion can be used to describe bacterial kinetic effects that are sometimes attributed to "K-strategists" and in fact the physics of the system is the dominant mechanism affecting the apparent (extant) $K_{S}$; not biological selection, in real treatment systems. The authors use the "porter-diffusion" model to make their case.
\end{abstract}

Keywords

$\mathrm{K}_{\mathrm{S}}$, Monod, diffusion, strategists, half-saturation

\section{BACKGROUND}

It is commonly held that populations of bacteria can be subdivided into " $\mu$-strategists" (also termed "r-strategists") that thrive in conditions of high substrate concentrations, and "K-strategists" that somehow predominate under low substrate conditions. This paper offers the opinion - backed by measurements and plausible theory - that in some cases, observations attributed to different "strategist" populations can, more simply, be attributed to diffusion effects due to a boundary layer thickness and/or substrate concentration levels. In other words, different kinetic rates can be described adequately using the physical phenomenon of diffusion without reverting to the somewhat subjective approach of selecting different kinetic parameters in order to match different observed removal rates for the same biomass types under different substrate conditions.

\section{$K_{S}$ is a function of Maximum Rate}

Over the past several years, several researchers have shown that half-saturation coefficients $\left(K_{S}\right)$ in many biological treatment systems are not constant but in fact are functions of the maximum removal rates. Wilson et al. (2012) showed that the $K_{S}$ for acetic acid in anaerobic systems was a linear function of the maximum removal rate. Shaw et al. (2013) showed that the extant $K_{S}$ for nitrate in denitrification is a function of the maximum denitrification rate. Further, they showed that the apparent extant $K_{N O 3}$ value can be described by using a diffusion model with a very small intrinsic $K_{\mathrm{NO}}$.

Figure 1 shows data from Shaw et al. (2013), Wilson et al. (2012) and data from two other wastewater treatment systems. Despite the systems being very different and for different substrates, all 4 show a strong correlation between $K_{S}$ and the maximum substrate removal rate. 


\section{WWTmod}

Shaw et al.

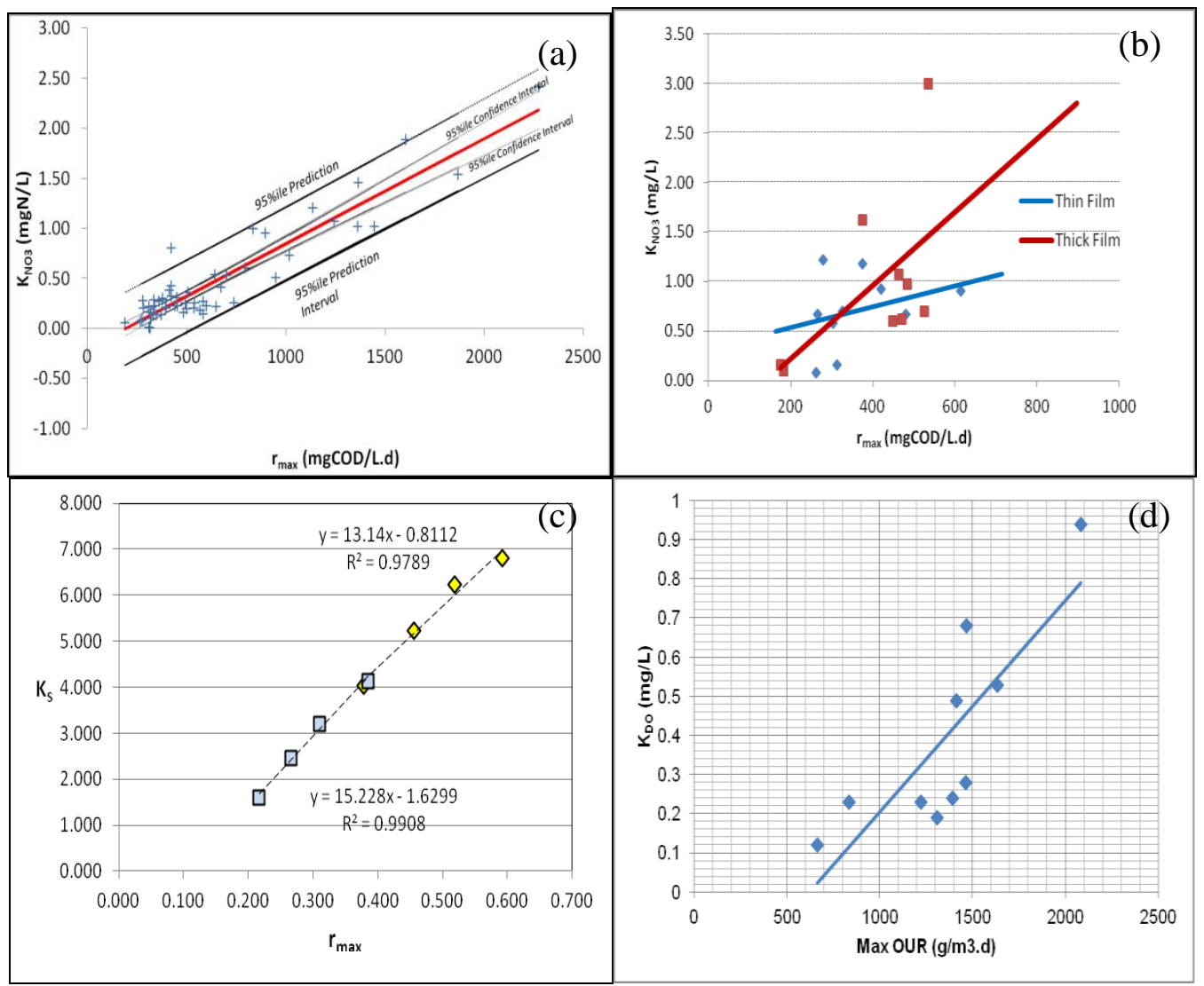

Figure 1: $K_{S}$ as a function of $\boldsymbol{r}_{\max }$ for 4 different biological systems: (a) nitrate limit for denitrification in an activated sludge system (Shaw 2013); (b) nitrate limit in an MBBR; (c) mesophilic anaerobic digestion (Wilson 2012); (d) DO limit for nitrification in activated sludge. Note, maximum rates shown with respect to biomass growth in cases (a), (b) and (c) but with respect to dissolved oxygen for case (d).

Many researchers have used the concept of "strategists" to explain the apparently different kinetics including substrate affinity under different operating conditions. For example, Wett et al. (2011) used the concept of " $\mu$-strategists" to explain different overall rates and growth kinetics that had to be used in a model to match overall observed performance when adjusting mixed liquor recycle rates (Figure 2).

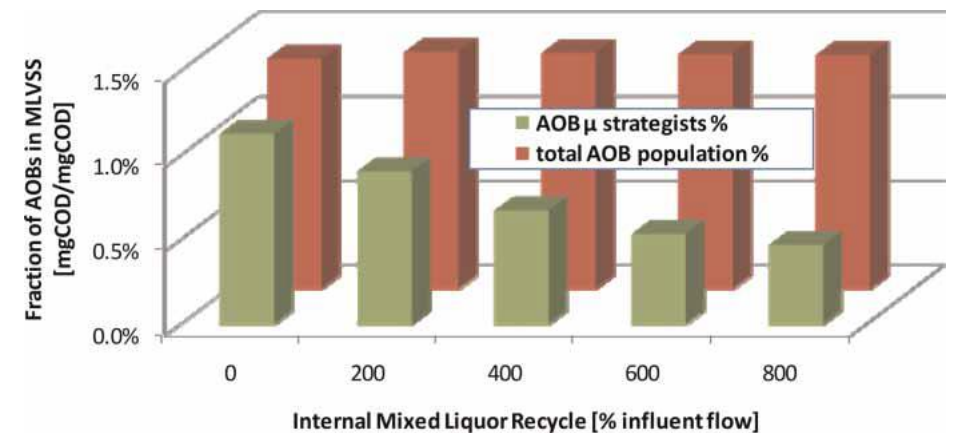

Figure 2: Graph from Wett et al. (2011) showing the Model Fraction of " $\mu$ strategists" Based on Internal Mixed Liquor Recycle 
In their discussions of nitrite modelling, Sin et al (2010) used higher $K_{D O}$ values for ammonia oxidizing bacteria (AOB) and nitrite oxidizing bacteria (NOB) to model sidestream treatment (nonsubstrate limited conditions) than the rest of the plant. Other researchers have used the $\mu$ vs K strategist concept to explain observed differences between high rate and low rate systems for methylotrophs (e.g. Purtschert and Gujer, 1999) and anaerobic digestion (Wett et al., 2012). Though these systems are for different types of biological treatment, they use the common approach of shifting kinetic parameters - especially $K_{S}$ - for low substrate conditions from those used for high substrate conditions.

\section{EXPLAINING $K_{S}$ USING THE PORTER-DIFFUSION CONCEPT}

The Monod equation that is used to describe biological kinetics is empirical and does not have a mechanistic basis. It does have the same form as the Michaelis-Menten equation that is used to describe enzyme kinetics and does have a mechanistic basis. Researchers in the field of natural aquatic systems have developed a mechanistic model using a "porter-diffusion" concept that couples enzyme kinetics (Michaelis-Menten) with a diffusion model for a spherical cell. A conceptual sketch of the model, first presented by Pasciak and Gavis (1974) and refined further by several authors (Armstrong, 2008; Aksnes et al., 2011; Fiksen et al., 2013) is shown in Figure 3. Substrate in the bulk liquid $\left(S_{\infty}\right)$ passes through a boundary layer where the transport is governed by diffusion until it reaches the cell wall at a concentration $S_{0}$. At this point, a porter enzyme transports the substrate into the cell using Michaelis-Menten kinetics. Figure 3 shows a curve in blue to represent a high bulk liquid concentration of $S$ and a red curve to show a low concentration for $S_{\infty}$, demonstrating that, although the overall rate is higher with a higher bulk liquid concentration, diffusion limitations are more pronounced (i.e. greater curvature).

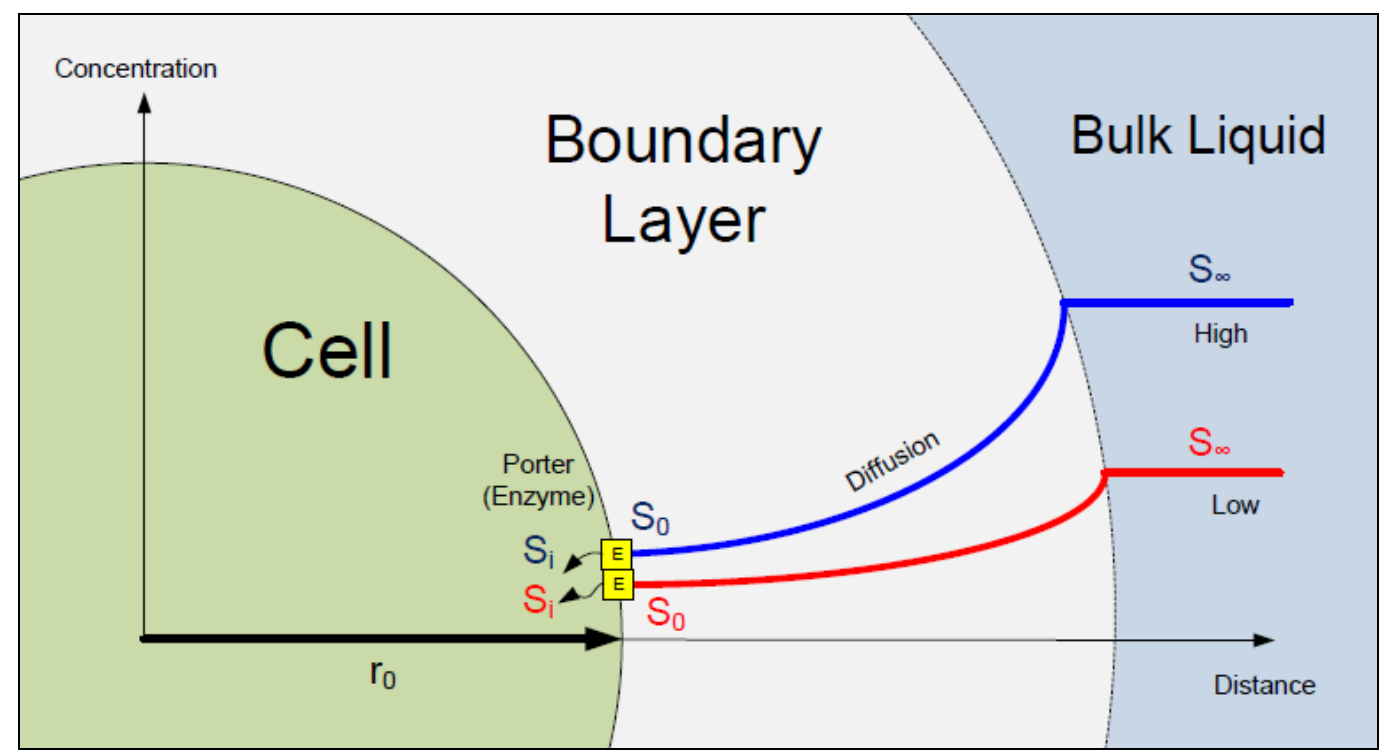

Figure 3: Conceptual sketch of the porter-diffusion model for an individual spherical cell with radius $r_{0}$. The blue line shows the decrease in substrate concentration through the boundary layer by diffusion from an initially high substrate concentration $\left(S_{\infty}\right)$ in the bulk liquid, to a lower concentration at the cell surface $\left(S_{0}\right)$. At the surface the substrate is then transferred into the cell $\left(S_{i}\right)$ by a porter enzyme $(E)$. The red line shows a similar transport but for an initially lower substrate concentration $\left(S_{\infty}\right)$ in the bulk liquid. 
The solution to the porter-diffusion model is a quadratic equation. However, Armstrong (2008), Aksnes et al. (2011) and Fiksen et al. (2013) show that it can also be approximated to a Monod (or Michaelis-Menten) type of equation such as Equation 1 (nomenclature based on Fiksen et al (2013).

\section{Equation 9}

$$
V=V_{\max } \frac{S_{\infty}}{K_{0}+V_{\max }\left(4 \pi r_{0} D\right)^{-1}+S_{\infty}}
$$

Where: $\quad V$ is the substrate uptake rate per cell (mols cell ${ }^{-1} \mathrm{~s}^{-1}$ )

$V_{\text {max }}$ is the maximum uptake rate per cell $\left(\right.$ mols cell $\left.{ }^{-1} \mathrm{~s}^{-1}\right)$

$S_{\infty}$ is the bulk liquid concentration $\left(\right.$ mols $\mathrm{m}^{-3}$ )

$K_{0}$ is the Michealis-Menten half-saturation coefficient with $S_{0}$ as the reference concentration $\left(\mathrm{mol} \mathrm{m}^{-3}\right)$

$r_{0}$ is the cell radius $(\mathrm{m})$

$D$ is the diffusivity of the substrate $\left(\mathrm{m}^{2} \mathrm{~s}^{-1}\right)$

Comparing Equation 1 to the Monod equation, an expression can be given to the extant (observed) half-saturation coefficient, $K_{S}$.

\section{Equation 10}

$K_{S}=K_{0}+V_{\max }\left(4 \pi r_{0} D\right)^{-1}$

In this expression, the extant half-saturation coefficient, $K_{S}$, is made up of two parts summed. The first part is $K_{0}$ which is the Michaelis-Menten half-saturation coefficient, or the intrinsic halfsaturation coefficient. The second part of the expression incorporates the observed effect of diffusion on the extant $K_{S}$ and is a function of the maximum substrate removal rate, cell radius and diffusivity. The fact that $K_{S}$ is proportional to the maximum substrate removal rate, matches the observations of the four wastewater treatment systems shown in Figure 1.

Armstrong (2008) proposed modifications to Equation 2 to include a shape factor, $\Phi$, to account for diffusion to non-spherical cells and a dimensionless Sherwood number, $S h$, to account for convective mass transport compared to molecular or diffusive mass transport, resulting in Equation 3 as shown.

\section{Equation 11}

$$
K_{S}=K_{0}+V_{\max }\left(4 \pi r_{0} D \Phi S h\right)^{-1}
$$

Finally, the $V_{\max }$ in Equation 3 is expressed as the rate per cell. In order to use rates based on overall cell volume or mass, $V_{\max }$ has to be multiplied by the cell volume. Assuming the cell is a sphere with volume $4 / 3 \pi r^{3}$, equation 3 then becomes Equation 4 , where $R_{\max }$ is a volumetric rate.

\section{Equation 12}

$$
K_{S}=K_{0}+R_{\max } r_{0}^{2}(3 D \Phi S h)^{-1}
$$


Equation 4 was fitted to the data for each of the four different systems shown in Figure 1, and the results are shown in Figure 4. In each case, the "cell radius", $r_{0}$, was adjusted until the model line gave a reasonable fit to the data; $K_{0}$ was assumed to be 0 ; diffusivity was selected based on substrate; the shape factor was assumed to be 1; Sh was assumed to be 1.0 for cases (a), (b), and (d) but was adjusted for case (c). The final point is discussed further below. Table 1 is a summary of the parameters used for each case.

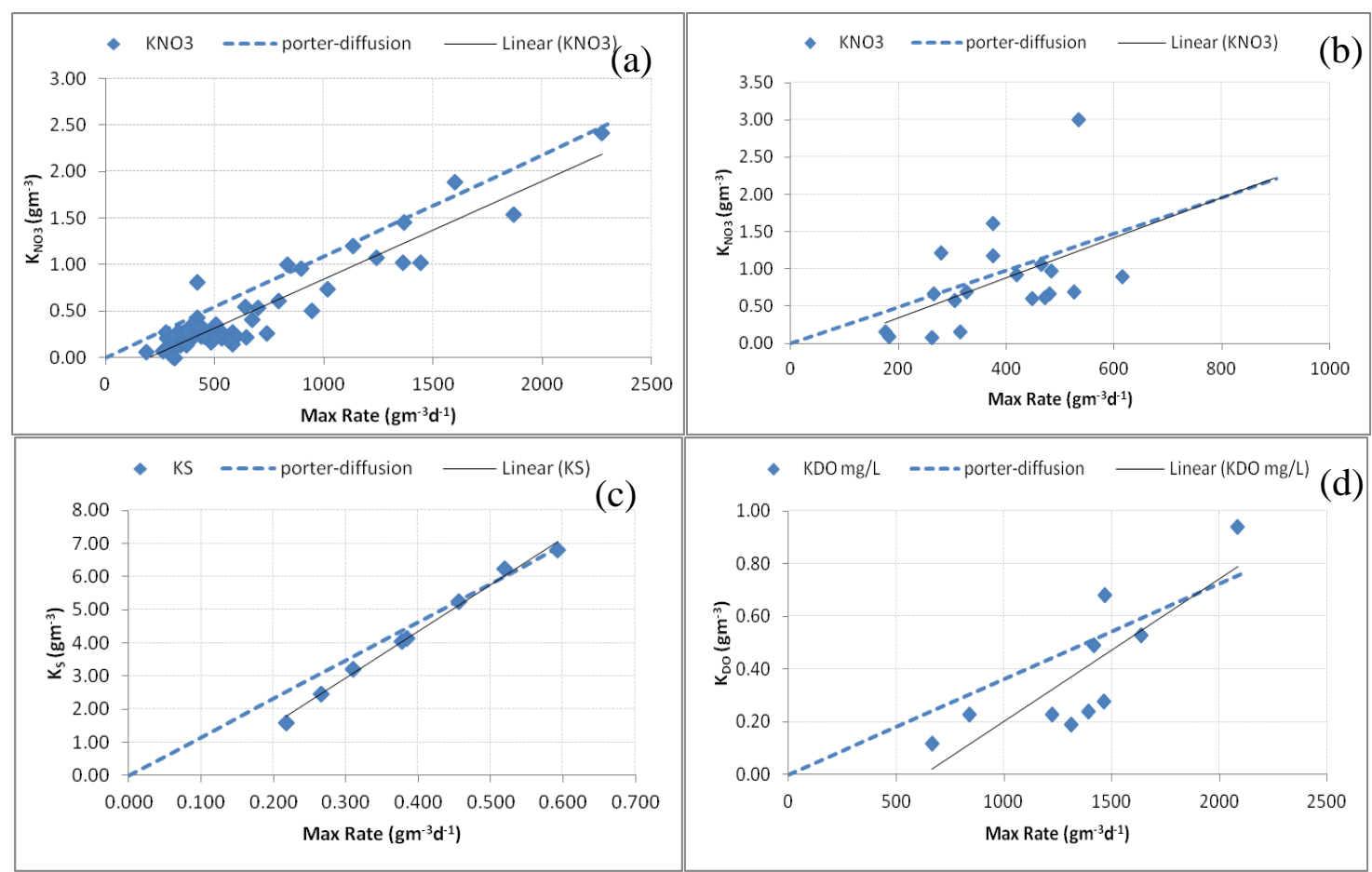

Figure 4: Porter-diffusion model (dotted) and simple linear regression (solid) for the 4 different biological systems shown in Figure 1: (a) nitrate limit for denitrification in an activated sludge system (Shaw 2013); (b) nitrate limit in an MBBR; (c) mesophilic anaerobic digestion (Wilson 2012); (d) DO limit for nitrification in activated sludge.

TABLE 1: Porter-diffusion model parameters used in Figure 4

\begin{tabular}{l|c|ccccc}
\hline \multicolumn{2}{c}{} & $K_{0}$ & $\Phi$ & $D$ & $S h$ & $r_{0}$ \\
\cline { 3 - 7 } \multicolumn{2}{c}{ System } & $g m^{-3}$ & - & $m^{2} d^{-1}$ & - & $\mu m$ \\
\hline (a) & Denite AS & 0 & 1 & $1.47 \mathrm{E}-04$ & 1.0 & 400 \\
(b) & Denite MBBR & 0 & 1 & $1.47 \mathrm{E}-04$ & 1.0 & 600 \\
(c) & MAD & 0 & 1 & $8.64 \mathrm{E}-05$ & 0.001 & 1000 \\
(d) & Nitrification & 0 & 1 & $1.73 \mathrm{E}-04$ & 1.0 & 250 \\
\hline
\end{tabular}

The following observations are made from Figure 4 and Table 1: 
1. The porter-diffusion model was developed for individual cells, whereas the systems shown in Figure 4 contain most of the biomass within flocs or biofilms. However the logic applied to developing the cellular model can be extended to a larger grouping of cells within a floc or biofilm, with $r_{0}$ a representative diffusive distance to give the equivalent effect.

2. In cases (a), (b), and (d) it was possible to select a value for $r_{0}$ that is within the realms of a plausible dimension for an equivalent floc size or biofilm thickness. No other parameters had to be adjusted.

3. In case (c), $r_{0}$ was adjusted up to a maximum of $1000 \mu \mathrm{m}$ but the model line did not match the data by several orders of magnitude. Sh was then adjusted to match the data better. A lower $S h$ may indicate that diffusion is severely restricted in this system.

4. In all systems, the linear line fit did not have a positive intercept on the $\mathrm{Y}$-axis which would be necessary to provide an estimate for $K_{0}$. The inference from this is that $K_{0}$ is insignificant (near zero) in comparison to mass transfer effects for these particular systems.

In the derivation of Equation 1, Fiksen et al. (2013) show that $V_{\max }=n h^{-1}$, where $n$ is the number of porter sites and $h$ is the handling time for each site. All of the systems analyzed in this paper have a relatively high concentration of biomass or low food to microorganism ratio (F/M) and hence the number of porter sites is not limiting. This may explain why $K_{0}$ (porter effect) is not significant for these systems, but diffusion dominates the formulation of $K_{S}$.

\section{Significance of the Porter-Diffusion Model}

In Equation $4, K_{S}$ is proportional to $r^{2}, D^{-1}$ and $S h^{-1}$. On first glance, $K_{S}$ being proportional to $S h^{-1}$ seems logical because when diffusion controls (over convection), $K_{S}$ will be larger (low affinity). Strictly speaking, however, the modification proposed by Armstrong (2008) relating $K_{S}$ to $S h$ is valid only for single cells, and may not hold for flocs because there is little or no convection inside the flocs. This is similar to pore diffusion in carbon adsorption where advection is zero. Assuming that convection is zero inside the flocs, then diffusion always controls. $K_{S}$ is proportional to $D^{-1}$ and therefore at high diffusivity, $K_{S}$ is low and vice versa. When $r_{0}$ (indicative of diffusion distance) is small, the inter-cell tubes are smaller both in diameter and length reducing the impact of diffusion.

In practical terms, Equation 4 can be used to give a reasonable first estimate of $K_{S}$ depending on the overall maximum rate $\left(R_{\max }\right.$, measured or modelled), floc size ( $r_{0}$, measured or used as a calibration parameter), and diffusivity ( $D=$ physical constant, dependent on substrate and temperature). If this estimate does not match observations, $\Phi$ (shape factor) and/or $S h$ can be used as adjustment factors to provide a better fit. This equation can be coupled with the approach outlined in Shaw et al. (2013) to provide an estimate for $K_{S}$ that can be incorporated into a process simulator to provide a better model fit for a wider range of substrate concentrations than the typical current approach of assuming a fixed $K_{S}$.

A final observation from fitting data to the porter-diffusion model is that $K_{0}$ was near zero for all of the modelled systems in Figure 4. It is posited that this is due to the systems all having a relatively low F/M and hence an abundance of porter sites. It is plausible that high F/M systems would exhibit a greater influence from the lower number of porters and hence $K_{0}$ would be higher for these systems. A practical consequence of this is that $K_{S}$ should not be estimated from high F/M tests and then applied to low F/M systems. Ideally, $K_{S}$ should be estimated using tests carried out at a similar $\mathrm{F} / \mathrm{M}$ to that of the modelled or real-world system. 
Shaw et al.

\section{A NEW PARADIGM}

As noted in the paper by Shaw et al. (2013) and as explained by the porter-diffusion model discussed in this paper, at low substrate concentrations diffusion becomes more important and can be used to explain the reduction in observed kinetic rates, rather than adjusting kinetic parameters to fit data. This reduction in rate will cause a shift in biomass populations that appear to be due to "Kstrategists" but in fact may be caused by diffusion and other physical effects. Another way to view this is that the apparent "K-strategists" gain their advantage by somehow exploiting an ability to overcome diffusion limitations, such as growing in filamentous form outside of the floc which shortens diffusion distances.

Figure 5 shows a comparison of processes types and the importance of diffusion effects versus maximum rates. For systems operating at or near maximum rates the impact of $K_{S}$ is not significant and diffusion is not important. However, for treatment processes where substrate concentrations are low it is important to consider shifting to variable extant $K_{S}$ values or explicitly modelling the effects of diffusion. Additionally it is important to consider diffusion effects or variable extant $K_{S}$ for systems where biomass is moved from high rate to low rate systems such as the case when waste activated sludge from a high-rate, sidestream treatment facility is used to bioaugment a mainstream system (e.g. Wett, 2011).

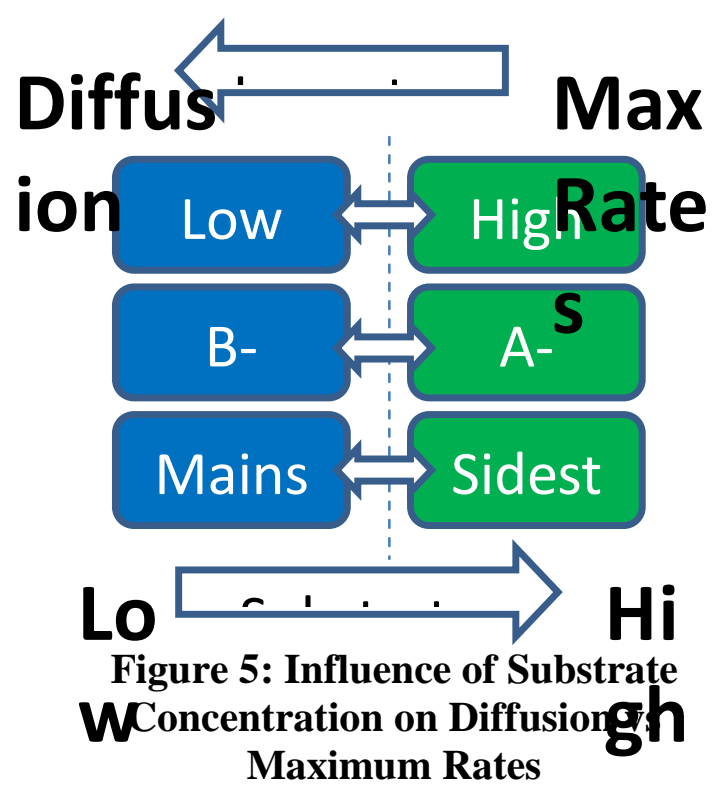

Variable $K_{S}$ is an important consideration wherever low substrate concentrations are important such as facilities with low effluent limits, systems with competing organisms (e.g. anammox vs NOBs in deammonification, Stinson et al. 2013) or models dependent on transitional species (e.g. $\mathrm{NO}_{2}$ for models of $\mathrm{N}_{2} \mathrm{O}$ emissions, Guo et al. 2012). 


\section{References}

Aksnes, D.L. and Cao, F.J. (2011) "Inherent and apparent traits in microbial nutrient uptake” Mar Ecol Prog Ser, Vol 440:41-51, 2011

Armstrong, R.A. (2008) "Nutrient uptake rate as a function of cell size and surface transporter density: A Michaelis-like approximation to the model of Pasciak and Gavis" Deep-Sea Research I, 55 (2008) 1311-1317

Fiksen, O, Follows, M.J., and Aksnes, D.L. (2013) "Trait-based models of nutrient uptake in microbes extend the Michaelis-Menten framework” Limnol. Oceanogr., 58(1), 2013, 193-202

Guo, L., Porro,J., Sharma, K. R., Amerlinck, Y., Benedetti, L., Nopens, I., Shaw, A., Van Hulle, S. W. H., Yuan, Z., and Vanrolleghem, P.A. (2012) "Towards a benchmarking tool for minimizing wastewater utility greenhouse gas footprints" Water Science and Technology, 66 (11) pp 2483-2495

Pasciak, W.J. and Gavis, J. (1974) “Transport limitation of nutrient uptake in phytoplankton” Limnology and Oceanography, 19 (6), pp881-888

Sin, G., Kaelin, D., Kampschreur, M.J., Takács, I., Wett, B., Gernaey, K.V., Rieger, L., Siegrist, H. and van Loosdrecht M.C.M. (2010) "Modelling nitrite in wastewater treatment systems: A discussion of different modelling concepts" Proceedings of WWTmod2010, Mont Sainte Anne, Quebec, Canada

Purtschert, I and Gujer, W (1999) "Population dynamics by methanol addition in denitrifying wastewater treatment plants" Water Science and Technology 39 (1) pp 43-50

Shaw, A.R., Takács, I., Pagilla, K.R. and Murthy, S. (2013) “A new approach to assess the dependency of extant halfsaturation coefficients on maximum process rates and estimate intrinsic coefficients" Water Research, 47 (16), 15 October 2013, pp 5986-5994

Stinson, B., Murthy, S., Bott, C., Wett, B, Al-Omari, A., Bowden, G., Mokhyerie, Y., and De Clippeleir, H. (2013) "Roadmap Toward Energy Neutrality \& Chemical Optimization at Enhanced Nutrient Removal Facilities" Proceedings of WEF/IWA Nutrient Removal and Recovery 2013, Vancouver, BC, July 2013

Wett,B., Jimenez, J.A., Takács, I., Murthy, S., Bratby, J.R., Holm, N.C., and Rönner-Holm, S.G.E. (2011) "Models for nitrification process design: one or two AOB populations?" Water Science \& Technology, 64(3), 2011

Wett, B., Takács, I., Batstone, D., Wilson, C. and Murthy, S. (2012) “Anaerobic model for high-loaded or hightemperature digestion- additional pathway of acetate oxidation” Proceedings of WWTmod2012, Mont St Anne, Quebec, Canada.

Wilson, C.A., Novak, J., Takács, Wett, B. and Murthy, S. (2012) "The kinetics of process dependent ammonia inhibition of methanogenesis from acetic acid" Water Research 46 (2012) 6247-6256 


\title{
Evaluating two concepts for the modelling of biological denitrification
}

\author{
Yuting Pan ${ }^{1}$, Bing-Jie Ni ${ }^{1}$, Huijie $\mathrm{Lu}^{2}$, Kartik Chandran ${ }^{3}$, David Richardson ${ }^{1,4}$, Zhiguo Yuan ${ }^{1}$ * \\ ${ }^{1}$ Advanced Water Management Centre (AWMC), The University of Queensland, St Lucia, Brisbane, \\ QLD 4072, Australia \\ ${ }^{2}$ Department of Civil and Environmental Engineering, University of Illinois-Urbana Champaign, \\ Urbana, IL 61801, USA \\ ${ }^{3}$ Department of Earth and Environmental Engineering, Columbia University, New York, NY 10027, \\ USA \\ ${ }^{4}$ Centre for Molecular Structure and Biochemistry (CMSB), School of Biological Sciences, University \\ of East Anglia, Norwich, NR4 7TJ, UK
}

\begin{abstract}
The accumulation of the denitrification intermediates in wastewater treatment can be highly undesirable, since both nitrite and $\mathrm{NO}$ are known to be toxic to bacteria, and $\mathrm{N}_{2} \mathrm{O}$ is a potent greenhouse gas and an ozone depleting substance. The four steps in denitrification could exert influence on each other through electron competition, leading to denitrification intermediates accumulation. To date, two distinct concepts for the modelling of the four-step denitrification process have been proposed, with one of them adopting the "directly coupling" and the other adopting the "indirectly coupling" approach in linking the carbon oxidation and nitrogen oxides reduction processes. In this study, these two models are examined and compared based on their ability to describe the experimental denitrification dynamics reported in literature. The modelling results show that the "indirectly coupling approach" could predict all the data from the three cases studied, while the "directly coupling approach" was able to reproduce two of the three datasets. The results suggest that separating the kinetics of carbon oxidation from the nitrogen oxides reduction is advantageous in modelling the electron competition in denitrification.
\end{abstract}

Keywords

denitrification; modelling; electron competition; nitrous oxide; nitrate; nitrite

\section{INTRODUCTION}

Denitrification is an important part of the global nitrogen cycle. Nitrate reduction consists of four consecutive reduction steps, with nitrite $\left(\mathrm{NO}_{2}{ }^{-}\right)$, nitric oxide $(\mathrm{NO})$ and nitrous oxide $\left(\mathrm{N}_{2} \mathrm{O}\right)$ as three obligatory intermediates (Zumft 1997). Each reduction step is catalysed by one or more specific reductase enzymes, namely nitrate reductase (Nar), nitrite reductase (Nir), NO reductase (Nor) and $\mathrm{N}_{2} \mathrm{O}$ reductase (Nos). In the wastewater treatment process, denitrification is a key process to remove the nitrogen pollutants from wastewater. Tremendous efforts have been made to optimise the performance of this process, including avoiding the accumulation of reaction intermediates.

The accumulation of the denitrification intermediates can be highly undesirable. For example, nitrite and NO are known to be toxic to bacteria (Schulthess R. V. 1995, Tan et al. 2008), while $\mathrm{N}_{2} \mathrm{O}$ is a potent greenhouse gas with a 300 -fold stronger radiative force than carbon dioxide, and is also a primary ozone depleting substance in the 21 century (IPCC 2007, Ravishankara et al. 2009). Previous work clearly demonstrated that the four denitrification steps could exert influence on each other through electron competition, leading to denitrification intermediates accumulation (Pan et al. 2013a, Schalk-Otte et al. 2000). The fundamental cell physiology responsible for the electron competition phenomenon is that all denitrifying enzymes require electrons from a common source, the ubiquinol pool of the respiratory electron transport chain (Richardson et al. 2009). 
Mathematical modelling has been widely applied to predict nitrogen removal during wastewater treatment (Henze et al. 2000). Previous modelling efforts have primarily focussed on the prediction of the removal of nitrate, and in some case nitrite as well. However, it is increasingly recognised that $\mathrm{NO}$ and $\mathrm{N}_{2} \mathrm{O}$ accumulation should also be modelled, especially due to their potent and vicious influence on atmosphere. This can be achieved through modelling denitrification as a four-step process that use nitrate, nitrite, nitric oxide, and nitrous oxide, respectively, as the terminal electron acceptor (Hiatt and Grady 2008, Schulthess and Gujer 1996, Vonschulthess et al. 1994). With each step being modelled with individual, reaction-specific kinetics, the accumulation of nitrite, NO and $\mathrm{N}_{2} \mathrm{O}$ can be, theoretically, predicted.

To date, two distinct concepts have been proposed in the development of multi-step denitrification models, with their structure schemes shown in Figure 1.

Model A: the "direct coupling approach"(Hiatt and Grady 2008), with which the carbon oxidation and nitrogen reduction processes are directly coupled in the model. This type of model describes each of the four steps as a separate and independent oxidation-reduction reaction (Figure 1a), with the kinetics of each step modelled according to the reduction reaction kinetics.

Model B: the "indirect coupling approach"(Pan et al. 2013b), with which the carbon oxidation and nitrogen reduction processes are indirectly coupled. Electron carriers are introduced as a new component in this model to link carbon oxidation to nitrogen oxides reduction (Figure 1b). In this way, the reactions can be regulated by both the reduction and the oxidation processes.

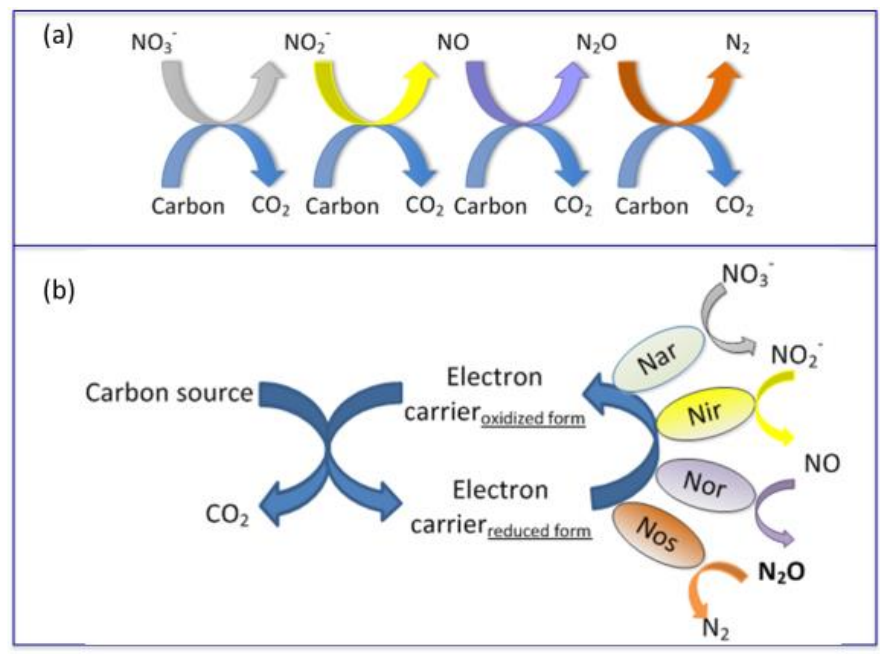

Figure 1. Simplified reaction schemes used in the two 4-step denitrification models evaluated in this study: Model A - Using the "direct coupling approach" to model the carbon oxidation and nitrogen reduction processes during denitrification; Model B - Using the "indirect coupling approach" to model the carbon oxidation and nitrogen reduction processes during denitrification.

It is of importance to know the prediction ability of these two different types of models, by conducting parallel comparison with existing data reported for different cultures and under different conditions. However, such comparison has not been done to date. Therefore, the aim of this work is to reveal how the structures of the two models presented in Figure 1 would affect their ability to reproduce experimental data reported in literature. Three distinctive denitrifying cultures were used 
in this examination, which included one pure culture (Paracoccus denitrijkans (N.C.1.B. 8944)) and two mixed denitrifying cultures fed with acetate and methanol, respectively. In particular, their ability in predicting electron competition during denitrification is assessed. The findings are expected to provide guidance to both future experimental studies and modelling practice aimed to get better understanding of denitrification.

\section{MATERIALS AND METHODS}

\section{Mathematical models for denitrification}

The kinetic and stoichiometric matrices describing the nitrogen reduction and the carbon oxidation processes for the two mathematical models are presented in Table 1. Nomenclature for all state variables used slightly differs from the original publications. We employ the following symbols for concentration: heterotrophic biomass $(X)$, nitrate $\left(\mathrm{S}_{\mathrm{NO} 3}\right)$, nitrite $\left(\mathrm{S}_{\mathrm{NO} 2}\right)$, nitric oxide $\left(\mathrm{S}_{\mathrm{NO}}\right)$, nitrous oxide $\left(\mathrm{S}_{\mathrm{N} 2 \mathrm{O}}\right)$, readily biodegradable carbon source $\left(\mathrm{S}_{\mathrm{S}}\right)$, reduced form of electron carriers $\left(\mathrm{S}_{\mathrm{Mred}}\right)$, oxidized form of electron carriers $\left(\mathrm{S}_{\mathrm{Mox}}\right)$. Other processes involved in denitrification, such as death and lysis of heterotrophs, hydrolysis of particulate organic nitrogen are included in both models with standard ASM kinetic expressions and parameter values taken from published literature (Hiatt and Grady 2008, Ni et al. 2011, Schulthess and Gujer 1996). Table 2 listed the definitions, values and units of the parameters used in the two models.

As show in Table 1, in Model A, the reduction of a nitrogen oxide compound (e.g. nitrate) and the oxidation of organic carbon are "directly coupled" in a single oxidation-reduction reaction with a stoichiometric relationship obtained through electron balance. However, in Model B, the carbon oxidation process $\left(R_{1}\right)$ is decoupled from the nitrogen reduction processes $\left(R_{2}\right.$ to $\left.R_{5}\right)$. Electron carriers $\left(\mathrm{S}_{\mathrm{Mred}}\right.$ and $\left.\mathrm{S}_{\mathrm{Mox}}\right)$ are introduced as a new component in this model to link carbon oxidation and nitrogen oxides reduction.

\section{Testing the predictive abilities of the models}

Experimental data from three cases (KuČEra et al. 1983, McMurray 2008, Pan et al. 2012) concerning denitrification dynamics were used for testing the predictive abilities of the two mathematical models.

Case 1: The nitrogen conversion dynamics by a pure denitrifying culture of Paracoccus denitrijkans (N.C.1.B. 8944) were measured in two batch tests by KuČEra et al. (1983). In the first batch test, nitrate was added to a concentration of $14 \mathrm{mg} \mathrm{N} / \mathrm{L}$ at the beginning of the tests, followed by nitrite addition to around $5 \mathrm{mg} \mathrm{N} / \mathrm{L}$ at 0.5 hour. In the second batch test, the nitrate reduction rate was measured under the following condition: 1) with only $\mathrm{NO}_{3}^{-}$being present; 2) with $\mathrm{NO}_{2}{ }^{-}$being present simultaneously with $\mathrm{NO}_{3}^{-} ; 3$ ) with $\mathrm{N}_{2} \mathrm{O}$ being present with $\mathrm{NO}_{3}{ }^{-}$; 4) with $\mathrm{NO}_{2}^{-} \& \mathrm{~N}_{2} \mathrm{O}$ \& antimycin (an inhibitor for nitrite and $\mathrm{N}_{2} \mathrm{O}$ reduction) being present with $\mathrm{NO}_{3}{ }^{-}$. Glucose was used as the carbon source and was in excess throughout all the tests. They found that the extent of electron flow to nitrate depends on the activity of electron flows to nitrite and $\mathrm{N}_{2} \mathrm{O}$. By quantitatively evaluating the extent of electron flows, they revealed that the nitrate, nitrite and $\mathrm{N}_{2} \mathrm{O}$ reductases competed for a constant limited flow of redox equivalents supplied from dehydrogenases, although the external carbon source (glucose) was in excess.

Case 2: McMurray (2008) investigated denitrification dynamics by a full-scale activated sludge fed with acetate. In a batch test, nitrate, nitrite were added at the beginning of the test, to initial 
concentrations around $5.2 \mathrm{mg} \mathrm{N} / \mathrm{L}, 8.9 \mathrm{mg} \mathrm{N} / \mathrm{L}$, respectively. Acetate was also added at the same time, and was present in excess during the entire experiment. The conversion of nitrate, nitrite and acetate was monitored throughout the test, along with the production rate of nitrogen gas $\left(\mathrm{N}_{2}\right)$.

Case 3: By studying a enriched denitrifying culture fed with methanol, Pan et al. (2013a) carried out extensive investigations to study electron competition during denitrification. Four batch tests were chosen in this paper to evaluate the two types of models, which include 1) nitrate being added as the sole externally-supplied electron acceptor; 2) nitrite being added as the sole externallysupplied electron acceptor; 3) $\mathrm{N}_{2} \mathrm{O}$ being added as the sole externally-supplied electron acceptor; 4) nitrate, nitrite and $\mathrm{N}_{2} \mathrm{O}$ being added simultaneously. The initial concentration of the nitrogen compounds were between 30 and $50 \mathrm{mg} \mathrm{N} / \mathrm{L}$. Methanol was in excess in all these four tests, with its initial concentration at approximately $300 \mathrm{mg} \mathrm{N} / \mathrm{L}$.

Parameter estimation were performed with AQUASIM for aquatic systems (Reichert et al. 1995). Not all the parameters were identifiable from the experimental data; however, some of the parameters have been extensively studied and well established in previous studies (Hiatt and Grady 2008, Pan et al. 2013b). Therefore, literature values were adopted for these parameters (see Table $2)$. Only parameters specific for each model $\left(\eta_{g 1}, \eta_{g 2}, \eta_{g 4}\right.$ for Model A and $r_{C O D \text {, max }}, K_{M r e d, 1}$, $K_{\text {Mred }, 2}$ and $K_{\text {Mred,4 }}$ for Model B) were calibrated in this modelling practice, with their values presented in Table 2 as well.

\section{RESULTS}

\section{Evaluation of the Mathematical Models: Case 1}

In the first case, the two denitrification models in Figure 1 were evaluated based on their ability to capture the nitrogen conversion by a pure culture of Paracoccus denitrijkans (N.C.1.B. 8944), reported in KuČEra et al. (1983). The influence of nitrite and $\mathrm{N}_{2} \mathrm{O}$ on nitrate reduction was extensively investigated in this study. The experimental data along with the model predictions are presented in Figure 2.

In the first batch test, after nitrite was added externally at 0.5 hour, the nitrate reduction rate was significantly lowered (Figure 2 (a) \& (b), phases $1 \& 2$ ). However, when nitrite depleted, the activity of nitrate reductase recovered to its original level (Figure 2 (a) \& (b), phase 3). Model B was able to reproduce both the nitrate and nitrite profiles (Figure 2b). In contrast, Model A failed to predict the dynamic nitrate profile although the nitrite profile was correctly reproduced (Figure 2a).

The results from the second batch are shown in Figure $2 \mathrm{c}$ and $2 \mathrm{~d}$. With the initial nitrate reduction rate, measured with nitrate as the sole externally-supplied electron acceptor, being accounted as $100 \%$, the nitrate reduction rate reduced to $32 \%$ after the nitrite addition. The value lowered even further to $6 \%$ after the addition of $\mathrm{N}_{2} \mathrm{O}$. However, when $\mathrm{N}_{2} \mathrm{O}$, nitrite and antimycin (a chemical which inhibits nitrite and $\mathrm{N}_{2} \mathrm{O}$ reduction) were added together, the nitrate reduction rate increased to 233\%. Model A completely failed to predict the experimentally observed variations in the nitrate reduction rate after the addition of nitrite, $\mathrm{N}_{2} \mathrm{O}$ and antimycin (Figure 2c). The results clearly indicate that Model A is not able to reflect the influence of nitrite and $\mathrm{N}_{2} \mathrm{O}$ on nitrate reduction. On the contrary, as shown in Figure 2d, Model B successfully captured the influence of nitrite, $\mathrm{N}_{2} \mathrm{O}$ and antimycin on nitrate reduction, with $38 \%$ of nitrate reduction activity left after nitrite addition (in comparison to the $32 \%$ experimentally observed), $7 \%$ left after $\mathrm{N}_{2} \mathrm{O}$ addition (in comparison the experimental data of $6 \%$ ). With the inhibition of nitrite and $\mathrm{N}_{2} \mathrm{O}$ reduction using antimycin, the 
model also correctly predicted the substantial increase $(240 \%$ vs. the experimentally observed $233 \%$ ) in the nitrate reduction rate (Figure 2d).
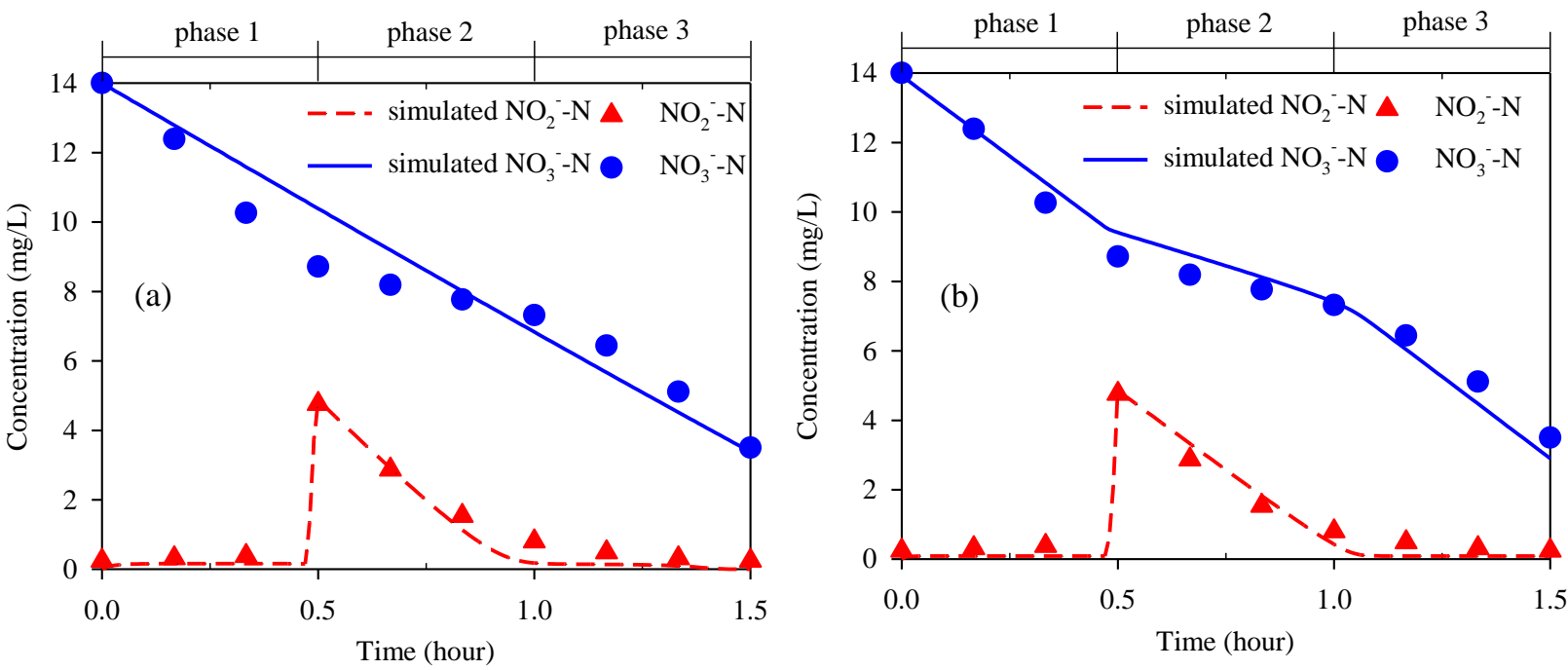

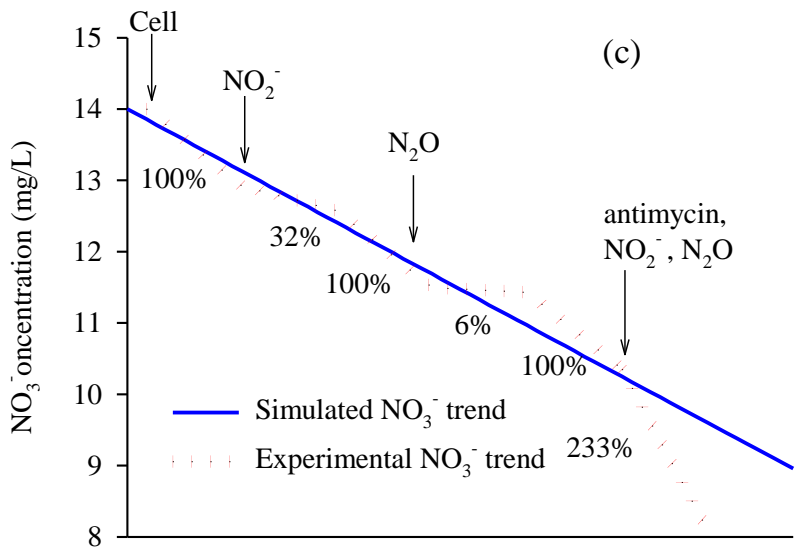

Time (hour)

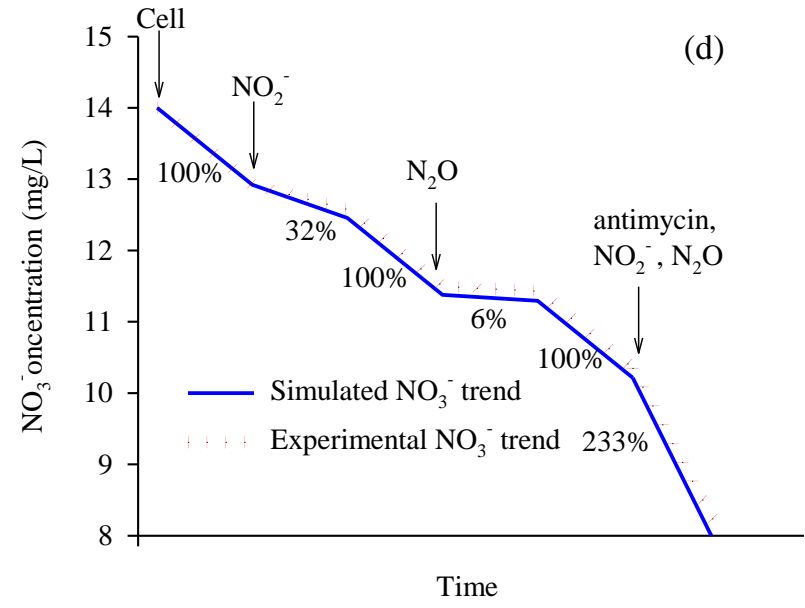

Figure 2: Experimental results and model predictions for Case 1 (KuČEra et al. 1983). (a) \& (c) Evaluation of Model A; (b) \& (d) - Evaluation of Model B.

\section{Evaluation of the Mathematical Models: Case 2}

In the second case, the denitrification dynamics by a full-scale activated sludge fed with acetate was studied by McMurray (2008). The experimental data along with the model predictions are presented in Figure 3. While not shown in the figure, no $\mathrm{N}_{2} \mathrm{O}$ accumulation was observed during the entire experiment. Both models predicted negligible $\mathrm{N}_{2} \mathrm{O}$. 

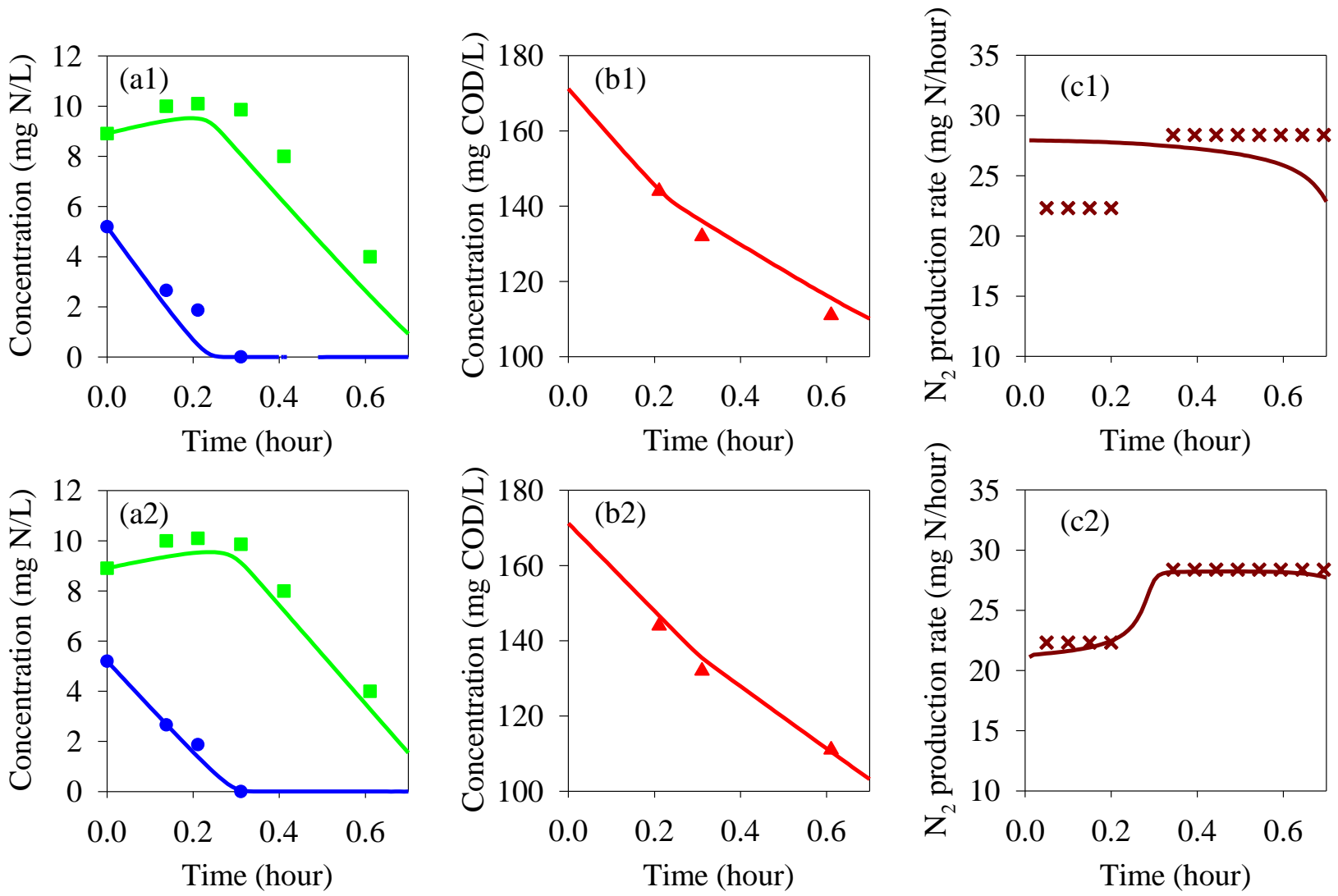

- $\mathrm{NO}_{2}$

- $\mathrm{NO}_{3}{ }^{-}$

$\triangle \mathrm{COD} \times \mathrm{N}_{2}$

Figure 3: Experimental results and model predictions in Case 2 (McMurray 2008): (a1), (b1) \& (c1) - Evaluation of Model A; (a2), (b2) \& (c2) - Evaluation of Model B

Nitrite accumulated during nitrate reduction, and declined after the depletion of nitrate (Figure 3 (a1) \& (a2)). COD was consumed during nitrate and nitrite reduction (Figure 3 (b1) \& (b2)). The $\mathrm{N}_{2}$ production was around $22 \mathrm{mg} \mathrm{N} / \mathrm{hour}$ when both nitrate and nitrite were present, and increased to around $28 \mathrm{mg} \mathrm{N} /$ hour when only nitrite was present.

Model A roughly captured the trend of nitrate and nitrite reduction (Figure 3 (a1)), and the trend of acetate (Figure 3 (b1)). However, the fit between the model predictions and experimental data was relatively poor. The consequence of the poor fit can be clearly seen from the incorrect prediction of the $\mathrm{N}_{2}$ production rate (Figure 3 (c1)). In comparison, Model B successfully reproduced all the data observed including the $\mathrm{N}_{2}$ data (Figure (a2), (b2) \& (c2)).

\section{Evaluation of the Mathematical Models: Case 3}

In the third case, the two denitrification models in Figure 1 were evaluated based on their ability to capture the nitrogen conversions by an enriched denitrifying culture fed with methanol as the carbon source (Pan et al. 2013a). The experimental data points along with the model predictions are presented in Figure 4. 

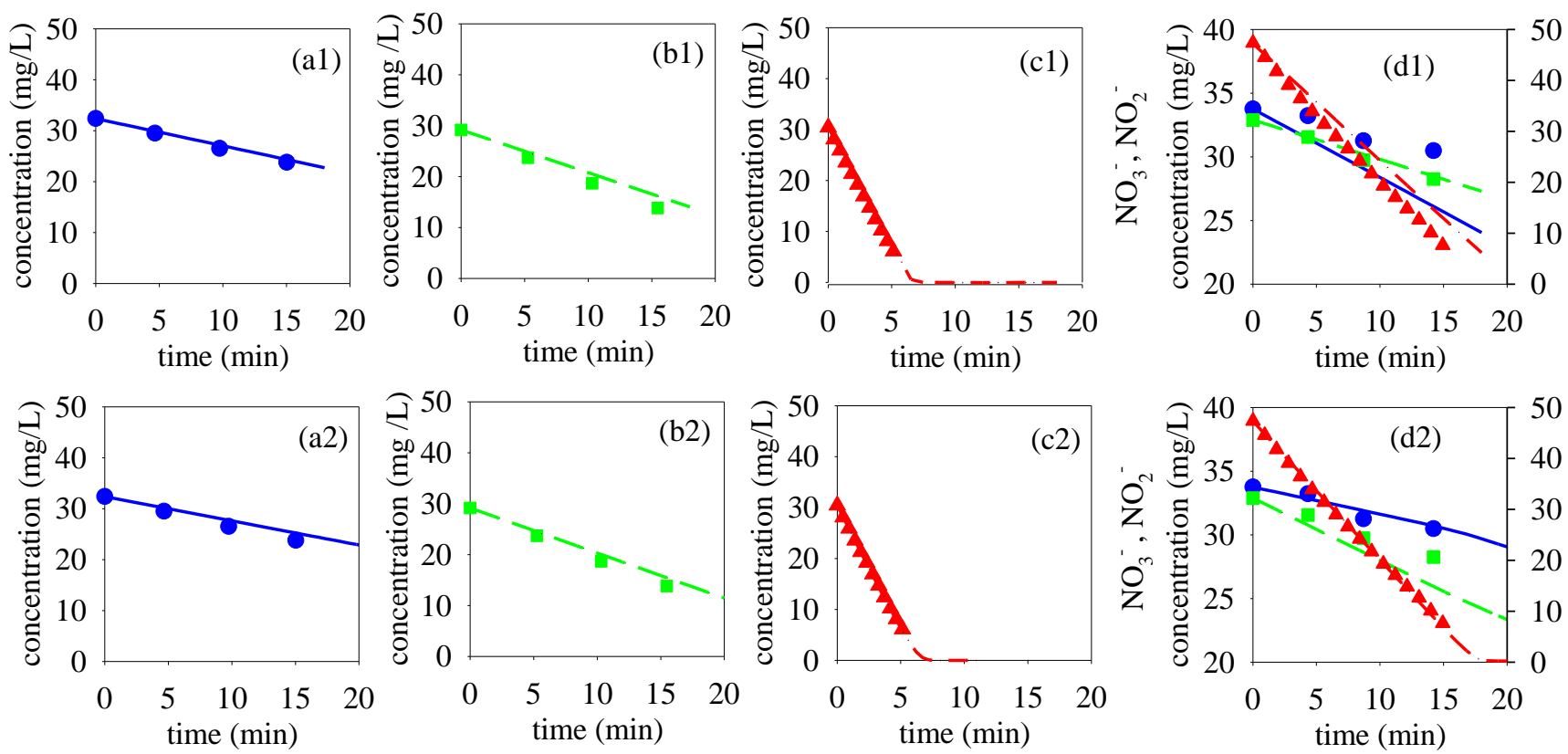

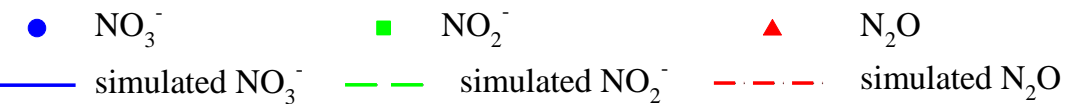

Figure 4: Experimental results and model predictions Case 3(Pan et al. 2013a): (a1) \& (b1) \& (c1) \& (d1) - Evaluation of Model A; (a2) \& (b2) \& (c2) \& (d2) - Evaluation of Model B

In the tests when only one nitrogen oxide specie was added (Figure 4 (a1) to (c1) and (a2) to (c2)), the nitrate, nitrite and $\mathrm{N}_{2} \mathrm{O}$ reduction rate was 45,74 and $370 \mathrm{mg} \mathrm{N} /(\mathrm{gVSS} \times \mathrm{h})$, respectively. However, when nitrate, nitrite and $\mathrm{N}_{2} \mathrm{O}$ were added simultaneously, the reduction rate of all the nitrogen oxides decreased, valued at 19, 39 and $256 \mathrm{mg} \mathrm{N} /(\mathrm{gVSS} \times \mathrm{h})$, respectively (Figure 4 (d1) and (d2)).

Generally, both Model A and Model B were able reproduce the nitrate, nitrite and $\mathrm{N}_{2} \mathrm{O}$ profiles in Figure 4 (a1) to (c1) and Figure 4 (a2) to (c2). However, Model A failed to reproduce the experimental results in Figure (d1). The predicted nitrate reduction rate is significantly higher than the predicted nitrite reduction rate, which is opposite to the experimental observation. In addition, the predicted $\mathrm{N}_{2} \mathrm{O}$ reduction rate is significantly lower than the experimentally observed $\mathrm{N}_{2} \mathrm{O}$ reduction rate. In comparison, Model $\mathrm{B}$ reproduced all experimental data reasonably well despite a slight mismatch between the model-predicted and experimentally observed nitrite data (Figure 4 $(\mathrm{d} 2))$.

\section{DISSCUSSION}

In this work, the two types of 4-step denitrification models were evaluated for their ability to predict denitrification dynamics in three cases. One common feature for all three cases is that interactions between the reduction of two or three nitrogen compounds during denitrification were observed (i.e. nitrate \& nitrite \& $\mathrm{N}_{2} \mathrm{O}$ in Case 1 and Case 3; nitrate \& nitrite in Case 2). Model $\mathrm{B}$ was able to describe the experimental data in all the three cases, while Model A failed to reproduce the experimental data in any of the three cases.

The question arising herein is why the two models performed differently. The answer to this question lies in their consideration of the electron competition process during denitrification. All 
the denitrifying enzymes derive their electrons from a common electron supply source, the ubiquinol pool of the respiratory electron transport chain (Richardson et al. 2009). The electron competition among the nitrogen reduction steps could happen if the electron supply rate becoming the rate limiting process during denitrification, leading to dynamic changes in the electron allocation and hence the nitrogen conversions. A closer examination of the experiment results of the three cases studied reveals that the electron competition process played a key role in determining the denitrification dynamics in all the cases studied. Below, we will comment on Case 1 in detail to illustrate the differences between the two models. Case 2 and Case 3 will be briefly discussed, further highlighting the key features of the two models.

For Case 1, electron supply was the rate limiting process in all the batch tests. This is evident from the fact the nitrate reduction rate increased by around $233 \%$ when both nitrite and $\mathrm{N}_{2} \mathrm{O}$ reduction was inhibited by antimycin (Figure 2d). Herein, the experimental results revealed that nitrate reductase did not achieve its maximum turnover rate even when only nitrate was added, due to the fact that the reduction of the continuously produced downstream denitrification intermediates (nitrite, $\mathrm{NO}, \mathrm{N}_{2} \mathrm{O}$ ) were deriving their electrons from the same electron source. Similarly, the reason for the decline of the nitrate reduction rate with nitrite or $\mathrm{N}_{2} \mathrm{O}$ addition (Figure $2 \mathrm{~d}$ ) was due to the electron competition between the different denitrification steps. In Model B, by modeling the carbon oxidation process (II- $R_{1}$ ) and the nitrogen reduction processes (II-R $\mathrm{R}_{2}$ to II- $\mathrm{R}_{5}$ ) separately, the model was able to predict both the electron supply rate and the electron consumption rate. The electron supply rate was reflected by II- $\mathrm{R}_{1}$, while the electron consumption rate was reflected by II$\mathrm{R}_{2}$ to II- $\mathrm{R}_{5}$. The relative ability of each denitrification step to compete for electrons was reflected by the calibration of $\mathrm{k}_{\text {Mred,1 }}, \mathrm{k}_{\mathrm{Mred}, 2}, \mathrm{k}_{\mathrm{Mred}, 3}, \mathrm{k}_{\mathrm{Mred}, 4}$ in Model $\mathrm{B}$. Therefore, the model predicts that the electron flow to nitrate reduction (II- $\mathrm{R}_{2}$ ) was affected by the electron flows to nitrite (II- $\left.\mathrm{R}_{3}\right)$ and $\mathrm{N}_{2} \mathrm{O}$ (II- $\mathrm{R}_{5}$ ). When nitrite reduction (II- $\mathrm{R}_{3}$ ) and $\mathrm{N}_{2} \mathrm{O}$ reduction (II- $\mathrm{R}_{5}$ ) were stopped, nitrate reduction (II- $\mathrm{R}_{2}$ ) received more electrons and thus a higher reduction rate was achieved. When external nitrite or nitrous oxide were added, the overall rate of nitrite reduction $\left(\right.$ II- $\left.\mathrm{R}_{3}\right)$ or $\mathrm{N}_{2} \mathrm{O}$ reduction (II- $\mathrm{R}_{5}$ ) increased due to higher substrate concentrations, leading to a smaller electron flow to nitrate reduction (II- $\mathrm{R}_{2}$ ). In comparison, in Model A, the carbon oxidation process is not modeled independently, but directly lumped into the four nitrogen reduction steps ( $I-R_{1}$ to $\left.I-R_{4}\right)$. Therefore, the structure of Model $\mathrm{A}$ is intrinsically unable to distinguish the electron flow regulation between the carbon oxidation and the four-step nitrogen reduction processes. Thus, a constant nitrate reduction rate was predicted by Model A when all the tests were used to calibrate the relevant parameters. This constant nitrate reduction rate is essentially a compromise of the model to simulate the nitrate dynamics observed in all the tests in Case 1. One possible argument might be that by adding an inhibition terms to describe nitrite and $\mathrm{N}_{2} \mathrm{O}$ inhibition on nitrate reduction (e.g. $\left(\frac{K_{N O 2}}{K_{N O 2}+S_{N O 2}}\right)\left(\frac{K_{N 2 O}}{K_{N 2 O}+S_{N 2 O}}\right)$ in I-R1, with $K_{N O 2}$ and $K_{N 2 O}$ defined as nitrite inhibition and $\mathrm{N}_{2} \mathrm{O}$ inhibition coefficient on nitrate reduction), Model A may be able to predict the nitrate reduction dynamics presented in Figure 2. This is proven to be infeasible. Even such terms are added, the 233\% nitrate reduction rate achieved after the addition of antimycin $\&$ nitrite $\& \mathrm{~N}_{2} \mathrm{O}$ would still not be predicted.

For Case 2, the nitrogen production rate, which essentially reflected the turnover rate of the nitrite reductase since there was no accumulation of $\mathrm{NO}$ or $\mathrm{N}_{2} \mathrm{O}$, increased after nitrate depletion (Figure 3 (c1), (c2)). This phenomenon indicates that there was electron competition between nitrate reduction and nitrite reduction when both of them were present. The competition was relaxed after the depletion of nitrate, resulting in a higher nitrite reduction rate (nitrogen production rate). Again, Model B captured the two distinctive nitrite reduction rates through regulating the electron flow to 
II- $R_{2}$ to II- $R_{5}$. Model A failed to do so since the nitrite reduction rate was determined by I- $R_{2}$, and the depletion of nitrate could not exert any influence to the nitrite reduction rate predicted by $\mathrm{I}_{2}$.

For Case 3, the decline of the reduction rates of nitrate, nitrite and $\mathrm{N}_{2} \mathrm{O}$ when all of them were added was due to electron competition (Figure 4 (d1) \& (d2)). Model B captured this trend through calibrating the relatively ability of the three steps to compete for electrons, reflected by $\mathrm{k}_{\text {Mred,1 }}$, $\mathrm{k}_{\text {Mred,2, }}, \mathrm{k}_{\text {Mred,4 }}$. The prediction by Model $\mathrm{A}$ accelerated the nitrate reduction rate $\left(\mathrm{I}-\mathrm{R}_{1}\right)$ and reduced the $\mathrm{N}_{2} \mathrm{O}$ reduction rate to give its best fit of all the batches. It is worth to note that, unlike Case 1 in which the carbon oxidation process was rate limiting in all the cases, nitrate or nitrite reduction in Case 3 was rate limiting by the turnover rate of nitrate reductase or nitrite reductase rather than by the electron supply rate (Pan et al. 2013a) (Figure 4 (a1) \& (a2), (b1) \& (b2)). Thus, the electron competition process did not play a key role in determining the denitrification dynamics when only nitrate or nitrite was present.

To date, the presentence of denitrification intermediates during wastewater treatment has been widely reported. The ability of models to predict electron competition during the denitrification process enables the model to predict denitrification intermediates. It has been revealed by this work that Model B could give satisfactory prediction of the dynamics of the intermediates during denitrification. Further investigation should be made by upgrading the current ASM models widely used by wastewater treatment plants by incorporating the concept of the "indirect coupling approach".

\section{CONCLUSIONS}

In this work, two distinct mathematical model structures were compared for their ability to predict nitrogen conversion dynamics in one pure culture and two mixed culture studies. It was demonstrated that the model based on the indirect coupling of the carbon oxidation and nitrogen oxides reduction processes was able to describe the experimental data in all three cases studied, while the ASMN model, directly couples the carbon oxidation and nitrogen oxides reduction processes, failed to describe the experimental data. The results suggest that the 'indirect coupling' approach is advantageous over the 'direct coupling' approach in describing the electron competition between the four steps of denitrification and in predicting the accumulation of denitrification intermediates.

\section{ACKNOWLEDGMENT}

This study was funded by the Australian Research Council, Western Australia Water Corporation and Melbourne Water Corporation through projects LP0991765 and DP0987204. Bing-Jie Ni acknowledges the supports of Australian Research Council Discovery Early Career Researcher Award (DE130100451). 


\section{WWTmod}

\section{Pan et al.}

Table 1: process matrices for the two types of denitrification models evaluated in this study Model components

\begin{tabular}{llllllllll} 
& \multicolumn{10}{c}{} & & & \\
\cline { 2 - 6 } & Processes & $\mathrm{S}_{\mathrm{NO} 3}$ & $\mathrm{~S}_{\mathrm{NO} 2}$ & $\mathrm{~S}_{\mathrm{NO}}$ & $\mathrm{S}_{\mathrm{N} 2 \mathrm{O}}$ & $\mathrm{S}_{\mathrm{S}}$ & $\mathrm{S}_{\mathrm{Mox}}$ & $\mathrm{S}_{\mathrm{Mred}}$ & $\mathrm{X}$
\end{tabular}

Model A - the "direct coupling appraoch" adapted from Hiatt and Grady (2008)

\begin{tabular}{|c|c|c|c|c|c|c|c|}
\hline $\mathrm{I}-\mathrm{R}_{1}$ & $-A$ & $+\mathrm{A}$ & & & $-1 /\left(Y_{H} \cdot \eta_{Y}\right)$ & 1 & $R 1=\mu_{H} \cdot \eta_{g 1} \cdot X\left(\frac{S_{S}}{K_{S 1}+S_{S}}\right)\left(\frac{S_{N O 3}}{K_{N O 3}^{H B}+S_{N O 3}}\right)$ \\
\hline $\mathrm{I}-\mathrm{R}_{2}$ & & $-B$ & $+\mathrm{B}$ & & $-1 /\left(Y_{H} \cdot \eta_{Y}\right)$ & 1 & $R 2=\mu_{H} \cdot \eta_{g 2} \cdot X\left(\frac{S_{S}}{K_{S 2}+S_{S}}\right)\left(\frac{S_{N O 2}}{K_{N O 2}^{H B}+S_{N O 2}}\right)\left(\frac{K_{N O, 2}}{K_{N O, 2}+S_{N O}}\right)$ \\
\hline $\mathrm{I}-\mathrm{R}_{3}$ & & & $-B$ & $+\mathrm{B}$ & $-1 /\left(Y_{H} \cdot \eta_{Y}\right)$ & 1 & $R 3=\mu_{H} \cdot \eta_{g 3} \cdot X\left(\frac{S_{S}}{K_{S 3}+S_{S}}\right)\left(\frac{S_{N O}}{K_{N O}^{H B}+S_{N O}+S_{N O}^{2} / K_{N O, 3}}\right)$ \\
\hline $\mathrm{I}-\mathrm{R}_{4}$ & & & & $-B$ & $-1 /\left(Y_{H} \cdot \eta_{Y}\right)$ & 1 & $R 2=\mu_{H} \cdot \eta_{g 4} \cdot X\left(\frac{S_{S}}{K_{S 4}+S_{S}}\right)\left(\frac{S_{N 2 O}}{K_{N 2 O}^{H B}+S_{N 2 O}}\right)\left(\frac{K_{N O, 4}}{K_{N O, 4}+S_{N O}}\right.$ \\
\hline
\end{tabular}

Model B - the "indirect coupling approach" adapted from Pan et al.(2013b)

\begin{tabular}{|c|c|c|c|c|c|c|c|c|c|}
\hline II-R ${ }_{1}$ & & & & & -1 & $-\left(1-Y_{H}\right)$ & $1-Y_{H}$ & $Y_{H}$ & $R 1=r_{C O D, \max } X\left(\frac{S_{S}}{K_{S}+S_{S}}\right)\left(\frac{S_{M o x}}{K_{M o x}+S_{M o x}}\right)$ \\
\hline II-R ${ }_{2}$ & -1 & 1 & & & & 1 & -1 & & $R 2=r_{N O 3, \max } X\left(\frac{S_{N O 3}}{K_{N O 3}^{H B}+S_{N O 3}}\right)\left(\frac{S_{\text {Mred }}}{K_{\text {Mred }, 1}+S_{\text {Mred }}}\right)$ \\
\hline II-R ${ }_{3}$ & & -1 & 1 & & & $\frac{1}{2}$ & $-\frac{1}{2}$ & & $R 3=r_{N O 2, \max } X\left(\frac{S_{N O 2}}{K_{N O 2}^{H B}+S_{N O 2}}\right)\left(\frac{S_{\text {Mred }}}{K_{\text {Mred }, 2}+S_{\text {Mred }}}\right)$ \\
\hline II-R 4 & & & -1 & $\frac{1}{2}$ & & $\frac{1}{2}$ & $-\frac{1}{2}$ & & $R 4=r_{N O, \max } X\left(\frac{S_{N O}}{K_{N O}^{H B}+S_{N O}}\right)\left(\frac{S_{\text {Mred }}}{K_{\text {Mred }, 3}+S_{\text {Mred }}}\right)$ \\
\hline II-R ${ }_{5}$ & & & & -1 & & 1 & -1 & & $R 5=r_{N 2 O, \max } X\left(\frac{S_{N 2 O}}{K_{N 2 O}^{H B}+S_{N 2 O}}\right)\left(\frac{S_{\text {Mred }}}{K_{\text {Mred }, 4}+S_{\text {Mred }}}\right.$ \\
\hline
\end{tabular}

$\mathrm{A}=\frac{1-Y_{H} \cdot \eta_{Y}}{1.143 \cdot Y_{H} \cdot \eta_{Y}}, \mathrm{~B}=\frac{1-Y_{H} \cdot \eta_{Y}}{0.571 \cdot Y_{H} \cdot \eta_{Y}}, \mathrm{~S}_{\mathrm{Mred}}+\mathrm{S}_{\mathrm{Mox}}=\mathrm{C}_{\mathrm{tot}}$ 


\section{WWTmod}

Pan et al.

Table 2: Best-fit parameters of the two models describing denitrification dynamics

\begin{tabular}{|c|c|c|c|c|c|}
\hline Parameter & Definition & Case 1 & Case 2 & Case 3 & Source \\
\hline \multicolumn{6}{|c|}{ Model A - the "direct coupling approach" adapted from Hiatt and Grady (2008) } \\
\hline$\mu_{H}$ & Maximum specific growth rate $\left(\right.$ hour $^{-1}$ ) & 0.26 & 0.26 & 0.26 & Hiatt and Grady (2008) \\
\hline$Y_{H}$ & Heterotrophic yield (g COD/g COD) & $0.6^{\mathrm{a}}$ & $0.6^{\mathrm{a}}$ & $0.5^{\mathrm{b}}$ & $\begin{array}{l}\text { a: Hiatt and Grady (2008) } \\
\text { b: Pan et al. (2013b) }\end{array}$ \\
\hline$\eta_{Y}$ & Anoxic yield factor (dimensionless) & 0.9 & 0.9 & 0.9 & Hiatt and Grady (2008) \\
\hline$\eta_{g 1}$ & Anoxic growth factor, R1 (dimensionless) & 0.029 & 0.14 & 0.18 & Estimated \\
\hline$\eta_{g 2}$ & Anoxic growth factor, R2 (dimensionless) & 0.024 & 0.058 & 0.15 & Estimate \\
\hline$\eta_{g 3}$ & Anoxic growth factor, R3 (dimensionless) & 0.35 & 0.35 & 0.35 & Hiatt and Grady (2008) \\
\hline$\eta_{g 4}$ & Anoxic growth factor, R4 (dimensionless) & $0.35^{\mathrm{a}}$ & $0.35^{\mathrm{a}}$ & $0.81^{\mathrm{b}}$ & $\begin{array}{l}\text { a: Hiatt and Grady (2008) } \\
\text { b: Estimated }\end{array}$ \\
\hline$K_{S 1}$ & Affinity constant for Ss, R1 (mgCOD/L) & 20 & 20 & 20 & Hiatt and Grady (2008) \\
\hline$K_{S 2}$ & Affinity constant for Ss, R2 (mgCOD/L) & 20 & 20 & 20 & Hiatt and Grady (2008) \\
\hline${ }^{K_{S 3}}$ & Affinity constant for Ss, R3 (mgCOD/L) & 20 & 20 & 20 & Hiatt and Grady (2008) \\
\hline${ }^{K_{S 4}}$ & Affinity constant for $\mathrm{Ss}, \mathrm{R} 4$ (mgCOD/L) & 40 & 40 & 40 & Hiatt and Grady (2008) \\
\hline$K_{N O 3}^{H B}$ & Affinity constant for nitrate-nitrogen (mg N/L) & 0.2 & 0.2 & 0.2 & Hiatt and Grady (2008) \\
\hline$K_{\mathrm{NO} 2}^{\mathrm{HB}}$ & Affinity constant for nitrite-nitrogen (mg N/L) & 0.2 & 0.2 & 0.2 & Hiatt and Grady (2008) \\
\hline$K_{N O}^{H B}$ & Affinity constant for nitric oxide-nitrogen (mg N/L) & 0.05 & 0.05 & 0.05 & Hiatt and Grady (2008) \\
\hline$K_{N 2 O}^{H B}$ & Affinity constant for nitrous oxide-nitrogen (mg N/L) & 0.05 & 0.05 & 0.05 & Hiatt and Grady (2008) \\
\hline$K_{N O, 2}$ & NO inhibition coefficient, R2 (mg N/L) & 0.5 & 0.5 & 0.5 & Hiatt and Grady (2008) \\
\hline$K_{N O, 3}$ & NO inhibition coefficient, R3 (mg N/L) & 0.3 & 0.3 & 0.3 & Hiatt and Grady (2008) \\
\hline$K_{N O, 4}$ & NO inhibition coefficient, R4 (mg N/L) & 0.075 & 0.075 & 0.075 & Hiatt and Grady (2008) \\
\hline \multicolumn{6}{|c|}{ Type II Model- the "indirect coupling approach" adaped from Pan et al.(2013b) } \\
\hline$r_{C O D, \max }$ & Maximum carbon source oxidation rate (mmol COD/(L*hour) & 0.064 & 0.090 & 0.34 & Estimated \\
\hline$r_{N O 3, \max }$ & Maximum nitrate reduction rate $\left(\mathrm{mmol} \mathrm{NO}_{3}{ }^{-} / \mathrm{mmol}\right.$ biomass*hour) & 0.045 & 0.045 & 0.045 & Pan et al. (2013b) \\
\hline$r_{N O 2, \max }$ & Maximum nitrite reduction rate ( $\mathrm{mmol} \mathrm{NO}_{2}^{-} / \mathrm{mmol}^{\text {biomass* }}{ }^{*}$ hour) & 0.059 & 0.059 & 0.059 & Pan et al. (2013b) \\
\hline
\end{tabular}




\section{WWTmod}

Pan et al.

\begin{tabular}{|c|c|c|c|c|c|}
\hline$r_{N O, \max }$ & Maximum nitric oxide reaction rate (mmol NO/mmol biomass*hour) & 0.56 & 0.56 & 0.56 & Pan et al. (2013b) \\
\hline$r_{N 2 O, \max }$ & $\begin{array}{l}\text { Maximum nitrous oxide reaction rate }\left(\mathrm{mmol}_{2} \mathrm{O} / \mathrm{mmol}\right. \\
\text { biomass*hour) }\end{array}$ & 0.23 & 0.23 & 0.23 & Pan et al. (2013b) \\
\hline$K_{S}$ & Affinity constant for Ss ( mmol COD/L) & 0.1 & 0.1 & 0.1 & Pan et al. (2013b) \\
\hline$K_{N O 3}^{H B}$ & Affinity constant for nitrate-nitrogen $\left(\mathrm{mmol} \mathrm{NO}_{3}{ }^{-} / \mathrm{L}\right)$ & 0.018 & 0.018 & 0.018 & Pan et al. (2013b) \\
\hline$K_{N O 2}^{H B}$ & Affinity constant for nitrite-nitrogen $\left(\mathrm{mmol} \mathrm{NO}_{2}^{-} / \mathrm{L}\right)$ & 0.0041 & 0.0041 & 0.0041 & Pan et al. (2013b) \\
\hline$K_{N O}^{H B}$ & Affinity constant for nitric oxide-nitrogen (mmol NO/L) & 0.000011 & 0.000011 & 0.000011 & Pan et al. (2013b) \\
\hline$K_{N 2 O}^{H B}$ & Affinity constant for nitrous oxide-nitrogen $\left(\mathrm{mmol} \mathrm{N}_{2} \mathrm{O} / \mathrm{L}\right)$ & 0.0025 & 0.0025 & 0.0025 & Pan et al. (2013b) \\
\hline$K_{M o x}$ & Affinity constant for $S_{M o x}, R 1 \mathrm{mmol} /(\mathrm{mmol}$ biomass $)$ & 0.0001 & 0.0001 & 0.0001 & Pan et al. (2013b) \\
\hline$K_{\text {Mred,1 }}$ & Affinity constant for $S_{\text {Mred }}, \mathrm{R} 2$ & $0.0015^{\mathrm{a}}$ & $0.0068^{\mathrm{a}}$ & $0.0046^{\mathrm{b}}$ & Estimated \\
\hline$K_{\text {Mred,2 }}$ & Affinity constant for $S_{\text {Mred }}, \mathrm{R} 3$ & $0.00058^{\mathrm{a}}$ & $0.016^{\mathrm{a}}$ & $0.00040^{\mathrm{b}}$ & Estimated \\
\hline$K_{\text {Mred,3 }}$ & Affinity constant for $S_{M r e d}, R 4$ & 0.000010 & 0.000010 & 0.000010 & Pan et al. (2013b) \\
\hline$K_{\text {Mred }, 4}$ & Affinity constant for $S_{\text {Mred }}, \mathrm{R} 5$ & $0.00024^{\mathrm{a}}$ & $0.0032^{\mathrm{b}}$ & $0.0032^{b}$ & $\begin{array}{l}\text { a: Estimated } \\
\text { b: Pan et al. (2013b) }\end{array}$ \\
\hline$Y_{H}$ & Heterotrophic yield & $0.6^{\mathrm{a}}$ & $0.6^{\mathrm{a}}$ & $0.5^{\mathrm{b}}$ & $\begin{array}{l}\text { a: Hiatt and Grady (2008) } \\
\text { b: Pan et al. (2013b) }\end{array}$ \\
\hline$C_{t o t}$ & Total electron carrier concentration $\mathrm{mmol} / \mathrm{mmol}$ biomass & 0.01 & 0.01 & 0.01 & Pan et al. (2013b) \\
\hline
\end{tabular}




\section{REFERENCES:}

Henze, M., Gujer, W., Mino, T. and van Loosdrecht, M.C.M., 2000. Activated sludge models ASM1, ASM2, ASM2d, and ASM3. IWA Scientific and Technical Report No. 9. IWA Publishing, London, UK.

Hiatt, W.C. and Grady, C.P.L. 2008. An updated process model for carbon oxidation, nitrification, and denitrification. Water Environment Research 80 (11), 2145-2156.

IPCC (2007) Climate Change 2007: The Physical Science Basis. Contribution of Working Group I to the Fourth Assessment Report of the Intergovernmental Panel on Climate Change [Solomon, S., D. Qin, M. Manning, Z. Chen, M. Marquis, K.B. Averyt, M.Tignor and H.L. Miller (eds.)]. Cambridge University Press, Cambridge, United Kingdom and New York, NY, USA.

KuČEra, I., DadÁK, V. and DobrÝ, R. 1983. The distribution of redox equivalents in the anaerobic respiratory chain of paracoccus denitrificans. European Journal of Biochemistry 130 (2), 359-364.

McMurray, S.H. (2008) Formation of denitrification intermediates and their impact on process performance. PhD Thesis, The University of Queensland, Brisbane.

Ni, B.-J., Ruscalleda, M., Pellicer-Nàcher, C. and Smets, B.F. 2011. Modeling nitrous oxide production during biological nitrogen removal via nitrification and denitrification: extensions to the general ASM models. Environmental Science \& Technology 45 (18), 7768-7776.

Pan, Y., Ni, B.-J., Bond, P.L., Ye, L. and Yuan, Z. 2013a. Electron competition among nitrogen oxides reduction during methanol-utilizing denitrification in wastewater treatment. Water Research 47 (10), 3273-3281.

Pan, Y., Ni, B.-J. and Yuan, Z. 2013b. Modeling electron competition among nitrogen oxides teduction and $\mathrm{N}_{2} \mathrm{O}$ sccumulation in denitrification. Environmental Science \& Technology 47 (19), 11083-11091.

Pan, Y., Ye, L., Ni, B.-J. and Yuan, Z. 2012. Effect of $\mathrm{pH}$ on $\mathrm{N}_{2} \mathrm{O}$ reduction and accumulation during denitrification by methanol utilizing denitrifiers. Water Research 46 (15), 4832-4840.

Ravishankara, A.R., Daniel, J.S. and Portmann, R.W. 2009. Nitrous oxide $\left(\mathrm{N}_{2} \mathrm{O}\right)$ : The dominant ozone-depleting substance emitted in the $21^{\text {st }}$ century. Science 326 (5949), 123-125.

Reichert, P., von Schulthess, R. and Wild, D. 1995. The use of AQUASIM for estimating parameters of activated sludge models. Water Science and Technology 31 (2), 135147.

Richardson, D., Felgate, H., Watmough, N., Thomson, A. and Baggs, E. 2009. Mitigating release of the potent greenhouse gas $\mathrm{N}_{2} \mathrm{O}$ from the nitrogen cycle - could enzymic regulation hold the key? Trends in Biotechnology 27 (7), 388-397.

Schalk-Otte, S., Seviour, R.J., Kuenen, J.G. and Jetten, M.S.M. 2000. Nitrous oxide $\left(\mathrm{N}_{2} \mathrm{O}\right)$ production by Alcaligenes faecalis during feast and famine regimes. Water Research 34 (7), 2080-2088.

Schulthess R. V., K.M., Gujer, W. 1995. Release of nitric and nitrous oxides from denitrifying activated sludge. Water Research 29 (1), 215-226.

Schulthess, R.V. and Gujer, W. 1996. Release of nitrous oxide $\left(\mathrm{N}_{2} \mathrm{O}\right)$ from denitrifying activated sludge: Verification and application of a mathematical model. Water Research 30 (3), 521-530. 


\section{WWTmod}

Tan, N.C.G., Kampschreur, M.J., Wanders, W., van der Pol, W.L.J., van de Vossenberg, J., Kleerebezem, R., van Loosdrecht, M.C.M. and Jetten, M.S.M. 2008. Physiological and phylogenetic study of an ammonium-oxidizing culture at high nitrite concentrations. Systematic and Applied Microbiology 31 (2), 114-125.

Vonschulthess, R., Wild, D. and Gujer, W. 1994. Nitric and nitrous oxides from denitrifying activated-sludge at low-oxygen concentration. Water Science and Technology 30 (6), 123-132.

Zumft, W.G. 1997. Cell biology and molecular basis of denitrification. Microbiology and Molecular Biology Reviews 61 (4), 533-616. 


\title{
Calibration of nitrous oxide production models with continuous long-term process data
}

\author{
Mathieu Spérandio ${ }^{1}$, Mathieu Pocquet ${ }^{1}$, Lisha Guo $^{2}$, Peter A. Vanrolleghem ${ }^{2}$, Bing-Jie $\mathrm{Ni}^{3}$, Zhiguo \\ Yuan $^{3}$ \\ ${ }^{1}$ LISBP-INSA, Université de Toulouse, 135 av. de rangueil, 31077, Toulouse (Email: \\ sperandio@insa-toulouse.fr) \\ ${ }^{2}$ modelEAU, département de génie civil et de génie des eaux, Université Laval, Québec, QC G1V \\ 0A6, Canada \\ ${ }^{3}$ Advanced Water Management Centre, The University of Queensland, St Lucia, QLD 4072, Australia
}

\begin{abstract}
In this study five activated sludge models including $\mathrm{N}_{2} \mathrm{O}$ emission were compared to four different long-term process data sets. Different production pathways and kinetics were considered for $\mathrm{N}_{2} \mathrm{O}$ production by ammonium oxidising bacteria (AOB). Satisfying calibration could be obtained but none of the models based on a unique pathway of $\mathrm{AOB}$ were able to describe all the $\mathrm{N}_{2} \mathrm{O}$ data obtained in the different systems with similar parameter set. The dependence of pathways with nitrite (or FNA) concentration is confirmed by this confrontation of models and experiences. These results suggest that efforts should be deployed to create a model in which the two main $\mathrm{N}_{2} \mathrm{O}$ pathways active in AOBs are represented together.
\end{abstract}

\section{Keywords}

Calibration, modelling; $\mathrm{N}_{2} \mathrm{O}$, NO, nitrification, denitrification, wastewater treatment

\section{INTRODUCTION}

$\mathrm{N}_{2} \mathrm{O}$ is a powerful greenhouse gas that can be emitted from wastewater treatment plants (WWTP). The emission varies with the design and operation of a WWTP. Experimental campaigns demonstrated that the fraction of influent nitrogen load emitted as $\mathrm{N}_{2} \mathrm{O}$ shows high temporal and spatial variability in a range $0.01 \%$ to more than $10 \%$ (Kampschreur et al., 2009; Ahn et al., 2010). Both denitrification and nitrification processes can produce $\mathrm{N}_{2} \mathrm{O}$. However, recent measurement campaigns have conclusively shown that ammonium oxidising bacteria (AOB) in most cases contribute significantly more to $\mathrm{N}_{2} \mathrm{O}$ production than heterotrophic denitrification (Daelman et al., 2013; Guo et al., 2013a; Wunderlin et al., 2012), whereas heterotrophic denitrification may be important in removal of the produced $\mathrm{N}_{2} \mathrm{O}$ (Guo and Vanrolleghem, 2014a).

In order to evaluate the influence of process configuration and operation on the $\mathrm{N}_{2} \mathrm{O}$ emission a significant effort in mathematical modelling has been recently developed. For dynamic modelling of $\mathrm{N}_{2} \mathrm{O}$ production, new model components have been proposed to enhance the currently commonly used nitrification and denitrification models to include various reaction intermediates such as nitrous oxide $\left(\mathrm{N}_{2} \mathrm{O}\right)$, nitric oxide $(\mathrm{NO})$ and hydroxylamine $\left(\mathrm{NH}_{2} \mathrm{OH}\right)$.

Concerning heterotrophic denitrification $\mathrm{N}_{2} \mathrm{O}$ and $\mathrm{NO}$ are known to be intermediary compounds. These compounds were included in the ASMN model proposed by Hiatt and Grady (2008) considering four successive steps in denitrification. The accumulation rate of $\mathrm{NO}$ and $\mathrm{N}_{2} \mathrm{O}$ depends on the respective ratio between rates of successive steps. More recently Pan et al. (2013) also proposed to consider the electron transport and the competition between the different electron acceptors. 
The mechanisms responsible for $\mathrm{N}_{2} \mathrm{O}$ production by AOB are more controversial. The two widely accepted mechanisms are the AOB denitrification pathway, through which AOB produce $\mathrm{N}_{2} \mathrm{O}$ via NO by reducing nitrite (Chandran et al., 2011), and the hydroxylaminerelated pathway. In this second mechanism, incomplete oxidation of hydroxylamine could form $\mathrm{NO}$ or $\mathrm{NOH}$ (nitroxyl radical) as intermediates for $\mathrm{N}_{2} \mathrm{O}$ production (Stein, 2011; Law et al., 2012). Several mathematical models have been proposed based on these hypothesised pathways (Ni et al., 2011, Ni et al., 2013a, Ni et al., 2013b; Mampaey et al., 2013). Ni et al. (2013a) evaluated four different models by calibrating these models with data reported in literature, obtained in batch experiments with activated sludge samples. As none of the models tested could reproduce all results, $\mathrm{Ni}$ et al., 2013a suggested that a regulation between the two main pathways probably occurs, and called for more work to further identify the specific conditions under which each of the models would be applicable, and to also develop a unified model by integrating various pathways. One issue related to model calibration with batch experiments is that the sludge history may impact the physiological state of the sludge, potentially leading to transient behaviour due to metabolic regulation, especially after a sudden change from biomass cultivation conditions to the batch condition. For this reason models confronting to long term operational data measured in situ is necessary.

In the last years, measurement campaigns have been performed by many research groups including the authors' groups. The objective of this study is to evaluate the calibration of different models with the collected continuous long-term data from different systems to reveal the performance of these models under various process conditions. This could shed light on the conditions under which each of the models would be suitable, and would also facilitate the development of a unified model by combining different pathways.

\section{METHODS}

\section{Experimental data}

As detailed in Table 1, four different continuous biological systems were considered in this study: a UCT process (University of Cape Town), an oxidation ditch, two sequencing batch reactors (SBR). Three of them were full-scale processes treating real domestic wastewater, whereas one was a lab-scale pilot treating high strength wastewater. The $\mathrm{N}_{2} \mathrm{O}$ concentration in the off-gas was monitored using online continuous Infra-Red spectroscopy for three of these systems (UCT and SBRs). In the oxidation ditch dissolved $\mathrm{N}_{2} \mathrm{O}$ was measured in liquid samples using gas chromatography (GC) at different times and in different zones. In addition NO concentration in the off-gas was also collected in the SBR(1) by means of an IR analyser. In the different systems daily average $\mathrm{N}_{2} \mathrm{O}$ emission factors varied from 0.12 to $5 \%(\mathrm{gN}$ $\mathrm{N}_{2} \mathrm{O} / \mathrm{gTN}$ removed), the highest emission being obtained with the SBR process working at the highest loading rate $\left(0.267 \mathrm{kgN} / \mathrm{m}^{3} / \mathrm{d}\right)$ with the highest nitrite variation $(0-50 \mathrm{mgN} / \mathrm{L})$. More details concerning each system and measurement campaigns can be found in dedicated articles and communications (Ni et al., 2013b; Guo and Vanrolleghem, 2014b; Pocquet et al., 2013).

\section{Mathematical models}

In this study mathematical models based on ASMN framework (Hiatt and Grady, 2008) were used with additional modification for considering production of $\mathrm{NO}$ and $\mathrm{N}_{2} \mathrm{O}$ by AOB. As nitrification was supposed to be the main contributor to $\mathrm{N}_{2} \mathrm{O}$ emission and because of the different possible pathways involved in, different $\mathrm{AOB}$ models were compared. The reaction stoichiometry and kinetics of five $\mathrm{N}_{2} \mathrm{O}$ models related to AOB are summarized in Table 2. 
Two models were based on the AOB denitrification pathway: namely the $\mathrm{Ni}$ et al. (2011) model which does include $\mathrm{NH}_{2} \mathrm{OH}$ as an intermediate in ammonium oxidation (model A), and the Mampaey et al. (2013) model, which does not (model B). Another key difference is the influence of oxygen: model A includes DO inhibition whereas oxygen is a substrate in the model $\mathrm{B}$. The third model was based on the hydroxylamine incomplete oxidation pathway (model $\mathrm{C}$ ). In this last model $\mathrm{NO}$ is considered as an intermediary compound during the oxidation of $\mathrm{NH}_{2} \mathrm{OH}$ into nitrite ( $\mathrm{Ni}$ et al., 2013a, $\mathrm{Ni}$ et al., 2013b). $\mathrm{N}_{2} \mathrm{O}$ is then produced by reduction of $\mathrm{NO}$ with the same reaction as in model A. Note that a modification has been made in model $\mathrm{C}$ compared to the initial model of $\mathrm{Ni}$ et al. (2013b): as growth was considered in two processes originally, here biomass production was removed from process 3 in order to use the conventional value for the growth yield $\left(\mathrm{Y}_{\mathrm{AOB}}\right)$. Consequently the value of the new maximal rate $\mu_{\mathrm{AOB}, \mathrm{HAO}, 2}$ is here calculated as $\mu_{\mathrm{AOB}, \mathrm{HAO}, 1} / \mathrm{Y}_{\mathrm{AOB}}$.

Two modifications of the original AOB denitrification models (A and B) have also been considered (Pocquet et al., 2013; Guo and Vanrolleghem, 2014b) (model A1 and B1). In the model A1 the oxygen inhibition function on AOB reduction pathway was not considered. In addition free ammonia (FA) and free nitrous acid (FNA) were considered as the substrate for $\mathrm{AOB}$ reaction rates, in order to consider the effect of variation of $\mathrm{pH}$. In the model $\mathrm{B} 1$, oxygen limitation and inhibition was considered as a Haldane function in both the kinetics of $\mathrm{NO}_{2}{ }^{-}$reduction and NO reduction (Guo and Vanrolleghem, 2014a, Guo and Vanrolleghem, 2014b). Inhibition by FNA and FA were also considered in models A1 and B.

The gas liquid transfers for oxygen, $\mathrm{NO}$ and $\mathrm{N}_{2} \mathrm{O}$ were also included. The transfer coefficients (Kla) for both $\mathrm{NO}$ and $\mathrm{N}_{2} \mathrm{O}$ were calculated with measured oxygen transfer coefficient and respective diffusivity ratio (Ye et al., 2014). Simulations were performed using AQUASIM software (Reichert, 1998) and WEST (Vanhooren et al., 2003).

\section{Parameters calibration}

Calibration was performed in two different steps considering first the major rates and components (ammonia, nitrate, nitrite) and secondly the $\mathrm{N}_{2} \mathrm{O}$ and $\mathrm{NO}$ data. Our approach has been to use typical parameter values reported in literature (Hiatt and Grady, 2008) for most of heterotrophic and autotrophic processes whenever possible. Parameter estimation was first realised manually and in a second time, for sensitive parameters, automatic minimisation of the mean square root error was used. The results of the sensitivity analysis on $\mathrm{N}_{2} \mathrm{O}$ and $\mathrm{NO}$ for parameters involved in the AOB models are presented in the table 3. Parameters influencing ammonium, nitrite, nitrate as well as $\mathrm{NO}$ and $\mathrm{N}_{2} \mathrm{O}$ were first calibrated during the first step (ex: $\left.\mu_{\mathrm{AOB}, \mathrm{AMO}}, \mu_{\mathrm{AOB}}, \mathrm{K}_{\mathrm{NH} 4, \mathrm{AOB}}\right)$. Secondly the parameters influencing only and mostly the $\mathrm{N}_{2} \mathrm{O}$ emission and $\mathrm{NO}$ emission were adapted. In this second phase the reduction factor $\eta_{\mathrm{AOB}}$ as well as affinity constants (ex: $\mathrm{K}_{\mathrm{NO} 2, \mathrm{AOB}}$ ) were focused upon due to their high influence (table 3). The $\mathrm{NO}$ emission is also very sensitive to the parameter $\mathrm{K}_{\mathrm{NO} \text {,AOB }}$ which was estimated with the NO measurements performed on the system SBR(1).

\section{RESULTS}

\section{$\operatorname{SBR}(1)$}

The models were examined with the data collected from the $\operatorname{SBR}(1)$ operated at a high ammonia load $\left(0.25\right.$ to $1.10 \mathrm{kgN} / \mathrm{m}^{3}$.d) with transient nitrite accumulation. This process was operated during more than six months treating wastewater with high ammonium concentration $\left(500 \mathrm{mgN} . \mathrm{L}^{-1}\right)$. Cycles were composed of five phases: feeding, aerobic period, anoxic period with secondary COD feeding, settling, withdraw. The system was controlled for nitrification over nitrite by controlling the aerobic period time. Very low nitrate 
concentrations were observed in the reactor and more than $97 \%$ of the ammonia was converted to nitrite during the aerobic period. Different volumetric exchange ratios and various anoxic times were imposed in order to modify the ammonium concentration as well as the nitrite concentration in the reactor. Heterotrophic denitrification was performed during the anoxic period with a suitable COD: $\mathrm{N}$ ratio by means of organic influent feeding. $\mathrm{N}_{2} \mathrm{O}$ and $\mathrm{NO}$ peaks were only observed during the aerobic period whereas no production was observed during the anoxic phase. The models were calibrated on a series of data (5 cycles) and model predictions were also validated with other cycles collected at different times in contrasted conditions. Figure 1 shows examples of simulated and experimental data $\left(\mathrm{NH}_{4}{ }^{+}, \mathrm{NO}_{2}{ }^{-}, \mathrm{DO}\right.$, $\mathrm{NO}$ and $\mathrm{N}_{2} \mathrm{O}$ ) during the aerobic periods. Oxygen varied from 2 to $6 \mathrm{mg} \mathrm{O}_{2} \mathrm{~L}^{-1}$, initial ammonium from 10 to $40 \mathrm{mg} \mathrm{N} \mathrm{L}^{-1}$, and final nitrite ranged from 30 to $135 \mathrm{mg} \mathrm{N} \mathrm{L}^{-1}$.

For all models the predicted profiles of ammonium, nitrite and DO match the observed experimental trends. The four models based on nitrite denitrification (A, A1, B, B1) also describe the observed $\mathrm{N}_{2} \mathrm{O}$ peak well. In contrast, the last model (C) could not predict the variation of $\mathrm{N}_{2} \mathrm{O}$ peaks for the different cycles, with high discrepancies at high nitrite concentrations. In those conditions with relatively high DO, simulations indicate that $\mathrm{N}_{2} \mathrm{O}$ was mainly related to $\mathrm{AOB}$ processes with an insignificant contribution of heterotrophic denitrification.

The order of magnitude of NO peaks was correctly predicted with the four models based on AOB denitrification whereas model $\mathrm{C}$ could not predict the NO peaks variations. Based on simultaneous monitoring of $\mathrm{N}_{2} \mathrm{O}$ and $\mathrm{NO}$ an accurate calibration of the $\mathrm{K}_{\mathrm{NO}}$ value was possible in this work. However despite this calibration effort model $\mathrm{C}$ was unable to predict the experimentally observed change in the $\mathrm{NO}$ to $\mathrm{N}_{2} \mathrm{O}$ ratio (table A1). Finally, the best predictions for $\mathrm{NO}$ and $\mathrm{N}_{2} \mathrm{O}$ were observed with model $\mathrm{A} 1, \mathrm{~B}$ and $\mathrm{B} 1$. This could be explained by a better description of the $\mathrm{pH}$ effect on the kinetics through the use of FA and FNA in their formulation. Corrections made on the oxygen effect (inhibition) could also impact the simulation. But in the data used the oxygen concentration was relatively high and the constant for oxygen inhibition was not really identifiable. It should be pointed out that this system has high transient nitrite accumulation and the data indicates a clear correlation between FNA and the $\mathrm{N}_{2} \mathrm{O}$ production rate. It was possible to predict this phenomenon with AOB denitrification models but not with the $\mathrm{NH}_{2} \mathrm{OH} / \mathrm{NO}$ model (model C).

Figure 2 compares the predicted and experimental emission factor (EF) for $\mathrm{N}_{2} \mathrm{O}$ and $\mathrm{NO}$ for 11 different cycles (calibration and validation). This result confirms that models A1, B and B1 show the best prediction of $\mathrm{N}_{2} \mathrm{O}$ and $\mathrm{NO}$ emissions, model $\mathrm{A}$ also being relatively good. The predictions of $\mathrm{NO}$ fluctuations are less accurate than for $\mathrm{N}_{2} \mathrm{O}$ but the ratio between both gases is relatively well predicted by the models based on the AOB denitrification pathway (Table 5). Model $\mathrm{C}$ based on $\mathrm{NH}_{2} \mathrm{OH} / \mathrm{NO}$ pathway could not predict the experimental data as it is unable to predict the effect of nitrite accumulation on $\mathrm{N}_{2} \mathrm{O}$ and $\mathrm{NO}$ production. This leads to an underestimation of $\mathrm{N}_{2} \mathrm{O}$ at high FNA concentration whereas $\mathrm{NO}$ emission is overestimated.

\section{Oxidation ditch}

The AOB denitrification models (Models $\mathrm{A}$ and $\mathrm{B}$ ) and the $\mathrm{NH}_{2} \mathrm{OH} / \mathrm{NO}$ model (Model C) were examined with the experimentally observed continuous $\mathrm{N}_{2} \mathrm{O}$ data from the full-scale oxidation ditch (OD) plant with surface aerators. The OD system receives domestic wastewater at approximately 4 mega liter (ML) per day. The plant consists of primary clarifier and an activated sludge system. After primary sedimentation, wastewater is introduced into the activated sludge unit, which is an oxidation ditch with a working volume of $8750 \mathrm{~m}^{3}$. The average hydraulic retention time (HRT) in the oxidation ditch is approximately $48 \mathrm{~h}$. The 
mixed liquor from the oxidation ditch flows into a secondary settler. The solids retention time (SRT) is approximately $10 \mathrm{~d}$.

The models were calibrated using the extensive monitoring data from the three-day intensive sampling campaigns at the three different locations (OD4, OD5 and OD2) of the ditch. Figure 3 shows the simulated and experimental data $\left(\mathrm{NH}_{4}{ }^{+}, \mathrm{NO}_{2}{ }^{-}, \mathrm{NO}_{3}{ }^{-}\right.$and $\left.\mathrm{N}_{2} \mathrm{O}\right)$ at the three locations along the ditch. For the three models the predicted profiles of ammonium, nitrite and nitrate match the observed experimental trends. However, the results indicated that Model A could not predict the $\mathrm{N}_{2} \mathrm{O}$ data. Indeed, Model A predicted a dependency of $\mathrm{N}_{2} \mathrm{O}$ production on DO that is opposite to that observed at the OD plant (Figure 3A-F). The kinetic structure of Model B ensures that the $\mathrm{N}_{2} \mathrm{O}$ production rate is dependent on oxygen availability, resulting in a $\mathrm{N}_{2} \mathrm{O}$ dynamic trend similar to that shown by the experimental data (Figure 3G-L). In order to reasonably predict the $\mathrm{N}_{2} \mathrm{O}$ production rate when nitrite accumulation in the OD system is very low $(<0.67 \mathrm{mg}-\mathrm{N} / \mathrm{L})$, a relatively high anoxic reduction factor had to be employed due to the fact that the $\mathrm{N}_{2} \mathrm{O}$ production rate is dependent on nitrite concentrations in Model B. In contrast, Model $\mathrm{C}$ achieved a good fit between the model-predicted and measured $\mathrm{N}_{2} \mathrm{O}$ data. It is important to note that the OD system has low nitrite accumulation. The $\mathrm{N}_{2} \mathrm{O}$ emissions occurred mainly during aerobic zones (OD4) with high ammonium concentrations but low nitrite accumulation. The $\mathrm{NH}_{2} \mathrm{OH}$ pathway of Model $\mathrm{C}$ captures all these trends.

\section{$\operatorname{SBR}(2)$}

Models A, B and C were also evaluated with the experimentally observed continuous cycling $\mathrm{N}_{2} \mathrm{O}$ data from the full-scale SBR plant (SBR2). The average daily flow of the plant is 120 ML. The plant is commissioned with a primary sedimentation tank followed by secondary treatment. The biological nutrient removal component of the plant comprises a circular tank that is evenly quartered into four basins. Each basin operates as a separate SBR. At the time of this study, each SBR cycle consisted of the following phases in sequence: 90 min continuous feeding and aeration, 35 min settling and 55 min decanting. The average exchange volume per cycle in each SBR was approximately 5 ML. Each SBR had a working volume of $28 \mathrm{ML}$, and hence the average HRT was $17 \mathrm{~h}$. The total airflow to the three SBRs was fixed at $45000 \mathrm{~m}^{3}$ $\mathrm{h}^{-1}$ throughout the aeration phase with equal distribution among the three reactors. The SRT was maintained at 19 days.

The models were calibrated using the monitoring data collected from SBR2 during the threecycle continuous intensive sampling campaign. Figure 4 shows the simulated and experimental data (effluent $\mathrm{NH}_{4}{ }^{+}, \mathrm{NO}_{2}{ }^{-}, \mathrm{NO}_{3}{ }^{-}$and $\mathrm{N}_{2} \mathrm{O}$ ) during the three cycles. Again, for the three models the predicted profiles of ammonium, nitrite and nitrate match the observed experimental trends. Model A predicted an opposite $\mathrm{N}_{2} \mathrm{O}$ trend to that observed. Models $\mathrm{B}$ and $\mathrm{C}$ achieved a good fit between the model-predicted and measured $\mathrm{N}_{2} \mathrm{O}$ data. Similar to the OD system, SBR2 also has low nitrite accumulation. In SBR2, $\mathrm{N}_{2} \mathrm{O}$ emissions occurred mainly during aerated periods. The $\mathrm{N}_{2} \mathrm{O}$ production rate increased with the increase of $\mathrm{DO}$ concentration during the cycles of SBR2. Model C captures all these trends in general.

\section{UCT process}

The Eindhoven WWTP, in the Netherlands, has a capacity of 750,000 PE. It treats wastewater using a University Cape Town (UCT) process which consists of three rings. The inner ring is an anaerobic tank, the middle ring is an anoxic tank and the outer ring is a partially aerated tank. The outer ring is equipped with two aeration packages. The summer aeration package is open all year round, but the winter aeration package is turned on occasionally under certain conditions, e.g. under rain events. 
Model A1, Model B1 and Model C were implemented to this plant and their parameters were calibrated based on a 1-month measurement campaign. Figure 5 compares the simulated $\mathrm{NH}_{4}{ }^{+}, \mathrm{NO}_{3}{ }^{-}$and DO concentrations with the on-line sensor data near the outlet of the summer aeration package. Figure 6 compares the simulation and the measurement data of $\mathrm{N}_{2} \mathrm{O}$ emissions at three different locations along the summer aeration package. The conclusion is that all models can be calibrated to the same level of fit. They have similar performance and can follow the dynamic variations in the measurement data.

There was a rain event encountered on August 25th - 26th. All models showed better simulation performance under dry-weather conditions than wet-weather conditions (Figure 5). Results show that there was less $\mathrm{N}_{2} \mathrm{O}$ emission under wet-weather conditions compared to dry-weather conditions (Figure 6).

\section{DISCUSSION}

\section{Capabilities of the models and comparison of parameter sets}

The data monitored on the four continuous systems considered in this study confirm that the $\mathrm{N}_{2} \mathrm{O}$ emission factors varied very significantly from 0.1 to $5.2 \%$ of the nitrogen removed. Data and simulations also confirmed that major $\mathrm{N}_{2} \mathrm{O}$ productions were related to nitrification. For instance, $\mathrm{N}_{2} \mathrm{O}$ emission were negligible in $\mathrm{SBR}(1)$ during anoxic period (even if nitrogen gas was injected) whereas 0.5 to $5 \%$ of nitrogen was converted into $\mathrm{N}_{2} \mathrm{O}$ during aerobic nitrification depending on the FNA concentration (DO from 2 to $6 \mathrm{ppm}$ ). In OD the overall $\mathrm{N}_{2} \mathrm{O}$ emission factor over a full month was $0.52 \%$ of the nitrogen load to the plant, with over $90 \%$ contribution from the aeration zone (DO of over $5 \mathrm{ppm}$ ), $8 \%$ contribution from the DO zone at $1 \mathrm{ppm}$, and less than $2 \%$ contribution from DO zone at $0.05 \mathrm{ppm}$. Similarly, the $\mathrm{N}_{2} \mathrm{O}$ emission factor of the SBR2 over the month is around 1.0-1.5\% of the nitrogen load, with $\mathrm{N}_{2} \mathrm{O}$ emissions occurring mainly during aerated periods. In the UCT process the average contribution of each species to the $\mathrm{N}_{2} \mathrm{O}$ production has been quantified with the model $\mathrm{B} 1$. $\mathrm{N}_{2} \mathrm{O}$ is produced through the AOB pathway but is consumed by heterotrophic denitrification. Finally, the total $\mathrm{N}_{2} \mathrm{O}$ production by $\mathrm{AOB}$ is $291.83 \%$ of the net production while the heterotrophs contribute by $-191.83 \%$ (Guo and Vanrolleghem, 2014b). Hence these results clearly confirm the need of a good prediction for AOB-related $\mathrm{N}_{2} \mathrm{O}$ production as well as heterotrophic denitrification by $\mathrm{N}_{2} \mathrm{O}$.

The modelling results indicate that all five models can correctly describe the ammonium, nitrite and nitrate measurements. However none of these models was able to accurately predict all measured $\mathrm{N}_{2} \mathrm{O}$ data sets. On the one hand this confirms the first conclusion made by $\mathrm{Ni}$ et al. (2013a) in their previous evaluation with batch data. On the other hand, new information (summarized below) is provided by this comparison on long-term data. Table 6 summarizes the models' capabilities for the different case-studies.

Satisfying predictions were observed with AOB denitrification models for the SBR(1) in which the nitrite concentration varied significantly, the best results being obtained with models based on FNA instead of total nitrite (A1, B, B1). At the contrary it was not possible to predict the data with model $\mathrm{C}$ based on hydroxylamine incomplete oxidation as the effect of nitrite (or FNA) was not considered in this model. In addition, NO emissions were also predicted more accurately with the AOB denitrification model. For the UCT and SBR(2) processes in which nitrite did not accumulate, the simulations with model $\mathrm{C}$ were in agreement with the observations whereas a model based on AOB denitrification (model $\mathrm{A}$ ) failed. Finally, for the UCT process models A1, B1 and C gave similar trends after an important calibration effort. 
On figure 7, the FNA influence on simulated NO production rate is represented for two models (A1 and B1) calibrated on the SBR(1) and UCT processes. It should be pointed out that the $\mathrm{N}_{2} \mathrm{O}$ production rate is correlated to this rate as $\mathrm{NO}$ is the precursor for $\mathrm{N}_{2} \mathrm{O}$ in these models. In the system with low nitrite concentration (UCT), a high value for $\eta_{\text {AOB }}(0.3-0.5)$ and a low value for $\mathrm{K}_{\mathrm{HNO}, \mathrm{AOB}}\left(10^{-5}-10^{-6}\right)$ are obtained in order to satisfy the observed $\mathrm{NO}$ and $\mathrm{N}_{2} \mathrm{O}$ emission data. With these parameter values the $\mathrm{N}_{2} \mathrm{O}$ production rate is poorly influenced by the nitrite concentration except at very low concentrations. In comparison a lower value for $\eta_{\mathrm{AOB}}(0.1-0.2)$ and a higher value for $\mathrm{K}_{\mathrm{HNO} 2 \text { АОВ }}\left(2.10^{-3}\right)$ were obtained with the calibration on the SBR(1) process. In that case the variation of FNA concentration influences the NO and $\mathrm{N}_{2} \mathrm{O}$ production rates significantly. On the one hand these large variations of parameters from one system to another could possibly be explained by adaptation of enzymatic activity (NirK). This would mean that the calibration realised at low nitrite concentrations is not valuable in a system with high nitrite accumulation (and vice-versa). On the other hand another explanation is that the second pathway based on hydroxylamine incomplete oxidation is also present next to the denitrification pathway. For this reason a model which would consider both pathways would be useful as it would probably be able to predict a larger range of experimental conditions with the same parameter set.

\section{Relation between FNA concentration and AOB pathways}

From the models comparison it can be concluded that the AOB denitrification models (especially A1, B, B1) were able to describe the process with high nitrite variation whereas the $\mathrm{NH}_{2} \mathrm{OH} / \mathrm{NO}$ model (C) fits well with the data collected on low loaded systems with low nitrite accumulation. In addition, Models $\mathrm{B}$ and $\mathrm{C}$ could both predict the trend of the $\mathrm{N}_{2} \mathrm{O}$ production dependency on DO at high DO level. These observations may help the future development of a unified model involving both the AOB denitrification and the $\mathrm{NH}_{2} \mathrm{OH}$ pathways. Considering the model structures, only the models based on AOB denitrification correlate the $\mathrm{N}_{2} \mathrm{O}$ production rate to nitrite, whereas the $\mathrm{NH}_{2} \mathrm{OH}$ oxidation models correlate the $\mathrm{N}_{2} \mathrm{O}$ production to the ammonium uptake rate.

The maximal nitrite concentration in the OD and $\mathrm{SBR}(2)$ are respectively around $0.67 \mathrm{ppm}$ and $1.53 \mathrm{ppm}$, with $\mathrm{pH}$ close to 7.1 . It corresponds to 0.16 and $0.32 \mu \mathrm{gN}-\mathrm{FNA} / \mathrm{L}$ respectively at $15^{\circ} \mathrm{C}$. It was also observed in $\operatorname{SBR}(1)$ that below a concentration around $0.5 \mu \mathrm{gN}-\mathrm{FNA} / \mathrm{L}$ the production of $\mathrm{N}_{2} \mathrm{O}$ decreases very significantly. It could be concluded that this value could be the limit under which the AOB denitrification processes become less significant compare to the hydroxylamine oxidation pathway. On the other hand, in the UCT process the maximal nitrite was only $0.11 \mathrm{mgN} / \mathrm{L}$ and the model $\mathrm{A} 1, \mathrm{~B} 1$ and $\mathrm{C}$ were able to predict the observed trends.

These results are in accordance with the results of the quantification of $\mathrm{N}_{2} \mathrm{O}$ origin during nitrification based on isotopes signature (Toyoda et al., 2011; Wunderlin et al., 2013; Rathnayake et al., 2013). Wunderlin et al. (2013) demonstrated that the nitrite reduction pathway was the major mechanism responsible for $\mathrm{N}_{2} \mathrm{O}$ production during batch feeding with ammonium and nitrite. In contrast the hydroxylamine oxidation pathways became the major process as soon as hydroxylamine accumulated or was injected. The contribution of $\mathrm{N}_{2} \mathrm{O}$ reduction was also observed to increase with the nitrite accumulation over time (Wunderlin et al., 2013; Rathnayake et al., 2013).

\section{CONCLUSIONS}

The continuous long-term data sets collected from four different full-scale wastewater treatment plants and the calibration results obtained for five different model structures compiled in this paper lead to the conclusion that neither of the proposed models presented so 
far in literature were capable to properly describe all observations. More specifically none of the models were able to describe with similar parameter set the data obtained in systems with high and low nitrite concentration.

This is consistent with the conclusions drawn from a previous exercise based on short-term laboratory scale data and the suggestion is that efforts should be deployed to create a model in which the two main $\mathrm{N}_{2} \mathrm{O}$ pathways active in $\mathrm{AOBs}$ are represented and their interaction described by their dependence on the reactor conditions.

\section{ACKNOWLEDGEMENTS}

The research project and fellowship of Mathieu Pocquet was supported by the National French Research Agency (ANR). Bing-Jie Ni acknowledges the supports of Australian Research Council Discovery Project (DP130103147). The work at Universite Laval benefitted from the financial support obtained through the TECC project of the Québec Ministry of Economic Development, Innovation and Exports (MDEIE) and the research project funded by the Flemish Fund for Scientific Research (FWO_-G.A051.10). Peter Vanrolleghem holds the Canada Research Chair in Water Quality Modelling.

\section{REFERENCES}

Ahn, J.H., Kim, S., Park, H., Rahm, B., Pagilla, K., Chandran, K., 2010. N2O emissions from activated sludge processes, 2008-2009: results of a national monitoring survey in the United States. Environ. Sci. Technol. 44, 4505-4511.

Chandran, K., Stein, L.Y., Klotz, M.G., Van Loosdrecht, M.C.M., 2011. Nitrous oxide production by lithotrophic ammonia-oxidizing bacteria and implications for engineered nitrogen-removal systems. Biochem. Soc. Trans. 39, 1832-1837.

Daelman, M.R.J., Van Voorthuizen, E.M., Van Dongen, U.G.J.M., Volcke, E.I.., Van Loosdrecht, M.C.M., 2013. Full-scale evaluation of process conditions leading to the emission of nitrous oxide from municipal wastewater treatment plants. In: Proceedings WEF/IWA Nutrient Removal and Recovery 2013: Trends in Resource Recovery and Use. Vancouver, British Columbia, Canada, July 28-31.

Guo, L., Lamaire-Chad, C., Bellandi, G., Daelman, M., Amerlinck, Y., Maere, T., Nous, J., Flameling, T., Weijers, S., Van Loosdrecht, M.C.M., Volcke, E.I.P., Nopens, I., Vanrolleghem, P.A., 2013a. High-Frequency Field Measurement of Nitrous oxide (N2O) Gas Emissions and Influencing Factors at WWTPs under Dry and Wet Weather Conditions. In: Proceedings WEF/IWA Nutrient Removal and Recovery 2013: Trends in Resource Recovery and Use. Vancouver, British Columbia, Canada, July 28-31.

Guo, L., Vanrolleghem, P.A., 2014a. Calibration and validation of an activated sludge model for greenhouse gases no. 1 (ASMG1): Prediction of temperature-dependent N2O emission dynamics. Bioprocess and Biosystems Engineering 37, 151-163.

Guo, L., Vanrolleghem, P.A., 2014b. Full-scale simulation of N2O emissions with ASMG2d and elucidation of its different production and emission sources in nitrogen $(\mathrm{N})$ and phosphorus (P) removed systems. Under review.

Hiatt, W.C., Grady, C.P.L., 2008. An Updated Process Model for Carbon Oxidation, Nitrification, and Denitrification. Water Environment Research 80, 2145-2156.

Kampschreur, M.J., Temmink, H., Kleerebezem, R., Jetten, M.S.M., Van Loosdrecht, M.C.M., 2009. Nitrous oxide emission during wastewater treatment. Water Research 
43, 4093-4103.

Law, Y., Ni, B.-J., Lant, P., Yuan, Z., 2012. N2O production rate of an enriched ammoniaoxidising bacteria culture exponentially correlates to its ammonia oxidation rate. Water Research 46, 3409-3419.

Mampaey, K.E., Beuckels, B., Kampschreur, M.J., Kleerebezem, R., Van Loosdrecht, M.C.M., Volcke, E.I.P., 2013. Modelling nitrous and nitric oxide emissions by autotrophic ammonia-oxidizing bacteria. Environ Technol 34, 1555-1566.

Ni, B.-J., Ruscalleda, M., Pellicer-Nàcher, C., Smets, B.F., 2011. Modeling nitrous oxide production during biological nitrogen removal via nitrification and denitrification: extensions to the general ASM models. Environ. Sci. Technol. 45, 7768-7776.

Ni, B.-J., Ye, L., Law, Y., Byers, C., Yuan, Z., 2013b. Mathematical Modeling of Nitrous Oxide (N2O) Emissions from Full-Scale Wastewater Treatment Plants. Environ. Sci. Technol. 47, 7795-7803.

Ni, B.-J., Yuan, Z., Chandran, K., Vanrolleghem, P.A., Murthy, S., 2013a. Evaluating four mathematical models for nitrous oxide production by autotrophic ammonia-oxidizing bacteria. Biotechnology and Bioengineering 110, 153-163.

Pan, Y., Ni, B.-J., Yuan, Z., 2013. Modeling Electron Competition among Nitrogen Oxides Reduction and N2O Accumulation in Denitrification. Environ. Sci. Technol. 47, $11083-11091$.

Pocquet, M., Queinnec, I., Spérandio, M., 2013. Adaptation and identification of models for nitrous oxide (N2O) production by autotrophic nitrite reduction. In: Proceedings 11th IWA Conference on Instrumentation, Control and Automation (ICA2013). Narbonne, France, September 18-20.

Rathnayake, R.M.L.D., Song, Y., Tumendelger, A., Oshiki, M., Ishii, S., Satoh, H., Toyoda, S., Yoshida, N., Okabe, S., 2013. Source identification of nitrous oxide on autotrophic partial nitrification in a granular sludge reactor. Water Research 47, 7078-7086.

Reichert, P., 1998. User manual, computer program for the identification and simulation of aquatic systems.

Stein, L.Y., 2011. Surveying N2O-producing pathways in bacteria. Meth. Enzymol. 486, 131152.

Toyoda, S., Suzuki, Y., Hattori, S., Yamada, K., Fujii, A., Yoshida, N., Kouno, R., Murayama, K., Shiomi, H., 2011. Isotopomer analysis of production and consumption mechanisms of $\mathrm{N} 2 \mathrm{O}$ and $\mathrm{CH} 4$ in an advanced wastewater treatment system. Environ. Sci. Technol. 45, 917-922.

Vanhooren H., Meirlaen J., Amerlinck Y., Claeys F., Vangheluwe H. and Vanrolleghem P.A. 2003. WEST: Modelling biological wastewater treatment. Journal of Hydroinformatics, 5, 27-50.

Wunderlin, P., Lehmann, M.F., Siegrist, H., Tuzson, B., Joss, A., Emmenegger, L., Mohn, J., 2013. Isotope signatures of $\mathrm{N} 2 \mathrm{O}$ in a mixed microbial population system: constraints on $\mathrm{N} 2 \mathrm{O}$ producing pathways in wastewater treatment. Environ. Sci. Technol. 47, 1339-1348.

Wunderlin, P., Mohn, J., Joss, A., Emmenegger, L., Siegrist, H., 2012. Mechanisms of N2O production in biological wastewater treatment under nitrifying and denitrifying conditions. Water Res 46, 1027-37.

Ye, L., Ni, B.-J., Law, Y., Byers, C., Yuan, Z., 2014. A novel methodology to quantify nitrous oxide emissions from full-scale wastewater treatment systems with surface aerators. Water Research 48, 257-268. 
Table 1. Comparison of the experimental systems and operating conditions

\begin{tabular}{|c|c|c|c|c|}
\hline Process type & AS - UCT & $\operatorname{SBR}(1)$ & $\begin{array}{c}\text { AS - Oxidation } \\
\text { ditch }\end{array}$ & $\operatorname{SBR}(2)$ \\
\hline Country & Netherlands & France & Australia & Australia \\
\hline Wastewater type & Domestic & Agro-industry & Domestic & Domestic \\
\hline $\mathrm{COD} / \mathrm{N}(\mathrm{gCOD} / \mathrm{gN})$ & 9 & 5 & 10 & 10 \\
\hline SRT (d) & 10 & 15 & 10 & 19 \\
\hline $\operatorname{HRT}(\mathrm{h})$ & 19 & 35 & 48 & 17 \\
\hline DO aerobic $\left(\mathrm{mgO}_{2} / \mathrm{L}\right)$ & $0.1-5.4$ & $2-6.0$ & $0.1-8.0$ & $0.1-4.0$ \\
\hline Aerobic fraction (of time or volume) & $0.2-0.5$ & 0.55 & $<0.5^{*}$ & 0.5 \\
\hline Temperature during campaign $\left({ }^{\circ} \mathrm{C}\right)$ & $21+/-0.5$ & $28+/-0.5$ & $25+/-0.5$ & $25+/-0.5$ \\
\hline MLSS (g/L) & $2.5-3.5$ & $5.3-6.3$ & $2.9-3.7$ & $4-4.5$ \\
\hline Reactor $\mathrm{N}-\mathrm{NH}_{4}^{+}(\mathrm{mgN} / \mathrm{L})$ & $0-45$ & $0-45$ & $0-5$ & $0-25$ \\
\hline Reactor $\mathrm{N}-\mathrm{NO}_{2}^{-}(\mathrm{mgN} / \mathrm{L})$ & $0-0.1$ & $0-50$ & $0-0.5$ & $0-2.5$ \\
\hline Reactor $\mathrm{N}-\mathrm{NO}_{3}^{-}(\mathrm{mgN} / \mathrm{L})$ & $0-6.5$ & $0-10$ & $0-1$ & $0-5$ \\
\hline Nitrogen load $\left(\mathrm{kgN} / \mathrm{m}^{3} / \mathrm{d}\right)$ & 0.070 & 0.267 & 0.045 & 0.074 \\
\hline $\mathrm{N}_{2} \mathrm{O}$ emission factor $\left(\mathrm{gN}-\mathrm{N}_{2} \mathrm{O} / \mathrm{gTN}\right)$ & $\begin{array}{c}0.12 \%- \\
3.10 \% \\
\end{array}$ & $\begin{array}{c}1.0 \%- \\
5 \% \\
\end{array}$ & $\begin{array}{c}0.36 \%- \\
0.68 \% \\
\end{array}$ & $\begin{array}{l}1.0 \%- \\
1.5 \% \\
\end{array}$ \\
\hline
\end{tabular}


Table 5. Processes stoechiometry and kinetics of the models for AOB.

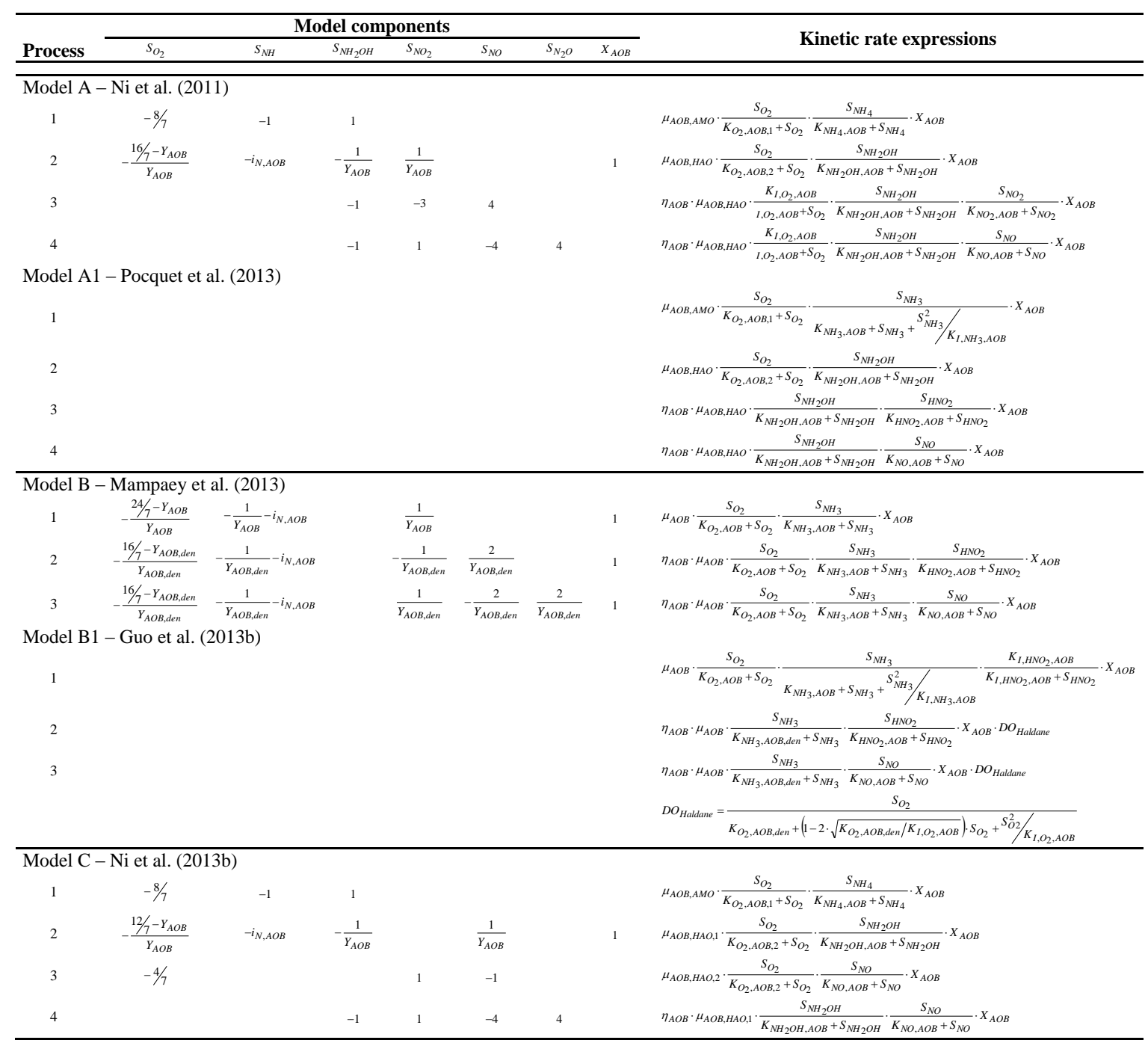


Table 6. Sensitivity of state variables $\mathrm{NO}$ and $\mathrm{N}_{2} \mathrm{O}$ (in liquid) to stoichiometric and kinetic parameters for the five models. The classification is related to the root mean square of the

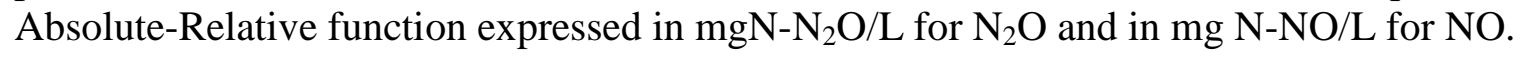

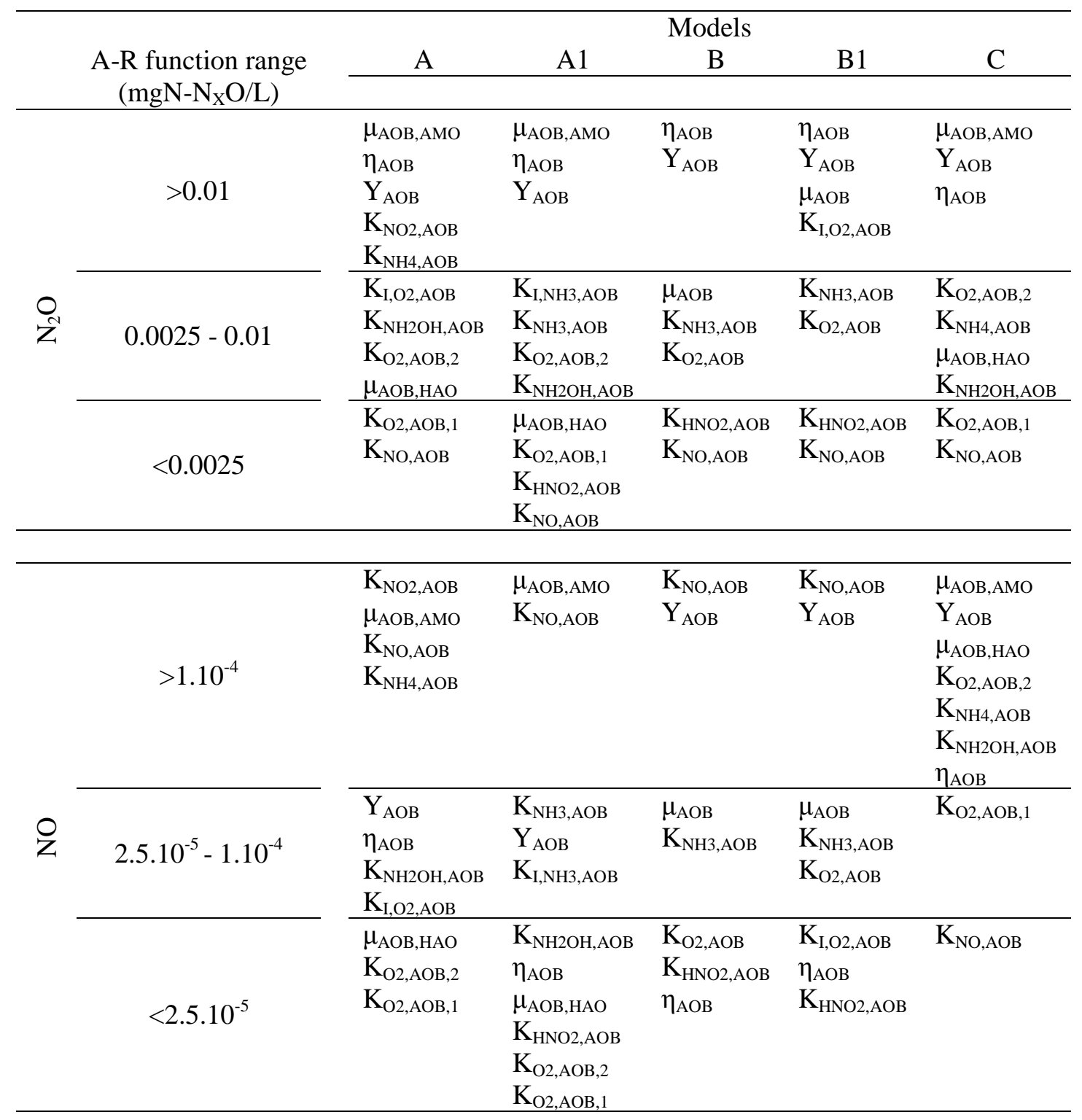


Table 7. Parameters of the models A, A1, B, B1, C calibrated with four case studies.

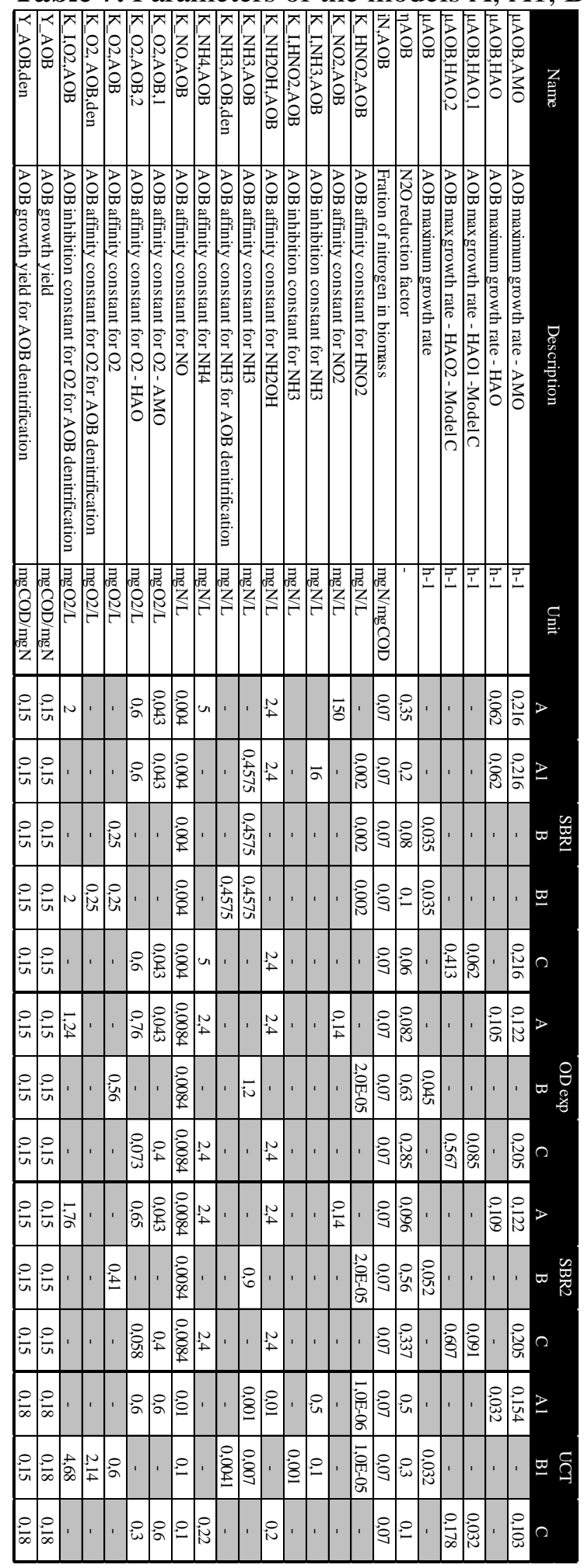


Table 8. Comparison between experimental and simulated $\mathrm{NO}$ to $\mathrm{N}_{2} \mathrm{O}$ ratio from $\mathrm{SBR}(1)$.

\begin{tabular}{lccccc}
\hline & \multicolumn{5}{c}{$\mathrm{NO} / \mathrm{N}_{2} \mathrm{O}$ ratio $\left(\mathrm{gN}-\mathrm{NO} / \mathrm{gN}-\mathrm{N}_{2} \mathrm{O}\right)$} \\
\hline Experiment/cycle & 1 & 2 & 3 & 4 & 5 \\
\hline Data & $4.4 \%$ & $4.6 \%$ & $4.9 \%$ & $2.9 \%$ & $3.5 \%$ \\
\hline Model A & $5.3 \%$ & $7.5 \%$ & $9.2 \%$ & $2.9 \%$ & $8.6 \%$ \\
Model A1 & $3.6 \%$ & $4.2 \%$ & $4.9 \%$ & $2.5 \%$ & $4.6 \%$ \\
Model B & $3.5 \%$ & $4.2 \%$ & $4.7 \%$ & $2.4 \%$ & $2.4 \%$ \\
Model B1 & $4.1 \%$ & $4.2 \%$ & $5.2 \%$ & $2.7 \%$ & $4.5 \%$ \\
Model C & $12.0 \%$ & $10.7 \%$ & $11.6 \%$ & $10.5 \%$ & $10.8 \%$ \\
\hline
\end{tabular}

Table 6. Comparison of models capabilities on the different case-studies

\begin{tabular}{lcccc}
\hline & \multicolumn{4}{c}{ System } \\
\hline Experiment/cycle & AS-UCT & SBR(1) & OD & SBR(2) \\
\hline Model A & & $+/-$ & - & - \\
Model A1 & + & + & & + \\
Model B & & + & + & + \\
Model B1 & + & + & & \\
Model C & + & - & + & + \\
\hline
\end{tabular}




\section{WWTmod}

Spérandio et al.
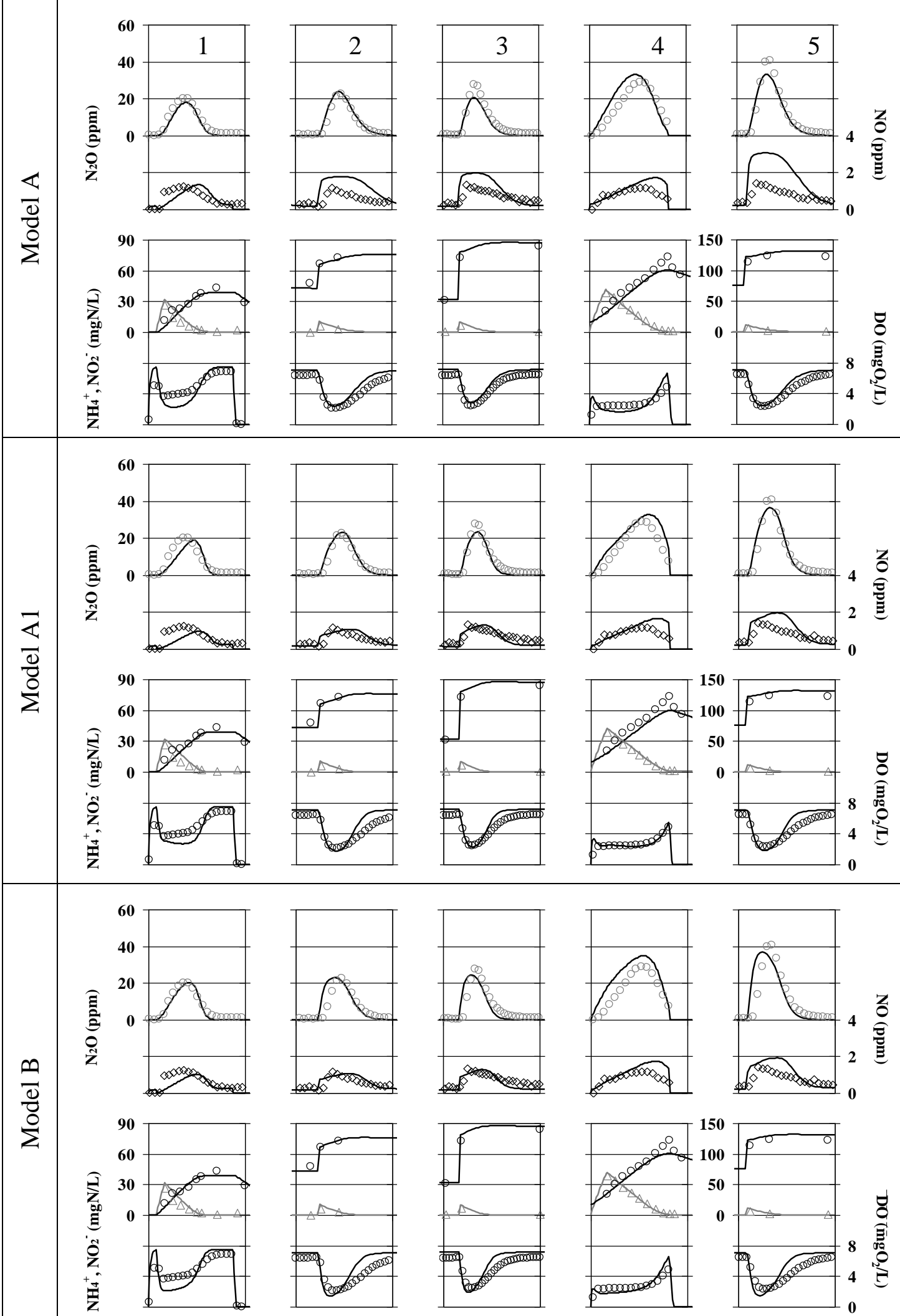


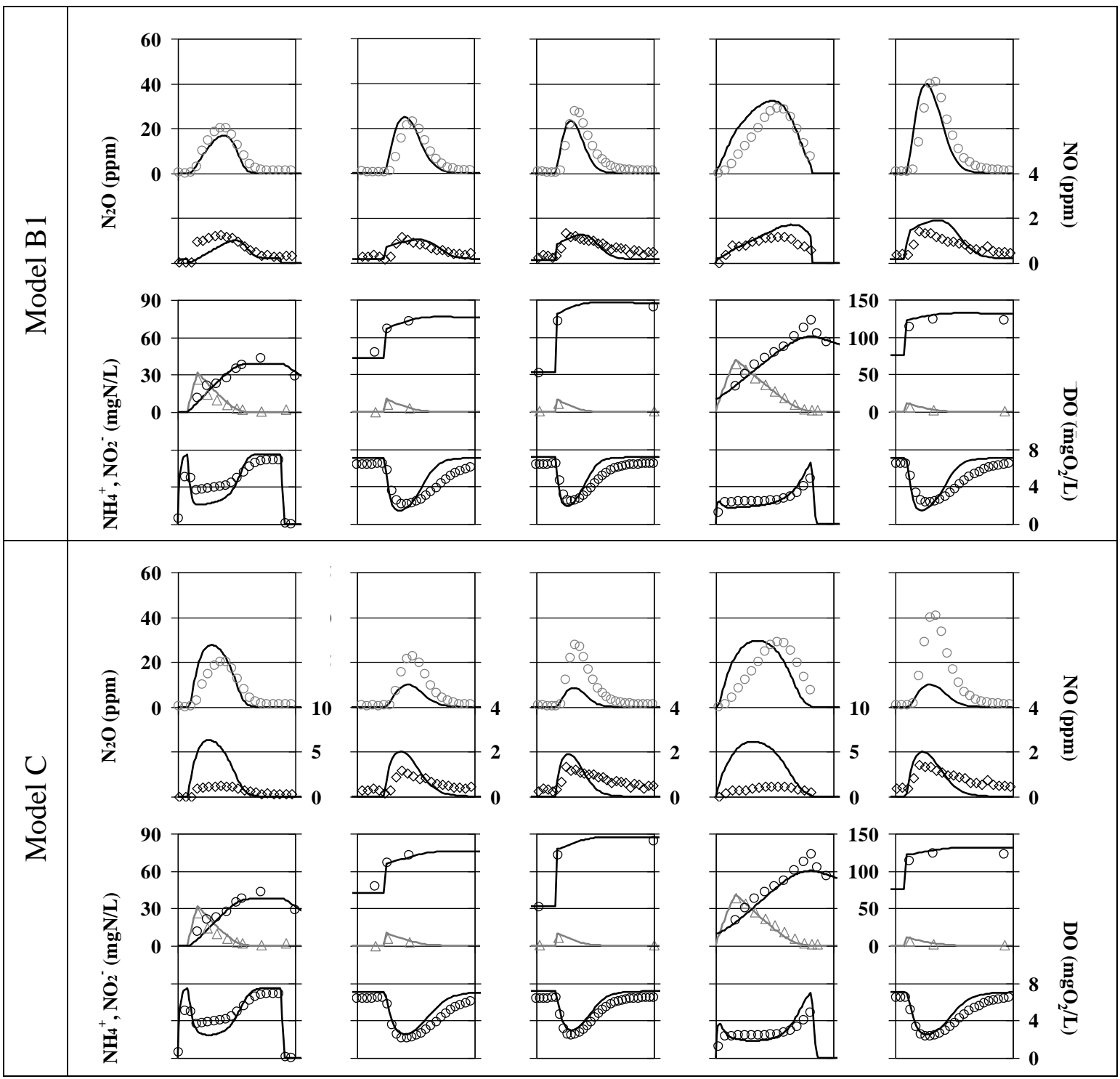

Figure 8. Dynamic of nitrification and $\mathrm{NO}$ and $\mathrm{N}_{2} \mathrm{O}$ production for 5 experiment obtained with the SBR. Experimental results (in points) are confronted to modelling results (in line) for the five models. The dynamic of $\mathrm{NO}(\diamond)$ (secondary axis) and $\mathrm{N}_{2} \mathrm{O}(\circ)$ (primary axis) production are presented in the first line of each models whereas ammonium $(\Delta)$, nitrite $(0)$ (primary axis) and dissolved oxygen $(0)$ (secondary axis) are presented in the second line. Duration of experiment 1 to $5: 1 \mathrm{~h}, 0.4 \mathrm{~h}, 0.4 \mathrm{~h}, 1 \mathrm{~h}$, $0.4 \mathrm{~h}$. Experimental $\mathrm{N}_{2} \mathrm{O}$ emission factor for experiment 1 to 5 (in $\mathrm{gN}-\mathrm{N}_{2} \mathrm{O} / \mathrm{gN}-\mathrm{NH}_{4}{ }^{+}$removed): $1.39 \%$, $2.58 \%, 3.86 \%, 1.83 \%, 4.52 \%$. 


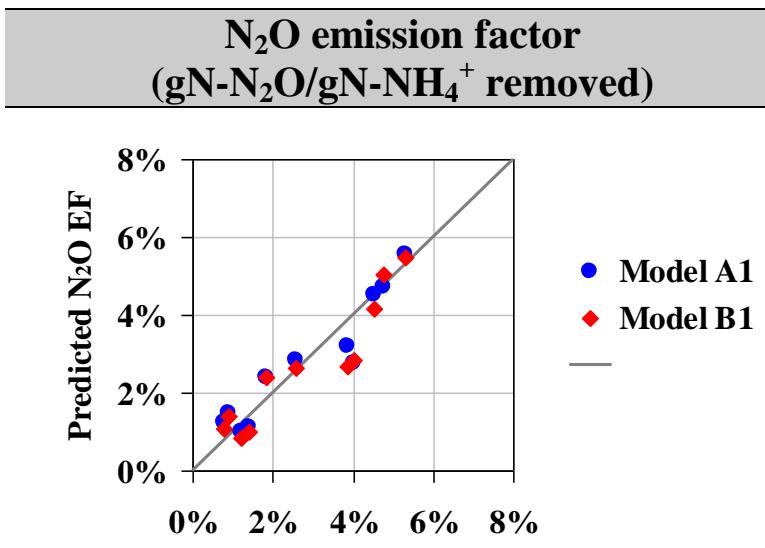

Experimental $\mathrm{N}_{2} \mathrm{O}$ EF
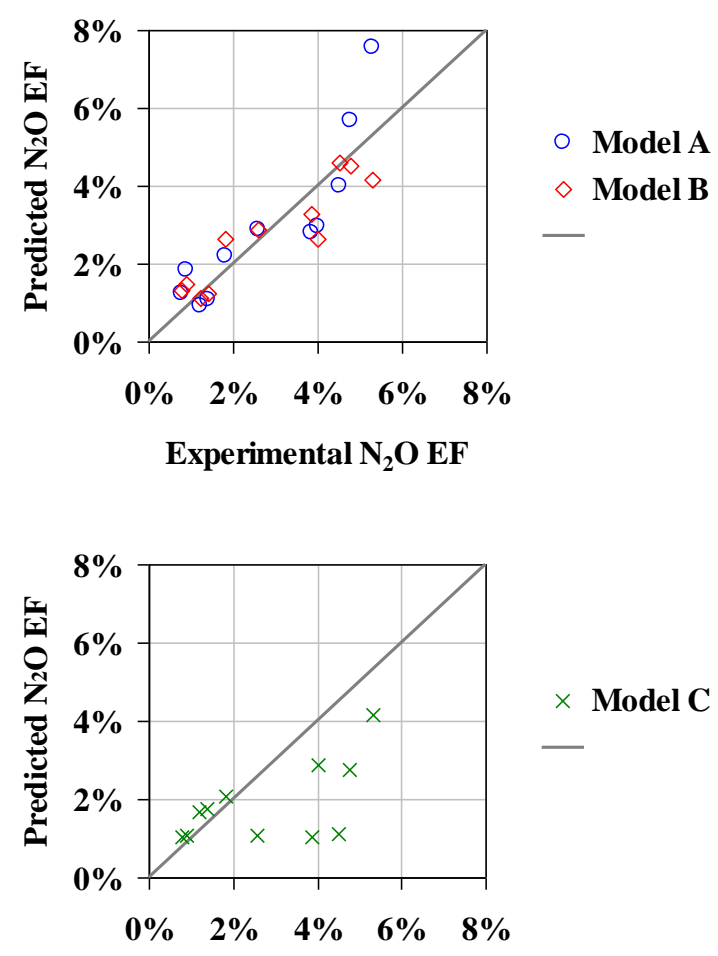

Experimental $\mathrm{N}_{2} \mathrm{O}$ EF
NO emission factor (gN-NO/gN-NH ${ }_{4}{ }^{+}$removed)

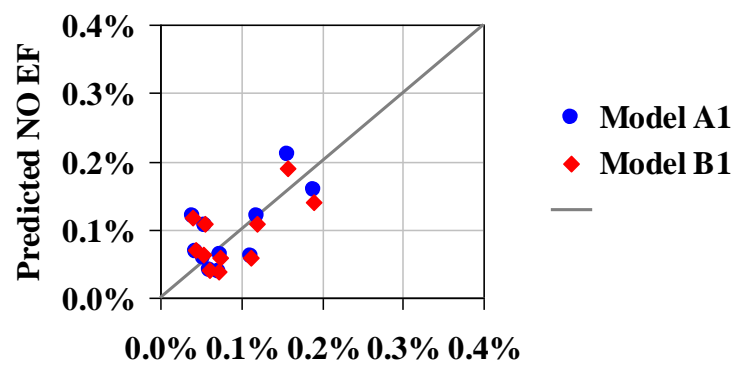

Experimental NO EF

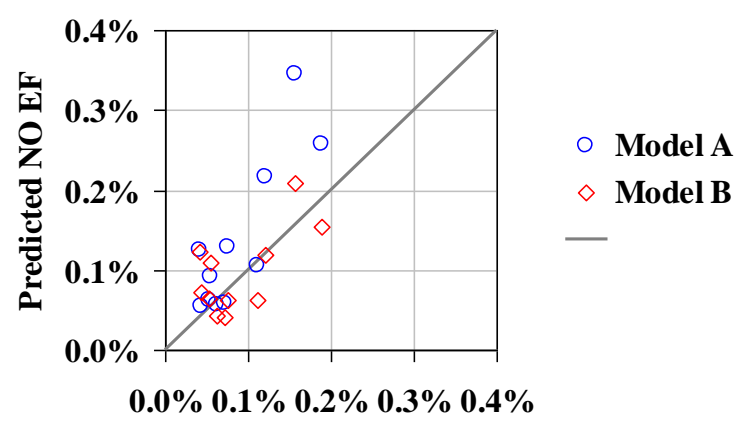

Experimental NO EF

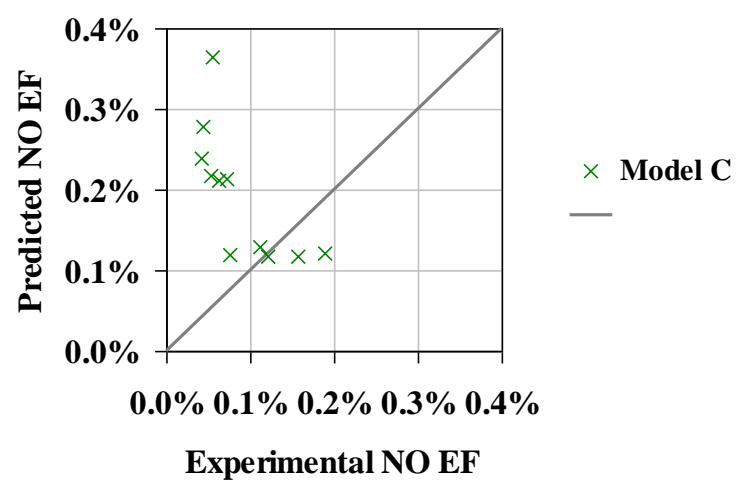

Figure 9. Comparison between experimental and predicted $\mathrm{N}_{2} \mathrm{O}$ and $\mathrm{NO}$ emission factors for 11 cycles obtained with the SBR(1). 


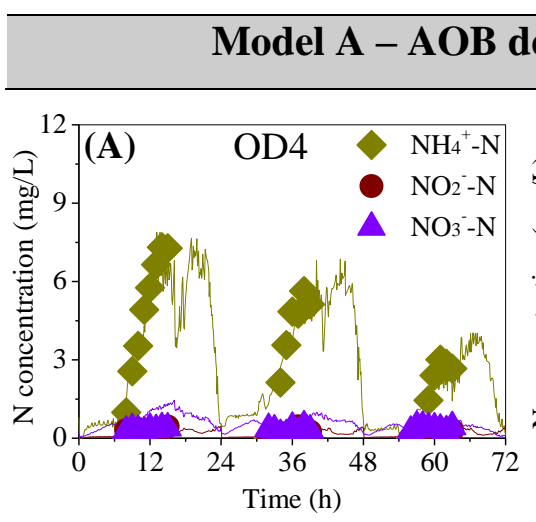

\section{Model A - AOB denitrification pathway (Ni et al., 2011; Table 2)}
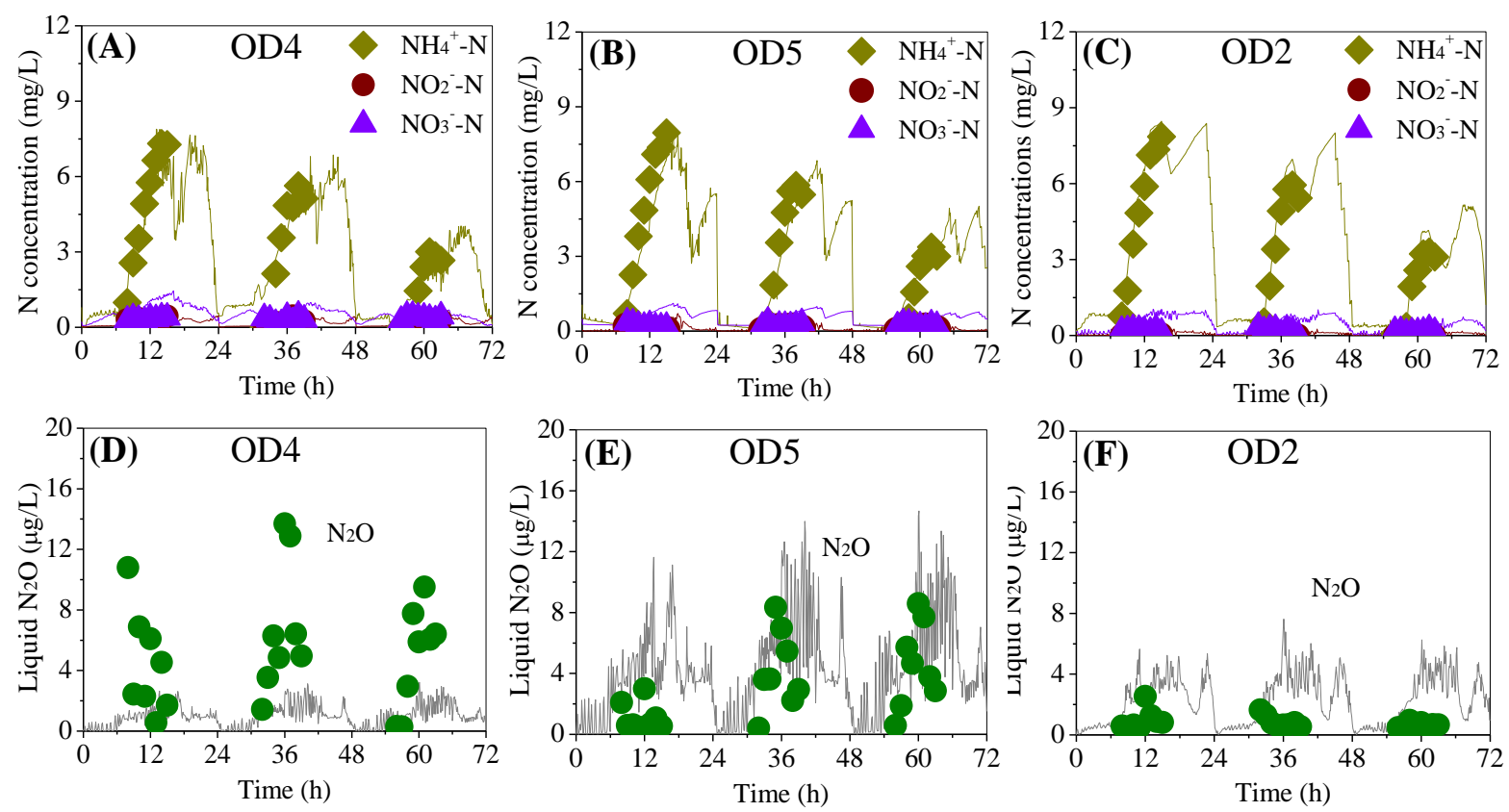

\section{Model B - AOB denitrification pathway (Mampaey et al., 2013; Table 2)}
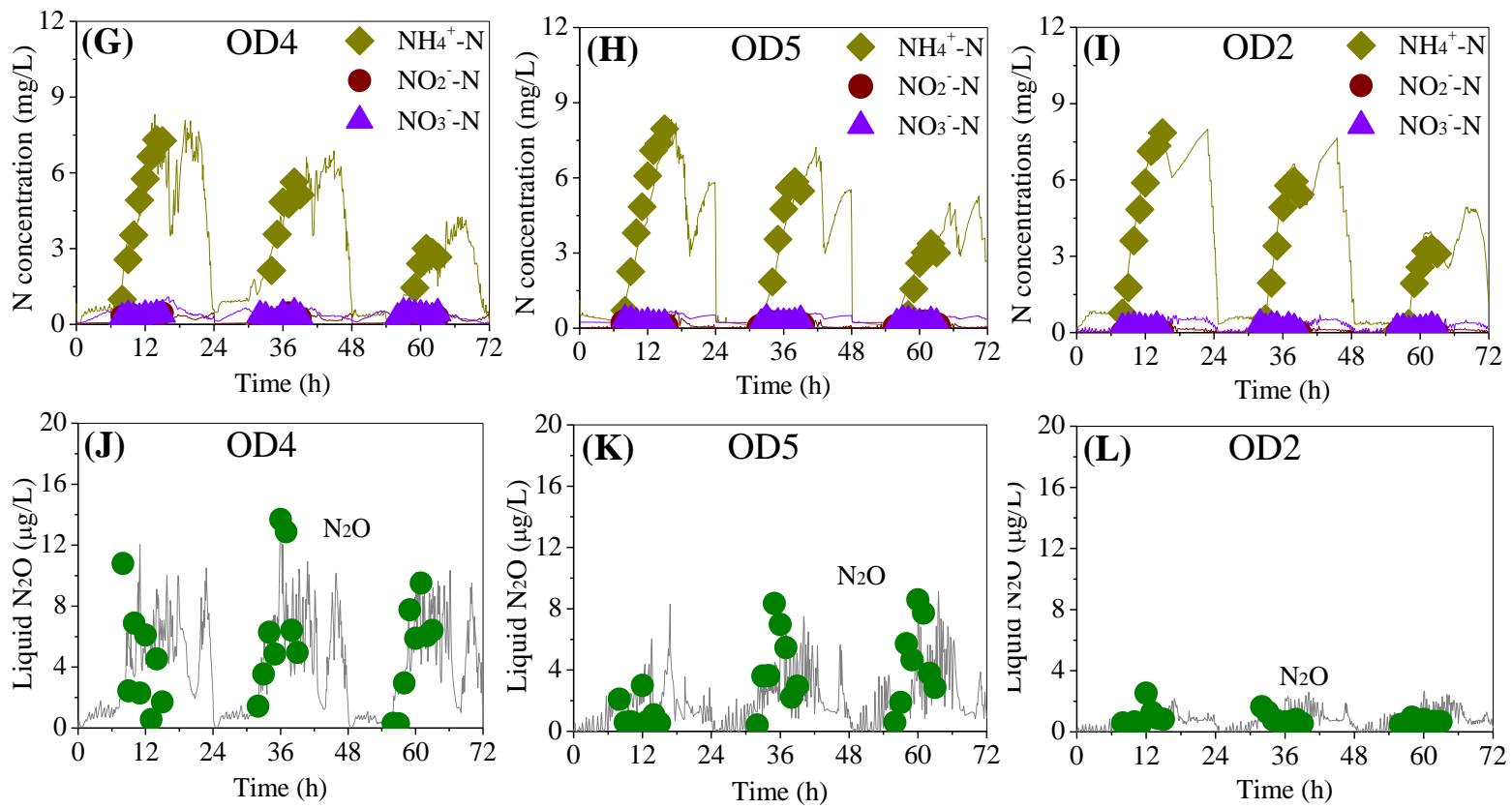


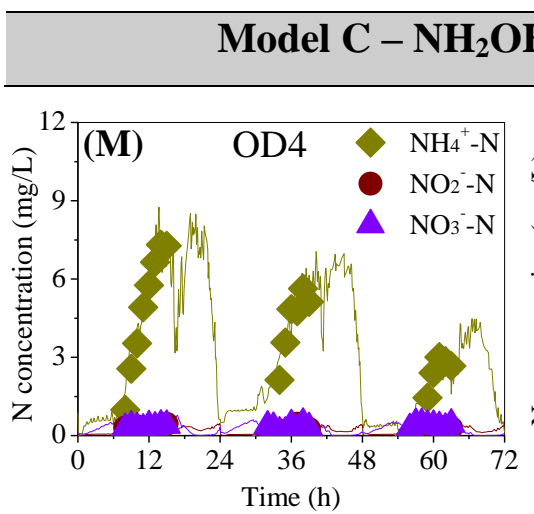

\section{Model $\mathrm{C}-\mathrm{NH}_{2} \mathrm{OH}$ oxidation pathway (Ni et al., 2013b; Table 2)}
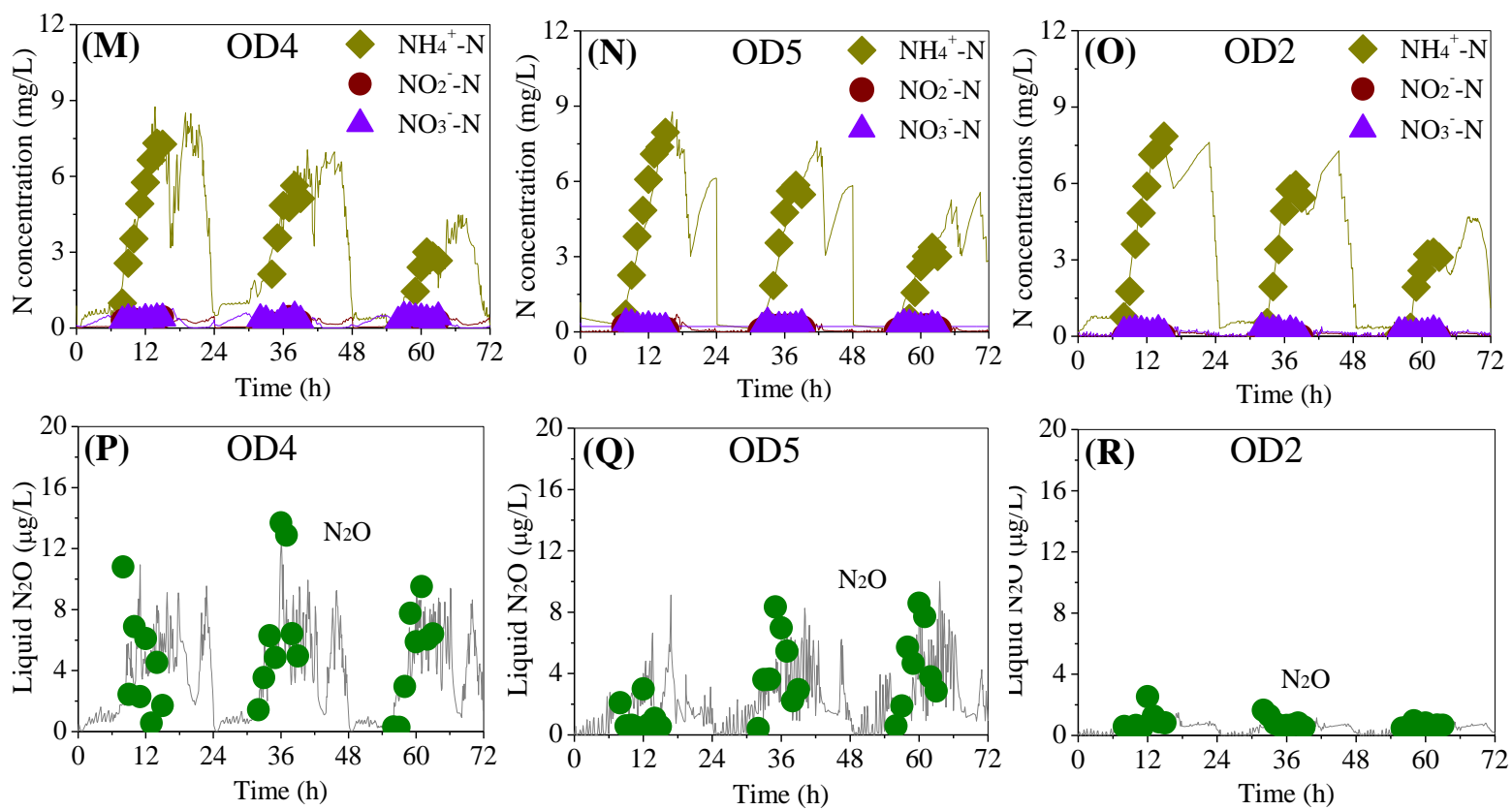

Figure 3. Model evaluation results of the three-day $\mathrm{N}_{2} \mathrm{O}$ production data from the Oxidation Ditch WWTP (experimental data: symbols; model predictions: lines) with ammonium, nitrite, nitrate, and liquid phase $\mathrm{N}_{2} \mathrm{O}$ profiles at the different sampling locations (OD4, OD5 and OD2): (A-F) Model A; (GL) Model B; and (M-R) Model C. 

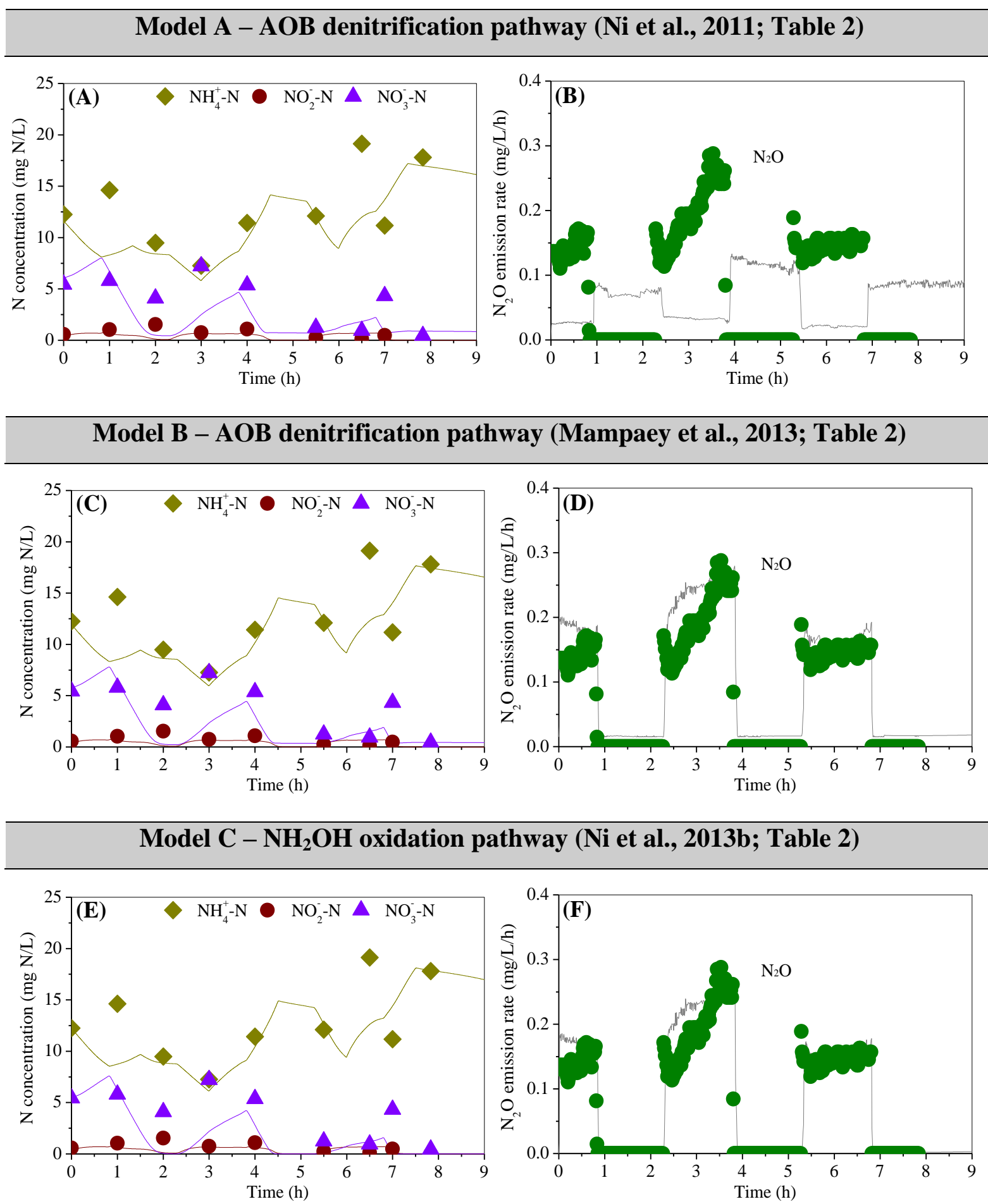

Figure 4. Model evaluation results of the $\mathrm{N}_{2} \mathrm{O}$ production data from SBR (2) WWTP (experimental data: symbols; model predictions: lines) with ammonium, nitrite, nitrate, and $\mathrm{N}_{2} \mathrm{O}$ emission rate profiles: (A-B) Model A; (C-D) Model B; and (E-F) Model C. 
a.

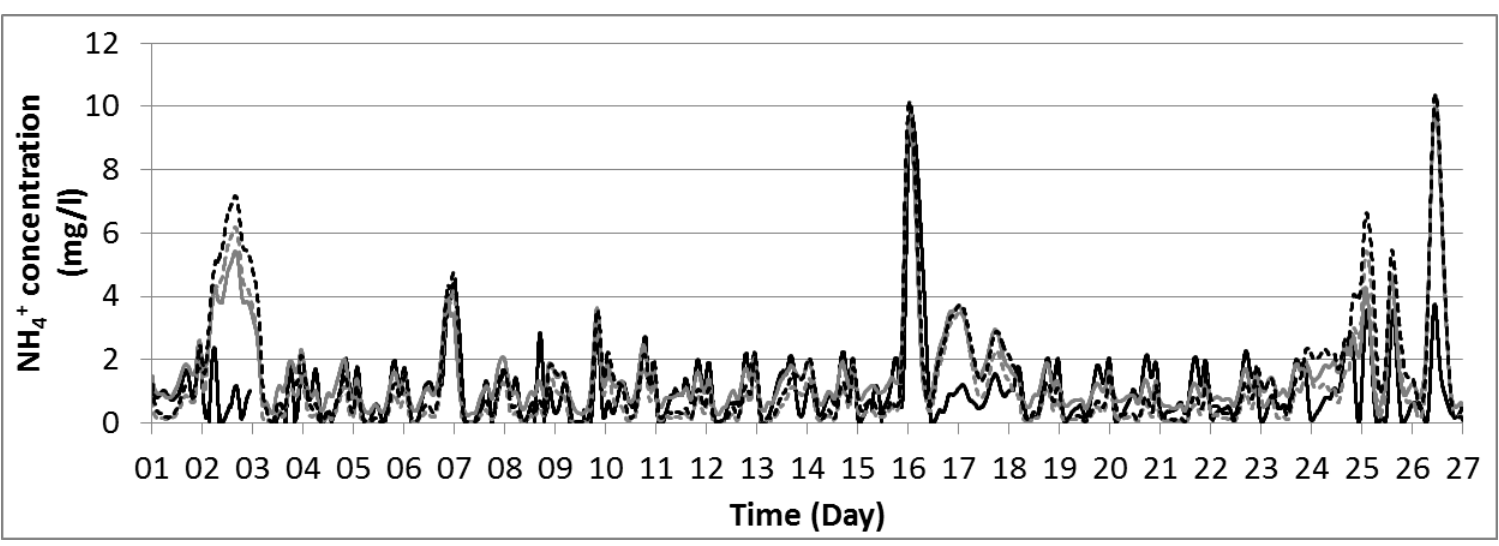

b.

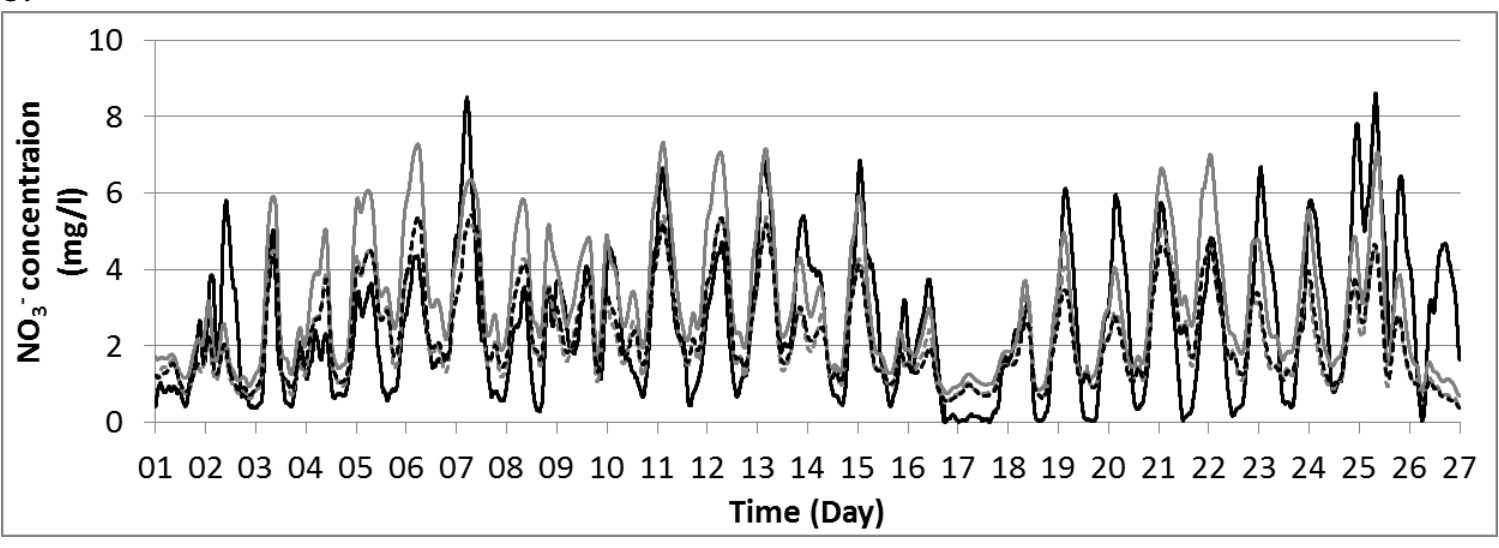

c.

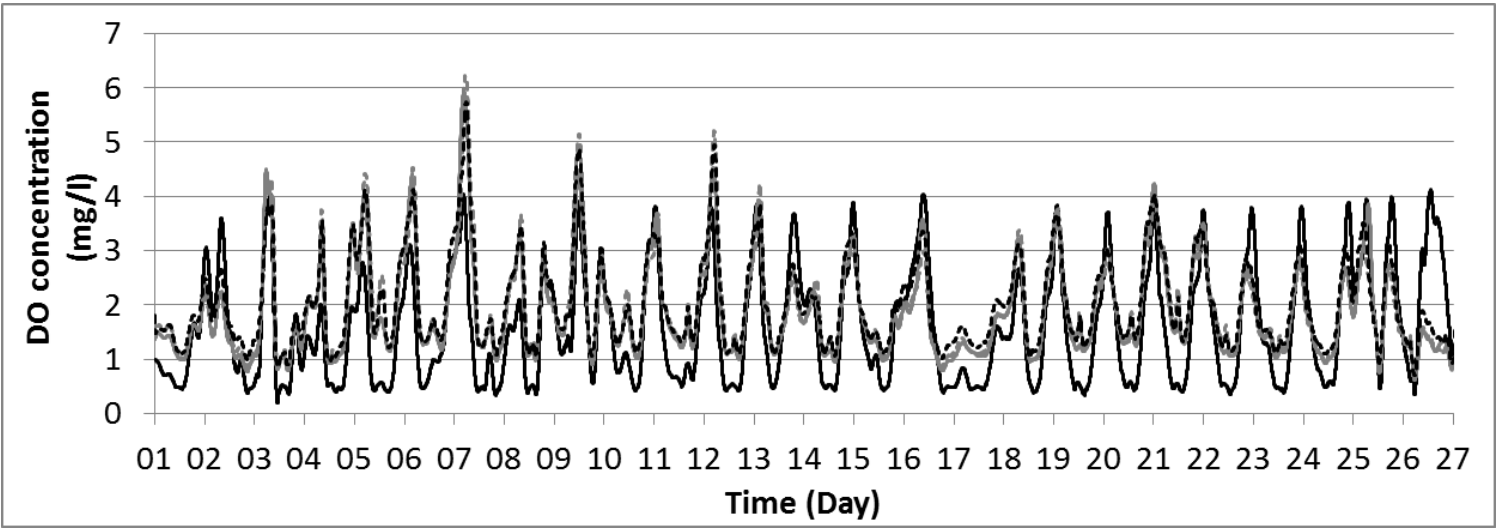

$\begin{array}{lll}\text { Measurement } & ---\cdot & \text { Simulation by Model A1 } \\ \text { Simulation by Model B1 } & \text {---.. } & \text { Simulation by Model C }\end{array}$

Figure 5. Comparison of the measurement results with the simulation results of $\mathrm{NH}_{4}{ }^{+}$(a), $\mathrm{NO}_{3}{ }^{-}$(b) and $\mathrm{DO}$ (c) near the outlet of the summer aeration package 
a.

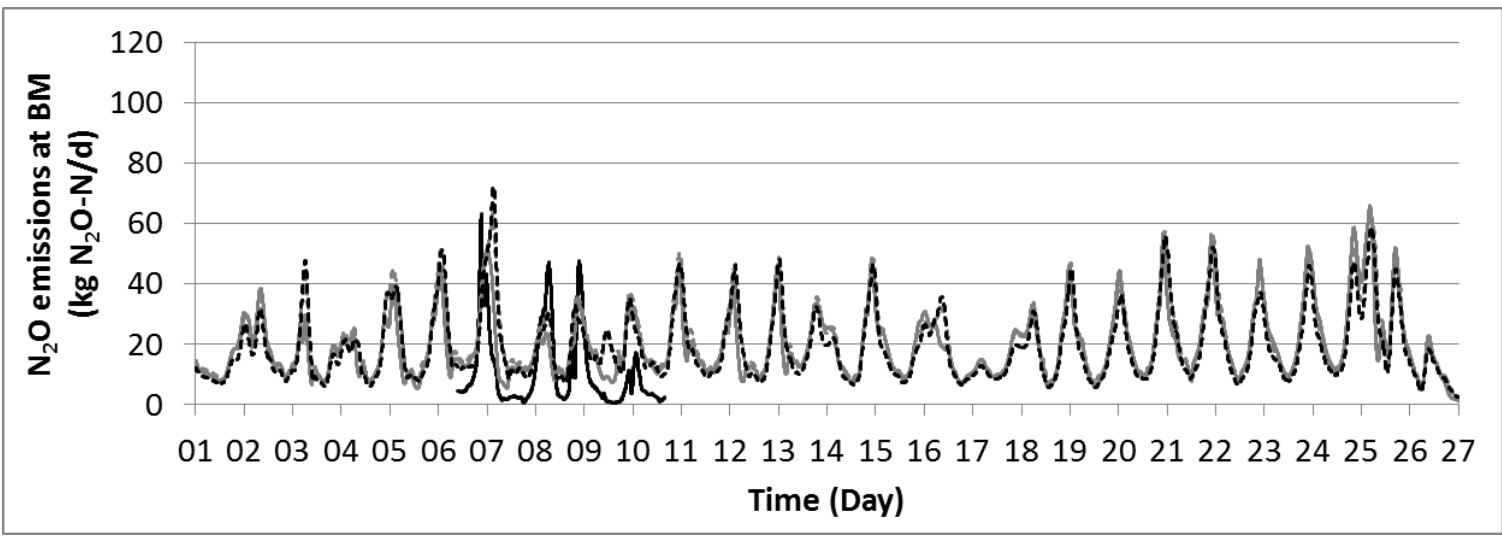

b.

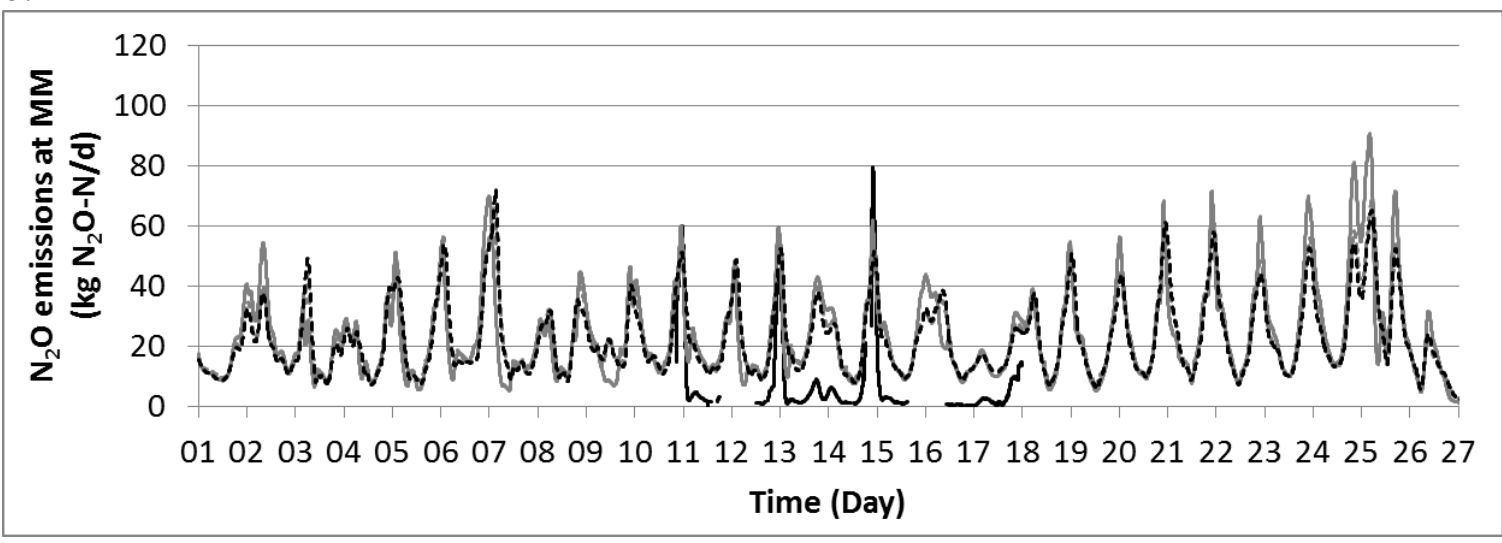

c.

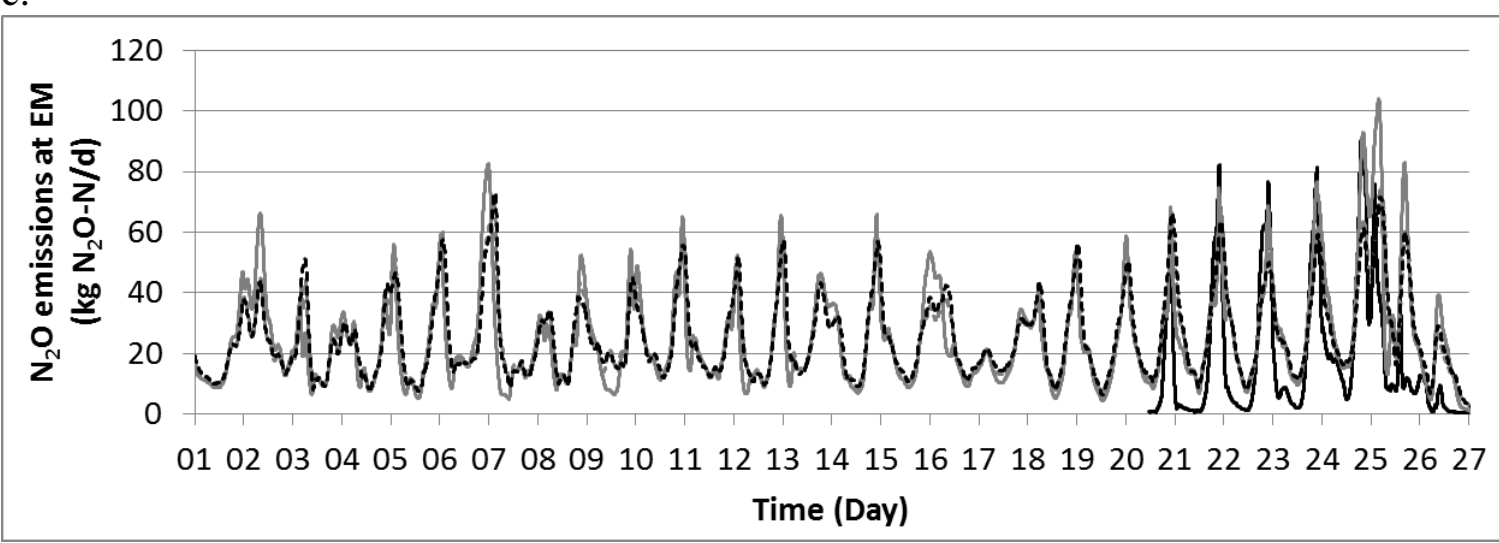

Measurement - - $\quad$ Simulation by Model A1

Simulation by Model B1 _.... Simulation by Model C

Figure 6. Comparison of simulated and measured $\mathrm{N}_{2} \mathrm{O}$ emissions at the beginning (BM) (a), the middle (MM) (b) and the end section (EM) (c) of the summer aeration package 


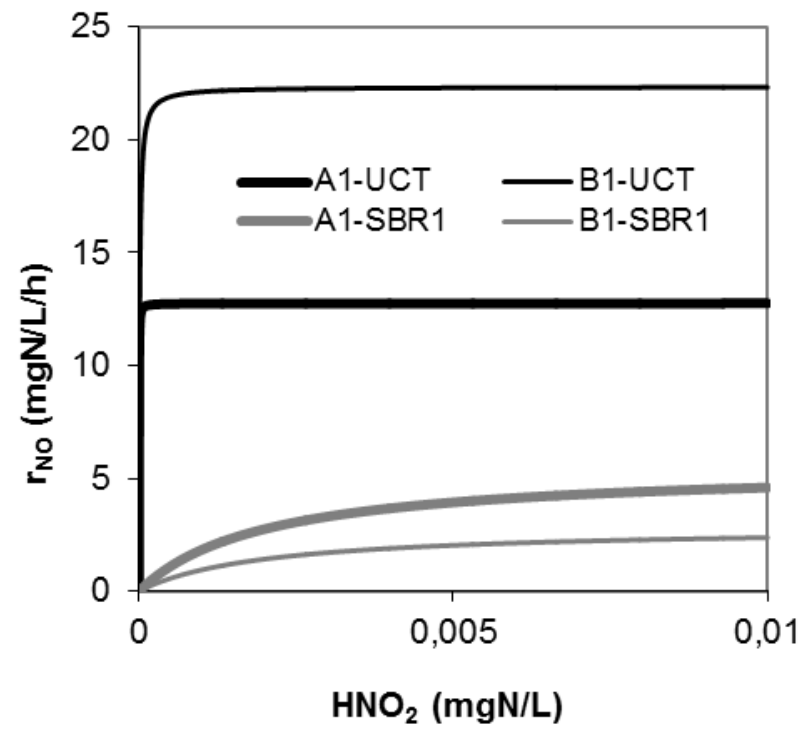

Figure 7. Relation between NO production rate and FNA concentration with AOB denitrification models (A1, B1) calibrated on different data (UCT, SBR(1)). 


\title{
Towards BSM2-GPS-X: A plant-wide benchmark simulation model not only for carbon and nitrogen, but also for greenhouse gases $(G)$, phosphorus $(\mathbf{P})$, sulphur (S) and micropollutants $(X)$, all within the fence of WWTPs/WRRFs
}

\author{
Peter Vanrolleghem ${ }^{1}$, Xavier Flores-Alsina ${ }^{2}$, Lisha Guo ${ }^{1}$, Kimberly Solon ${ }^{3}$, David Ikumi ${ }^{4}$, Damien \\ Batstone $^{5}$, Chris Brouckaert ${ }^{6}$, Imre Takács ${ }^{7}$, Paloma Grau ${ }^{8}$, George Ekama ${ }^{4}$, Ulf Jeppsson ${ }^{3}$ and Krist \\ V. Gernaey ${ }^{2}$ \\ ${ }^{1}$ modelEAU, Département de génie civil et de génie des eaux, Université Laval, Québec, QC, Canada, G1V \\ 0 A6. \\ ${ }^{2}$ Department of Chemical and Biochemical Engineering, Technical University of Denmark (DTU), \\ Building 229, DK-2800 Kgs. Lyngby, Denmark. \\ ${ }^{3}$ Department of Measurement Technology and Industrial Electrical Engineering, Division of Industrial \\ Electrical Engineering and Automation (IEA), Lund University, Box 118, SE-221 00 Lund, Sweden. \\ ${ }^{4}$ Water Research Group, Department of Civil Engineering, University of Cape Town, Rodenbosh, South \\ Africa. \\ ${ }^{5}$ Advanced Water Management Centre (AWMC), University of Queensland, St Lucia, QLD 4072, \\ Australia. \\ ${ }^{6}$ Pollution Research Group, School of Chemical Engineering, University KwaZulu-Natal, Durban, South \\ Africa \\ ${ }^{7}$ Dynamita, 7 Lieu-dit Eoupe, F-26110 Nyons, France. \\ ${ }^{8}$ Department of Environmental Engineering, CEIT, Paseo Manuel Lardizábal 15, 20018 Donostia, Spain.
}

\section{INTRODUCTION}

Benchmarking WWTP/WRRF control strategies has turned out to be a very successful vehicle for development of consolidated models of whole facilities (Gernaey et al., 2014). Over the last year, a comprehensive think tank of researchers involved in this development has put together a wish list of future developments in WWTP/WRRF modelling. They want these ideas to be exposed to the modelling community gathered at WWTmod2014. Starting from a summary ongoing work on extending the Benchmark Simulation Model No.2 (BSM2) and the wish to extend the current BSM2 to include phosphorus aspects, the think tank presents this abstract to outline the options lying ahead. The lecture will present arguments for choosing among the identified modelling options, in order to get informed feedback from the WWTmod2014 audience and define a roadmap for future modelling efforts. It is believed that this way of guiding the combined (voluntary) efforts of model development will be beneficial to the WWTP/WRRF modelling community at large.

The developments in the benchmarking area this paper will discuss relate to:

G. Greenhouse gases (GHG): Next to methane and $\mathrm{CO}_{2}$ that are intrinsically part of the plant-wide benchmark simulation model, recent work has focused significantly on $\mathrm{N}_{2} \mathrm{O}$ emissions, leading to considerable extensions to the details of the N-removal submodel of the BSM2;

P. Phosphorus: P-removal has been a focus of WWTP/WRRF design and operation, but its inclusion in whole plant models is lagging behind that of $\mathrm{N}$-removal, due to the difficulties in modelling the precipitation processes that $\mathrm{P}$ is involved in, especially in the sludge train. 
S. Sulphur: This element is receiving increased attention not only because of its role as a competitor for $\mathrm{P}$ and the related impact on P-complexation and release, but also because new processes in seawater-based systems (e.g. the SANI-process) take advantage of sulphur as a reactive element. Efforts to control $\mathrm{H}_{2} \mathrm{~S}$ emissions and induced corrosion in sewer systems will benefit from such S-focused modelling efforts as well.

X. Micropollutants: Recent interest in micropollutants has led to a diversity of model developments that would benefit from consensus-building and inclusion in the benchmark simulation platform. The diversity of micropollutants remains a challenge, but consensus can probably be found regarding models of the overall fate-determining mechanisms (sorption, biodegradation, volatilization, hydrolysis, photolysis ...).

With these four themes combined, the name to be given to this extension of the plant-wide Benchmark Simulation Model No.2 coincidentally turns out to be BSM2-GPS-X, a nice wink to one of the important WWTP/WRRF simulators.

To reach this goal, the following topics will have to be addressed: 1) new evaluation criteria; 2) new chemical and biochemical processes that should be taken into account; 3) new components involved in these processes that thus need to be modelled; 4) new influent wastewater characteristics; 5) modifications to the original BSM2 physical plant layout and new unit process models; 6) model integration; and, 7) new control handles and opportunities. These are shortly presented below.

The intention at the WWTmod2014 seminar is to select a number of the more clear-cut topics developed below, and get the opinion from the audience by presenting them with a clear choice and a way to directly provide feedback (colored cards or a SurveyMonkey on-line voting system).

\section{DISCUSSION TOPICS}

\section{New evaluation criteria}

With the ambition to use benchmark simulation models to evaluate the control and monitoring performance of WWTP/WRRFs that go beyond what could be achieved with BSM1/2 (COD/N-removal) a new set of evaluation criteria needs to be put forth:

$\checkmark$ Is the approach for GHG-emission evaluation appropriate (Flores-Alsina et al., 2014)?

$\checkmark$ P-related criteria should be added, such as its contribution to the effluent quality index.

$\checkmark$ Given the interest in nutrient recovery, should evaluation criteria be developed that highlight production of (high quality) recovered nutrients from WWTPs/WRRFs?

$\checkmark$ Should sulphur compounds be considered in performance evaluation and in what way?

$\checkmark$ Do we consider ecotoxicity to evaluate micropollutant removal (Clouzot et al., 2013)?

\section{New chemical and biochemical transformations}

Upgrading the BSM WWTP with the GPS-X related transformations inevitably leads to a wide range of processes that need to be added. The level of complexity remains an open question though:

$\checkmark$ GHG-modelling has matured significantly over the last two years, but finalization is still required (e.g. Ni et al., 2013);

$\checkmark$ In GHG-models temperature dependency of reaction kinetics has been modelled using the Ratkowsky equation instead of Arrhenius, enabling to model decreasing rates at high temperatures. Is this to be applied to all kinetics?

$\checkmark$ Is ASM2d still adequate for modelling enhanced biological P-removal or do we need modifications, e.g. electron acceptor dependent decay, denitrification by specific 
substrates with special kinetics (methanol, glycerol, methane, ...), role of the ions $\mathrm{Mg}^{2+}, \mathrm{Ca}^{2+}, \mathrm{K}^{+}$

$\checkmark$ Is it important to model the behaviour of PAO's when they enter the anaerobic digester with a consequent P-release, VFA uptake, storage? How must ADM1 be upgraded to account for phosphorus and treatment of Bio-P sludge? Is ADM1 the proper model (Ikumi et al., 2011)?

$\checkmark$ Is it important to include anoxic-aerobic digestion of Bio-P wastage sludge with lime or $\mathrm{Mg}$ dosing for P-precipitation (to achieve low $\mathrm{N}$ and $\mathrm{P}$ dewatering liquors) for sludge treatment and how must ASM2/2d be modified to also model this (Vogts and Ekama, 2012)?

$\checkmark$ Which precipitation reactions to model and which numerical approach to use?

$\checkmark$ How will the physico-chemical model look like (precipitation, acid-base reactions, $\mathrm{pH}$, ion-pairing, ion activity, etc.) in terms of level of detail, equation structure and solver requirements, etc. (Batstone et al., 2012);

$\checkmark$ If S-containing components are considered important for P-modelling (e.g. competition for iron), which of the (bio)chemical S-related oxido-reduction processes need to be included and how is the competition with methanogens to be modelled?

$\checkmark$ Do the traditional micropollutant fate models (volatilization, sorption, photolysis, biodegradation) that only require standard chemical properties (Henry coefficients, Kow, ...) suffice or are dedicated models necessary for each micropollutant (Clouzot et al., 2013)?

\section{New components}

If the above list of processes is all considered important, a wide range of new components will have to be added to the current set of components considered in the next generation of BSM models:

$\checkmark$ Inert inorganic suspended solids need to be added for proper TSS accounting;

$\checkmark$ P-related components (phosphate, PAO's, poly-P, PHA) and the components related to precipitation and PAO hydrolysis (calcium, magnesium, potassium, iron, struvite, $\mathrm{K}$ struvite, newberyite, calcium phosphate, iron phosphate, iron hydroxide, calcite, magnesite);

$\checkmark$ S-related components (sulphate, sulphite, sulphur, $\mathrm{H}_{2} \mathrm{~S}$, poly-S, iron and other sulphides);

$\checkmark$ A multitude of micropollutants (and their transformation products);

$\checkmark$ Components related to GHG emissions (methane, $\mathrm{CO}_{2}, \mathrm{NO}, \mathrm{N}_{2} \mathrm{O}, \mathrm{NH}_{2} \mathrm{OH}$ ).

\section{New influent wastewater characteristics}

Evidently, when the set of state variables is extended, the inputs to the system will have to be extended too, including methane $(\mathrm{G})$, phosphorus $(\mathrm{P})$, sulphur $(\mathrm{S})$, micropollutants $(\mathrm{X})$. Are colloids important for the proposed configuration? Multiple approaches could be proposed:

$\checkmark$ Can we just use correlations with the traditional wastewater components in the influent files used so far (using TSS, COD and $\mathrm{N}$-fractions as independent variables to correlate with)?

$\checkmark$ Do we need to develop new influent generation models that include, for instance, methane formation in the sewer (Guisasola et al., 2009), micropollutant release patterns (De Keyser et al., 2010), sulphur conversions, etc.?

$\checkmark$ Do we need to provide detailed information on influent $\mathrm{pH}$-dynamics and acid-base / ion composition to support the physico-chemical model that is required?

\section{New physical layout and unit process models}


The suggested layout of the BSM2-GPS-X WWTP is shown in Figure 1. The activated sludge section has been modified by including an anaerobic section for Bio-P removal. The actual volumes are still open for discussion and will be obtained through application of standard design guidelines, just as for BSM2 (Gernaey et al., 2014). Some proposals have been made to extend the layout that this paper will put to the floor for feedback:

$\checkmark$ Should we include a P-recovery unit process in the sludge train and how do we model it? Evidently, including such a process in the layout would attract a lot of attention to the BSM work, and in principle the physico-chemical model contains the necessary processes, but is there a "typical" P-recovery process that industry would accept as being representative?

$\checkmark$ Sludge reject water treatment was already tried out in the BSM2 process layout (Volcke et al., 2006). Is it time to make this an integral part of a whole plant model?

$\checkmark$ Should we also model the pumping station, screens and grit chamber so as to really model all processes within the fence? A storage tank prior to the digester could allow for more control options and the addition of external organic material input to the system.

$\checkmark$ The primary clarifier is receiving increased attention due to its potential role in separating organics for energy recovery by anaerobic digestion (Flores-Alsina et al., 2014). Should chemical enhancing of primary treatment (CEPT) be added to the BSMs (Tik et al., 2013)?

$\checkmark$ Is a "fermenter" that generates VFA's out of primary sludge a unit process to be included in the whole plant configuration to be studied in the future? It certainly would provide an interesting possibility for control.

$\checkmark$ Should the secondary settler model be upgraded to include compression and dispersion and to make its numerical solution consistent (Bürger et al., 2011)?

$\checkmark$ Is it now time to explicitly consider the reactions in the secondary clarifier, in particular denitrification, and how would that best be done (fully reactive settler, separate reactor, etc. (Gernaey et al., 2006))? Must the same hold for thickeners and storage tanks?

$\checkmark$ Should we add effluent polishing systems for nutrients (denitrifying filters) and TSS (disk filters) and can consensus on a representative technology and appropriate models be found?

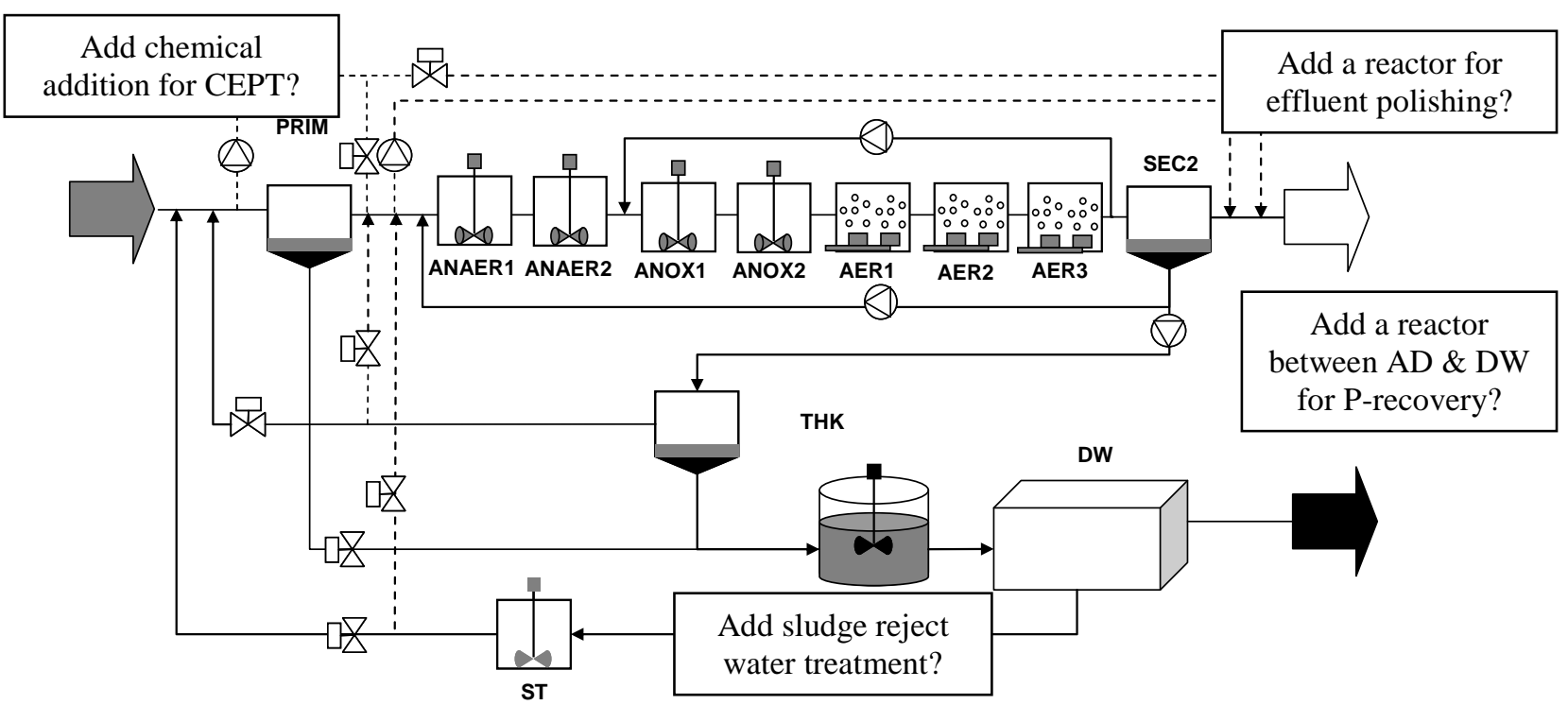

Figure 1. Plant layout of the proposed BSM2-GPS-X. Some questions remain to be answered. 


\section{Model integration}

So far, BSMs have used interfaces to integrate the submodels. As complexity increases, the following questions can be asked:

$\checkmark$ Do we stick to the approach with submodels with individual component sets and mass continuity interfaces to connect them or do we move towards the plant-wide modelling concept or the Supermodel approach (Grau et al., 2009)?

$\checkmark$ If we stick to interfaces, do we extend the existing ad hoc interface or do we rigorously apply the continuity-based-interfacing-of-models (CBIM) approach (Vanrolleghem et al., 2005)?

\section{New sensors and control handles}

Control of WWTP/WRRFs will require a new set of sensor models to measure, for example, phosphate concentrations. Also, more control handles will become available compared to BSM2.

$\checkmark$ Models for P-analysers and nitrite measurement devices need to be established;

$\checkmark$ Will off-gas analysis (methane, $\mathrm{H}_{2} \mathrm{~S}, \mathrm{O}_{2}, \mathrm{~N}_{2} \mathrm{O}, \mathrm{CO}_{2} \ldots$ ) break through and how will we model these measuring systems with gas sample preparation?

$\checkmark$ Models for actuators needed for CEPT may have to be developed, given their particularities;

$\checkmark$ Which sensors and control handles can be modelled for nutrient recovery systems? For instance one could imagine on-line particle size distribution measurement combined with seeding as a control handle for struvite formation.

\section{CONCLUSION}

This contribution intends to allow the control benchmarking community to get feedback from the wastewater modelling community regarding the requirements for the next generation benchmark simulation model. Given the many spin-offs that the benchmark modelling efforts have generated for the wastewater modelling community at large (Gernaey et al., 2013), it is believed that streamlining the work that is planned to occur in this benchmarking framework should be guided by the specialists in the discipline.

Efforts have and will be deployed to make the interaction with the audience as efficient as possible by presenting only a selected number of key topics presented above and providing clear choices that can be answered during the time allocated for the presentation.

\section{ACKNOWLEDGEMENTS}

Peter Vanrolleghem holds the Canada Research Chair in Water Quality Modelling.

\section{REFERENCES}

Batstone D.J., Amerlinck Y., Ekama G., Goel R., Grau P., Johnson B., Kaya I., Steyer J.-P., Tait S., Takács I., Vanrolleghem P.A., Brouckaert C.J. and Volcke E. (2012) Towards a generalized physicochemical framework. Wat. Sci. Tech., 66(6), 1147-1161.

Bürger R., Diehl S. and Nopens I. (2011) A consistent modelling methodology for secondary settling tanks in wastewater treatment. Water Res., 45, 2247-2260.

Clouzot L., Choubert J.-M., Cloutier F., Goel R., Love N.G., Melcer H., Ort C., Patureau D., Plósz B.G., Pomiès M. and Vanrolleghem P.A. (2013) Perspectives on modelling micropollutants in wastewater treatment plants. Wat. Sci. Tech., 68(2), 448-461.

De Keyser W., Gevaert V., Verdonck F., De Baets B. and Benedetti L. (2010) An emission time series generator for pollutant release modelling in urban areas. Environ. Modell. Softw., 25, 554-561.

Flores-Alsina X., Arnell M., Amerlinck Y., Corominas Ll., Gernaey K.V., Guo L., Lindblom E., Nopens I., Porro J., Shaw A., Snip L., Vanrolleghem P.A. and Jeppsson U. (2014) Balancing effluent quality, economic cost and greenhouse gas emissions during the evaluation of (plant-wide) control/operational strategies in WWTPs. Sci. Total Environ. 466-467, 616-624.

Gernaey K.V., Jeppsson U., Batstone D.J. and Ingildsen P. (2006) Impact of reactive settler models on simulated 
WWTP performance. Wat. Sci. Tech., 53(1), 159-167.

Gernaey K.V., Jeppsson U., Vanrolleghem P.A. and Copp J.B. (2014) Benchmarking of Control Strategies for Wastewater Treatment Plants. IWA Scientific and Technical Report, IWA Publishing, London, UK.

Gernaey K.V., Copp J.B., Vanrolleghem P.A. and Jeppsson U. (2013) Lessons learned from the WWTP benchmarking exercise. In: Proceedings $11^{\text {th }}$ IWA Conference on Instrumentation, Control and Automation (ICA2013), Narbonne, France, September 18-20 2013.

Grau P., Copp J., Vanrolleghem P.A., Takács I. and Ayesa E. (2009) A comparative analysis of different approaches for integrated WWTP modelling. Wat. Sci. Tech., 59(1), 141-147.

Guisasola A., Sharma K.R., de Haas D., Keller J. and Yuan Z. (2009) Development of a model for assessing methane formation in rising main sewers. Water Res., 43, 2874-2884.

Ikumi D.S., Brouckaert C.J. and Ekama G.A. (2011) A 3 phase anaerobic digestion model. In: Proceedings $8^{\text {th }}$ IWA International Symposium on Systems Analysis and Integrated Assessment in Water Management (Watermatex2011). San Sebastian, Spain, June 20-22 2011.

Ni B.-J., Yuan Z., Chandran K., Vanrolleghem P.A. and Murthy S. (2013) Evaluating four mathematical models for nitrous oxide production by autotrophic ammonia-oxidizing bacteria. Biotechnol. Bioeng., 110, 153163.

Tik S., Langlois S. and Vanrolleghem P.A. (2013) Establishment of control strategies for chemically enhanced primary treatment based on online turbidity data. In: Proceedings $11^{\text {th }}$ IWA Conference on Instrumentation, Control and Automation (ICA2013). Narbonne, France, September 18-20 2013.

Vanrolleghem P.A., Rosen C., Zaher U., Copp J., Benedetti L., Ayesa E. and Jeppsson U. (2005) Continuitybased interfacing of models for wastewater systems described by Petersen matrices. Wat. Sci. Tech., 52(12), 493-500.

Vogts M. and Ekama G.A. (2012) Anoxic-aerobic digestion of waste activated sludge from biological nitrogen and phosphorus removal systems. In: Proceedings $12^{\text {th }}$ biennial WISA conference and exhibition, Cape Town, South Africa, May 7-10 2012. Paper 1154-164.

Volcke E.I.P., Gernaey K.V., Vrecko D., Jeppsson U., van Loosdrecht M.C.M. and Vanrolleghem P.A. (2006) Plant-wide (BSM2) evaluation of reject water treatment with a SHARON-Anammox process. Wat. Sci. Tech., 54(8), 93-100. 


\title{
Population Balance Models: A useful complementary modelling framework for future WWTP modelling
}

\author{
Ingmar Nopens ${ }^{1}$, Elena Torfs ${ }^{1}$, Joel Ducoste $^{2}$, Peter A. Vanrolleghem ${ }^{3}$ and Krist V. Gernaey ${ }^{4}$ \\ ${ }^{1}$ BIOMATH, Department of Mathematical Modelling, Statistics and Bio-informatics, Ghent University, \\ Coupure Links 653, 9000 Gent, Belgium (Email: Ingmar.Nopens@UGent.be) \\ ${ }^{2}$ NC State University, 208 Mann Hall Raleigh, NC USA (Email: jducoste@ ncsu.edu) \\ 3 modelEAU, Département de génie civil et de génie des eaux, Université Laval, 1065 av. de la Médecine, \\ Québec, QC, Canada, G1V 0A6 (Email : peter.vanrolleghem@gci.ulaval.ca) \\ ${ }^{4}$ Department of Chemical and Biochemical Engineering, Technical University of Denmark (DTU), \\ Building 229, DK-2800 Lyngby, Denmark (Email: kvg@kt.dtu.dk)
}

\begin{abstract}
Population Balance Models (PBMs) represent a powerful modelling framework for the description of the dynamics of properties that are characterised by statistical distributions. This has been demonstrated in many chemical engineering applications. Modelling efforts of several current and future unit processes in WWTPs could potentially benefit from this framework, especially when distributed dynamics have a significant impact on the overall unit process performance. In these cases, current models that rely on average properties cannot sufficiently captured the true behaviour. Examples are bubble size, floc size, crystal size or granule size,... PBMs can be used to provide new insights that can be embedded in our current models to improve their predictive capability. This paper provides an overview of current applications and the future potential of PBMs in the field of WWT modelling, introducing new insights and knowledge from other scientific disciplines.
\end{abstract}

\section{Keywords}

bubble size, distributed properties, floc size, granule size, heterogeneity, PBM, product specifications, quality by design

\section{INTRODUCTION TO POPULATION BALANCE MODELS (PBM)}

Many natural systems consist of populations of individual entities (e.g. flocs, bubbles, granules, crystals, bacterial cells) with specific properties (e.g. size, composition, density, activity). The individual entities interact with their environment (e.g. dissolved chemical precipitation, oxygen transfer from air bubble to liquid phase, shear-induced breakup) or with one another (e.g. aggregation, coalescence). Typically, these interactions are a function of one or more properties, which may vary within a population of entities. In this context, we can refer to this variation as "distributed properties" as they can be represented by a distribution instead of a scalar (i.e. one single value). A simple example of the use of a distribution would be to characterize the variation in floc size in an activated sludge system. This distributed feature implies that the behaviour of distinct entities can be significantly different, and can deviate substantially from 'average' non-distributed behaviour.

Current modelling frameworks usually assume non-distributed scalar properties (e.g. using a single particle size or bubble size), implying that all individuals behave in exactly the same way. In some cases, this is sufficient, but in others this assumption is too strict and will lead to predictions that deviate significantly from the real system. The required level of detail is clearly governed by the modelling objective. Yet, few researchers and design engineers in WWT are aware of alternative modelling approaches that can account for the impact of these distributed properties on unit process performance.

A framework to achieve distributed behaviour by considering distributed population properties already exists and is called Population Balance Models (PBMs). PBMs have been 
extensively used in chemical engineering for a myriad of applications (Ramkrishna, 2000; Marchisio, 2012). The governing equation in its most general form is given as

$$
\frac{\partial f_{1}(x, t)}{\partial t}+\frac{\partial}{\partial x}\left(\dot{X}(x, t) f_{1}(x, t)\right)=h(x, t)
$$

where $x$ is the internal coordinate (i.e. the distributed property), $f_{l}(x, t)$ is the number density function (i.e. the distribution of the distributed property), $\dot{X}(x, t)$ is the continuous growth term of $x$ and $h(x, t)$ is the PBM reaction term (through discrete events). Table 1 provides some examples in regard to wastewater treatment applications.

Table 1 - Some examples of wastewater treatment processes and related internal coordinates (x), number density functions $\left(f_{l}(x, t)\right)$ and continuous $(\dot{X}(x, t))$ and discrete $(h(x, t))$ governing mechanisms

\begin{tabular}{|c|c|c|c|c|}
\hline Process & X & $f_{1}(x, t)$ & $\dot{X}(x, t)$ & $h(x, t)$ \\
\hline \multirow[b]{2}{*}{ (De)flocculation* } & Floc size & Floc size distribution & Microbial growth & Aggregation, breakage \\
\hline & Size/Density** & $\begin{array}{l}\text { Size/Density } \\
\text { distribution }\end{array}$ & - & Aggregation \\
\hline Coalescence & Bubble size & Bubble size distribution & - & Coalescence \\
\hline Granulation & Granule size & \begin{tabular}{|ll}
$\begin{array}{l}\text { Granule } \\
\text { distribution }\end{array}$ & size \\
\end{tabular} & Microbial growth & Granulation \\
\hline Crystallization & Crystal size & Crystal size distribution & Crystal growth & Aggregation, breakup \\
\hline Bio P-removal & Poly-P & Poly-P distribution & $\begin{array}{l}\text { Poly-P storage, } \\
\text { release }\end{array}$ & Cell division, cell birth \\
\hline PHB production & PHB & PHB distribution & $\begin{array}{l}\text { PHB storage, } \\
\text { release }\end{array}$ & Cell division, cell birth \\
\hline Growth & $\mu_{\max }$ & $\mu_{\max }$ distribution & $\begin{array}{ll}\begin{array}{l}\text { Growth } \\
\text { gradient }\end{array} & \text { rate }\end{array}$ & Cell division, cell birth \\
\hline Affinity & $\mathrm{Kx}$ & Kx distribution & Affinity gradient & Cell division, cell birth \\
\hline
\end{tabular}

*Note that this mechanism is driving the settling processes in primary and secondary sedimentation

$* *$ In this case a $2 \mathrm{D}$ PBM is obtained

The first term on the left hand side of Eq. 1 represents the accumulation term. Distribution dynamics that can be described are either governed by continuous processes (e.g. biomass growth, crystal growth, particle drying - represented by the second term on the left hand side of Eq. 1) or discrete processes (e.g. aggregation, breakage, coalescence, granulation represented by the term on the right hand side). The latter term usually consists of a birth and a death term, where the birth rate describes the rate at which particles of property $\mathrm{x}$ are being formed and the death rate describes the rate at which they are being removed. In crystallisation, a nucleation term needs to be added in the smallest size class mimicking the nucleus that is formed in an oversaturated solution.

The internal coordinate $x$ can be either a scalar (i.e. a single independent variable) or a vector resulting in a 1 dimensional or multi-dimensional PBM, respectively. The use of multidimensional PBMs means that the distribution of one or more material properties can be described. It should be noted that formulating and solving multidimensional PBMs is a challenging task.

The nature of the resulting equation depends on the presence of the $\dot{X}(x, t)$ and $h(x, t)$ terms. If only the former is present, a partial differential equation (PDE) is obtained for which solution methods are available. The latter term usually contains integral terms expressing the interactions between members of the distribution, turning the equation into an integro-partial differential equation. Several numerical methods have been reported in the literature to solve this type of equations (Ramkrishna, 2000). 
Applications of PBMs to wastewater treatment processes are rather scarce. The first application was introduced by Fukushi et al. (1995), where a PBM model was used for modelling the dissolved air flotation process in water and wastewater treatment. The authors described the attachment process of bubbles to flocs during the flocculation process in a turbulent flow. Gujer (2002) investigated the impact of lumped average cell composition versus distributed composition in the context of ASM2 and ASM3 and concluded that this indeed had a significant impact. Schuler (2005) demonstrated that lumped state (= averaged) assumptions in EBPR system performance models produced large errors due to the difference in individual residence times of organisms in different zones. This was found to be related to process hydraulics (Schuler, 2006) and to impact the endogenous respiration as the latter was found to be more important when distributed models were applied (Schuler and Jassby, 2007). Finally, several PBM references can be found in the field of activated sludge flocculation ranging from very simple formulations (Parker et al., 1972) to more elaborate ones (Biggs et al., 2003; Nopens et al., 2002) and papers focussing on experimental validation (Nopens et al., 2005) and model structure analysis (Nopens et al., 2007; Torfs et al., 2012).

PBMs can serve the purpose of building process understanding. The result of such a detailed modelling exercise can be included in next generation simplified WWTP models that go beyond the currently used paradigms (i.e. ASM using averaged biomass behaviour combined with residence time distribution models and oversimplified aeration and settling models). Hence, PBM models should not be considered as replacement of current WWTP models, but as enhancement tools to improve the future quality of their unit process predictions. This paper intends to outline the potential of PBMs in the field of wastewater treatment through several examples of different WWTP unit processes.

\section{APPLICATIONS OF PBMS IN THE FIELD OF WWT}

\section{Improved flocculation to better exploit primary and secondary settling}

The current settling models are either based on removal efficiencies or settling velocities correlated with particle concentration. Settler models are still receiving attention as there is still room for improvement in their use (e.g. storage function during wet weather) and operation. This is especially true for primary settlers as they can be an important asset for energy recovery through the maximisation of the primary sludge that is sent to the anaerobic digester. Their behaviour has not been widely studied thus far (Bachis et al., 2014). Moreover, the primary treatment process is often chemically enhanced (CEPT) which creates an optimal dosage problem. As particle concentrations are low in primary settlers, the settling regime is not hindered but rather discrete, i.e. Stokian, and a function of particle size, shape and density, leading to a wide distribution of settling velocities as evidenced by Bachis et al. (2014). The discrete settling assumption is also true for the zone just above the sludge blanket of a secondary settler. In discrete settling, settling velocities are directly related to size, shape and density and, hence, the particle size distribution (PSD). The PSD depends on the original flocculation state as well as on actions undertaken to improve the flocculation state (e.g. turbulent shear, coagulant). Note that the flocculation state also depends on the particle's history (e.g; a sludge can have the same PSD, but it can react very different if the floc strength is different caused by a different flocculation history).

Flocculation of particles is probably the most straightforward application of PBMs. Since biological growth occurs on a much longer time scale compared to aggregation and breakage 
it can be ignored when studying short-term flocculation behaviour of activated sludge. The $\mathrm{PBM}$ reaction term is then defined as

$h(x, t)=B(x)_{\text {agg }}-D(x)_{a g g}+B(x)_{b r e a k}-D(x)_{b r e a k}$

Eq. 2

in which the birth (B) and death (D) terms occur for both aggregation and breakage as shown in Figure 1. Through these different mechanisms flocs of any size can be formed or removed. Furthermore, it becomes clear that aggregation is a particle-particle interaction process, whereas shear-induced breakage is not. The rates of all these processes are, hence, governed by the number of flocs present $(\mathrm{N})$ as well as an aggregation rate $(\beta)$, an aggregation efficiency $(\alpha)$ and a breakage rate $(S)$ and distribution of resulting particles (the so-called daughter size distribution). Aggregation and breakage rates are in their turn a function of the mechanisms that drive the aggregation or breakup. Traditionally, shear and polymer addition are accounted for which is likely sufficient for the application in the context of primary and secondary settling. More details on these rates and their dependencies on shear and flocculant addition can be found elsewhere (Nopens et al., 2002; Nopens et al., 2005). An example of a model prediction along with measured size distribution during a batch sludge flocculation process is shown in Fig. 2.

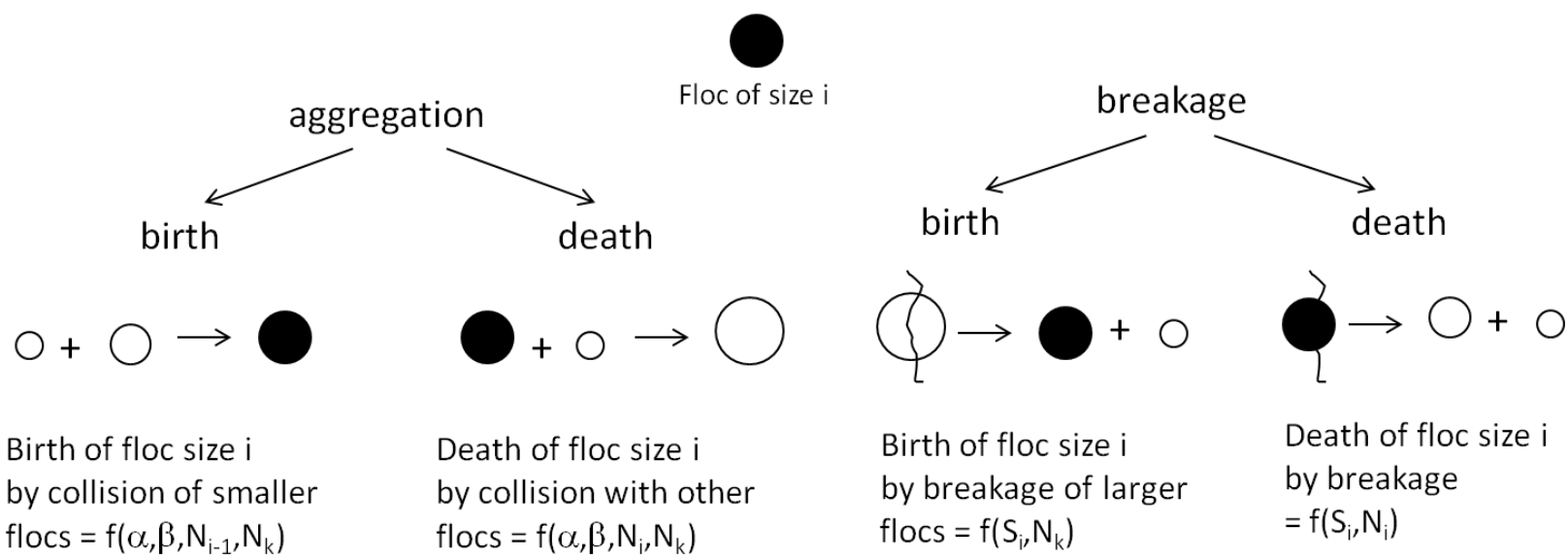

Fig. 1 - Illustration of mechanisms involved in flocculation (after Nopens et al., 2002)

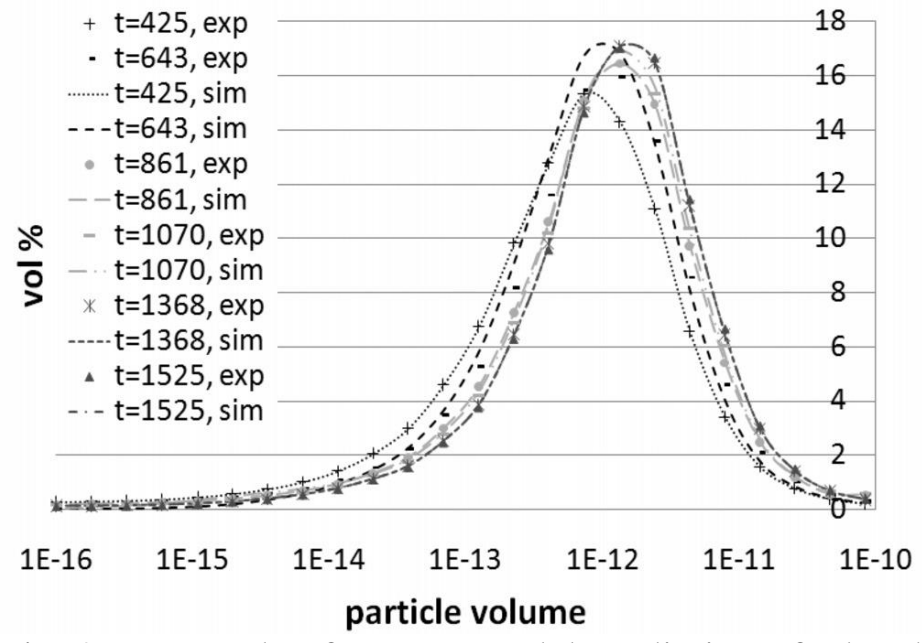

Fig. 2 - Example of a PBM model prediction of a batch sludge flocculation process (time unit $=$ seconds; unit on size axis $=\mu \mathrm{m}^{3}$ ) after Torfs et al., 2012) 
Flocculation usually takes place in the process units prior to the actual settling tank as well as in the settling tanks (if conditions are good). Flocculation models as described above can be used for both. Currently, the flocculation process in primary and secondary clarifiers is not studied in detail and its effects are incorporated using rules of thumb. However, understanding the contribution of flocculation would improve their design and operation which can significantly improve the settling performance and control. Indeed, being able to predict and control the size distribution of a population of particles arriving to either the primary or secondary settler would be a useful input to settler models that can handle a distribution of settling velocities, calculated from the size distribution derived with the PBM (Bachis et al., 2014).

In a secondary settler, exposure of flocs to elevated shear during transport from the bioreactor to the centre well of the settler will induce reflocculation and impact the floc size distribution and the floc strength. An appropriate application of PBM for settler induced flocculation would specify size as the internal coordinate.

In primary settler applications, the availability of particle settling velocity distributions as the internal coordinate of the suspended solids has generated a more accurate prediction of the load to the secondary treatment model, reduced the need for calibration (Bachis et al., 2012, 2014), and produced more accurate and optimal control of chemical dosage that lead to cost savings. In addition to particle size, particle density can be included as a second internal coordinate when density varies significantly with floc size. This additional internal coordinate can be accomplished using a 2D PBM approach but comes with an increased computational and parameter estimation cost since the rate expressions need to be extended to include density, which will require a detailed investigation of the process. Another interesting route for further research is the coupling of PBMs to computational fluid dynamics models as the latter can predict local shear which then serves as input for the PBM model. Research that couples PBM with CFD in WWT has been reported already (Griborio et al., 2006; Gong et al., 2011), but needs further attention. Here, again, it should be clear that coupling a 1D PBM to a CFD model is a challenging task, typically resulting in models which need very long simulation times. One strategy to reduce the computational burden is to reduce the PBM model before coupling it to a CFD model (Mortier et al., 2013).

\section{More accurate aeration modelling for better design and energy optimisation}

For a long time, $\mathrm{k}_{\mathrm{L}} \mathrm{a}$-based models have been used to capture mass transfer between the gas and adjacent phases during aeration. More recently, models taking air flow rate as input were proposed. Despite the inclusion of somewhat more complexity and the resulting improved model performance, the variability of the $\alpha$ "fudging factor" in space could still not be entirely related to process variables such as sludge concentration and sludge age, i.e. a lot of unexplained variance remains. Moreover, $\alpha$ was shown to vary spatially in a reactor (Rosso et al., 2011). This spatial variation introduces a significant amount of uncertainty in the model prediction when a single $\alpha$ value is used, resulting in locally different dissolved oxygen concentrations and, hence, aerobic process rates.

To date, a key assumption in all aeration models is assigning a single average bubble size. This assumption is not very apparent, but resides in the gas-liquid interface surface area (a) of $\mathrm{K}_{\mathrm{L}} \mathrm{a}$ and is hidden in the $\alpha$ value in oxygen transfer efficiency (OTE) based models. This constant bubble size assumption is unrealistic and can be very restrictive for the model since the bubble's interfacial area drives the oxygen transfer process. In reality, bubble size is spatially distributed (Fig. 3, left) from the point of injection to the top of a reactor due to the process of coalescence leading to a significantly different bubble size distribution near the reactor top. Another factor that plays a role here is the fact that there are pressure differences 
when moving from the bottom to the top of the reactor, and these will also influence bubble size. Increased viscosity (due to the presence of sludge) further promotes coalescence compared to clean water (Fig. 4) (Fabiyi and Novak, 2008; Ratkovich et al., 2013). A PBM using bubble size as the internal coordinate and including coalescence as a PBM reaction process can significantly improve the local prediction of oxygen mass transfer (and hence $\alpha$ ) as well as improve the design of aeration systems to maximize the oxygen transfer (in combination with Computational Fluid Dynamics - CFD). It should be noted that the current work in CFD linked to aeration also uses a fixed bubble size (Fayolle et al., 2007). The use of PBMs in aeration systems with suspended solids has not been widely studied in WWT. PBMs applied to bubble columns are widespread in fermentation systems and the chemical engineering literature (Wang, 2011; Dhanasekharan et al., 2005; Sanyal et al., 2005) and can serve as a solid examples of improved benefits to these process models.

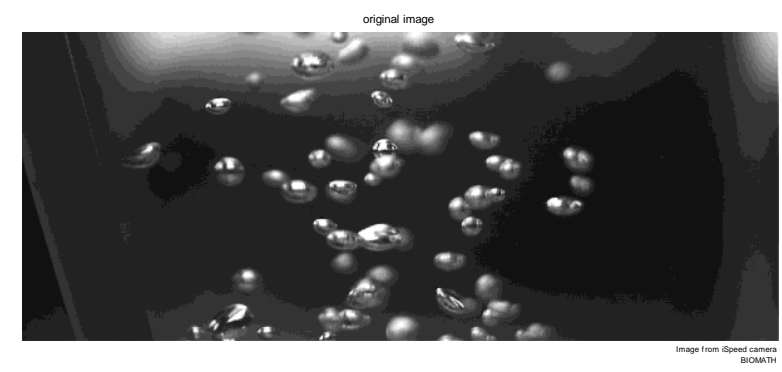

Fig. 3 - Illustration of variability in bubble size as measured with a high speed camera

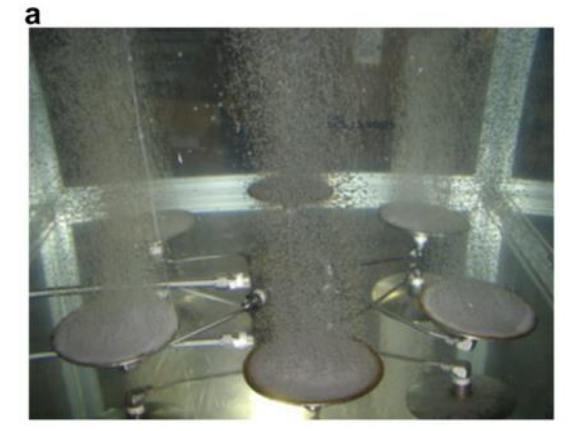

b

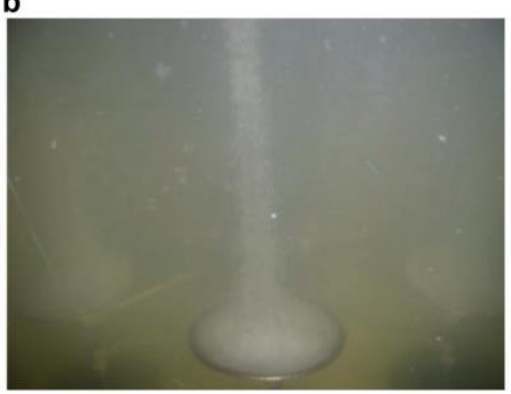

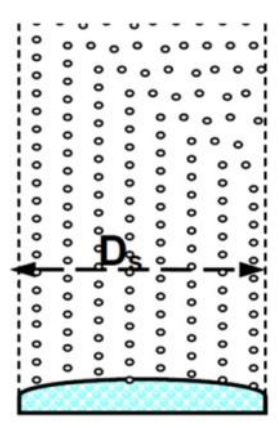

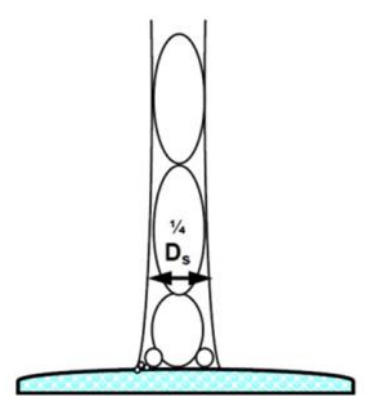

Fig.4 - Impact of viscosity on bubble coalescence

Typical mechanisms taking place in bubble breakup and coalescence are shown in Fig. 5. The kernels used in a PBM describing bubble breakup and coalescence are very similar as those used in a flocculation PBM. 

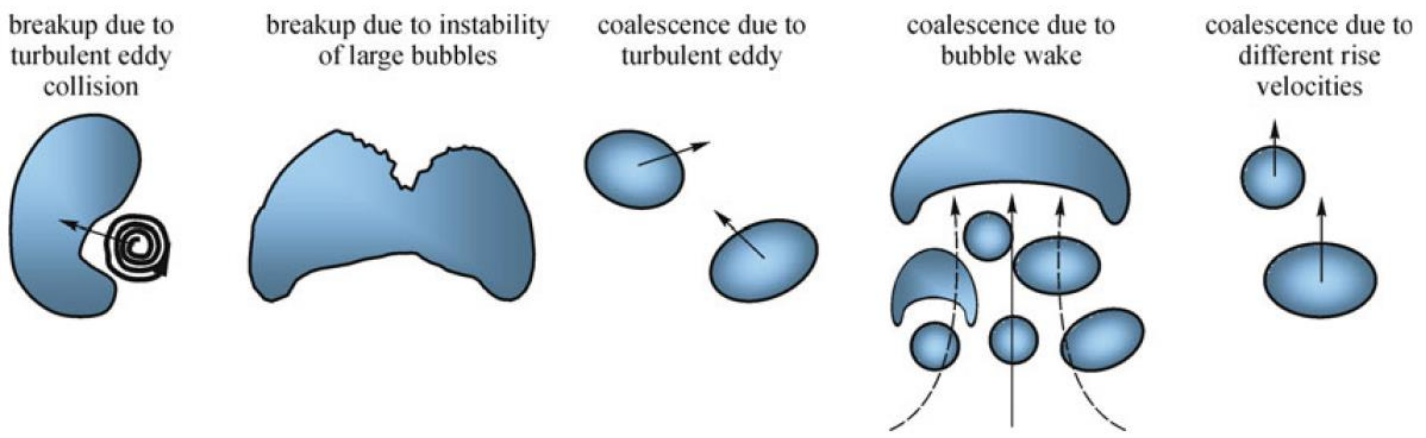

Fig. 5 - Bubble breakup and coalescence due to different mechanisms (after Wang, 2011).

\section{Floc and granule size: distributed kinetics}

Despite the fact that the size of agglomerates, be it flocs or granules, can play an important role in biological activity (e.g. different microbial consortia at different locations in the agglomerate), the simulation of such a microenvironment within a macro-scale fluid transport environment has hardly been performed. Recently, Volcke et al. (2012) demonstrated the significant impact of granule size distribution on the performance of an Anammox-based granular sludge reactor. The authors used a fixed size distribution for this analysis. It is clear that size distribution can be impacted by shear and, hence, will further influence the system behaviour. The absence of size and size dynamics in the currently used models indicates that the activity loss caused by particle size (causing transport limitation due to stratification, e.g. Vangsgaard et al., 2012) cannot be predicted by current models. Again, when experimental data are confronted with these models, other degrees of freedom (i.e. parameters or input variables) will be calibrated for inappropriate reasons.

Sobremisana et al. (2011) demonstrated that including floc size can result in significantly deviating reactor performance since kinetics can be quite different depending on reactor location and the size of the biological floc. The authors used an integrated PBM-CFD approach to simulate the carbon and nitrogen removal process at both the reactor scale and internal floc scale. The effect of size was introduced by means of an effectiveness factor (i.e. ratio of rate with and without diffusional resistance) based on floc size for all different processes. For a simple baffled reactor the treatment performance deviated by $13 \%$ for COD, $10 \%$ for $\mathrm{NH}_{4}$ and $56 \%$ for $\mathrm{NO}_{3}$ compared to the same simulation not accounting for influence of size. However, further validation is required.

Knowledge arising from this can be useful to (partially) decouple affinity constants in kinetic rate expressions and reduce their requirement for calibration. Understanding the interaction between size and reaction kinetics can inform researchers and engineers on how to better design and operate these processes (e.g. avoid or promote certain shear zones and account for imposed shear of mixing and aeration intensities). Moreover, it will reduce the need to adjust parameters unnecessarily to improve the model fit.

Apart from size heterogeneity, incomplete mixing can lead to spatial heterogeneous concentrations in biomass and substrates that ultimately result in locally different kinetics. Integrating the effects of spatial variations in macroscale mixing as well as the biomass and substrate concentration is another avenue for further model development. Lencastre Fernandes et al. (2013) demonstrated the effect of this heterogeneity for a budding yeast population using a multi-scale modelling approach that included PBM. A similar approach could be used for WWT modelling, and could be helpful in developing an improved understanding of 
biomass population dynamics. A complicating factor for the WWT compared to a yeast fermentation is that multiple species have to be considered to model the WWT appropriately.

Models including floc size and local heterogeneities could also be helpful in developing technologies to select between wanted and unwanted microbial communities, which is a recently developing topic in view of mainstream Anammox application. Microbial selection can be done on a physical basis (size) or through selection by creating favourable growth conditions for the targeted microbial consortium (Al-Omari et al., 2014).

\section{Precipitation/crystallisation for better quality marketable products}

WWTPs are transforming into WRRFs (Water Resource Recovery Facilities) leading to new modelling challenges (Vanrolleghem, 2013). One important aspect in product recovery, driving their market value, will be the specifications of the recovered material. These comprise both composition and size. Crystallisation has been extensively modelled in the field of chemical engineering and pharmaceutical engineering to produce crystals with tailormade specs (e.g. Aamir et al., 2009, Nagy and Braatz, 2012). A PBM with crystal size as an internal coordinate and inclusion of nucleation (function of supersaturation) and crystal growth can be used as a first approximation. If needed, more internal coordinates can be added to deal with composition or crystal shape (e.g. 2-D compared to 1-D, Samad et al., 2011). Additional phenomena such as agglomeration and breakage can be added. Interestingly, describing crystallisation with a PBM also allows describing phenomena such as size-dependent crystal growth (Samad et al., 2011).

The use of a PBM modelling framework is widely accepted when studying crystallization processes. However, in a WRRF context the PBM framework has not really been used thus far, with the exception of a recent manuscript by Galbraith and Schneider (2014) where a discretized PBM was used to describe the chemical precipitation of phosphorus. The most important discussion points, when implementing a PBM, are usually related to the model assumptions (which phenomena should be included?), and to the selection of the kinetic expressions for each phenomenon that is included in the PBM. The main phenomena are nucleation, crystal growth and dissolution (= negative growth), agglomeration and breakage. However, it happens frequently that the PBM only considers growth and nucleation (Fujiwara et al., 2005). Another important variable that needs to be included in these models is the super-saturation, which will vary as a function of temperature and is usually represented as a polynomial describing the super-saturation curve as a function of temperature.

\section{CONCLUSIONS}

Many processes in WWTPs are governed by population dynamics of materials characterised by variation in property dynamics. These potential complexities in system behaviour are lost or significantly suppressed when only average behaviour is characterized or simulated. Population Balance Models can deal with these process complexities and have already demonstrated their benefits in the field of (bio)chemical engineering. The majority of the models in WWT modelling that need more rigour are physical-chemical processes. Hence, more than ever we need to look over the fence and integrate available (bio)chemical engineering knowledge into WWTP models. Some examples are described in this paper, but potentially many more applications of PBM in WWT exist and can be exploited. The intention of this paper is to make WWT modellers aware of this framework and its potential applications, challenges, and pitfalls. 


\section{ACKNOWLEDGEMENT}

Peter Vanrolleghem holds the Canada Research Chair on Water Quality Modelling.

\section{REFERENCES}

Aamir E., Nagy Z.K., Rielly C.D., Kleinert T. and Judat B. (2009). Combined quadrature method of moments and method of characteristics approach for efficient solution of Population Balance Models for dynamic modeling and crystal size distribution control of crystallization processes. Ind. Eng. Chem. Res., 48, 8575-8584.

Al-Omari A., Wett B., Nopens I., De Clippeleir H., Han M., Regmi P., Bott C. and Murthy S. (2014). Modelbased evaluation of mechanisms and benefits of mainstream shortcut nitrogen removal processes. In: Proceedings 4th IWA/WEF Wastewater Treatment Modelling Seminar (WWTmod2014). Spa, Belgium, March 30 - April 22014.

Bachis G., Vallet B., Maruejouls T., Clouzot L., Lessard P. and Vanrolleghem P.A. (2012). Particle classesbased model for sedimentation in urban wastewater systems. In: Proceedings IWA Particle Separation Conference. Berlin, Germany, June 18-20 2012.

Bachis G., Maruéjouls T., Tik S., Amerlinck Y., Nopens I., Lessard P. and Vanrolleghem P. (2014) Modelling and characterisation of primary settlers in view of whole plant and resource recovery modelling. In: Proceedings 4th IWA/WEF Wastewater Treatment Modelling Seminar (WWTmod2014). Spa, Belgium, March 30 - April 22014.

Biggs C.A., Lant P.A., Hounslow M.J. (2003). Modelling the effect of shear history on activated sludge flocculation.

Water Sci. Technol., 47(11), 251-257.

Dhanasekharan K.M., Sanyal J., Jain A. and Haidari A. (2005). A generalized approach to model oxygen transfer in bioreactors using population balances and computational fluid dynamics, Chem. Sci. Eng., 60, 213218.

Fabiyi M.E. and Novak R. (2008). Evaluation of the factors that impact successful membrane biological reactor operations at high solids concentration. In: Proceedings of the $81^{\text {st }}$ Water Environment Federation Annual Conference and Exposition (WEFTEC2008), Chicago, IL, USA, October 18-22, 2008. 503-512.

Fayolle Y., Cockx A., Gillot S., Roustan M. and Heduit A. (2007). Oxygen transfer prediction in aeration tanks using CFD. Chem. Eng. Sci., 62(24), 7163-7171.

Fujiwara M., Nagy Z.K., Chew, J.W. and Braatz R.D. (2005). First-principles and direct design approaches for the control of pharmaceutical crystallization. J. Proc. Control, 15, 493-504.

Fukushi K., Tambo N. and Matsui Y. (1995). A kinetic model for dissolved air flotation in water and wastewater treatment. Water Sci. Technol., 31(3-4), 37-47.

Galbraith S.C. and Schneider P.A. (2014) Modelling and simulation of inorganic precipitation with nucleation, crystal growth and aggregation: A new approach to an old method. Chem. Eng. J., 240, 124-132.

Griborio A. and McCorquodale J.A., 2006. Optimum design of your center well: .use of a CFD model to understand the balance between flocculation and improved hydrodynamics. Proceedings of the $79^{\text {th }}$ Water Environment Federation Annual Conference and Exposition (WEFTEC2006), Dallas, TX, USA, October 21-25, 2006. 5735-5746.

Gong M., Xanthos S., Ramalingam K., Fillos J., Beckmann K., Deur A. and McCorquodale J.A. (2011). Development of a flocculation sub-model for a 3-D CFD model based on rectangular settling tanks. Water Sci. Technol., 63(2), 213-219.

Gujer W. (2002). Microscopic versus macroscopic biomass models in activated sludge systems. Water Sci. Technol., 45(6), 1-11.

Lencastre Fernandes R., Carlquist M., Lundin L., Heins A-L, Dutta A., Sørensen S.J., Jensen A.D., Nopens I., Eliasson Lantz A. and Gernaey K.V. (2013). Cell mass and cell cycle dynamics of an asynchronous budding yeast population: experimental observations, flow cytometry data analysis and multi-scale modeling. Biotechnol. Bioeng., 110(3), 812-826.

Mortier S.T.F.C., Van Daele T., Gernaey K.V., De Beer T. and Nopens I. (2013). Reduction of a single granule drying model: An essential step in preparation of a Population Balance Model with a continuous growth term. AIChE J., 59(4), 1127-1138.

Nagy Z.K. and Braatz R. (2012). Advances and new directions in crystallization control. Annu. Rev. Chem. Biomol. Eng., 3, 55-75.

Nopens I., Biggs C.A., De Clercq B., Govoreanu R., Wilén B.-M., Lant P. and Vanrolleghem P.A. (2002). Modelling the activated sludge flocculation process combining laser diffraction particle sizing and population balance modelling (PBM). Water Sci. Technol., 45(6), 41-49. 
Nopens I., Koegst T., Mahieu K. and Vanrolleghem P.A. (2005). Population Balance Model and activated sludge flocculation: from experimental data to a calibrated model. AIChE J., 51(5), 1548-1557.

Nopens I., Nere N., Vanrolleghem P.A. and Ramkrishna D. (2007). Solving the inverse problem for aggregation in activated sludge flocculation using a population balance framework. Water Sci. Technol., 56(6), 95103.

Parker, D., Kaufman, W. and Jenkins, D. (1972). Floc breakup in turbulent flocculation processes. J. Sanitary Div. A.S.C.E., 1, 79-99.

Ramkrishna, D. (2000). Population Balances: Theory and Applications to Particulate Systems in Engineering. Academic Press, London (UK), 355p.

Ratkovich N., Horn W., Helmus F.P., Rosenberger S., Naessens W., Nopens I. and Bentzen T. (2013). Activated sludge rheology: A critical review on data collection and modeling. Water Res., 47(2), 463-482.

Rosso D., Lothman S.E., Jeung M.K., Pitt P., Gellner W.J., Stone A.L. and Howard D. (2011). Oxygen transfer and uptake, nutrient removal, and energy footprint of parallel full-scale IFAS and activated sludge processes. Water Res., 45(18), 5987-5996.

Samad N.A.F.A., Singh R., Sin G., Gernaey K.V. and Gani R. (2011). A generic multi-dimensional model-based system for batch cooling crystallization processes. Computers Chem. Eng., 35, 828-843.

Sanyal J., Marchisio D., Fox R. and Dhanasekharan K. (2005). On the comparison between Population Balance Models for CFD simulation of bubble columns. Ind. Eng. Chem. Res., 44 (14), 5063-5072

Schuler A.J. (2005). Diversity matters: Dynamic simulation of distributed bacterial states in suspended growth biological wastewater treatment systems. Biotechnol. Bioeng., 91(1), 62-74.

Schuler A.J. (2006). Process hydraulics, distributed bacterial states, and biological phosphorus removal from wastewater. Biotechnol. Bioeng., 94(5), 909-920.

Schuler A.J. and Jassby D. (2007). Distributed state simulation of endogenous processes in biological wastewater treatment. Biotechnol. Bioeng., 97(5), 1087-1097.

Sobremisana, A., de los Reyes III F.L. and Ducoste J.J. (2011). Combined CFD, floc aggregation, and microbial growth kinetics modeling for carbon and nitrogen removal. In: Proceedings $84^{\text {th }}$ Water Environment Federation Annual Conference and Exposition (WEFTEC 2011), Los Angeles, CA, USA, October 15-19, 2011.

Torfs E., Dutta A. and Nopens I. (2012). Investigating kernel structures for shear and Ca-induced activated sludge aggregation using an inverse problem methodology. Chem. Eng. Sci., 70, 176-187.

Vangsgaard A.K., Mauricio-Iglesias M., Gernaey K.V., Smets B.F. and Sin G. (2012). Sensitivity analysis of an autotrophic granular biofilm process: significance of mass transfer vs. microbial kinetics on nitrogen removal. Bioresource Technol., 123, 230-241.

Vanrolleghem P.A. (2013). Water resource recovery facilities: Modelling and control challenges. Keynote lecture at workshop "Emerging Challenges for a sustainable and integrates urban water system management", $10^{\text {th }}$ IWA Leading Edge Technology (LET) conference on water and wastewater technologies, Bordeaux, France, 2-6 June.

Wang, T. (2011). Simulation of bubble column reactors using CFD coupled with a population balance model, Chem. Sci. Eng., 5, 162-172. 


\title{
Modelling and characterisation of primary settlers in view of whole plant and resource recovery modelling
}

Giulia Bachis ${ }^{1}$, Thibaud Maruéjouls ${ }^{1}$, Sovanna Tik $^{1}$, Youri Amerlinck ${ }^{2}$, Henryk Melcer ${ }^{3}$, Ingmar Nopens $^{2}$, Paul Lessard ${ }^{1}$, Peter A. Vanrolleghem ${ }^{1}$

\author{
${ }^{1}$ Département de génie civil et de génie des eaux, Université Laval, 1065 av. de la Médecine, Québec, QC, \\ Canada, G1V 0A6 (Email: peter.vanrolleghem@gci.ulaval.ca) \\ ${ }^{2}$ BIOMATH, Department of Mathematical Modelling, Statistics and Bioinformatics, Ghent University, \\ Coupure Links 653, B-9000 Ghent, Belgium \\ ${ }^{3}$ Brown and Caldwell, 999 Third Avenue, Suite 500, Seattle, WA 98104, USA
}

\begin{abstract}
Characterisation and modelling of primary settlers have been neglected pretty much to date. However, whole plant and resource recovery modelling require primary settler model development, as current models lack detail. This paper focuses on the improved modelling and experimental characterisation of primary settlers. First, a new modelling concept based on particle settling velocity distribution is proposed which is then applied for the development of an improved primary settler model as well as for its characterisation under addition of chemicals (Chemically Enhanced Primary Treatment, CEPT). Second, another basic primary settler model, developed for control under chemicals addition, is presented. Third, the variation of the COD fractionation produced by primary settling is investigated, showing that typical wastewater ratios are modified by primary treatment. The latter provides a further argument for more detailed primary settler models in view of whole plant modelling as they clearly impact the downstream processes.
\end{abstract}

\section{Keywords}

Primary clarification model, particle settling velocity distribution, CEPT, ASM fractionation.

\section{INTRODUCTION}

The role of primary settling in wastewater treatment has often been neglected and very few efforts have been made for its optimisation and modelling (Lessard and Beck, 1988; Gernaey et al., 2001; Ribes et al., 2002). It has been neglected either because primary settling is not considered very influential for modelling purposes, or because the simple models proposed earlier were considered sufficiently robust to describe the primary settling tanks (PSTs) behaviour (Otterpohl and Freund, 1992). In many modelling case studies, the boundaries of the wastewater treatment plant (WWTP) are defined from the primary effluent onwards, i.e. using the primary effluent as model input, hereby keeping the primary settler out of the modelling scope. However, a better understanding and modelling of the processes taking place in PST result in a more accurate description of the primary effluent characterisation and sludge wastage. As such, it results in improved operation of the subsequent treatment phases, i.e. water and sludge treatment.

Improved primary settler models are also essential ingredients of whole WWTP descriptions. In this respect, Choubert et al. (2013) stated that based on combined expertise of modellers (Phillips et al. 2009) and sensitivity analysis (Petersen et al. 2002) profound effects of wastewater characterisation on modelling outputs (Henze et al. 2000) have been shown:

- Sludge production is influenced by the estimated inert particulate COD.

- Oxygen demand is influenced by the estimated total biodegradable COD.

- Anoxic denitrification rate and anaerobic phosphorus release are influenced by the estimated readily biodegradable COD. 
- Effluent COD is influenced by the estimated inert soluble COD.

The importance of providing reliable wastewater characterisation, enabling the link with the industry-standard activated sludge models (ASM) (Henze et al., 2000), was also highlighted. Hence, the function of PSTs under the ASM framework should be reconsidered since the impact of primary treatment on wastewater fractionation may be significant.

In this context, the simulation study of Flores-Alsina et al. (2014) illustrated the considerable advantages given by the enhancement of the TSS removal in a PST on final effluent quality and operational costs. This enhancement can be obtained by addition of chemicals (combined or not with lamellar settling) in the primary treatment, which may increase TSS removal efficiency up to $90 \%$ (Tchobanoglous et al., 2003).

Chemically enhanced primary treatment (CEPT) by addition of coagulants/flocculants, which is often operated under wet weather conditions, may be also pursued for maximising the organic material directed to biogas production and other resource recovery. It thus becomes directly involved in the design of the energy self-sufficient WWTP. CEPT can be applied to achieve many different objectives in wastewater treatment facilities: to increase the TSS removal performance of PST in primary only plants; to reduce organic loading rates thereby reducing demand on aerobic biological treatment facilities; lastly, it can permit increased hydraulic loading rates to existing PST, thus favouring plants that receive high wet weather flows. The first most significant application of CEPT was in the 1960s by Canadian and U.S. engineers to address eutrophication of the Great Lakes through chemical precipitation of phosphorus. Galil and Rebhun (1990) showed that the reduction in organic load using CEPT significantly reduced aeration tank volume in the downstream activated sludge process. More recently, in the U.S., with increased emphasis on CSO and SSO controls, agencies are seeking for inexpensive and compact solutions to manage wet weather flows, other than just increasing secondary treatment hydraulics and process capacity. CEPT has been extensively evaluated because of the minimal investment in new infrastructure. Indeed, hydraulic capacities of existing primary settlers can be increased by a factor of up to three, which is often sufficient to manage peak wet weather flows. Bench-scale (Melcer et al., 2005, 2009) and pilot-scale (Melcer et al., 2012; Newman et al., 2013) demonstrations of wet weather treatment using CEPT have been conducted. These have led to the application of CEPT at full scale.

Most of the existing settling models make use of a unique settling velocity for all the particles, even though the particles are heterogeneous and the assumption of a single settling velocity is a too simplistic approach. Introducing the concept of particle settling velocity distribution (PSVD) in the model provides a better description of the behaviour of the particles in the PST. Moreover, even though little literature exists on the topic, a few studies have highlighted that a link exists between particle physical properties and particle biodegradation properties (Chebbo and Bachoc, 1992; Hvitved-Jacobsen et al., 1998; Morgenroth et al., 2002), emphasizing the need to focus more on how primary settler models and subsequent biological reaction models have to be complementary. Hence, models of an adequate complexity need to be developed for a more accurate description of the PST behaviour and the chemical/biological phenomena that may affect particles, their settling velocity and, as a consequence, their removal. Indeed, the efficiency of the PST directly influences the performance of the subsequent treatment units in WWTPs, since during settling organic matter and suspended solids of the influent, as well as pollutants associated with them, are removed. Not only does this determine the load to the downstream treatment steps, 
it is also critical in the evaluation of the benefits that the sludge treatment train will be able to accomplish (energy and nutrient recovery).

This work presents different ongoing developments related to the improved modelling and experimental characterisation of primary settlers. The paper is organised in four sections: (1) a new primary settling model based on particle settling velocity distribution (PSVD) is first proposed; (2) it is briefly illustrated how PSVD can also be used to characterise and model a CEPT process; (3) a simple primary settling model for CEPT is presented and (4) ASM fractionation in primary settlers is discussed.

\section{PSVD FOR PRIMARY SETTLER MODEL DEVELOPMENT}

A new dynamic primary settler model, based on the PSVD approach and inspired by the work of Maruéjouls et al. (2012) on retention tanks, was initially presented by Bachis et al. (2012). This model allows improved predictions in terms of effluent TSS compared to previous primary settling models. It was shown that by creating a number of particle classes that cover the settling velocity distribution, a vertical gradient of the concentration of each of the particle classes and the pollutants associated to them can be calculated.

The ViCAs (Vitesses de Chute en Assainissement) batch settling protocol developed by Chebbo and Gromaire (2009) is an excellent method to feed this type of PSVD-model, as it allows to experimentally determine the fraction of the different settling velocity classes, each characterised by a distinct settling velocity $\mathrm{V}_{\mathrm{s}}$. A ViCAs experiment consists in filling a settling column $(\mathrm{H}=60 \mathrm{~cm}, \varnothing=7 \mathrm{~cm})$ with a homogenized suspension. Solids settled during predefined time intervals are recovered at the bottom of the column and weighed for TSS. From the time evolution of the cumulated mass of particles settled since the beginning of the experiment one can calculate the distribution of settling velocities.

The PSVD model was implemented on the modelling and simulation environment WEST (mikebydhi.com). To describe the vertical gradient of particle class concentrations the settler is divided into a number of layers and a mass balance is calculated around each layer for each of the classes. Five particle classes with different (constant) settling velocities make up the core of the model.

\section{Influent TSS fractionation into particle classes}

Each particle class is assigned a fraction of the influent TSS. Given the dynamics of the wastewater composition, this assignment is, however, not constant. To assign the fraction of influent TSS to the classes, advantage is taken from the observation from multiple ViCAs experiments that the ViCAs curves are located higher for low TSS concentration and lower for high TSS. This means that high TSS samples contain a larger fraction of rapidly settling particles. Therefore, the assignment is made by interpolating the PSVD curve between two boundary curves (continuous lines on Figure 1). These are the boundaries delimiting the zone where most of the observed influent PSVD curves for the particular plant under study were located (results not shown). The upper limit of this zone is the ViCAs representing low influent TSS concentrations, while the lower limit is given for high influent TSS concentrations. The assignment for a sample with a certain TSS concentration is performed as follows: for a certain settling velocity (on the x-axis), the two corresponding limiting TSS fractions are determined (y-axis) and a linear interpolation is made between them from the influent TSS-value. Thus, the observed relation between PSVD and TSS concentration is used to define the fraction of each class of the influent TSS. The settling velocities characterising 
each class were calculated as the geometrical mean of the settling velocity boundaries of the class.

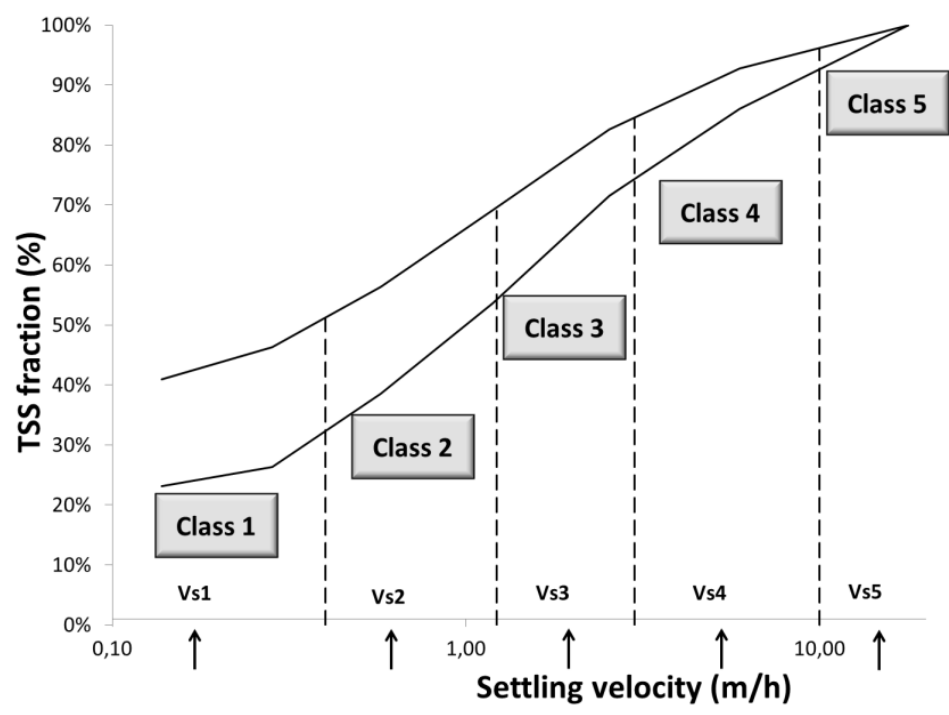

Figure 1 Fractionation of the ViCAs zone into 5 classes and upper and lower limits of the zone where most of the PSVD curves observed for the case study were found. Settling velocities characterising each class were calculated as the geometrical mean of the settling velocity boundaries of the class

\section{Primary settler data}

The performance of the five classes PSVD model was evaluated through the simulation of the data from the Eastern wastewater treatment plant of Québec City (Canada). Two series of data were available: one was the TSS 24h-evolution of the influent and effluent collected at the full-scale primary settlers during a sampling campaign conducted in 2010 (three days under dry weather flow conditions); the other data set contained online TSS values measured by turbidity sensors on a pilot-scale primary settler (2013) (one day under dry weather flow conditions).

The PSTs of the Eastern WWTP of Québec City are lamellar settlers, with a total surface of $27,000 \mathrm{~m}^{2}$, treating a mean flow rate of $236,600 \mathrm{~m}^{3} / \mathrm{d}$ during dry weather conditions. The $5 \mathrm{~m}^{3}$ pilot-scale PST was installed in the same WWTP and it received the influent from the fullscale PSTs, treating a mean flow rate of $192 \mathrm{~m}^{3} / \mathrm{d}$.

\section{Evaluation of the model performance}

Model parameters were estimated by fitting the model to the data sets. The goodness-of-fit of the model was statistically evaluated through the calculation of the chi-squared criterion (weighted least squares). The assumption of independent and normally distributed measurement errors is made.

$$
\chi^{2}(\theta)=\sum_{i=1}^{\mathrm{n}}\left(\frac{1}{\sigma_{i}}\left(y_{i}-\hat{y}_{i}(\theta)\right)^{2}\right.
$$

where $y_{i}$ is the observed value; $\hat{y}_{i}(\theta)$ is the simulated value for the parameter set $\theta$; $\sigma_{i}$ is the standard measurement error of the observation $\mathrm{y}_{\mathrm{i}}$ and $\mathrm{n}$ represents the number of data points to which the model was fitted. The computed $\chi^{2}$ is then compared to tabulated values of the chi- 
squared distribution for $n-1-n_{\theta}$ degrees of freedom $\left(n_{\theta}\right.$ stands for the number of estimated parameters), to decide whether the model is justified by the data or not (Gujer, 2008).

\section{Calibration results}

The parameters estimated during the calibration consisted on the location of the five settling velocity class boundaries (see Figure 1, lower limit not visible). During the calibration of the model different settling velocities and, consequently, different sets of fractions were tested until a good model fit to the measured effluent TSS time series was achieved. Two of the four full-day data sets were used for the calibration, visibly resulting in a good fit for the effluent TSS concentrations (Figure 2). The calculated $\chi^{2}$ for the two events is respectively 11 (Figure $2 a)$ and 34 (Figure 2b). For 18 degrees of freedom $\left(n=24\right.$ and $\left.n_{\theta}=5\right)$ the observed $\chi^{2}$ is in $99 \%$ of the cases smaller than the critical value 34.8. This means that the model is justified by the data, especially for the first simulation. The PSVD model's $\mathrm{V}_{\mathrm{s}}$ values and limit TSS fractions resulting from the calibration are given in Table 1.
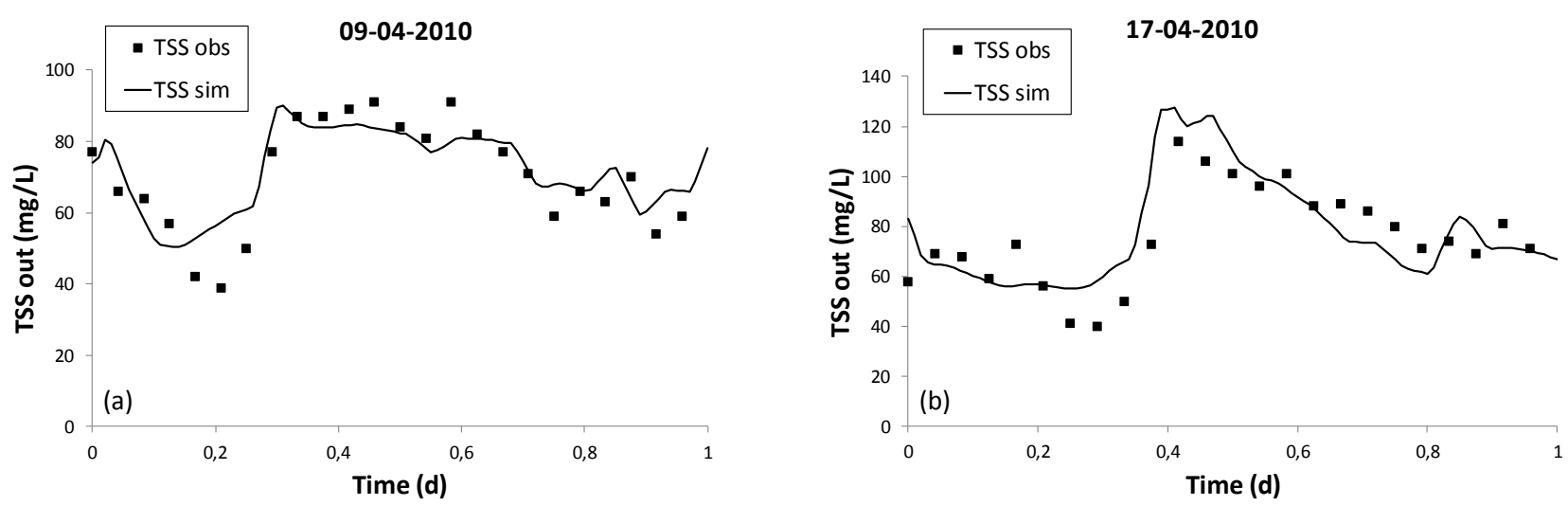

Figure 2 Model fit for effluent TSS concentrations during the calibration phase.

Table 1. Settling velocity (Vs) and boundary TSS fractions (F) associated to each of the 5 classes in the PSVD model and settling velocities used in the primary settling model from Lessard and Beck (1988).

\begin{tabular}{cccccc}
\hline & Class 1 & Class 2 & Class3 & Class 4 & Class 5 \\
\hline Class-characterising Vs (m/h) & 0.06 & 0.70 & 1.91 & 5.48 & 13.36 \\
F (high TSS-low TSS) (\%) & $32-51$ & $22-19$ & $20-15$ & $18-11$ & $8-4$ \\
\hline & \multicolumn{2}{c}{$\begin{array}{c}\text { Dry } \\
\text { weather }\end{array}$} & $\begin{array}{c}\text { Wet } \\
\text { weather }\end{array}$ & $\begin{array}{c}\text { Return } \\
\text { liquors }\end{array}$ \\
\hline Vs Lessard\&Beck (1988) (m/h) & 1 & 2 & 10 \\
\hline
\end{tabular}

\section{Validation results}

The remaining two full-day data sets were used to validate the model. One of the data sets was collected at the full-scale PST, the other was the data set with on-line turbidity data collected at the pilot primary settler treating the same wastewater. 
Full-scale PST. The TSS concentrations were simulated quite well (Figure 3a). The $\chi^{2}$-test resulted in an acceptable value (23) since in $90 \%$ of the cases the sum of squares is smaller than 26.

Pilot-scale PST. The pilot-scale PST was modelled in the same way as the full-scale PST, with adjusted dimensions. The PSVD-model was fed with influent TSS data obtained through a linear correlation from NTU data provided by the turbidity sensor located at the inlet of the pilot-scale PST. The PSVD-model parameters estimated above were applied as such. Figure $3 \mathrm{~b}$ confronts the simulated effluent TSS concentrations with the observations from the turbidity sensor located at the effluent of the pilot-scale PST. Even if it failed the $\chi^{2}$-test, it can be stated that, given the difference in configuration of the settler, a remarkably good fit is obtained.
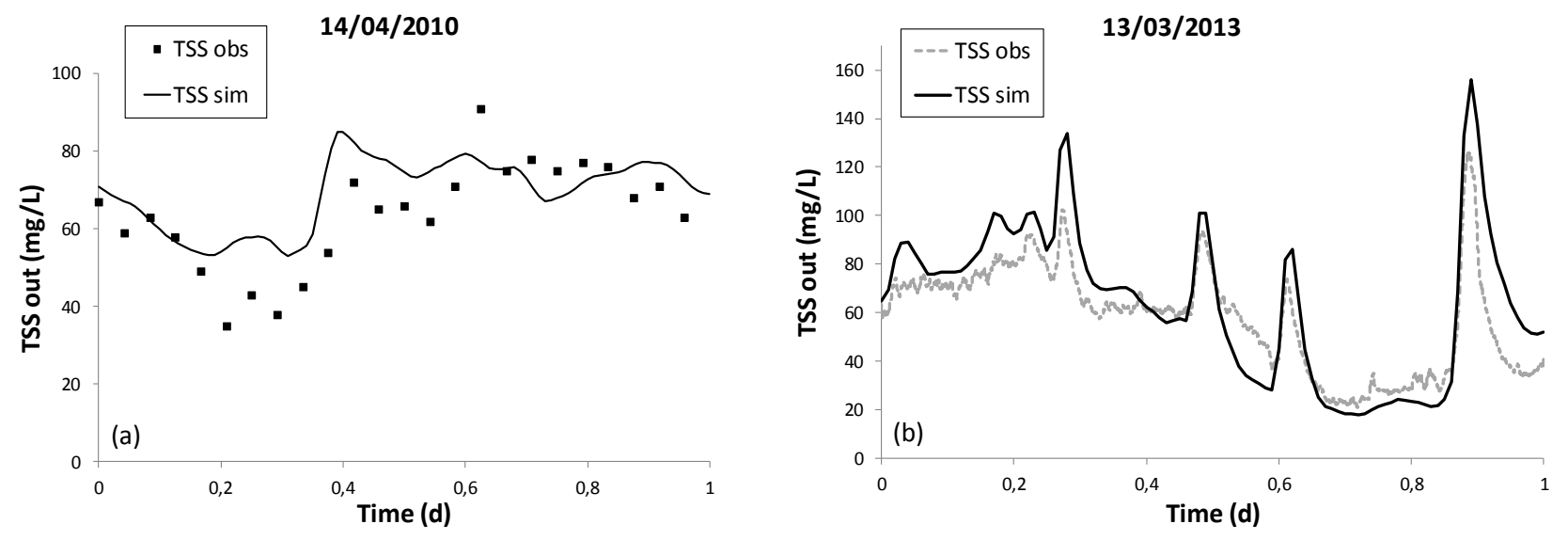

Figure 3 Model fit for effluent TSS concentrations during the validation phase of the full-scale PST (a) and the pilot-scale PST simulated with the same PSVD model and model parameters (b).

Further tests of the model were conducted by applying it to simulate TSS concentrations obtained from a 10-day sampling campaign at the Norwich (UK) treatment plant (Lessard and Beck, 1988). Supernatant liquors from the sludge treatment were returned to the primary inlet two or three times a day. Return of supernatant sludge liquors and storm sewage to the influent stream affected the wastewater composition, producing peaks of TSS concentrations that were reproduced in the effluent as well. Therefore, Lessard and Beck distinguished in their model three different streams: crude sewage, storm sewage and crude sewage with return liquors and attributed different settling velocities to them $(1,2$ and $10 \mathrm{~m} / \mathrm{h}$ respectively) (Table 1). Hence, in their work the unique settling velocity of their model had to be changed each time one of the three mentioned events occurred (Figure 4a). When applying the dynamic PSVD model no such changes are needed as it just needs the observed influent TSS concentrations. It is not only capable of taking into account these sudden changes, but also proves to better simulate the data (Figure $4 \mathrm{~b}$ ), especially with regard to the time delay of the peaks. Please note the remarkable finding that the ViCAs curves obtained in Québec City could be applied as such to the Norwich treatment plant with excellent predictive capabilities. The only calibration performed was the estimation of the class settling velocity boundaries.

In conclusion, a new dynamic primary settler model based on particle classes has been developed, showing to be effective in predicting effluent TSS concentration and providing increased accuracy in simulating the TSS dynamics at the outlet of a primary settler compared to existing dynamic settling models. The approach of taking into account the PSVD of the 
particles in the influent provides a type of primary settler model with very good prediction power for different sewages and weather conditions.

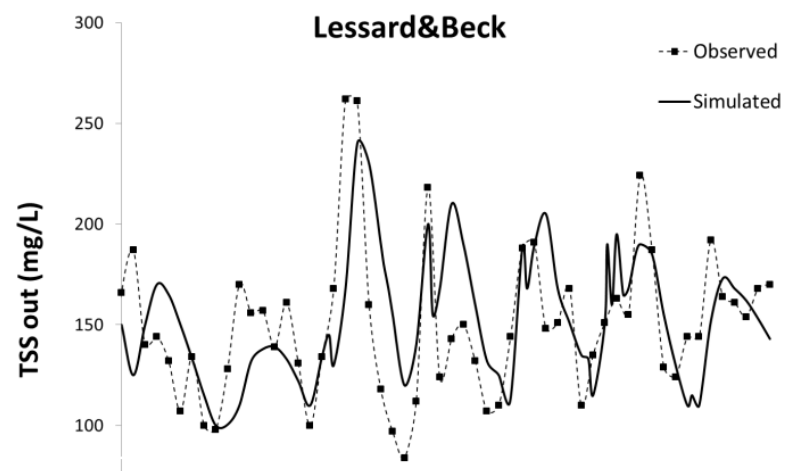

(a)

50

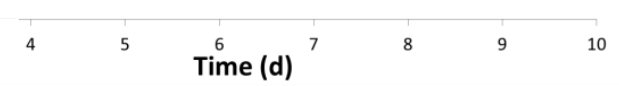

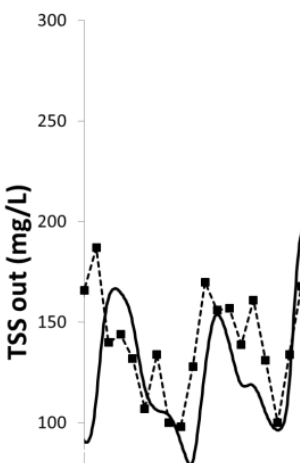

(b)

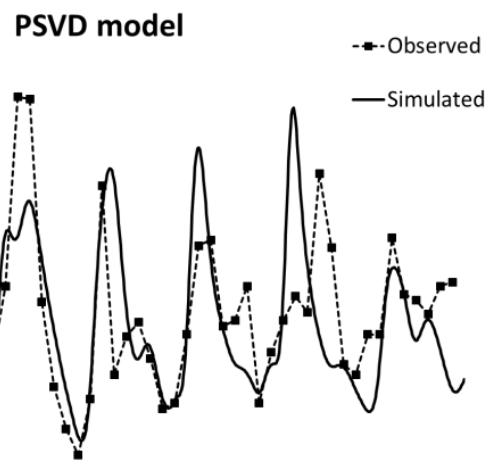

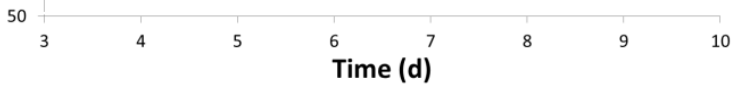

Figure 4 Model fit for effluent TSS concentrations in (a) the Lessard\&Beck model and (b) the PSVD model.

\section{PSVD FOR CEPT MODELLING}

The effect of CEPT on the PSVD can also be characterised by means of ViCAs tests. To illustrate this, samples taken at the inlet of the pilot-scale PST after addition of coagulants/flocculants were subjected to the ViCAs test. Figure 5a illustrates that the inlet PSVD after chemical addition is shifted towards higher settling velocities and outside the typical reference zone of the primary settler influent without CEPT (Maruéjouls et al., 2011). The effect is more pronounced for slow settling particles, which is the logical consequence of the aggregation of the particles produced by the addition of chemicals, making them grow in size and increase in settling velocity.

This experimental approach may thus be very well suited to model the effect of the addition of coagulation/flocculation chemicals on primary settling. Indeed, the curve with the appropriate PSVD (with or without chemical addition) may be used directly as input to the model, fractionating the TSS in the appropriate better settling fractions. Applying the model using the PSVD with chemical addition results in a significantly better TSS removal, as illustrated with the simulation of CEPT applied to the same influent situation (Figure 5b). Further confirmations are under study.
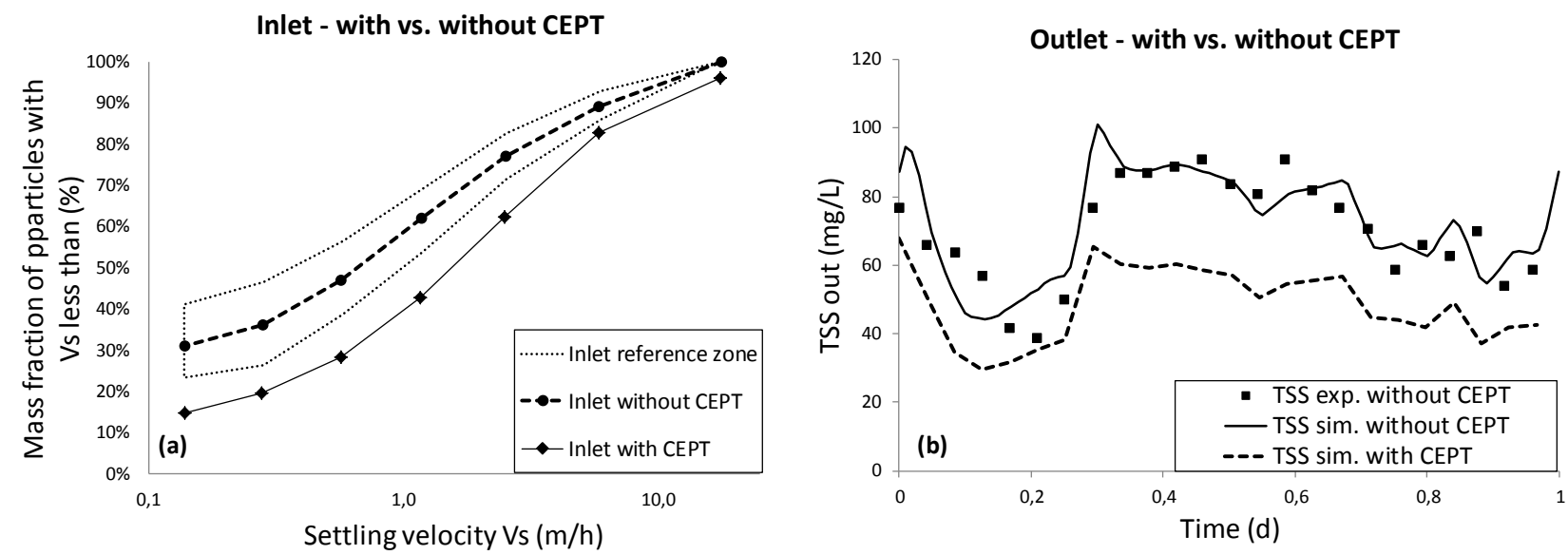
Figure 5 (a) Comparison of PSVD observed at the PST inlet during operation without CEPT and with CEPT.

The reference zone illustrates typical PSVD observed at the PST inlet in Québec City, Canada, without CEPT (Maruéjouls et al., 2011). (b) PSVD model fit for effluent TSS concentrations without CEPT and simulation with

\section{SIMPLIFIED CEPT MODEL}

CEPT.

As an alternative to the relatively complex PSVD model, a simple model for chemically enhanced primary settlers was developed by Tik et al. (2013) with the dedicated objective of having a model that can be used for the development of a controller for chemical addition. Without the need for ViCAs characterisation, the effect of alum addition was modelled by varying two settling characteristics in the settling velocity function: the overall particle settling velocity $\left(\mathrm{V}_{0}\right)$ (Figure 6a) and the fraction of non-settleable suspended solids $\left(\mathrm{f}_{\mathrm{ns}}\right)$ (Figure 6b). The proposed model allows the primary settlers' outlet concentration of TSS to be properly simulated during an experiment of full-scale alum addition with step concentration changes (Figure 6c) and seems sufficiently robust to satisfactorily describe dry weather conditions as well as wet weather conditions. Further validation on other case studies is required to confirm the usefulness of the model for this type of control development and tuning studies.
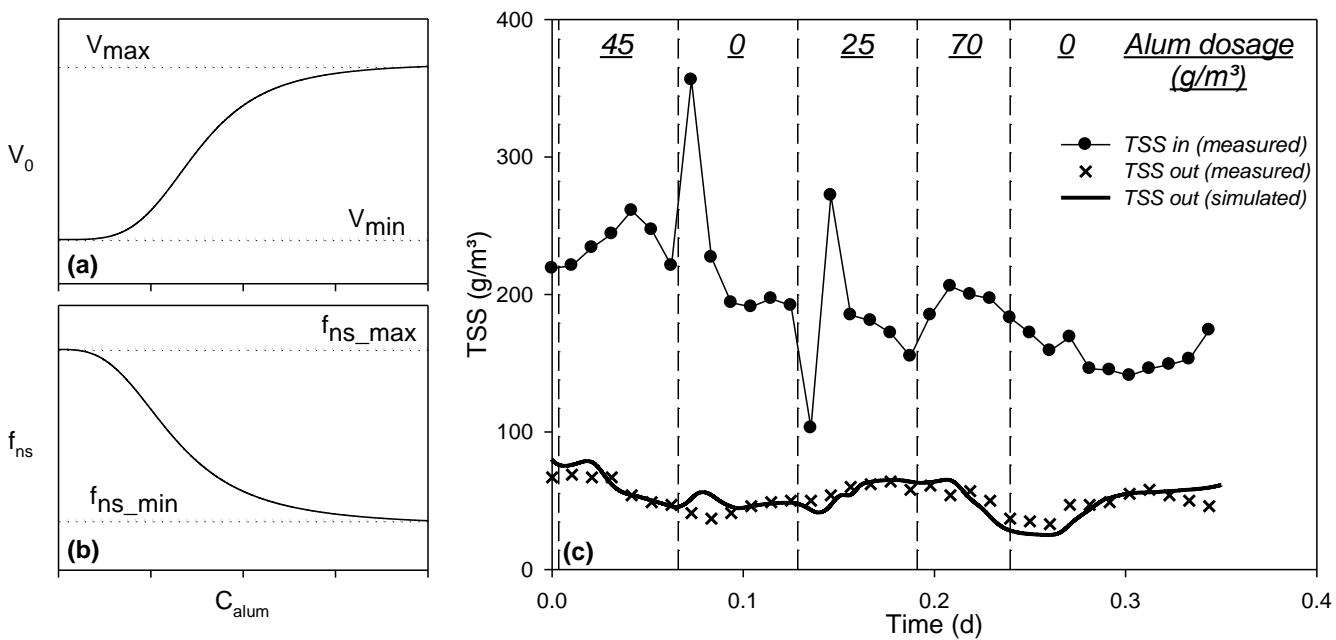

Figure 6 Dependency of (a) the non-settleable fraction of TSS $\left(\mathrm{f}_{\mathrm{ns}}\right)$ and (b) settling velocity, $\mathrm{V}_{0}$, on alum concentration $\left(\mathrm{C}_{\mathrm{alum}}\right)$; (c) experimental (inlet and outlet) and simulated (outlet) TSS concentrations of a full-scale experiment in Québec City, Canada, on August $25^{\text {th }}, 2011$. The flow rate was approximately constant at 9,300 $\mathrm{m}^{3} / \mathrm{h}$ (Tik et al., 2013).

With this model Tik et al. (2013) developed a successful control loop using effluent turbidity measurements that could reduce alum addition by $30 \%$ compared to a constant alum addition and yielding the same performance in terms of maximum TSS concentration in the primary effluent.

\section{ASM FRACTIONATION IN PRIMARY TREATMENT}

Primary treatment removes particles from the wastewater and as such changes its composition. These changes can be expressed in terms of several calculated ratios of traditional pollutant characteristics (Table 2). Fractionation of wastewater is thus affected by primary settling, thus impacting the subsequent treatment processes (Kristensen et al. 1992, Pasztor et al. 2009). 
For the correct model-based evaluation of a WWTP in which ASMs are used to describe the subsequent bioreactor models, a proper prediction of the primary effluent into the ASM input fractions is required. However, the effect of the PST on these fractions is frequently overlooked or oversimplified (the fractions in the PST are assumed to remain constant under all conditions). Note that the models described above only focus on TSS removal prediction and do not consider variations in fractionation in the primary settler.

Table 2. Differences in typical ratios of traditional wastewater characteristics of raw influent and primary effluent of municipal wastewater treatment (redrafted after Rieger et al., 2012) and comparison with Québec City ratios.

\begin{tabular}{|c|c|c|c|c|c|c|c|c|c|c|}
\hline & & Ratio & Unit & $\mathrm{n}^{1)}$ & mean & $\operatorname{Std} \%{ }^{2}$ & & $\mathrm{n}$ & Mean & Std\% \\
\hline \multirow{10}{*}{ 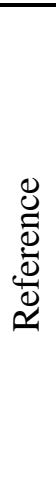 } & $\bar{\Xi}$ & Ntot/CODtot & g N/g COD & 12 & 0.095 & $17 \%$ & \multirow{10}{*}{ 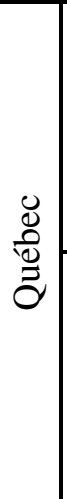 } & 8 & 0.089 & $1 \%$ \\
\hline & $\stackrel{\bar{g}}{\Xi}$ & N-NHx/TKN & g N/g N & 13 & 0.684 & $8 \%$ & & 8 & 0.482 & $13 \%$ \\
\hline &.$\Xi$ & CODtot/BOD5 & $\mathrm{g} \mathrm{COD} / \mathrm{g}$ BOD & 12 & 2.060 & $11 \%$ & & 8 & 2.062 & $32 \%$ \\
\hline & 芯 & TSS/CODtot & g TSS/g COD & 12 & 0.503 & $18 \%$ & & 11 & 0.573 & $14 \%$ \\
\hline & $\ddot{\simeq}$ & BOD5/BOD $\infty$ & g BOD/g BOD & 7 & 0.655 & $7 \%$ & & 8 & 0.859 & $6 \%$ \\
\hline & \multirow{5}{*}{ 苞苞 } & Ntot/CODtot & g N/g COD & 9 & 0.134 & $35 \%$ & & 8 & 0.123 & $2 \%$ \\
\hline & & N-NHx/TKN & g N/g N & 11 & 0.755 & $4 \%$ & & 8 & 0.509 & $9 \%$ \\
\hline & & CODtot/BOD5 & $\mathrm{g} \mathrm{COD} / \mathrm{g}$ BOD & 9 & 1.874 & $31 \%$ & & 8 & 1.931 & $20 \%$ \\
\hline & & TSS/CODtot & g TSS/g COD & 9 & 0.380 & $21 \%$ & & 11 & 0.426 & $9 \%$ \\
\hline & & BOD5/BOD $\infty$ & $\mathrm{g} \mathrm{BOD} / \mathrm{gBOD}$ & 6 & 0.644 & $10 \%$ & & 8 & 0.894 & $4 \%$ \\
\hline
\end{tabular}

Therefore, to better describe the subsequent biological treatment by providing a good fractionation, primary settling was also studied from an ASM point of view, taking inlet and outlet samples from primary settlers at three different WWTPs (Eindhoven, Roeselare and Québec City). The samples were analysed in terms of COD fractions into four components: the readily biodegradable $\mathrm{COD}, \mathrm{S}_{\mathrm{B}}$; the slowly biodegradable $\mathrm{COD}, \mathrm{XC}_{\mathrm{B}}$; the inert soluble COD, $\mathrm{S}_{\mathrm{U}}$; the inert particulate COD, $\mathrm{X}_{\mathrm{U}, \mathrm{Inf}}$ (notation from Corominas et al., 2010). For the Québec City samples, these fractions were determined by combining a respirometric protocol together with total (COD) and soluble (sCOD) COD analysis and ultimate BOD (UBOD) measurements (Petersen et al., 2003). $S_{B}$ directly resulted from the respirometric test on the wastewater sample, while $\mathrm{XC}_{\mathrm{B}}, \mathrm{S}_{\mathrm{U}}$ and $\mathrm{X}_{\mathrm{U}, \mathrm{Inf}}$ were calculated as follows: $\mathrm{XC}_{\mathrm{B}}=\mathrm{UBOD}-\mathrm{S}_{\mathrm{B}}$; $\mathrm{S}_{\mathrm{U}}=\mathrm{sCOD}-\mathrm{S}_{\mathrm{B}} ; \mathrm{X}_{\mathrm{U}, \mathrm{Inf}}=\mathrm{COD}-\mathrm{sCOD}-\mathrm{XC}_{\mathrm{B}}$. For the WWTPs of Eindhoven and Roeselare both a respirometric evaluation and the STOWA method (Roeleveld and van Loosdrecht, 2002) were applied. The two protocols resulted in different COD fractions for the same wastewater sample. Nevertheless, both showed that primary treatment has a significant impact on the ASM1 fractions. Primary treatment yielded a significant variation of the particulates ratio $\left(\mathrm{XC}_{\mathrm{B}} / \mathrm{X}_{\mathrm{U}, \mathrm{Inf}}\right)$ (on average 1.9 to 1.2 for the Eindhoven, 1.1 to 0.5 for the Roeselare and 1.5 to 1.8 for the Québec City experiments), while, as expected, the soluble ratio $\left(\mathrm{S}_{U} / \mathrm{S}_{\mathrm{B}}\right)$ was not affected by the primary settler (Figure 7). 

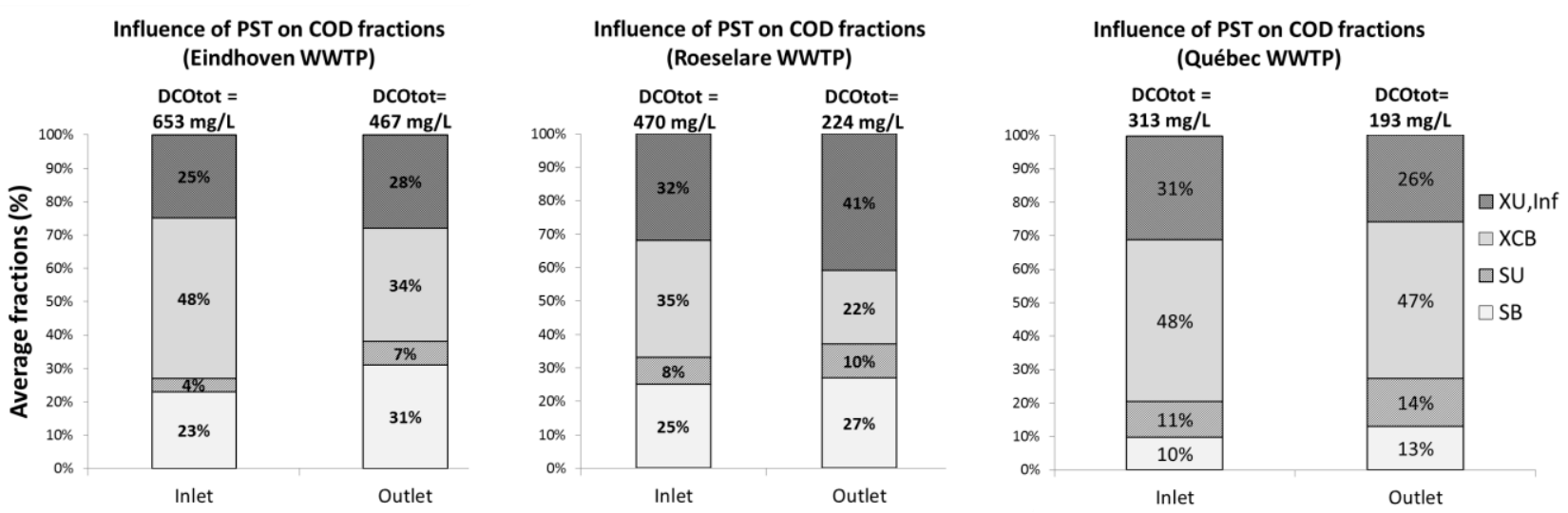

Figure 7 Evolution of COD fractions before and after a PST at three WWTPs: Eindhoven (The Netherlands), Roeselare (Belgium) (both by the STOWA protocol) and Québec City (respirometric protocol).

In the Québec WWTP a more detailed analysis was conducted. The wastewater samples could be classified into two types: a low loaded one (sampled at nighttime) and a heavily loaded one (sampled at daytime). According to this classification, by comparing inlet and outlet samples different trends in the aforementioned ratios were noticed: the particulate ratio $\left(\mathrm{XC}_{\mathrm{B}} / \mathrm{X}_{\mathrm{U} \text {,Inf }}\right)$ systematically decreases for daytime samples, but increases for wastewaters collected at nighttime. Moreover, some work was also conducted on samples collected after addition of chemicals. It was found that both the particulate and the soluble ratios tend to increase, i.e. the primary effluent contains relatively more biodegradable material than the influent.

These first results, although requiring further investigation, show that primary treatment has a significant impact on the ASM1 fractions. Therefore, the influence of the PST on the wastewater characterisation cannot be neglected and a proper COD fractionation into model variables can significantly improve simulation results. For instance, by applying the PSVD model concept to the mass balances of the ASM fractions in the primary settler model, i.e. having five classes for each of the ASM fractions, it will be possible to make them settle at different velocities, allowing the observed increase in ratios to be predicted properly. This will result in an appropriate fractionation at the primary settler effluent.

\section{CONCLUSIONS}

All studies presented in this contribution focus on primary settlers. The authors believe that PSTs need to be properly modelled and characterised in view of whole plant and resource recovery modelling. A new experimental and modelling approach, based on Particle Settling Velocity Distributions (PSVD), is proposed, and was shown to successfully predict TSS effluent concentrations on the basis of influent TSS time series and a number of ViCAs characterisation experiments. Simulation results under calibration and validation of the model were presented. It is illustrated that this approach can also be used to characterise primary influent under addition of chemicals, representing a potentially useful tool for the modelling of PSTs under CEPT. In addition, a simpler settler model, in view of controller development for CEPT, was presented as an alternative to the particle classes-based model. Finally, wastewater fractionation results obtained for the influent and the effluent of PSTs have shown, for the first time, that the primary settler produces a significant change in the wastewater composition ratios and, as a consequence, in the ASM fractionation of the wastewater. Hence, it can be anticipated that a more detailed primary settler model with explicit consideration of ASM fractions may be needed to properly feed the subsequent bioreactor models of a whole plant and resource recovery model. 


\section{ACKNOWLEDGEMENT}

The authors would like to acknowledge the whole primEAU team for its contribution. Funding for this work comes from the Natural Sciences and Engineering Research Council of Canada (NSERC/CRSNG), John Meunier and Québec City. Peter Vanrolleghem holds the Canada Research Chair on Water Quality Modelling. The authors also want to acknowledge Aquafin N.V. and Water board the Dommel for giving the opportunity to and assisting in the set-up of the sampling, testing and modelling.

\section{REFERENCES}

Bachis G., Vallet B., Maruéjouls T., Clouzot L., Lessard P. and Vanrolleghem P.A. (2012) Particle classes-based model for sedimentation in urban wastewater systems. In: Proceedings IWA Particle Separation Conference. June 18-20, 2012, Berlin, Germany.

Chebbo G. and Bachoc, A. (1992) Characterization of suspended solids in urban wet weather discharges. Water Sci. Technol., 25(8), 171-179.

Chebbo G. and Gromaire M.-C. (2009) VICAS - An operating protocol to measure the distributions of suspended solid settling velocities within urban drainage samples. J. Environ. Eng., 135(9), 768-775.

Choubert J.M., Rieger L., Shaw A., Copp J., Spérandio M., Sørensen K., Rönner-Holm S., Morgenroth E., Melcer H. and Gillot S. (2013) Rethinking wastewater characterisation methods for activated sludge systems - a position paper. Water Sci. Technol., 67, 2363-2373.

Corominas L., Rieger L., Takács I., Ekama G., Hauduc H., Vanrolleghem P.A., Oehmen A., Gernaey K.V., van Loosdrecht M.C.M. and Comeau Y. (2010) New framework for standardized notation in wastewater treatment modelling. Water Sci. Technol., 61, 841-857.

Flores-Alsina X., Arnell M., Amerlinck Y., Corominas L., Gernaey K., Guo L., Lindblom E., Nopens I., Porro J., Shaw A., Snip L., Vanrolleghem P.A. and Jeppsson U. (2014) Balancing effluent quality, economic cost and greenhouse gas emissions during the evaluation of (plant-wide) control/operational strategies in WWTPs. Sci. Total Environ. 466-467, 616-624.

Galil N. and M. Rebhun (1990) Primary chemical treatment minimizing dependence on bioprocesses in small treatment plants. Water Sci. Technol., 22 (3-4), 203-210.

Gernaey K., Vanrolleghem P.A. and Lessard P. (2001) Modeling of a reactive primary clarifier. Water Sci. Technol., 43(7), 73-82.

Gujer W. (2008) Systems Analysis for Water Technology. Springer-Verlag Berlin Heidelberg, Germany. pp. 462.

Hvitved-Jacobsen T., Vollertsen J. and Tanaka N. (1998) Wastewater quality changes during transport in sewers - an integrated aerobic and anaerobic model concept for carbon and sulfur microbial transformations. Water Sci. Technol., 38(10), 257-264.

Henze M., Gujer W., Mino T. and van Loosdrecht M.C.M. (2000) Activated Sludge Models ASM1, ASM2, ASM2d and ASM3. Scientific and Technical Report No. 9. IWA Publishing, London, UK.

Kristensen G.H, Jorgensen P.E. and Henze M (1992) Characterization of functional groups and substrate in activated sludge and wastewater by AUR, NUR and OUR. Water Sci. Technol., 25(6), 43-57.

Lessard P. and Beck M.B. (1988) Dynamic modeling of primary sedimentation. J. Environ. Eng., 114, 753-769.

Maruéjouls T., Lessard P., Wipliez B., Pelletier G. and Vanrolleghem P.A. (2011) Characterization of the potential impact of retention tank emptying on wastewater primary treatment: a new element for CSO management. Water Sci. Technol., 64, 1898-1905.

Maruéjouls T., Vanrolleghem P.A., Pelletier G. and Lessard P. (2012) A phenomenological retention tank model using settling velocity distributions. Water Res., 46, 6857-6867.

Melcer H., Krugel S., Butler R., Carter P. and Land G. (2005) Alternative Operational Strategies to Control Pollutants in Peak Wet Weather Flows. In: Proceedings of WEFTEC 2005, Washington DC. Water Environment Federation, Alexandria VA.

Melcer H., Ciolli M., Lilienthal R., Ott G., Land G., Dawson D., Klein A. and Wightman D. (2010) Bringing CEPT Technology into the 21st Century. In: Proceedings of WEFTEC 2010, New Orleans, LA. Water Environment Federation, Alexandria VA.

Melcer H., Davis D.P., Xiao S., Shaposka H., Ifft J., Bucurel N. and G. Land (2012). Wet weather flow treatment with a difference: Novel ideas for applying Chemically Enhanced Primary Treatment with high rate disinfection. In: Proceedings of WEFTEC 2012, Los Angeles, CA. Water Environment Federation, Alexandria VA. 
Newman D., Melcer H., Davis D.P., Pepe L., Winn R., Nascimento D. and Tyler T. (2013) At the nexus of process and design: Optimizing a wet weather treatment system. In: Proceedings of WEFTEC 2013, Chicago IL. Water Environment Federation, Alexandria, VA.

Morgenroth E., Kommedal R. and Harremoës P. (2002) Processes and modeling of hydrolysis of particulate organic matter in aerobic wastewater treatment - a review. Water Sci. Technol., 45(6), 25-40.

Otterpohl R. and Freund M. (1992) Dynamic models for clarifiers of activated sludge plants with dry and wet weather flows. Water Sci. Technol., 26(5-6), 1391-1400.

Pasztor I., Thury P. and Pulai, J. (2009) Chemical oxygen demand fractions of municipal wastewater for modeling of wastewater treatment. Int. J. Environ. Sci. Te., 6(1), 51-56.

Petersen B., Gernaey K., Henze M. and Vanrolleghem, P. A. (2002) Evaluation of an ASM1 model calibration procedure on a municipal-industrial wastewater treatment plant. J. Hydroinformatics, 4, 15-38.

Petersen B., Gernaey K., Henze M. and Vanrolleghem P.A. (2003) Calibration of activated sludge models: a critical review of experimental designs. In: Agathos, S.N., Reineke, W. (Eds.), Biotechnology for the Environment: Wastewater Treatment and Modeling, Waste Gas Handling. Kluwer Academic Publishers, Dordrecht, The Netherlands, pp. 101-186.

Phillips H.M., Sahlstedt K.E., Frank K., Bratby J., Brennan W., Rogowski S., Pier D., Anderson W., Mulas M., Copp J.B. and Shirodkar N. (2009) Wastewater treatment modelling in practice: A collaborative discussion of the state of the art. Water Sci. Technol., 59, 695-704.

Ribes J., Ferrer J., Bouzas A. and Seco A. (2002) Modelling of an activated primary settling tank including the fermentation process and VFA elutriation. Environ. Technol., 23, 1147-1156.

Rieger L., Gillot S., Langergraber G. and Shaw A. (2012) Good Modelling Practice: Guidelines for Use of Activated Sludge Models. IWA Publishing, London, UK.

Roeleveld P. J. and van Loosdrecht M.C.M. (2002) Experience with guidelines for wastewater characterisation in The Netherlands. Water Sci. Technol., 45(6), 77-87.

Tchobanoglous G., Burton F.L. and Stensel H.D. (2003) Wastewater Engineering: Treatment and Reuse. 4th edition, Metcalf \& Eddy, Inc, US.

Tik S., Langlois S. and Vanrolleghem P.A. (2013) Establishment of control strategies for chemically enhanced primary treatment based on online turbidity data. In: Proceedings $11^{\text {th }}$ IWA Conference on Instrumentation, Control and Automation (ICA2013). September 18-20, 2013, Narbonne, France. 


\title{
Modelling the Impact of Filamentous Bacteria Abundance in a Secondary Settling Tank: CFD Sub-models Optimization Using Long-term Experimental Data
}

\author{
Elham Ramin ${ }^{1, *}$, Dorottya S. Wágner ${ }^{1, *}$, Lars Yde ${ }^{2}$, Peter Szabo ${ }^{3}$, Michael R. Rasmussen ${ }^{4}$, Arnaud \\ Dechesne $^{1}$, Barth F. Smets ${ }^{1}$, Peter Steen Mikkelsen ${ }^{1}$, Benedek Gy. Plósz ${ }^{1}$ \\ ${ }^{1}$ Department of Environmental Engineering (DTU Environment), Technical University of Denmark, \\ Miljøvej, Building 113, DK-2800 Kgs. Lyngby, Denmark (E-mail: elhr@env.dtu.dk, dosaw@env.dtu.dk, \\ bfsm@env.dtu.dk,psmi@env.dtu.dk, beep@env.dtu.dk). \\ ${ }^{2}$ DHI Water \& Environment (S) Pte Ltd, Singapore 637141 (E-mail: lay@dhigroup.com). \\ ${ }^{3}$ Department of Chemical and Biochemical Engineering, Technical University of Denmark, Building 229, \\ DK-2800 Kgs. Lyngby (E-mail: ps@kt.dtu.dk) \\ ${ }^{4}$ Department of Civil Engineering, Aalborg University, DK-9000 Aalborg, Denmark (mr@ civil.aau.dk) \\ *Joint first authors
}

\begin{abstract}
The objective of this work was to assess the impact of filamentous bacteria on the settling velocity and rheological behaviour of activated sludge. We then identified the relevant settling and rheological model parameters to account for the impact of filamentous bulking on the prediction of sludge mixing and transport in secondary settling tanks by a computational fluid dynamics (CFD) model. We identified the relevant settling velocity and rheology model parameters influenced by the filamentous bacteria content of activated sludge. The hindered, transient, and settling parameters of the settling velocity model proposed in our previous study were estimated using measurements from batch settling tests with a novel column setup. Additionally, the rheological measurements from experiments with a rotational viscometer were used to calibrate the Herschel-Bulkley rheology model including the rheology correlations with the sludge concentration obtained in our previous study. Both settling and rheological tests were performed with sludge samples collected biweekly from the Lundtofte wastewater treatment plant in a four-month measurement campaign. Quantitative fluorescent in-situ hybridisation (qFISH) analysis was carried out on the sludge samples to quantify the volume fraction of filamentous bacteria. Based on the correlations of settling and rheological model parameter values with the volume fraction of filamentous bacteria, we identify the significant impact of filamentous bacteria on the hindered settling of activated sludge. However, no significant impact on the transient and compression settling model parameters was observed. This study also finds that microbial filaments residing inside the microbial flocs can significantly alter the rheological behaviour of activated sludge. A two-dimensional, axi-symmetrical CFD was used to assess the impact of calibration scenarios for settling and rheology under low and high abundance of filamentous bacteria on the CFD predictions. Results obtained suggest that the influence of filamentous bulking on the settling and rheology of activated sludge can affect the solids distribution and transport in the SSTs.
\end{abstract}

\section{Keywords}

Activated sludge; filamentous bulking; compression settling; computational fluid dynamics; rheology; secondary settling tank

\section{INTRODUCTION}

Secondary settling tanks (SSTs) are located after the biological reactors in wastewater treatment plants (WWTPs) to separate the treated water from the microbial mass by means of 
gravity sedimentation. The clarification and thickening performance of SSTs depend on their hydraulic features as well as the settleability of activated sludge. A malfunctioning SST with a poor quality effluent in terms of suspended solids and insufficiently thickened sludge for recycling to the reactors, impacts the sludge retention time (SRT) in the system, and potentially deteriorates the performance efficiency of the biological processes. Moreover, SSTs are the hydraulic bottlenecks of WWTPs. The efficiency of SSTs can limit the maximum flow rate entering the WWTPs under wet-weather conditions.

A common operational problem in SSTs is the poor settling of activated sludge resulting from the excessive growth of filamentous bacteria, which prevents the formation of well-settling sludge (Wanner, 1994). Activated sludge flocs have a very heterogeneous structure, which consists of a variety of microorganisms as well as organic and inorganic particles and dead cells surrounded by extracellular polymeric substances (Wilén et al., 2008). The operational and seasonal variations in activated sludge units, such as dissolved oxygen concentration, nutrient deficiency and substrate limiting conditions, influence the structure of the growing flocs in bioreactors (Comas et al., 2008). However, the exact cause of filamentous bulking can be very diverse (Jenkins et al., 1993), and is not fully understood (Mielczarek et al., 2012). A common approach to identify filamentous bulking is to detect and quantify the content of filamentous bacteria in activated sludge samples by performing quantitative fluorescent insitu hybridisation (qFISH) analysis (Nielsen et al., 2009).

In WWTP modelling, conventionally, the influence of filamentous bulking is accounted for by modifying the hindered settling parameters in the settling velocity formulation in the SST models (Ekama et al., 1997). Several studies have shown the relation between the morphology of bulking sludge and settling parameters (Grijspeerdt and Verstraete, 1997; Jin et al., 2003; Wilén et al., 2008). However, the question arises whether filamentous bulking can also affect the transient and compression settling as well as the rheology of activated sludge and how these effects influence the sludge distribution in the SSTs.

Computational Fluid Dynamic (CFD) models have been used to predict the internal flow and solids transport in SSTs (Deininger et al., 1998; Lakehal et al., 1999; De Clercq, 2003; Weiss et al., 2007). CFD models are computationally heavy, and they are thus mainly used for the purpose of design of new SSTs, or optimization and trouble shooting of existing SSTs. However, validated CFD models can replace expensive field experiments to calibrate and validate one-dimensional models (De Clercq, 2003; Plósz et al., 2007). The non-Newtonian behaviour of activated sludge as well as its hindered and compression settling behaviour have significant impacts on the overall solids transport in the SSTs (Ekama et al., 1997). Thus, the accurate CFD prediction of hydrodynamics and solids distribution in the tank requires inclusion of optimized setting and rheology models.

In this study, we used a validated two-dimensional, axi-symmetrical CFD model with settling velocity and rheology models developed in our previous study (Ramin et al., 2014) to simulate the sludge distribution in the SST at Lundtofte WWTP. Additionally, we used the long-term settling and rheological measurements, as well as qFISH analysis performed by Wágner et al. (2014) on the sludge samples from Lundtofte WWTP.

The main objectives of this study are (i) to assess the impact of filamentous bacteria on hindered, transient and compression settling as well as the rheological behaviour of activated sludge based on the measurements with activated sludge of varying filamentous bacteria abundance, (ii) to identify the relevant settling and rheological model parameters, and finally 
(iii) to investigate how the effect of filamentous bulking on the model parameters can influence the prediction of sludge mixing and transport in SSTs by the CFD model.

\section{MATERIAL AND METHODS}

\section{Laboratory experiments}

In this section, the settling and rheology experiments, as well as qFISH analysis (Fig. 1) performed by Wágner et al. (2014) are briefly described.
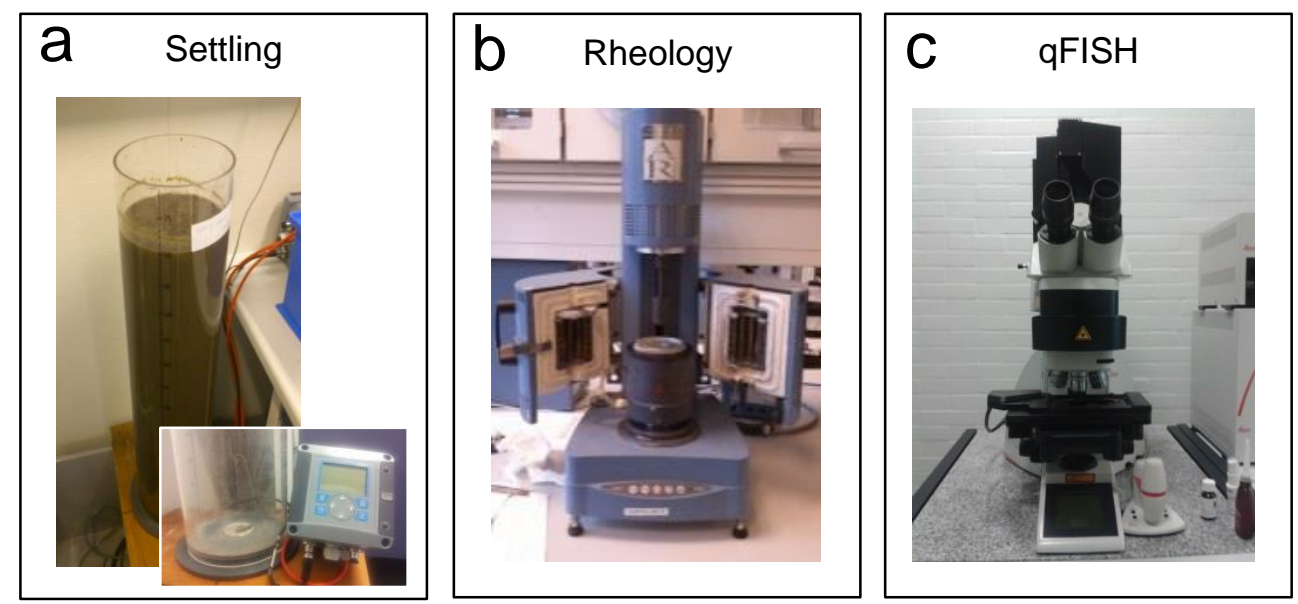

Figure 1. The laboratory set-ups for the settling (a), rheology (b), and qFISH (c) experiments performed by Wágner et al. (2014) .

Sampling. Activated sludge samples were collected biweekly for four months from the combined recycle flow at Lundtofte WWTP (Lyngby, Denmark). The samples were used on the same day of collection for settling experiments, and some were stored in $4{ }^{\circ} \mathrm{C}$ for rheology experiments on the next day. The concentration of sampled sludge was determined using method 2540 D of Standard Methods (APHA, 1995). Additionally, some sludge was pretreated and fixed with $4 \%$ paraformaldehyde to preserve its initial state, and then stored at -20 ${ }^{\circ} \mathrm{C}$ until the qFISH analysis.

Settling tests. Settling tests were performed using the newly developed settling column set-up (Ramin et al., 2014), consisting of a large settling column (diameter $=20 \mathrm{~cm}$, Height $=80 \mathrm{~cm}$ ) with a total suspended solids (TSS) sensor (Solitax ${ }^{\circledR}$, Hach Lange, Germany) installed at the bottom of the settling column (Fig. 1a). Prior to each settling test, the sludge sample was diluted with the SST effluent in the settling column and homogenized with coarse-bubble aeration. During each 60-minute settling test, the evolution of sludge blanket height $(S B H)$ and the sludge concentration at the bottom $\left(X_{b}\right)$ were recorder. Next, the settled sludge was rehomogenized and diluted to a lower concentration. Overall, the settling tests were performed at sludge concentration in the range $1.5-4.5 \mathrm{~g} / \mathrm{l}$.

Rheology measurements. The rheological experiments were performed using a standard rotational rheometer (TA Instruments AR2000, USA) with a conical single-gap cylindrical geometry (Fig. 1b). The experiments were performed on sludge samples diluted with SST effluent over the concentration range of 5-12.8 g/l under shear-stress controlled conditions to obtain shear rates in the range of $0.001-250 \mathrm{~s}-1$. The shear stress was applied from high to low values to minimize the sludge settling problem in the sample during the tests. 
FISH analysis. The qFISH procedure was conducted using $1 \mu \mathrm{l}$ of fixed sample taken from the activated sludge used in the settling and rheological experiments. The total amount of bacteria and the specific filamentous bacteria were targeted with two different fluorescentlylabelled probes (MPA mix: MPA 645, MPA 223, MPA 60). A confocal laser scanning microscope (LEICA SP5®, Leica, Germany) was used to assess the samples with 20x magnification and 2x zoom (Fig. 1c). 15-20 randomly chosen images were taken using the confocal microscope (Nielsen et al., 2009). The images were analysed using the daime (digital image analysis in microbial ecology) software (Daims et al., 2006). We note that, in the study of Wágner et al. (2014), the two dominant microbial species namely Chloroflexi spp. (CFX) and Microthrix parvicella (MPA) were identified in the activated sludge samples. In the present paper, we only present results obtained on the impact of the MPA volume fraction on the settling and rheological behaviour of activated sludge. For further information on the study, readers are kindly referred to Wágner et al. (2014).

\section{Numerical modelling}

Description of the SST. The SST under study is part of the Lundtofte WWTP (Lyngby, Denmark). It is a circular centre-feed conical tank with a diameter of $24.5 \mathrm{~m}$ and an average depth of $4 \mathrm{~m}$.

CFD simulations of the SST. The CFD simulations of the SST was executed in OpenFOAM CFD toolbox (OpenCFD, 2012) and using the settlingFoam solver (Brennan, 2001). The physics of the solver is based on the average Eulerian two-phase flow combined with the modified k- $\varepsilon$ model, accounting for density stratification. To predict the distribution of solids, a convection-diffusion equation derived from the continuity equation for the solid phase (drift flux model) is coupled with the momentum and turbulence equations in the solver.

To reduce the computation effort, the flow in the SST is assumed to be axi-symmetric, and only a radial segment of the tank is considered for CFD modelling. The geometry is discretised with around 6000 polyhedral grids in depth and radial directions (Fig. 2). The imposed boundary conditions are as follows. The water-surface is modelled as a symmetryplane, i.e. normal gradients to the surface are zero. The inclined bottom is considered as a frictionless boundary to simulate the effect of an ideal scraper facilitating the sludge flow to the hopper by overcoming the wall stress, as proposed by Deininger et al. (1998). The rest of the walls were considered as no-slip with standard wall-functions to approximate the mean velocity near the wall.

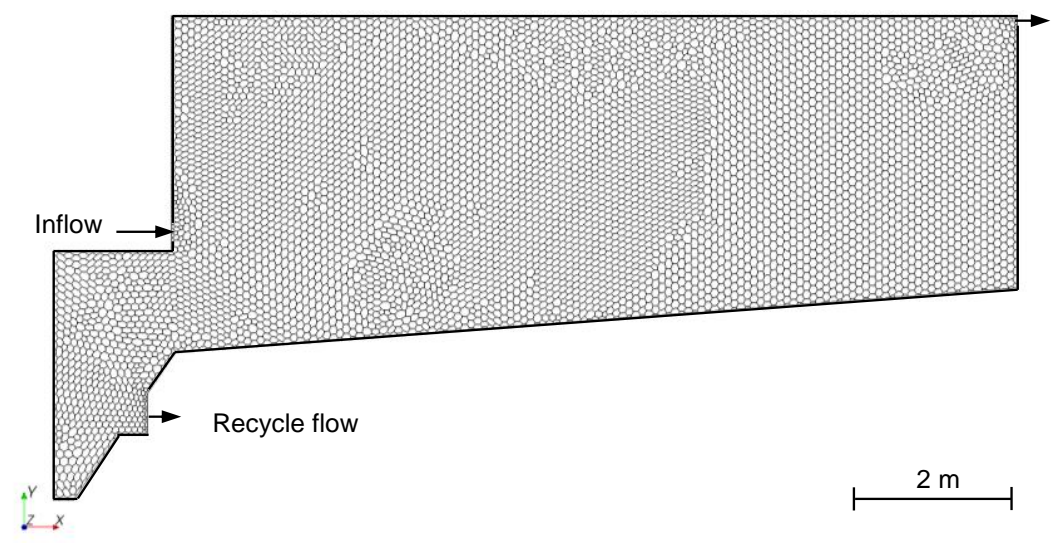


Figure 2. The 2-D axi-symmetric mesh with about 6000 polyhedral grids generated in STAR$\mathrm{CCM}+{ }^{\circledR}$ and implemented in OpenFOAM for CFD simulations of the circular SST at Lundtofte WWTP.

\section{THE SETTLING VELOCITY AND RHEOLOGICAL MODEL The settling velocity model}

The settling velocity model developed by Ramin et al. (2014) accounts for hindered, transient and compression settling regimes that are typically observed in the activated sludge batch settling tests. This model was developed based on an evaluation of state-of-the-art settling velocity models with measurements from the simple, novel batch settling experimental set-up explained in the previous section.

The widely used double-exponential hindered settling velocity model developed by Takács et al. (1991) and the mechanistic compression settling velocity model based on phenomenological sedimentation-consolidation theory (Bürger, 2000; Kinnear, 2002; De Clercq, 2006) with the empirical effective solids stress formulation developed by De Clercq et al. (2008) were evaluated based on their predictions of the $S B H$ and $X_{b}$ measurements. To evaluate these settling velocity models, the different models were implemented in a dynamic 1-D model of the settling column, developed based on a modified form of the 1-D SST model by Plósz et al. (2007), i.e. using a discretisation level of 60 layers and the numerical fluxes treated with the Godunov scheme (Bürger et al., 2011). The differential mass conservation equation is

$$
\frac{\partial X}{\partial t}-\frac{\partial\left(v_{s} X\right)}{\partial z}=0
$$

(1)

where $X$ is the sludge concentration, $t$ denotes time, $v_{s}$ is the settling velocity model, and $z$ is the depth in the column. We note that using the compression formulation in Eq. 1 yields a second order partial differential equation.

Results obtained by Ramin et al. (2014) show that, using Takács hindered settling velocity model with the hindered parameter $\left(v_{0}\right.$, and $r_{H}$, Vesilind, 1968) estimated directly from the SBH measurements the predictions were shown to diverge from the $S B H$ and $X_{b}$ measurements during the transient and compression regime. Furthermore, including the mechanistic compression settling velocity model with the effective solids stress formulation of De Clercq et al. (2008) was shown to over-predict the $X_{b}$ data when it was calibrated to the $S B H$ data only. Consequently, a new power formulation for the effective solids stress was developed to improve the predictions of $X_{b}$. Finally, by applying an exponential transition formulation in the compression zone, the best prediction of $X_{b}$ data could be achieved. The formulation of the settling velocity model is

$$
v_{S}=\left\{\begin{array}{cc}
v_{0} e^{-r_{H} \cdot X}-v_{0} e^{-r_{P} \cdot X} & X<X_{C} \\
v_{0, t} e^{-r_{t} X}\left(1-\frac{\rho_{S}}{\left(\rho_{S}-\rho_{f}\right) g X}\left(\frac{X-X_{C}}{C_{1}}\right)^{C_{2}} \frac{d X}{d z}\right) & X \geq X_{C}
\end{array}\right.
$$


where $v_{0}$ is the maximum settling velocity; $r_{H}$ and $r_{P}$ are the hindered and low concentration indices, respectively; $v_{0, t}$ and $r_{t}$ are the transient settling parameters; $C_{1}$ and $C_{2}$ are parameters in the compression settling model; $\rho_{s}$ and $\rho_{f}$ are the sludge and water density, respectively; $g$ denotes the gravity constant; $C_{1}$ and $C_{2}$ are compression parameters; and $X_{C}$ is the threshold compression concentration. Fig. 3 illustrates the prediction of the sludge profile in the settling column by simultanously calibrating it to the $S B H$ and $X_{b}$ measurements using the adaptive Markov Chain Monte Carlo (MCMC) Bayesian global optimization method DREAM (ZS) (Laloy and Vrugt, 2012). Using the $\operatorname{DREAM}_{(\mathrm{ZS})}$ optimization algorithm, the prediction uncertainty of the settling velocity model for the estimated parameters can be obtained from the posterior parameter distributions. Fig. 4 shows the prediction accuracy of the settling velocity model for the measurements with sludge samples taken from two WWTPs in Denmark, Lundtofte $(\mathrm{PE}=135.000, \mathrm{SRT}=31 \mathrm{~d})$ and Lynetten $(\mathrm{PE}=750.000, \mathrm{SRT}=29 \mathrm{~d})$.

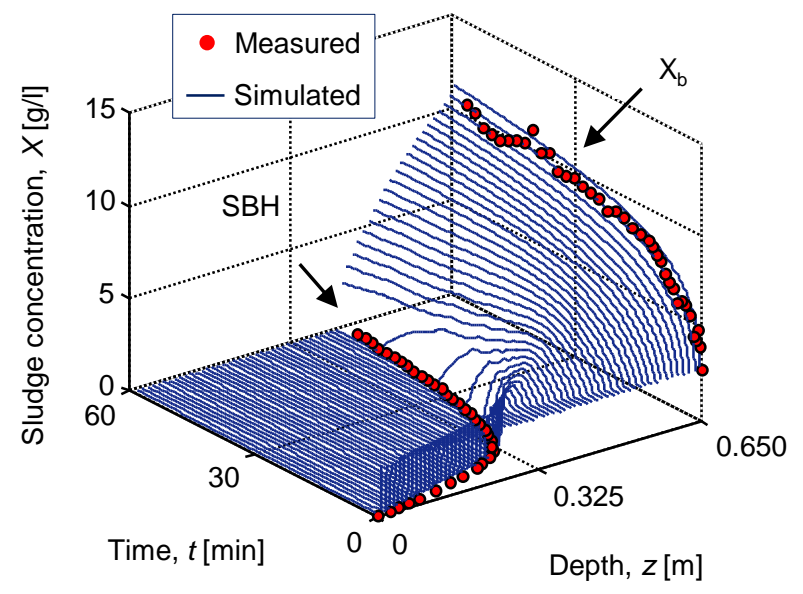

Figure 3. Prediction of the settling velocity model (Ramin et al., 2014) calibrated to the $S B H$ and $X_{b}$ measurements by implementing it in a 1-D settling column model (with 60 layers discretization). The lines correspond to the simulated evolution of sludge concentration in each layer. 

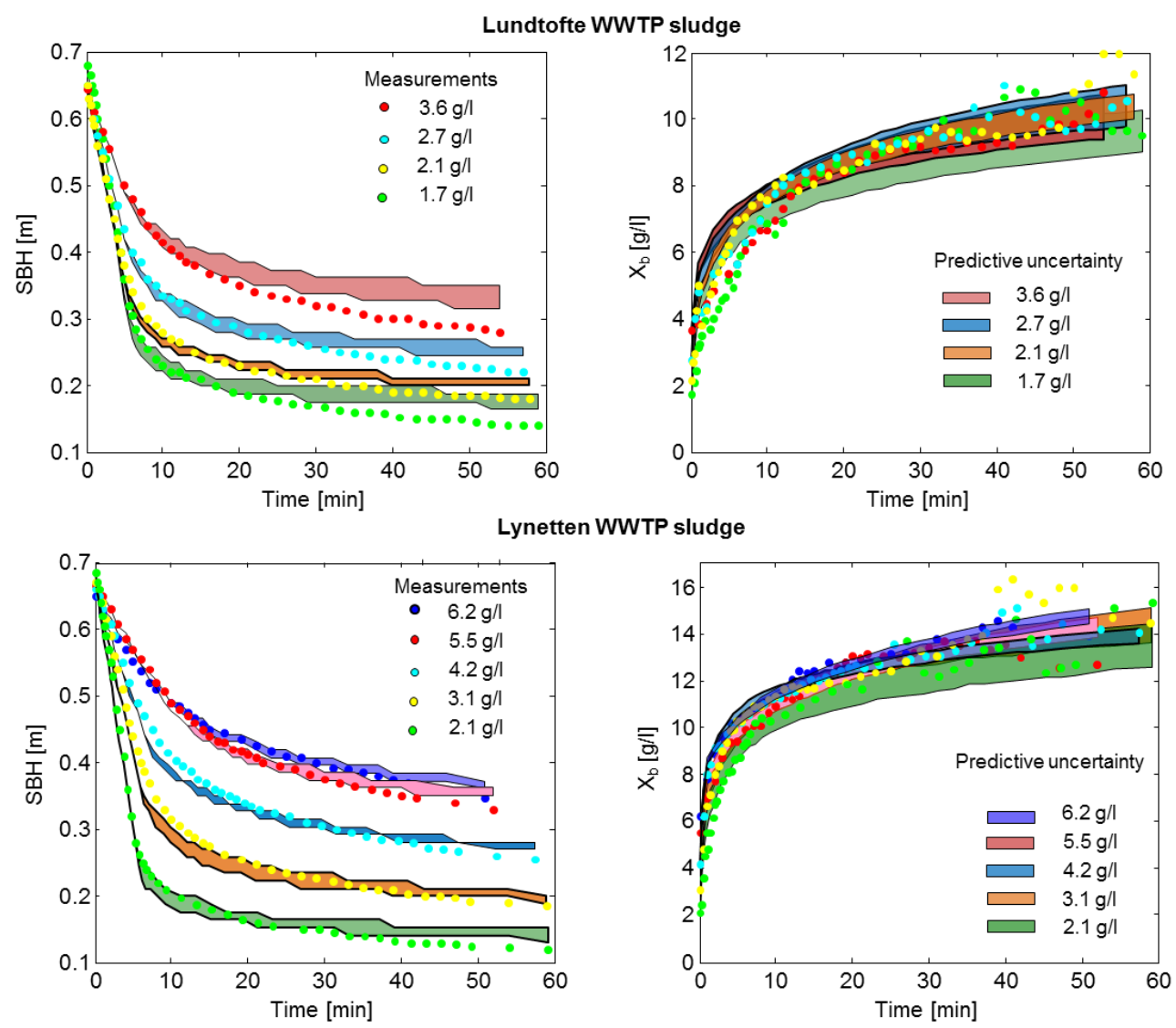

Figure 4. Predictive uncertainty ( $95 \%$ confidence intervals of the model prediction due to parameter uncertainty) of the settling velocity model calibrated to the measurements with the Lundtofte and Lynetten WWTP sludge using the DREAM $_{(\mathrm{ZS})}$ optimization algorithm.

The Rheological model

The rheological measurements can very accurately described with the yield-pseudoplastic type Herschel-Bulkley rheology model (e.g., Ratkovich et al., 2013):

$$
\eta=\frac{\tau_{0}}{\gamma}+K \gamma^{n-1}
$$

where $\eta$ is the apparent viscosity, $\tau_{0}$ is the yield stress, $\gamma$ is the shear rate, $K$ is the consistency index, and $n$ is the flow behaviour index. Fig. 5 shows the predictions of the Herschel-Bulkley model for one set of measurements with different sludge concentrations. A constraint of maximum viscosity was set for the Herschel-Bulkley model for the shear rates of below $0.01 \mathrm{~s}^{-}$ ${ }^{1}$ to avoid unrealistic prediction of apparent viscosity values at very low shear rate conditions. 


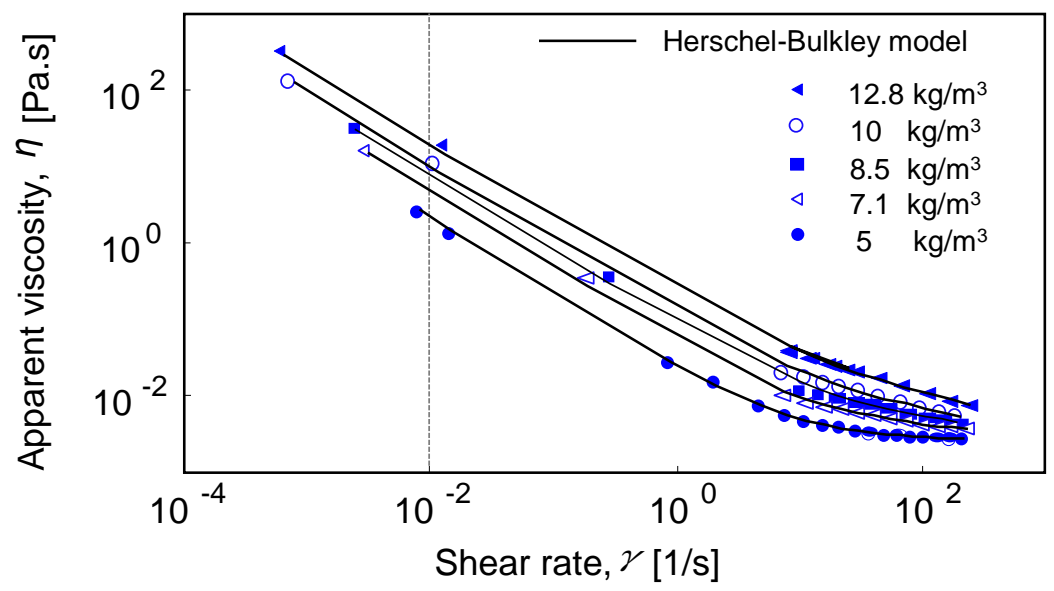

Figure 5. An example on the prediction of activated sludge apparent viscosity with the Herschel-Bulkley model (Eq. 3), shown for one set of the rheological measurements (out of eight) with different sludge concentrations.

The estimated parameters of the Herschel-Bulkley model $\left(\tau_{0}, K\right.$, and $\left.n\right)$ were correlated to the sludge concentration with the correlations presented in our previous study (Ramin et al., 2014), e.g. for yield stress, the following power formulation was used:

$$
\tau_{0}=A X^{B}
$$

where $A$ and $B$ are the yield stress correlation parameters.

\section{RESULTS}

\section{Impact of filamentous bulking on settling}

Hindered settling. Fig. 6a illustrates the relation between the ratio of hindered settling parameters $\left(\mathrm{v}_{0} / \mathrm{r}_{\mathrm{H}}\right)$ estimated from the settling measurement sets, and the volume fraction of Microthrix parvicella (MPA) filamentous bacteria. The decreasing trend of $\mathrm{v}_{0} / \mathrm{r}_{\mathrm{H}}$ with increasing filament volume fraction (i.e. development of filamentous bulking sludge) is in line with conventional theory (Ekama et al., 1997). 

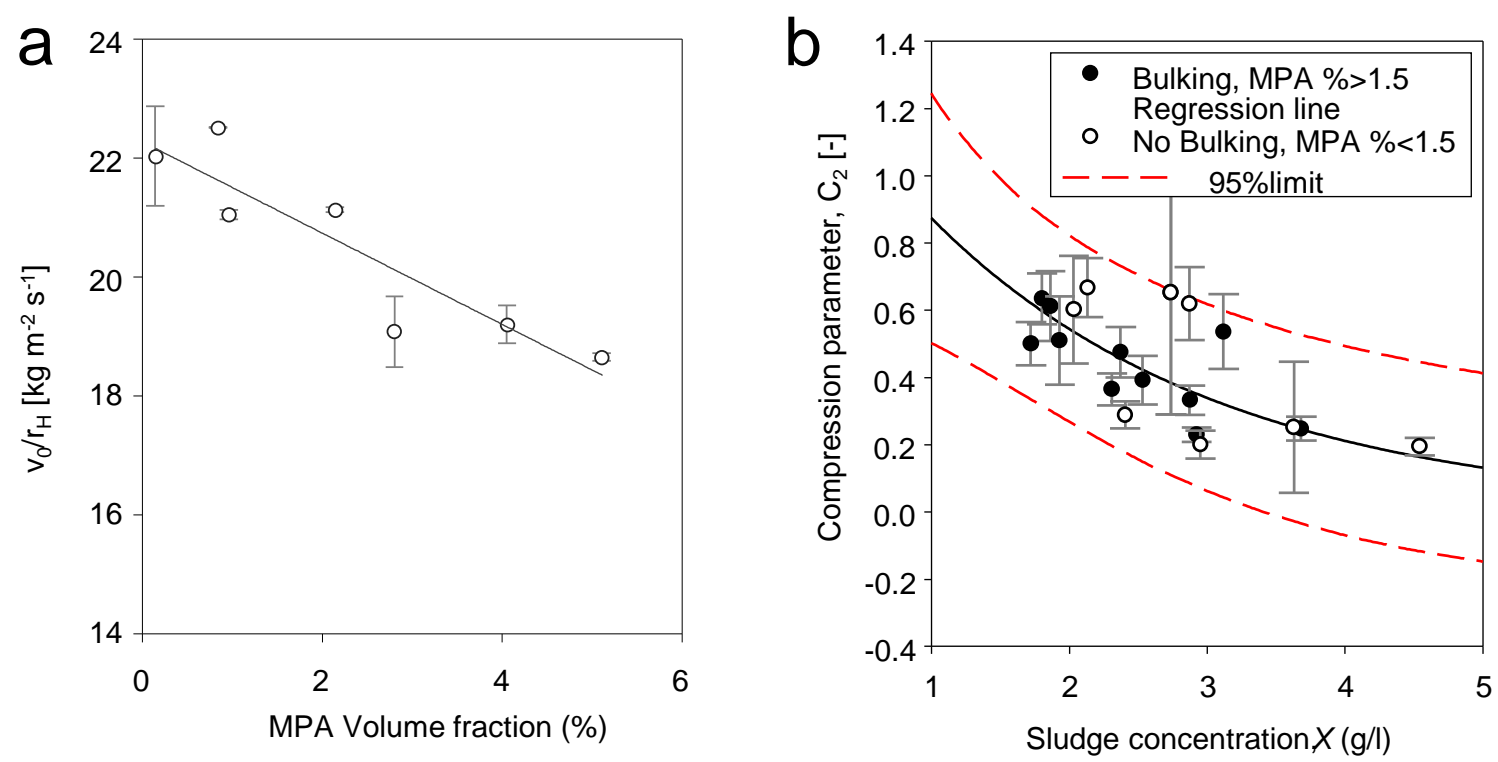

Figure 6. Correlation of the ratio between the hindered settling parameters with the volume fraction of MPA filamentous bacteria in the activated sludge (a), and dependency of the compression settling parameter on the initial sludge concentration in each settling test (b). The hindered parameters were estimated for the eight measurement sets (one outlier), and the compression settling parameter were estimated for each settling test ( 3 to 4 tests for each measurement set).

Transient and compression settling. We further assessed the influence of filamentous bacteria on the transient and compression settling processes, characterised by parameters $r_{t}$ and $C_{2}$, respectively (Eq. 2). No clear relation between these parameters and the volume fraction of filamentous bacteria was observed (data shown by Wágner et al., 2014). The estimated values of $r_{t}$ were obtained in a narrow range $(0.6-1.0 \mathrm{l} / \mathrm{g})$ regardless of the sludge concentration and the filamentous bacteria content. On the other hand, the estimated values of $C_{2}$ were scattered in a wider range $\left(0.1-0.8\right.$, dimensionless). The dependency of $C_{2}$ on the sludge concentration is further investigated in Fig. 3b, showing $C_{2}$ as a function of the initial sludge concentration in the settling column tests.

Based on Fig. 3b, no effective correlation between $C_{2}$ and $X$ or distinct tendencies under bulking and no-bulking conditions (defined based on the MPA volume fraction - cut-off value: $1.5 \%$ ) can be observed. The $95 \%$ confidence interval (defined by the dashed lines in Fig. 4) shows a relatively high uncertainty in estimating the value of $\mathrm{C}_{2}$ based on the initial sludge concentration of the settling tests. For the CFD model (Ramin et al., 2014), the value of $C_{2}$ is determined based on the SST feed concentration $(3 \mathrm{~g} / \mathrm{l})$. Therefore, later in this paper it is investigated if the variability of $C_{2}$ parameter values (see Fig. 3b) causes any significant variation in the CFD simulation results of an SST.

\section{Impact of filamentous bulking on rheology}

We investigated how the rheological behaviour of activated sludge is influenced by the presence of filamentous bacteria. The Herschel-Bulkley rheology model (Eq. 3) was calibrated to the eight rheology measurement sets (each set includes measurements with four to five different sludge concentrations). The estimated rheology parameters $\left(\tau_{0}, K\right.$, and $\left.n\right)$ were then correlated to the volume fraction of MPA filamentous bacteria in the sludge. Among the 
three parameters, only the estimated yield stress values showed a higher degree of association with the volume fraction of MPA (see Fig. 7a). No significant influence on the behaviour index $(n)$ and consistency index $(K)$ was observed (data not shown). Fig. 7a shows a decreasing tendency in the value of yield stress with the increase in MPA volume fraction at high sludge concentrations (only the concentration range of 7.5-9.5 g/l is shown here).

a

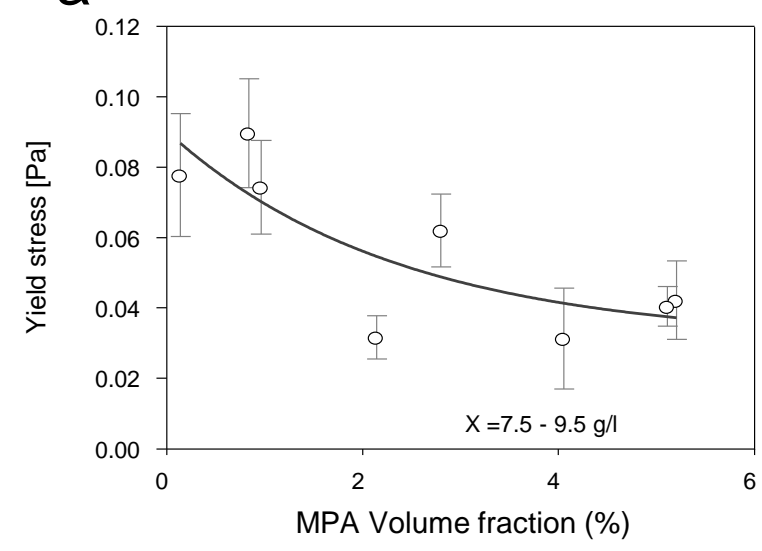

b

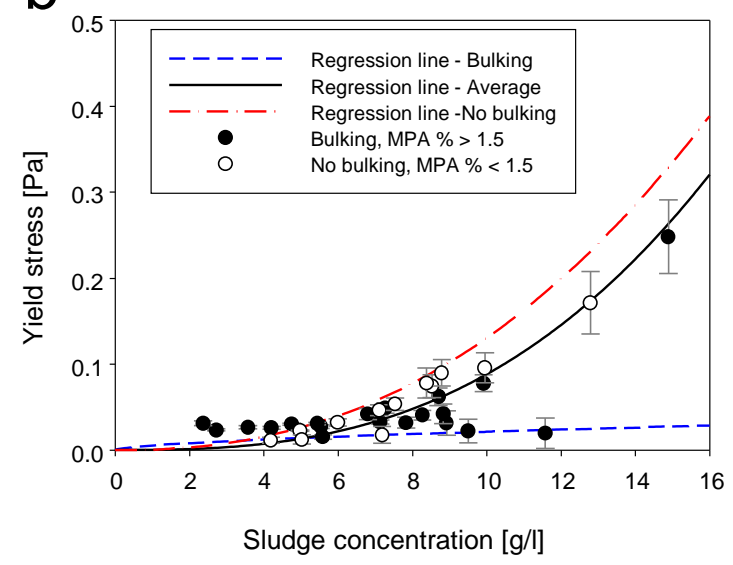

Figure 7. Dependency of yield stress of activated sludge on (a) the MPA filamentous bacteria (MPA) volume fraction for the concentration range of 7.5-9.5 g/l.

The reasoning behind the correlations shown in Fig. 7a can be explained as follows. The increase in the content of MPA filament, residing inside the flocs as the backbone of the flocs, influences the bulkiness of the flocs. As stated by Eshtiaghi et al. (2013), the increase of sludge water content can decrease the sludge viscosity. Since MPA resides in the floc, a high abundance of this filament can result in high bound water content, which would explain the observed decrease of yield stress. These results suggest that the filaments residing in the flocs mixture can impact the rheology of activated sludge.

Based on Fig. 7b, the estimated yield stress values shows an overall dependency on the sludge concentration (solid line in Fig. 7b), which confirms the proposed correlation (Eq. 4) in our previous study. However, the values are more scattered with the increase in the sludge concentration. Consequently, two power formulations are fitted to the lower and upper data points to determine the interval of the estimated yield stress values (the two dash lines in Fig. 7b). Based on Fig. 7b, good settling (or no bulking) condition, characterised with low MPA filament presence $(<1.5 \%)$ can increase the yield stress, which then corresponds to values closer to the upper curve (dashed-dotted line in Fig. 7b). On the other hand, bulking conditions (MPA volume fraction > $1.5 \%$ ) can decrease yield stress, which corresponds to values closer to the lower curve (dashed line in Fig. 7b).

\section{CFD simulations}

Based on the above observations, it is investigated here how the influence of filaments filamentous organisms can impact on the hindered settling and yield stress of sludge, and thus the sludge distribution and hydrodynamics in the SST can influence the using CFD scenario simulations. Moreover the influence of uncertainty in estimating the compression parameter $\left(C_{2}\right)$ on the variation of the CFD simulation results is assessed. Table 1 summarizes the CFD simulation cases and includes only the values of the parameters under investigation that were 
obtained from Figures $6 \mathrm{a}, 6 \mathrm{~b}$ and $7 \mathrm{~b}$. In the first case, the impact of $C_{2}$ is assessed by keeping the hindered and rheology parameters to the average values, and changing $C_{2}$ to the minimum and maximum values at the feed flow sludge concentration to the SST $\left(X_{F}=3 \mathrm{~g} / \mathrm{l}\right)$ based on Fig. 6b. In the second case, the no bulking and bulking conditions are imposed to the CFD model by applying the minimum (0.1\% MPA) and maximum (extrapolated to 9\% MPA) values of the hindered settling velocity parameter values based on Fig. 6a and the correlations of $\tau_{0}$ to sludge concentration with the upper and lower curves in Fig. $7 \mathrm{~b}$.

Table 1. The CFD simulation cases and the values of the settling and rheological model parameters under investigation.

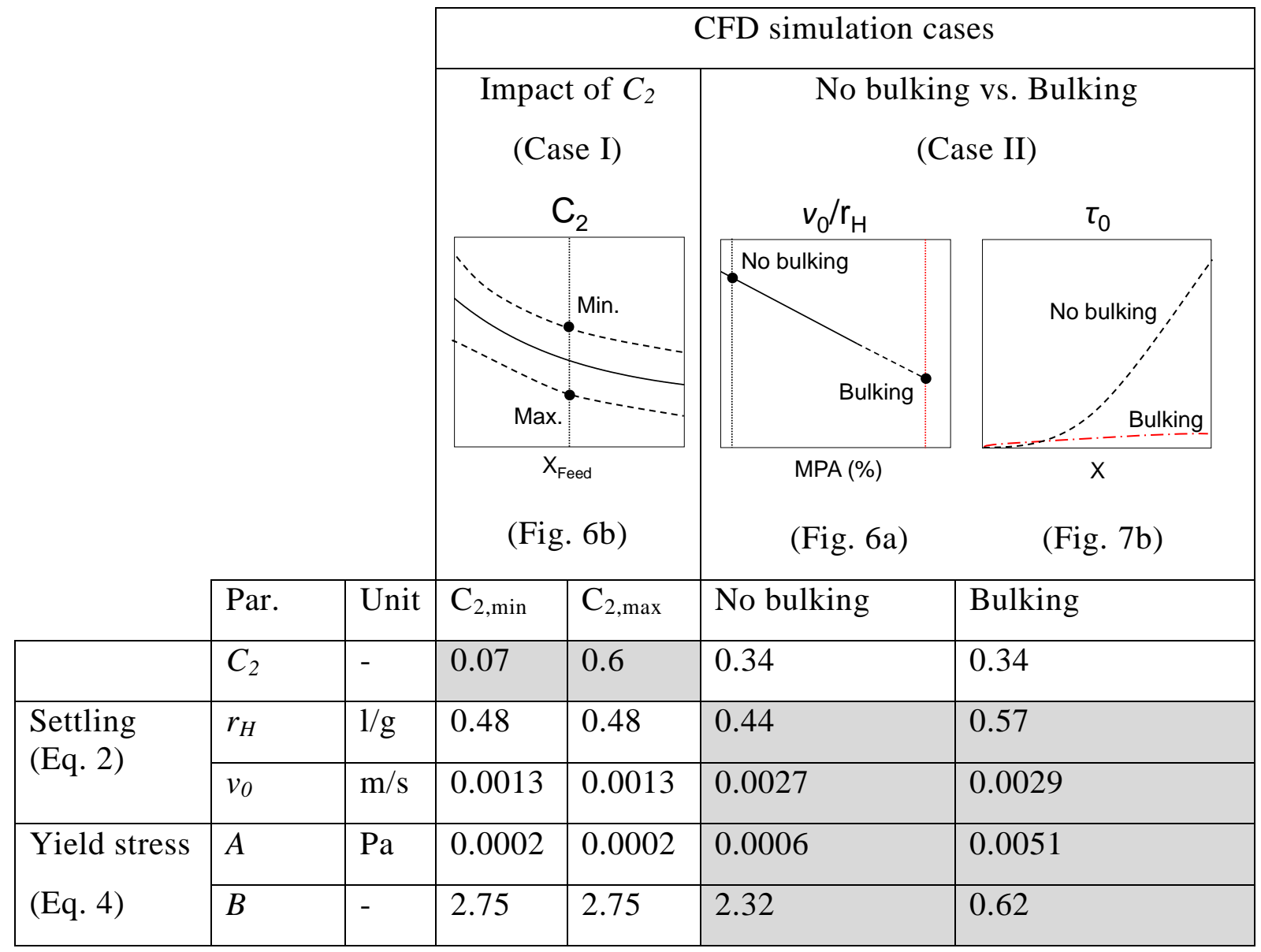

Case I. Fig. 8 illustrates the impact of the uncertainty in correlating the compression settling parameter $C_{2}$ with the initial sludge concentration (Fig. 6b) on the CFD simulation results. The uncertainty of $C_{2}$ is shown to result in about 10-30\% variation in the pre-diction of sludge blanket height (Fig. 8a) and up to 50\% in the maximum radial velocity in the density current (Fig. 8b). These results imply that the uncertainty in estimating $C_{2}$ needs to be reduced probably by performing additional settling measurements. 

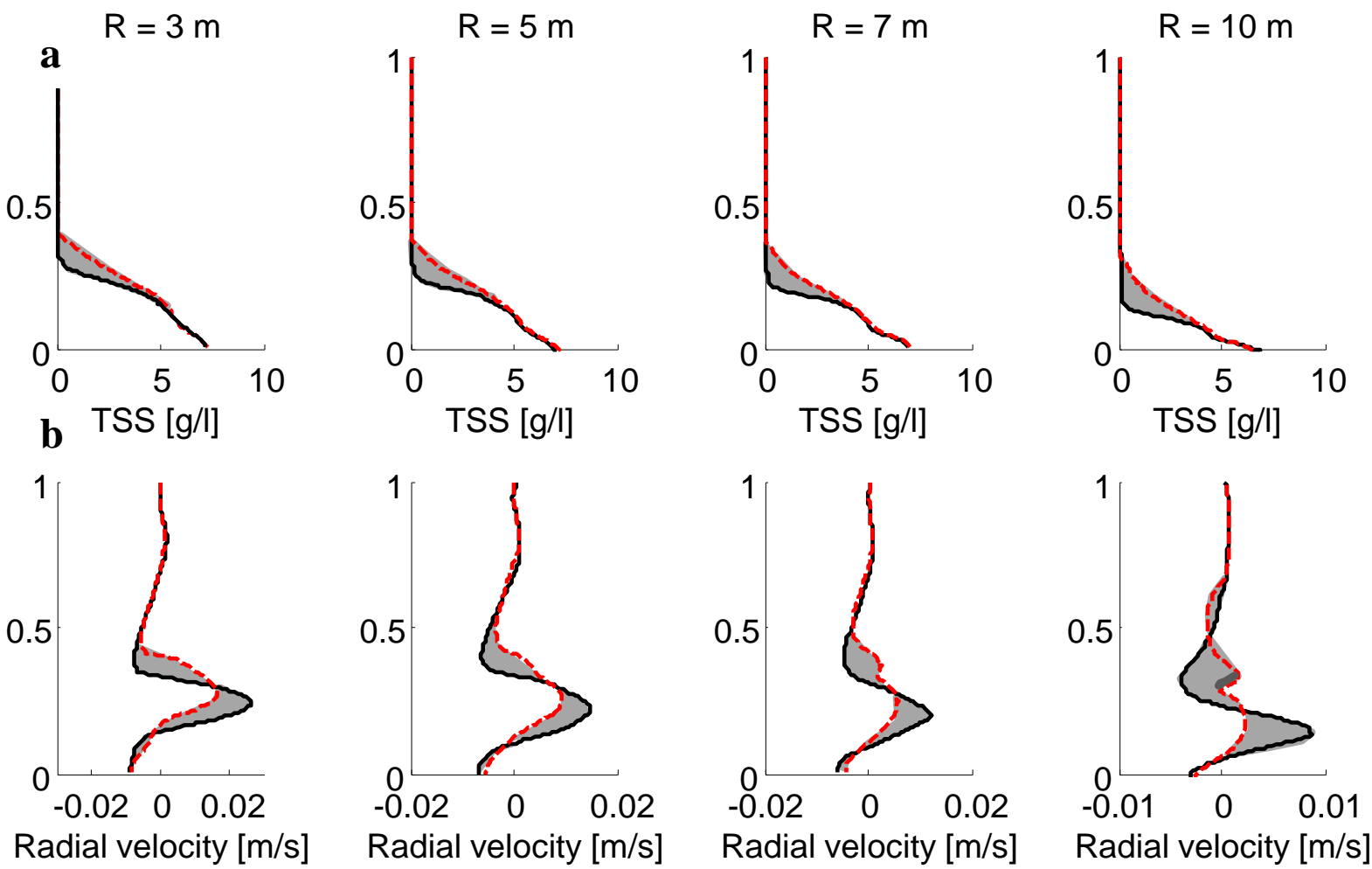

Figure 8. The variation in the prediction of the vertical profiles of sludge distribution (a), and radial velocity (b) by the CFD model due to uncertainty in the compression parameter $\left(C_{2}\right)$. The profiles with solid lines are predicted with $C_{2, \max }$, and the profiles with dashed lines are predicted with $C_{2, \min }$. Profiles are shown with normalized height at four different radial distances from the centre of the tank.

Case II. To assess the impact of yield stress and hindered settling on CFD model prediction, the value of $C_{2}$ is set constant to an average value of 0.34. Fig. 9 illustrates the CFD simulations based on the impact of filamentous bulking on the hindered settling parameters and yield stress. The rise in the sludge blanket height is up to $20 \%$ under the bulking condition (dashed lines in Fig. 9). Moreover, the slight increase in the flow of thickened sludge over the inclined bottom to the hopper can be observed in Fig. 9b due to the reduced yield stress under bulking condition. In general, the impact of hindered settling velocity parameter values and yield stress under bulking is not straight forward. This is because these parameters influence the complex interaction between the sludge distribution and hydrodynamics of the tank. 

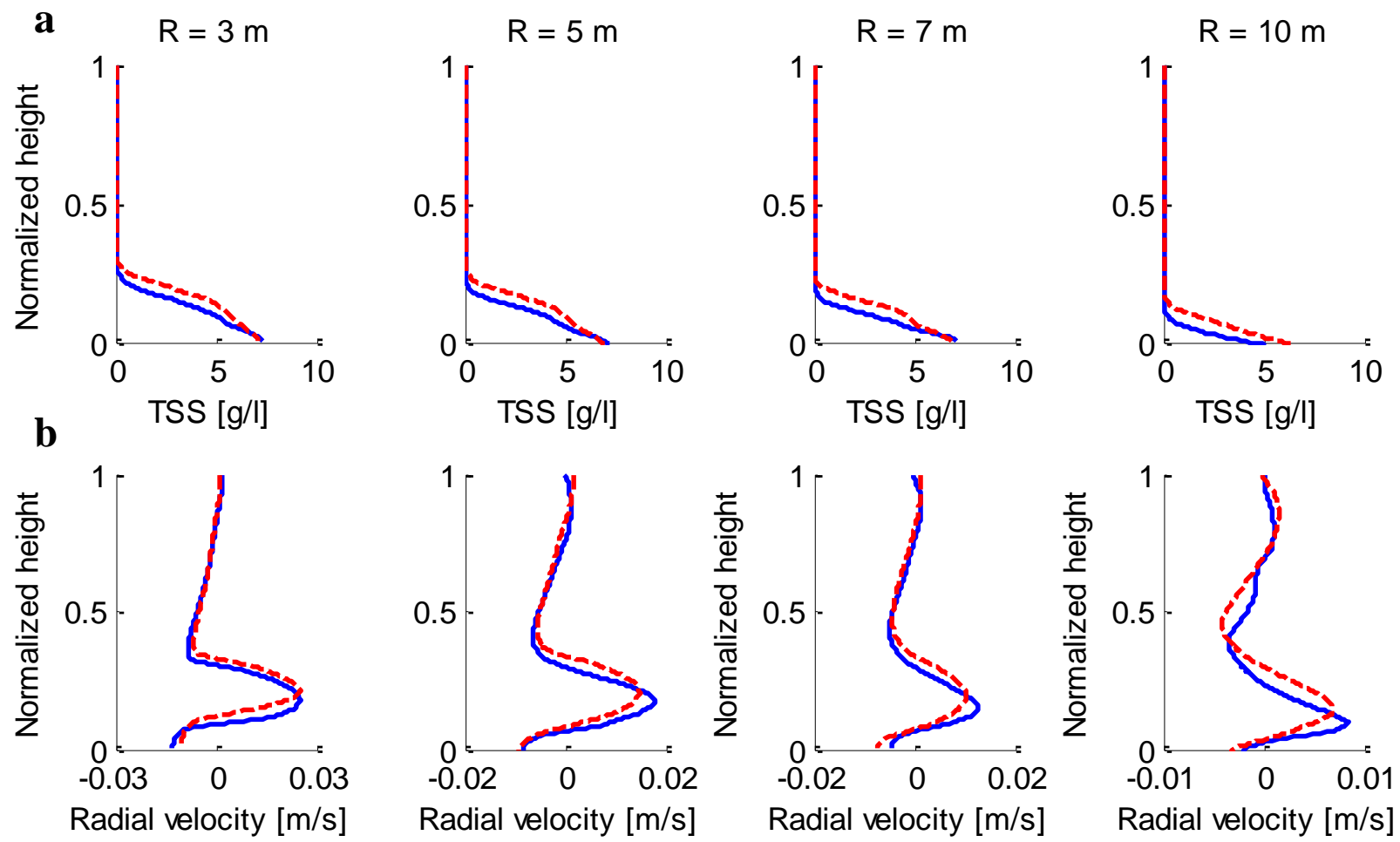

Figure 9. The predicted sludge distribution (a), and radial velocity (b) with the CFD model considering the influence of bulking (dashed line) and no bulking (solid line) on the estimated yield stress and hindered settling parameters. For more information see caption of Fig. 8.

\section{CONCLUDING REMARKS}

This study investigated whether the volume fraction of Microthrix parvicella (MPA) filamentous bacteria, as quantified with qFISH analysis, can influence the settling and rheology of activated sludge as characterized by settling and rheology measurements. The activated sludge was sampled biweekly, during a period of four months from Lundtofte WWTP. The model parameters were estimated for the settling velocity model (Eq. 2) including hindered, transient and compression settling, and rheology (Hershel-Bulkley model, Eq. 3). Results obtained suggest that the abundance of MPA - identified as a species residing predominantly inside the microbial flocs - associates with hindered settling velocity and yield stress parameters. The obtained compression and transient settling parameters show high and low variability, respectively, in the four-month period; and, the filamentous bacteria are found not to directly relate to any of these parameters. The generality of the observations made in this study may be limited by the variability of the abundance of the filamentous bacteria during the four-month period. Therefore, future research on the association of microbial structure with functional characteristics will require higher filamentous bacteria levels than those shown in this contribution. Additionally, the impact of model structure and functionality of the events on the estimation of sludge retention time in the system should be evaluated in the future. Numerical simulations were performed using a validated CFD model from our previous study with full scale profile measurements under normal operational conditions. To further improve the prediction of the filamentous bulking effect on the sludge distribution of SSTs with the CFD model, full-scale profile measurements on the SST under filamentous 
bulking conditions could possibly yield more insight into the solids mixing and transport in SSTs, and would therefore be desirable to further investigate in the future.

\section{ACKNOWLEDGMENTS}

The research was financially supported by the Danish Council for Strategic Research, Programme Commission on Sustainable Energy and Environment, as part of the Storm and Wastewater Informatics (SWI) project (http://www.swi.env.dtu.dk).

\section{REFERENCES}

APHA, A., 1995. WPCF. Standard methods for the examination of water and wastewater , 19th Ed., Washington, D.C.

Brennan, D., 2001. The Numerical Simulation of Two-Phase Flows in Settling Tanks. Ph.D. Dissertation, Imperial College, London.

Bürger, R., 2000. Phenomenological foundation and mathematical theory of sedimentationconsolidation processes. Chemical Engineering Journal 80, 177-188.

Bürger, R., Diehl, S., Nopens, I., 2011. A consistent modelling methodology for secondary settling tanks in wastewater treatment. Water Research 45 (6), 2247-2260.

De Clercq, B., 2003. Computational fluid dynamics of settling tanks: development of experiments and rheological, settling, and scraper submodels.

De Clercq, J., 2006. Batch and continuous settling of activated sludge: in-depth monitoring and 1D compression modelling. Ph.D. Dissertation, University of Gent, Belgium.

De Clercq, J., Nopens, I., Defrancq, J., Vanrolleghem, P.A., 2008. Extending and calibrating a mechanistic hindered and compression settling model for activated sludge using in-depth batch experiments. Water Research 42 (3), 781-791.

Comas, J., Rodríguez-Roda, I., Gernaey, K.V., Rosen, C., Jeppsson, U., Poch, M., 2008. Risk assessment modelling of microbiology-related solids separation problems in activated sludge systems. Environmental Modelling \& Software 23 (10-11), 1250-1261.

Daims, H., Lücker, S., Wagner, M., 2006. Daime, a Novel Image Analysis Program for Microbial Ecology and Biofilm Research. Environmental Microbiology 8 (2), 200-213.

Deininger, A., Holthausen, E., Wilderer, P.A., 1998. Velocity and solids distribution in circular secondary clarifiers: full scale measurements and numerical modelling. Water research 32 (10), 2951-2958.

Ekama, G.A., Barnard, G.L., Gunthert, F.W., Krebs, P., McCorquodale, J.A., Parker, D.S., Wahlberg, E.J., 1997. Secondary settling tanks: theory, modelling, design and operation. International Association on Water Quality, London, UK.

Grijspeerdt, K., Verstraete, W., 1997. Image analysis to estimate the settleability and concentration of activated sludge. Water Research 31 (5), 1126-1134.

Jenkins, D., Richard, M.G., Daigger, G.T., 1993. Manual on the causes and control of activated sludge bulking and foaming,, 2nd ed. Lewis Publisher, Boca Raton, Fla.

Jin, B., Wilén, B.-M., Lant, P., 2003. A comprehensive insight into floc characteristics and their impact on compressibility and settleability of activated sludge. Chemical Engineering Journal 95 (1-3), 221-234.

Kinnear, D.J., 2002. Biological solids sedimentation: a model incorporating fundamental settling parameters. PhD dissertation, University of Utah, Salt Lake City, UT.

Lakehal, D., Krebs, P., Krijgsman, J., Rodi, W., 1999. Computing shear flow and sludge blanket in secondary clarifiers. Journal of Hydraulic Engineering 125 (3), 253-262. 
Laloy, E., Vrugt, J.A., 2012. High-dimensional posterior exploration of hydrologic models using multiple-try DREAM (ZS) and high-performance computing. Water Resources Research 48 (1), W01526.

Liao, B.Q., Allen, D.G., Droppo, I.G., Leppard, G.G., Liss, S.N., 2000. Bound Water Content of Activated Sludge and Its Relationship to Solids Retention Time, Floc Structure, and Surface Properties. Water Environment Research 72 (6), 722-730.

Mielczarek, A.T., Kragelund, C., Eriksen, P.S., Nielsen, H.P., 2012. Population dynamics of filamentous bacteria in Danish wastewater treatment plants with nutrient removal. Water Research 46 (12), 3781-3795.

Nielsen, P.H., Daims, H., Lemmer, H., 2009. FISH Handbook for Biological Wastewater Treatment. Identification and quantification of microorganisms in activated sludge and biofilms by FISH. IWA Publishing, London, UK.

Plósz, B.G., Weiss, M., Printemps, C., Essemiani, K., Meinhold, J., 2007. One-dimensional modelling of the secondary clarifier-factors affecting simulation in the clarification zone and the assessment of the thickening flow dependence. Water Research 41 (15), 3359-3371.

Ramin, E., Wágner, D.S., Yde, L., Binning, P.J., Rasmussen, M.R. Mikkelsen, P.S., Plósz, B.G., 2014. A new settling and rheological model for secondary settling tank modelling. Submitted to Environmental Science \& Technology.

Ratkovich, N., Horn, W., Helmus, F.P., Rosenberger, S., Naessens, W., Nopens, I., Bentzen, T.R., 2013. Activated sludge rheology: a critical review on data collection and modelling. Water Research 47 (2), 463-482.

Takács, I., Patry, G., Nolasco, D., 1991. A dynamic model of the clarification-thickening process. Water Research 25 (10), 1263-1271.

Wágner, D.S., Ramin, E., Dechesne, A., Smets, B.F., Szabo, P., Plósz, B.G., 2014. Impact of filamentous bacteria on the settling and rheology of activated sludge (In preparation).

Wanner, J., 1994. Activated sludge bulking and foaming control. CSC Press.

Weiss, M., Plósz, B.G., Essemiani, K., Meinhold, J., 2007. Suction-lift sludge removal and nonNewtonian flow behaviour in circular secondary clarifiers: Numerical modelling and measurements. Chemical Engineering Journal 132 (1-3), 241-255.

Wilén, B.-M., Lumley, D., Mattsson, A., Mino, T., 2008. Relationship between floc composition and flocculation and settling properties studied at a full scale activated sludge plant. Water Research 42 (16), 4404-18.

Wilén, B.-M., Lumley, D., Mattsson, A., Mino, T., 2010. Dynamics in Flocculation and Settling Properties Studied at a Full-Scale Activated Sludge Plant. Water Environment Research 82 (2), $155-168$. 


\title{
Model-based evaluation of mechanisms and benefits of mainstream shortcut nitrogen removal processes
}

\author{
Ahmed Al-Omari ${ }^{1,2}$, Bernhard Wett ${ }^{3}$, Ingmar Nopens ${ }^{1}$, Haydee De Clippeleir ${ }^{4}$, Mofei Han ${ }^{5}$, Pusker \\ Regmi $^{6}$, Charles Bott ${ }^{7}$, Sudhir Murthy ${ }^{2}$. \\ ${ }^{1}$ Department of Mathematical Modelling, Statistics and Bio-informatics, Coupure Links 653, 9000, \\ Ghent Univ, Ghent, Belgium \\ ${ }^{2}$ DC Water and Sewer Authority, 20032 Washington DC, USA \\ ${ }^{3}$ ARAconsult, Unterbergerstr.1, A-6020 Innsbruck, Austria \\ ${ }^{4}$ Department of Earth and Environmental Engineering, Columbia University, New York \\ ${ }^{5}$ Laboratory of Microbial Ecology and Technology (LabMET), Ghent University, Gent, Belgium \\ ${ }^{6}$ Civil and Environment Engineering Department, Old Dominion University, Norfolk, VA 23529 \\ ${ }^{7}$ Hampton Roads Sanitation District, Virginia Beach, VA, USA
}

\begin{abstract}
For implementing short cut nitrogen removal processes for mainstream wastewater treatment, out-selection of nitrite oxidizing bacteria (NOB) to limit nitrate production is the main challenge. A model-based approach was utilized to simulate the impact of individual features of process control strategies to achieve the $\mathrm{NO}_{2}^{-}-\mathrm{N}$ shunt via NOB out-selection. Simulations were conducted using a two step nitrogen removal model from the literature. Nitrogen shortcut removal processes from two case studies were modelled to illustrate the contribution of NOB out-selection mechanisms. The paper highlights a comparison between two control schemes, i.e. ammonia-based control and the novel AVN [AOB Vs. NOB] control, recently described in the literature. Results indicate that the AVN controller possesses unique features that promote a better management of incoming organics and bicarbonate, which optimizes both $\mathrm{NH}_{4}^{+}-\mathrm{N}$ removal rates as well as $\mathrm{NO}_{2}^{-}-\mathrm{N} \& \mathrm{NO}_{3}^{-}-\mathrm{N}$ removal. Moreover, it allows for a more efficient NOB out-selection. Finally, the model was used in a scenario analysis, simulating hypothetical optimized performance of the pilot process. An estimated potential saving of $60 \%$ in carbon addition for nitrogen removal by implementing full-scale mainstream deammonification was found.
\end{abstract}

\section{Keywords}

Anammox, AOB seeding, mainstream deammonification, $\mathrm{NO}_{2}{ }^{-} \mathrm{N}$ shunt, $\mathrm{NOB}$ outselection, online control, transient anoxia

\section{INTRODUCTION}

Traditional nitrogen removal processes are high energy consumers and not cost-effective which is a serious downside of currently used technology where cost and energy reduction are pursued. Shortcut nitrogen removal processes provide a superior alternative to conventional processes used in municipal wastewater treatment (i.e. nitrification-denitrification) since they significantly reduce oxygen demand and external carbon requirements (Vlaeminck et al., 2012). These processes involve the creation of unique conditions to steer the biological conversion of oxidizable nitrogen (i.e. $\mathrm{NH}_{4}{ }^{+} \mathrm{N}$ and organic nitrogen) to nitrogen gas by taking a 2-step pathway shortcut and thus conserve energy. There are two main shortcut nitrogen removal processes i.e. nitritation/denitritation and deammonification via anammox. In both processes, the first step of the nitrogen shortcut pathway is converting $\mathrm{NH}_{4}{ }^{+} \mathrm{N}$ via ammonia oxidizing bacteria (AOB) to $\mathrm{NO}_{2}^{-}-\mathrm{N}$ only. This requires repressing the NOB population to avoid producing $\mathrm{NO}_{3}{ }^{-}-\mathrm{N}$. The second step can either be converting $\mathrm{NO}_{2}{ }^{-}-\mathrm{N}$ to $\mathrm{N}_{2}$ gas via heterotrophic bacteria using organic carbon or via anammox bacteria without the need for organic carbon. These processes have already been implemented and controled successfully for sidestream (i.e. nitrogen rich warm streams) treatment and the operational savings were 
reported in the literature (Wett, 2007). In contrast, the potential operational savings using shortcut nitrogen removal processes can be significantly higher for mainstream applications with $40 \%$ savings for nitritation/denitritation process and $84 \%$ saving for the deammonificaiton process compared to conventional nitrification/denitrification (De Clippeleir, 2012). In addition, potential savings in capital costs for new systems and increase in capacity for converted systems are anticipated. The savings are associated with low sludge production of $0.6 \mathrm{~kg} / \mathrm{kgN}$ for nitritation/denitritation and $0.1 \mathrm{~kg} / \mathrm{kgN}$ for deammonification compared to $1 \mathrm{~kg} / \mathrm{kgN}$ for conventional nitrification/denitrification (De Clippeleir, 2012). These potential benefits however come with challenges associated with NOB outselection under cold and diluted loading conditions. While the operational strategies are well documented for sidestream applications (Gut et al., 2005; Hellinga et al., 1998; van de Graaf et al., 1996; van Loosdrecht and Salem, 2005; Wett et al., 2007), many recent studies were conducted to address these challenges for mainstream applications using nitritation/denitritation (Blackburne et al., 2008; Gao et al., 2009, 2014; Peng et al., 2004, 2012; Regmi et al., 2013) and deammonification (Al-Omari et al., 2012; Hu et al., 2013; Lotti et al., 2013; Wett et al., 2013; Winkler et al., 2011) or both (Vlaeminck et al., 2012; Stinson et al., 2013). Several mechanisms were identified by which the three autotrophic groups, i.e. AOB, NOB \& anammox, compete. These mechanisms include (1) operating at low dissolved oxygen (DO) concentration, (2) operating at high DO concentration, (3) operating with high residual ammonia, (4) transient anoxia where the process reactor is intermittently aerated either by turning the air flowrate on and off or by creating a spatial sequence of anoxic and oxic zones in the reactor. The use of transient anoxia is a commonly used approach for NOB out-selection (Li et al., 2012, Ling, 2009, Pollice et al., 2002, Rosenwinkel et al., 2005, Zekker et al., 2012). In the context of controlling the nitrogen process towards out-selection of NOB, transient anoxia provides means to control the aerobic SRT, as well as to introduce a lag-time for NOB to transition from the anoxic to aerobic environment, either due to $\mathrm{NO}_{2}{ }^{-} \mathrm{N}$ limitation (Knowles et al., 1966; Chandran and Smets, 2000) or by an enzymatic lag (Kornaros and Dokianakis, 2010). Aggressive SRT (i.e. near minimum SRT) is applied based on target removal rates and is controlled via manipulating wasting rates, aerobic volumes and DO setpoints to maximize NOB outselection potential (Regmi et al., 2013; Wett et., 2013). For this study two case studies were selected for both shortcut processes using the HRSD pilot for nitritation/denitritation (Regmi et al., 2014) and the DC Water pilot for mainstream deammonification (Al-Omari et al., 2012). Figure 1 illustrates, using Monod functions of utilization rates as a function of $\mathrm{DO}, \mathrm{NH}_{4}{ }^{+}-\mathrm{N}$ and $\mathrm{NO}_{2} \mathrm{~N}$, some of the concepts used in these two case studies to maximize the rate differential between AOB and NOB to facilitate NOB out-selection.
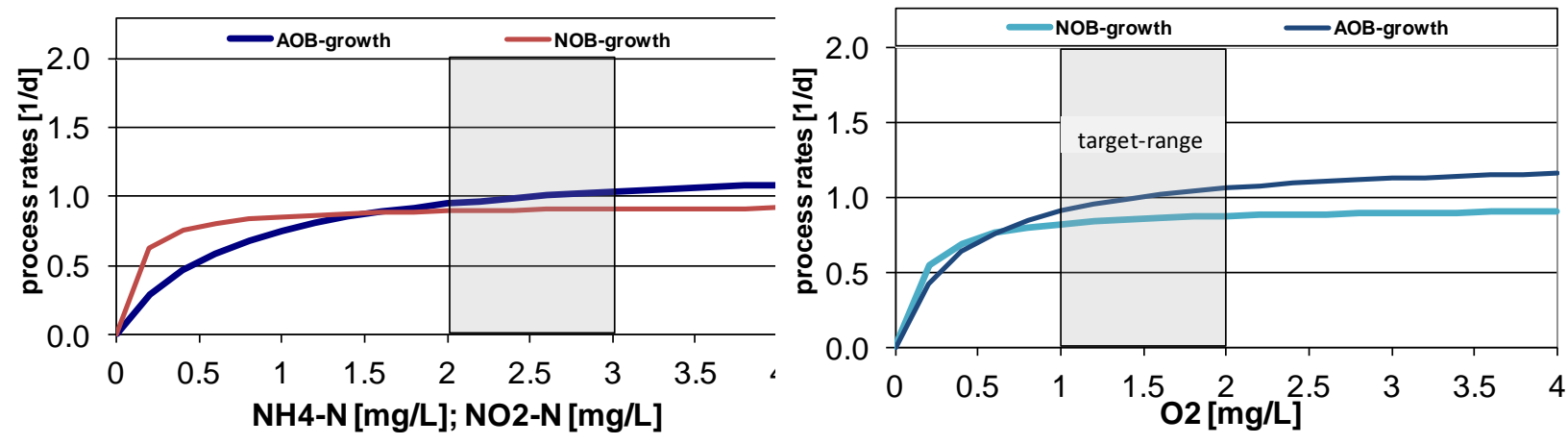

Figure 1. Monod functions describing rate and substrate affinities and competition under pilot target operational conditions for $\mathrm{NH}_{4}{ }^{+}-\mathrm{N}, \mathrm{NO}_{2}{ }^{-}-\mathrm{N}$ and DO. Target ranges are those where AOB rates are higher than NOB rates 


\section{MATERIALS AND METHODS}

\section{Long term experimental pilot tests HRSD Pilot Study - AVN Controller}

This pilot process is part of a larger configuration including a high rate activated sludge Astage for COD removal providing the influent for the AVN controlled reactor (Miller et al., 2012) and a post-anoxic anammox moving bed bioreactor after the AVN controlled reactor allowing for a final polishing of the treated sewage. The reader is referred to Regmi et al. (2014) for detailed description of the pilot setup and operation. The AVN controlled process includes a single $340 \mathrm{~L}$ aeration tank operated as a continuously stirred tank reactor (CSTR) followed by a clarifier. Return activated sludge (RAS) from the clarifier is returned to the AVN reactor at $100 \%$ of the influent flow rate. SRT is controlled by wasting solids from the bioreactor with a programmable digital peristaltic pump. The reactor is equipped with sensors for $\mathrm{NO}_{3}{ }^{-}-\mathrm{N}, \mathrm{NO}_{2}{ }^{-}-\mathrm{N}$ (s::can Spectro::lyser, Austria), DO (Hach LDO, CO, USA), and $\mathrm{NH}_{4}{ }^{+}-\mathrm{N}$ (WTW VARiON, Germany). $\mathrm{NH}_{4}{ }^{+}-\mathrm{N}, \mathrm{NO}_{3}{ }^{-} \mathrm{N}, \mathrm{NO}_{2}{ }^{-}-\mathrm{N}$ signals are used to control aeration (Figure 2).

To impose conditions favorable for NOB out-selection and to provide an effluent suitable for anaerobic ammonia oxidation (AMX) polishing, an aeration controller was developed which uses online in-situ DO, $\mathrm{NH}_{4}{ }^{+}, \mathrm{NO}_{2}^{-}$and $\mathrm{NO}_{3}{ }^{-}$sensors. The first component of the AVN control is the aerobic duration controller with the goal of maintaining equal effluent $\mathrm{NH} 4+-\mathrm{N}$ and NOx-N (i.e. $\mathrm{NH}_{4}{ }^{+}-\mathrm{N} / \mathrm{NO}_{\mathrm{x}}-\mathrm{N}=1$ ) in the AVN CSTR at all times. The latter would guarantee a treatable effluent for the final polishing step with AMX. The other component of the AVN control is the DO controller, which maintains the DO at a desired set-point during the aerated period (Figure 2).

Under the AVN strategy, $\mathrm{NH}_{4}{ }^{+}-\mathrm{N}$ was compared to the sum of $\mathrm{NO}_{2}{ }^{-}-\mathrm{N}$ and $\mathrm{NO}_{3}{ }^{-}-\mathrm{N}^{-}\left(\mathrm{NO}_{\mathrm{x}}-\mathrm{N}\right)$. Firstly, the cycle duration (aerobic duration + anoxic duration) had a defined minimum and maximum aerobic duration. The cycle duration was kept constant at 12 minutes during the entire experiment. As the AVN controller aims at ammonium concentrations equal to $\mathrm{NO}_{\mathrm{x}}$, aerobic duration is increased up to a predetermined maximum aeration time set-point, while maintaining the cycle duration constant at $\mathrm{NH}_{4}{ }^{+}-\mathrm{N}$ over $\mathrm{NO}_{\mathrm{x}}-\mathrm{N}$ ratios greater than 1 . When $\mathrm{NH}_{4}{ }^{+}-\mathrm{N}$ was less than $\mathrm{NO}_{\mathrm{x}}-\mathrm{N}$, aerobic duration was decreased until it reached the minimum aeration time. When aerated, the proportional-integral-derivative (PID) controller steered a mechanically operated valve (MOV) to maintain the target DO set-point of $1.6 \mathrm{mg} / \mathrm{L}$. Figure 3 shows the effluent quality of the pilot reactor under AVN control in terms of the nitrogen species measured in daily grab samples. The chart demonstrates the balance between ammonia and NOx as a result of the AVN control strategy.

\section{Water Case Study - Ammonia based control}

The objective of the pilot process at DC Water is to evaluate the mainstream deammonification feasibility at the Blue Plains Advanced Wastewater Treatment Plant (AWTP) with an emphasis on process controls. This process appears specifically appropriate for the existing process configuration with high up-stream carbon removal (primaries followed by a high-rate process achieving $85 \%$ overall efficiency) and chemical carbon 
dosing downstream to meet the extremely low total nitrogen limit of $3.9 \mathrm{mgN} / \mathrm{L}$. The deammonification pilot consists of a 200L activated sludge tank divided into 10 sequential aerobic and anoxic zones (D1 - D10) with dissolved oxygen and ammonia based controls. Oxygen level is maintained at $1.5 \mathrm{mg} / \mathrm{L}$ in the aerated cells where oxygen is measured using an LDO sensor (HACH, Düsseldorf, Germany). $\mathrm{NH}_{4}{ }^{+}-\mathrm{N}$ is measured using an NH4D sc ammonium sensor (HACH, Düsseldorf, Germany). $\mathrm{NH}_{4}{ }^{+}-\mathrm{N}$ concentration in the
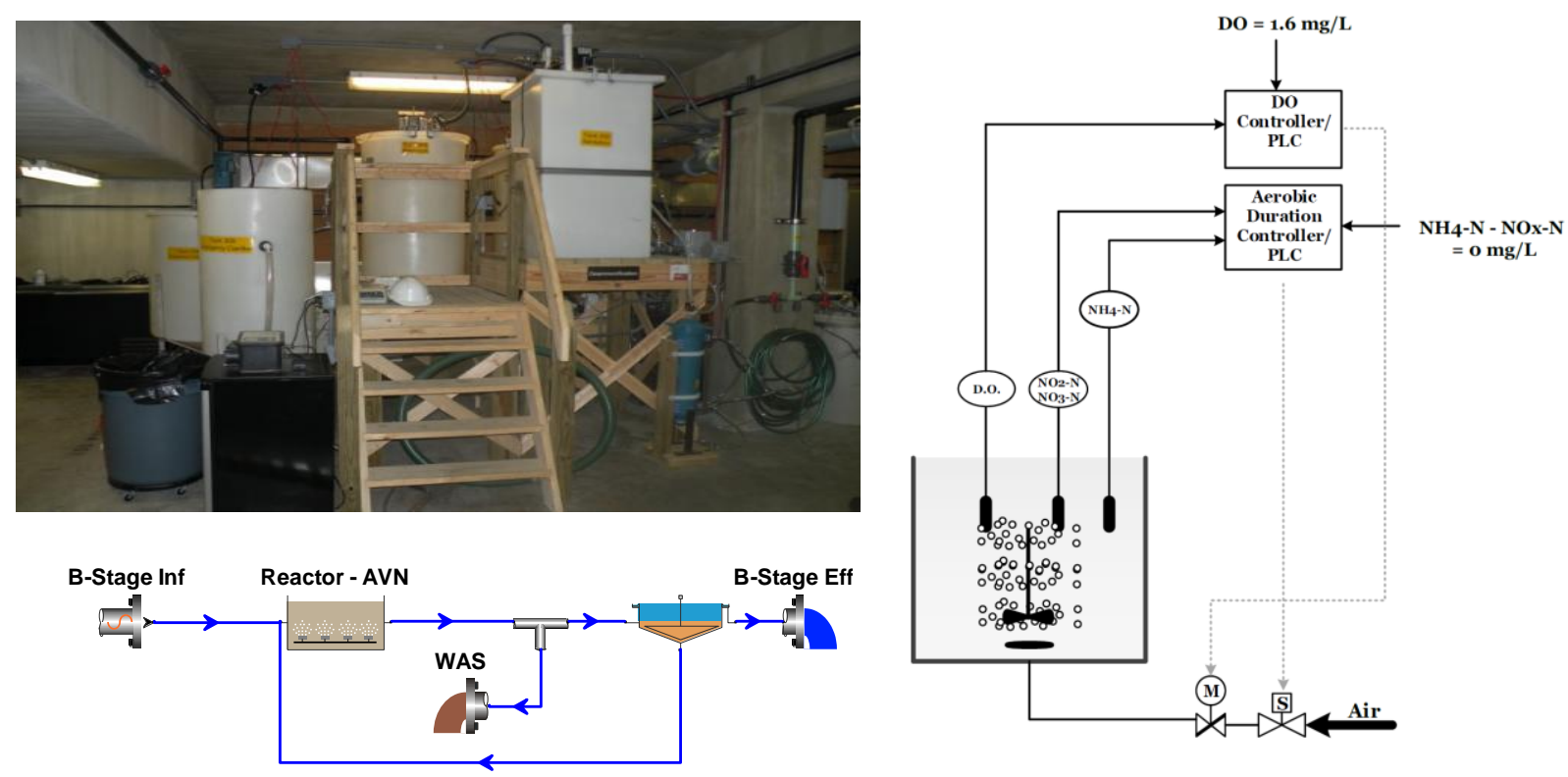

Figure 2. Photo of the HRSD nitrogen removal pilot, Virginia [left-top], and pilot model configuration [left-bottom], schematic diagram of AVN process control elements [right].

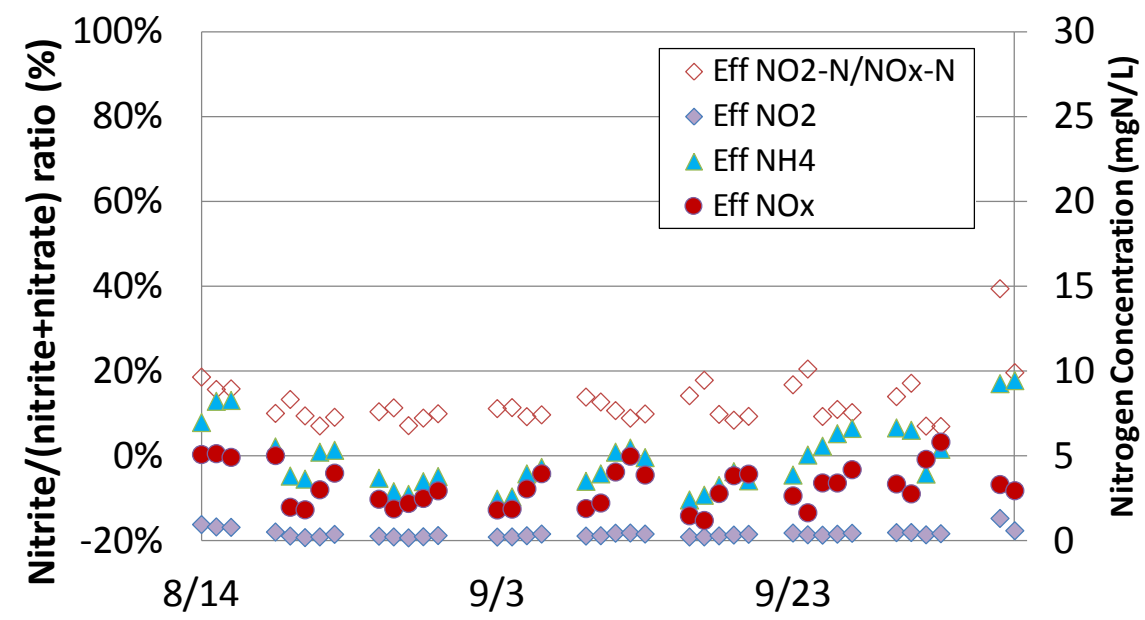

Figure 3. HRSD pilot reactor effluent quality when applying AVN control

last cell (D10) is maintained above $2 \mathrm{mg} / \mathrm{L}$. Data acquisition and control is performed using Labview (National Instruments, USA). The aerobic SRT is modified by turning downstream swing zones into aerobic or anoxic zones and by adjusting wasting rates as needed to maintain target $\mathrm{NH}_{4}{ }^{+}-\mathrm{N}$ concentration in cell D10. During the period selected for the simulation runs 
the aerobic SRT was adjusted by modifying the wasting rates. Anammox seed is added to the reactor on a daily basis using sludge from a full-scale sidestream DEMON reactor and AOBs are seeded using the waste sludge from a bench-scale sidestream reactor that is proportional to the full-scale sidestream DEMON facility. Anammox is selectively retained in the system using sieves with mesh size No.70 and No.120 (or 212 micron and 125 micron). Downstream of the deammonification cells a polishing step is present which consists of 8 anoxic zones (P1 - P8). The post anoxic step is not discussed in this paper. Figure 4 shows the pilot reactor and the model configuration of the pilot.

Figure 5 shows the effluent quality of the pilot reactor under ammonia based control. One interesting observation from the chart is that $\mathrm{NO}_{2}{ }^{-} \mathrm{N}$ accumulation was observed when $\mathrm{NH}_{4}{ }^{+}-$ $\mathrm{N}$ and $\mathrm{NO}_{3}{ }^{-} \mathrm{N}$ (or NOx) were in equilibrium. The figure also shows a profile across the reactor from cell D1 until $\mathrm{P} 8 . \mathrm{NO}_{2}^{-}-\mathrm{N}$ is produced in the aerobic cells in the deammonification reactor (D2, D5, D8 \& D10). However, NOB out-selection is not effective as observed from the relative $\mathrm{NO}_{3}{ }^{-}-\mathrm{N}$ produced compared to $\mathrm{NH}_{4}{ }^{+}-\mathrm{N}$ removed $(>85 \%)$.
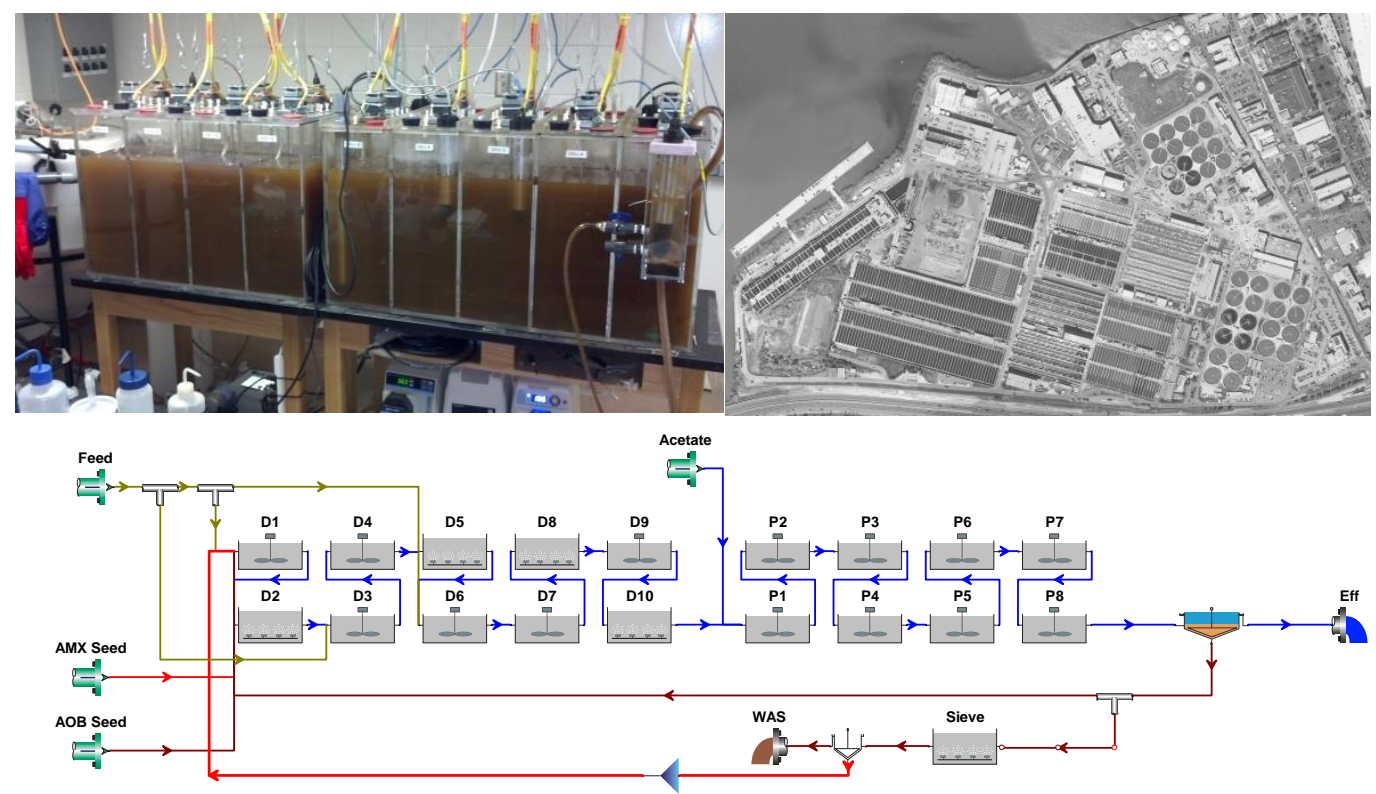

Figure 4. Aerial view of Blue Plains AWTP, Washington DC [right], pilot-scale mainstream deammonification reactor [left], and pilot model configuration [bottom].
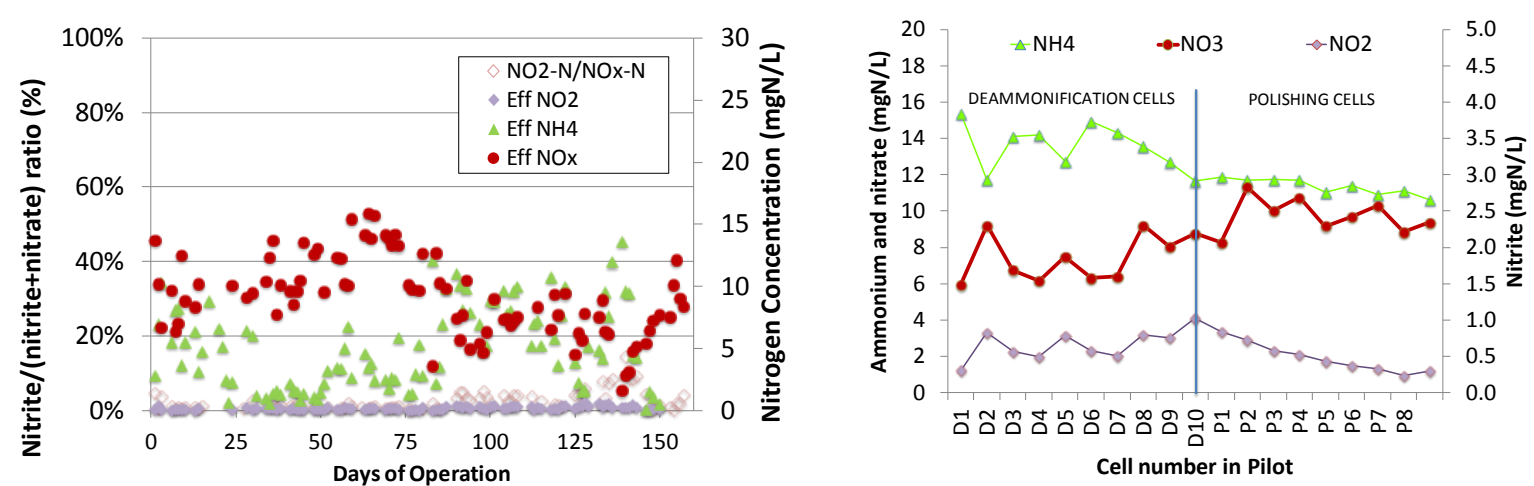

Figure 5. DC Water pilot reactor effluent quality [left] and profile [right] 


\section{Case study pilot influent characteristics}

Average influent characteristics for the pilot reactors are given in Table 1. One major difference is the carbon to nitrogen ratios which is much higher in the HRSD pilot reactor. Another difference is the aerobic fraction of the reactor volume.

Table 1. Case studies pilots reactors and average influent characteristics

\begin{tabular}{lcc}
\hline Parameter & $\begin{array}{c}\text { HRSD } \\
\text { AVN Control }\end{array}$ & $\begin{array}{c}\text { DC Water } \\
\text { Ammonia Control }\end{array}$ \\
\hline Flow, L/d & 2,722 & 1,840 \\
Total COD, mgCOD/L & 303.0 & 40.3 \\
Total Kjeldahl Nitrogen, mgN/L & 39.0 & 24.5 \\
$\mathrm{NH}_{4}{ }^{-}, \mathrm{mgN} / \mathrm{L}$ & 29.3 & 21.7 \\
$\mathrm{NO}_{3}{ }^{-}, \mathrm{mgN} / \mathrm{L}$ & 0.0 & 0.5 \\
$\mathrm{NO}_{2}{ }^{-}, \mathrm{mgN} / \mathrm{L}$ & 0.0 & 0.2 \\
Total P, mgP/L & 4.7 & 1.1 \\
Alkalinity, mmol/L & 3.6 & 4.5 \\
Reactor Type & CSTR & Step Feed-Plug Flow \\
Transient Anoxia & Time & Space \\
Total SRT (AVG), day & 6.5 & 8.7 \\
aerobic fraction (AVG), \% & 64 & 22 \\
\hline
\end{tabular}

\section{Modelling approach}

The model used for the simulations is based on the two step nitrogen model proposed by Jones et al. (2007) (Table 2). The oxygen half saturation concentrations for AOB, NOB and anammox were modified using the calibrated values reported by Al-Omari et al. (2012), which were based on actual measurements using SBR reactors.

Table 2. Autotrophic Biomass Model Parameters (Default Parameters - Jones et al., 2007).

\begin{tabular}{lccc} 
Parameter & AOB & NOB & Anammox \\
\hline Max. spec. growth rate [1/d] & $(0.9)$ & $(0.7)$ & $(0.1)$ \\
Arrhenius on max. spec. growth rate & $(1.072)$ & $(1.06)$ & $(1.1)$ \\
Substrate (NH4) half sat. [mgN/L] & $(0.7)$ & - & $(2)$ \\
Substrate (NO2) half sat. [mgN/L] & - & $(0.05)$ & $(1)$ \\
Aerobic decay rate [1/d] & $(0.17)$ & $(0.17)$ & $(0.019)$ \\
Anoxic/anaerobic decay rate [1/d] & $(0.08)$ & $(0.08)$ & $(0.0095)$ \\
Nitrous acid inhibition constant [mmol/L] & $(0.005)$ & $(0.075)$ & - \\
$\mathrm{NO}_{2}^{-}-\mathrm{N}$ inhibition constant [mgN/L] & - & - & $(1000)$ \\
$\mathrm{NO}_{2}^{-}-\mathrm{N}$ toxicity constant [L/(d mgN)] & & & $(0.016)$ \\
DO half sat. [mgO2/L] & $\mathbf{0 . 4}(0.25)$ & $\mathbf{0 . 1 4}(0.5)$ & $\mathbf{0 . 0 5}(0.01)$ \\
bicarbonate switch [mmol/L] & $\mathbf{0 . 7 5}(0.1)$ & $(0.1)$ & $(0.1)$ \\
Yield [mgCOD/mgN] & $(0.15)$ & $(0.09)$ & $(0.114)$ \\
\hline
\end{tabular}

Notes: values in brackets are default values. Values in bold are modified values. 
The default inorganic carbon half saturation concentration for all autotrophic biomass reported in the Jones model was set at $0.1 \mathrm{mmol} / \mathrm{L}$ while for sidestream application the recommended parameter was $4 \mathrm{mmol} / \mathrm{L}$ for $\mathrm{AOB}$ in line with recommendations by Wett et al. (2005). The reasoning is to eliminate the impact of inorganic carbon at typical residual concentrations observed in nitrogen removal systems. However, in bench-scale shallow reactors, $\mathrm{CO}_{2}$ stripping is much greater than full-scale reactors and may become limiting to autotrophic bacteria (Wett et al., 2003). In addition, Guisasola et al. (2007) suggested that AOB were limited by inorganic carbon availability at concentrations as low as $3 \mathrm{mmol} / \mathrm{L}$ while the NOB were not limited even at concentrations below $0.1 \mathrm{mmol} / \mathrm{L}$. In this modelling exercise, a value of $0.75 \mathrm{mmol} / \mathrm{L}$ was used based on a calibration using ammonia removal profiles in the DC Water deammonification reactor (not shown). It was observed that the removals were lower at these conditions than expected by the model using the default inorganic carbon half saturation concentration (i.e. $0.1 \mathrm{mmol} / \mathrm{L}$ ). To model the different impact of inorganic carbon on $\mathrm{AOB}$ and $\mathrm{NOB}$, new equations describing the growth and decay of $\mathrm{AOB}$ were introduced to the model in a Gujer matrix format. A user defined parameter was used to represent $\mathrm{AOB}$ biomass while the growth rate of $\mathrm{AOB}$ (Xaob) in the global model was set to zero. Table 3 presents the modified AOB growth and decay rate equations and stoichiometric expressions.

\section{RESULTS AND DISCUSSION}

\section{AVN vs. ammonia based control - Modelling}

A simulation of the HRSD pilot reactor operation using average loading conditions to reach steady state was used as the starting point for any dynamic simulation. The model was able to predict average $\mathrm{NH}_{4}{ }^{+}-\mathrm{N}, \mathrm{NO}_{3}{ }^{-} \mathrm{N}$ and $\mathrm{NO}_{2}{ }^{-} \mathrm{N}$. To allow controller performance comparison, the HRSD simulation of the pilot was operated with both strategies (i.e. AVN vs Ammoniabased control) side by side. Lacking an advanced controller simulator that is able to mimic the AVN control strategy for dynamic input, the reactor influent flowrate was modified to reflect a step change in influent mass loading [kg/d] by $+25 \%$ of the average loading rate for 12 hours and $-25 \%$ of the mass loading rate for the following 12 hours. In an AVN control mode, the aeration time was manually adjusted as the loading changed so that the balance between $\mathrm{NH}_{4}{ }^{+}-\mathrm{N}$ and $\mathrm{NOx}-\mathrm{N}$ was maintained. The aeration time was reduced by 0.49 min during the low loading step and increased by 0.65 min during the high loading step. Under the ammoniabased control, the aeration time was manually adjusted as the load changes so that effluent $\mathrm{NH}_{4}{ }^{+}-\mathrm{N}$ concentration was maintained constant. The aeration time was reduced by $1.63 \mathrm{~min}$ during the low loading step and increased by 3.25 min during the high loading step. Figure 6 shows the simulation output of the AVN and ammonia control modes.

Comparing the two simulation outputs reveals that nitrogen removal efficiency was increased by approximately $17.5 \%$ under the AVN control. Also, the stable alkalinity level in the reactor for the AVN simulation compared to that for the ammonia-based control is noteworthy. It is important to realize that the controller under the AVN strategy controls the $\mathrm{NH}_{4}{ }^{+}-\mathrm{N}$ removal rate based on denitrification capacity. I.e. the aerobic SRT is adjusted so that $\mathrm{NH}_{4}{ }^{+}-\mathrm{N}$ is nitrified only if the same amount of nitrogen can be removed via denitrification. This balancing action allows for more efficient use of the biodegradable carbon for nitrogen removal, recovery of alkalinity, and applying SRT pressure on NOB. In the ammonia-based control simulation, aeration is increased during high loading conditions to maintain the effluent $\mathrm{NH}_{4}{ }^{+}-\mathrm{N}$ level. By increasing aeration time, more COD is 


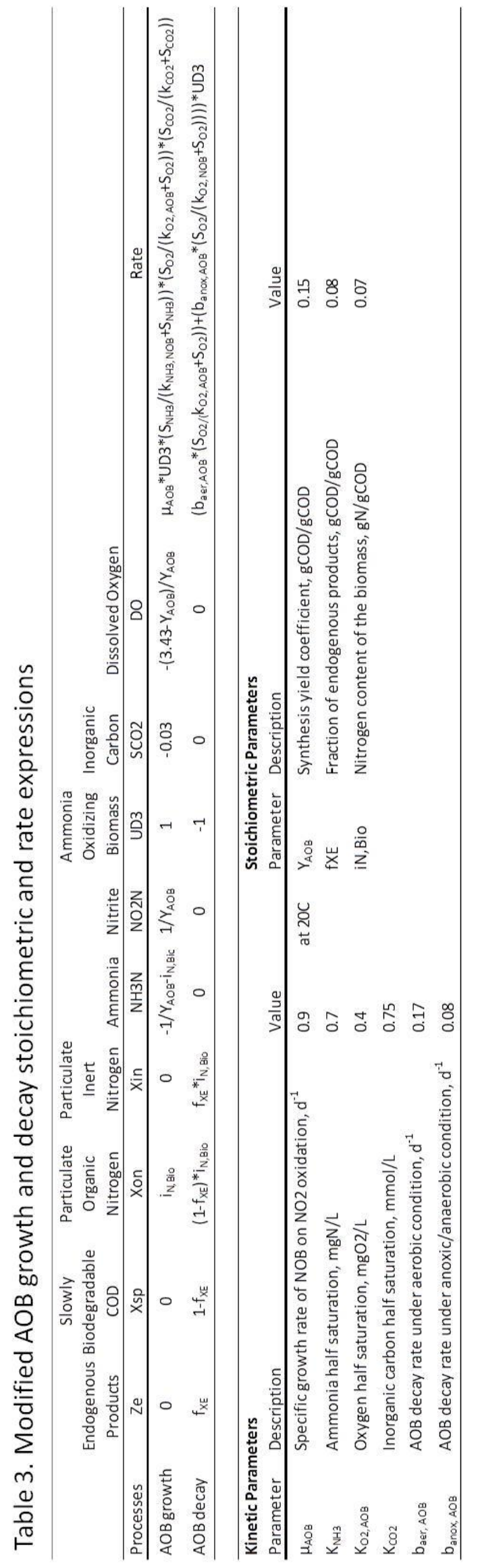


aerobically oxidized and more alkalinity is consumed. As alkalinity is consumed, $\mathrm{NH}_{4}{ }^{+}-\mathrm{N}$ oxidation rates slowdown due to inorganic carbon limitation. In return, the controller increases the aeration time even further, which again will cause further COD oxidation and alkalinity suppression. This continues until the $\mathrm{NH}_{4}{ }^{+}-\mathrm{N}$ concentration cannot be reduced any further. Table 4 summarises the comparison between AVN and ammonia-based controls with regard to nitrogen removal, oxygen demand and NOB levels. The comparison reveals that an 8.6\% reduction in NOB concentration was achieved. This incremental reduction can be significant when combined with other incremental reductions due to other mechanisms such as AOB seed, which is discussed in the following section.

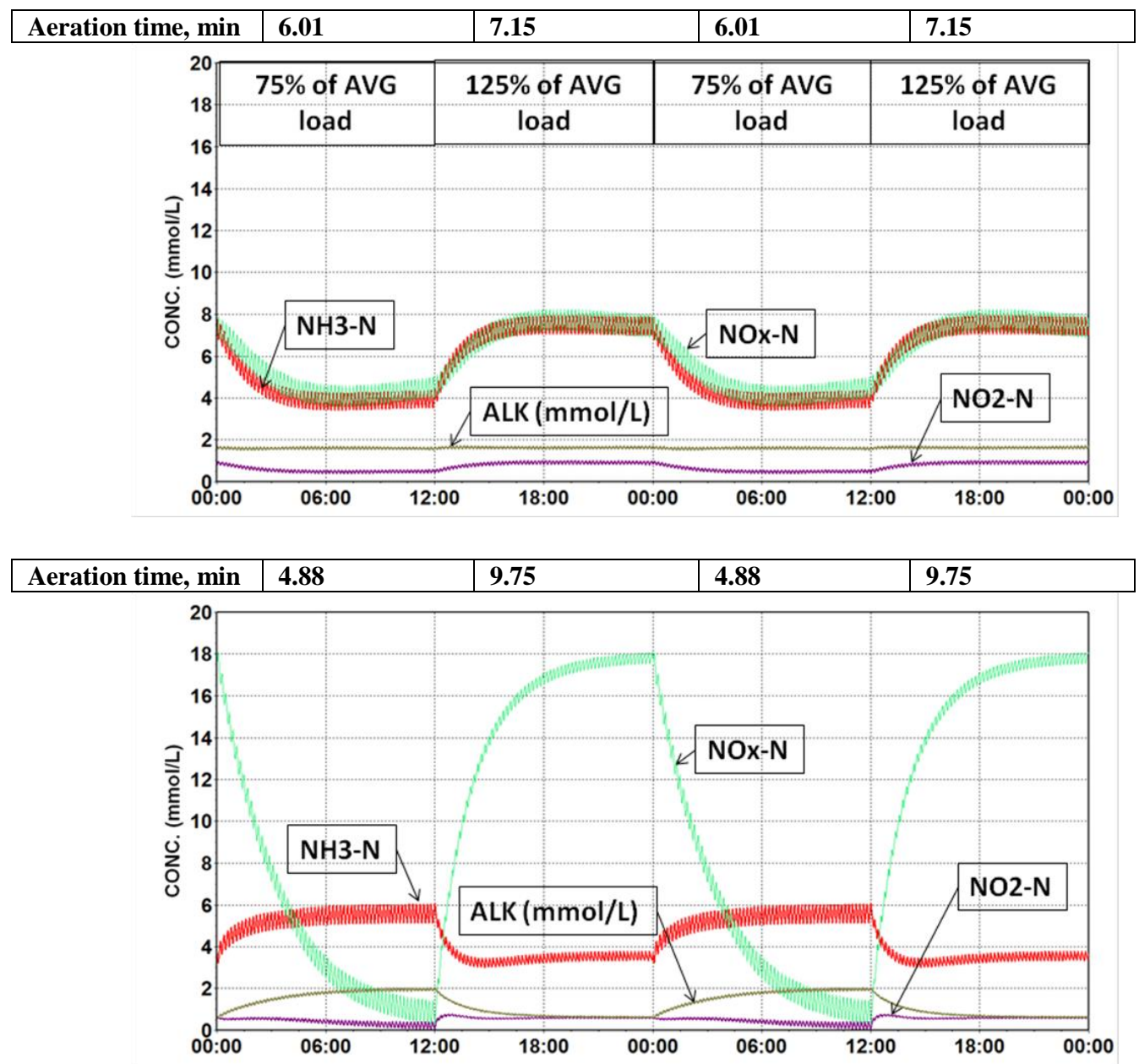

Figure 6. Simulation output for AVN (top) and ammonia based (bottom) controls for HRSD pilot reactor with 12 min cycle.

\section{Impact of $A O B$ seeding and SRT}

Seeding AOB from a sidestream process that utilizes a shortcut nitrogen removal process can be beneficial to enhance NOB repression in mainstream processes with the aim of achieving the $\mathrm{NO}_{2}{ }^{-}-\mathrm{N}$ shunt. Figure 7 illustrates the concept of seeding a tank with AOB rich waste from a sidestream process where the difference in critical SRT between AOB and NOB increases with the AOB seed mass introduced to the mainstream tank. 
Al-Omari et al.

Table 4. Comparison between AVN and ammonia based control strategies in terms of total nitrogen removal, oxygen demand and NOB supression for simulated HRSD pilot reactor.

\begin{tabular}{|c|c|c|c|}
\hline & $\begin{array}{c}\text { (1) } \\
\text { AVN } \\
\text { Control }\end{array}$ & $\begin{array}{c}(2) \\
\text { Ammonia } \\
\text { Control } \\
\end{array}$ & $\begin{array}{c}\% \\
\text { Change } \\
{[(1-2) / 2]}\end{array}$ \\
\hline Total Nitrogen Removal, (mgN/L) & 28.9 & 24.6 & $17.5 \%$ \\
\hline Oxygen consumed, (mg/L) & 936 & 1024 & $-8.6 \%$ \\
\hline NOB Concentration, (mg/L) & 39.1 & 42.8 & $-8.6 \%$ \\
\hline
\end{tabular}

It is assumed that $20 \%$ of the influent load is recycled back and treated in the sidestream process. Simulations of the HRSD pilot reactor with (1) $50 \%$ seeding activity assuming that $50 \%$ of activity is lost due to the difference in temperature between sidestream and mainstream processes (Wett et al., 2011) and (2) 100\% seeding activity assuming no loss of activity were examined. The system SRT was reduced to maintain the same $\mathrm{NH}_{4}{ }^{+}-\mathrm{N}$ removal rate in the system. Figure 8 shows the simulation output for AOB and NOB under both seeding conditions. The simulation showed that the gap between AOBs and NOBs widened with increased $\mathrm{AOB}$ seeding rate as evident by the $\mathrm{AOB} / \mathrm{NOB}$ ratios.

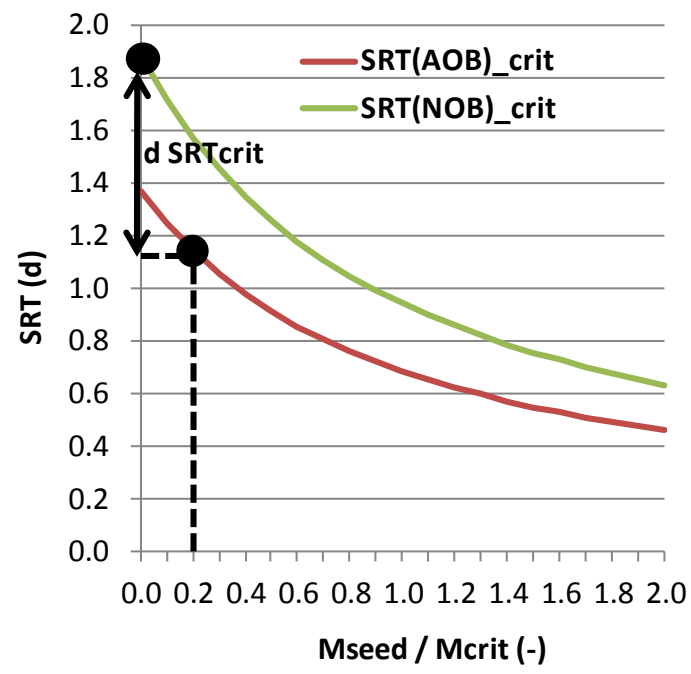

$$
S R T_{\text {critical }}=\frac{1}{(\mu m-b)} *\left(\frac{M_{\text {critical }}}{M_{\text {seed }}+M_{\text {critical }}}\right)
$$

Assumptions:

$\mathrm{SRT}_{\text {Side }}=\mathrm{SRT}$ side-stream $=10$ days

AOB maximum specific growth rate $(\mu \mathrm{m}, \mathrm{AOB})=0.9 \mathrm{~d}^{-1}$

NOB maximum specific growth rate $(\mu \mathrm{m}, \mathrm{NOB})=0.7 \mathrm{~d}^{-1}$

Decay rate $(b)=0.17 \mathrm{~d}^{-1}$

Neglect SRT impacts by seeding

Neglect AOB-activity loss due to Temperature-gap

Figure 7. Bioaugmentation versus SRT conceptual model

\section{Impact of retention efficiency and shallow reactors on $\mathrm{NOB}$ outselection}

Simulations of the DC Water pilot reactor are compared to the actual measurement in Figure 9. The overall profiles of $\mathrm{NH}_{4}^{+}{ }_{-} \mathrm{N}, \mathrm{NO}_{2}{ }^{-} \mathrm{N}, \mathrm{NO}_{3}{ }^{-} \mathrm{N}$ were closely predicted by the model. The model utilized activity measurement of retained sludge from the sieve mechanisms to assess AOB, NOB and anammox retention efficiency. The model predicted minor improvement in NOB out-selection with an $\mathrm{AOB} / \mathrm{NOB}$ ratio of 1.8 compared to 1.6 for fully nitrifying systems. One explanation for the low NOB outselection efficiency would be the effect of the inorganic carbon limitation switch on AOB growth and the other would be the NOB retention by the sieve where NOBs may have attached onto the anammox granules. To address the impact of these two factors, the retention of the various organisms was modified in the model to reflect an ideal separation of granular anammox bacteria and AOBs and NOBs and the depth of the reactors in the model was adjusted to mimic that of full-scale tank depth. Table 5 
presents a comparison between the pilot reactor performance with and without these hypothetical improvements. The model predicted an AOB/NOB ratio of 7.1 when both retention and tank depth are optimized. The optimized model was then used to determine the potential savings in carbon addition in the form of acetate between conventional nitrification/denitrification system and a system with nitrogen shortcut (i.e. repressed NOB). The model showed that for nitrogen removal efficiency of approximately $90 \%$ and effective (i.e. $70 \%$ ) NOB out-selection, the acetate saving due to nitrogen shortcut was $60 \%$ compared to conventional nitrification/denitrification. However, a validation of the model will be required either with full scale or with modified pilot reactor to confirm the hypotheses introduced.

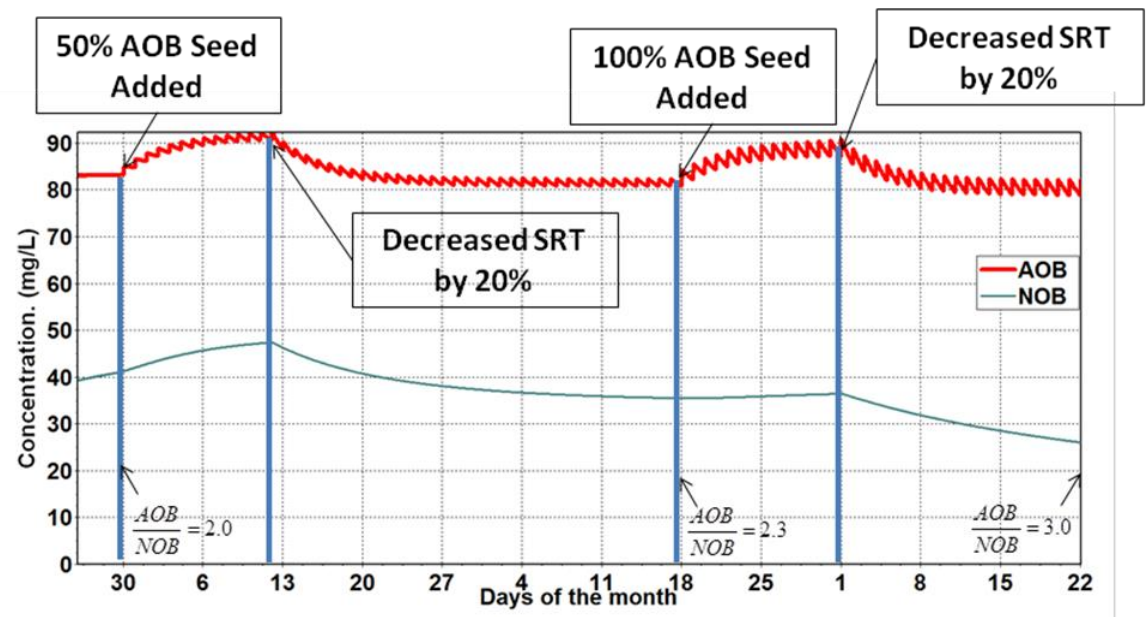

Figure 8. Simulation of HRSD pilot reactor with AVN control showing $100 \%$ and $50 \%$ seed mass rates and SRT variation

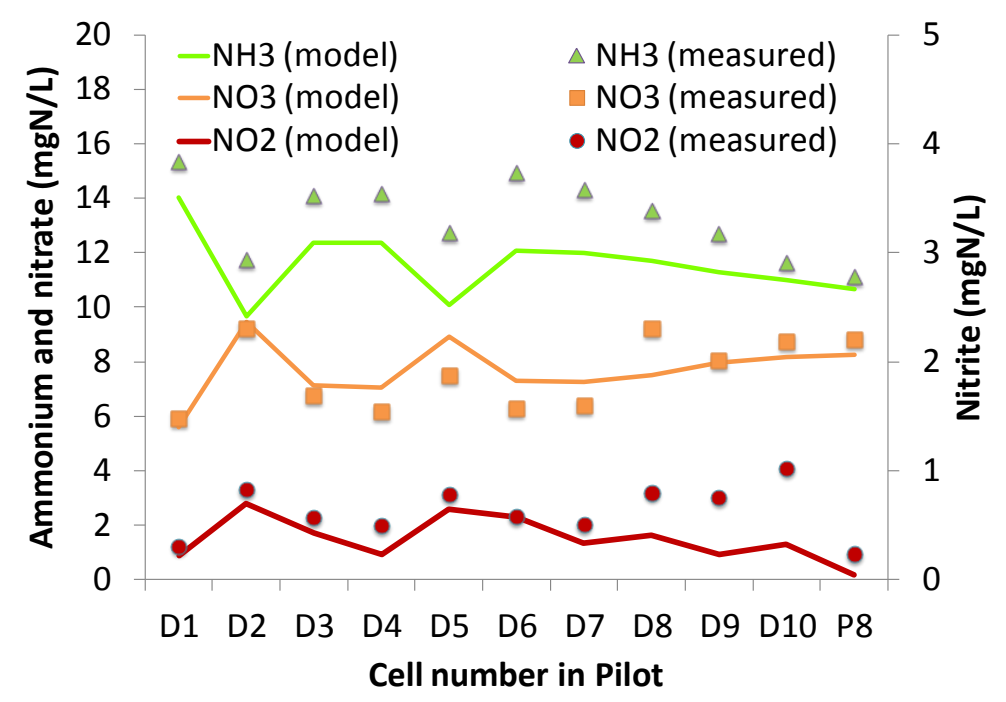

Figure 9. Simulation and measured profile of the DC Water pilot reactor 
Al-Omari et al.

Table 5. Impact of selective retention and tank depth on NOB outselection

Improved Selective

\begin{tabular}{lccc} 
Parameter & Pilot & Improved Selective Retention & Retention+Deep Tankage \\
\hline Anammox retention Efficiency, \% & 73 & 90 & 90 \\
AOB retention Efficiency, \% & 35 & 20 & 20 \\
NOB retention Efficiency, \% & 52 & 20 & 20 \\
Tank Depth, m & 0.3 & 0.3 & 9.0 \\
AOB/NOB ratio & 1.8 & $\mathbf{2 . 9}$ & $\mathbf{7 . 1}$ \\
\hline
\end{tabular}

\section{CONCLUSIONS}

In this paper, a model based approach of the key mechanisms for shortcut nitrogen removal to facilitate mainstream deammonification was presented. The modelling served as a useful tool to separate the impact of individual mechanisms on NOB out-selection and to identify artifacts associated with bench-scale reactors. The model illustrated the benefits of using the novel AVN controller over the ammonia-based control by managing carbon removal and recovering alkalinity. It also demonstrated the impact of AOB seeding and SRT on NOB outselection and showed the importance of applying aggressive SRT for effective NOB outselection. In addition, the model was used in a hypothetical scenario analysis to demonstrate the potential external carbon savings of $60 \%$ that would be realized by converting a conventional nitrification-denitrification system to mainstream deammonification exemplified by the Blue Plains WWTP case study.

\section{REFERENCES}

Al-Omari A., Wett B., Han M., De Clippeleir H., Bott C., Nopens I., Murthy S. (2013) Competition Over Nitrite in Single Sludge Mainstream. In: Proceedings of IWA Conf. On Nutrient Removal and Recovery, Vancouver, Canada, July 2013.

Al-Omari A., Wett B., Han H., Hell M., Bott C., Murthy S. (2012) Full-plant deammonification based on NOBrepression, AOB seeding, anammox seeding and successful retention. In: Proceedings of IWA Conf. On Nutrient Removal, Harbin, China, September 2012.

Al-Omari A., Han M., Wett B., Dockett N., Stinson B., Okogi S., Bott C., Murthy S. (2012) Mainstream Deammonification Evaluation at Blue Plains Advanced Wastewater Treatment Plant (AWTP). In: Proceedings of WEFTEC, New Orleans, LA, USA, October 2012.

Blackburne R., Yuan Z., Kelle J. (2008) Demonstration of nitrogen removal via nitrite in a sequencing batch reactor treating domestic wastewater. Water Research 42, $2166-2176$

Chandran K., and Smets B.F. (2000) Single-step nitrification models erroneously describe batch ammonia oxidation profiles when nitrite oxidation becomes rate limiting. Biotechnology \& Bioengineering 68: 396406.

De Clippeleir H. (2012) Microbial resource management of OLAND focused on sustainability. PhD Thesis, Ghent University.

Gao D., Lu J., Liang H. (2014) Simultaneous energy recovery and autotrophic nitrogen removal from sewage at moderately low temperatures. Applied Microbiology and Biotechnology, 98 (6), 2637-2645.

Gao D., Peng Y., Li B., Liang H. (2009) Shortcut nitrification-denitrification by real-time control strategies. Bioresource Technology, 100(7), 2298-300.

Guisasola A., Petzet S., Baeza J. A., Carrera L., Lafuente J. (2007) Inorganic carbon limitations on nitrification: Experimental assessment and modeling. Water Research, 41, $277-286$.

Gut L., Plaza E., Trela J., Hultman, B., Bosander J. (2005) Combined Partial Nitritation/Anammox System for Treatment of Digester Supernatant. In: Proceedings of the IWA Specialty Conference: Nutrient Management in Wastewater Treatment Processes and Recycle Streams, Krakow, Poland, September 2005. 
Hellinga C., Schellen A. A. J. C., Mulder J. W., van Loosdrecht M., Heijnen J. J. (1998) The SHARON Process: an Innovative Method for Nitrogen Removal from Ammonium-Rich Waste Water. Water Science and Technology, 37 (9), 135.

Hu Z., Lotti T., de Kreuk M., Kleerebezem R., van Loosdrecht M.C.M., Kruit J. (2013) Nitrogen Removal by a Nitritation-Anammox Bioreactor at Low Temperature. Applied and Environmental Microbiology, 79(8), 2807-2812.

Jones R.M., Dold P., Takács I., Chapman K., Wett B., Murthy S. \& O'Shaughnessy M. (2007) Simulation for operation and control of reject water treatment processes. In: Proceedings of WEFTEC, San Diego, CA, USA, October 2007.

Knowles G., Downing A.L., Barrett M.J. (1965) Determination of kinetic constants for nitrifying bacteria in mixed culture, with the aid of an electronic computer. Journal of General Microbiol 38: 263-278.

Kornaros M., \& Dokianakis S. (2010). Partial nitrification/denitrification can be attributed to the slow response of nitrite oxidizing bacteria to periodic anoxic disturbances. Environmental Science and Techonlogy. 44, 7245-7253.

Li H., Zhou S., Huang G., Xu B. (2012) Partial nitritation of landfill leachate with varying influent composition under intermittent aeration conditions. Process Safety and Environmental Protection. 91, 4, $285-294$.

Ling D. (2009) Experience from commissioning of full-scale DeAmmon® plant at Himmerfjärden (Sweden). In: Proceedings of 2nd IWA specialized Conference on nutrient Management in Wastewater treatment Processes. Lemtech Konsulting, Krakow, Poland, September 2009.

Miller M., Bunce R., Regmi P., Hingley D., Kinnear D., Murthy S., Wett B., Bott C. (2012) A/B process pilot optimized for nitrite shunt: High rate carbon removal 438 followed by BNR with ammonia-Based cyclic aeration control. In: Proceedings of the 85th Annual Water Environment Federation Technical Exposition and Conference, New Orleans, LA, USA, October 2012.

Peng Y., Chen Y., Peng C., \& Liu M. (2004) Nitrite accumulation by aeration controlled in sequencing batch reactors treating domestic wastewater. Water Science and Technology. 50(10):35-43

Peng Y., Guo J., Horn H., Yang X., Wang S. (2012) Achieving nitrite accumulation in a continuous system treating low-strength domestic wastewater: switchover from batch start-up to continuous operation with process control. Applied Microbiology and Biotechnology, 94(2), 517-526.

Pollice, A., Tandoi, V., \& Lestingi, C. (2002). Influence of aeration and sludge retention time on ammonium oxidation to nitrite and nitrate. Water research, 36 (10), 2541-2546.

Regmi P., Holgate B., Miller M. W., Bunce R., Park H., Chandran C., Wett B., Murthy S., Bott C. (2013) NOB out-selection in mainstream makes two-stage deammonification and nitrite-shunt possible. Proc. IWA/WEF Conf. On Nutrient Removal and Recovery, Vancouver, Canada, July 2013.

Regmi R., Miller M., Holgate B., Bunce R., Park H., Chandran K., Wett B., Murthy S., Bott C. (2014) Control of aeration, aerobic SRT and COD input for mainstream nitritation/denitritation. Final revision was submitted to Water Research.

Rosenwinkel K., Cornelius A., Thöle D. (2005). Full scale application of the deammonification process for the treatment of sludge water. In: Proceedings of IWA specialized Conference on nutrient Management in Wastewater treatment Processes and Recycle streams, Krakow, Poland, September 2005.

Stinson B.M., Murthy S., Bott C., Wett B., Al-Omari A., Bowden G., Mokhyerie Y., De Clippeleir H. (2013) Roadmaps Toward Energy Neutrality Chemical Optimization at Enhanced Nutrient Removal Facilities. In: Proceedings of WEF/IWA Nutrient removal and recovery trends in resource recovery and use, Vancouver, Canada, July 2013.

van de Graaf A. A., Mulder A., de Bruijn P., Jetten M.S.M., Robertson L.A., Kuenen J.G. (1996) Autotrophic Growth of Anaerobic Ammonium Oxidizing Micro-Organisms in a Fluidized Bed Reactor. Microbiology. 142, 2187-2196.

van Loosdrecht M.C.M., Salem S. (2005) Biological Treatment of Sludge Digester Liquids. In: Proceedings of the IWA Specialty Conference: Nutrient Management in Wastewater Treatment Processes and Recycle Streams, Krakow, Poland, September 2005.

Vlaeminck S.E., De Clippeleir H., Verstraete W. (2012) Microbial resource management of one-stage partial nitritation/anammox. Microbial Biotechnology, 5(3), 433-448.

Wett B., Omari A., Podmirseg S. M., Han M., Murthy S., Bott C., Hell M., Takács I., Nyhuis G., Gómez Brandón M., O'Shaughnessy M. (2013) Going for mainstream deammonification from bench- to full-scale for maximized resource efficiency. Water Science \& Technology, 68 (2), 283-289.

Wett B., Jimenez J. A., Takacs I., Murthy S., Bratby J. R., Holm N. C., Ronner-Holm S. G. (2011) Models for nitrification process design: one or two AOB populations? Water Science and Technology, 64 (3) 568-78.

Wett B., Hell M., Nyhuis G., Puempel T., Takács I., Murthy S. (2010) Syntrophy of aerobic and anaerobic ammonia oxidisers. Water Science and Technology, 61 (8), 1915-1922 
Wett B. (2007) Development and implementation of a robust deammonification process. Water Science and Technology, 56, 81-88

Wett B. (2005) Solved upscaling problems for implementing deammonification of rejection water. Water Science and Technology 53 (12), 121-128.

Wett B., Rauch W. (2003) The role of inorganic carbon limitation in biological nitrogen removal of extremely ammonia concentrated wastewater. Water Research 37, 1100-1110.

Winkler M.K.H., Kleerebezem R., Kuenen J.G., Yang J., van Loosdrecht M.C.M (2011) Segregation of Biomass in Cyclic Anaerobic/Aerobic Granular Sludge Allows the Enrichment of Anaerobic Ammonium Oxidizing Bacteria at Low Temperatures. Environ. Sci. Technol. 2011, 45, 7330-7337.

Zekker I., Rikmann E., Tenno T., Saluste A., Tomingas M., Menert A., Loorits L. (2012). Achieving nitritation and anammox enrichment in a single moving-bed biofilm reactor treating reject water. Environmental technology, 33(4-6), 703-710. 


\title{
Modelling of Organic Substrate Transformation in the High-Rate Activated Sludge Process: Why Current Models Don't Work and a Recommended Unified Model Approach
}

\author{
Thomas Nogaj ${ }^{1}$, Andrew Randall ${ }^{1}$, Jose Jimenez ${ }^{2}$, Imre Takacs ${ }^{3}$, Charles Bott ${ }^{4}$, Mark Miller ${ }^{5}$, Sudhir \\ Murthy ${ }^{6}$ and Bernhard Wett $^{7}$ \\ ${ }^{1}$ Department of Civil and Environmental Engineering, University of Central Florida, Orlando, FL, USA \\ ${ }^{2}$ Brown and Caldwell, 850 Trafalgar Court, Suite 300, Maitland, FL, USA \\ ${ }^{3}$ Dynamita SARL, Nyons, France \\ ${ }^{4}$ Hampton Roads Sanitation District, 1436 Air Rail Ave., Virginia Beach, VA, USA \\ ${ }^{5}$ Civil and Environment Engineering Department, Virginia Tech, Blacksburg, VA, USA \\ ${ }^{6}$ DC Water Authority, 5000 Overlook Ave. SW, Washington DC, USA \\ ${ }^{7}$ ARA Consult GmbH, Unterbergerstraße 1, Innsbruck, Austria
}

\begin{abstract}
This paper describes the development of a modified ASM1 model framework to describe the organic substrate transformation in the high-rate activated sludge (HRAS) process. New state variables and process rate equations were incorporated. New process mechanims for dual soluble substrate, extracellular polymeric substances (EPS) production, production of storage polymers, and adsorption of colloidal substrate were included in the modified model. Data from two HRAS pilot plants were used to calibrate and to validate the proposed model framework for HRAS systems. A dual substrate model for soluble biodegradable substrate transformation was adopted since it described the pilot plant data. The modified model incorporates EPS production as part of the aerobic growth process on the soluble substrate and flocculation of colloidal COD to particulate COD. The adsorbed organics are then converted through hydrolysis to the slow fraction of soluble readily biodegradable substrate. The proposed model framework was able to predict the performance of the pilot plants and provided better overall results than the ASM1 model.
\end{abstract}

\section{Keywords}

A-stage, adsorption, ASM, EPS, flocculation, high-rate activated sludge, organic substrate, oxidation, process modeling, storage

\section{INTRODUCTION}

The high-rate activated sludge (HRAS) process for carbon removal uses high food-tomicroorganism ratios and low solids and hydraulic retention times (SRT and HRT) for the biological substrate (COD) transformation from wastewater. When a HRAS system is the first step in the Adsorption-Bio-oxidation (AB) process (Böhnke et al., 1980), the general objectives are to maximize the removal of organics through adsorption rather than oxidation and to produce large amounts of waste sludge that can be converted to biogas by anaerobic digestion (Schulze-Rettmer et al., 1998). Hence, accurate modelling of this system is of importance to design, control, optimization and prediction of the performance of not only HRAS systems but of the $\mathrm{AB}$ process as a whole.

The modelling of activated sludge processes, particularly the COD transformations, has significantly evolved towards fundamental principles in the past decades from simple singlesubstrate models to more complex multiple-substrate models involving the processes of oxidation, hydrolysis and storage (Dold et al., 1980; Sin et al., 2005). However, these models have evolved to describe COD removal in systems operating at long SRT (i.e. 3 days or longer) where the biodegradable organic substrate $\left(\mathrm{S}_{\mathrm{B}}\right)$ can be modelled as a single substrate with a single kinetic expression. In addition, flocculation and adsorption of colloidal and particulate substrate $\left(\mathrm{C}_{\mathrm{B}}\right.$ and $\left.\mathrm{X}_{\mathrm{B}}\right)$ are assumed to be complete and instantaneous; hence, it can be ignored in the models (Haider et al., 2003; Jimenez et al., 2003). However, in high-rate 
systems such as those employed in the AB process (where the SRT is well below 1 day), these assumptions with respect to organic substrate transformation are no longer applicable.

Full-scale and pilot-scale results from high-rate systems (Haider et al., 2000 and 2003; Miller et al., 2012; Jimenez et al. 2014) show that very low SRT (i.e. 1 day or less) may result in a selection of fast growing bacteria, which can use only part of the influent substrate. Haider et al. (2003) showed that the inert soluble COD fraction (SU) of the wastewater was always significantly higher if the wastewater was added to a HRAS with an SRT of 0.5 days in comparison to systems with an SRT of 20 days. Hence, they recommended that for modelling, the $S_{B}$ fraction of the wastewater should be split into two distinct biodegradable fractions. Jimenez et al. (2005) recognized that in effluent from these systems, some of the particulate and (especially) colloidal COD may not be removed by flocculation and adsorption resulting in incomplete enmeshment and hydrolysis of $X_{B}$ and $C_{B}$. Jimenez et al. (2005) recommended that flocculation kinetics should be considered as an important mechanism from a modelling perspective. Hence, for these reasons the existing model's assumption of organic substrate transformation kinetic parameters based on instantaneous flocculation/adsorption becomes questionable and should be addressed to properly model low SRT systems.

This paper discusses a modelling approach which evaluates the organic substrate transformations as it pertains to HRAS systems. This approach uses the Activated Sludge Model No.1 (ASM1) (Henze et al., 2000) as the initial framework. The original framework was modified to describe the proper mechanisms required to accurately describe the performance of the HRAS system.

\section{MATERIALS AND METHODS}

Historical operating data from two pilot systems were evaluated to understand the organic substrate transformation mechanisms in HRAS and used to calibrate and validate the proposed process model. The data used during this study includes operating data from an AStage pilot plant owned and operated by the Hampton Roads Sanitation District (HRSD) (Miller et al., 2013) and from a HRAS pilot plant operated by the University of New Orleans (Jimenez et al., 2014).

HRSD's pilot plant, located at the Chesapeake-Elizabeth Treatment Plant in Virginia Beach, Virginia, uses an A-Stage process for carbon removal followed by a B-Stage system for nitrogen removal. Currently, the A-Stage includes three reactors in series (170 L per reactor), operated at an aggregate 0.2-day SRT and 0.5-hour HRT, and is fed screened and degritted raw municipal wastewater at a constant flow rate of $24.5 \mathrm{~m}^{3} / \mathrm{d}$.

The HRAS pilot plant operated by the University of New Orleans consists of a rotating screen, a complete mix aeration tank, and a secondary clarifier. The unit was designed for a flow rate of $7.5 \mathrm{~m} 3 / \mathrm{d}$ and a HRT in the bioreactor of approximately 0.5 hours. The pilot plant was operated at a range of SRT conditions, ranging from 0.3 days to 2 days.

\section{MODEL DESCRIPTION}

To describe the behavior of the HRAS pilot plants, the ASM model framework was modified to incorporate non-steady state material balance equations for dual soluble substrate $\left(\mathrm{S}_{\mathrm{Bf}}, \mathrm{S}_{\mathrm{Bs}}\right)$, EPS production $\left(\mathrm{X}_{\mathrm{EPS}}\right)$, production of storage polymers $\left(\mathrm{X}_{\mathrm{STO}}\right)$, and adsorption of inert and biodegradable colloidal substrate $\left(\mathrm{C}_{\mathrm{U}}\right.$ and $\left.\mathrm{C}_{\mathrm{B}}\right)$. A partial list of state variables used in the modified model framework is shown in Table 1. 
Table 9 Partial list of state variables

\begin{tabular}{lll}
\hline Symbol & Name & \multicolumn{1}{c}{ Units } \\
\hline$S_{\mathrm{U}}$ & Soluble non-biodegradable organics & $\mathrm{g} \mathrm{COD} \cdot \mathrm{m}^{-3}$ \\
$S_{\mathrm{Bf}}$ & Readily soluble biodegradable organics & $\mathrm{g} \mathrm{COD} \cdot \mathrm{m}^{-3}$ \\
$S_{B s}$ & Slowly soluble biodegradable organics & $\mathrm{g} \mathrm{COD} \cdot \mathrm{m}^{-3}$ \\
$C_{\mathrm{U}}$ & Colloidal non-biodegradable organics & $\mathrm{g} \mathrm{COD} \cdot \mathrm{m}^{-3}$ \\
$C_{\mathrm{B}}$ & Colloidal biodegradable organics & $\mathrm{g} \mathrm{COD} \cdot \mathrm{m}^{-3}$ \\
$X_{\mathrm{U}}$ & Particulate non-biodegradable organics & $\mathrm{g} \mathrm{COD} \cdot \mathrm{m}^{-3}$ \\
$X_{\mathrm{B}}$ & Particulate biodegradable organics & $\mathrm{g} \mathrm{COD} \cdot \mathrm{m}^{-3}$ \\
$X_{\mathrm{OHO}, \mathrm{ACT}}$ & Active ordinary heterotrophic organisms & $\mathrm{g} \mathrm{COD} \cdot \mathrm{m}^{-3}$ \\
$X_{\mathrm{E}}$ & Particulate non-biodegradable endogenous products & $\mathrm{g} \mathrm{COD} \cdot \mathrm{m}^{-3}$ \\
$X_{E P S}$ & Extracellular polymeric substances & $\mathrm{g} \mathrm{COD} \cdot \mathrm{m}^{-3}$ \\
$X_{S T O}$ & Intracellular storage polymeric substances & $\mathrm{g} \mathrm{COD} \cdot \mathrm{m}^{-3}$ \\
\hline
\end{tabular}

Review of the literature (Jimenez, 2002; Laspidou et al., 2002b; Miller et al., 2013) has led to a modification of the model framework. Figure 1 illustrates the flow of electrons in the modified ASM1 model framework (Nogaj et al., 2013). Details are discussed in the following sections.

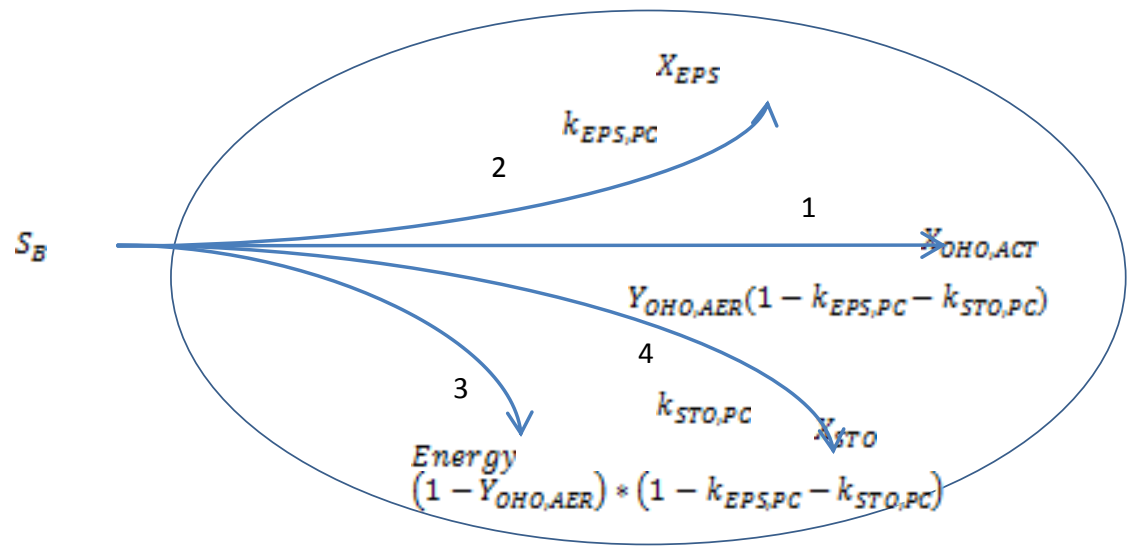

Figure 1 Electron flow for soluble substrate

\section{Fate of Soluble Substrate}

Results from the pilot plants show a higher effluent soluble COD from the A-stage than the Bstage (where it is removed at a significantly longer SRT) (Nogaj et al., 2013). Conventionally, the method to quantify the non-biodegradable soluble COD is to operate a laboratory or pilot scale activated sludge system at an SRT longer than 3 days (Ekama et al., 1986) and use the effluent soluble COD as the non-biodegradable fraction. However, at the low SRT (and low HRT) of the HRAS system, there is a fraction of the effluent COD that is biodegradable in the higher SRT, B-Stage process but not biodegradable in the conditions in the A-Stage. This has led to the establishment of two state variables for $S_{B}$ designated as $S_{B f}\left(S_{B}\right.$ fast) and $S_{B s}\left(S_{B}\right.$ slow). $\mathrm{S}_{\mathrm{Bf}}$ corresponds to the soluble COD that is biodegradable in the HRAS system at low 
SRT by a fast growing population of $\mathrm{X}_{\mathrm{OHO}, \mathrm{ACT}}$ (Haider et al., 2003; Pala-Ozkok et al., 2013). $\mathrm{S}_{\mathrm{Bs}}$ is the soluble COD fraction that is non-biodegradable in the HRAS system, but is

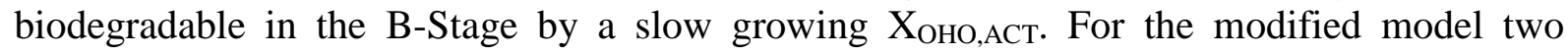
frameworks were evaluated. In one framework, $S_{\mathrm{Bf}}$ is biodegraded first, and it is only when $\mathrm{S}_{\mathrm{Bf}}$ is fully utilized that biodegradation of $\mathrm{S}_{\mathrm{Bs}}$ becomes significant. This is analogous to diauxic growth in which one substrate is biodegraded immediately by constitutive enzymes, and only when it runs out are enzymes induced for metabolism of the second substrate. The data does not show, or disprove, this mechanism, but this framework is at least plausible mechanistically. This framework is referred to as the Diauxic model. In the second framework $\mathrm{S}_{\mathrm{Bf}}$ and $\mathrm{S}_{\mathrm{Bs}}$ are utilized simultaneously with the growth on $\mathrm{S}_{\mathrm{Bf}}$ occurring at a higher maximum specific substrate utilization rate than on $S_{\mathrm{Bs}}$. This framework is referred to as the Dual Substrate model.

\section{EPS Production}

Extracellular polymeric substances (EPS) production impacts the bioflocculation removal efficiency for particulate and colloidal substrate (Jimenez, 2002). Past models assume instantaneous enmeshment whereas the data from Jimenez (2005, 2007 and 2014) shows that this assumption may not be valid for high rate systems. The EPS data produced by Jimenez et al. (2013) was used as calibration data for this study. This dataset shows a linear correlation between substrate utilization rate and EPS production and an increase in EPS production with SRT (thus decrease with growth) over a range of 0.3 to 2.0 days. In addition, EPS increased with the DO concentration over the same range of SRT values. Laspidou et al. (2002a) indicated that the net EPS concentration is a function of the portion of influent soluble substrate (substrate electron pool) shunted to EPS formation versus the EPS hydrolysis rate. Hence, the modified model incorporates EPS production as part of the aerobic growth process on $\mathrm{S}_{\mathrm{Bf}}$ and $\mathrm{S}_{\mathrm{Bs}}$. The proportionality coefficient $\mathrm{k}_{\mathrm{EPS}, \mathrm{PC}}$ quantifies the portion of influent electrons shunted to EPS formation. The portion of substrate electrons that are shunted to EPS formations $\left(\mathrm{k}_{\mathrm{EPS}, \mathrm{PC}}\right)$ are then subtracted from the biomass yield coefficient $\mathrm{Y}_{\mathrm{OHO}}$, i.e. $\left.\mathrm{Y}_{\mathrm{OHO}} *\left(1-\mathrm{k}_{\mathrm{EPS}, \mathrm{PC}}\right)\right)$, reducing the electrons available for biomass synthesis. In the Diauxic model, EPS formation is first driven by $S_{B f}$ during aerobic growth. EPS formation on $S_{B s}$ does not occur until $\mathrm{S}_{\mathrm{Bf}}$ starts to run out. In contrast, the EPS formation in the Dual Substrate model occurs simultaneously on both soluble substrate fractions. In both models EPS formation driven by influent $S_{\mathrm{Bs}}$ does not have to compete with the formation of storage products (which only occurs through $\mathrm{S}_{\mathrm{Bf}}$ ). Additional $\mathrm{S}_{\mathrm{Bs}}$ becomes available through hydrolysis of $\mathrm{X}_{\mathrm{B}}$ (Carucci et al., 2001). $\mathrm{K}_{\mathrm{O} \text {,EPS }}$ was estimated using a nonlinear regression analysis of the EPS production data vs DO concentration data provided by Jimenez et al. (2000). The value $\mathrm{k}_{\mathrm{EPS}, \mathrm{MAX}}$ (maximum EPS production) was determined by developing a least square logarithmic fit of the dataset provided by Jimenez et al. (2014) resulting in an estimated $\mathrm{k}_{\mathrm{EPS}, \mathrm{MAX}}$ value of 0.25 (g COD $\left.\mathrm{EPS}_{\mathrm{g}} / \mathrm{g} \mathrm{VSS}\right)$ and $\mathrm{K}_{\mathrm{O}, \mathrm{EPS}}$ value of $0.55\left(\mathrm{gSO}_{2} / \mathrm{m}^{3}\right)$.

$\mathrm{k}_{\mathrm{EPS}, \mathrm{PC}}=\left(\frac{\mathrm{k}_{\mathrm{EPS}, \mathrm{MAx}}}{\mathrm{i}_{\mathrm{CB}}}\right) \times\left(\frac{\mathrm{S}_{\mathrm{O}_{2}}}{\left(\mathrm{~K}_{\mathrm{O}_{2} \mathrm{EPS}}+\mathrm{S}_{\mathrm{O}_{2}} \mathrm{j}\right.}\right)$

Equation 1 shows how $\mathrm{k}_{\mathrm{EPS}}$, PC is calculated. The term $i_{\mathrm{CB}}\left(1.48 \mathrm{gCOD} \mathrm{Vss}_{\mathrm{gVSS}}\right)$ is a stoichiometric conversion factor that converts $\mathrm{k}_{\mathrm{EPS}, \mathrm{MAX}}$ from units of $\mathrm{gCOD}_{\mathrm{EPS}} / \mathrm{gVSS}$ to $\mathrm{gCOD}_{\mathrm{EPS}} / \mathrm{gCOD}_{\mathrm{Vss}}$.

Production of Storage Polymers 
Review of the literature suggests that systems operated at low DO concentrations $(<0.9 \mathrm{mg} / \mathrm{L}$; according to Third et al., 2003), typical of a HRAS system, the microbial uptake of readily biodegradable COD $\left(\mathrm{S}_{\mathrm{Bf}}\right)$ could result in the formation of storage polymers. Third et al. (2003) found using acetate as the substrate for COD, the microbial uptake of acetate and its conversion to storage polymers was strictly oxygen dependent. At low DO, the flow of electrons is used for acetate uptake and production of storage polymers. Higher DO supply rates resulted in higher growth rates with the flow of electrons to biomass production and approximately $20 \%$ of the substrate is oxidized, independent of the DO concentration. The following expression was added to the modified model framework to simulate the flow of electrons to storage as a function of DO concentration.

$\mathrm{f}_{\text {STO }}=\left(f_{\text {shunt } \max }\right) \times\left(\frac{\mathrm{S}_{\mathrm{O}_{2}}}{\left(\mathrm{~K}_{\mathrm{O}, 3 \mathrm{SO}}+\mathrm{S}_{\mathrm{O}_{2}} \mathrm{j}\right.}\right)$

Where $f_{\text {STO }}$ represents the fraction of storage products in the active biomass, $f_{\text {Shunt,max }}$ represents the maximum flow electrons as a function of dissolved oxygen concentration and $\mathrm{K}_{\mathrm{O}, \mathrm{STO}}$ is the half-saturation coefficient for $\mathrm{S}_{\mathrm{O} 2}$. These two values were determined from a plot of $\mathrm{f}_{\mathrm{STO}} \mathrm{vs} \mathrm{DO}$ which represented a Monod type curve. The extrapolated values were 0.15 for $\mathrm{f}_{\text {Shunt,max }}$ and $0.7 \mathrm{gSO}_{2} / \mathrm{m}^{3}$ for $\mathrm{K}_{\mathrm{O}, \mathrm{STO}}$.

The diversion of substrate electrons to storage in the modified model is represented by the proportionality constant $\mathrm{k}_{\mathrm{STO}, \mathrm{PC}}$. The portion of electrons that are shunted to $\mathrm{k}_{\mathrm{STO}, \mathrm{PC}}$ are also subtracted from the biomass yield coefficient $\mathrm{Y}_{\mathrm{OHO}}$, i.e. $\left(\mathrm{Y}_{\mathrm{OHO}} *\left(1-\mathrm{k}_{\mathrm{EPS}, \mathrm{PC}}-\mathrm{k}_{\mathrm{STO}, \mathrm{PC}}\right)\right.$ ), for aerobic growth using $\mathrm{S}_{\mathrm{Bf}}$, further reducing the electrons available for biomass synthesis.

\section{Adsorption of Colloidal COD}

Typical characteristics for any municipal wastewater include both soluble and particulate organics. Before developing modifications to the mathematical model it is important to define the soluble, particulate and colloidal fractions of the influent COD. Total chemical oxygen demand (tCOD) can be defined as the sum of particulate COD (pCOD) and soluble COD (sCOD) present in the wastewater. For the purpose of this investigation, the pCOD consists of organic suspended solids (ssCOD) and organic colloids (cCOD) in the wastewater $(\mathrm{pCOD}=\mathrm{ssCOD}+\mathrm{cCOD})$. The state variable $\mathrm{X}_{\mathrm{B}}$ represents $\mathrm{pCOD}$ in the modified model. The dissolved COD excluding colloids is the truly soluble organic material in the wastewater and this was quantified by coagulation/flocculation followed by filtration (i.e. ffCOD) (Mamais et al., 1993). The truly sCOD is defined in the modified model as the sum of the state variables $S_{\mathrm{Bf}}, \mathrm{S}_{\mathrm{Bs}}$ and $\mathrm{S}_{\mathrm{U}}$. The most important aspect is the differentiation of particulate and colloidal COD, which has not traditionally been done in most studies nor in the previous ASM derived models(Henze et al., 2000). The reason for this is that in higher HRT and SRT systems there is plenty of time for both colloidal and particulate COD to flocculate completely and to be degraded. However, in low HRT/SRT systems (HRAS) there is not always time for this to occur. This also means that effluent CODs in HRAS systems are higher than would be predicted by existing models.

\section{Modified model Solution}

The ASM models use matrix notation for the presentation of kinetic models. The matrix approach summarizes the components (state variables) and the transformation processes 
which are to be considered in the model. The stoichiometric coefficients and process rate equations are presented in the matrix. The matrix is often referred to as the Gujer matrix and will be referred to as such throughout this paper. Using the ASM1 Gujer matrix as the reference model, proposed modifications to the framework for the modified model are defined in Figure 10.

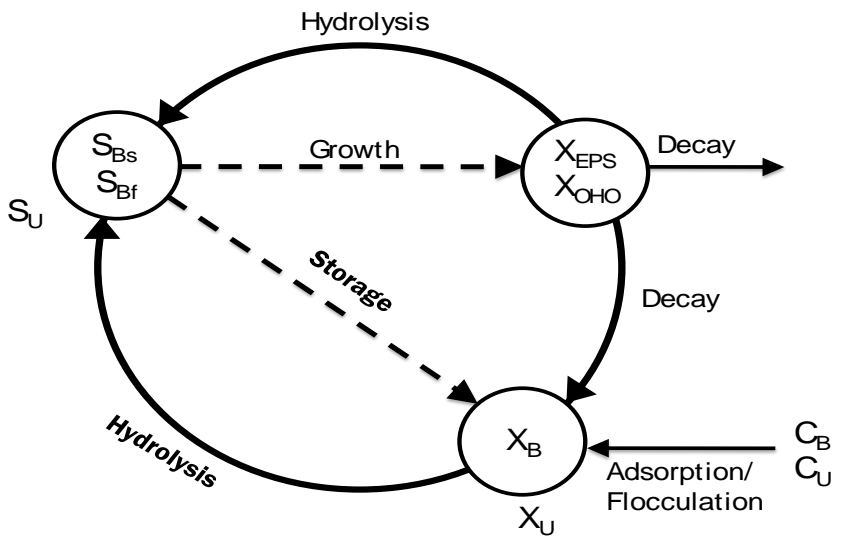

Figure 10 Proposed Mathematical Model modifications for the HRAS carbon removal model The modified stoichiometric matrix is shown in Table with the associated process rate

The modified stoichiometric matrix is shown in Table with the associated process rate equations shown in Table. In the Diauxic model it must be noted that the pathways emanating from $S_{\mathrm{Bf}}$ and $\mathrm{S}_{\mathrm{Bs}}$ will never both be significant at the same time. This is due to the model kinetic equations being such that $S_{\mathrm{Bs}}$ transformations will not be significant until $\mathrm{S}_{\mathrm{Bf}}$ runs out (i.e. when $\mathrm{S}_{\mathrm{Bf}}<\mathrm{K}_{\mathrm{Bf}}$;).

The colloidal substrate $\left(C_{B}\right)$ is added as a new state variable. The $C_{B}$ and slowly biodegradable particulate $\mathrm{COD}\left(\mathrm{X}_{\mathrm{B}}\right)$ are enmeshed which represents the colloidal and particulate COD adsorbed through the bioflocculation mechanism. The adsorbed organics are then converted through hydrolysis to the slow fraction of soluble readily biodegradable substrate $\mathrm{S}_{\mathrm{Bs}}$. The modified model framework incorporates two new process components; flocculation of $\mathrm{C}_{\mathrm{B}}$ and flocculation of $\mathrm{C}_{\mathrm{U}}$ as follows:

$$
\begin{aligned}
& \frac{d C_{B}}{d t}=-\left(\mathrm{q}_{\mathrm{ADS}} \times \mathrm{C}_{\mathrm{B}} \times\left(\mathrm{X}_{\mathrm{OHO}}+\mathrm{X}_{\mathrm{ANO}}\right) \times\left(\frac{\mathrm{K}_{\mathrm{SL}}}{\left.\frac{C_{\mathrm{B}}}{\left(\mathrm{X}_{\mathrm{OHO}}+\mathrm{X}_{\mathrm{ANO}}\right)}\right)+\mathrm{K}_{\mathrm{SL}}}\right) \times\left(\frac{\mathrm{x}_{\mathrm{EPS}}}{\mathrm{K}_{\mathrm{EPS}}+\mathrm{X}_{\mathrm{BPS}}}\right)\right. \\
& \frac{d C_{U}}{d t}=-\left(\mathrm{q}_{\mathrm{ADS}} \times \mathrm{C}_{\mathrm{U}} \times\left(\mathrm{X}_{\mathrm{OHO}}+\mathrm{X}_{\mathrm{ANO}}\right) \times\left(\frac{\mathrm{K}_{\mathrm{SL}}}{\left.\frac{C_{\mathrm{U}}}{\left(\mathrm{X}_{\mathrm{OHO}}+\mathrm{X}_{\mathrm{ANO}}\right)}\right)+\mathrm{K}_{\mathrm{SL}}}\right) \times\left(\frac{\mathrm{x}_{\mathrm{EPS}}}{\mathrm{K}_{\mathrm{EPS}}+\mathrm{X}_{\mathrm{BPS}}}\right)\right.
\end{aligned}
$$

The kinetic rate expression for each process is a first-order rate expression with respect to the colloidal concentration. The kinetic parameter $\mathrm{q}_{\mathrm{ADS}}$ is the adsorption rate constant and $\mathrm{K}_{\mathrm{SL}}$ is the surface limitation coefficient. The $\mathrm{C}_{\mathrm{B}}$ is flocculated onto the $\mathrm{X}_{\mathrm{B}}$, becoming part of that category of organics. The adsorbed organics are then converted through hydrolysis to $\mathrm{S}_{\mathrm{Bs}}$ which can then be oxidized or converted to EPS or biomass by the microorganisms. The $\mathrm{C}_{\mathrm{U}}$ is flocculated onto the $\mathrm{X}_{\mathrm{U}}$ and removed from the system through wasting. 


\section{WWTmod}

Nogaj et al.

Table 2 Partial Gujer matrix processes and stoichiometric coefficients for the HRAS model

\begin{tabular}{|c|c|c|c|c|c|c|c|c|}
\hline & Name & $\mathrm{S}_{\mathrm{Bf}}$ & $\mathrm{S}_{\mathrm{Bs}}$ & $\mathrm{C}_{\mathrm{B}}$ & $\mathrm{X}_{\mathrm{B}}$ & ХОНО, АСТ & $\mathrm{X}_{\mathrm{EPS}}$ & $\mathrm{X}_{\mathrm{STO}}$ \\
\hline $\mathrm{r} 1$ & $\begin{array}{l}\text { Aerobic growth of } \mathrm{X}_{\mathrm{OHO}} \mathrm{S}- \\
\text { Fast }\end{array}$ & $\begin{array}{l}-1 /\left(\mathrm{Y}_{\mathrm{OHO}, \mathrm{AER}} *\left(1-\mathrm{k}_{\mathrm{EPS}, \mathrm{PC}}{ }^{-}\right.\right. \\
\left.\left.\mathrm{k}_{\mathrm{STO} . \mathrm{PC}}\right)\right)\end{array}$ & & & & 1 & $\begin{array}{l}\mathrm{k}_{\mathrm{EPS}, \mathrm{PC}} /\left(\mathrm{Y}_{\mathrm{OHO}, \mathrm{AER}} *\left(1-\mathrm{k}_{\mathrm{EPS}, \mathrm{PC}}{ }^{-}\right.\right. \\
\left.\left.\mathrm{k}_{\mathrm{STO}, \mathrm{PC}}\right)\right)\end{array}$ & $\begin{array}{l}\mathrm{k}_{\mathrm{STO}} /\left(\mathrm{Y}_{\mathrm{OHO}, \mathrm{AER}} *(1-\right. \\
\left.\left.\mathrm{k}_{\mathrm{EPS}, \mathrm{PC}}-\mathrm{k}_{\mathrm{STO}, \mathrm{PC}}\right)\right)\end{array}$ \\
\hline $\mathrm{r} 2$ & $\begin{array}{l}\text { Aerobic growth of } \mathrm{X}_{\mathrm{OHOs}}- \\
\text { Slow }\end{array}$ & & $-1 /\left(\mathrm{Y}_{\mathrm{OHO}, \mathrm{AER}} *\left(1-\mathrm{k}_{\mathrm{EPS}, \mathrm{PC}}\right)\right)$ & & & 1 & $\mathrm{k}_{\mathrm{EPS}, \mathrm{PC}} /\left(\mathrm{Y}_{\mathrm{OHO}, \mathrm{AER}} *\left(1-\mathrm{k}_{\mathrm{EPS}, \mathrm{PC}}\right)\right)$ & \\
\hline r3 & Decay of heterotrophs & & & & $1-f_{\mathrm{U}}$ & -1 & & \\
\hline $\mathrm{r} 4$ & $\begin{array}{l}\text { Hydrolysis of entrapped } \\
\text { organics }\end{array}$ & & 1 & & -1 & & & \\
\hline r5 & $\begin{array}{l}\text { flocculation of colloidal } \\
\text { substrate }\end{array}$ & & & -1 & 1 & & & \\
\hline r6 & $\begin{array}{l}\text { flocculation of colloidal } \\
\text { inerts }\end{array}$ & & & & & & & \\
\hline $\mathrm{r} 7$ & $\begin{array}{l}\text { Hydrolysis of storage } \\
\text { products }\end{array}$ & 1 & & & & & & -1 \\
\hline r8 & EPS hydrolysis & 1 & & & & & -1 & \\
\hline
\end{tabular}

$\underline{\text { Table } 3 \text { Partial Gujer matrix process rate equations for the HRAS model }}$

\begin{tabular}{|c|c|c|}
\hline & Name & Rate expression $\left(r_{j}\right)$ \\
\hline $\mathrm{r} 1$ & Aerobic growth of heterotrophs - Fast & $\mu_{\mathrm{OHO}} *\left(\mathrm{~S}_{\mathrm{B} f} /\left(\mathrm{K}_{\mathrm{Bf}}+\mathrm{S}_{\mathrm{Bf}}\right)\right) *\left(\mathrm{~S}_{\mathrm{O} 2} /\left(\mathrm{K}_{\mathrm{O}, \mathrm{OHO}}+\mathrm{S}_{\mathrm{O} 2}\right)\right) *\left(\mathrm{~S}_{\mathrm{NHx}} /\left(\mathrm{K}_{\mathrm{NH} \mathrm{nut}}+\mathrm{S}_{\mathrm{NHx}}\right)\right) * \mathrm{X}_{\mathrm{OHO}}$ \\
\hline $\mathrm{r} 2 \mathrm{a}$ & Aerobic growth of heterotrophs - Slow & $\mu_{\mathrm{OHO}, \mathrm{SLOW}} *\left(\mathrm{~S}_{\mathrm{Bs}} /\left(\mathrm{K}_{\mathrm{Bs}}+\mathrm{S}_{\mathrm{Bs}}\right)\right) *\left(\mathrm{~K}_{\mathrm{Bl}} /\left(\mathrm{K}_{\mathrm{Bf}}+\mathrm{S}_{\mathrm{Bf}}\right)\right) *\left(\mathrm{~S}_{\mathrm{O} 2} /\left(\mathrm{K}_{\mathrm{O}, \mathrm{OHO}}+\mathrm{S}_{\mathrm{O} 2}\right)\right) *\left(\mathrm{~S}_{\mathrm{NHx}} /\left(\mathrm{K}_{\mathrm{NHx}, \mathrm{nut}}+\mathrm{S}_{\mathrm{NHx}}\right) * \mathrm{X}_{\mathrm{OHO}}\right.$ \\
\hline $\mathrm{r} 2 \mathrm{~b}$ & Aerobic growth of heterotrophs - Slow & $\mu_{\mathrm{OHO}, \mathrm{SLOW}} *\left(\mathrm{~S}_{\mathrm{Bs}} /\left(\mathrm{K}_{\mathrm{Bs}}+\mathrm{S}_{\mathrm{Bs}}\right) *\left(\mathrm{~S}_{\mathrm{O} 2} /\left(\mathrm{K}_{\mathrm{O}, \mathrm{OHO}}+\mathrm{S}_{\mathrm{O} 2}\right)\right) *\left(\mathrm{~S}_{\mathrm{NHx}} /\left(\mathrm{K}_{\mathrm{NH} \mathrm{xut}}+\mathrm{S}_{\mathrm{NHx}}\right)\right) * \mathrm{X}_{\mathrm{OHO}}\right.$ \\
\hline r3 & Decay of heterotrophs & $\mathrm{b}_{\mathrm{OHO}} * \mathrm{X}_{\mathrm{OHO}, \mathrm{ACT}}$ \\
\hline r4 & Hydrolysis of entrapped organics & $\begin{array}{l}\mathrm{q}_{\mathrm{XB}, \mathrm{HYD}} *\left(\left(\mathrm{X}_{\mathrm{B}} / \mathrm{X}_{\mathrm{OHO}}\right) /\left(\mathrm{K}_{\mathrm{B}, \mathrm{HYD}}+\mathrm{X}_{\mathrm{B}} / \mathrm{X}_{\mathrm{OHO}}\right)\right) *\left(\left(\mathrm{~S}_{\mathrm{O} 2} /\left(\mathrm{K}_{\mathrm{O}, \mathrm{OHO}}+\mathrm{S}_{\mathrm{O} 2}\right)\right)+\eta_{\mathrm{HYD}} *\left(\mathrm{~K}_{\mathrm{O}, \mathrm{OHO}} /\left(\mathrm{K}_{\mathrm{O}, \mathrm{OHO}}+\mathrm{S}_{\mathrm{O} 2}\right)\right) *\left(\mathrm{~S}_{\mathrm{NOX}} /\left(\mathrm{K}_{\mathrm{NOX}}+\mathrm{S}_{\mathrm{NOx}}\right)\right)\right) * \mathrm{X} \\
\text { оно }\end{array}$ \\
\hline r5 & flocculation of colloidal substrate & $\mathrm{q}_{\mathrm{ADS}} * \mathrm{C}_{\mathrm{B}} *\left(\mathrm{X}_{\mathrm{OHO}}+\mathrm{X}_{\mathrm{ANO}}\right) *\left(\mathrm{~K}_{\mathrm{SL}} /\left(\left(\mathrm{C}_{\mathrm{B}} /\left(\mathrm{X}_{\mathrm{OHO}}+\mathrm{X}_{\mathrm{ANO}}\right)\right)+\mathrm{K}_{\mathrm{SL}}\right)\right) *\left(\mathrm{X}_{\mathrm{EPS}} /\left(\mathrm{K}_{\mathrm{EPS}}+\mathrm{X}_{\mathrm{EPS}}\right)\right)$ \\
\hline r6 & flocculation of colloidal inerts & $\mathrm{q}_{\mathrm{ADS}} * \mathrm{C}_{\mathrm{U}} *\left(\mathrm{X}_{\mathrm{OHO}}+\mathrm{X}_{\mathrm{ANO}}\right) *\left(\mathrm{~K}_{\mathrm{SL}} /\left(\left(\mathrm{C}_{\mathrm{U}} /\left(\mathrm{X}_{\mathrm{OHO}}+\mathrm{X}_{\mathrm{ANO}}\right)\right)+\mathrm{K}_{\mathrm{SL}}\right)\right) *\left(\mathrm{X}_{\mathrm{EPS}} /\left(\mathrm{K}_{\mathrm{EPS}}+\mathrm{X}_{\mathrm{EPS}}\right)\right)$ \\
\hline r7 & Hydrolysis of storage products & $\mathrm{q}_{\mathrm{STO}, \mathrm{HYD}} *\left(\mathrm{X}_{\mathrm{STO}} / \mathrm{X}_{\mathrm{OHO}} /\left(\mathrm{K}_{\mathrm{STO}, \mathrm{HYD}}+\mathrm{X}_{\mathrm{STO}} / \mathrm{X}_{\mathrm{OHO}}\right)\right) *\left(\mathrm{~K}_{\mathrm{Bl}} /\left(\mathrm{K}_{\mathrm{Bf}}+\mathrm{S}_{\mathrm{Bf}}\right)\right) *\left(\mathrm{~K}_{\mathrm{Bs}} /\left(\mathrm{K}_{\mathrm{Bs}}+\mathrm{S}_{\mathrm{Bs}}\right) *\left(\mathrm{~S}_{\mathrm{O} 2} /\left(\mathrm{K}_{\mathrm{O}, \mathrm{OHO}}+\mathrm{S}_{\mathrm{O} 2}\right)\right) * \mathrm{X}_{\mathrm{OHO}}\right.$ \\
\hline r8 & EPS hydrolysis & $\mathrm{q}_{\mathrm{EPA}, \mathrm{HYD}} * \mathrm{X}_{\mathrm{EPS}}$ \\
\hline
\end{tabular}

r2a corresponds to the Diauxic model, r2b corresponds to the Dual Substrate model 


\section{RESULTS AND DISCUSSION}

The modified ASM1 frameworks were analyzed using the process simulator software SUMO version 0.9.15.0 developed by Dynamita (Nyons, France). The experimental datasets from New Orleans and HRSD were used to calibrate and validate the modified model frameworks.

\section{Model Calibration}

The dataset used to calibrate the modified framework model was collected by Jimenez et al. (2014). The SRT and DO concentration were varied in order to evaluate the effect of these operating parameters on the production of EPS and the removal of organic substrate. The average influent concentrations used for the model calibration are summarized in Table .

Table 4 Influent wastewater concentrations

\begin{tabular}{llll} 
Symbol & Description & Value & Units \\
\hline $\mathrm{S}_{\mathrm{I}}$ & Soluble unbiodegradable organics & 10 & $\mathrm{~g} \mathrm{COD} \cdot \mathrm{m}^{-3}$ \\
$\mathrm{~S}_{\mathrm{Bf}}$ & Soluble biodegradable organics & 60 & $\mathrm{~g} \mathrm{COD} \cdot \mathrm{m}^{-3}$ \\
$\mathrm{~S}_{\mathrm{Bs}}$ & Slowly biodegradable organics & 30 & $\mathrm{~g} \mathrm{COD} \cdot \mathrm{m}^{-3}$ \\
$\mathrm{C}_{\mathrm{U}}$ & Colloidal unbiodegradable organics & 20 & $\mathrm{~g} \mathrm{COD} \cdot \mathrm{m}^{-3}$ \\
$\mathrm{C}_{\mathrm{B}}$ & Colloidal biodegradable organics & 40 & $\mathrm{~g} \mathrm{COD} \cdot \mathrm{m}^{-3}$ \\
$\mathrm{X}_{\mathrm{U}}$ & Particulate unbiodegradable organics & 30 & $\mathrm{~g} \mathrm{COD} \cdot \mathrm{m}^{-3}$ \\
$\mathrm{X}_{\mathrm{B}}$ & Particulate biodegradable organics & 150 & $\mathrm{~g} \mathrm{COD} \cdot \mathrm{m}^{-3}$ \\
$\mathrm{X}_{\mathrm{OHO}, \mathrm{ACT}}$ & Active Ordinary heterotrophic organisms & 10 & $\mathrm{~g} \mathrm{COD} \cdot \mathrm{m}^{-3}$ \\
$\mathrm{X}_{\mathrm{EPS}}$ & Extracellular Polymer Substances & 1 & $\mathrm{~g} \mathrm{COD} \cdot \mathrm{m}^{-3}$ \\
$\mathrm{X}_{\mathrm{STO}}$ & Storage Polymer Substances & 1 & $\mathrm{~g} \mathrm{COD} \cdot \mathrm{m}^{-3}$ \\
\hline
\end{tabular}

A partial list of the kinetic parameter values established through model calibration is summarized in Table. The kinetic parameters shown were added to represent the pathways incorporated to the modified model framework for soluble substrate, EPS production, adsorption/flocculation and the production of storage polymers.

Figure presents a comparison of the model results as a function of SRT and DO. These predictions are compared to the corresponding experimental data from Jimenez et al., 2014. Overall, the model predictions described the experimental trends.

Colloidal substrate removal was calibrated by adjusting the adsorption rate parameter $\mathrm{q}_{\mathrm{ADS}}$ $\left(0.08 \mathrm{~d}^{-1}\right)$ and the surface limitation parameter $\mathrm{K}_{\mathrm{SL}}(0.002)$ until the HRAS model results trended well with the experimental date. Future research includes experiments to further validate these parameter values. 
Table 5 Partial list of default parameter values for the mass-balance equations

\begin{tabular}{|c|c|c|c|}
\hline Symbol & Name & Value & Unit \\
\hline$\overline{\mathrm{K}_{\mathrm{B}, \mathrm{HYD}}}$ & Saturation coefficient for $\mathrm{X}_{\mathrm{B}} / \mathrm{X}_{\mathrm{OHO}}$ & 0.03 & $\mathrm{~g} \mathrm{X} \mathrm{X}_{\mathrm{B}} / \mathrm{g} \mathrm{X}_{\mathrm{OHO}}$ \\
\hline$\mu_{\mathrm{OHO}}$ & Maximum growth rate of $\mathrm{X}_{\mathrm{OHO}}$ on $\mathrm{S}_{\mathrm{Bf}}$ & 7.0 & $\mathrm{~d}^{-1}$ \\
\hline $\mathrm{K}_{\mathrm{Bf}}$ & Half-saturation coefficient for $\mathrm{S}_{\mathrm{Bf}}\left(\mathrm{X}_{\mathrm{OHO}}\right)$ & 2.0 & $\mathrm{~g} \mathrm{~S} \mathrm{Bf}_{\mathrm{Bf}} \cdot \mathrm{m}^{-3}$ \\
\hline $\mathrm{K}_{\mathrm{Bs}}$ & Half-saturation coefficient for $\mathrm{S}_{\mathrm{Bs}}\left(\mathrm{X}_{\mathrm{OHO}}\right)$ & 15.0 & $\mathrm{~g} \mathrm{~S}_{\mathrm{Bs}} \cdot \mathrm{m}^{-3}$ \\
\hline $\mathrm{b}_{\mathrm{OHO}}$ & Decay rate for $\mathrm{X}_{\mathrm{OHO}}$ & 0.62 & $\mathrm{~d}^{-1}$ \\
\hline $\mathrm{K}_{\mathrm{O}, \mathrm{OHO}}$ & Half-saturation coefficient for $\mathrm{S}_{\mathrm{O} 2}\left(\mathrm{X}_{\mathrm{OHO}}\right)$ & 0.1 & $\mathrm{~g} \mathrm{~S}_{\mathrm{O} 2} \cdot \mathrm{m}^{-3}$ \\
\hline $\mathrm{K}_{\text {Nox }}$ & Half-saturation coefficient for $\mathrm{S}_{\mathrm{NOx}}\left(\mathrm{X}_{\mathrm{OHO}}\right)$ & 0.5 & $\mathrm{~g} \mathrm{~S}_{\mathrm{NOx}} \cdot \mathrm{m}^{-3}$ \\
\hline $\mathrm{K}_{\mathrm{NHx} \text {,nut }}$ & Nutrient half-saturation coefficient & 0.05 & $\mathrm{~g} \mathrm{~S}_{\mathrm{NHx}} \cdot \mathrm{m}^{-3}$ \\
\hline $\mathrm{q}_{\mathrm{ADS}}$ & Rate constant for adsorption & 0.08 & $\mathrm{~d}^{-1}$ \\
\hline $\mathrm{K}_{\mathrm{SL}}$ & Half-saturation coefficient for surface limitation & 0.002 & - \\
\hline qSTO & Rate constant for growth on $\mathrm{X}_{\mathrm{STO}}\left(\mathrm{X}_{\mathrm{OHO}}\right)$ & 2.0 & $\mathrm{~d}^{-1}$ \\
\hline $\mathrm{k}_{\mathrm{EPS}, \mathrm{MAX}}$ & EPS formation coefficient & 0.25 & $\mathrm{~g} \mathrm{COD}_{\mathrm{EPS}} \cdot \mathrm{gVSS}^{-1}$ \\
\hline $\mathrm{q}_{\mathrm{EPS}, \mathrm{HYD}}$ & EPS hydrolysis & 0.12 & $\mathrm{~d}^{-1}$ \\
\hline $\mathrm{K}_{\mathrm{EPS}}$ & Half-saturation coefficient for EPS $\left(\mathrm{X}_{\mathrm{OHO}}\right)$ & 100 & $\mathrm{gX}_{\mathrm{EPS}} \cdot \mathrm{m}^{-3}$ \\
\hline $\mathrm{q}_{\mathrm{XB}, \mathrm{HYD}}$ & Particulate COD Hydrolysis Rate Constant & 2.75 & $\mathrm{~d}^{-1}$ \\
\hline $\mathrm{k}_{\mathrm{STO}, \mathrm{MAX}}$ & Maximum Production Yield for Storage Polymers & 0.58 & $\mathrm{~g} \mathrm{X}_{\mathrm{STO}} \cdot \mathrm{gS}_{\mathrm{Bf}}^{-1}$ \\
\hline $\mathrm{f}_{\mathrm{STO}}$ & Fraction of $\mathrm{X}_{\mathrm{STO}}$ in the Active Biomass & 0.15 & - \\
\hline $\mathrm{q}_{\mathrm{STO}, \mathrm{HYD}}$ & Storage Hydrolysis Rate Constant & 3.0 & $\mathrm{~d}^{-1}$ \\
\hline $\mathrm{K}_{\mathrm{STO}, \mathrm{HYD}}$ & Hydrolysis Half-saturation coefficient for $\mathrm{X}_{\mathrm{STO}}\left(\mathrm{X}_{\mathrm{OHO}}\right)$ & 0.15 & $\mathrm{gX}_{\mathrm{STO}} \cdot \mathrm{gX}_{\mathrm{OHO}}{ }^{-1}$ \\
\hline $\mathrm{K}_{\mathrm{O}, \mathrm{EPS}}$ & Half-saturation coefficient for $\mathrm{S}_{\mathrm{O} 2}$ & 0.55 & $\mathrm{~g} \mathrm{~S}_{\mathrm{O} 2} \cdot \mathrm{m}^{-3}$ \\
\hline$\mu_{\mathrm{OHO}, \mathrm{SLOW}}$ & Maximum growth rate of $\mathrm{X}_{\mathrm{OHO}}$ on $\mathrm{S}_{\mathrm{Bs}}$ & 3.0 & $d^{-1}$ \\
\hline
\end{tabular}




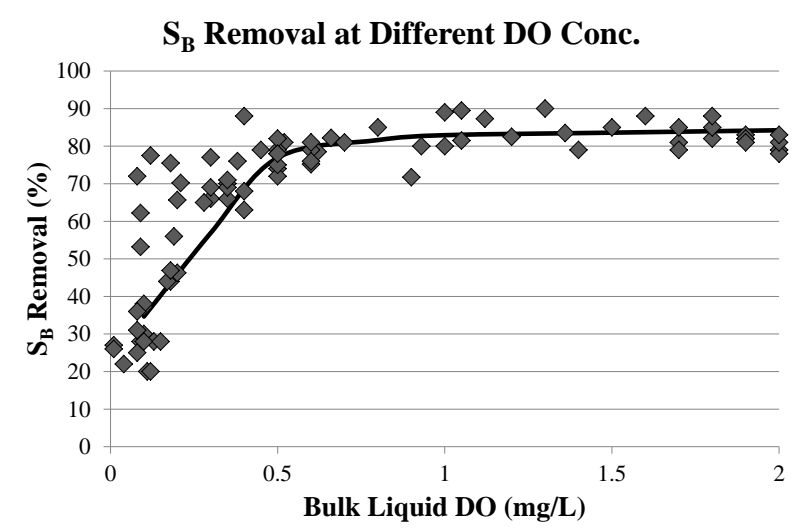

-Model $\diamond$ ffCOD

EPS Production at Different DO Conc.

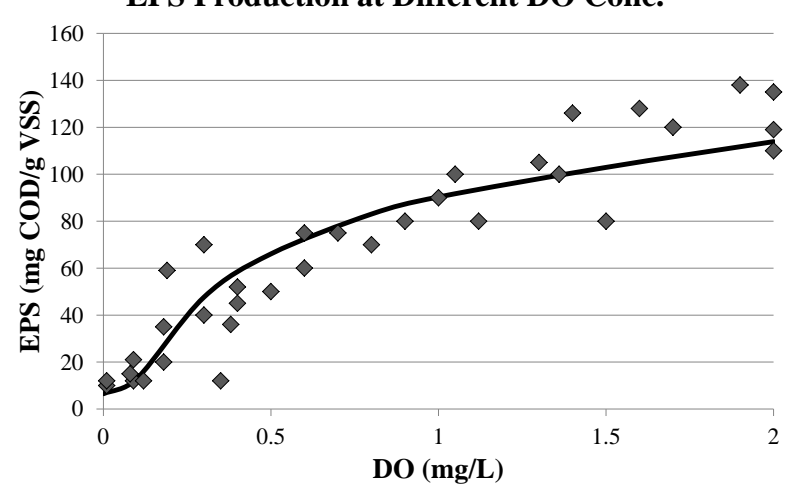

-Model $\diamond$ Obs.

CB Removal at Different DO Conc.
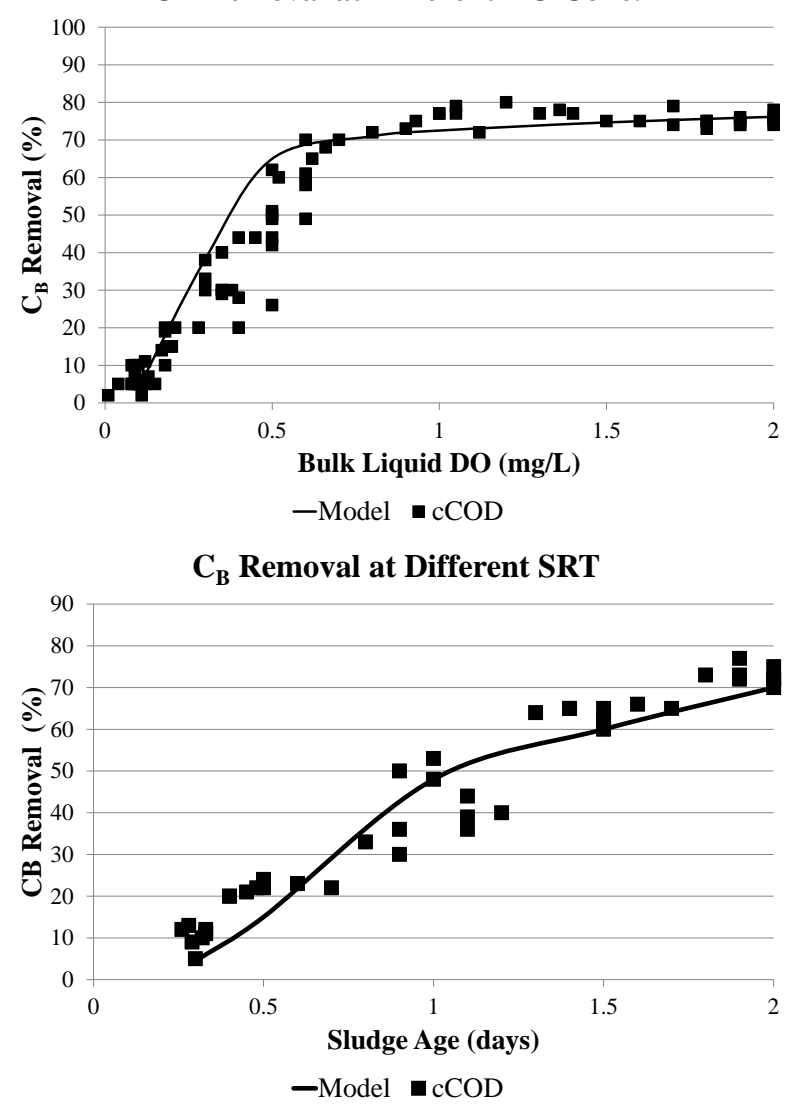
Figure 3 Model calibration results compared to the University of New Orleans pilot plant

results

\section{Modified Model Validation}

The HRSD dataset used in this analysis spanned a five week period where the pilot plant had reached steady-state operating condition. Weakly averages were calculated for that period and the data reduced to a format compatible with the model framework. Influent values for select state variables are shown in Table. State variables not shown in Table 5 are generated by the model as such, they were not measured. The measured values were input into the model representing daily values for each corresponding week resulting in a dynamic analysis period of 35 days. Dynamic input data for model validation also included DO profiles, and return and waste activated sludge (RAS and WAS) flow.

Table 6 Influent state variables for model validation

\begin{tabular}{cccccccc}
\hline $\begin{array}{c}\text { Time } \\
(\mathbf{w e e k})\end{array}$ & $\begin{array}{c}\mathbf{Q} \\
\left(\mathbf{m}^{\mathbf{3}} \mathbf{\mathbf { d }}\right)\end{array}$ & $\begin{array}{c}\mathbf{S}_{\mathbf{U}} \\
\left(\mathbf{g} / \mathbf{m}^{\mathbf{3}}\right)\end{array}$ & $\begin{array}{c}\mathbf{S}_{\mathbf{B f}} \\
\left(\mathbf{g} / \mathbf{m}^{\mathbf{3}}\right)\end{array}$ & $\begin{array}{c}\mathbf{S}_{\mathbf{B s}} \\
\left(\mathbf{g} / \mathbf{m}^{\mathbf{3}}\right)\end{array}$ & $\begin{array}{c}\mathbf{C}_{\mathbf{B}} \\
\left(\mathbf{g} / \mathbf{m}^{\mathbf{3}}\right)\end{array}$ & $\begin{array}{c}\mathbf{X}_{\mathbf{B}} \\
\left(\mathbf{g} / \mathbf{m}^{\mathbf{3}}\right)\end{array}$ & $\begin{array}{c}\mathbf{X}_{\mathbf{O H O}} \\
\left(\mathbf{g} / \mathbf{m}^{\mathbf{3}}\right)\end{array}$ \\
\hline 1 & 24.84 & 28.00 & 78.00 & 43.00 & 44.45 & 312 & 10.00 \\
2 & 24.84 & 19.80 & 79.00 & 58.20 & 40.30 & 278 & 10.00 \\
3 & 24.84 & 19.00 & 113.00 & 31.00 & 32.28 & 355 & 20.00 \\
4 & 24.84 & 27.00 & 99.00 & 26.00 & 53.03 & 362 & 10.00 \\
5 & 24.84 & 26.30 & 112.00 & 24.70 & 57.40 & 299 & 18.00 \\
\hline
\end{tabular}

Dynamic simulations were performed in order to compare the predicted effluent values with those of the HRSD pilot plant. As previously mentioned, two different framework models were developed for this study and Figure 4 presents a model comparison of the effluent soluble biodegradable fraction $\left(\mathrm{S}_{\mathrm{Bf}}+\mathrm{S}_{\mathrm{Bs}}\right)$. Based on these results, the Dual Substrate model predicts better the performance of the HRSD pilot plant. In general, the Dual Substrate model provided a better prediction of the HRSD pilot plant results. Figure 5 shows the model predicted values for $S_{B f}$ and $S_{B s}$. Based on the definition of the $S_{B f}$ and $S_{B s}$ fractions discussed previously, the model predicts almost full removal of $S_{B f}$ whereas the $S_{B s}$ fraction passes through the biological reactor operated at an SRT of approximately 0.2 days.

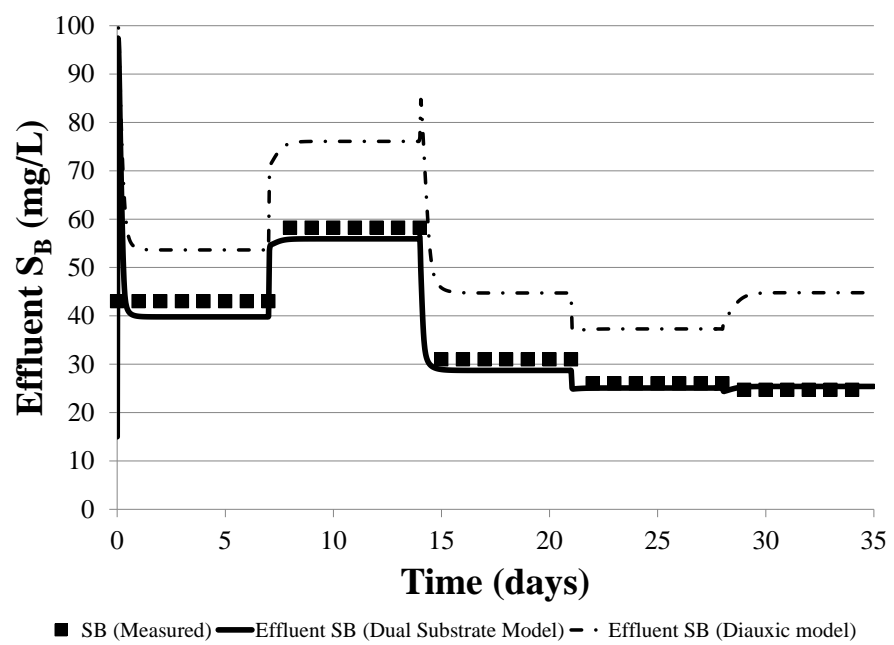


Figure 4 Comparison of the effluent $S_{B}$ concentration from the Diauxic and Dual Substrate models

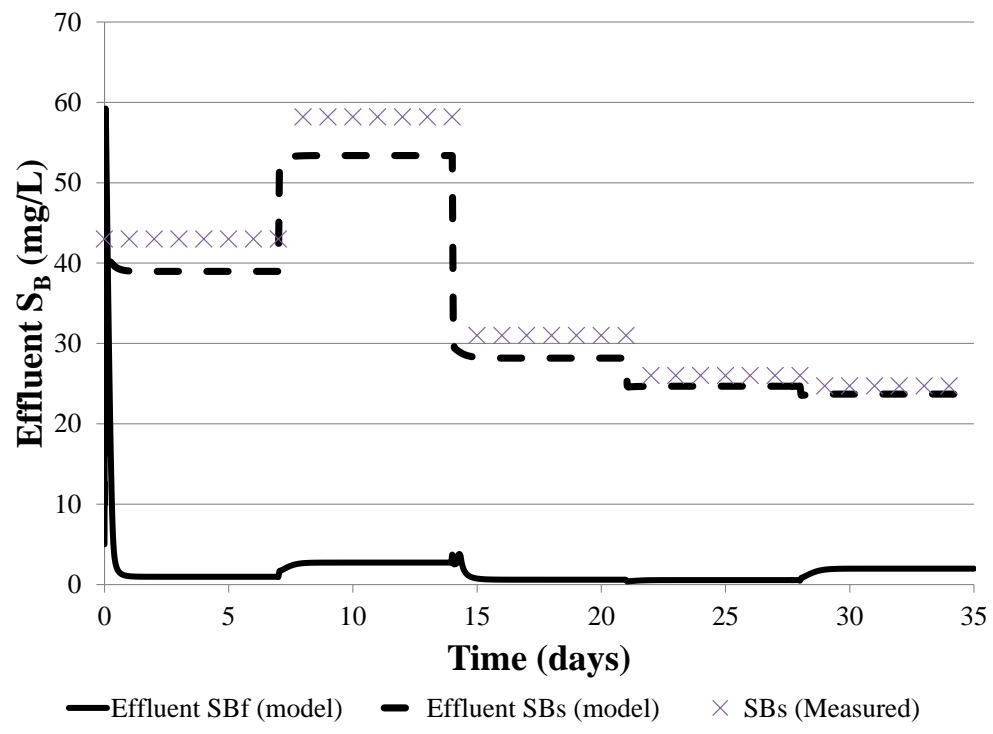

Figure 5 Dual Substrate model prediction for $\mathrm{S}_{\mathrm{Bf}}$ and $\mathrm{S}_{\mathrm{Bs}}$ concentrations

A carbon balance performed around the HRSD pilot plant shows a total COD balance distribution of approximately $55 \%$ of the COD is in the effluent, $30 \%$ of the COD is the WAS and $15 \%$ of COD was allocated to mineralization. The Dual Substrate model predicts (using a $\mathrm{k}_{\mathrm{STO}, \mathrm{PC}}$ in range 0.40 to 0.45 ) a COD distribution of $38 \%$ of the COD in the effluent, $46 \%$ of the COD in the WAS and $16 \%$ of COD to mineralization. The difference in the COD distribution between the HRSD pilot plant and the model predictions can be attributed to excess solids carryover in the effluent of the pilot plant which was not predicted by the model. Therefore, the model predicted more COD being removed from the liquid and being directed to the WAS stream. It is important to note that the percent COD mineralized is well predicted by the model.

Figure 6 presents the model predictions and measured values for effluent colloidal COD concentration. The adsorption/flocculation model added to the model predicts negligible removal of the colloidal COD fraction. In fact, at the conditions that the pilot plant was operated, at increase in colloidal COD concentration through the reactor was predicted by the model and observed in the HRSD pilot plant data. The model suggests that because of the unfavorable flocculation conditions, a major fraction of the EPS being generated through biological activity are ending up in the effluent and being recorded as colloidal COD. At the conditions simulated, the model predicted approximately $15 \mathrm{mg} \mathrm{COD} \mathrm{EPS}_{\mathrm{gX}} / \mathrm{g}_{\mathrm{VSS}}$. 


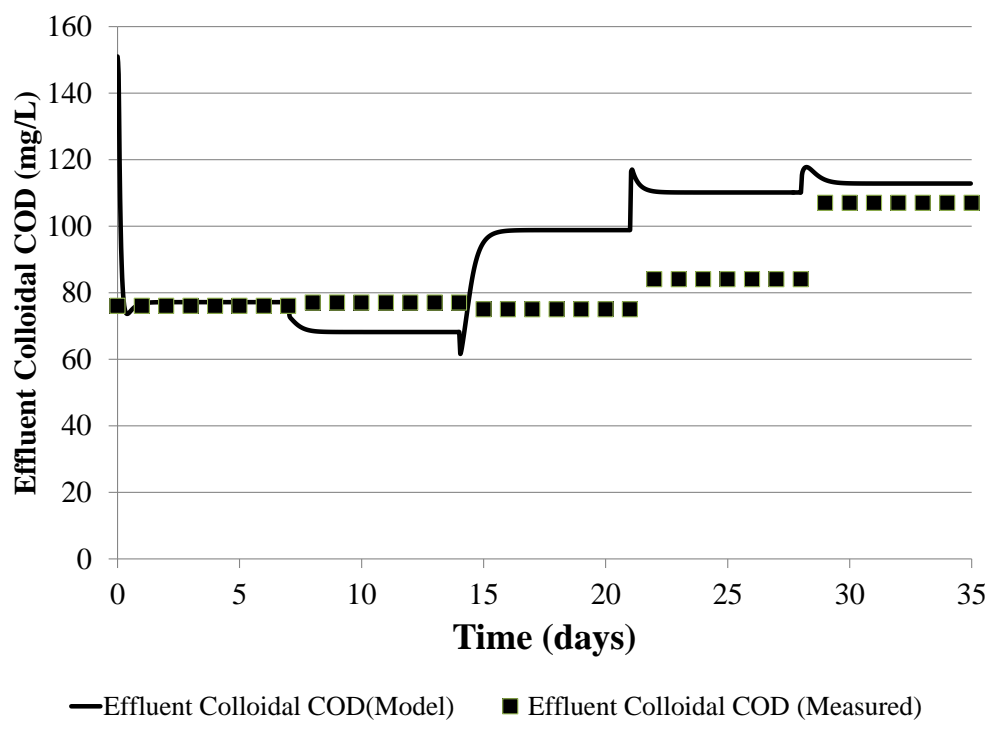

Figure 6 Comparison of HRSD pilot plant and model predicted effluent colloidal COD

\section{ASM1 Model}

The ASM1 model was developed to simulate the aerobic and anoxic treatment of domestic wastewater based on typical operating conditions, e.g. SRT greater than 3 days. These models were not developed to model activated sludge systems with very high organic loads or low SRTs (less than 1 day) where bioflocculation/adsorption of particulate and colloidal (slowly biodegradable) substrate and storage may become rate limited (Henze et al., 2000). In addition, the very short HRT of some HRAS systems may result in differences in predicted performance since the implicit assumption that substrate reactions can proceed to completion may no longer be valid. These models assume a two-step process for the removal of slowly biodegradable substrate (primarily particulate substrate and colloidal substrate): instantaneous bioflocculation and complete hydrolysis of particulate and colloidal substrate followed by oxidation of soluble biodegradable substrate. However, researchers have overlooked the effect of the kinetics of bioflocculation on the overall particulate and colloidal substrate removal process and have concentrated their attention on the kinetics of hydrolysis and oxidation when modeling carbon removal in activated-sludge systems.

Using the ASM1 framework (Henze et al., 2000) and default parameter values, e.g. $\mu_{\max }=6.0$ $\mathrm{d}^{-1}$ and $\mathrm{Ks}=20 \mathrm{mg} / \mathrm{L}$, the ASM1 model generated the results shown in Figure 7. This figure shows both the ASM1 model results and the HRSD pilot plant experimental data. These results clearly shows that the ASM1 framework when used at low SRT and high F:M ratios as those employed in HRAS systems, the model does not properly predicts the removal of organic substrate. In addition, it should be noted as previously mentioned, the structure of the framework for the ASM1 model does not describe properly the removal of slowly biodegradable substrate. It should be noted that the ASM1 model does not include colloidal $\mathrm{COD}$ as a state variable so no comparison was possible. Overall, the ASM1 model over predicts the performance of the HRAS system. 


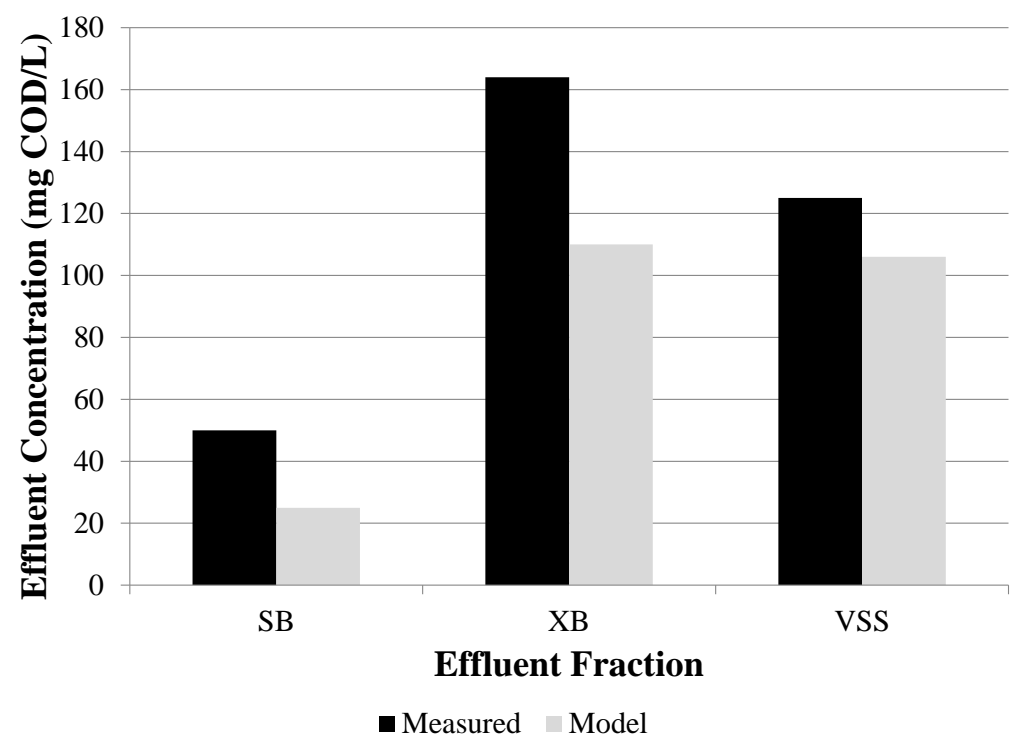

Figure 7 Comparison of HRSD pilot plant effluent quality and ASM1 model predictions

\section{CONCLUSIONS}

A new proposed model framework was developed to describe the organic substrate transformation in the high-rate activated sludge (HRAS) process. Data from two HRAS pilot plants were used to calibrate and to validate the proposed model framework for HRAS systems. Two soluble substrate models were evaluated during this study including a Dual Substrate and a Diauxic model. Both model frameworks used two state variables for biodegradable soluble susbtrate $\left(\mathrm{S}_{\mathrm{Bf}}\right.$ and $\left.\mathrm{S}_{\mathrm{Bs}}\right)$ and two biomass populations (fast growing and slow growing $\mathrm{X}_{\mathrm{OHO}, \mathrm{ACT}}$ with maximum growth rates of 7.0 and $3.0 \mathrm{~d}^{-1}$, respectively). Overall, the Dual Substrate model provided better results than the Diauxic model; and therefore, it was adopted during this study. The modified model framework described sucesfully the production of EPS at low SRT and variable DO conditions using a $\mathrm{k}_{\mathrm{EPS}, \mathrm{MAX}}$ value of $0.15 \mathrm{~g}$ $\mathrm{COD}_{\mathrm{EPS}} / \mathrm{g}$ VSS and a $\mathrm{K}_{\mathrm{O} \text {,EPS }}$ value of $0.55 \mathrm{~g} \mathrm{~S}_{\mathrm{O} 2} / \mathrm{m}^{3}$. This provided valuable information to relate EPS production and bioflocculation/adsorption of colloidal susbtrate. Colloidal substrate removal was calibrated by adjusting the $\mathrm{q}_{\mathrm{ADS}}$ to $0.08 \mathrm{~d}^{-1}$ and $\mathrm{K}_{\mathrm{SL}}$ to 0.002 . Finally, the proposed model framework accurately predicted substrate utilization and mass balances, transformation and redicrection of COD in the HRAS system.

\section{REFERENCES}

Böhnke, B., Diering, B., and Zuckut, S. (1997) Cost-effective wastewater treatment process for removal of organics and nutrients. Water Engineering \& Management 7, 30-35.

Böhnke, B., \& Diering, B. C. (1980). Two-stage activated sludge process. Canada.

Carucci, A., Dionisi, D., Majone, M., Rolle, E., \& Smurra, P. (2001). Aerobic storage by activated sludge on real wastewater. Water Research, 35(16), 3833-3844.

Ekama, G. A., Dold, P. L., \& Marais Van, G. R. (1986). Procedure for determining influent COD fractions and the maximum specific growth rate of heterotrophs in activated sludge systems. Water Science \& Technology, 18(6), 91-114.

Haider, S., Svardal, K., Vanrolleghem, P. A., \& Kroiss, H. (2003). The effect of low sludge age on wastewater fractionation (SS, SI).

Henze, M., Gujer, W., Mino, T., \& Vanloosdrecht, M. (2000). Activated Sludge Models ASM1, ASM2, ASM2d and ASM3

Jimenez, J. A. (2002). Kinetics of COD Removal in the Activated Sludge Process Including Bioflocculation. (Doctor of Philosophy), University of New Orleans. 
Jimenez, J. A., Bott, C. B., Murthy, S., Nogaj, T., Randall, A., \& Wett, B. (2013). Removal of organic substrate in the high-rate activated sludge process and its kinetics implications. Submitted to Water Research.

Jimenez, J. A., La Motta, E. J., \& Parker, D. S. (2005). Kinetics of removal of particulate chemical oxygen demand in the activated-sludge process. Water Environment Research, 77(5), 437-446.

Laspidou, C. S., \& Rittmann, B. E. (2002a). Non-steady state modeling of extracellular polymeric substances, soluble microbial products, and active and inert biomass. Water Research, 36(8), 1983-1992.

Laspidou, C. S., \& Rittmann, B. E. (2002b). A unified theory for extracellular polymeric substances, soluble microbial products, and active and inert biomass. Water Research, 36(11), 2711-2720.

Mamais, D., Jenkins, D., \& Pitt, P. (1993). A rapid physical-chemical method for he determination of readily biodegradable soluble COD in municipal wastewater. Water Research, 27(1), 195-197.

Miller, M. W., Jimenez, J., Murthy, S., Kinnear, D., Wett, B., \& Bott, C. B. (2013). Mechanisms of Cod Removal in the Adsorption Stage of the A/B Process. Paper presented at the Weftec 2013, Chicago, Illinois.

Nogaj, T. M., Randall, A. A., Jimenez, J. A., Takacs, I., Bott, C. B., Miller, M. W., .. Wett, B. (2013). Mathematical Modeling of Carbon Removal in the High-Rate Activated Sludge System: Model Presentation and Application. Water Environment Federation, 20.

Pala-Ozkok, I., Rehman, A., Kor-Bicakci, G., Ural, A., Schilhabel, M. B., Ubay-Cokgor, E., ... Orhon, D. (2013). Effect of sludge age on population dynamics and acetate utilization kinetics under aerobic conditions. Bioresource Technology, 143, 68-75.

Schulze-Rettmer, R., \& Zuckut, S. W. (1998). Treatment of textile dyeing wastewater by adsorption/biooxidation process. Textile Chemist and Colorist, 30(5), 19-23.

Third, K. A., Newland, M., \& Cord-Ruwisch, R. (2003). The effect of dissolved oxygen on PHB accumulation in activated sludge cultures. Biotechnology and Bioengineering, 82(2), 238-250. 
WWTmod

2 201 


\section{POSTER}

CONTRIBUTIONS 


\title{
Towards Calibration of Phosphorus (P) Removal Plant- Wide Models
}

\author{
D. S. Ikumi ${ }^{1}$, P.A. Vanrolleghem ${ }^{2}$, C.J. Brouckaert ${ }^{3}$, M.B. Neumann ${ }^{2,4,5}$ and G.A. Ekama ${ }^{1}$ \\ ${ }^{1}$ Water Research Group, Dept of Civil Engineering, University of Cape Town, \\ Rondebosch 7701, Cape Town, South Africa. (E-mail david.ikumi@uct.ac.za, \\ george.ekama@uct.ac.za) \\ ${ }^{2}$ modelEAU, Département de génie civil et de génie des eaux, Université Laval, 1065 av. de la Médecine, \\ Québec (QC), G1V 0A6, Canada. (E-mail: peter.vanrolleghem@gci.ulaval.ca) \\ ${ }^{3}$ Pollution Research Group, School of Chemical Engineering, University of KwaZulu-Natal, \\ Durban 4041, South Africa. (E-mail: brouckae@ukzn.ac.za) \\ ${ }^{4}$ Basque Centre for Climate Change, Alameda Urquijo $4-4^{\circ}$, 48008, Bilbao, Spain \\ (E-mail: marc.neumann@bc3research.org) \\ ${ }^{5}$ IKERBASQUE, Basque Foundation for Science, 48011, Bilbao, Spain
}

Keywords

Plant-wide modelling; biological phosphorus removal; calibration protocol; wastewater treatment

\begin{abstract}
INTRODUCTION
Over the past years, wastewater treatment plant (WWTP) mathematical models have been advancing towards their widespread application for sizing and operation of treatment plants to minimize energy consumption and cost while maximizing nutrient recovery and effluent quality. Effective utilisation of these models requires that they are well calibrated. However, difficulties (with important parameters not identified and uncertainties in intepretation of model output results) can be experienced in model calibration, especially due to (i) the intricate relationships of model output variables with model input factors (where parameters are inter-related to various model outputs), resulting in non-linearity, and (ii) the limitations (due to expensive and/or time consuming experimental methods) experienced in procuring and reconciling data required for determination of the model input factors (especially when the model has significantly large numbers of unknown parameters and model components).

The BIOMATH protocol, developed by Vanrolleghem et al. (2003), provides a systematic approach for calibration. The main objective of this paper is to apply the BIOMATH protocol in providing a guidance towards calibration of a plant-wide model that includes phosphorus. The three phase (aqueous-gas-solid) University of Cape Town plant wide (UCT-PW) model (Ikumi et al., 2013) that was calibrated against the experimental layout described below is used as a case study for this calibration procedure.
\end{abstract}

\section{EXPERIMENTAL SYSTEM LAYOUT}

The experimental layout of Ikumi (2011) is used in this study. It replicates at laboratory scale three WWTP schemes, comprising (1) a Modified Ludzack - Ettinger (MLE) nitrificationdenitrification (ND) activated sludge (AS) system treating raw sewage (MLE 1) with anaerobic digestion (AD) of its waste activated sludge (WAS) in AD system number 1 (i.e., AD1), (2) an identical MLE system (MLE 2) treating settled sewage with AD of its WAS in AD2 and (3) a membrane (MBR) University of Cape Town (UCT) ND enhanced biological P removal (NDEBPR) system treating settled sewage with (i) AD of its WAS in AD3 and (ii) anoxic/aerobic digestion (AAD) of its WAS in two intermittently aerated (3hour air on, 3hour air off) aerobic digesters, AAD1 fed with concentrated WAS (2x, 20gTSS/l) and AAD2 fed with dilute WAS $(1 / 3,3.3 \mathrm{gTSS} / 1)$. 


\section{MODEL DESCRIPTION}

The UCT three phase plant wide model was developed for simulating the biological processes to track and predict the output of materials (COD, carbon $(\mathrm{C})$, hydrogen $(\mathrm{H})$, oxygen $(\mathrm{O})$, nitrogen $(\mathrm{N})$, phosphorus $(\mathrm{P})$, magnesium $(\mathrm{Mg})$, potassium $(\mathrm{K})$ and calcium $(\mathrm{Ca}))$ along the unit processes of a WWTP. It comprises three sub-models, integrated for simulation of the entire WWTP under various configurations (e.g. NDBEPR AS system linked to an AD or an anoxic-aerobic digestion (AAD) for WAS stabilisation). These sub-models include:

1. The ionic speciation model (Brouckaert et al., 2010). This model includes pairing of ionic components (the set of model ionic species is given in Table 3) and inter-phase transfers of component species. Table 4 gives an example of a set of equilibrium and mass balance equations used in the ionic speciation subroutine.

2. The ASM2-3P model: This is the Activated Sludge Model No. 2 (ASM2, Henze et al., 1995), modified to include the ionic speciation model (Brouckaert et al., 2010), the Inorganic Settleable Solids (ISS) model of Ekama and Wentzel (2004) and including multiple mineral precipitation according to Musvoto et al. (2000a,b).

3. The ADM3P Model: This is the University of Cape Town Anaerobic Digestion Model (UCTADM; Sötemann et al., 2005), modified to include the hydrolysis of multiple organic sludge types (PS, ND WAS, NDBEPR WAS and PS-WAS blends), the Ekama and Wentzel (2004) ISS model, multiple mineral precipitation processes according to Musvoto et al. (2000a, b) and the Brouckaert et al. (2010) aqueous speciation model which facilitates ionic speciation (Ikumi et al., 2011).

For their compatibility, the ASM2-3P and ADM3P models have the same comprehensive set of model components (supermodel approach, Volcke et al., 2006; model components given in Table 1 and applied stoichiometric processes in Table 4), including parameterized stoichiometry for the bioprocesses and sharing the same ionic speciation subroutine model (1).

\section{MODEL EVALUATION PROCESS}

1. Model Verification: To initiate the evaluation of the UCT-PW model (Ikumi et al., 2013), the systematic method proposed by Hauduc et al. (2010) was applied to verify that material $(\mathrm{COD}, \mathrm{C}, \mathrm{H}, \mathrm{O} \mathrm{N}, \mathrm{P}, \mathrm{Mg} \mathrm{K}$ and $\mathrm{Ca}$ ) balances were achieved in the determination of all stoichiometric processes.

2. Parameter Values: The initial values for suitable kinetic and stoichiometric parameters as obtained experimentally or from literature were entered, and given the typical value range, determined according to the methods proposed by Brun et al. (2002).

3. Senitivity Analysis: The parameters were subsequently evaluated using two different methods of global sensitivity analysis: (1) Standardised Regression Coefficients (SRC) and (2) Morris Screening. The results obtained using these methods are used to identify important parameters (prioritisation of those with greatest effects), non-influential parameters (those that can be 'fixed' at any value within their range without effecting outputs) and interacting parameters (Neumann, 2012).

4. Model Calibration and Validation: Non-influential parameters were set at their default values and random samples were drawn from the remaining subset of parameters. For the 
sampled parameter sets simulations were conducted and predicted model outputs were compared with observed outputs. During this calibration phase, a consistent set of parameter values was used to simulate all experimental systems/periods, and detailed explanations of observed discrepancies (if any) were reported by Ikumi (2011). The sensitivity analysis together with intuitive observation on a steady state stoichiometric model was a significant role in selecting the 'best' set of parameters. It was noted that influent sewage and sludge characterisation, and determination of hydrolysis kinetic rates were important requirements prior to any simulation. In $\mathrm{AD}$, the hydrolysis process is the slowest one and requires the best possible calibration. The hydrolysis kinetic constants were fit to match experimental data by Ikumi et al. (2013), using the non-linear regression. The biological reactions following hydrolysis are limited by the hydrolysis rate, hence their kinetic parameters are not identifiable from these experimental data, and so were adopted without adjustment from literature. Most of the parameterised influent and sludge characteristics could be obtained or calculated from directly measured results (Ikumi, 2011). This is because the unbiodegradable fractions of influent organics and the characteristics of the biodegradable feed components have a significant effect on the quality of model predictions, but are usually specific to the feed source.

5. Model Performance Results: Below are observations in experimental behaviour of $\mathrm{P}$ removal systems, replicated by the UCT-PW model as required to promote confidence in its application:

i. Applying the ASM2-3P model to an MLE system with ND does not stimulate EBPR (i.e., there is no PAO growth, hence no polyphosphate (PP) storage) and its effluent $\mathrm{P}$ comprises mainly the OP not utilized by the biomass (mainly OHOs) for growth.

ii. For MLE systems with little or no nitrification taking place, high quantities of $\mathrm{P}$ and acetate in the un-aerated ('anoxic') zone will result in the growth of phosphorus accumulating organisms (PAOs) rather than ordinary heterotrophic organisms (OHOs) only as expected in fully aerobic or nitrogen $(\mathrm{N})$ removal systems. The concentration of acetate available for this PAO growth (and associated EBPR) depends on the rate of fermentation of biodegradable soluble organics (BSO) that occurs and the concentration of nitrate that gets recycled to the anaerobic reactor in these systems. Moreover, as noted in 3 and 5-stage Bardenpho systems - in winter, when denitrification is lower, the nitrates recycled to the anaerobic reactor can be sufficiently high to suppress EBPR. The ASM2-3P model predicts this behaviour qualitatively well.

iii. Applying ASM2-3P for sludge treatment with anoxic-aerobic digestion (AAD), the absence of VFA and an anaerobic period renders the PAOs unable to compete with the OHOs. Consequently, the PAOs do not grow and undergo endogenous respiration and die, releasing their stored PP as magnesium $(\mathrm{Mg})$, calcium $(\mathrm{Ca})$, potassium $(\mathrm{K})$ and orthophosphate (OP). Struvite $\left(\mathrm{MgNH}_{4} \mathrm{PO}_{4}\right)$ precipitation occurs when the concentration of $\mathrm{Mg}$, ammonia and $\mathrm{OP}$ is high enough (i.e., the struvite is supersaturated) in the mixed liquor. If the ammonia is low $(<1 \mathrm{mg} / \mathrm{N} / l)$, due to nitrification, K-struvite $\left(\mathrm{MgKPO}_{4}\right)$ forms.

iv. In $\mathrm{AD}$, organically bound $\mathrm{N}$ is released with the hydrolysis of biodegradable organics in the non-ionic $\mathrm{NH}_{3}$ form, which are non-reference species (reference species of a weak acid system being one that, when added to pure water, creates a solution state, relative to which the alkalinity of the weak acid system is measured) for the ammonia weak $\mathrm{acid} / \mathrm{base}$ system. Therefore, the aqueous alkalinity increases by the concentration of $\mathrm{NH}_{3}$ transferred from the organics (the $\mathrm{NH}_{3}$ being an intrinsic alkalinity content of the organics) to the aqueous phase. This is the main aqueous $\mathrm{H}_{2} \mathrm{CO}_{3}{ }^{*}$ alkalinity generation 
process in an AD treating PS or WAS that is not P-rich. For P-rich systems with PP, the aqueous $\mathrm{H}_{2} \mathrm{CO}_{3}{ }^{*}$ alkalinity increase also depends on $\mathrm{PP}$ and cell bound $\mathrm{P}$ release because $\mathrm{PP}$ is released as $\mathrm{H}_{2} \mathrm{PO}_{4}$ and biomass $\mathrm{P}$ is released as $\mathrm{H}_{3} \mathrm{PO}_{4}$, which interact with the other weak acid/base systems and influence $\mathrm{pH}$. The ADM3P model predicts the $\mathrm{pH}$ for both these systems (P- rich or not) really well.

v. In the dynamic model of the $\mathrm{AD}$, initially, PP release and poly-hydroxy-alkanoate (PHA) storage by PAOs takes place with the uptake of acetate, as would happen in the anaerobic part of the parent NDEBPR system. This results in increased alkalinity because the $\mathrm{PP}$ is released as $\mathrm{H}_{2} \mathrm{PO}_{4}{ }^{-}$. Because the PAOs also require alternating aerobic conditions for their growth, they cannot grow in the AD. Therefore, the PAOs are modelled to "die" in AD at a rate faster than their endogenous respiration; releasing their PHA and the remainder of their stored PP, adding more $\mathrm{H}_{2} \mathrm{PO}_{4}{ }^{-}$and alkalinity. Depending on the charge/proton balance requirements, some of the $\mathrm{H}_{2} \mathrm{PO}_{4}{ }^{-}$species become $\mathrm{HPO}_{4}{ }^{2-}$ species by reacting with $\mathrm{HCO}_{3}{ }^{-}$to form $\mathrm{HPO}_{4}{ }^{2-}, \mathrm{H}_{2} \mathrm{O}$ and $\mathrm{CO}_{2}$. The increase in $\mathrm{CO}_{2}$ gas increases the partial pressure of the gas phase, which influences the aqueous speciation. The split between the OP species co-dependent on the inorganic carbon (IC) system (and any other weak acid/base system that may be present), which together establish the $\mathrm{AD} \mathrm{pH}$.

vi. Because $\mathrm{H}_{3} \mathrm{PO}_{4}$ is the reference species for the $\mathrm{OP}$ weak acid/base system, the total alkalinity does not change with the slower release of organically bound $\mathrm{P}$, but the species that represent it do.

vii. The rapid release of PP and associated $\mathrm{Mg}^{2+}$ and the slow release of biomass $\mathrm{N}$ and $\mathrm{P}$ generate high concentrations of $\mathrm{P}, \mathrm{NH}_{4}{ }^{+}$and $\mathrm{Mg}^{2+}$ species in the $\mathrm{AD}$ liquor, which promotes struvite precipitation. This struvite precipitation decreases the total alkalinity and so results in re-speciation of the IC system, which increases the $\mathrm{CO}_{2}$ partial pressure and decreases $\mathrm{AD} \mathrm{pH}$.

\section{COMPARISON OF EXPERIMENTAL AND PREDICTED RESULTS}

Figures 1a to $\mathrm{f}$ show a comparison between the data measured and simulated by the three phase AD dynamic model for the AD1 fed with the NDBEPR WAS (i.e. the AD effluent from UCT NDBEPR linked to AD in a plant wide setting). Considering the general complexities in characterization of the AD influent (i.e. the NDBEPR WAS; Ikumi et al., 2013), the simulated results match quite well for COD removal (Fig 1a, which it should because the hydrolysis rates were calibrated on to the experimental results), and FSA (Fig1b) and OP (Fig 1c) release. Because all the $\mathrm{AD}$ products, including the $\mathrm{H}_{2} \mathrm{CO}_{3}$ and $\mathrm{H}_{3} \mathrm{PO}_{4}$ alkalinities and gas $\mathrm{CO}_{2}$ partial pressure $\left(\mathrm{p}_{\mathrm{CO} 2}\right)$, are entirely dependent on the composition of the biodegradable organics ( $\mathrm{x}, \mathrm{y}, \mathrm{z}, \mathrm{a}, \mathrm{b}, \mathrm{q}, \mathrm{c}, \mathrm{d}, \mathrm{e}$ in $\mathrm{C}_{\mathrm{x}} \mathrm{H}_{\mathrm{y}} \mathrm{O}_{\mathrm{z}} \mathrm{N}_{\mathrm{a}} \mathrm{P}_{\mathrm{b}} \mathrm{qMg}_{\mathrm{c}} \mathrm{K}_{\mathrm{d}} \mathrm{Ca}_{\mathrm{e}} \mathrm{PO}_{3}$ ), if the organics' composition entered into the model is not "correct", then the simulated and measured results will not match, even with $100 \%$ experimental material balances (which of course were not achieved on the UCT and AD systems). Improving the comparison between predicted and measured results is a complex exercise because multiple processes act on single compounds. For instance under-predicted FSA (Fig 1b) means the determined $\mathrm{N}$ content of the biodegradable part of the $\mathrm{OHO}$ and PAO biomass is too low, but this does not mean that the $\mathrm{H}_{2} \mathrm{CO}_{3}$ alkalinity also has to be under-predicted (through $\mathrm{NH}_{3}+\mathrm{H}_{2} \mathrm{CO}_{3} \rightarrow \mathrm{NH}_{4}{ }^{+}+\mathrm{HCO}_{3}{ }^{-}$, as it would for an AD fed with low P organics, Sötemann et al., 2005a,b) because the release of PP also produces alkalinity (through $\mathrm{MePO}_{3}+\mathrm{H}_{2} \mathrm{O} \rightarrow \mathrm{Me}^{+}+\mathrm{H}_{2} \mathrm{PO}_{4}{ }^{2-}$ ). 
Ikumi et al.

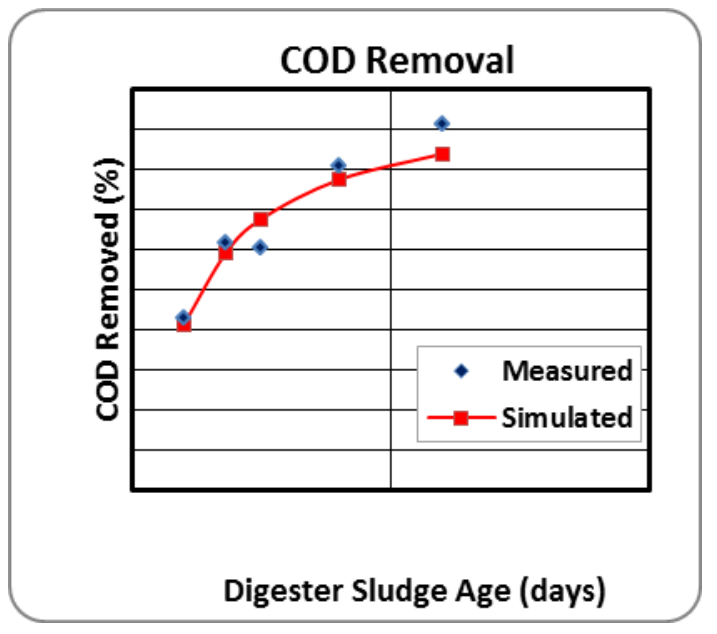

Figure 1a

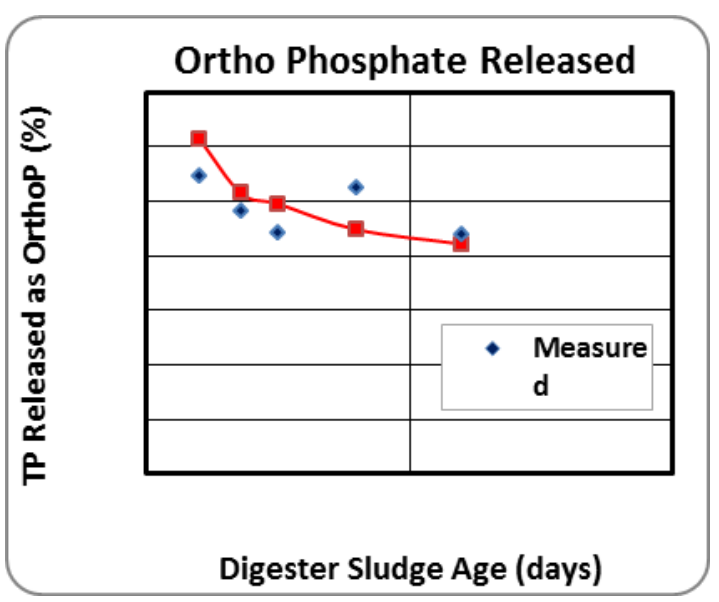

Figure 1c

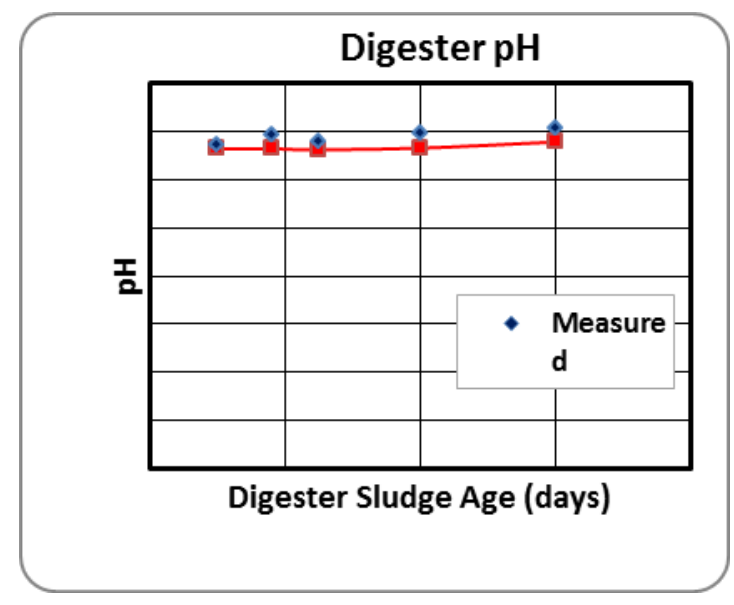

Figure 1e

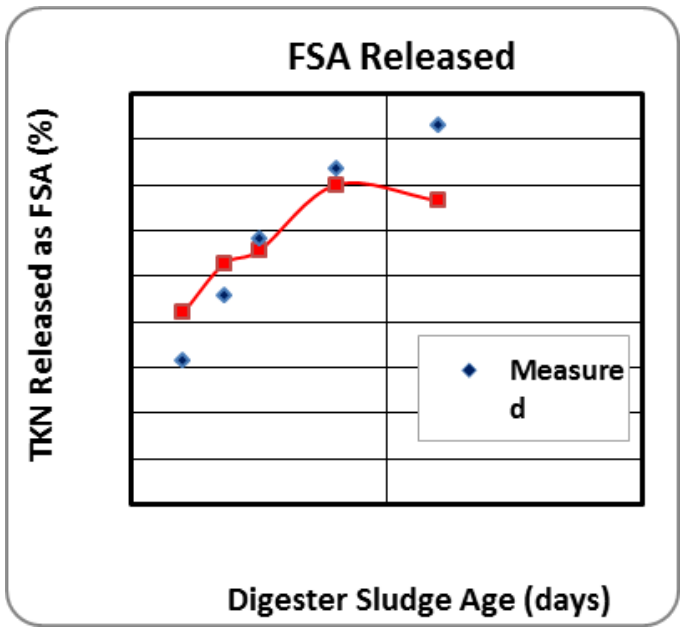

Figure 1b

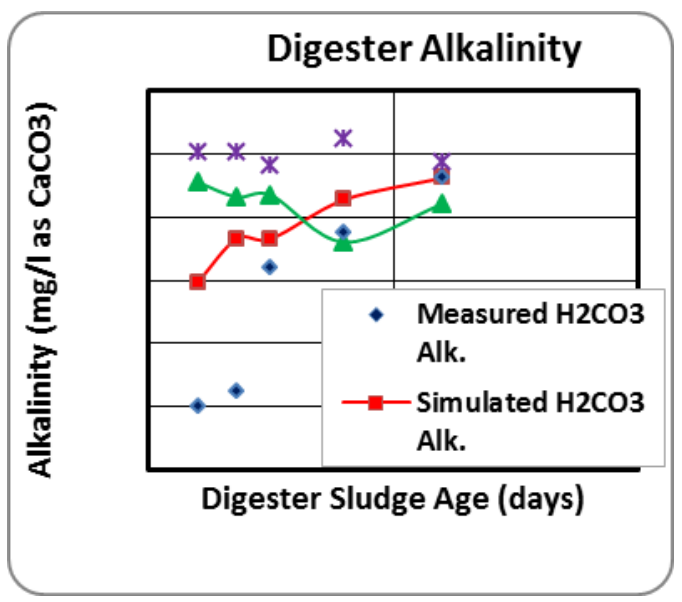

Figure 1d

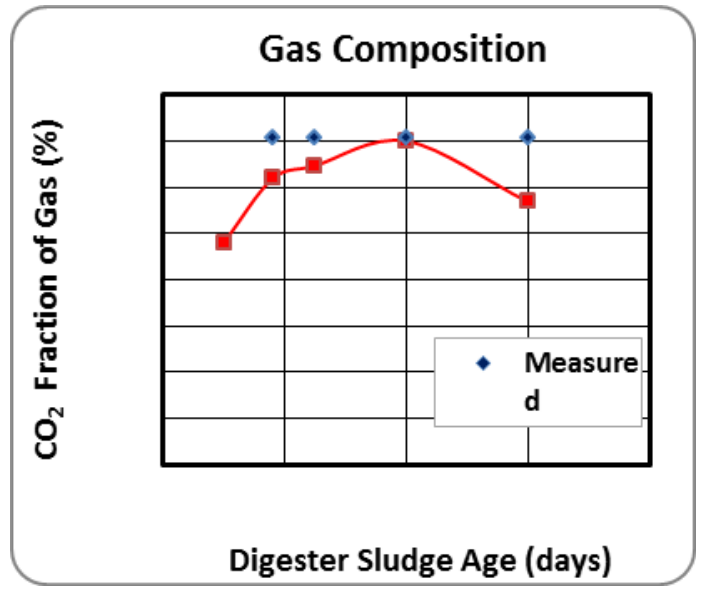

Figure 1f

Figure 1: Comparison between simulated and measured results for $A D$ of WAS from the laboratory scale UCT NDBEPR system fed with settled WW and added acetate to increase BEPR. 


\section{CLOSURE}

The BIOMATH protocol was applied for the calibration of the UCT-PW model, for promotion of its widespread utilisation in a reproducible way. However, it is noted that the effective calibration of this model requires a further step - from modelling the laboratory scale systems (under controlled and completely mixed environments) to assessment of model predictions for full-scale wastewater treatment plant systems, interlinked to plant-wide configurations. This prospective work may be of particular interest to the IWA group on benchmarking of control strategies for WWTPs who are including P into an extended BSM model.

\section{ACKNOWLEDGEMENTS}

Peter Vanrolleghem holds the Canada Research Chair in Water Quality Modelling

\section{REFERENCES}

1. Brouckaert C.J., Ikumi D.S. and Ekama G.A. (2010). A 3-phase anaerobic digestion model. In: Proceedings. $12^{\text {th }}$ IWA Anaerobic Digestion Conference (AD12), Guadalajara, Mexico, 1-4 Nov, 2010.

2. Brun R., Kühni M., Siegrist H., Gujer W. and Reichert P. (2002). Practical identifiability of ASM2d parameters - systematic selection and tuning of parameter subsets. Water Research, 36, 4113-4127.

3. Ekama G.A. and Wentzel M.C. (2004). A predictive model for the reactor inorganic suspended solids concentration in activated sludge systems. Water Research, 38, 4093-4106.

4. Hauduc H., Rieger L., Takács I., Héduit A., Vanrolleghem P.A. and Gillot S. (2010). A systematic approach for model verification: Application on seven published activated sludge models. Water Science and Technology, 61 (4), 825-839.

5. Henze M., Gujer W., Mino T., Matsuo T., Wentzel M.C. and Marais G.v.R. (1995). Activated Sludge Model No.2 (ASM2). IWA Scientific and Technical Report No.3, IWA Publishing, London, U.K.

6. Ikumi D.S., Brouckaert C.J. and Ekama G.A. (2011). A 3 phase anaerobic digestion model. In: Proceedings $8^{\text {th }}$ IWA Watermatex conference, San Sebastian, Spain, 20-22 June, 2011.

7. Ikumi D.S. (2011). The Development of a Three Phase Plant-Wide Mathematical Model for Sewage Treatment. Water Research Group (WRG). Department of Civil Engineering. University of Cape Town, South-Africa.

8. Ikumi D.S., Harding T.H., Brouckaert C.J. and Ekama G.A. (2013). Plant-wide integrated biological, chemical and physical bioprocesses modelling of wastewater treatment plants in 3 phases (aqueous-gassolid). Research Report W136, Department of Civil Engineering. University of Cape Town, South Africa.

9. Musvoto E..V. Wentzel M.C., Loewenthal R.E. and Ekama G.A. (2000a). Integrated chemical, physical and biological processes modelling Part I - Development of a kinetic based model for weak acid/base systems. Water Research, 34, 1857-1867.

10. Musvoto E.V., Wentzel M.C. and Ekama G.A. (2000b). Integrated chemical, physical and biological processes modelling Part II - Modelling aeration treatment of anaerobic digester supernatants. Water Research, 34, 1868-1880..

11. Sötemann S.W., van Rensburg P., Ristow N.E., Wentzel M.C., Loewenthal R.E. and Ekama G.A. (2005). Integrated chemical, physical and biological processes modelling Part 2 : Anaerobic digestion of sewage sludges. Water SA., 31, 545-568.

12. Neumann M.B. (2012). Comparison of sensitivity analysis methods for pollutant degradation modelling: A case study from drinking water treatment. Science of the Total Environment, 433, 530-537.

13. Sötemann S.W., Ristow N.E., Wentzel M.C. and Ekama G.A. (2005a) A steady-state model for anaerobic digestion of sewage sludges. Water SA, 31, 511-527.

14. Sötemann S.W., van Rensburg P., Ristow N.E., Wentzel M.C., Loewenthal R.E. and Ekama G.A. (2005b) Integrated chemical, physical and biological processes modelling Part 2 - Anaerobic digestion of sewage sludges. Water SA, 31, 545-568.

15. Vanrolleghem P.A., Insel G., Petersen B., Sin G., De Pauw D., Nopens I., Weijers S. and Gernaey K. (2003). A comprehensive model calibration procedure for activated sludge models. In: Proceedings WEF $26^{\text {th }}$ Annual Technical Exhibition and Conference (WEFTEC, 2003), Los Angeles, CA, USA, 11-15 Oct, 2003.

16. Volcke E.I.P., van Loosdrecht M.C.M. and Vanrolleghem P.A. (2006). Continuity-based model interfacing for plant-wide simulation : A general approach. Water Research, 40, 2817-2828. 
Ikumi et al. 
Table 1: Universally selected model components for UCT three phase plant wide model (UCT-PW)

\begin{tabular}{|c|c|c|c|}
\hline Name & Empirical formula & Description & Units \\
\hline $\mathrm{H} 2 \mathrm{O}$ & $\mathrm{H} 2 \mathrm{O}$ & Water & $\mathrm{m}^{3} / \mathrm{d}$ \\
\hline S_H & $\mathrm{H}^{+}$ & Hydrogen ion & $\mathrm{gH} / \mathrm{m}^{3}$ \\
\hline $\mathrm{S} \_\mathrm{Na}$ & $\mathrm{Na}^{+}$ & Sodium & $\mathrm{gNa} / \mathrm{m}^{3}$ \\
\hline S_K & $\mathrm{K}^{+}$ & Potassium & $\mathrm{gK} / \mathrm{m}^{3}$ \\
\hline S_Ca & $\mathrm{Ca}^{2+}$ & Calcium & $\mathrm{gCa} / \mathrm{m}^{3}$ \\
\hline S_Mg & $\mathrm{Mg}^{2+}$ & Magnesium & $\mathrm{gMg} / \mathrm{m}^{3}$ \\
\hline $\mathrm{S} \_\mathrm{NH}_{\mathrm{x}}$ & $\mathrm{NH}_{4}^{+}$ & Ammonium & $\mathrm{gNH}_{4} / \mathrm{m}^{3}$ \\
\hline S_Cl & $\mathrm{Cl}^{-}$ & Chloride & $\mathrm{gCl} / \mathrm{m}^{3}$ \\
\hline S_VFA & $\mathrm{CH}_{3} \mathrm{COO}^{-}$ & Acetate & $\mathrm{gAc} / \mathrm{m}^{3}$ \\
\hline S_Pr & $\mathrm{CH}_{3} \mathrm{CH}_{2} \mathrm{COO}^{-}$ & Propionate & $\mathrm{gPr} / \mathrm{m}^{3}$ \\
\hline $\mathrm{S} \_\mathrm{CO}_{3}$ & $\mathrm{CO}_{3}^{2-}$ & Carbonate & $\mathrm{gCO}_{3} / \mathrm{m}^{3}$ \\
\hline $\mathrm{S} \_S O_{4}$ & $\mathrm{SO}_{4}^{2-}$ & Sulphate & $\mathrm{gSO}_{4} / \mathrm{m}^{3}$ \\
\hline $\mathrm{S} \_\mathrm{PO}_{4}$ & $\mathrm{PO}_{4}^{3-}$ & Phosphate & $\mathrm{gPO}_{4} / \mathrm{m}^{3}$ \\
\hline $\mathrm{S} \_\mathrm{NO}_{\mathrm{x}}$ & $\mathrm{NO}_{3}^{-}$ & Nitrate & $\mathrm{gNO}_{3} / \mathrm{m}^{3}$ \\
\hline $\mathrm{S} \_\mathrm{H} 2$ & $\mathrm{H}_{2}$ & Dissolved hydrogen & $\mathrm{gH}_{2} / \mathrm{m}^{3}$ \\
\hline S_O2 & $\mathrm{O}_{2}$ & Dissolved oxygen & $\mathrm{gO}_{2} / \mathrm{m}^{3}$ \\
\hline S_U & $\mathrm{CH}_{\mathrm{Yu}} \mathrm{O}_{\mathrm{Zu}} \mathrm{N}_{\mathrm{Au}} \mathrm{P}_{\mathrm{Bu}}$ & Unbiodegradable Soluble Organics & $\mathrm{g} / \mathrm{m}^{3}$ \\
\hline S_F & $\mathrm{CH}_{\mathrm{Yf}} \mathrm{O}_{\mathrm{Zf}} \mathrm{N}_{\mathrm{Af}} \mathrm{P}_{\mathrm{Bf}}$ & $\begin{array}{l}\text { Fermentable Biodegradable Soluble } \\
\text { Organics }\end{array}$ & $\mathrm{g} / \mathrm{m}^{3}$ \\
\hline S_Glu & $\mathrm{C}_{6} \mathrm{H}_{12} \mathrm{O}_{6}$ & Glucose & $\mathrm{g} / \mathrm{m}^{3}$ \\
\hline X_U_inf & $\mathrm{CH}_{\text {Yup }} \mathrm{O}_{\text {Zup }} \mathrm{N}_{\text {Aup }} \mathrm{P}_{\text {Bup }}$ & $\begin{array}{l}\text { Unbiodegradable particulate } \\
\text { organics }\end{array}$ & $\mathrm{g} / \mathrm{m}^{3}$ \\
\hline X_B_Org & $\mathrm{CH}_{\mathrm{Ybp}} \mathrm{O}_{\mathrm{zbp}} \mathrm{N}_{\mathrm{Abp}} \mathrm{P}_{\mathrm{Bbp}}$ & Biodegradable particulate organics & $\mathrm{g} / \mathrm{m}^{3}$ \\
\hline X_B_Inf & $\mathrm{CH}_{\text {Ybps }} \mathrm{O}_{\text {Zbps }} \mathrm{N}_{\text {Abps }} \mathrm{P}_{\mathrm{Bbps}}$ & $\begin{array}{l}\text { Influent biodegradable particulate } \\
\text { organics }\end{array}$ & $\mathrm{g} / \mathrm{m}^{3}$ \\
\hline X_PAO_PP & $\mathrm{K}_{\mathrm{kp}} \mathrm{Mg}_{\mathrm{mp}} \mathrm{Ca}_{\mathrm{cp}} \mathrm{PO}_{3}$ & Polyphosphate & $\mathrm{g} / \mathrm{m}^{3}$ \\
\hline X_PAO_Stor & $\mathrm{C}_{4} \mathrm{H}_{6} \mathrm{O}_{2}$ & Poly-hydroxy-alkanoate & $\mathrm{g} / \mathrm{m}^{3}$ \\
\hline X_Str_NH4 & $\mathrm{MgNH}_{4} \mathrm{PO}_{4} \cdot 6 \mathrm{H}_{2} \mathrm{O}$ & Struvite & $\mathrm{g} / \mathrm{m}^{3}$ \\
\hline X_ACP & $\mathrm{Ca}_{3}\left(\mathrm{PO}_{4}\right)_{2}$ & Calcium Phosphate & $\mathrm{g} / \mathrm{m}^{3}$ \\
\hline X_Str_K & $\mathrm{MgKPO}_{4} \cdot 6 \mathrm{H}_{2} \mathrm{O}$ & K-struvite & $\mathrm{g} / \mathrm{m}^{3}$ \\
\hline X_Cal & $\mathrm{CaCO}_{3}$ & Calcite & $\mathrm{g} / \mathrm{m}^{3}$ \\
\hline X_Mag & $\mathrm{MgCO}_{3}$ & Magnesite & $\mathrm{g} / \mathrm{m}^{3}$ \\
\hline X_Newb & $\mathrm{MgHPO}_{4}$ & Newberyite & $\mathrm{g} / \mathrm{m}^{3}$ \\
\hline X_ISS & & Influent inorganic settleable solids & $\mathrm{gISS} / \mathrm{m}^{3}$ \\
\hline X_OHO & $\mathrm{CH}_{\mathrm{Yo}} \mathrm{O}_{\mathrm{Zo}} \mathrm{N}_{\mathrm{Ao}} \mathrm{P}_{\mathrm{Bo}}$ & Ordinary heterotrophic organisms & $\mathrm{g} / \mathrm{m}^{3}$ \\
\hline X_PAO & $\mathrm{CH}_{\mathrm{Yo}} \mathrm{O}_{\mathrm{Zo}} \mathrm{N}_{\mathrm{Ao}} \mathrm{P}_{\mathrm{Bo}}$ & Phosphate accumulating organisms & $\mathrm{g} / \mathrm{m}^{3}$ \\
\hline X_ANO & $\mathrm{CH}_{\mathrm{Yo}} \mathrm{O}_{\mathrm{Zo}} \mathrm{N}_{\mathrm{Ao}} \mathrm{P}_{\mathrm{Bo}}$ & Autotrophic nitrifying organisms & $\mathrm{g} / \mathrm{m}^{3}$ \\
\hline X_ZAD & $\mathrm{CH}_{\mathrm{Yo}} \mathrm{O}_{\mathrm{Zo}} \mathrm{N}_{\mathrm{Ao}} \mathrm{P}_{\mathrm{Bo}}$ & Acidogens & $\mathrm{g} / \mathrm{m}^{3}$ \\
\hline X_ZAC & $\mathrm{CH}_{\mathrm{Yo}} \mathrm{O}_{\mathrm{Zo}} \mathrm{N}_{\mathrm{Ao}} \mathrm{P}_{\mathrm{Bo}}$ & Acetogens & $\mathrm{g} / \mathrm{m}^{3}$ \\
\hline X_ZAM & $\mathrm{CH}_{\mathrm{Yo}} \mathrm{O}_{\mathrm{Zo}} \mathrm{N}_{\mathrm{Ao}} \mathrm{P}_{\mathrm{Bo}}$ & Acetoclastic Methanogens & $\mathrm{g} / \mathrm{m}^{3}$ \\
\hline X_ZHM & $\mathrm{CH}_{\mathrm{Yo}} \mathrm{O}_{\mathrm{Zo}} \mathrm{N}_{\mathrm{Ao}} \mathrm{P}_{\mathrm{Bo}}$ & Hydrogenotrophic methanogens & $\mathrm{g} / \mathrm{m}^{3}$ \\
\hline X_U_Org & $\mathrm{CH}_{\mathrm{ye}} \mathrm{O}_{\mathrm{ze}} \mathrm{N}_{\mathrm{ae}} \mathrm{P}_{\mathrm{be}}$ & Endogenous residue & $\mathrm{g} / \mathrm{m}^{3}$ \\
\hline $\mathrm{G}_{-} \mathrm{CO}_{2}$ & $\mathrm{CO}_{2}$ & Carbon dioxide & $\mathrm{gCO}_{2} / \mathrm{m}^{3}$ \\
\hline $\mathrm{G}_{-} \mathrm{CH}_{4}$ & $\mathrm{CH}_{4}$ & Methane & $\mathrm{gCH}_{4} / \mathrm{m}^{3}$ \\
\hline
\end{tabular}


Ikumi et al.

Table 2: Ionic species selected for the UCT three phase model (UCT-PW)

\begin{tabular}{|c|c|c|c|c|c|}
\hline & Formula & Description & & Formula & Description \\
\hline 1 & $\mathrm{H}^{+}$ & $\begin{array}{l}\text { Hydrogen } \\
\text { ion }\end{array}$ & 23 & $\mathrm{NH}_{4} \mathrm{SO}_{4}^{-}$ & Ammonium sulphate \\
\hline 2 & $\mathrm{Na}^{+}$ & Sodium & 24 & $\mathrm{MgPO}_{4}^{-}$ & $\begin{array}{l}\text { Magnesium } \\
\text { phosphate }\end{array}$ \\
\hline 3 & $\mathrm{~K}^{+}$ & Potassium & 25 & $\mathrm{CaCH}_{3} \mathrm{COO}^{+}$ & Calcium acetate \\
\hline 4 & $\mathrm{Ca}^{2+}$ & Calcium & 26 & $\mathrm{CaCH}_{3} \mathrm{CH}_{2} \mathrm{COO}^{+}$ & Calcium propionate \\
\hline 5 & $\mathrm{Mg}^{2+}$ & Magnesium & 27 & $\mathrm{CaHCO}_{3}^{+}$ & Calcium bi-carbonate \\
\hline 6 & $\mathrm{NH}_{4}^{+}$ & Ammonium & 28 & $\mathrm{NaSO}_{4}^{-}$ & Sodium sulphate \\
\hline 7 & $\mathrm{Cl}^{-}$ & Chloride & 29 & $\mathrm{MgHPO}_{4}$ & $\begin{array}{l}\text { Magnesium } \\
\text { hydrogen phosphate }\end{array}$ \\
\hline 8 & $\mathrm{CH}_{3} \mathrm{COO}^{-}$ & Acetate & 30 & $\mathrm{CH}_{3} \mathrm{COONa}$ & Sodium Acetate \\
\hline 9 & $\mathrm{CH}_{3} \mathrm{CH}_{2} \mathrm{COO}^{-}$ & Propionate & 31 & $\mathrm{H}_{2} \mathrm{CO}_{3}$ & $\begin{array}{l}\text { Di-hydrogen } \\
\text { carbonate }\end{array}$ \\
\hline 10 & $\mathrm{CO}_{3}{ }^{2-}$ & Carbonate & 32 & $\mathrm{MgSO}_{4}$ & Magnesium sulphate \\
\hline 11 & $\mathrm{SO}_{4}^{2-}$ & Sulphate & 33 & $\mathrm{HPO}_{4}^{2-}$ & Hydrogen phosphate \\
\hline 12 & $\mathrm{PO}_{4}{ }^{3-}$ & Phosphate & 34 & $\mathrm{NH}_{3}$ & Ammonia \\
\hline 13 & $\mathrm{NO}_{3}^{-}$ & Nitrate & 35 & $\mathrm{MgCO}_{3}$ & $\begin{array}{l}\text { Magnesium } \\
\text { carbonate }\end{array}$ \\
\hline 14 & $\mathrm{OH}^{-}$ & $\begin{array}{l}\text { Hydroxide } \\
\text { ion }\end{array}$ & 36 & $\mathrm{ACPO}_{4}^{-}$ & Calcium Phosphate \\
\hline 15 & $\mathrm{CH}_{3} \mathrm{COOH}$ & Acetic acid & 37 & $\mathrm{MgHCO}_{3}^{+}$ & $\begin{array}{l}\text { Magnesium } \\
\text { hydrogen carbonate }\end{array}$ \\
\hline 16 & $\mathrm{CH}_{3} \mathrm{CH}_{2} \mathrm{COOH}$ & $\begin{array}{l}\text { Propionic } \\
\text { acid }\end{array}$ & 38 & $\mathrm{CaHPO}_{4}^{-}$ & $\begin{array}{l}\text { Calcium hydrogen } \\
\text { phosphate }\end{array}$ \\
\hline 17 & $\mathrm{HCO}_{3}^{-}$ & Bi-carbonate & 39 & $\mathrm{NaCO}_{3}{ }^{-}$ & Sodium carbonate \\
\hline 18 & $\mathrm{CaSO}_{4}$ & $\begin{array}{l}\text { Calcium } \\
\text { sulphate }\end{array}$ & 40 & $\mathrm{MgH}_{2} \mathrm{PO}_{4}^{+}$ & $\begin{array}{l}\text { Magnesium di- } \\
\text { hydrogen phosphate }\end{array}$ \\
\hline 19 & $\mathrm{H}_{2} \mathrm{PO}_{4}^{-}$ & $\begin{array}{l}\text { Di-hydrogen } \\
\text { phosphate }\end{array}$ & 41 & $\mathrm{NaHCO}_{3}$ & $\begin{array}{l}\text { Sodium hydrogen } \\
\text { carbonate }\end{array}$ \\
\hline 20 & $\mathrm{MgCH}_{3} \mathrm{COO}^{+}$ & $\begin{array}{l}\text { Magnesium } \\
\text { acetate }\end{array}$ & 42 & $\mathrm{NaHPO}_{4}^{-}$ & $\begin{array}{l}\text { Sodium hydrogen } \\
\text { phosphate }\end{array}$ \\
\hline 21 & $\mathrm{MgCH}_{3} \mathrm{CH}_{2} \mathrm{COO}^{+}$ & $\begin{array}{l}\text { Magnesium } \\
\text { propionate }\end{array}$ & 43 & $\mathrm{CaOH}^{+}$ & Calcium hydroxide \\
\hline 22 & $\mathrm{CaCO}_{3}$ & $\begin{array}{l}\text { Calcium } \\
\text { carbonate }\end{array}$ & 44 & $\mathrm{MgOH}^{+}$ & $\begin{array}{l}\text { Magnesium } \\
\text { hydroxide }\end{array}$ \\
\hline
\end{tabular}

Table 3: Example for equilibrium and mass balance equations for ionic speciation

\begin{tabular}{ccc} 
Weak Acid Sub-System & $\begin{array}{c}* \text { Equeous Phase Equilibrium } \\
\text { Equations }\end{array}$ & Mass Balance Equation \\
\hline & {$\left[\mathrm{NH}_{3}\right]=\frac{\mathrm{K}_{\mathrm{NH}_{4}} \cdot\left[\mathrm{NH}_{4}^{+}\right]}{\left(\mathrm{H}^{+}\right)}$} & $\mathrm{NH}_{\mathrm{x}}=\left[\mathrm{NH}_{4}^{+}\right]+\left[\mathrm{NH}_{3}\right]+\left[\mathrm{NH}_{4} \mathrm{SO}_{4}^{-}\right]$ \\
Ammonia & {$\left[\mathrm{NH}_{4} \mathrm{SO}_{4}^{-}\right]=\frac{\left[\mathrm{SO}_{4}^{2-} \llbracket \mathrm{NH}_{4}^{+}\right]}{\mathrm{K}_{\mathrm{NH}_{4} \mathrm{SO}_{4}}}$} &
\end{tabular}

*Where $\left(\mathrm{H}^{+}\right)$is the hydrogen ion activity, $[\mathrm{X}]$ the molar concentrations of species $\mathrm{X}$ and $\mathrm{K}_{\mathrm{X}}$, is the thermodynamic equilibrium constant for species X, adjusted for Debye Hückel effects to account for the activity of ions in low salinity water (Stumm and Morgan, 1996). 
Table 4: Processes used in the application of UCT three phase plant wide model

\begin{tabular}{|c|c|}
\hline Name & Description \\
\hline AerHydrol & Aerobic hydrolysis of biodegradable particulate organics (BPO) \\
\hline AnHydrol & Anoxic hydrolysis of BPO \\
\hline AnaerHydrol & Anaerobic hydrolysis of BPO \\
\hline AerGrowthOnSf & Aerobic OHO growth on fermentable soluble organics (FBSO) \\
\hline AerGrowthOnSa & Aerobic OHO growth on Acetate \\
\hline AnGrowthOnSfDenitrif & Anoxic OHO growth on FBSO \\
\hline AnGrowthOnSaDenitrif & Anoxic OHO growth on Acetate \\
\hline Fermentation & Fermentation of FBSO \\
\hline LysisOfAuto & Storage of poly-hydroxy-alkanoate (PHA) by PAOs \\
\hline StorageOfXPP & Aerobic storage of PP with PHA uptake \\
\hline AerGrowthOnXPHA & Aerobic growth of PAOs \\
\hline LysisOfXPP & Release and hydrolysis of polyphosphate (PP) \\
\hline LysisOfXPHA & Release and hydrolysis of PHA \\
\hline GrowthOfAuto & Aerobic growth of ANOs with nitrification \\
\hline OHO_Lysis & Lysis of OHOs in aerobic systems \\
\hline LysisOfXPAO & Lysis of PAOs in aerobic systems \\
\hline LysisOfAuto & Lysis of ANOs in AS system \\
\hline Aeration & Oxygen supply to aerobic reactor \\
\hline FSO_Hydrolysis & Hydrolysis of FBSO in AD system \\
\hline BPO_Hydrolysis & Hydrolysis of BPO produced by dead biomass \\
\hline BPO_PS_Hydrolysis & Hydrolysis of BPO from primary sludge (PS) \\
\hline OHO_Lysis_AD & Lysis of OHOs in AD system \\
\hline PAO_Lysis_AD & Lysis of PAOs in AD system \\
\hline PP_Release & Release of PP with uptake of PHA in AD system \\
\hline PP_Hydrolysis & Release and hydrolysis of PP in AD system \\
\hline PHA_Hydrolysis & Release and hydrolysis of PHA in AD system \\
\hline Acidogenesis_L & Low hydrogen partial pressure $\left(\mathrm{p}_{\mathrm{H} 2}\right)$ Acidogenesis \\
\hline Acidogenesis_H & High $\mathrm{p}_{\mathrm{H} 2}$ Acidogenesis \\
\hline AD_decay & Lysis of acidogens \\
\hline Acetogenesis & Growth of acetogens in AD system \\
\hline AC_decay & Lysis of acetogens \\
\hline Acet_methanogenesis & Growth of acetoclastic methanogens in $\mathrm{AD}$ system \\
\hline AM_decay & Lysis of acetoclastic methanogens \\
\hline Hyd_methanogenesis & Growth of hydrogenotrophic methanogens in AD system \\
\hline HM_decay & Lysis of hydrogenotrophic methanogens \\
\hline
\end{tabular}




\title{
Modelling the behaviour of Polyphosphate Accumulating Organisms (PAOs) in biological nutrient removal processes in the presence of external carbon
}

\author{
Xiang $\mathrm{Hu}^{1}$, Dominika Sobotka ${ }^{2}$, Krzysztof Czerwionka ${ }^{2}$, Qi Zhou ${ }^{1}$, Li Xie ${ }^{1}$, Giulio Munz ${ }^{3}$, Jan A \\ Oleszkiewicz $^{4}$ and Jacek Makinia ${ }^{2}$ \\ ${ }^{1}$ College of Environmental Science and Engineering, Tongji University, 1239 Siping Road, Shanghai, \\ People's Republic of China (Email: xiangh1215@outlook.com) \\ ${ }^{2}$ Faculty of Civil and Environmental Engineering, Gdansk University of Technology, ul. Narutowicza \\ 11/12, 80-233 Gdansk, Poland (Email: jmakinia@pg.gda.pl) \\ ${ }^{3}$ Department of Civil and Environmental Engineering, University of Florence, Via S. Marta n. 3, 50139 \\ Florence, Italy (Email: giulio@dicea.unifi.it) \\ ${ }^{4}$ Department of Civil Engineering, University of Manitoba, Winnipeg, Manitoba, Canada R3T 5V6 (Email: \\ oleszkie@cc.umanitoba.ca)
}

\begin{abstract}
The aim of this study was to develop extensions of Activated Sludge Model No. 2d (ASM2d) for modelling the behaviour of Polyphosphate Accumulating Organisms (PAOs) in biological nutrient removal (BNR) activated sludge systems with addition of an external carbon source. Two significant modifications for the expanded ASM2d were included: (1) a switch function for the inhibitory effect of dissolved oxygen on storage of poly- $\beta$-hydroxyalkanoates (PHA); (2) a new process describing the direct growth of PAOs on fermentation products $\left(\mathrm{S}_{\mathrm{A}}\right)$ under aerobic conditions. These modifications improved the model predictions of $\mathrm{P}$ release and uptake processes in one- and two-phase batch experiments with acetate as an external carbon source.
\end{abstract}

\section{Keywords}

ASM2d; biological nutrient removal; EBPR; external carbon; PAOs

\section{INTRODUCTION}

External carbon sources are readily biodegradable compounds which are usually added to enhance the denitrification process and improve the overall nitrogen removal efficiency in biological nutrient removal (BNR) processes. In combined N/P removal systems, the external carbon sources may also interact with the enhanced biological $\mathrm{P}$ removal (EBPR) process accomplished by Polyphosphate Accumulating Organisms (PAOs). However, the possibility that PAOs may oxidize the fermentation products $\left(\mathrm{S}_{\mathrm{A}}\right)$ has been ignored in activated sludge models (ASMs) because, as noted by Henze et al. (2000), "it is unlikely that such substrates ever become available under aerobic or anoxic conditions in a biological nutrient removal (BNR) plant'. This assumption is likely to fail for systems with external carbon addition, as the readily biodegradable compounds may become available for direct utilization by PAOs under anoxic and aerobic conditions. Few studies focused specifically on modelling the effects of external carbon addition on PAOs with the ASMs so far. Swinarski et al. (2012) developed a new model as an expansion of ASM2d to predict the effect of adding a readily biodegradable substrate to a combined N/P system. The aim of this study was to further modify the ASM2d with regard to the behaviour of PAOs under aerobic conditions in the presence of external carbon (acetate).

\section{MATERIAL AND METHODS}

\section{Model development and simulation platform}

A conceptual model of the expanded ASM2d under aerobic conditions is presented in Figure 1.The following extensions were considered: (1) the inhibitory effect of dissolved oxygen on storage of poly- $\beta$-hydroxyalkanoates $\left(\mathrm{X}_{\mathrm{PHA}}\right)$ by PAOs $\left(\mathrm{X}_{\mathrm{PAO}}\right)$ under aerobic conditions, (2) direct aerobic growth of $\mathrm{X}_{\mathrm{PAO}}$ on fermentation products $\left(\mathrm{S}_{\mathrm{A}}\right)$. Therefore, the kinetic expressions for storage of $X_{\mathrm{PHA}}$ and aerobic growth of $\mathrm{X}_{\mathrm{PAO}}$ on $\mathrm{S}_{\mathrm{A}}$ in the expanded ASM2d 
become as follows:

$$
\begin{gathered}
q_{P H A} \frac{S_{A}}{K_{S A, P A O}+S_{A}} \frac{K_{O 2, P A O}}{K_{O 2, P A 0}+S_{O 2}} \frac{S_{A L K}}{K_{A L K, P A O}+S_{A L K}} \frac{X_{P P} / X_{P A O}}{K_{P P}+X_{P P} / X_{P A O}} X_{P A O} \\
\mu_{\mathrm{PAO} 2} \frac{S_{O 2}}{K_{O 2, P A O}+S_{O 2}} \frac{S_{A}}{K_{S A, P A O}+S_{A}} \frac{S_{N H 4}}{K_{N H 4, P A O}+S_{N H 4}} \frac{S_{P O 4}}{K_{P O 4, P A O}+S_{P O 4}} \frac{S_{A L K}}{K_{A L K, P A O}+S_{A L K}} X_{P A O}
\end{gathered}
$$

The stoichiometric matrix for these two processes is presented in Table 1. GPS-X ver. 5.0.2 (Hydromantis, Canada) was used as a simulator environment for implementing the developed models and running simulations. For model calibration, a special utility called "Optimizer" was used. Parameters were estimated based on the Nelder-Mead simplex method with the maximum likelihood as an objective function. Three different scenarios were conducted for optimizing prediction of the external carbon addition (Table 2).

\section{Batch experiments}

In order to examine the effects of external carbon addition and the $\mathrm{PO}_{4}-\mathrm{P}$ concentration at the beginning of aerobic conditions on the PAOs behavior, two-phase (anaerobic/aerobic) and one-phase (aerobic) experiments were carried out with fresh mixed liquor withdrawn from the aerobic zone of a large BNR activated sludge plant in Gdansk (northern Poland). At the beginning of the aerobic phase, sodium acetate $\left(\mathrm{CH}_{3} \mathrm{COONa}\right)$ and nitrification inhibitor (ATU) were added. Samples were filtered and analyzed for $\mathrm{PO}_{4}-\mathrm{P}$ and $\mathrm{COD}$. In addition, oxygen uptake rate (OUR) was measured in a cyclic (3 minutes) mode.

\section{RESULTS AND CONCLUSIONS}

In the two kinds of batch experiments, the external carbon addition at the beginning of aerobic condition resulted in a significantly different PAO behavior. In the two-phase experiment, no $\mathrm{PO}_{4}-\mathrm{P}$ release was observed (Figure $2 \mathrm{a}$ ), which indicated that the addition of external carbon had no or a minor effect on P uptake by PAOs. In this case, predictions of ASM2d which assume the growth of PAOs on PHA only and ignore the aerobic growth of PAOs on $S_{A}$, could not match the experiment data accurately. Therefore, the ASM2d required appropriate extensions to better predict the effect of external carbon addition on PAOs. Model predictions for Scenario 1, which considered oxygen inhibition of storage of PHA, appeared to be consistent. Results from one-phase experiment showed that $\mathrm{PO}_{4}-\mathrm{P}$ was released in the initial 45 min of the experiment (Figure 2b). This confirmed the process of storage of PHA can occur under aerobic conditions provided that readily biodegradable substrates are available and that $\mathrm{PO}_{4}-\mathrm{P}$ is not completely released. In this case, $\mathrm{P}$ release and uptake data were matched accurately by the model predictions of Scenario 2 which considered the direct aerobic growth of $\mathrm{X}_{\mathrm{PAO}}$ on the external carbon. Scenario 3, considering both the aerobic inhibition of storage of PHA and aerobic growth of PAOs on $\mathrm{S}_{\mathrm{A}}$, can generally predict the behaviour of $\mathrm{PO}_{4}-\mathrm{P}, \mathrm{COD}$ and OUR for both experiments (Figure 2a-d). Further extensions of ASM2d for better predictions of the aerobic and anoxic behaviors of PAOs in the presence of external carbon addition will be presented in the full paper.

\section{ACKNOWLEDGEMENT}

This work was carried out under the project "CARbon BALAncing for nutrient control in wastewater treatment (CARBALA)".

\section{REFERENCES}

Henze M., Gujer W., Mino T., van Loosdrecht M. (Eds.) (2000). Activated Sludge Models ASM1, ASM2d and ASM3. Scientific and Technical Report No. 9, IWA Publishing: London.

Swinarski M., Makinia J., Stensel H. D. Czerwionka K., Drewnowski, J. (2012). Modeling external carbon addition in biological nutrient removal processes with an extension of the International Water Association activated sludge model. Water Environ. Res., 84(8), 646-655. 
$\mathrm{Hu}$ et al.

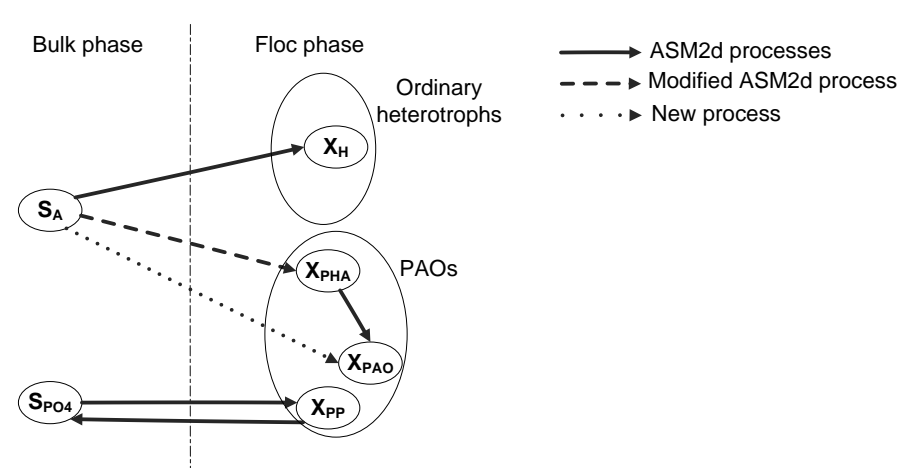

Figure 1 Model Concept in the expanded ASM2d under aerobic conditions
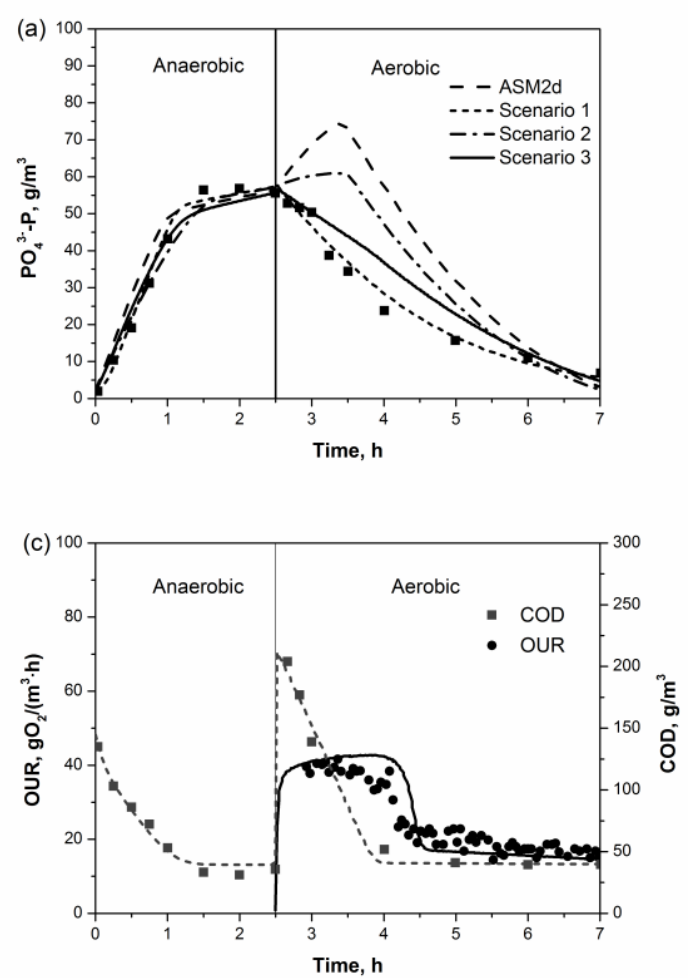
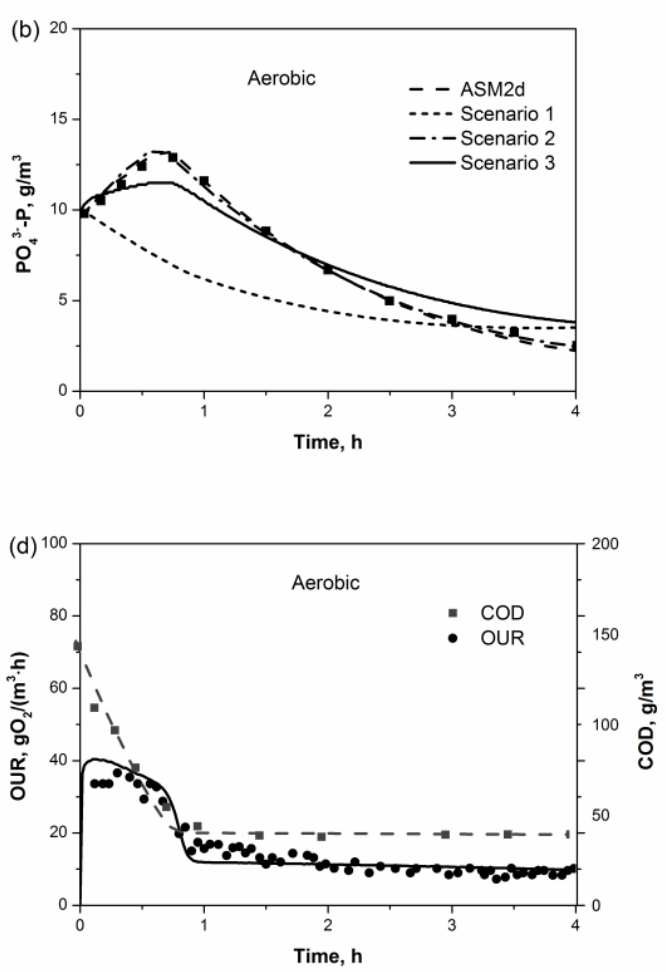

Figure 2 Measured data vs. model predictions in the batch experiments in different scenarios of the expanded ASM2d: (a) $\mathrm{PO}_{4}-\mathrm{P}$ in the anaerobic/aerobic experiment, (b) $\mathrm{PO}_{4}-\mathrm{P}$ in the aerobic experiment, (c) COD and OUR in the anaerobic/aerobic experiment (Scenario 3), (d) COD and OUR in the aerobic experiment (Scenario 3).

Table 1 Stoichiometric matrix for the expanded ASM2d

\begin{tabular}{|c|c|c|c|c|c|c|c|c|}
\hline \begin{tabular}{|l|} 
Process \\
Component \\
\end{tabular} & $\mathrm{S}_{\mathrm{O} 2}$ & $\mathrm{~S}_{\mathrm{A}}$ & $\mathrm{S}_{\mathrm{NH} 4}$ & $\mathrm{~S}_{\mathrm{PO} 4}$ & $\mathrm{~S}_{\mathrm{ALK}}$ & $\mathrm{X}_{\mathrm{PAO}}$ & $\mathrm{X}_{\mathrm{PP}}$ & $\mathrm{X}_{\mathrm{PHA}}$ \\
\hline Storage of $\mathrm{X}_{\mathrm{PHA}}$ by $\mathrm{X}_{\mathrm{PAO}}$ & & -1 & & $\mathrm{Y}_{\mathrm{PO} 4}$ & $v_{\mathrm{ALK}}$ & & $-\mathrm{Y}_{\mathrm{PO} 4}$ & 1 \\
\hline Aerobic growth of $\mathrm{X}_{\mathrm{PAO}}$ on $\mathrm{S}_{\mathrm{A}}$ & $\frac{1-Y_{P A O}}{Y_{P A O}}$ & $-\frac{1}{Y_{P A O}}$ & $-\mathrm{i}_{\mathrm{N}, \mathrm{BM}}$ & $-\mathrm{i}_{\mathrm{P}, \mathrm{BM}}$ & $v_{\mathrm{ALK}}$ & 1 & & \\
\hline
\end{tabular}

Table 2 Three different scenarios considered and adjusted parameters in the expanded ASM2d

No. Extensions of the expanded ASM2d Adjusted parameters and their values

Scenario $1 \quad$ Aerobic inhibition of storage of $\mathrm{X}_{\mathrm{PHA}}$ (Eq. 1) $\quad \mathrm{K}_{\mathrm{O} 2 \text {, PAO }}=0.20 \mathrm{~g} \mathrm{O}_{2} / \mathrm{m}^{3}$

Scenario 2 Aerobic growth of $\mathrm{X}_{\mathrm{PAO}}$ on $\mathrm{S}_{\mathrm{A}}$ (Eq. 2) $\quad \mu_{\mathrm{PAO} 2}=0.21 \mathrm{~d}^{-1}, \mathrm{~K}_{\mathrm{SA}, \mathrm{PAO}}=4.12 \mathrm{~g} \mathrm{COD} / \mathrm{m}^{3}$

Scenario 3 Both scenario 2 and scenario 3 (Eq. 1 \& Eq. $\quad \mathrm{K}_{\mathrm{O} 2, \mathrm{PAO}}=1.11 \mathrm{~g} \mathrm{O}_{2} / \mathrm{m}^{3}, \mu_{\mathrm{PAO} 2}=0.14 \mathrm{~d}^{-1}$, 2)

$\mathrm{K}_{\mathrm{SA}, \mathrm{PAO}}=5.05 \mathrm{~g} \mathrm{COD} / \mathrm{m}^{3}$ 


\title{
A DYNAMIC MODEL FOR PHYSICOCHEMICAL PHOSPHORUS REMOVAL: VALIDATION AND INTEGRATION IN ASM2d
}

\author{
Hélène Hauduc ${ }^{1,2,3}$, Imre Takacs ${ }^{4},{\text { Scott } \text { Smith }^{5} \text {, Anita Szabo }}^{6}$, Sudhir Murthy ${ }^{7}$, Glen T. Daigger ${ }^{8}$, \\ Mathieu Sperandio ${ }^{1,2,3 *}$ \\ ${ }^{1}$ Université de Toulouse; INSA, UPS, INP; LISBP 135 Avenue de Rangueil, F-31077 Toulouse, France \\ (Email :Helene.Hauduc@Insa-Toulouse.Fr,Mathieu.Sperandio@insa-toulouse.fr) \\ ${ }^{2}$ INRA, UMR792 Ingénierie des Systèmes Biologiques et des Procédés F-31400 Toulouse, France \\ ${ }^{3}$ CNRS, UMR5504 F-31400 Toulouse, France \\ ${ }^{4}$ Dynamita, Nyons, France (Email imre@dynamita.com) \\ ${ }^{5}$ Wilfrid Laurier University, Waterloo, Ontario, Canada (Email Ssmith@wlu.ca) \\ ${ }^{6}$ Inno-Water Ltd., Budapest, Hungary (Email Szabo.Anita@innowater.hu) \\ ${ }^{7}$ DCWater, Washington DC, USA (Email Sudhir.Murthy@dcwater.com) \\ ${ }^{8}$ CH2MHill, Denver, Colorado, USA (Email Glen.Daigger@ch2m.com)
}

\section{Keywords}

Chemical phosphorus removal, physicochemical modeling, ferric chloride, hydrous ferric oxide, phosphate adsorption

\section{INTRODUCTION}

Achieving phosphorus removal from wastewater to very low levels is often performed by metal salt addition. The engineering stakes for this process consist of ensuring compliant effluent phosphorus concentrations, while at the same time saving chemicals and limiting chemical sludge production. Modeling is a commonly used engineering tool to handle such issues. However, metal salt precipitation such as iron dosing is not well described in current wastewater process models. Existing chemical phosphorus removal models are mainly focused on ferric phosphate precipitation (de Haas et al., 2001; Fytianos et al., 1998; Luedecke et al., 1989). However, this pathway occurs only at acidic $\mathrm{pH}$ (below $\mathrm{pH}$ 5) as demonstrated by Smith et al. (2008) and based on experiments and literature solubility data (NIST 2001). De Haas et al. (2001) published a series of articles presenting a pilot-plant operated with different conditions of metal salts dosage, including a study dedicated to the application of an ASM2 model combined with an iron phosphate precipitation process similar to Luedecke et al. (1989) model on their case study. The authors showed several pitfalls of their model: the Fe:P precipitate stoichiometry has to be changed depending on the sludge age (the model does not predict storage effects) and the model does not adequately predict behavior at low $\mathrm{P}$ concentration, high and low iron doses. Furthermore the authors underlined the necessity to improve such precipitation model by including chemical equilibria, $\mathrm{pH}$ and alkalinity prediction.

The combination of very fast (chemical equilibrium) and slow (kinetic precipitation/adsorption) reactions, variable molar ratios, multiple dosage points, the effect of mixing, colloidal material conversions and multiple precipitates requires a new modeling framework. This framework can then be applied to full plant process models to optimize doses and dosage locations and increase the safety of effluent compliance. A dynamic physicochemical model for chemical phosphorus removal has been developed and calibrated based on previous studies and experimental data from Smith et al (2008) and Szabo et al. (2008) (Hauduc et al., 2013). The objectives of this study are threefold: first the model is extended for a better prediction of batch data at low $\mathrm{pH}$ and high metal salt dosing, then it is 
combined with a biokinetic model and finally compare the model with continuous data obtained by de Haas et al. (2001) in pilot plant with real wastewater.

\section{MODEL DEVELOPMENT}

Iron dosage leads to rapid hydrous ferric oxide (HFO, including amorphous minerals such as 2-line ferrihydrite) precipitation. Phosphorus may then be removed from the bulk solution through different pathways: 1) adsorption of phosphates onto HFO by sharing an oxygen atom with iron; 2) co-precipitation of phosphate molecules into the HFO structure; 3) precipitation of ferric phosphate and 4) precipitation of mixed cation phosphates (Smith et al., 2008). These processes are taken into account in the new model, which predicts kinetically the precipitation of hydrous ferric oxides (HFO), the phosphates adsorption, and the coprecipitation mechanisms. It is combined with chemical equilibrium and physical precipitation reactions in order to model observed bulk dynamics in term of $\mathrm{pH}$. The model is calibrated based on previous studies and experimental data from Smith et al (2008) and Szabo et al. (2008). The simulations results showed that the structure of the model overall describes adequately the mechanisms of adsorption and co-precipitation of phosphates onto HFO and that the model is robust to experimental conditions: Fe/P dose, time, $\mathrm{HFO}$ aging and mixing intensity, as illustrated on Figure for Fe/P dose and mixing effect.
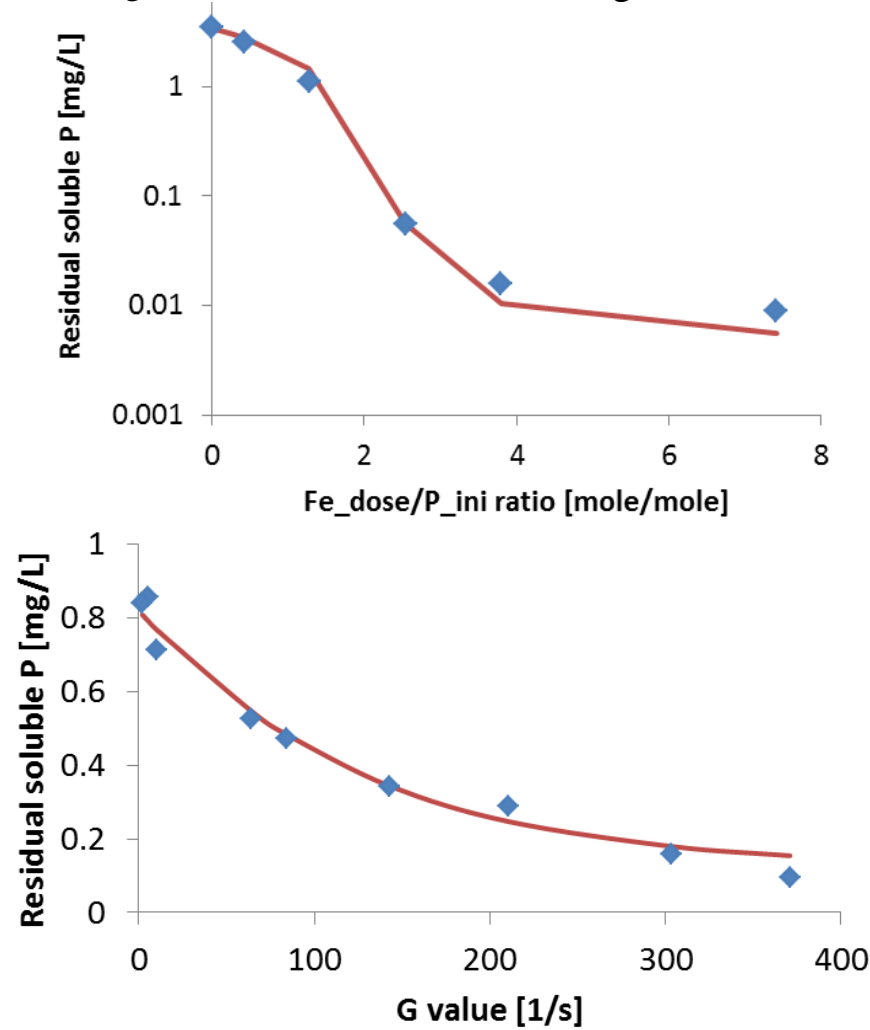

Figure 1. Left: Effect of initial Fe/P molar ratio on residual soluble phosphorus and on final $\mathrm{pH}$ of the batch: experimental results from Szabo et al. (2008) (batch tests of $11 \mathrm{~min}, \mathrm{P}_{\text {ini }}=3.5 \mathrm{mg} / \mathrm{L}, \mathrm{pH}_{\mathrm{ini}}=6.5$, $\mathrm{Alk}_{\text {ini }}=125 \mathrm{mg} \mathrm{CaCO} / \mathrm{L}, \mathrm{G}=425 \mathrm{~s}^{-1}$ ). Right: Effect of $\mathrm{G}$ value (mixing intensity) on residual soluble phosphorus: experimental results from Szabo et al. (2008) (batch tests of $11 \mathrm{~min}, \mathrm{P}_{\mathrm{in}}=4.1 \mathrm{mg} / \mathrm{L}$, $\left.\mathrm{Fe} / \mathrm{P}_{\text {ini }}=1.8 \mathrm{~mole} / \mathrm{mole}\right)$.

\section{COMBINING FeP MODEL WITH ASM2D}

To combine the FeP model with a biological model, the biological model has first to be adapted to insure continuity in charge and carbon of the processes and to the ionic species 
consumed or released by biomasses. In bio-P models, at least five state variables are involved in acid-base systems: acetate, phosphate, carbonate, nitrate and ammonia. These state variables comprise all the acid-base species. However, to introduce chemical equilibrium processes and to correctly model the $\mathrm{pH}$, a choice has to be made in the species consumed or produced by the biological reactions.

According to Sotemann et al. (2005) microorganisms are taken up the non-ionic form of acetate, propionic acid, ammonia and phosphate for cell synthesis. In the case of polyphosphate storage, phosphate is taken in the form $\mathrm{H}_{2} \mathrm{PO}_{4}{ }^{-}$linked to counter ions $\mathrm{K}^{+}$and $\mathrm{Mg}^{2+}$ (Barat et al., 2005). However at typical $\mathrm{pH}$ range of our system (7 to 8.2), the most abundant species are $\mathrm{NH}_{4}{ }^{+}$and $\mathrm{HPO}_{4}{ }^{2-}$. Consequently we chose to use $\mathrm{NH}_{4}{ }^{+}$and $\mathrm{HPO}_{4}{ }^{2-}$ as state variables in the biokinetic model and protons are corrected accordingly.

Inorganic carbon uptake and release is considered to be the non-ionic form $\mathrm{H}_{2} \mathrm{CO}_{3}$. The stoichiometry of $\mathrm{H}_{2} \mathrm{CO}_{3}$ is based on the difference in carbon content of substrate and product, calculated with their $\mathrm{COD} / \mathrm{C}$ ratio.

\section{CASE-STUDY APPLICATION}

De Haas et al (2000a) published a series of articles presenting a three-stage Phoredox (Table ) operated with different conditions of metal salts dosage: alum or ferric chloride (for this study only data on ferric chloride addition are considered), doses of metal salt, sludge age and dosage points. The Table summarized the 9 experimental periods considered in this study and their respective conditions. An identical control pilot-plant is operated in parallel without metal salt addition. Therefore a new model is required to predict HFO precipitation, $\mathrm{pH}$ modifications through chemical equilibrium and phosphate adsorption, and co-precipitation onto/into HFO.

Table 1. Experimental periods of $\mathrm{FeCl}_{3}$ dosing from de Haas et al. (2000b) with effluent phosphorous concentration obtained in the control pilot-plant (R2) and the pilot-plant with metal salt addition (R1). The figure represents the pilot-plant configuration.

\begin{tabular}{ccc|c|ccc}
\hline & & & R2 & \multicolumn{3}{|c}{ R1 } \\
\hline $\begin{array}{c}\text { Period } \\
\text { name }\end{array}$ & $\begin{array}{c}\text { Nb of } \\
\text { days }\end{array}$ & $\begin{array}{c}\text { Sludge } \\
\text { age (d) }\end{array}$ & $\begin{array}{c}\text { Pe R2 } \\
(\mathrm{mg} / \mathrm{L})\end{array}$ & $\begin{array}{c}\text { Dosage } \\
\text { point }\end{array}$ & $\begin{array}{c}\text { Fe/ } \\
\text { PeR2 }\end{array}$ & $\begin{array}{c}\text { Pe R1 } \\
(\mathrm{mg} / \mathrm{L})\end{array}$ \\
\hline 1 & 62 & 20 & 26.95 & 2 & 0.21 & 22.73 \\
2 & 34 & 20 & 26.98 & 2 & 0.42 & 15.05 \\
3 & 27 & 20 & 28.34 & 1 & 0.20 & 23.8 \\
4 & 18 & 10 & 21.27 & 1 & 0.27 & 18.88 \\
5 & 21 & 10 & 25.29 & 1 & 0.45 & 16.03 \\
6 & 20 & 10 & 21.64 & 2 & 0.53 & 15.94 \\
7 & 45 & 10 & 0.43 & 2 & 13.31 & 0.45 \\
8 & 64 & 10 & 0.62 & 2 & 9.23 & 0.62 \\
9 & 12 & 10 & 11.15 & 2 & 0.51 & 10.42 \\
\hline \multicolumn{4}{c}{}
\end{tabular}

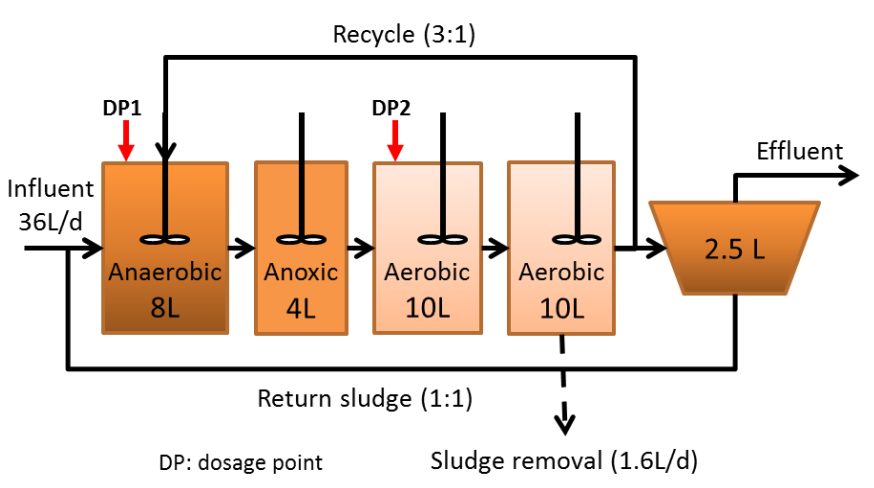

The ability of the new FeP model to reproduce the behavior of a simple three-stage Phoredox pilot plant operated under different conditions will be evaluated and compare to a simple iron phosphate precipitation model. The model combined to ASM2d is implemented in Aquasim software (version 2.1g, EAWAG, Dübendorf, Switzerland) (Reichert, 1994). The behavior of the biological model will first be compared with the control pilot data. The full model will then be applied to the different periods of the pilot-plant operation. 


\section{REFERENCES}

Barat, R., Montoya, T., Seco, A., Ferrer, J., 2005. The Role of Potassium, Magnesium and Calcium in the Enhanced Biological Phosphorus Removal Treatment Plants. Environ. Technol. 26, 983-992.

De Haas, D.W., Wentzel, M.C., Ekama, G.A., 2000a. The use of simultaneous chemical precipitation in modified activated sludge systems exhibiting biological excess phosphate removal Part 3: Experimental periods using alum. Water Sa 26, 467-483.

De Haas, D.W., Wentzel, M.C., Ekama, G.A., 2000b. The use of simultaneous chemical precipitation in modified activated sludge systems exhibiting biological excess phosphate removal Part 4: Experimental periods using ferric chloride. Water Sa 26, 485-504.

De Haas, D.W., Wentzel, M.C., Ekama, G.A., 2001. The use of simultaneous chemical precipitation in modified activated sludge systems exhibiting biological excess phosphate removal - Part 7: Application of the IAWQ model. Water Sa 27, 151-168.

Fytianos, K., Voudrias, E., Raikos, N., 1998. Modelling of phosphorus removal from aqueous and wastewater samples using ferric iron. Environ. Pollut. 101, 123-130.

Hauduc, H., Tackacs, I., Smith, S., Szabo, A., Murthy, S., Daigger, G.T., Sperandio, M., 2013. A Dynamic Physicochemical Model for Chemical Phosphorus Removal. Presented at the WEF/IWA Nutrient Removal and Recovery 2013, Vancouver, BC, Canada.

Luedecke, C., Hermanowicz, S., Jenkins, D., 1989. Precipitation of Ferric Phosphate in Activated-Sludge - a Chemical-Model and Its Verification. Water Sci. Technol. 21, 325-337.

Reichert, P., 1994. Aquasim - A tool for simulation and data analysis of aquatic systems. Water Sci. Technol. 30, 21-30.

Smith, S., Takacs, I., Murthy, S., Daigger, G.T., Szabo, A., 2008. Phosphate complexation model and its implications for chemical phosphorus removal. Water Environ. Res. 80, 428-438.

Sotemann, S., Musvoto, E., Wentzel, M., Ekama, G., 2005. Integrated biological, chemical and physical processes kinetic modelling Part 1 - Anoxic-aerobic $\mathrm{C}$ and $\mathrm{N}$ removal in the activated sludge system. Water Sa 31, 529-544.

Szabo, A., Takacs, I., Murthy, S., Daigger, G.T., Licsko, I., Smith, S., 2008. Significance of design and operational variables in chemical phosphorus removal. Water Environ. Res. 80, 407-416. 


\title{
A control-based approach to achieve efficient biological phosphorus removal in WWTPs: design, simulation, optimisation and experimental validation
}

\author{
Javier Guerrero, Albert Guisasola and Juan Antonio Baeza \\ Departament d'Enginyeria Química, Escola d'Enginyeria, Universitat Autònoma de Barcelona, \\ 08193, Bellaterra, Barcelona, Spain. Corresponding Author: JuanAntonio.Baeza@uab.cat
}

\section{MOTIVATIONS}

The increasingly stricter legislation for WWTP has raised the need of a more efficient management and hence, successful biological $\mathrm{P}$ removal has become a short term aim. The implementation of enhanced biological phosphorus removal (EBPR) is considered the most sustainable approach to meet $\mathrm{P}$ discharge levels, and the current knowledge gained on this process has raised the opportunity of developing new control structures to specifically control P effluent concentration (Guerrero et al., 2011b; Machado et al., 2009; Ostace et al., 2013). However, despite EBPR is nowadays a quite known technology, its interaction with biological nitrogen removal may still lead to P removal failures in full-scale WWTP (Guerrero et al., 2011a).

The research presented in this work integrates a modelling-based study of a novel control strategy to minimize the existing detrimental interactions between biological $\mathrm{N}$ and $\mathrm{P}$ removal together with its experimental validation in a $170 \mathrm{~L}$ pilot plant with on-line monitoring of nutrients (phosphate, nitrate and ammonium). The model calibrated and validated was an extension of ASM2d that also includes nitrite as state variable and corrected settler reactivity (Guerrero et al., 2013). This research, thus, embraces the design, implementation, simulation, optimization, systematic evaluation and experimental validation of conventional and innovative control strategies under normal operation and stress operation (i.e. influent disturbances, ammonium, nitrate and nitrite peaks...) for two different WWTP configurations with P-removal ( $\mathrm{A}^{2} \mathrm{O}$ and Johannesburg). A systematic approach was conducted to assess the performance of the control strategy based on classical benchmark indexes related to the operational costs and related to the possibility of developing microbial-related problems in the settler.

Several studies reported that the presence of VFA in the wastewater is mandatory to obtain a high P removal capacity. Unfortunately, an external VFA addition is not usually cost-effective and it increases the overall plant carbon footprint. A promising and very attractive alternative would be focused on the utilization of waste materials that could be fermented to VFA (Guerrero et al., 2012). Glycerol is a good example of such wastes materials since it is a byproduct of biodiesel fuel production: about $1 \mathrm{~L}$ of glycerol is generated for every $10 \mathrm{~L}$ of produced biodiesel fuel produced. Taking into account all these considerations, crude glycerol could be a very practical and cost-effective external carbon source to reduce the detrimental effect of nitrate under anaerobic conditions since it could be used in both $\mathrm{N}$ a $\mathrm{P}$ removal processes. However, there are not previous studies about crude glycerol utilization as carbon source for improving EBPR. 


\section{RESULTS AND DISCUSSION}

The control strategy presented is based on external carbon addition to mitigate the detrimental effect on EBPR of nitrate entering to the anaerobic phase. The procedure used involved four steps:

1) Model calibration under open-loop conditions including experimental data when the plant is operated with different disturbances (see Figure 1). The effect of these disturbances should be minimised once the control strategy is active. The parameters were calibrated following the guidelines of GMP and using the FIM approach to define confidence intervals (see example in Table 1). Data from a high ammonium disturbance (HAD) were used for calibration and data from a high nitrite disturbance (HND) for validation.

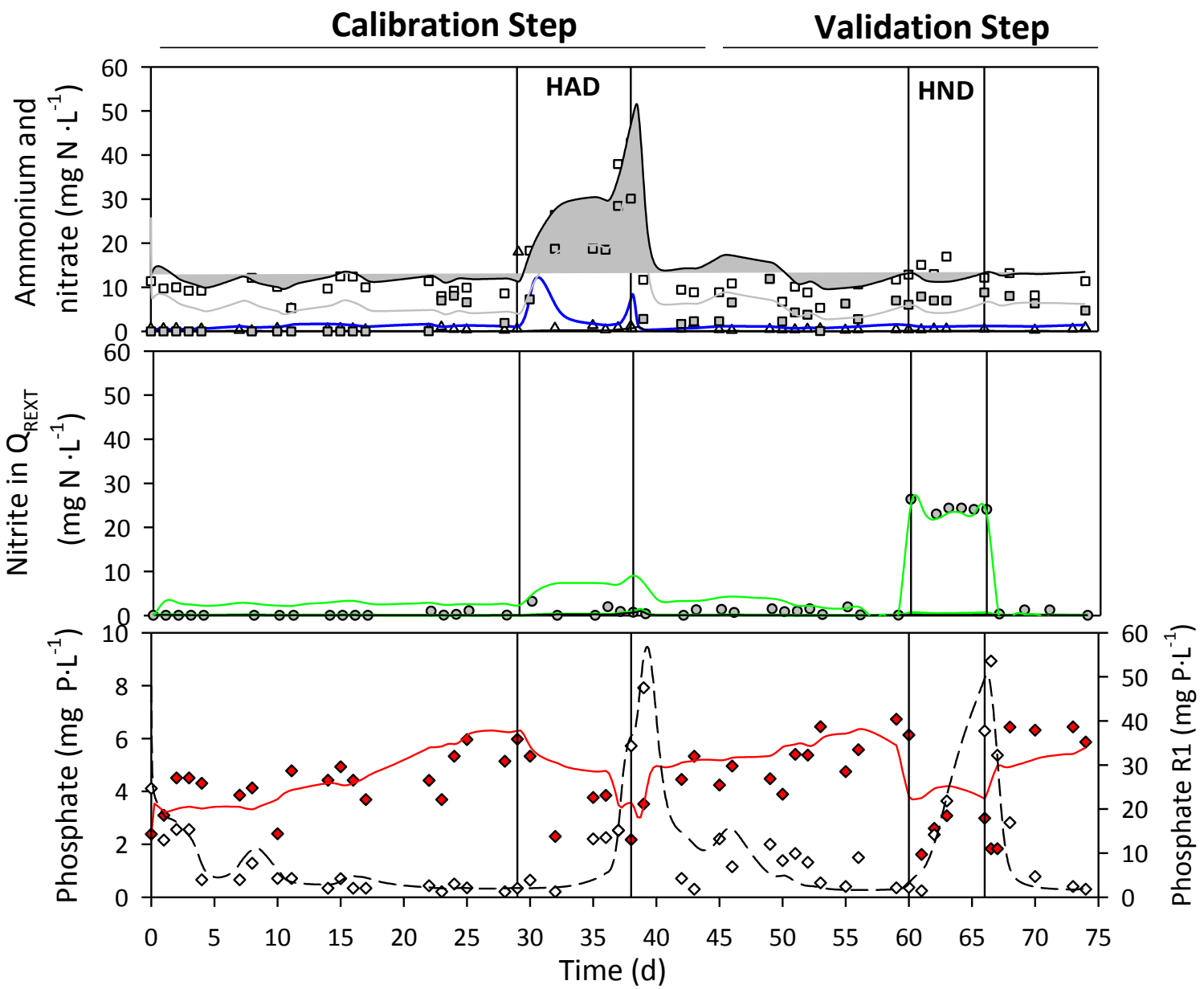

Figure 1 Model calibration and validation. $\mathrm{A}^{2} / \mathrm{O}$ pilot plant behaviour and model predictions. $\triangle$ represents ammonium, $\square$ nitrate, $\bigcirc$ nitrite and $\diamond$ phosphorus. Red colour belongs to R1 (anaerobic reactor) concentrations, white colour to R3 (effluent), grey colour to $\mathrm{Q}_{\mathrm{REXT}}$. Model predictions: blue line indicates simulation ammonium results in R3, black line belongs to nitrate in R3, grey line to nitrate in $\mathrm{Q}_{\mathrm{REXT}}$, green line to nitrite in $\mathrm{Q}_{\mathrm{REXT}}$, red line to phosphate in $\mathrm{R} 1$ and black dashed line to phosphate in $\mathrm{R}$.

Table 1 Parameters obtained in the model calibration by using the experimental data of $\mathrm{AD}$ in $\mathrm{A}^{2} / \mathrm{O}$ configuration. RSF: Reduction factor applied to the reactive settler capacity.

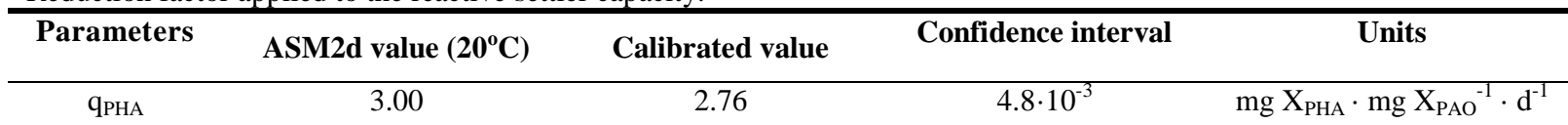




\begin{tabular}{|c|c|c|c|c|}
\hline $\mathrm{q}_{\mathrm{PP}}$ & 1.50 & 1.70 & $2.4 \cdot 10^{-3}$ & 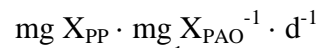 \\
\hline$\mu_{\mathrm{HET}}$ & 6.00 & 4.10 & $3.6 \cdot 10^{-3}$ & $\mathrm{~d}^{-1}$ \\
\hline $\mathrm{y}_{\mathrm{NO} 3}$, оно & 0.80 & 0.32 & $5.7 \cdot 10^{-3}$ & . \\
\hline $\mathrm{y}_{\mathrm{NO} 2, \text { оно }}{ }^{2}$ & - & 0.48 & $2.5 \cdot 10^{-2}$ & - \\
\hline RFS* & - & 0.59 & $2.6 \cdot 10^{-3}$ & - \\
\hline
\end{tabular}

* These parameters do not appear in ASM2d model (Henze et al., 2000)

2) Design the adequate control strategy for the existing problem. This is a theoretical step where the know-how of the designers and the previous experimental data is essential. Figure 2 shows, in this example, the description of the control strategy based on crude glycerol addition under anaerobic conditions to reduce the nitrate concentration in the anaerobic reactor. The same control strategy was proposed and evaluated for both $\mathrm{A}^{2} \mathrm{O}$ and Johannesburg configurations. The parameters of the digital controllers were tuned according to ITAE criteria.

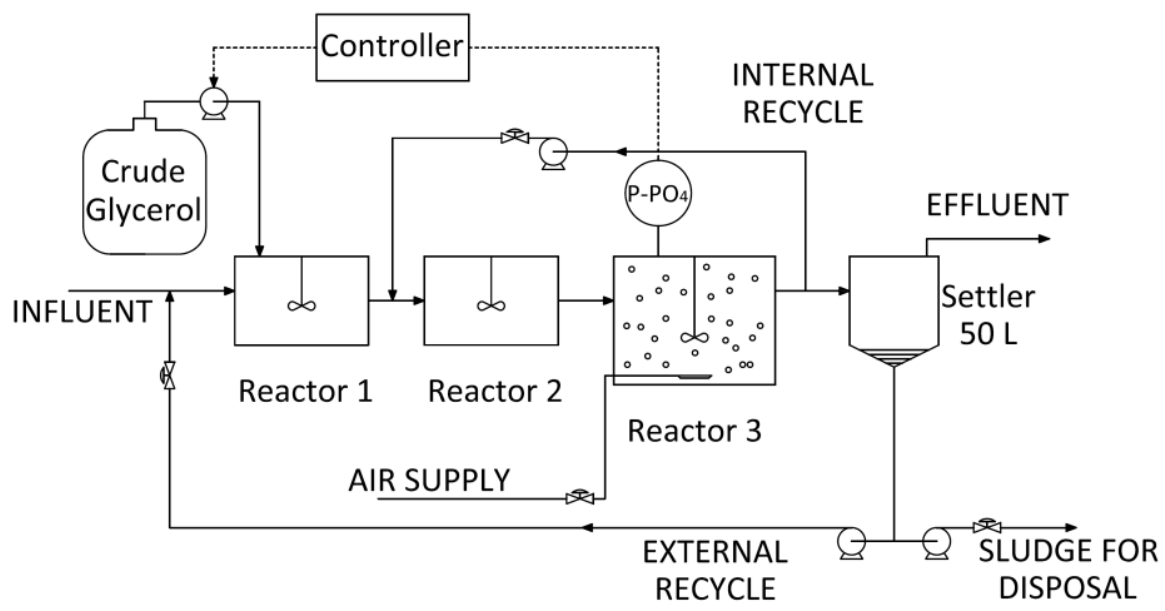

Figure 2 Scheme of the control structure proposed to reduce nitrate effect on EBPR in an A2/O configuration.

3) Simulation-based assessment of the proposed control strategy under different scenarios. Once the control design is implemented, several scenarios (influent variability, disturbances, parameter changes, $\mathrm{T}, \mathrm{pH} . .$.$) can be tested depending on the model prediction$ capability. In this case, the optimised and tuned control strategy was implemented in $\mathrm{A}^{2} / \mathrm{O}$ and JHB pilot plants to prevent EBPR failure for different ammonium and nitrite disturbances (see Figure 3).

4) Experimental validation. Once the model simulations are run, the control configuration with the optimised tuning is tested in the pilot plant. The maximum number of disturbances are applied to get even more insight on the process performance and to verify the simulationbased results. Figure 3 shows the validation step when the glycerol control strategy was applied. The model predicted very well the evolution of the main parameters and the control performance.

The results show that simple PI feed-back control for crude glycerol dosage could be very useful for easily maintain proper P-removal efficiency in full-scale WWTP, even operating under high nitrate anaerobic inlet conditions. However, some limitations on control performance could appear when treating influents with sudden changes in $\mathrm{N}$ influent concentration, because the controlled variable (P effluent concentration) has a slow response in front of manipulated variable changes (crude glycerol addition). Two different approaches are proposed to solve this behaviour: i) a simple modification of the control strategy by 
controlling the P concentration in R1 and ii) feed-forward control in combination with feedback control.

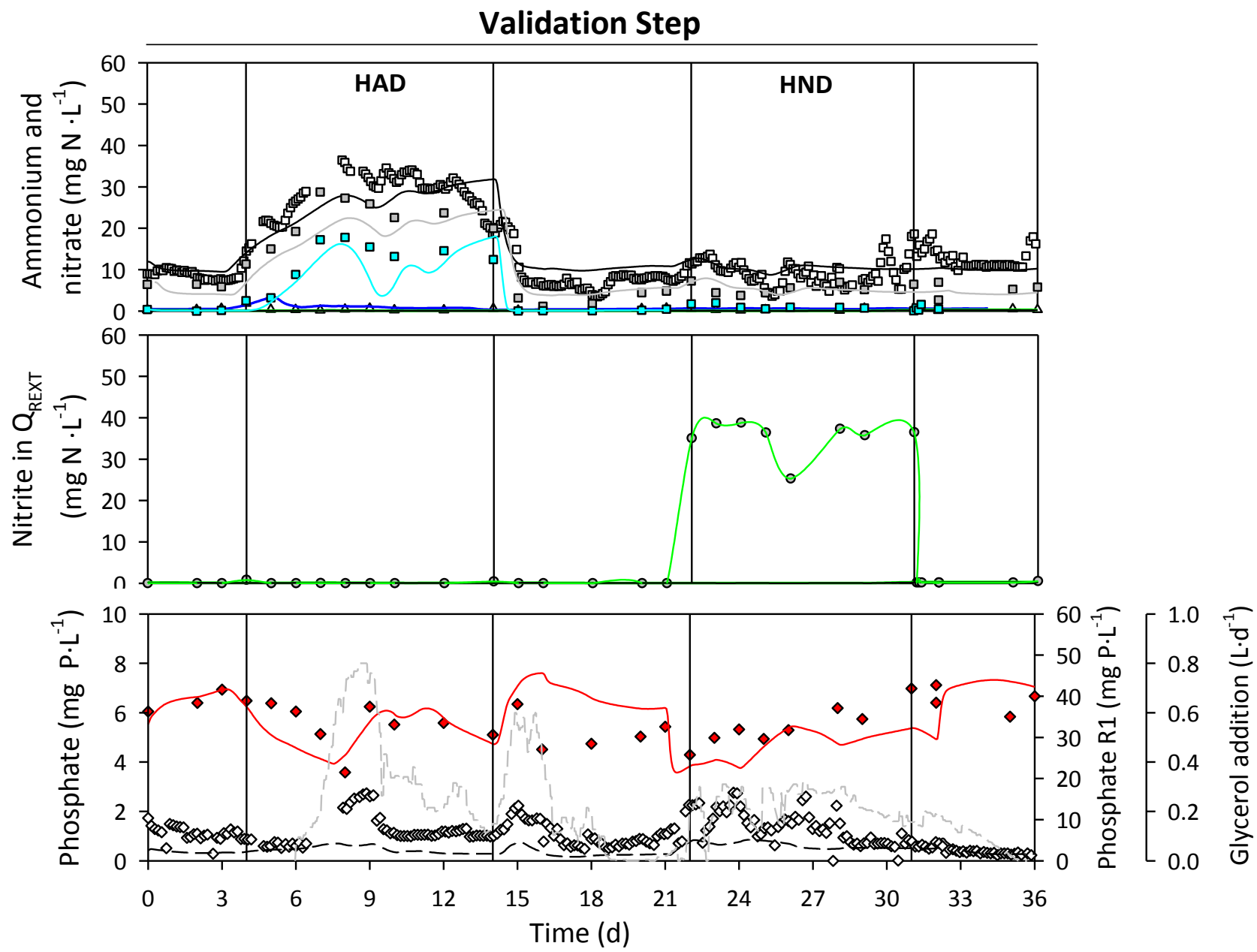

Figure 3 Experimental and model predictions when the control was applied in the JHB scenario. $\triangle$ represents ammonium, $\square$ nitrate, $\bigcirc$ nitrite and $\diamond$ phosphorus. Red filled colour belongs to R1 (anaerobic reactor), white colour to R3 (effluent) concentrations, grey colour to QREXT (external recycle) concentrations. Model predictions: blue line indicates simulation ammonium results in R3, black line belongs to nitrate in R3, green line belongs to nitrite in R3, grey line to nitrate in QREXT, grey dashed line to nitrite in QREXT, red line to phosphate in R1 and black dashed line to phosphate in R3.

\section{REFERENCES}

Guerrero J, Flores-Alsina X, Guisasola A, Baeza JA, Gernaey K V. 2013. Effect of nitrite, limited reactive settler and plant design configuration on the predicted performance of simultaneous $\mathrm{C} / \mathrm{N} / \mathrm{P}$ removal WWTPs. Bioresource Technol. 136:680-688.

Guerrero J, Guisasola A, Baeza JA. 2011a. The nature of the carbon source rules the competition between PAO and denitrifiers in systems for simultaneous biological nitrogen and phosphorus removal. Water Res. 45:4793-802.

Guerrero J, Guisasola A, Vilanova R, Baeza JA. 2011b. Improving the performance of a WWTP control system by model-based setpoint optimisation. Environ. Model. Softw. 26:492-497.

Guerrero J, Tayà C, Guisasola A, Baeza JA. 2012. Glycerol as a sole carbon source for enhanced biological phosphorus removal. Water Res. 46:2983-2991.

Machado VC, Gabriel D, Lafuente J, Baeza JA. 2009. Cost and effluent quality controllers design based on the relative gain array for a nutrient removal WWTP. Water Res. 43:5129-5141. 
Ostace GS, Baeza JA, Guerrero J, Guisasola A, Cristea VM, Agachi PŞ, Lafuente J. 2013. Development and economic assessment of different WWTP control strategies for optimal simultaneous removal of carbon, nitrogen and phosphorus. Comput. Chem. Eng. 53:164-177.

\title{
Validation of spontaneous mineral precipitation models
}

\author{
Christian Kazadi Mbamba, Damien Batstone, Stephan Tait \\ Advanced Water Management Centre, The University of Queensland, St Lucia, Brisbane, QLD 4072, \\ Australia (Email: c.kazadimbamba@awmc.uq.edu.au)
}

\begin{abstract}
Current process models used across the wastewater industry have inherent limitations due to limited physico-chemical models, especially with respect to description of mineral precipitation reactions. As part of the overall effort towards development of more general and robust physicochemical models, this paper uses acid-base titration experiments to examine an equilibrium-kinetic modelling approach. The approach was first validated without precipitation and was then tested for titration with precipitation of magnesium and calcium phosphate minerals. The results suggest that a single-parameter equilibrium-kinetic approach can provide good prediction of wastewater $\mathrm{pH}$ over a wide range and is generally expandable for inclusion of multiple minerals.
\end{abstract}

Keywords

Acid-base titration, physico-chemical modelling, precipitation, equilibrium, kinetics

\section{INTRODUCTION}

Mathematical models have been successfully used as tools in research, process design, training, control and optimization of wastewater treatment. However, to date the emphasis of wastewater process models has largely been on biological reactions (Batstone et al. 2002, Henze et al. 2000) rather than physico-chemistry which is also highly influential and important (Batstone et al. 2012). Consequently, physico-chemistry modelling of wastewater has become an important field of research and development during recent times (Musvoto et al. 2000a, Tait et al. 2009). To date different modelling approaches have been applied to study a particular class of influential physico-chemical reactions, namely chemical precipitation. These modelling approaches are typically equilibrium-based (time-independent, thermodynamically driven) or kinetic-based (time-dependent, reaction rate dependent).

Briefly, equilibrium-based models assume that sufficient time has passed with each model timestep so that precipitation reactions achieve thermodynamic equilibrium and solution thermodynamics then determine the final mineral phase composition (Loewenthal et al. 1995, Ohlinger et al. 1998). Thus, the equilibrium approach does not give consideration of metastability (the occurrence of a significant state of supersaturation without any spontaneous precipitation), does not give information on intermediate mineral phases along the way to achieving thermodynamic equilibrium, and does not account for precipitation interactions between various mineral phases (Valsami-Jones 2001). An equilibrium-based approach can however be computationally efficient when compared with a kinetic-based approach and does 
not require calibration of model parameters because these are all pre-defined by well-known electrolyte solution thermodynamics.

On the other hand, a kinetic-based model allows chemical precipitation or dissolution reactions to occur with each model timestep to achieve a resulting solution composition which may still have a significant super-or-undersaturation state. Kinetic-based modelling approaches are helpful to capture mechanistic aspects such as intermediate mineral phases (Barat et al. 2011) and inhibition of precipitation (Tait et al. 2009). However, model complexity can be an issue with kinetic-based models. As the number of minerals and precipitating ions increase, the complexity of the model also increases (Batstone et al. 2012). Each kinetic physico-chemical reaction has a dedicated kinetic rate expression with additional parameters to determine, and can introduce additional dynamic states for the solid mineral phases.

A likely optimum scenario is a combination of equilibrium and kinetic-based model structure to increase computational efficiency as well as capture mechanistic information. Such a model treats fast physico-chemical reactions (such as aqueous phase reactions) as equilibrium, and slow physico-chemical reactions (such as minerals precipitation) as slow dynamic processes. Combined equilibrium and kinetics may be a robust modelling approach that could be adopted across the wastewater industry as a general approach that would foster collaboration and communication and allow streamlined formulation of plant-wide models (Batstone et al. 2012). The equilibrium-kinetic modelling does however require testing and validation before widespread adoption, and towards this end, the present work uses acid-base titration with mineral precipitation to evaluate the modelling approach.

\section{Materials and Methods}

\section{Sample materials}

Stock solutions were prepared with analytical grade reagent chemicals dissolved in de-ionised water. The ionic strength of the stock solutions was typically adjusted to a desired value by adding sodium chloride. No mineral precipitate seed was used in the experiments, so any precipitation occurred spontaneously.

\section{Apparatus}

Solution $\mathrm{pH}$ was measured with a calibrated DGi115-SC pH probe (Mettler-Toledo, Greifensee, Switzerland). Potentiometric titrations were performed with a fully automated auto-titrator (T50, Mettler-Toledo, Greifensee, Switzerland). The titrator was equipped with a $10 \mathrm{~mL}$ burette to add titrant to the test solution. Titration vessels used in the experiments were (a) $100 \mathrm{~mL}$ beakers, with working volumes of $75 \mathrm{~mL}$ or b) a $1 \mathrm{~L}$ stirred glass crystallizer. During a titration, the test sample to which titrant was added was stirred with a $25 \mathrm{~mm}$ tefloncoated magnetic bar stirrer at $150 \mathrm{rpm}$. The volume of each titrant added, the $\mathrm{pH}$ and the temperature were recorded over time through a LabX Light Titration Software interface (Mettle-Toledo, Greifensee, Switzerland).

\section{Experimental procedure}

Acid-base titration tests were performed with and without precipitation to provide experimental data for model testing. All experiments were carried out at room temperature 
$\left(20-25^{\circ} \mathrm{C}\right)$. The $\mathrm{pH}$ probe was calibrated before each titration experiment using standard calibration buffers at $\mathrm{pH} 2, \mathrm{pH} 4, \mathrm{pH} 7$ and $\mathrm{pH} 10$. The $\mathrm{pH}$ of a 75-1000 mL aliquot of test solution (actual volume known accurately) test solution was adjusted to acidic $\mathrm{pH}$ with hydrochloric acid. About $10-20 \mathrm{~mL}$ of sodium hydroxide (or other titrant) was then quantitatively added at a set dosing rate and $\mathrm{pH}$ was continuously measured. For each experiment, the volume of titrant added over time and the corresponding $\mathrm{pH}$ were recorded together with the initial volume of the test solution. In some of the experiments a $1 \mathrm{~mL}$ sample of the crystallizer contents was collected at specified time intervals, immediately filtered through $0.2 \mu \mathrm{m}$ syringe filters (PES) membrane, diluted with deionized water to prevent post-precipitation and stored at $4^{\circ} \mathrm{C}$ until further analysis by Inductively Coupled Plasma Optical Emission Spectroscopy (ICP-OES) and flow injection analysis (FIA).

\section{Analytical techniques}

The major elements (Ca, Mg) in the synthetic aqueous solution were analysed with ICP-OES (Perkin Elmer Optima 7300DV, Waltham, MA, USA). Ionic concentrations ammonical nitrogen (NH4-N) and phosphate phosphorus (PO4-P) were quantified with a Lachat QuickChem 8500 flow injection analyser (Lachat Instruments, Loveland, CO, USA) as per the Lachat QuickChem Method 31-107-06-1-A.

\section{Modelling}

The model structure consisted of two parts, namely, an equilibrium part which described aqueous phase reactions such as the acid-base and soluble ion association and a kinetic part for the slower chemical precipitation or dissolution reactions.

\section{Modelling of aqueous equilibria}

The equilibrium part consisted of a number of non-linear algebraic equations which were formulated by substituting equilibrium relationships (mass action laws) into species contribution balances in accordance with the so-called Tableau Method (Morel and Morgan 1972, Morgan and Stumm 1996). This provides a reduced equation set to solve for a smaller subset of selected ingredients (the model "components") which have been arbitrarily selected to account for the total composition of the wastewater. This is a common approach used in the formulation of equilibrium models. The selected model components in this work were $\mathrm{H}^{+}$, $\mathrm{Na}^{+}, \mathrm{NH}_{4}{ }^{+}, \mathrm{Cl}^{-}, \mathrm{Ca}^{2+}, \mathrm{Mg}^{2+}, \mathrm{CO}_{3}{ }^{2-}, \mathrm{PO}_{4}{ }^{3-}$ and the selected species (wastewater ingredients that were not selected as components) were $\mathrm{H}_{2} \mathrm{CO} 3, \mathrm{H}_{2} \mathrm{PO}_{4}^{-}, \mathrm{H}_{3} \mathrm{PO}_{4}, \mathrm{HCO}_{3}^{-}, \mathrm{HPO}_{4}^{-2}, \mathrm{Mg}\left(\mathrm{NH}_{3}\right)_{2}{ }^{2+}$, $\mathrm{Mg}_{2} \mathrm{CO}_{3}{ }^{2+}, \mathrm{MgCl}^{+}, \mathrm{MgCO}_{3}, \mathrm{MgHCO}_{3}{ }^{+}, \mathrm{MgHPO}_{4}, \mathrm{MgOH}^{+}, \mathrm{MgPO}_{4}^{-}, \mathrm{Na}_{2} \mathrm{HPO}_{4}, \mathrm{Na}_{2} \mathrm{PO}_{4}^{-}$, $\mathrm{NaCl}, \mathrm{NaCO}_{3}^{-}, \mathrm{NaH}_{2} \mathrm{PO}_{4}, \mathrm{NaHCO}_{3}, \mathrm{NaHPO}_{4}^{-}, \mathrm{NaOH}, \mathrm{NaPO}_{4}{ }^{2-}, \mathrm{NH}_{3}, \mathrm{OH}^{-}$. The effects of temperature was corrected for using the constant-enthalpy van't Hoff approach and ionic strength was corrected for with the Davies approximation of activity coefficients also with separate temperature correction. In the development of the speciation model, one of the mainstream aqueous speciation programs, visual MINTEQ (Allison et al., 1991), was used as reference model to validate the simulation outputs of the developed equilibrium model, and thermodynamic constants that are in the MINTEQ database were also used in this study. Ionic strength and activity coefficient equations were solved with the main tableau equation set such that there were 10 implicit unknowns for a like number of equations. The full implicit algebraic equation set is given in the supplementary material. 
The potential for precipitation or dissolution to occur was predicted using the well-known parameter termed the saturation index (SI) which is defined as follows

$$
S I=\log _{10} \frac{I A P}{K_{s p}}
$$

where IAP is the product of the chemical activities of participating ions in the supersaturated solution and as $K_{s p}$ is the solubility product constant. For a given solution, three states exist, depending on the saturation index:

- If $S I<0$, the solution is undersaturated, and a solid phase can dissolve into solution;

- If $S I=0$, the solution is said to be saturated or at equilibrium;

- If $S I>0$, the solution is supersaturated or oversaturated with respect to the mineral in solution, and chemical precipitation can occur.

\section{Modelling of precipitation kinetics}

The second equation subset consisted of slow kinetic reactions with corresponding kinetic rate laws which in-turn influenced a dynamic state equation set via respective stoichiometric coefficients. The rates at which these slow reactions occur are dependent on the concentrations of specific ingredients which are modelled in the equilibrium equation set (that is, the participating ions for a precipitating mineral). Thus, the kinetic equation set is influenced by the model solution of the parallel algebraic equation set. The equation set included dynamic state equations for total dissolved species and each mineral phase considered and the kinetic rate laws listed in Table 1. Each rate law expression had a lumped empirical rate coefficient $k$, a supersaturation term between brackets which determined the thermodynamic driving force for precipitation or dissolution to occur based on the chemical activities of the respective ions and a $K_{\mathrm{sp}}$ value which is the solubility product constant. The power-exponent values on each of the respective supersaturation terms were set at 3 for struvite and 2 for newberyite based on the prior work of Gunn (1976) and Musvoto et al. (2000b), respectively. This model structure can be easily expanded to include the precipitation of many other minerals.

Table 1: Stoichiometric matrix for the precipitation/dissolution and liquid-gas transfer processes

\section{Chemical states}

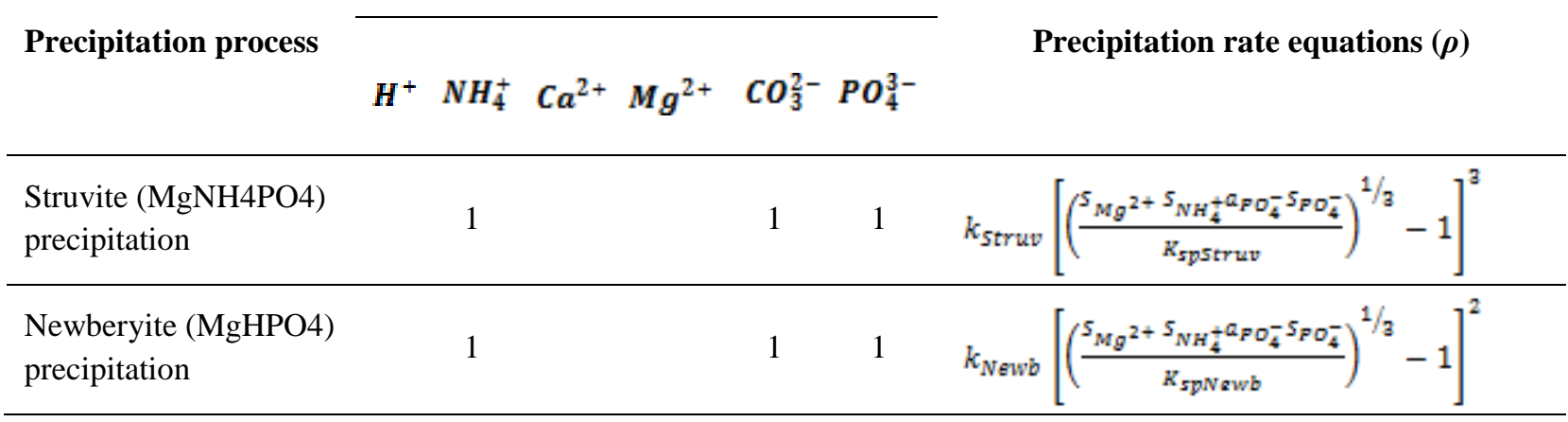

The $K_{s p}$ value for Struvite was assumed to be $10^{-13.26}$ (Kofina and Koutsoukos, 2005) and for Newberyite it was assumed to be $10^{-18.17}$ from the thermodynamic database in Visual Minteq (Allison et al. 1991). The model was implemented in MATLAB/SIMULINK (Version 8.1, 
MathWorks Inc.) and the code is available from the authors on request, including documentation of the code.

\section{RESULTS AND DISCUSSION}

\section{Equilibrium validation}

Figure 1 presents results for measured and modelled $\mathrm{pH}$ of preliminary validation experiments without any precipitation. The results show that the model reliably predicted $\mathrm{pH}$ in the absence of precipitation, thus indicating that the equilibrium portion of the model was correctly implemented. It is important to note that no parameter adjustment was required to achieve these predictions, because the equilibrium is based on well-established electrolyte solution thermodynamics.

(a)

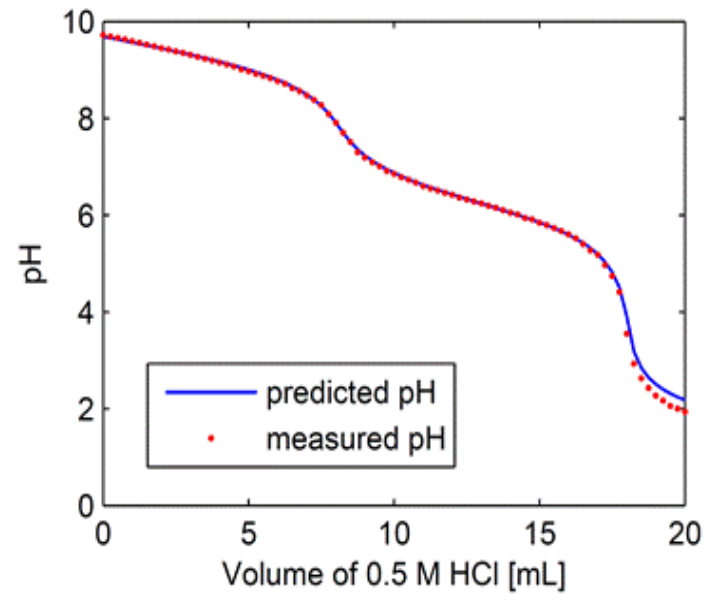

(b)

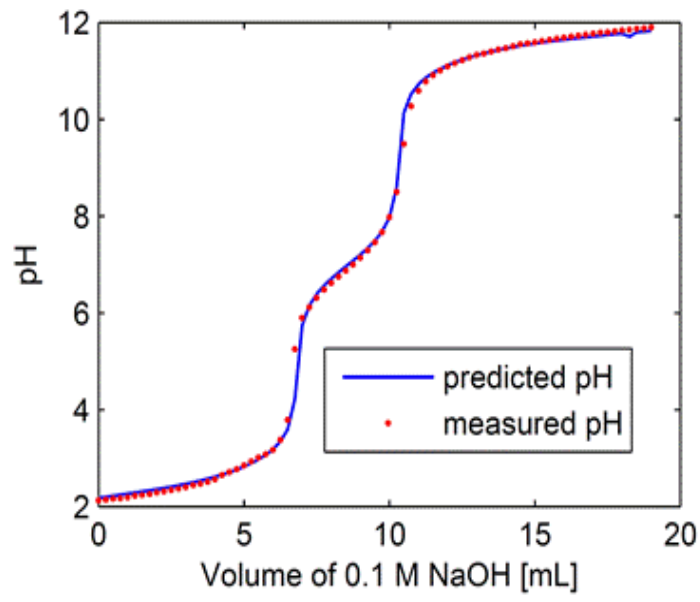

Figure 1: Model and experimental results for titration without precipitation and (a) $0.5 \mathrm{M} \mathrm{HCl}$ added to synthetic aqueous solution with $60 \mathrm{mM} \mathrm{Na}_{2} \mathrm{CO}_{3}, 50 \mathrm{mM} \mathrm{NH} \mathrm{NH}_{4} \mathrm{Cl}, 2.44 \mathrm{mM}$ sodium acetate and $5 \mathrm{mM} \mathrm{NaH}_{2} \mathrm{PO}_{4}$ (initial $\mathrm{pH}$ was increased with concentrated $\mathrm{NaOH}$ ); and (b) $0.1 \mathrm{M}$ $\mathrm{NaOH}$ added to synthetic aqueous solution with $5 \mathrm{mM} \mathrm{NaH}_{2} \mathrm{PO}_{4}$ (initial $\mathrm{pH}$ was decreased with concentrated $\mathrm{HCl}$ ).

\section{Titration with precipitation}

Figure 2 show modelled and measured $\mathrm{pH}$ values for experiments with precipitation. The model was able to describe the experimental behaviour well for a value of $k_{\text {Struv }}=0.7 \times 10^{-6}$ moles. $\min ^{-1}$ (Figure 2a). To attempt to simulate what would happen if struvite precipitation was occurring very fast (and thus the resulting solution would be forced to achieve a constant state of thermodynamic equilibrium) The value of $k_{S t r u v}$ was arbitrarily set at a high value of 0.1 moles. $\min ^{-1}$ and the simulation results for this model is presented in Figure $2 \mathrm{~b}$. The resulting quasi-equilibrium precipitation is clearly shown in Figure 2c by a Saturation Index value of 0 (at equilibrium) between a model time of about 100 minutes and 500 minutes. That is, a model with rate parameters which are set to be sufficiently rapid theoretically gives identical results to an equilibrium-based formulation of the same modelled system (Musvoto et al. 2000a). The marked discrepancy between modelled and measured $\mathrm{pH}$ shown in the 
quasi-equilibrium simulation (Figure $2 b$ ) is clear evidence that precipitation kinetics were influential over a titration time-scale of 600 minutes (10 hours). This is also shown in Figures $2 \mathrm{c}$ and $2 \mathrm{~d}$ by the highly positive Saturation Index values for struvite as calculated from the measured concentration of magnesium, ammonical nitrogen and phosphate phosphorus in the liquid phase (data points in Figure 2c and 2d).

(a)

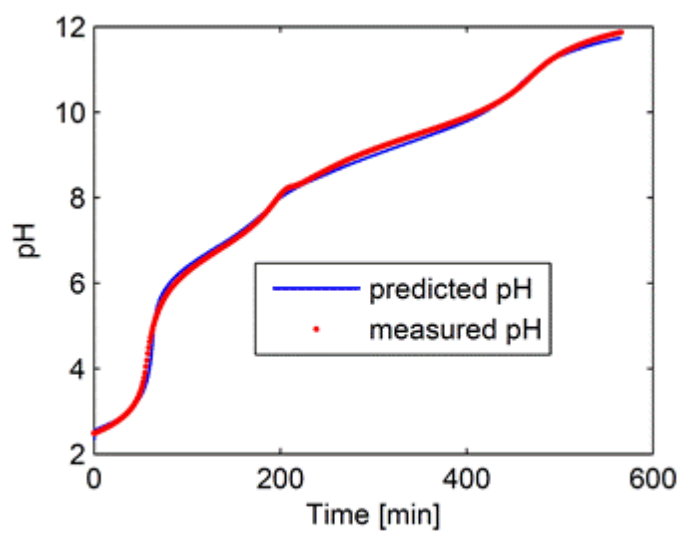

(c)

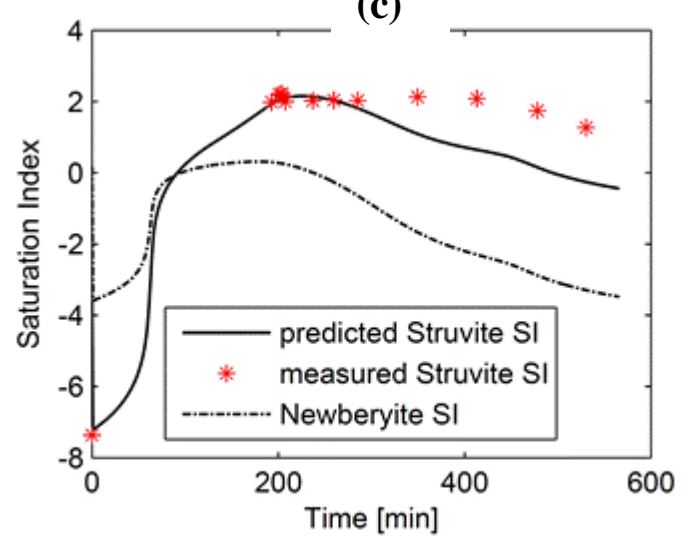

(b)

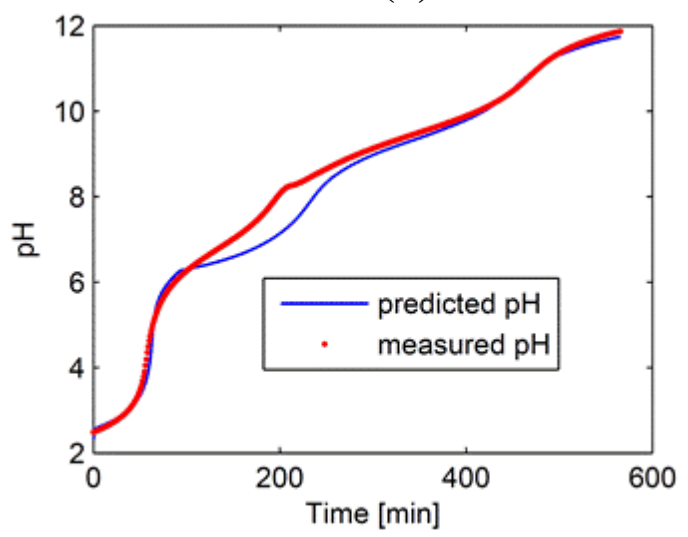

(d)

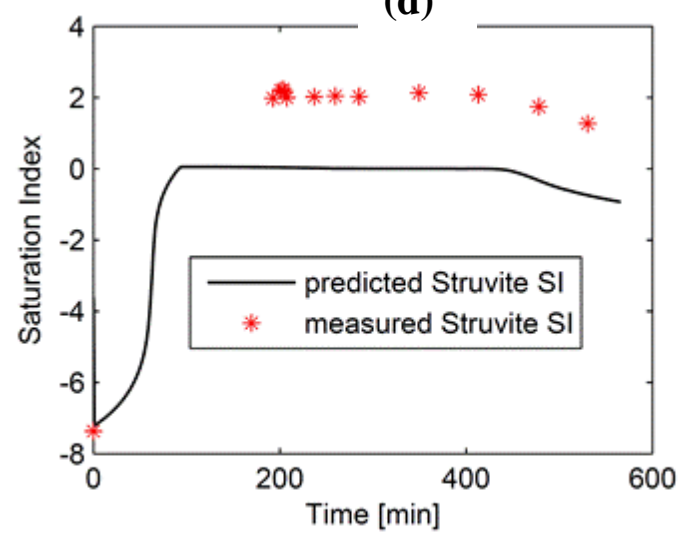

Figure 2: Results with precipitation - Measured $\mathrm{pH}$ for $2.1 \mathrm{M} \mathrm{NaOH}$ added at a rate of $5.35 \times$ $10^{-5} \mathrm{~mL} / \mathrm{min}$ to a synthetic aqueous solution with $14.27 \mathrm{mM} \mathrm{NaH}_{2} \mathrm{PO}_{4}, 29.84 \mathrm{mM} \mathrm{NH} \mathrm{NCl}_{4}$, $4.85 \mathrm{mM} \mathrm{MgCl}_{2}$ (initial $\mathrm{pH}$ was decreased with concentrated $\mathrm{HCl}$ ), presented with predicted $\mathrm{pH}$ for (a) $k=0.7 \times 10^{-6}$ moles. $\mathrm{min}^{-1}$ and (b) $k=0.7$ moles. $\mathrm{min}^{-1}$. Measured saturation index presented with predicted saturation index with (c) $k=0.7 \times 10^{-6}$ moles. $^{-1} n^{-1}$ and (d) $k=0.7$ moles. $\min ^{-1}$.

The precipitation kinetics only influenced predicted $\mathrm{pH}$ over the range of 6-9, which suggested that above $\mathrm{pH} 9$, the aqueous phase equilibrium dominated $\mathrm{pH}$ predictions. Below $\mathrm{pH} 6$ it was known that precipitation had not yet occurred, as observed from a clear test solution (no precipitates visually observed) and the negative Saturation Index values.

From the saturation index values in Figure 2 (c), it is clear that newberyite $\left(\mathrm{MgHPO}_{4} \cdot 3 \mathrm{H}_{2} \mathrm{O}\right)$ did not show a significant supersaturation state for the particular experimental conditions. Accordingly, it was expected that only struvite precipitated in the experiment. In general, 
struvite formation dominates, unless the supersaturation of newberyite is sufficiently greater than that of struvite (Abbona et al. 1982).

Figure 3 shows measured and predicted results for the aqueous-phase composition. As can be seen there is a good correlation between the experimental results and theoretical model predictions. The soluble concentrations of magnesium, total ammonical nitrogen and total phosphate phosphorus remain constant when the solution is undersaturated with respect to struvite as indicated by a negative Saturation Index value, and decreases when the solution becomes supersaturated with respect to struvite as shown by a positive Saturation Index value. These observations support the view that the saturation index can provide guidance to the modeller about which minerals may be precipitating under which conditions in a wastewater as previously suggested by others (Marti et al. 2008).

The model as a whole was considered to be highly identifiable due to the small number of fitting parameters (only the $k$-values for each of the minerals). The changing mineral surface area with continuous precipitation of struvite did not need to be considered in the model to give good predictions. These results suggested that a single-parameter ( $k$-value) equilibriumkinetic model (which is easily expandable to include other minerals) can provide good prediction of wastewater $\mathrm{pH}$ with precipitation.

(a)
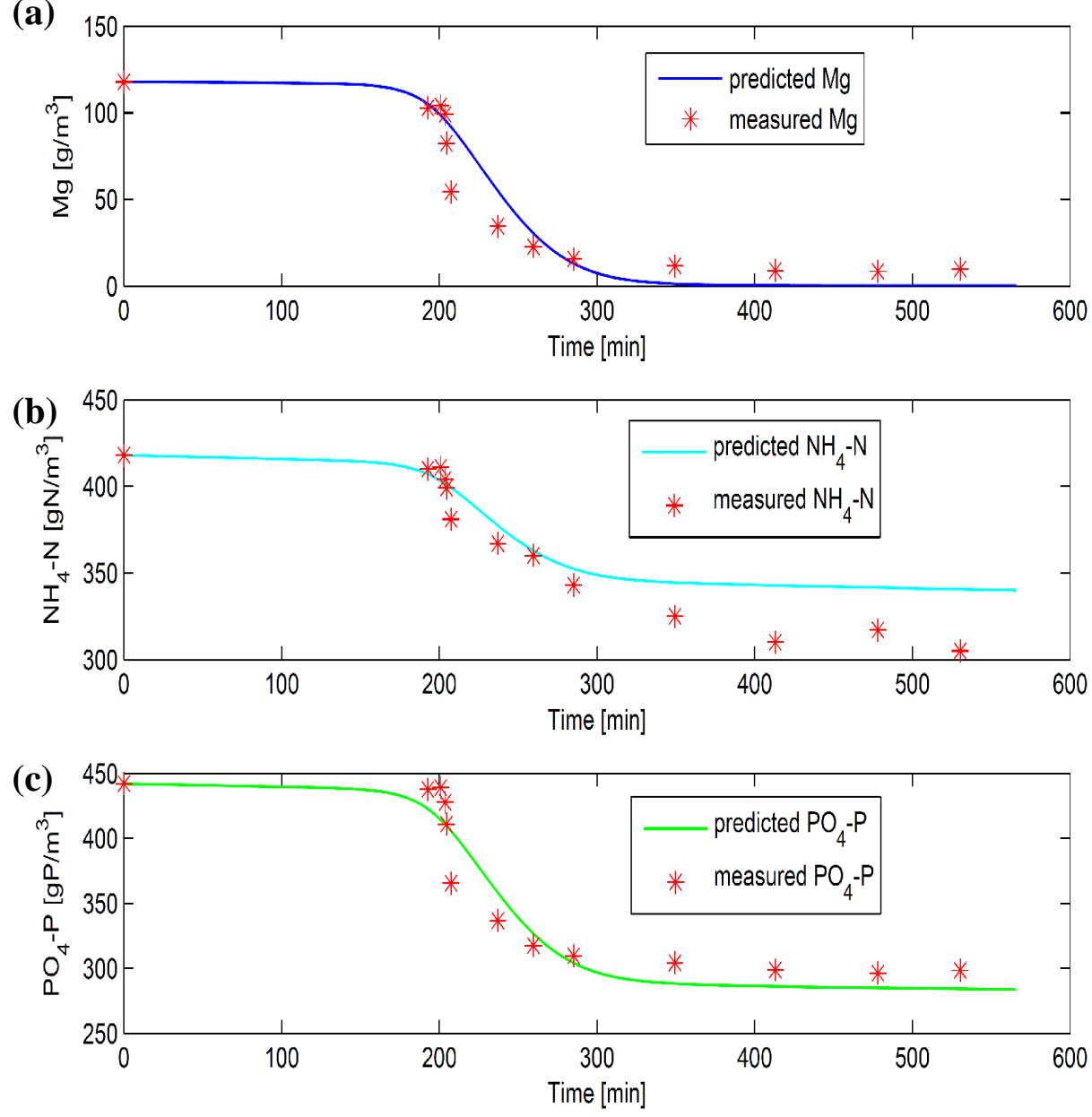

Figure 3: Predicted and measured soluble concentration for magnesium (top), ammonia (middle) and total phosphate (bottom) $\mathrm{pH}$ for $2.1 \mathrm{M} \mathrm{NaOH}$ added at a rate of $5.4 \times 10^{-5}$ 
$\mathrm{mL} / \mathrm{min}$ to synthetic aqueous solution with $14.27 \mathrm{mM} \mathrm{NaH2PO} 4,29.84 \mathrm{mM} \mathrm{NH} 4 \mathrm{Cl}, 4.85 \mathrm{mM}$ $\mathrm{MgCl} 2$ with a $\mathrm{k}=0.7 \times 10-6$ moles. $\mathrm{min}^{-1}$.

\section{Conclusion}

In this paper an equilibrium-kinetic based model is applied to simulate spontaneous mineral precipitation in wastewater treatment. In order to avoid computational inefficiency and due to timescale differences, in the reactions occurring in the aqueous phase are assumed in the model to be at equilibrium relative to reactions between the aqueous phase and a solid phase, whereas reactions between the aqueous phase and a solid phase are treated as slow dynamic processes, each with a dedicated rate expression. The model was validated both without and with precipitation of magnesium phosphate minerals. The model structure can be easily expanded to include the precipitation of many other minerals of interest in wastewater treatment. The results suggest that a single-parameter equilibrium-kinetic approach can provide good prediction of wastewater $\mathrm{pH}$ over a wide range and is generally expandable for inclusion of multiple minerals.

\section{Acknowledgement}

This research was supported financially by the University of Queensland through the UQ International Scholarships (UQI).

\section{REFERENCES}

Abbona, F., Lundager Madsen, H.E. and Boistelle, R. (1982) Crystallization of two magnesium phosphates, struvite and newberyite: Effect of $\mathrm{pH}$ and concentration. Journal of Crystal Growth 57(1), 6-14.

Allison, J.D., Brown, D.S. and Novo-Gradac, K.J. (1991) MINTEQA2/PRODEFA2, a Geochemical Assessment Model for Environmental Systems: Version 3.0 User's Manual, Environmental Research Laboratory, Office of Research and Development, U.S. Environmental Protection Agency.

Barat, R., Montoya, T., Seco, A. and Ferrer, J. (2011) Modelling biological and chemically induced precipitation of calcium phosphate in enhanced biological phosphorus removal systems. Water Research 45(12), 3744-3752.

Batstone, D.J., Amerlinck, Y., Ekama, G.A., Goel, R., Grau, P., Johnson, B., Kaya, I., Steyer, J.-P., Tait, S., Takács, I., Vanrolleghem, P.A., Brouchaert, C.J. and Volcke, E. (2012) Towards a generalised physicochemical modelling framework. Water Science and Technology 66(6), 1147-1161.

Batstone, D.J., Keller, J., Kalyuzhnyi, S.V., Pavlostathis, S.G., Rozzi, A., Sanders, W.T.M., Siegrist, H. and Vavilin, V.A. (2002) Anaerobic digestion model no. 1, IWA Publishing, London.

Gunn, D.J. (1976) Mechanism for the formation and growth of ionic precipitates from aqueous solution. Faraday Discussions of the Chemical Society 61(0), 133-140.

Henze, M., Gujer, W., Mino, T. and van Loosdrecht, M. (2000) Activated sludge models ASM1, ASM2, ASM2d and ASM3, IWA Publishing, London.

Loewenthal, R.E., Kornmüller, U.R.C. and van Heerden, E.P. (1995) Modelling struvite precipitation in anaerobic treatment systems. Water Science and Technology 30(12).

Marti, N., Bouzas, A., Seco, A. and Ferrer, J. (2008) Struvite precipitation assessment in anaerobic digestion processes. Chemical Engineering Journal 141(1-3), 67-74.

Morel, F. and Morgan, J. (1972) Numerical method for computing equilibriums in aqueous chemical systems. Environmental Science \& Technology 6(1), 58-67. 
Morgan, J.J. and Stumm, W. (1996) Aquatic chemistry: chemical equilibria and rates in natural waters, Wiley, New York.

Musvoto, E.V., Wentzel, M.C. and Ekama, G.A. (2000b) Integrated chemical-physical processes modelling-II. simulating aeration treatment of anaerobic digester supernatants. Water Research 34(6), 1868-1880.

Musvoto, E.V., Wentzel, M.C., Loewenthal, R.E. and Ekama, G.A. (2000a) Integrated chemical-physical processes modelling-I. Development of a kinetic-based model for mixed weak acid/base systems. Water Research 34(6), 1857-1867.

Ohlinger, K.N., Young, T.M. and Schroeder, E.D. (1998) Predicting struvite formation in digestion. Water Research 32(12), 3607-3614.

Tait, S., Clarke, W.P., Keller, J. and Batstone, D.J. (2009) Removal of sulfate from highstrength wastewater by crystallisation. Water Research 43(3), 762-772.

Valsami-Jones, E. (2001) Mineralogical controls on phosphorus recovery from wastewaters. Mineralogical Magazine 65(5), 611-620. 


\title{
Incorporating aquatic chemistry into wastewater treatment process models: A critical review of different approaches
}

\author{
Izaro Lizarralde ${ }^{1}$, Christopher J. Brouckaert ${ }^{2}$, Peter A. Vanrolleghem ${ }^{3}$, David S. Ikumi ${ }^{4}$, George A. \\ Ekama $^{4}$, Eduardo Ayesa ${ }^{1}$ and Paloma Grau ${ }^{1}$ \\ ${ }^{1}$ CEIT and Tecnun (University of Navarra) Manuel de Lardizábal 15, 20018 San Sebastián, Spain \\ (E-mail:ilizarralde@ceit.es; eayesa@ceit.es; pgrau@ceit.es) \\ ${ }^{2}$ Pollution Research Group, School of Chemical Engineering, University of KwaZulu-Natal, Durban 4041, \\ South Africa (E-mail: brouckae@ukzn.ac.za) \\ ${ }^{3}$ modelEAU, Département de génie civil et de génie des eaux, Université Laval, Canada \\ (E-mail: Peter.Vanrolleghem@gci.ulaval.ca) \\ ${ }^{4}$ Water Research Group, Department of Civil Engineering, University of Cape Town, Rondebosch, 7700, \\ Cape Town South Africa (E-mail: david.ikumi@uct.ac.za; george.ekama@uct.ac.za)
}

\section{Keywords}

Abiotic processes, mathematical modelling, $\mathrm{pH}$ calculation, water chemistry

\section{INTRODUCTION}

In recent years the awareness of the effect of abiotic processes in wastewater and sludge treatment technologies has increased rapidly (Batstone et al., 2012). In the field of municipal wastewater treatment for example, chemical processes play an important role in the performance of different technologies present throughout the plant. Besides, $\mathrm{pH}$ has an important impact on other processes that may take place in wastewater treatment such as precipitation or liquid gas transfer. Such processes will be critical in the future of these facilities as there are attempts to operate them in a sustainable and environmentally-friendly way. For example, there is a general concern about scarcity of some natural resources and the recovery processes of these resources often rely on physico-chemical processes (Vanrolleghem, 2013). Another example is the need to reduce greenhouse gas emissions that originate in the processes occurring at the wastewater treatment plant. Therefore, an appropriate description of the abiotic processes is of high importance in the development of models for the description of wastewater treatment technologies.

Traditional mathematical models, widely used for wastewater and sludge treatment, have different levels of detail for the calculation of $\mathrm{pH}$. In the models proposed for wastewater treatment (ASMs) it is considered that the buffering capacity is sufficient, therefore there is no rigorous calculation of $\mathrm{pH}$ included (Henze et al., 1987). In the case of models describing anaerobic digestion (ADM1) (Batstone et al., 2002), models describing river water quality (RWQM1) (Reichert et al., 2001) or the plant wide modelling (PWM) methodology proposed by Grau et al., (2007) pH calculation from chemical equilibria is included; however, these models do not consider ionic activity in the calculation, which makes the models only valid for dilute systems, with low ionic strength (Batstone et al., 2012). In the UCTADM of Sötemann et al., (2005a) ion pairing was included but the fast equilibrium and speciation processes were included as dynamic processes along with the slow biological and precipitation processes, causing slow calculation times and/or numerical instability. In the model proposed for biological nutrient removal N2 (BNRM2) by Barat et al., (2013) water chemistry is calculated using a commercial software tool MINTEQA2 (Allison et al., 2009). Finally, the UCTADMP, upgrade of the anaerobic digestion UCTADM proposed by Ikumi 
(2011) describes chemical reactions using an algebraic speciation model (Brouckaert et al., 2010).

As a result it has been recognised that these traditional models should be updated and rewritten in order to include abiotic phenomena. To address this, the IWA Physico-Chemical Framework Task Group (IWA PCF TG) has recently been constituted with the goal of developing guidelines and procedures to assist modellers to consider all these processes (Batstone et al., 2012). One particular aspect is that, from a numerical point of view, the inclusion of acid-base equilibrium and the subsequent $\mathrm{pH}$ calculation in biochemical models can lead to the appearance of some degree of stiffness, caused by the different conversion rates considered. This may introduce numerical instabilities and slow down the simulation speed. Tackling this problem requires analysis and testing of numerical methods that deal with combined algebraic and differential equations.

Based on these postulates, the work presented in this paper aims to introduce a methodology for incorporating aquatic chemistry into models representing wastewater treatment processes, based on different approaches found in literature for the description of aquatic chemistry and its numerical resolution. A simulation scenario has been defined in order to carry out a comparative analysis of the different approaches in terms of the accuracy of the results and the simulation time.

\section{METHODOLOGY TO INCORPORATE WATER CHEMISTRY INTO WASTEWATER TREATMENT PROCESS MODELS}

The construction of dynamic mathematical models comprises of: (1) the selection and description of the relevant biological and physico-chemical processes in the system under study and (2) the definition of water chemistry to predict $\mathrm{pH}$ tailored to the model defined in the first step (Figure 1).

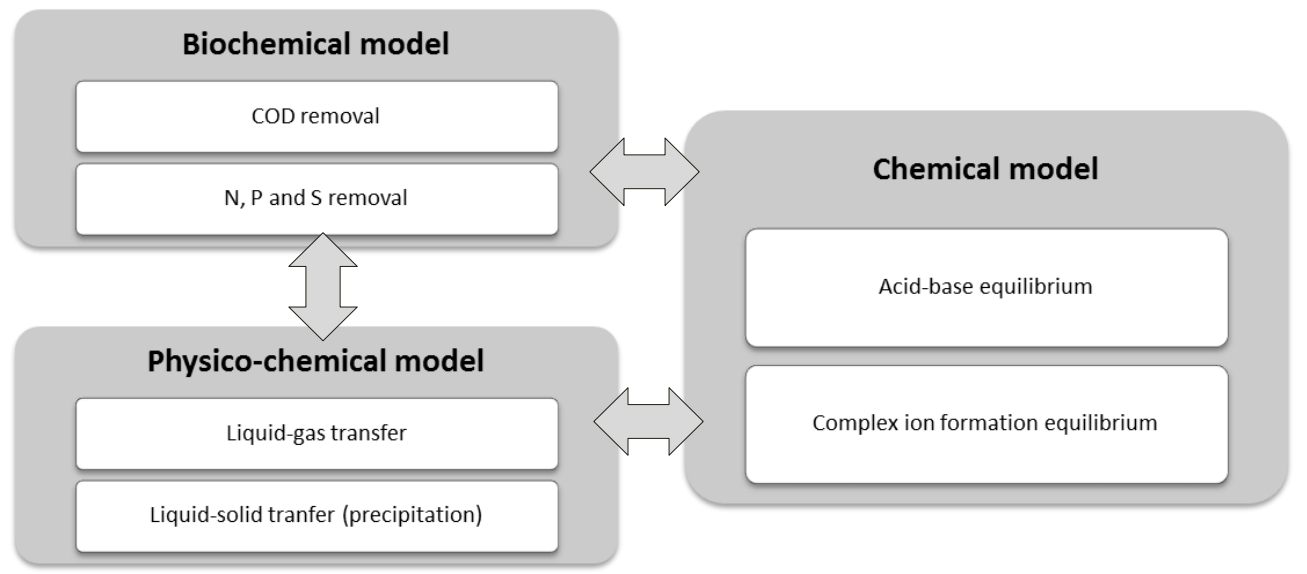

Figure 1. The selection of the chemical model depends on biological and physico-chemical model

The following sections focus on (1) the construction of the chemical model to describe chemical processes taking place in the system under study with the selected biological and physico-chemical processes; and (2) the introduction of different numerical resolution procedures to couple chemical models with biological and physico-chemical processes.

\section{Modelling water chemistry}

In order to describe the water chemistry in the system first the components and species have to be defined; as an example the 12 components and 38 species given in Table 1 were 
collected for the case study presented below. Species are chemical entities taken to be physically present in the system, and for the given set of species, components are selected so that all species can be expressed as linear combination of components, and no component can be written as combination of other components. Chemical equilibrium modelling consists basically of formulation of the material balance and mass action law which determine species concentrations from a mixture composition specified in terms of component concentrations.

Table 1. List of species and components selected

\begin{tabular}{|c|c|c|c|c|c|c|c|c|c|c|c|c|}
\hline \multicolumn{3}{|c|}{ Components } & \multicolumn{10}{|c|}{ Species } \\
\hline $\mathrm{S}_{\mathrm{h}}$ & $\mathrm{S}_{\mathrm{TVA}}$ & $\mathrm{S}_{\mathrm{Ca}}$ & $\mathrm{H}$ & $\mathrm{Mg}$ & $\mathrm{H}_{2} \mathrm{CO}_{3}$ & $\mathrm{H}_{2} \mathrm{PO}_{4}$ & $\mathrm{Hac}$ & Hpro & $\mathrm{CaH}_{2} \mathrm{PO}_{4}$ & $\mathrm{MgH}_{2} \mathrm{PO}_{4}$ & $\mathrm{MgCO}_{3}$ & $\mathrm{NaHPO}_{4}$ \\
\hline $\mathrm{S}_{\mathrm{IP}}$ & $\mathrm{S}_{\mathrm{TBU}}$ & $\mathrm{S}_{\mathrm{Mg}}$ & $\mathrm{Na}$ & $\mathrm{OH}$ & $\mathrm{HCO}_{3}$ & $\mathrm{HPO}_{4}$ & $\mathrm{Ac}$ & Pro & $\mathrm{CaHCO}_{3}$ & $\mathrm{MgHCO}_{3}$ & $\mathrm{MgPO}_{4}$ & $\mathrm{NaOH}$ \\
\hline $\mathrm{S}_{\mathrm{IN}}$ & $\mathrm{S}_{\mathrm{TPRO}}$ & $\mathrm{S}_{\mathrm{Na}}$ & $\mathrm{Ca}$ & $\mathrm{NH}_{3}$ & $\mathrm{CO}_{3}$ & $\mathrm{PO}_{4}$ & $\mathrm{Hbu}$ & Hva & $\mathrm{CaHPO}_{4}$ & $\mathrm{MgHPO}_{4}$ & $\mathrm{NaCO}_{3}$ & \\
\hline $\mathrm{S}_{\mathrm{IC}}$ & $\mathrm{S}_{\mathrm{TAC}}$ & $\mathrm{S}_{\mathrm{Cl}}$ & $\mathrm{Cl}$ & $\mathrm{NH}_{4}$ & $\mathrm{CaCO}_{3}$ & $\mathrm{CaOH}$ & $\mathrm{Bu}$ & $\mathrm{Va}$ & $\mathrm{CaPO}_{4}$ & $\mathrm{MgOH}$ & $\mathrm{NaHCO}_{3}$ & \\
\hline
\end{tabular}

The principles of water chemistry modelling are set out in Stumm and Morgan (1996). The equilibrium relationships are formulated in terms of species activities (e.g. Eq1), which are related to their concentrations by activity coefficients (e.g. Eq2). Activity coefficients were modelled using the Davies equation (Eq3 and Eq4). The mass conservation equation can be expressed either to (i) guarantee electroneutrality, i.e. guarantee the sum of cations equals the sum of anions (Eq5) or (ii) using the alkalinity-acidity continuity, this is formulating proton conservation equations (Eq6). Combining these mass conservation equations and the mass action relationships, a set of simultaneous equations is obtained which can be solved for all species concentrations.

$$
\begin{aligned}
& \mathrm{K}_{\mathrm{a}}=\frac{\left\{\mathrm{H}^{+}\left\{\mathrm{A}^{-}\right\}\right.}{\{\mathrm{HA}\}}(\mathrm{Eq} 1) \quad\left\{\mathrm{H}^{+}\right\}=\gamma_{\mathrm{H}^{+}} \cdot\left[\mathrm{H}^{+}\right](\mathrm{Eq} 2) \quad \mathrm{I}=\frac{1}{2} \sum_{\mathrm{i}} \mathrm{M}_{\mathrm{i}} \cdot \mathrm{z}_{\mathrm{i}}^{2} \quad(\mathrm{Eq} 3) \quad \log \left(\gamma_{\mathrm{i}}\right)=-\mathrm{Az}_{\mathrm{i}}^{2}\left[\frac{\sqrt{\mathrm{I}}}{1+\sqrt{\mathrm{I}}}-0.3 \mathrm{I}\right](\mathrm{Eq} 4) \\
& \text { Where, } \\
& \begin{array}{ll}
\mathrm{K}_{\mathrm{a}} \text { : equilibrium constant } & {[S] \text { : molality of species } S} \\
\{S\}: \text { activity of species } S & \gamma_{S} \text { : activity coefficient of } S
\end{array} \\
& \text { Where, } \\
& \sum \mathrm{S}_{\mathrm{C}_{+}-} \sum \mathrm{S}_{\mathrm{A}_{-}}=0 \text { (Eq5) } \quad \begin{array}{l}
S_{C_{+}} \text {: represents total cation equivalent concentration; and } \\
S_{A_{-}} \text {: represents total anion equivalent concentration. }
\end{array} \\
& \text { I: Ionic strength } \quad \mathrm{Z}_{\mathrm{i}} \text { : charge of species } \mathrm{i} \\
& \mathrm{M}_{\mathrm{i}} \text { : molality of species } \mathrm{i} \quad \text { A:Debye-Huckel constant } \\
& \text { Where, }
\end{aligned}
$$

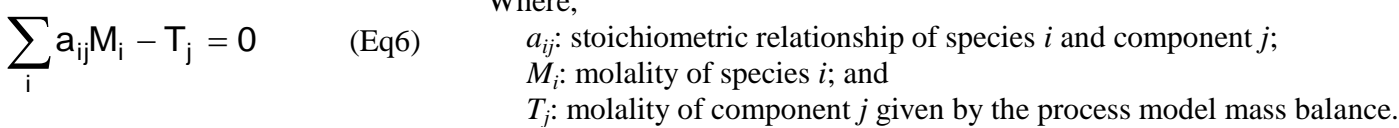

\section{Numerical resolution procedure}

When combining biological and chemical reactions, numerical resolution is a critical step, because of the stiffness that arises when considering reactions with very different conversion rates. In the case of dynamic models, there are two possible resolution procedures:

(i)

All reactions are calculated simultaneously using ordinary differential equations (ODE) as in Musvoto et al. (2000a,b) and Sötemann et al. (2005a,b).

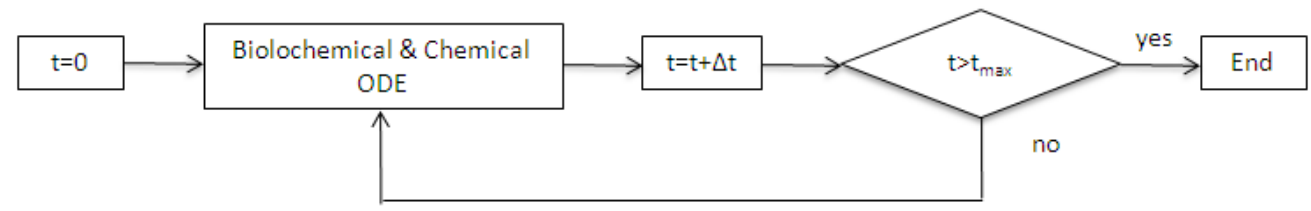

Figure 2. Model resolution procedure using the ordinary differential equations (ODE approach)

(ii) The slower reactions are represented by differential equations and the fast reactions are calculated algebraically (DAE) at each iteration step (Figure 3) as in ADM1 (Batstone 
et al., 2001), Volcke et al., (2005), Rosen et al. (2006) or UCTADMP (Brouckaert et al., 2010; Ikumi et al., 2011). In the DAE approach, the modeller can choose between having a tailored code to solve water chemistry or using an external software tool such as PhreeqC+ (Parkhurst and Appelo 2013) or MINTEQA2 (Allison et al., 2009; Barat et al., 2013) at each iteration step.

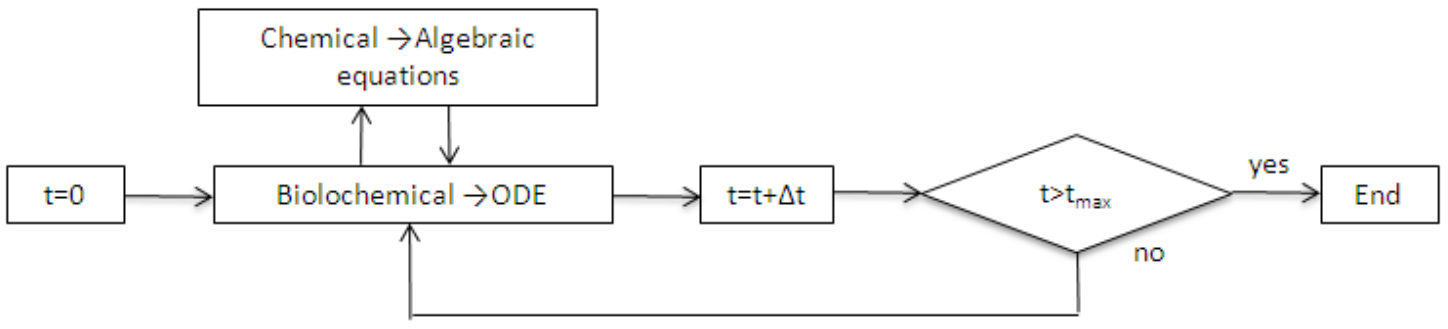

Figure 11. Model resolution combining differential and algebraic equations (DAE approach)

\section{COMPARISON OF DIFFERENT RESOLUTION APPROACHES}

The objective of this study was (i) to check the capability of the model presented in the previous section to calculate aquatic chemistry equilibrium, and (ii) to carry out a comparison of the different approaches in terms of results accuracy and simulation speed. For this purpose, the anaerobic reactor in the Benchmark Simulation Model No 2 (BSM2) (Jeppson et al., 2007) was selected as the simulation test case.

\section{Results and discussion}

Using the conditions presented above, a dynamic simulation for a period of 365 days was run in order to check the capabilities of the different models. When running the simulations, different integrators and kinetic parameters have also been tested. The comparison between results obtained with different approaches regarding simulation time, and accuracy of results is shown in Table 2. For the ODE approach, the CVODE solver was used, and two values for the equilibrium kinetic rate have been compared: $10^{12}$ and $10^{6}$. It is seen that when using the kinetic rate of $10^{12}$ the simulated results are more accurate, since the result is closer to equilibrium. Nevertheless, higher kinetic rates slow down the simulation speed. The DAE approach using a tailored code for equilibrium calculation showed the shortest simulation time. Finally, when simulating the scenario with the external software Phreeq $\mathrm{C}+$, the highest number of species was considered, but the simulations were the slowest (in designing the tailored code, species which had insignificant impact under the conditions found in anaerobic digesters were excluded to improve the simulation speed). A more detailed comparison using the full BSM2 will be presented in a forthcoming paper.

Table 2. Simulation of the anaerobic reactor in BSM2 under dynamic conditions for a period of 365 days

\begin{tabular}{|l|c|c|l|c|c|}
\hline \multirow{2}{*}{} & \multicolumn{2}{|c|}{ Evaluation criteria } & \multicolumn{2}{c|}{ Evaluation criteria } \\
\cline { 2 - 3 } \cline { 5 - 6 } & $\begin{array}{l}\text { Simulation } \\
\text { time (sec) }\end{array}$ & $\left.\sum\left(\mathrm{K}_{\mathrm{a}} \cdot\{\mathrm{HA}\}-\left\{\mathrm{H}^{+}\right\} \mathrm{A}^{-}\right\}\right\}^{2}$ & & $\begin{array}{l}\text { Simulation } \\
\text { time (sec) }\end{array}$ & $\left.\sum\left(\mathrm{K}_{\mathrm{a}} \cdot\{\mathrm{HA}\}-\left\{\mathrm{H}^{+}\right\} \mathrm{A}^{-}\right\}\right\}^{2}$ \\
\hline $\begin{array}{l}\text { ODE-Kab } \\
10^{12}\end{array}$ & 6.25 & $7.61^{-05}$ & $\begin{array}{l}\text { DAE- } \\
\text { Tailored }\end{array}$ & 0.96 & 0 \\
\hline $\begin{array}{l}\text { ODE-Kab } \\
10^{6}\end{array}$ & 3.82 & $7.85^{-05}$ & PhreeqC+ & 23.36 & 0 \\
\hline
\end{tabular}


The results show that the optimum resolution procedure depends on the objectives of the simulation study. However, this work shows that the methodology proposed using a tailormade equilibrium calculation using algebraic equations, and incorporating it into the biological ODE system gave the most effective methodology, based on the simulation times of the different approaches.

\section{CONCLUSIONS}

Different approaches for calculating chemical equilibrium have been presented and a critical review has been undertaken. Based on this comparison a methodology is proposed for incorporating water chemistry into biological models.

\section{ACKNOWLEDGEMENTS}

Peter Vanrolleghem holds the Canada Research Chair in Water Quality Modelling.

\section{REFERENCES}

Allison, J.D., Brown D.S. and Novo.Gradac K.J. (1991). MINTEQA2/PRODEFA2. A Geochemical Assessment Model for Environmental Systems: Version 3.0. EPA/600/3-91/021, USEPA Washington, D.C.

Barat, R., Serralta, J., Ruano, M.V., Jiménez, E., Ribes, J., Seco, A. and Ferrer, J. (2013). Biological Nutrionet Removal Model No2 (BNRM2): a general model for wastewater treatment plants. Water Science and Technology 67(7). 14819

Batstone D.J., Keller J., Angelidaki I., Kalyuzhnyi S.V., Pavlostathis S.G., Rozzi A., Sanders W.T.M., Siegrist H. and Vavilin V.A. (2002). Anaerobic Digestion Model No. 1. Scientific and Technical Report No. 13, IWA Publishing, London.

Batstone, D. J., Amerlinck, Y., Ekama, G., Goel, R., Grau, P., Johnson, B., Kaya, I., Steyer, J.P., Tait, S., Takács, I., Vanrolleghem, P. A., Brouckaert, C. J. and Volcke, E. (2012) Towards a generalized physicochemical framework. Water Science and Technology., 66(6), 1147-1161.

Brouckaert, C. J., Ikumi, D. S. Ekama, G. A. 2010. A Three Phase Anaerobic Digestion Model. Procs 12th IWA Anaerobic Digestion Conference (AD12). Guadalajara, Mexico.

Grau P., de Gracia M., Vanrolleghem P.A. and Ayesa E. (2007). A new plant-wide modelling methodology for WWTPs. Water Research. 41, 4357-4372.

Henze, M., Gujer, W., Mino, T., adn Van Loosdrecht, M. C. M (1987). Activated Sludge Models ASM1, ASM2, ASM2d and ASM3. Scientific and Technical Report No 9. IWA Publishing, London.

Ikumi D.S., Brouckaert C.J. and Ekama G.A. (2011) A 3 phase anaerobic digestion model. 8th IWA Watermatex conference, San Sebastian, Spain, 20-22 June.

Jeppsson U., Pons M. N., Nopens I., Alex J., Copp J. B., Gerneay K. V., Rosen C., Steyer J. P. and Vanrollenghem P.A. (2007). Benchmark simulation model No 2- general protocol and exploratory case studies. Way. Sci. Tech., 53(8), $67-78$.

Musvoto E..V. Wentzel M.C., Loewenthal R.E. and Ekama GA (2000a) Integrated chemical, physical and biological processes modelling Part I - Development of a kinetic based model for weak acid/base systems. Water Research34(6) $1857-1867$

Musvoto E.V., Wentzel M.C. and Ekama G.A. (2000b) Integrated chemical, physical and biological processes modelling Part II - Modelling aeration treatment of anaerobic digester supernatants. Water Research $\quad 34(6)$ 1868-1880.

Parkhurst, D.L., and Appelo, C.A.J., (2013) Description of input and examples for PHREEQC version 3-A computer program for speciation, batch-reaction, one-dimensional transport, and inverse geochemical calculations: U.S. Geological Survey Techniques and Methods, book 6, chap. A43, 497 p., available only at http://pubs.usgs.gov/tm/06/a43/.

Reichert, P., Borchardt, D., Henze, M., Rauch, W., Shanahan, P., Somlyódy, L. and Vanrolleghem, P. (2001). River Water Quality Model no. 1 (RWQM1) II. Biochemical process equations. Water Science and Technology 43(5), 11-30.

Rosen C., Vrecko D., Gernaey K.V., Pons M.N. and Jeppsson U. (2006) Implementing ADM1 for plant-wide benchmark simulations in Matlab/Simulink. Water Science \& Technology 40 (4), 11-19.

Sotemann S.W., van Rensburg P., Ristow N.E., Wentzel M.C., Loewenthal R.E. and Ekama G.A. (2005a) Integrated chemical, physical and biological processes kinetic modelling Part 2 - Anaerobic digestion of sewage sludges. Water SA 31(4) 545-568. 
Sotemann S.W., Musvoto E.V., Wentzel M.C. and Ekama GA (2005b) Integrated chemical, physical and biological processes kinetic modelling Part 1 - Anoxic and aerobic processes of carbon and nitrogen removal in the activated sludge system. Water SA 31(4) 529-544.

Stumm W. and Morgan J. J. 1996. Aquatic Chemistry: Chemical equilibria and rates in natural waters. John Wiley \& Sons Inc. New York.

Vanrolleghem P.A. (2013) Water Resource Recovery Facilities: Modelling and control challenges. Lecture held at the Workshop on Emerging Challenges for a Sustainable and Integrated Urban Water System Management at the 10th IWA Leading Edge Conference on Water and Wastewater Technologies (LET2013). Bordeaux, France, June 3-6 2013.

Volcke E.I.P., Van Hulle S., Deksissa T., Zhaer U. and Vanrolleghem P.A. (2005). Calculation of pH and concentration of equilibrium components during dynamic simulation by means of a chargebalance. BIOMATH Tech. Report, Ghent University, Ghent, Belgium. 


\title{
A protocol for the use of Computational Fluid Dynamics as a supportive tool for wastewater treatment plant modeling
}

\author{
J. Laurent ${ }^{1}$, R.W. Samstag ${ }^{2}$, J.M. Ducoste ${ }^{3}$, A. Griborio ${ }^{4}$, I. Nopens ${ }^{5}$, D.J. Batstone ${ }^{6}$, J.D. Wicks ${ }^{7}$, S. \\ Saunders ${ }^{8}$, O. Potier ${ }^{9}$ \\ ${ }^{1}$ Icube, UMR 7357, University of Strasbourg, Strasbourg, France (email: julien.laurent@icube.unistra.fr \\ ${ }^{2}$ Carollo Engineers, Seattle, WA US (email: rwsamstag@ stanfordalumni.org) \\ ${ }^{3}$ North Carolina State University, NC US (email: jducoste@ ncsu.edu) \\ ${ }^{4}$ Hazen and Sawyer, Hollywood, FL US (email: agriborio@ hazenandsawyer.com) \\ ${ }^{5}$ BIOMATH, Ghent University. Gent, Belgium (email: ingmar.nopens@ugent.be) \\ ${ }^{6}$ University of Queensland, Australia (email: damienb@awmc.uq.edu.au) \\ ${ }^{7}$ The Fluid Group, Oxford, UK (email: jim.wicks@thefluidgroup.com) \\ ${ }^{8}$ The Ibis Group, Florida, US (email: ibisgroup@bellsouth.net) \\ ${ }^{9}$ LRGP, UMR 7274, University of Lorraine, Nancy, France (email: olivier.potier@ univ-lorraine.fr)
}

\begin{abstract}
To date, computational fluid dynamics (CFD) models have been primarily used for the evaluation of hydraulic problems at wastewater treatment plants (WWTP). A potentially more powerful use, however, is to simulate integrated physical, chemical and/or biological processes involved in WWTP unit processes on a spatial scale and to use the gathered knowledge to accelerate improvement in plant models for everyday use. Evolving improvements in computer speed and memory and improved software for implementing CFD, as well as for integrated processes, has allowed for broader usage of this tool for understanding, troubleshooting, and optimal design of WWTP unit processes. This paper proposes a protocol for an alternative use of CFD in process modelling, i.e. as a way to gain insight into complex systems leading to improved modeling approaches used in combination with the IWA activated sludge models (ASM) and other kinetic models.
\end{abstract}

\section{Keywords}

CFD, biokinetic models, transport models, multi-phase flow, fluid motion, complex systems

\section{INTRODUCTION}

Wastewater treatment plants (WWTP) are complex systems with interacting hydraulic, biological, and chemical elements. Optimization of design and operation of WWTP unit processes is of interest, especially with respect to the challenge of highly dynamic influent flows. In this respect, mathematical modeling has proven to be a powerful tool. The main focus in the past has been on modeling chemical and biokinetic processes using simplified hydraulic assumptions. This approach has resulted in the development of the Activated Sludge Model (ASM) family of models (Henze et al., 2000) as well as the Anaerobic Digestion Model (ADM) (Batstone et al., 2002). These models typically assume a continuously stirred tank reactor (CSTR) or use relatively simplified hydraulic conceptualization, i.e. the tanks in series (TIS) approach. The latter was developed in the chemical engineering industry as a way to describe the level of macro-scale dispersion within the unit process while limiting the model's state numbers and avoiding use of spatiallyvarying partial differential equations as this complicates the numerical solution and slows down the simulation speed (Levenspiel, 1972). However, the industry has been well aware of 
the limitations of these hydraulic simplifications for many years: "If we know precisely what is happening within the vessel, thus if we have a complete velocity distribution map for the fluid, then we are able to predict the behavior of a vessel as a reactor. Though fine in principle, the attendant complexities make it impractical to use this approach." (Levenspiel, 1972, p 253). Considering current developments in the theory and practice of computational fluid dynamics (CFD) it is time to re-assess this conclusion.

CFD has become an accepted method for process analysis in a diverse range of industries from aeronautics to ocean engineering. It has been used for analysis and design of water and wastewater treatment plant process elements since Larsen's pioneering study presented the first CFD model for activated sludge sedimentation incorporating solids transport and settling (Larsen, 1977). Use of CFD as a full transport modeling approach for wastewater treatment tanks was already visualized over 20 years ago (Samstag et al., 1992), but has not been extensively or systematically applied until recently. CFD has evolved into a relatively accepted tool by consultants and practitioners for analysis of hydraulic problems in wastewater treatment plants, notably for outfalls and flow splitting devices, as well as for chemical mixing. With steadily increasing computational power over the past decades, it is no longer "impractical" to use CFD for process analysis.

In addition to CFD use in practice, it also keeps developing in academia. Initially, CFD had been used for evaluation of mixing and solids settling in sedimentation tanks and results from these models have been informative in improving process performance. Evolving promising areas of research include the use of CFD to simulate physical, chemical, and/or biological processes in WWTP tanks where fluid flow characteristics are important. This endeavor has long been hampered by lack of availability and high cost of CFD software (including pre and post-processing), steep learning curves for their use, and limitations in computational power. Hence, CFD was not used for integration with these kinetic models. Now that commercial and open-source software packages with a choice of turbulence models and graphical user interfaces for pre and post processing are available, however, researchers have been able to explore the CFD approach to investigate WWTP unit process performance.

These initial results have so increased confidence in CFD in helping elucidate the impact of the spatial variations in velocity profiles on process outcomes that it can now be used for prediction of performance of unit processes beyond sedimentation. For example, the insertion of bio-kinetic models into CFD simulations of WWTP processes as well as their validation (e.g. Le Moullec et al., 2010a; Gresch et al., 2011; Sobremisana et al., 2011) provided significant and reliable insights into complex contaminant removal performance in these processes.

As more experience is gained in CFD-based process modeling, researchers and engineers will achieve a better understanding of where and when simpler models are adequate and be able to suggest potential improvements in the TIS models themselves. Indeed, from these insights, simpler representations of these mechanisms can now be developed and used in significantly less computationally intensive unit process models. For example, Potier et al., (2005) proposed a dynamic TIS model with back-mixing which is able to simulate variations of the axial dispersion coefficient with the flowrate by taking into account a maximal fixed number of mixing cells and a variable backflow rate. This model derived from correlations with a large set of lab-scale and full-scale experiments. In the future, similar approaches could be performed using CFD to determine the appropriate number of tanks in a TIS model 
depending on the influent dynamics. The issue of biokinetic-hydraulic feedback can also be incorporated (e.g., gas production, changes in fluid properties) through iterative analysis.

The present paper aims at proposing a protocol for this alternative use of CFD modeling in order to get more insight into unit process description and improve simpler models conceptualization, calibration, and validation.

\section{PROTOCOL}

To date, it is not very clear to non-CFD model users what the exact role is that CFD is playing or can play in the field of wastewater treatment modeling. It is often perceived as an overly complex modeling tool that eats too much computational time and is therefore not considered. In this contribution we want to share our views on how this simulation tool can be used in the train of thought of wastewater process modeling apart from the current usage as stand-alone tool for unit processes design and troubleshooting. In this way, it can contribute to the further development of wastewater process models to its full extent.

Fig. 1 presents a schematic visualization of a protocol for CFD use in improvement of WWTP process modelling. The protocol suggests that CFD be used as a supportive tool for wastewater process modeling rather than as replacement for simpler modeling approaches. Indeed, dynamic simulation of a whole WWTP with CFD may not be feasible for another couple of decades. But in the meantime, CFD can still serve the community.

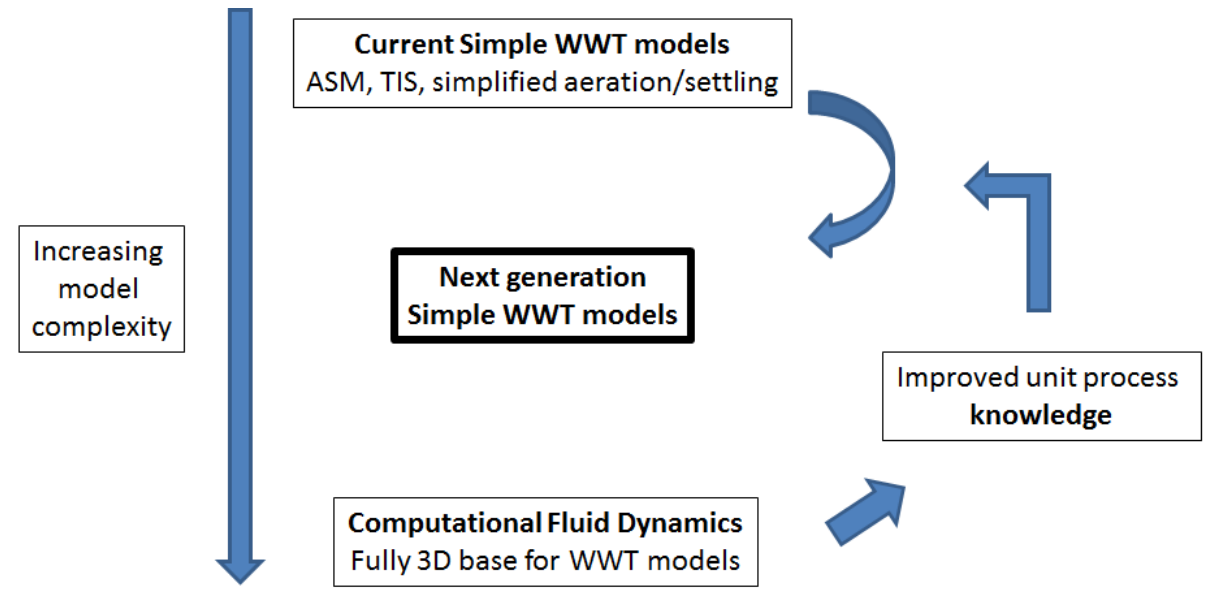

Figure 1. Conceptual map for use of CFD as a super-tool for process modeling.

The currently used "simple" WWT models are located at one end of the model spectrum (Fig. 1 - top), whereas the complex CFD models are at the other end (Fig. 1 - bottom). For certain model objectives, the former models are not adequate and slightly more complex models are required ("next generation simple WWT models"). In order to develop those, one needs improved process knowledge. It is especially in this respect that complex, validated CFD models can be of aid. 
We see this as a 5-step protocol for use of CFD as a tool for improving/developing simpler models:

1. CFD model formulation: Development of CFD models representing detailed features of the process tank geometry, as well as other ingredients such as turbulence, a coupled ASM biokinetics model, a full-fledged detailed aeration model, viscosity models, density couples, temperature gradients, solids gradients due to settling, etc.

2. Data collection: Lab or field test of appropriate process variables (velocity profiles, species concentration profiles, gas hold-up measurement, residence time distribution, etc.) to validate results of the CFD model

3. CFD model validation: Compare the CFD model prediction with the data. If match is insufficient, one should return to steps $1 \& 2$ and recheck model formulation and data quality.

4. Comparison to simpler model predictions: Detailed comparison to the results of simpler models for the same geometry and loading condition. Based on this, shortcomings can be pinpointed.

5. Improved simple model: These shortcomings lead the modeller in developing next generation models such as dynamic systemic models, compartmental, or other nonlinear macro-scale mixing models.

In the remainder of the paper we illustrate this train of thought through an example available in the literature that is actually an onset to this protocol, but not originally described in that way.

\section{CASE STUDY: MODELING A PILOT-SCALE BIOREACTOR}

The work in several papers of Le Moullec et al. $(2008,2010 \mathrm{a}, 2010 \mathrm{~b}, 2011)$ is representative of what could be seen as application of the protocol introduced above. While it was never presented in the form of a protocol, we feel that the approach is important in illustrating how this protocol could be implemented.

\section{STEP 1: CFD model formulation}

The unit process used for both experiments and modeling purposes was a bench scale channel reactor with a total length of $3.6 \mathrm{~m}$ with a rectangular cross section of width and height, respectively equal to 0.18 and $0.2 \mathrm{~m}$. One side of the walls of the reactor was fitted with stainless-steel tubes in which $1 \mathrm{~mm}$ holes had been drilled every centimeter for air sparging. Further description is presented in Le Moullec et al. (2008).

Development of the CFD model utilizing an Euler-Euler approach is described in detail in Le Moullec et al. (2008). CFD simulations were carried out with the CFD software FLUENT. Two turbulence models were tested: a two-phase k- $\varepsilon$ model and a Reynolds Stress Model (RSM). Boundary conditions were defined as presented in Table 1. 
Table 1. Boundary conditions used for CFD computations.

\begin{tabular}{cccc}
\hline Gas & \multicolumn{1}{c}{ Inlet } & Outlet & Top \\
\hline Liquid & $\begin{array}{c}\text { Specified velocity inlet } \\
\text { and phase fraction } \\
\text { Specified velocity inlet } \\
\text { and phase fraction }\end{array}$ & $\begin{array}{c}\text { Specified velocity } \\
\text { outlet and phase fraction }\end{array}$ & $\begin{array}{c}\text { Symmetry boundary } \\
\text { condition }\end{array}$ \\
ce & $\begin{array}{c}\text { Turbulence intensity } \\
(10 \%) \text { and inlet } \\
\text { hydraulic diameter }\end{array}$ & $\begin{array}{c}\text { Turbulence intensity } \\
(10 \%) \text { and outlet } \\
\text { hydraulic diameter }\end{array}$ & $\begin{array}{c}\text { Turbulence intensity } \\
(10 \%) \text { and outlet } \\
\text { hydraulic diameter }\end{array}$ \\
\hline
\end{tabular}

A second order discretization scheme (QUICK: Quadrative Upwind Interpolation for Convective Kinematics) was selected for the momentum equations, turbulent dissipation rate, and void fraction equations. The SIMPLE (Semi-Implicit Method for Pressure-Linked Equations) pressure-velocity coupling scheme was also used.

\section{STEP2: Data collection}

Experiments were carried out to validate the CFD model. Two types of data were gathered. First, laser Doppler velocimetry (LDV) allowed the axial (Ux), lateral (Uy) and vertical (Uz) time-averaged velocity fields to be measured (Le Moullec et al., 2008). Second, residence time distribution (RTD) data was obtained from multiple tracer experiments (Potier et al., 2005).

\section{STEP3: CFD model validation}

Mesh sensitivity was examined using different hexahedral cell sizes of $1 \mathrm{~cm}^{3}(130,000$ cells), $0.125 \mathrm{~cm}^{3}(1,000,000$ cells $)$, and $1 \mathrm{~cm}^{3}$ with a refinement near the walls $(350,000$ cells). This last grid offered the best compromise between precision and computational effort.

The results of the CFD model concerning velocity field and RTD simulation (with both passive scalar and particle tracking methods) were compared with the experimental values. The two simulated velocity fields are compared to experimental data in Fig. 2. Both models gave similar results and overall agreement was good. The observed discrepancies, respectively near the bubble injection position and near the free surface, were probably due to the simplification made for the gas inlet boundary conditions and the simplified representation of the surface, which was not planar in the experimental setup. In the RTD tests (Fig. 3), the RSM turbulence model coupled with the particle tracking method produced better fit to the experimental results than the $\mathrm{k}-\varepsilon$ model. 


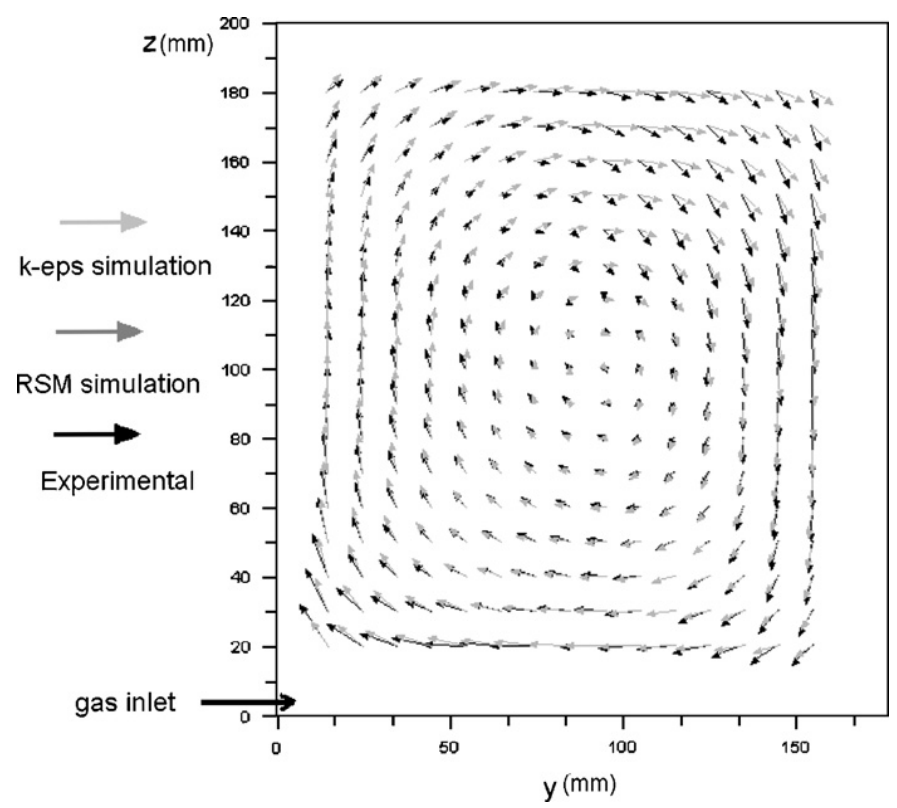

Figure 2. Overall representation of the experimental and simulated average velocity fields on a vertical plane $(y, z)$ for both turbulence models (source: Le Moullec et al., 2008)

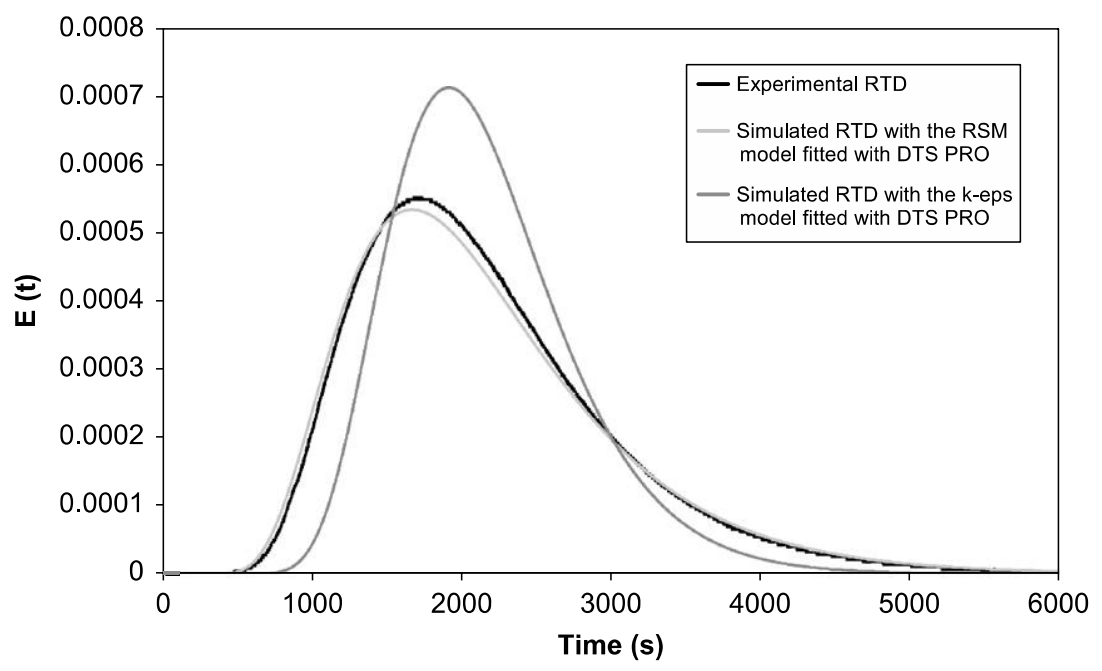

Figure 3. Comparison between experimental and simulated RTD data obtained with the RSM and the k- $\varepsilon$ turbulence models and the particle tracking method for a liquid flow rate of 3.6 L.min ${ }^{-1}$ and a gas flow rate of 15 L.min ${ }^{-1}$ (source: Le Moullec et al., 2008)

\section{STEP4: Comparison to simpler model predictions}

TIS and CFD hydrodynamic models were coupled with ASM1 biokinetic equations using standard parameters values in order to simulate biological reactions occurring in the pilot reactor. Comparison of both models prediction with experimental nitrate concentration profiles along reactor length is shown on Fig. 4. Even if biokinetic parameters were not at all calibrated in this study, one can observe that the CFD model provided a better prediction than the TIS model. 


\section{STEP5: Improved simple model}

In an effort to correct the shortcomings of the TIS model, a compartmental model was developed (Le Moullec et al., 2010a). This latter approach simulates the reactor as a network of spatially distributed functional compartments (Fig. 5). Definition of this kind of model relies on the results of the steady-state CFD hydrodynamics model (STEP 1-3). The number and spatial distribution of compartments are defined according to the homogeneous character of selected parameters with a given tolerance (e.g. gas fraction), as well as the exchange between them (convective flow rates and turbulent backflow rates). Fig. 4 shows that the fit of this somewhat more complex model is much better than the TIS approach and very close to the CFD-ASM1 model (STEP 4). This work (Le Moullec et al., 2011) demonstrated the possibility to accurately predict pollutant concentrations, not only with a detailed CFDbiokinetic model (STEP 4), but also with a simpler hydrodynamic model of which the structure is derived from the results of a single steady-state CFD simulation without biokinetics.

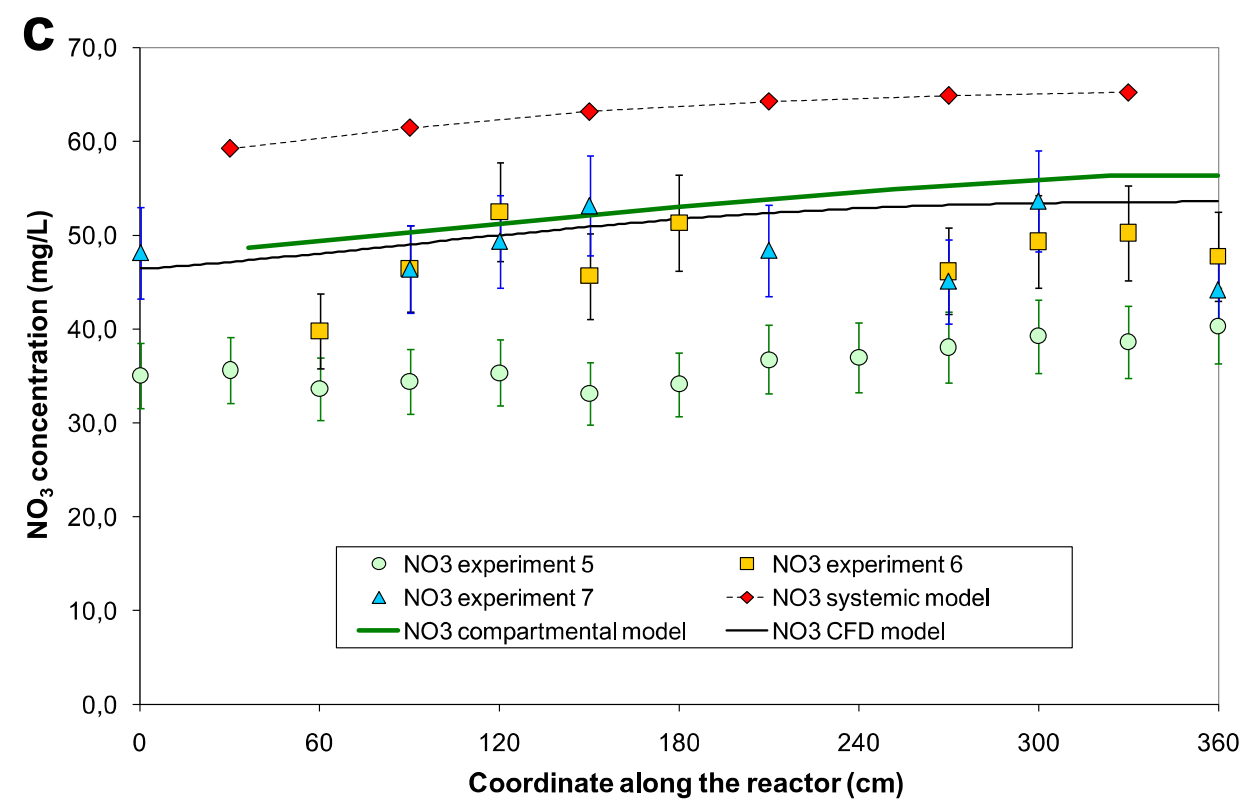

Figure 4. Nitrate concentration profile along the reactor for two experiments carried out on the pilot-scale bioreactor (source: Le Moullec et al., 2011) 


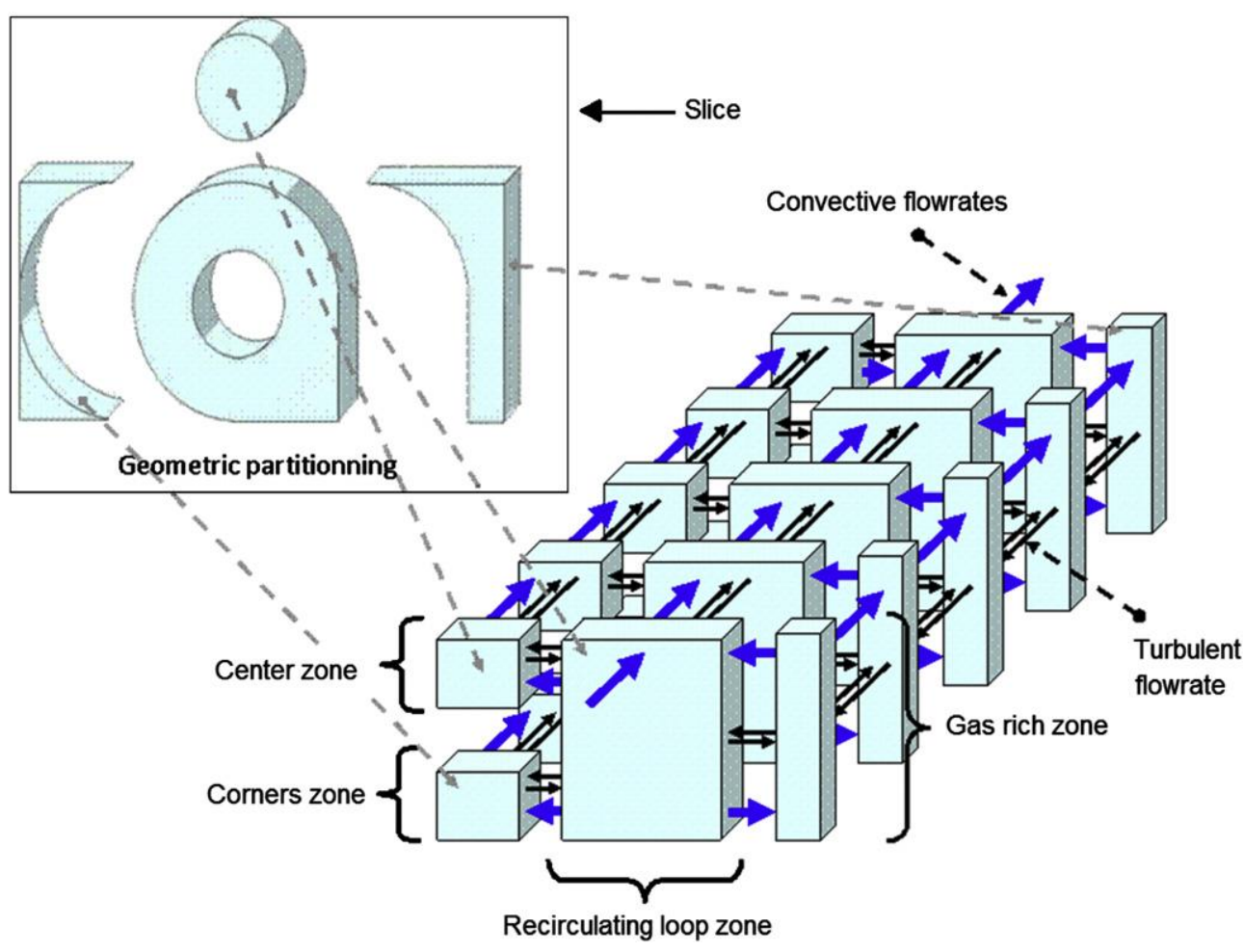

Figure 5. Structure of the compartmental model (source: Le Moullec et al., 2011)

\section{COMPARISON OF THE COMPUTATIONAL REQUIREMENTS OF CFD AND SIMPLER MODELS}

The compartmental model allowed the prediction of pollutant concentration within a pilotscale activated sludge reactor after a few minutes of calculation compared to 1 week of calculation for the CFD-biokinetic approach (Le Moullec et al. 2011). Compartmental modeling can be used where the incorporation of biokinetics within a CFD model would be computationally cost prohibitive and where the TIS model is not able to sufficiently describe the macro-scale mixing behavior of the complex system (Alvarado et al., 2012).

\section{PERSPECTIVES ON FUTURE APPLICATION}

The example illustrates the power of CFD as a supportive tool in developing improved "next generation" WWTP models. Further application of this protocol may even lead to certain recurrent patterns which exclude the necessity to build a CFD model in certain cases.

A logical next application for the protocol is in modeling the anaerobic digester process and more specifically, the mixing component of the process. Two-phase gas-liquid models have been performed of anaerobic digesters using CFD to help improve mixing performance. Yet, no CFD transport model of the anaerobic digester had been developed that captured both the biological processes and complete complex three phase fluid characteristics, until quite recently (Gaden, 2013). This type of model is needed to completely understand the impact of digester mixing systems and changes in digester influent characteristics on biogas production, or to assess the potential upgrades of digester capacity. TIS modeling is widely used to simulate tracer testing (Batstone et al., 2005), which may include feedback and bypass links, and it is a straightforward extension to validate these using CFD and to implement improved 
biokinetics for an assessment of how the hydraulic regime influences process stability. There is every reason to believe that this approach will also prove fruitful for evaluation of suspended growth treatment tanks.

One question that remains is when exactly one considers the CFD model to be sufficiently validated. What deviations are acceptable? Tools for evaluating this question need to be developed. Where the validation is determined to be inadequate, one needs to reiterate Steps 1 and 2. With regard to model formulation, recent work on process tank mixing (Samstag et al., 2012) has suggested an explanation for past failures to adequately size mixing devices resulting from ignoring density effects in the CFD analysis. In the future, this approach should be incorporated into CFD models and will, hence, be included in evaluation of simpler models.

Another major challenge that exists in biologically driven wastewater unit processes is due to the complex intersection between potential macro- and micro-scale reactions that occur outside and inside biological floc particles. These two-scale processes can be difficult to model and computationally expensive. Yet, the lack of modeling these two-scale processes can reduce the effectiveness of CFD in simulating specific phenomena in activated sludge systems such as the occurrence of simultaneous nitrification-denitrification processes, etc. Next to these examples, several other submodels can be added to the plain CFD models to further validate them and build even better process knowledge. This further knowledge development runs in parallel with the application of already gathered knowledge to build the next generation of practical models.

\section{CONCLUSION}

Direct use of CFD approaches that allow substantial expansion to include complex biokinetics or other behavior is currently challenging for practical use due to computing and numerical issues. However, CFD studies in the field of wastewater treatment can, next to their current application as design and troubleshooting tool, be used to develop the next generation of more practical, everyday models. A 5-step protocol was outlined describing how this can be done and was illustrated using an example from the literature. This shows the power of this approach and how it can lead to more reliable everyday models. Further perspectives were given as well as how current CFD model development fits into this train of thought.

\section{ACKNOWLEDGEMENTS}

This work has been completed as part of the work of IWA Working Group on Computational Fluid Dynamics.

\section{REFERENCES}

Alvarado, A., Vedantam, S., Goethals, P., and Nopens, I. (2012). A compartmental model to describe hydraulics in a full-scale waste stabilization pond. Water Research, 46(2), 521-530.

Batstone, D. J., Keller, J., Angelidaki, I., Kalyuzhnyi, S., Pavlovstathis, S. G., Rozzi, A., et al. (2002). Anaerobic Digestion Model No.1 (ADM1), Scientific \& Technical Report $n^{\circ} 13$, London, IWA Publishing.

Batstone, D.J., Hernandez, J.L.A. and Schmidt, J.E. (2005). Hydraulics of laboratory and full-scale UASB reactors Biotechnology and Bioengineering. 91(3):387-391

Gaden, D. (2013) Modelling Anaerobic Digesters in Three Dimensions: Integration of Biochemistry with Computational Fluid Dynamics. Ph.D Thesis, University of Manitoba.

Gresch, M., Armbruster, M., Braun, D. and Gujer, W. (2011). Effects of aeration patterns on the flow field in wastewater aeration tanks. Water Research, 45(2), 810-818.

Henze, M., Grady Jr, C. P., Gujer, W., Marais, G. v. R., Matsuo, T., Mino, T., et al. (2000) Activated sludge models ASM1, ASM2, ASM2d and ASM3, Scientific \& Technical Report $n^{\circ}$, London, IWA Publishing. 
Larsen, P. (1977). On the Hydraulics of Rectangular Settling Basins, Experimental and Theoretical Studies. Department of Water Resources Engineering. Lund Institute of Technology / University of Lund, Report No. 1001, Lund, Sweden.

Le Moullec, Y., Potier, O., Gentric, C., Leclerc, J.P. (2008) Flow field and residence time distribution simulation of a cross-flow gas-liquid wastewater treatment reactor using CFD. Chemical Engineering Science, 63(9), 2436-2449.

Le Moullec, Y., Gentric, C., Potier O. and Leclerc, J.P. (2010a). Comparison of systemic, compartmental and CFD modelling approaches: Application to the simulation of a biological reactor of wastewater treatment. Chemical Engineering Science 65(1), 343-350.

Le Moullec, Y., Gentric, C., Potier, O. and Leclerc, J.P. (2010b) CFD simulation of the hydrodynamics and reactions in an activated sludge channel reactor of wastewater treatment. Chemical Engineering Science, 65(1), 492- 498.

Le Moullec, Y., Potier, O., Gentric, C., and Leclerc, J.P. (2011) Activated sludge pilot plant: Comparison between experimental and predicted concentration profiles using three different modelling approaches. Water Research, 45(10), 3085-3097.

Levenspiel, O. (1972). Chemical Reaction Engineering. Wiley, New York.

Potier, O., Leclerc, J.P. and Pons, M.N. (2005). Influence of geometrical and operational parameters on the axial dispersion in an aerated channel reactor. Water Research, 39(18), 4454-4462.

Samstag, R., McCorquodale, J. and Zhou, S. (1992) Prospects for transport modeling of process tanks, Water Science and Technology, 26(5/6), 1401-1410.

Samstag, R.W., Wicklein, E.A., Reardon, R. D., Leetch, R. J., Parks, R. M., Groff, C. D. (2012) Field and CFD Analysis of Jet Aeration and Mixing. Water Environment Federation, WEFTEC Conference.

Sobremisana, A., Ducoste, J., de los Reyes III, F.L. (2011) Combining CFD, floc dynamics, and biological reaction kinetics to model carbon and nitrogen removal in an activated sludge system. Water Environment Federation, WEFTEC Conference. 


\title{
Dynamic Modelling of Diffused Aeration Systems
}

\author{
Lu-Man Jiang ${ }^{1}$, Riccardo Gori ${ }^{2}$, Thomas Gocke ${ }^{3}$, Diego Rosso ${ }^{3, *}$ \\ ${ }^{1}$ College of Environmental and Chemical Engineering, Shanghai University of Electric Power, Shanghai, \\ 200090, P. R. China (Email: jiangluman@shiep.edu.cn) \\ ${ }^{2}$ Department of Civil and Environmental Engineering - DICEA, University of Florence, Via S. Marta 3, \\ 50139 Florence, Italy (Email: riccardo.gori@ dicea.unifi.it) \\ ${ }^{3}$ Department of Civil and Environmental Engineering, University of California, Irvine, CA 92697-2175, \\ U.S.A \\ * Corresponding author (E-mail: bidui@uci.edu)
}

\begin{abstract}
Due to the elevated energy intensity inherent of fine-pore diffuser aeration within wastewater treatment, it is important to describe the real nature of aeration systems for improved design and optimization. We compiled two experimental datasets and developed a dynamic model to predict air flow for fine-pore diffuser aeration systems. The model was applied to two water reclamation plants, calibrated and validated with a time-sensitive database. Our model improves both prediction and description of field data with the introduction of an improved aeration model structure based on the organic load. Our results are a quantitative tool for prediction of energy wastage, and for minimizing aeration design uncertainty.
\end{abstract}

Keywords - Activated sludge; aeration; alpha factor; fine-pore diffusers; oxygen transfer

\section{INTRODUCTION}

Aeration is an essential and energy-intensive process for most wastewater treatment plants, and contributes 45 to $75 \%$ of process energy demand (Reardon, 1995). Fine-pore diffusers are preferred in the aeration of municipal wastewater treatment plants for their generally higher oxygen transfer efficiency (OTE, \%). OTE tested in clean water is corrected to standard conditions (i.e., zero dissolved oxygen, $1 \mathrm{~atm}, 20^{\circ} \mathrm{C}$, zero salinity) to produce the standard OTE, or SOTE (\%). OTE in process water is lower compared to clean water due to the effects of contamination, quantified by an $\alpha$ factor, which is the ratio of the oxygen transfer coefficients in process to clean water (Stenstrom and Gilbert, 1981). Thereby $\alpha$ SOTE (\%) is used to define standard oxygen transfer efficiency in process water. Evidence of the dynamic nature of $\alpha$ ranging from 0.25 to 0.55 was presented by Leu et al. (2009) with 48 off-gas tests over a $24 \mathrm{~h}$ cycle. Blower power is dependent on air flow rate (AFR), hence on $\alpha$ SOTE. Hence, a dynamic estimation of $\alpha$ and SOTE is key to curbing the uncertainty in modelling aeration energy and in aeration design. The goal of this research is to present a dynamic model to predict air flow from for diffused aeration accounting for the variation of $\alpha \mathrm{SOTE}$ with plant load.

\section{METHODS}

Process conditions. Both plants selected to test our model are located in California in areas characterized by a two-season climate with temperature oscillating between $19^{\circ} \mathrm{C}$ and $27^{\circ} \mathrm{C}$. The summary conditions for both plants are reported in the Table 1. Water reclamation plant 1 (WRP1) uses an activated sludge process that nitrifies and denitrifies using the modified Ludzack-Ettinger (MLE) configuration. WRP2 is a fully denitrifying water reclamation plant, operating biological nutrient removal in MLE configuration with tertiary operations. 
Jiang et al.

Table 1. Summary of average process conditions at the two plants studied.

\begin{tabular}{ccccc}
\hline & Q (MLD) & MCRT (days) & Diffuser Type & Diffuser Area $\left(\mathrm{m}^{2}\right)$ \\
\hline WRP1 & 37 & 7.0 & Polyurethane membrane strip & 0.61 \\
WRP2 & 57 & 8.5 & EPDM membrane disc & 0.041 \\
\hline
\end{tabular}

Field measurements. In order to estimate the specific SOTE, i.e. SOTE/Z or SSOTE $(\% / \mathrm{m})$, of new diffusers clean water, tests were performed beforehand following the American Society of Civil Engineers standard procedure (ASCE, 2007). The measurements of $\alpha$ SOTE and $\alpha \mathrm{SOTE} / \mathrm{Z}$ were performed according to the ASCE off-gas protocol (ASCE, 1996), which uses the same saturation depth correction as the ASCE clean water. Measurements at WRP1 were taken every 30 minutes for 24 hours. The ratio of the results from the off-gas tests and the clean water tests were used to calculate $\alpha$ factors.

Model characteristics. This model used the WRP1's $\alpha$ vs. COD set for calibration (Fig. 1). The calibration set of time-dependent data for $\alpha$ versus plant load (oxygen demand and flow rate) were previously presented by Leu et al. (2009). In short, instead of predicting the oxygen transfer rate OTR from the air flow multiplied by a constant $\alpha$, the model uses an $\alpha$ that is variable as a function of the COD. Thus, after selecting a reasonable initial guess value for AFR, the model iterates the re-calculation of AFR using $\alpha$ and SOTE as power fits functions of COD and AFR, respectively, until the DO target is met. This model is not aimed at substituting the ASMs, but is an extension of their structure to include dynamic modelling of $\mathrm{O}_{2}$ transfer.

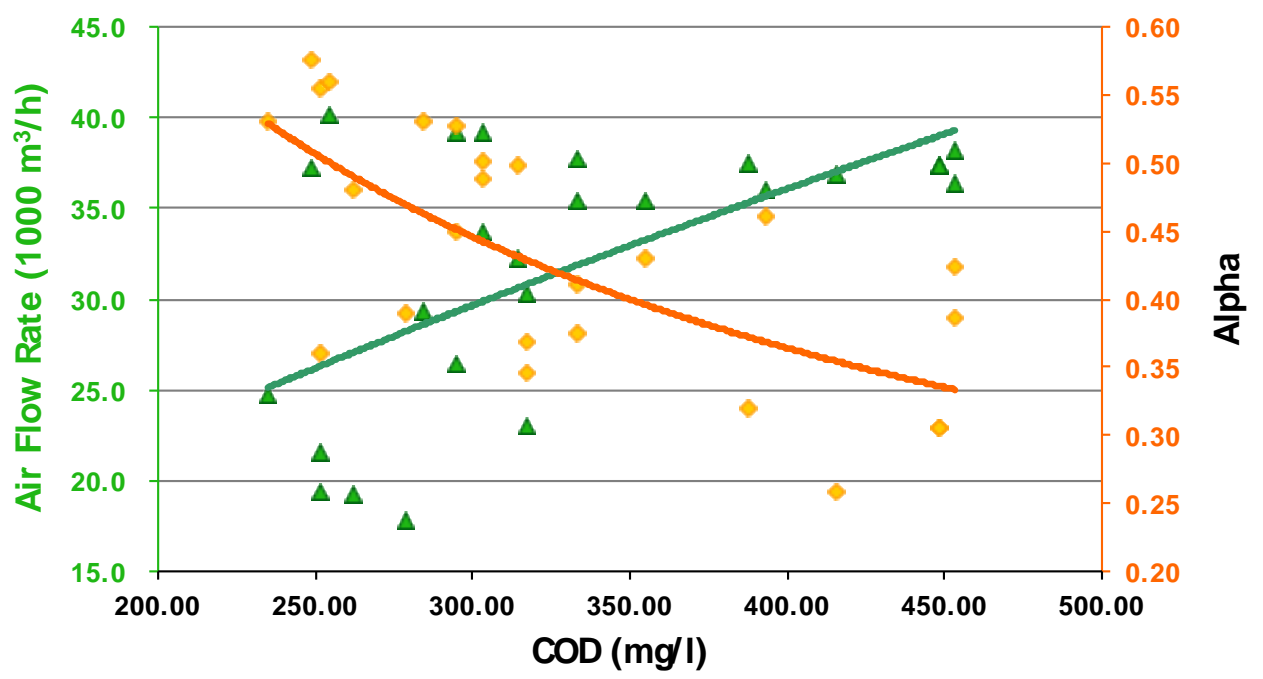

Figure 1. Case of WRP1, used as calibration set for alpha vs. COD.

\section{RESULTS AND DISCUSSION}

The model applied to WRP2 for validation. As showed in Fig. 2(a), the model on average describes well the actual trends of AFR. A comparative analysis of our dynamic $\alpha$ modelling vs. the traditional static modelling that is currently state-of-the-practice is also presented in Fig. 2. Due to the unavailability of a dynamic $\alpha$ model, aeration modelling is performed by selecting constant $\alpha$ values (typically based on one or a combination of recommendations from: diffuser manufacturers; modeller experience; design/process engineers) and using a dynamic function to compensate SOTE with different air flux. In Fig. $2 b$, the results from 
dynamic modelling (grey cross symbols) are compared with static modelling results, calculated with constant $\alpha$ values (blue trend lines). The linear trend that describes the data points is not plotted here as it has a slope of 0.961, and for clarity of plotting we use the diagonal instead. The coloured trend lines show the result of predicting air flux using static modelling: the region where the regression lines encounter the diagonal describes where (in terms of air flux) it is appropriate to use that $\alpha$ value. It appears evident that the range of alpha values to be employed in modelling is wide (0.4-0.8) depending on the air flux, as reported from previous field observations (inter alia, Rosso et al, 2005; Gillot and Héduit, 2008).

One should discuss here the implications of operating the plant at constant DO set point. Currently, new technologies in process control are gaining wider distribution, such as variable set point $\mathrm{DO} / \mathrm{NH}_{4}{ }^{+}$control systems. These allow an adaptive variation of DO controls based on the effluent ammonia levels, so to adjust the DO set point to the actually required value to meet effluent limit, thus curbing excess aeration and energy wastage. Neither plant modelled here had such system installed, but a next research step would be to model processes so equipped.
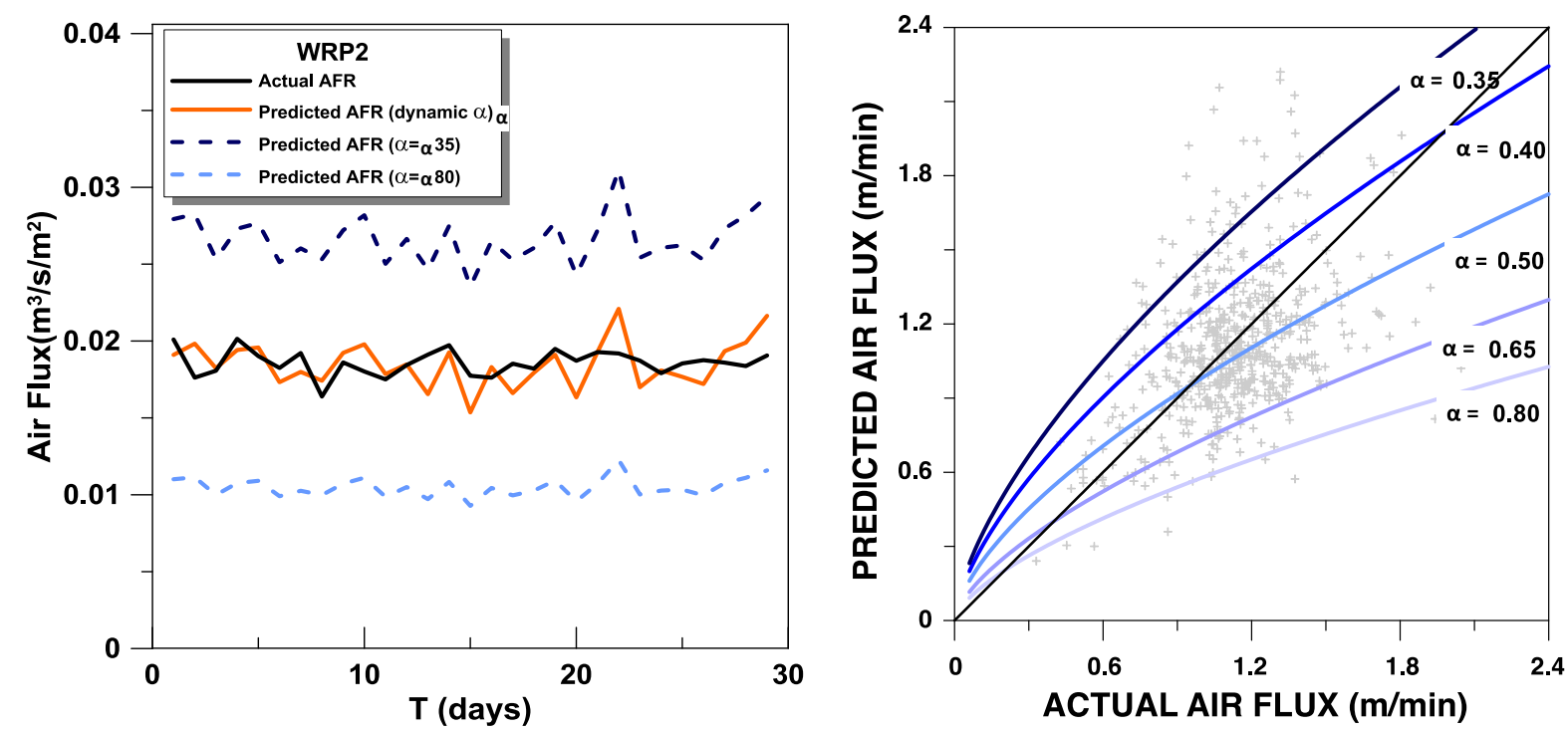

(a)

(b)

Figure 2. Predictive use of the dynamic model: simulation of WRP2. (a) Actual vs. predicted air flux for 1 month with dynamic $\alpha$ and with a constant $\alpha$ of 0.35 and 0.80; (b) Comparative results of 2 years of air flow rate (AFR) prediction using a dynamic $\alpha$ calculation from process parameters (described for plotting clarity by the diagonal in lieu of the actual linear fit with slope 0.961 ) versus using the classical approach of constant $\alpha$ (curves): the point where each curve meets the diagonal is the air flux point where the use of the curve's corresponding $\alpha$ factor is appropriate.

Some uncertainty is still evident in the data scatter of Fig. 2b. This may largely be attributed to the use of COD instead of its fraction rbCOD, which is expected to be descriptive of the surfactants present in municipal wastewater. These have been associated with the alteration of 
diffuser performance from clean water (i.e., $\alpha$ factors) since the 1980s (Zlokarnik, 1980). Future steps should include the model recalibration using fractionated COD, to test the hypothesis that rbCOD is indeed the variable needed for aeration modelling.

Also, the present model version does not include the effects of diffuser fouling. Therefore, the model extension presented here should be used by design engineers to reduce the design uncertainty. Current work is undergoing to include the effects of diffuser fouling on alpha. When these effects are embedded in future revisions of the model structure, the unified model can be applied also to predict future aeration performance.

\section{CONCLUSIONS}

A dynamic model to describe the load dependence of $\alpha$ factors in activated sludge diffused aeration is introduced. This is an extension to the ASM structure to include the dynamic recalculation of air flow using a dynamic $\alpha$ (i.e., as a function of COD). The results show the model adequacy to describe the true dynamic nature of the $\alpha$ factor and oxygen transfer. The calibration using a $24 \mathrm{~h}$ set of data from WRP1 allowed the validation on a 2-year data set from WRP2. We present here the discrepancy between the static alpha modelling practiced hitherto and our dynamic results. The results from current static modelling efforts are valid in a restricted domain of air flow, whereas our dynamic results transition smoothly from different regions of air flow.

\section{REFERENCES}

American Society of Civil Engineers (2007) Measurement of Oxygen Transfer in Clean Water. ASCE 2-07, New York.

Gillot, S. and Héduit, A. (2008) Prediction of alpha factor values for fine pore aeration systems, Wat. Sci. Technol. 57(8) 1265-1270.

Leu, S.-Y. et al (2009) Real-Time Aeration Efficiency Monitoring in the Activated Sludge Process and Methods to Reduce Energy Consumption and Operating Costs Wat. Environ. Res. 81, 2471-2481.

Metcalf \& Eddy, Inc. (2013) Wastewater Engineering: Treatment and Reuse - $5^{\text {th }}$ ed. McGraw-Hill, New York. Reardon, D.J. (1995) Turning down the power, Civ. Eng. 65(8) 54-56.

Stenstrom, M. K. and Gilbert, R. G. (1981) Effects of alpha, beta and theta factors in design, specification and operations of aeration systems, Wat. Res., 15 643-654

Zlokarnik, M. (1980) Koaleszenzphänomene im System gasfoerming, Korrespondenz Abwasser 27-11 728-734 


\title{
The application of removal coefficients for viruses in different wastewater treatment processes calculated using stochastic modelling
}

\author{
Edgard Dias ${ }^{1}$, James Ebdon ${ }^{1}$, and Huw Taylor ${ }^{1}$ \\ ${ }^{1}$ School of Environment and Technology, University of Brighton, Lewes Road, Brighton, United Kingdom, \\ BN2 4GJ (Email: e.dias@brighton.ac.uk; je3@brighton.ac.uk; h.d.taylor@brighton.ac.uk)
}

\begin{abstract}
The current study proposes the calculation and interpretation of removal coefficients $\left(\mathrm{K}_{20}\right)$ for bacteriophages in activated sludge (AS) and trickling filter (TF) systems using stochastic modelling. Initial results have demonstrated that the removal of somatic coliphages is higher than that of F-RNA coliphages in both AS and TF systems and that AS more effectively removes both phage groups than TF. The results also suggest that the obtained removal coefficient $\left(\mathrm{K}_{20}\right)$ values may be used to estimate the quality of final effluents in AS and TS systems using simplified models. Future work will include enumeration and modelling of specific enteric viral pathogens in order to develop practical predictive tools and to support integrated water and sanitation safety planning approaches to human health risk management.
\end{abstract}

\section{Keywords}

bacteriophages, viral particles, wastewater treatment, sanitation safety planning

\section{INTRODUCTION}

Human enteric viruses are commonly found in municipal wastewaters and many of them are capable of causing illnesses in humans (Bosch, 1998; Metcalf and Eddy, 2003). Viruses have been shown to be more resistant to wastewater treatment processes than other microorganisms. Furthermore, the limitations of traditional bacterial water quality indicators (e.g., faecal coliforms and E. coli), such as differences in their occurrence and persistence compared with enteric pathogenic microorganisms both in engineered and natural environments, have led to research into numerous novel viral indicators (Jofre et al., 1995; Purnell et al., 2011; Ebdon et al., 2012).

Emerging potential indicators include bacteriophages, or simply phages, which are a group of viruses capable of infecting prokaryotic organisms that are, as are all viruses, obligate intracellular parasites (Metcalf and Eddy, 2003; Withey et al., 2005). The phages used in water and wastewater quality monitoring fall into three main groups: (i) somatic coliphages phages that infect E. coli strains; (ii) phages infecting Bacteroides spp. - strict anaerobic bacteria comprising the major part of the human gastrointestinal microbiota; and (iii) malespecific F-RNA coliphages - phages commonly used as indicators of human enteric viruses (Grabow, 2001).

In order to estimate the concentration of physico-chemical or microbiological parameters in treated effluent from wastewater treatment plants (WWTP), different models are required, depending on the nature of the treatment hydraulics (e.g., plug-flow vs. mixed reactors) and the kinetics of such reactions (e.g., first or second order). Uncertainties around specific parameters can also be factored in using a 'stochastic modelling' approach, which assigns the 
input variables to value ranges according to their specific frequency or probability distributions (Morgan and Herion, 2007; Vose, 2008).

Therefore, this initial study (part of a larger evolving investigation that also considers enteric viruses and faecal indicator bacteria) demonstrates the potential application of stochastic modelling to improve our understanding of the removal of phages in activated sludge (AS) and trickling filter (TF) treatment systems, using data collected from four WWTPs situated in southern England. As such, it supports an effective multiple barrier approach to disease control, as part of a Sanitation Safety Plan (SSP).

\section{MATERIAL \& METHODS}

Samples of settled wastewater and post-secondary sedimentation wastewater were collected every two weeks from May to November 2013, from four WWTP situated in southern England, including two Activated Sludge (AS) and two Trickling Filter (TF) systems. The WWTP can be regarded as small to medium-scale, having population equivalents of 14,554 and 44,930 (for the AS systems) and 5,084 and 33,229 (for the TF systems). For all samples, somatic coliphage (WG-5) and F-specific coliphages (WG-49) were enumerated using standardised double-layer techniques (BSI, 2001; 2002) and expressed in terms of Plaque Forming Units (PFU) per 100mL.

To calculate the removal coefficients $\left(\mathrm{K}_{\mathrm{T}}\right)$, a completely-mixed model, based on hydraulic retention time (t), was used for AS systems (Eq. 1), while for TF, a plug-flow model, based on hydraulic loading rate (HLR), was adopted (Eq. 2). However, in order to be able to compare the removal coefficients obtained from both AS and TF systems, certain mathematical adjustments were made, from Eq. 3 to 5, to obtain Eq. 6. In this case, the unit for removal coefficient in TF systems is $\mathrm{d}^{-1}$, as with AS. What's more to standardise the removal coefficients according to an ambient temperature of $20^{\circ} \mathrm{C}$, the Arrhenius equation was used (Eq. 7). The final models used to calculate removal coefficients at $20^{\circ} \mathrm{C}\left(\mathrm{K}_{20}\right)$ for AS $\left(\mathrm{as} \mathrm{d}^{-1}\right)$ and TF (as $\mathrm{d}^{-1}$ and $\mathrm{m}^{3} \cdot \mathrm{m}^{-2} \cdot \mathrm{d}^{-1}$ ) systems are presented in Eq. 8, 9 and 10, respectively. All equations are presented in Table 1.

Table 1 - Equations used for the estimation of removal coefficients.

$$
\begin{aligned}
& \mathbf{N}=\frac{\mathbf{N}_{0}}{\left(\mathbf{1}+\mathbf{K}_{\mathrm{T}}, \mathbf{t}\right)} \quad \text { Eq. } 1 \quad \mathbf{N}=\mathbf{N}_{0} \cdot \exp \left(-\mathbf{K}_{\mathbf{T}}, \mathbf{t} / \mathbf{h} . \mathbf{n}\right) \quad \text { Eq.6 } \\
& \mathbf{N}=\mathbf{N}_{\mathbf{0}} \cdot \exp \left(-\mathbf{K}_{\mathrm{T}} / \mathbf{H L R}\right) \quad \text { Eq. } 2 \quad \mathbf{K}_{\mathbf{T}}=\mathbf{K}_{20} \cdot \boldsymbol{\theta}^{(\mathbf{T}-20)} \\
& \mathbf{t}=\mathbf{V o l} / \mathbf{Q} \quad \text { Eq. } 3 \quad \mathbf{K}_{\mathbf{2 0}}=\left(\frac{\mathbf{N}_{\mathbf{0}}}{\mathbf{N}}-\mathbf{1}\right) \cdot \frac{\mathbf{1}}{\mathbf{t . \boldsymbol { \theta } ^ { ( \mathbf { T } - 2 0 ) }}} \quad \text { Eq.8 } \\
& \mathbf{H L R}=\mathbf{Q} / \mathbf{A} \quad \text { Eq.4 } \quad \mathbf{K}_{20}=\ln \left(\frac{\mathbf{N}_{\mathbf{0}}}{\mathbf{N}}\right) \frac{\mathbf{H L R}}{\mathbf{n . h} \cdot \boldsymbol{\theta}^{(\mathbf{T}-20)}} . \quad \text { Eq.9 } \\
& \text { Vol = A.h.n Eq.5 } \quad \mathbf{K}_{20}=\ln \left(\frac{\mathbf{N}_{0}}{\mathbf{N}}\right) \frac{\text { HLR }}{\boldsymbol{\theta}^{(\mathbf{T}-20)}} \quad \text { Eq.10 }
\end{aligned}
$$

Where: $\mathrm{N}=$ final conc. $\left(\right.$ PFU.100mL $\left.\mathrm{m}^{-1}\right) ; \mathrm{N}_{0}=$ initial conc. $\left(\mathrm{PFU} .100 \mathrm{~mL}^{-1}\right) ; \mathrm{t}=$ hydraulic retention time $(\mathrm{d}) ; \mathrm{HLR}=$ hydraulic loading rate $\left(\mathrm{m}^{3} \cdot \mathrm{m}^{-2} \cdot \mathrm{d}^{-1}\right) ; \mathrm{Q}=$ flow $\left(\mathrm{m}^{3} \cdot \mathrm{d}^{-1}\right) ; \mathrm{Vol}=$ volume $\left(\mathrm{m}^{3}\right) ; \mathrm{Q}=$ flow $\left(\mathrm{m}^{3} \cdot \mathrm{d}^{-1}\right) ; \mathrm{A}=$ surface area $\left(\mathrm{m}^{2}\right) ; \mathrm{h}$ $=$ height $(\mathrm{m}) ; \mathrm{n}=$ empty space; $\mathrm{K}_{\mathrm{T}}=$ removal coefficient at temp $\mathrm{T}\left(\mathrm{d}^{-1}\right.$ or $\left.^{3} \cdot \mathrm{m}^{-2} \cdot \mathrm{d}^{-1}\right) ; \mathrm{K}_{20}=$ removal coefficient at 
$20^{\circ} \mathrm{C}\left(\mathrm{d}^{-1}\right.$ for $\mathrm{AS} ; \mathrm{m}^{3} \cdot \mathrm{m}^{-2} \cdot \mathrm{d}^{-1}$ for $\left.\mathrm{TF}\right) ; \theta=$ temp coefficient; $\mathrm{T}=$ temperature $\left({ }^{\circ} \mathrm{C}\right)$.

Probability density functions (PDF) were fitted for the database using the adherence test option present in the statistical software @ Risk version 5.5.0. The removal coefficients were then estimated by stochastic simulation (Eq. 8, 9 \& 10) with Latin Hypercube sampling and 100,000 iterations, again using the software @Risk version 5.5.0.

\section{RESULTS \& DISCUSSION}

Figure 1 presents the concentrations of somatic and F-specific coliphages in the settled wastewater $\left(\mathrm{N}_{\mathrm{o}}\right)$ and post-secondary sedimentation wastewater $\left(\mathrm{N}_{\mathrm{f}}\right)$ of both AS and TF systems. The initial and final concentrations of somatic coliphages were approximately $2 \log _{10}$ higher than those of F-specific coliphages in the systems monitored. Furthermore, it is apparent that, in general, the concentrations of somatic coliphages varied more than those of F-specific coliphages. With regard to the geometric mean values of initial and final concentrations, the removal of somatic coliphages in the AS systems was of the order $1.86 \log _{10}$, while for F-specific coliphages it was $1.41 \log _{10}$. In the TF systems, the removal of somatic and F-specific coliphages was of the order 0.44 and $0.46 \log _{10}$, respectively. These results demonstrate that the removal rate of somatic coliphages was higher than those of Fspecific coliphages in AS systems, whilst for TF the removal rate of both phage groups was very similar. Furthermore, AS systems appear to remove both phage groups more effectively than TF systems. Similar removal rates of somatic and F-specific coliphages in AS systems are presented in the literature (Zhang \& Farahbakhsh, 2007; De Luca et al, 2013). No study involving the removal of bacteriophages in TF systems has been found to date.

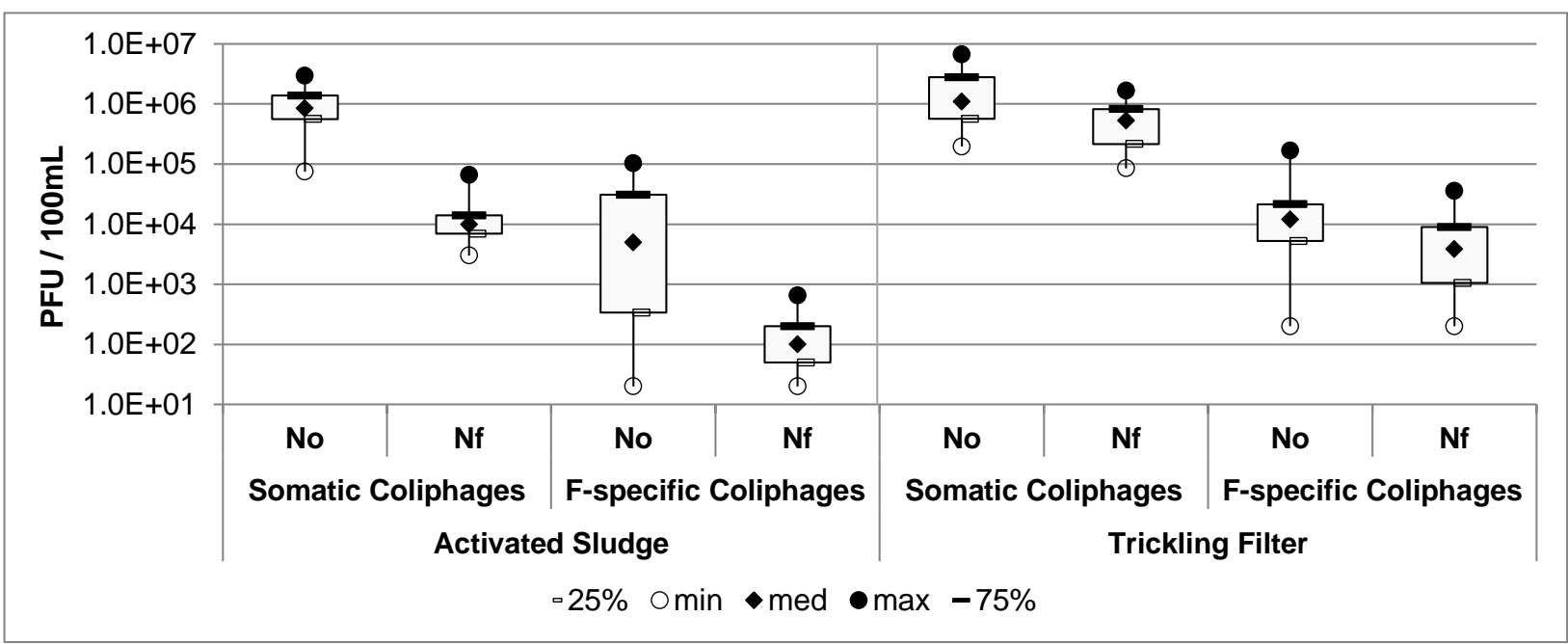

Figure 1 - Box-plot of the concentration of somatic and F-specific coliphages in the settled wastewater $\left(\mathrm{N}_{\mathrm{o}}\right)$ and post-secondary sedimentation wastewater $\left(\mathrm{N}_{\mathrm{f}}\right)$ of the Activated Sludge (AS) and Trickling Filter (TF) systems monitored.

Probability distribution functions (PDF) were adjusted for the initial and final concentrations $\left(\mathrm{N}_{0}\right.$ and $\left.\mathrm{N}\right)$ and temperature $(\mathrm{T})$ data collected using adherence tests. Hydraulic retention time (t) (uniform PDF: $\min =0.25$; $\max =0.33 \mathrm{~d}$ ), hydraulic loading rate (HLR) (uniform PDF: $\left.\min =1 ; \max =4 \mathrm{~m}^{3} \cdot \mathrm{m}^{-2} \cdot \mathrm{d}^{-1}\right)$, temperature coefficient $(\theta)$ (triangular PDF: $\min =1.00 ; \max =1.19$; mean=1.07), height $(\mathrm{h}$ ) (uniform PDF: $\min =1.8$; $\max =2.5 \mathrm{~m}$ ) an empty space (n) (uniform 
PDF: $\min =0.5 ; \max =0.6$ ) were assumed in accordance with the literature (Marais, 1974; Castagnino, 1977; Thomann and Mueller, 1987; Yanes, 1993; Metcalf and Eddy, 2003; von Sperling, 2007a; von Sperling, 2007c). Table 2 summarises the PDF fitted for each input variable of the model. The removal coefficients were then estimated by stochastic simulation (Eq. 8, 9 \& 10) with Latin Hypercube sampling and 100,000 iterations using the software @Risk version 5.5.0.

Table 2 - Probability density functions ${ }^{(a)}$ of the input variables of the models (Eq. $8,9 \& 10$ ) to estimate the removal coefficient at $20^{\circ} \mathrm{C}\left(\mathrm{K}_{20}\right)$ for somatic and F-specific coliphages in activated sludge (AS) and Trickling Filter (TF) systems.

\begin{tabular}{|c|c|c|c|}
\hline \multirow[b]{2}{*}{ System } & \multirow[b]{2}{*}{ Input Variable } & \multicolumn{2}{|c|}{ Microorganisms } \\
\hline & & Somatic Coliphages & F-Specific Coliphages \\
\hline \multirow{5}{*}{$\begin{array}{l}\text { Activated } \\
\text { Sludge }\end{array}$} & $\mathrm{N}$ & $\operatorname{LogN}\left(1.29 \times 10^{4} ; 9.78 \times 10^{3}\right)$ & $\operatorname{Exp}\left(1.74 \times 10^{2}\right)$ \\
\hline & $\mathrm{N}_{\mathrm{o}}$ & $\operatorname{Gamma}\left(1.78 ; 5.75 \times 10^{5}\right)$ & $\operatorname{Gamma}\left(0.35 ; 5.56 \times 10^{4}\right)$ \\
\hline & $\theta$ & Triang $(1.00 ; 1.07 ; 1.19)$ & Triang $(1.00 ; 1.07 ; 1.19)$ \\
\hline & $\mathrm{T}$ & Weibull(6.86;18.70) & Weibull(6.86;18.70) \\
\hline & $\mathrm{t}$ & Uniform $(0.25 ; 0.33)$ & Uniform $(0.25 ; 0.33)$ \\
\hline \multirow{7}{*}{ Trickling Filter } & $\mathrm{N}$ & Gamma(1.81;3.31x10 $)$ & Weibull $\left(0.77 ; 6.90 \times 10^{3}\right)$ \\
\hline & $\mathrm{N}_{\mathrm{o}}$ & $\operatorname{Exp}\left(1.91 \times 10^{6}\right)$ & $\log N\left(2.52 \times 10^{4} ; 6.19 \times 10^{4}\right)$ \\
\hline & $\theta$ & Triang(1.00;1.07;1.19) & Triang $(1.00 ; 1.07 ; 1.19)$ \\
\hline & $\mathrm{T}$ & Weibull( $6.88 ; 17.69)$ & Weibull(6.88;17.69) \\
\hline & HLR & Uniform $(1.0 ; 4.0)$ & Uniform $(1.0 ; 4.0)$ \\
\hline & $\mathrm{h}$ & Uniform $(1.8 ; 2.5)$ & Uniform $(1.8 ; 2.5)$ \\
\hline & $\mathrm{n}$ & Uniform $(0.5 ; 0.6)$ & Uniform $(0.5 ; 0.6)$ \\
\hline \multicolumn{4}{|c|}{$\begin{array}{l}\text { (a) Weibull }(\alpha ; \beta)=\text { Weibull distribution with shape parameter } \alpha \text { and scale parameter } \beta ; \operatorname{Gamma}(\alpha ; \beta) \text { Gamm } \\
\text { distribution with shape parameter } \alpha \text { and scale parameter } \beta ; \log N(\mu ; \sigma) \operatorname{lognormal} \text { distribution with specific mean } \\
\text { and standard deviation } \sigma ; \operatorname{Exp}(\lambda)=\text { exponential distribution with decay constant } \lambda \text {; Uniform }(\min ; \max )=\text { uniforn } \\
\text { distribution between minimum and maximum; Triang }(\min ; \operatorname{most} \text { likely;max })=\text { triangular distribution with definec } \\
\text { minimum, most likely and maximum values. }\end{array}$} \\
\hline
\end{tabular}

Table 3 presents descriptive statistics whilst Figure 2 presents the histograms and cumulative frequency curves for the removal coefficients at $20^{\circ} \mathrm{C}$, for somatic and F-RNA coliphages in activated sludge (AS) and trickling filters (TF).

Table 3 - Descriptive statistics of the removal coefficient at $20^{\circ} \mathrm{C}\left(\mathrm{K}_{20}\right)$ for somatic and F-specific coliphages in activated sludge (AS) and Trickling Filter (TF) systems.

\begin{tabular}{cccccccc}
\hline & \multicolumn{3}{c}{ Somatic Coliphages } & & \multicolumn{3}{c}{ F-Specific Coliphages } \\
\cline { 2 - 4 } Variable & $\mathbf{A S ~ K}_{\mathbf{2 0}}{ }^{(\mathbf{a})}$ & $\mathbf{T F ~ K}_{\mathbf{2 0}}{ }^{(\mathbf{a})}$ & $\mathbf{T F ~ K}_{\mathbf{2 0}}{ }^{(\mathbf{b})}$ & & $\mathbf{A S ~ K}_{\mathbf{2 0}}{ }^{(\mathbf{a})}$ & $\mathbf{T F ~ K}_{\mathbf{2 0}}{ }^{(\mathbf{a})}$ & $\mathbf{T F ~ K}_{\mathbf{2 0}}{ }^{(\mathbf{b})}$ \\
\hline Minimum & -5.48 & -56.29 & -69.70 & & -22.29 & -73.01 & -72.31 \\
$5 \%$ & 39.94 & -5.14 & -6.00 & & -3.64 & -6.58 & -7.73 \\
$10 \%$ & 67.86 & -2.78 & -3.28 & & -2.20 & -4.20 & -4.91 \\
$25 \%$ & 148.62 & -0.02 & -0.02 & & 22.43 & -0.98 & -1.17 \\
$50 \%$ & 324.44 & 2.33 & 2.76 & & 215.30 & 2.18 & 2.58 \\
$75 \%$ & 667.64 & 5.14 & 6.04 & & 1077.18 & 6.32 & 7.43 \\
$90 \%$ & 1241.95 & 8.64 & 10.06 & & 4032.01 & 11.74 & 13.71 \\
$95 \%$ & 1787.17 & 11.34 & 13.22 & & 9085.31 & 16.04 & 18.78 \\
Maximum & $3.56 \times 10^{4}$ & 90.41 & 89.42 & & $5.42 \times 10^{7}$ & 130.48 & 155.02 \\
\hline Mean & 549.93 & 2.65 & 3.10 & & 5822.11 & 3.18 & 3.71 \\
Std.Dev & 751.10 & 5.40 & 6.26 & & $2.36 \times 10^{5}$ & 7.50 & 8.71 \\
\hline
\end{tabular}

(a) Values in $\mathrm{d}^{-1}$; ${ }^{(\mathrm{b})}$ values in $\mathrm{m}^{3} \cdot \mathrm{m}^{-2} \cdot \mathrm{d}^{-1}$. 
Dias et al.
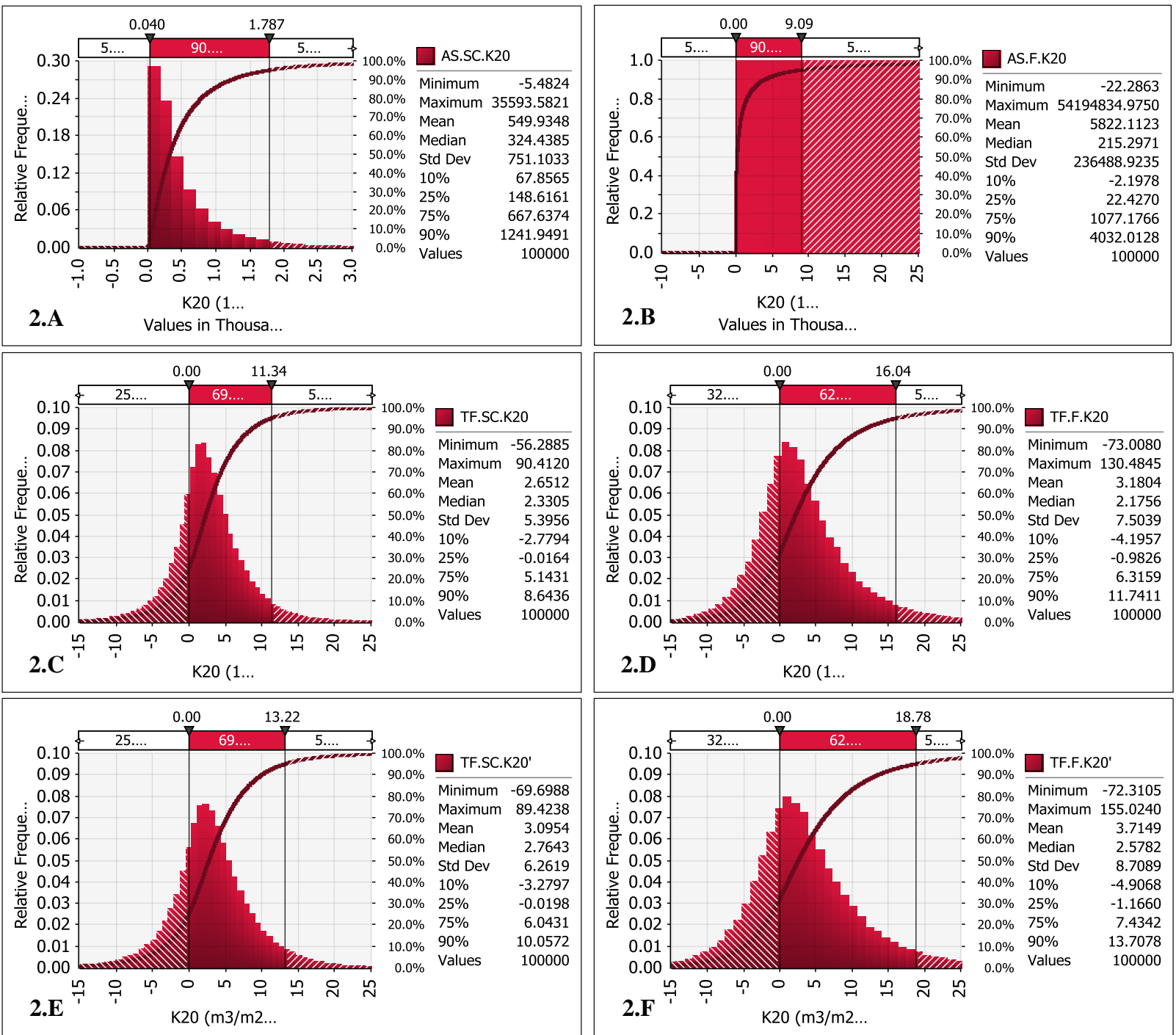

Figure 2 - Histogram and cumulative frequency curve of removal coefficients at $20^{\circ} \mathrm{C}\left(\mathrm{K}_{20}\right)$ for somatic coliphages and F-RNA coliphages, based on the hydraulic retention time ( $\mathrm{t}$ ) in AS systems (2.A \& 2.B, respectively) and in TF systems (2.C \& 2.D, respectively), and based on the hydraulic loading rate (HLR) in TF systems (2.E \& 2.F, respectively).

Stochastic modelling revealed that the $\mathrm{K}_{20}$ values for the AS systems were an order of magnitude higher than those of the TF systems, possibly as a result of the different models used for each system. Median $\mathrm{K}_{20}$ values for AS were $324.4 \mathrm{~d}^{-1}$ for somatic coliphages and $215.3 \mathrm{~d}^{-1}$ for F-specific coliphages (Figures 2.A \& 2.B; Table 3), while median $\mathrm{K}_{20}$ values for TF were 2.33 and $2.18 \mathrm{~d}^{-1}$ for somatic and F-specific coliphages, respectively (Figures 2.C \& 2.D; Table 3). With regard to the mean values of $\mathrm{K}_{20}$, the numbers were higher for both systems: 549.9 and $2.65 \mathrm{~d}^{-1}$ for somatic and F-specific coliphages, respectively, in AS systems (Figures 2.A \& 2.B; Table 3); 5822.1 and $3.18 \mathrm{~d}^{-1}$ for somatic and F-specific coliphages, respectively, in AS systems (Figures 2.C \& 2.D; Table 3).

The differences between $\mathrm{K}_{20}$ values obtained here for each system, and more specifically the considerably higher $\mathrm{K}_{20}$ values observed for TF systems compared with AS systems, could be explained by the inadequacy of the idealised models. As mentioned by von Sperling (2007b), even for the same conditions (initial and final concentrations, hydraulic retention time), the equations representing the plug-flow and complete-mix reactors would result in different 
removal coefficient $(\mathrm{K})$ values. This is because, in theory, the ideal complete-mix reactors are the least efficient reactors for first-order removal kinetics. In other words, the lower efficiency is compensated by a higher $\mathrm{K}$ value (von Sperling, 2007b). Conversely, ideal plug-flow reactors are the most efficient reactors, and the $\mathrm{K}$ value necessary to produce the same effluent quality is reduced (von Sperling, 2007b).

The $\mathrm{K}_{20}$ values for TF systems previously discussed, given as $\mathrm{d}^{-1}$, were obtained from Eq. 9 for comparison with the $\mathrm{K}_{20}$ of AS systems (Eq. 8). However, the parameter that is normally used for the design of biofilters is the hydraulic loading rate (HLR). Thus, Eq. 10 was also used to calculate $\mathrm{K}_{20}$ values for TF systems, given as $\mathrm{m}^{3} \cdot \mathrm{m}^{-2} \cdot \mathrm{d}^{-1}$. Using both Eq. $9 \& 10$, median $\mathrm{K}_{20}$ values were very similar for each microorganism: 2.33 and $2.18 \mathrm{~d}^{-1}$ for somatic and F-specific coliphages, respectively, from Eq. 9; and 2.76 and $2.58 \mathrm{~m}^{3} \cdot \mathrm{m}^{-2} \cdot \mathrm{d}^{-1}$ for somatic and F-specific coliphages, respectively, from Eq. 10 (Figures 2.C to 2.F; Table 3). Again, mean values of $\mathrm{K}_{20}$ were higher than median values, but were similar for each microorganism: 2.65 and 2.3.18 $\mathrm{d}^{-1}$ for somatic and F-specific coliphages, respectively, from Eq. 9; and 3.10 and $3.71 \mathrm{~m}^{3} \cdot \mathrm{m}^{-2} \cdot \mathrm{d}^{-1}$ for somatic and F-specific coliphages, respectively, from Eq. 10 (Figures 2.C to 2.F; Table 3). The reason for similar values of $\mathrm{K}$ obtained from Eq. 9 \& 10 results from the PDF assumed for the input variables height (h) (uniform PDF: $\min =1.8$; $\max =2.5 \mathrm{~m}$ ) and empty space (n) (uniform PDF: $\min =0.5$; $\max =0.6$ ) in Eq. 10.

Interestingly, the cumulative frequency curves for both AS and TF were markedly different in their appearance, as the output data are skewed towards the left side of the distribution for both phage groups in the AS systems (Figures 2.A \& 2.B), while in the TF systems, the data seem to follow a normal distribution (Figures 2.C to 2.F). With regards to the variation around the mean/median values, the standard deviation was higher for F-specific coliphages than for somatic coliphages, in both the AS and TF systems (Figure 2). Finally, comparison of the findings dicussed in this paper with the scientific literature are limited due to the paucity of data currently available in this area.

\section{CONCLUSION}

The work to date has demonstrated that appropriate forms of stochastic modelling may elucidate the behaviour of enteric bacteriophages in traditional biological wastewater treatment processes. Furthermore, using this approach it may be possible to export the results obtained from the monitored systems to other systems and predict final concentrations of coliphages using simple models.

Future work will compare the behaviour of these indices with the behaviour of specific enteric viral pathogens of human health significance. It is envisaged that the research will contribute new knowledge to inform better design and operation of wastewater treatment systems. At a time when greater emphasis is being placed on human health protection by minimising the transmission of pathogens at several points within the water cycle, this work will support more integrated water and sanitation safety planning approaches to human health risk management.

\section{REFERENCES}


BOSCH, A. 1998. Human enteric viruses in the water environment: a minireview. International microbiology: the official journal of the Spanish Society for Microbiology, 1, 191-6.

BSI - British Standards. BS EN ISO 10705-1:2001. Water quality - Detection and enumeration of bacteriophages - Part 1: Enumeration of F-specific RNA bacteriophages. London. 2002.

BSI - British Standards. BS EN ISO 10705-2:2001. Water quality - Detection and enumeration of bacteriophages - Part 2: Enumeration of somatic coliphages. London. 2001.

CASTAGNINO, W. A. 1997. Poluicion de agua. Modelos y control. Lima, 234 p. (in Spanish)

DE LUCA, G., SACCHETTI, R., LEONI, E. \& ZANETTI, F. 2013. Removal of indicator bacteriophages from municipal wastewater by a full-scale membrane bioreactor and a conventional activated sludge process: Implications to water reuse. Bioresource Technology, 129, 526-531.

EBDON, J., MUNIESA, M. \& TAYLOR, H. 2007. The application of a recently isolated strain of Bacteroides (GB-124) to identify human sources of faecal pollution in a temperate river catchment. Water Research, 41, 3683-90.

GRABOW, W. O. K. 2001. Bacteriophages: Update on application as models for viruses in water. Water SA, 27 , 251-268.

JOFRE, J., OLLE, E., RIBAS, F., VIDAL, A. \& LUCENA, F. 1995. Potential usefulness of bacteriophages that infect Bacteroides-fragilis as model organisms for monitoring virus removal in drinking-water treatment plants. Applied and Environmental Microbiology, 61, 3227-3231.

MARAIS, G. v. R. 1974. Faecal bacteria kinetics in stabilisation ponds. Journal of the Environmental Engineering Division, 100, 119-139.

METCALF \& EDDY. 2003. Wastewater Engineering: Treatment, and Reuse. $4^{\text {th }}$ ed. McGraw-Hill, New York, $1819 \mathrm{p}$.

MORGAN, M. G.; HERION, M. 2007. Uncertainty: a Guide to Dealing with Uncertainty in Quantitative Risk and Policy Analysis. Cambridge University Press, Cambridge. 332p.

PURNELL, S. E., EBDON, J. E. \& TAYLOR, H. D. 2011. Bacteriophage Lysis of Enterococcus Host Strains: A Tool for Microbial Source Tracking? Environmental Science \& Technology, 45, 10699-10705.

THOMANN, R. V.; MUELLER, J. A. 1987. Principles of surface water quality modeling and control. Harper \& Row. 644 p.

von SPERLING, M. 2007. Activated Sludge and Aerobic Biofilm Reactors. Biological Wastewater Treatment Series, Volume 5. IWA Publishing, London. 322 p.

von SPERLING, M. 2007. Waste Stabilisation Ponds. Biological Wastewater Treatment Series, Volume 3. IWA Publishing, London. 322 p.

von SPERLING, M. 2007. Wastewater Characteristics, Treatment and Disposal. Biological Wastewater Treatment Series, Volume 1. IWA Publishing, London. 292 p.

VOSE, D. 2008. Risk Analysis: A Quantitative Guide. $3^{\text {rd }}$ ed. John Wiley \& Sons, Chichester. 735 p.

WITHEY, S., CARTMELL, E., AVERY, L. M. \& STEPHENSON, T. 2005. Bacteriophages - potential for application in wastewater treatment processes. Science of the Total Environment, 339, 1-18.

YANES, F. 1993. Lagunas de estabilizacion. Teoria, diseño y mantenimiento. Cuenca. 421 p. (in Spanish)

ZHANG, K. \& FARAHBAKHSH, K. 2007. Removal of native coliphages and coliform bacteria from municipal wastewater by various wastewater treatment processes: Implications to water reuse. Water Research, 41, 2816-2824. 


\title{
Early-stage design of municipal wastewater treatment plants - presentation and discussion of an optimisation based concept
}

\author{
Hande Bozkurt $^{1}$, Alberto Quaglia ${ }^{1}$, Krist V. Gernaey ${ }^{2}$ and Gürkan $\operatorname{Sin}^{1}$ \\ ${ }^{1}$ Computer Aided Process Engineering Center (CAPEC), ${ }^{2}$ Center for Process Engineering and Technology \\ (PROCESS), Department of Chemical and Biochemical Engineering, Technical University of Denmark, \\ DK-2800 Lyngby, Denmark (Email: gsi@kt.dtu.dk)
}

\begin{abstract}
The number of alternative WWT technologies has grown steadily to meet increasingly stringent performance demands which increased the importance and complexity of earlystage decision making in WWTP design and retrofitting problems. Currently the conceptual design task is handled based on expert decisions and previous experiences. In this contribution, we propose a new approach based on mathematical programming to manage the complexity of the problem and generate novel and optimal WWTP network designs for domestic WWT. Within this context, a superstructure concept is used to represent the alternative WWT technologies described as a series of reaction and separation tasks at different treatment levels. Each process alternative is described by a generic model and the required data for both performance and economics of each alternative are collected and sorted in a multi-dimensional database. This database is embedded within the mixed integer nonlinear programming problem formulated and solved in GAMS for different objective functions (e.g. total annualized costs, etc.) and constraint definitions (e.g. effluent discharge limits). The developed framework is highlighted using the benchmark plant as a case study to generate and screen optimal concepts for retrofitting options under different scenarios.
\end{abstract}

\section{Keywords}

Design; Modelling; Superstructure optimization; Uncertainty; Wastewater treatment

\section{INTRODUCTION AND MOTIVATION}

Recently, the WWTP process selection and network design problem has evolved from being a simple technical design problem to a complex integrated decision making task, and this is mainly because of the numerous aspects that are being considered in the early decision making stage (Hamouda, 2009). Currently, the early stage decision making for WWTP design - i.e. which technology and treatment concept to employ - is mainly based on expert decisions and experiences accumulated internally in an engineering company from solving previous problems. This approach takes values like environmental issues, water reuse, byproduct recovery (if possible) and public impacts into account and identifies the alternatives based on experience, similar existing solutions and brainstorming to come up with the most viable WWTP network (Daigger, 2005). This study on the other hand, proposes a superstructure based optimization methodology which represents different aspects considered during the early stage decision making with the help of mathematical programming, and designs/retrofits the domestic WWTP network in a novel and optimal manner.

\section{FRAMEWORK FOR SYNTHESIS AND DESIGN OF WWTP NETWORKS}

The mathematical programming based optimization theory developed for chemical process synthesis and design by Quaglia et al. (2012) was modified and adapted in the context of the WWT design problem. The framework is seen in Figure 1 (Bozkurt et al., 2014). After defining the wastewater characterization, sink limitations and the objective function in the 
first step of the framework, one can define a superstructure where the treatment plant is defined as a sequence of treatment tasks (columns) and alternative technologies are placed in the rows, as seen in Figure 2. The superstructure is then formulated by defining connection streams between treatment tasks. Each process interval in the superstructure is structured using a generic model, which describes the treatment alternatives by input-output mass balances, as also illustrated in Figure 2.

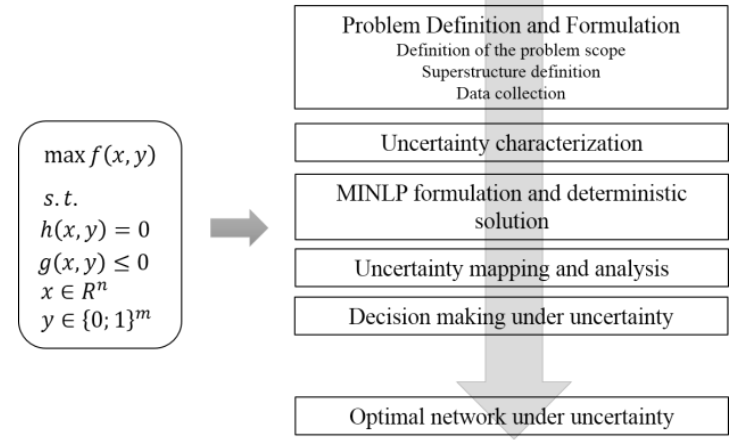

Figure 1. The superstructure based optimization methodology adapted for design of optimal WWT systems

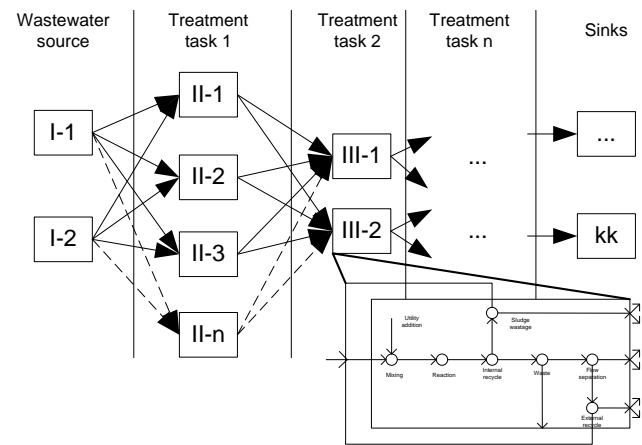

Figure 2. A representative superstructure for WWT networks and the generic process interval structure

The intervals are composed of a number of phenomena, namely: mixing the flows and the utilities added, reaction, separation of flow for internal recycle and sludge wastage, waste separation, flow separation for external recycle and sending the flow to the process intervals of the next column. The mathematical equations defining the intervals can be seen in Table 1 . A database lies behind the superstructure which contains the data to define the parameters of the generic model, e.g. process performances, utility consumptions, volumes, sludge production for each treatment alternative. To this end, a systematic data collection procedure was developed by integrating the procedures given by ATV design standards, Tchobanoglous (2003), WEF (2010) and Henze et al. (2008). The details of the procedure can be found elsewhere (Bozkurt et al., 2013). The optimal wastewater network problem is then formulated as a Mixed Integer (Non)Linear Programming (MI(N)LP) problem in GAMS and solved for different scenarios. The optimization model in GAMS consists of an objective function covering both operational and capital cost, logical constraints defining the topology of the solution and process constraints describing the process models of each treatment technology in the superstructure. The problem is also solved under uncertainty in order to identify the sensitivity of the optimal solutions to the data used in the optimization.

Table 1. Mathematical equations representing the process model in each interval

\begin{tabular}{|c|c|c|}
\hline Phenomena & Equation & Explanations \\
\hline Mixing & $\begin{array}{l}\operatorname{Fin}_{i, k k}=\sum_{k} F_{i, k, k k} \\
\text { Fmix }_{i, k k}=\text { Fin }_{i, k}+\alpha_{i, k k} * R_{i, k k}\end{array}$ & $\begin{array}{l}i, i i: \text { Component index } \\
k, k k: \text { Process interval index } \\
F_{i n}, k k: \text { Inflow to the process interval } \\
F_{i} \text {. Inflow of component } i \text { to process } k k \text { coming from } k\end{array}$ \\
\hline $\begin{array}{l}\text { Utility } \\
\text { addition }\end{array}$ & $R_{i, k k}=\sum_{i i}\left(\mu_{i, i i, k k} * F_{i i, k k}\right)$ & $\begin{array}{l}F m i x_{i, k k}: \text { Flow of component } i \text { after mixing } \\
R_{i, k k}: \text { Utility flow }\end{array}$ \\
\hline Reaction & Freac $_{i, k k}=$ Fmix $_{i, k k}+\sum_{r r, r e a c t}\left(\gamma_{i, k k, r r} * \theta_{r e a c t, k k, r r} * F_{r e a c t, k k}\right)$ & $\begin{array}{l}\alpha_{i, k k}: \text { Fraction of utility consumed } \\
\mu_{i, k}: \text { Specific consumption of utility }\end{array}$ \\
\hline $\begin{array}{l}\text { Waste } \\
\text { separation }\end{array}$ & $F w_{i, k k}=\operatorname{Freac}_{i, k k} *\left(1-W_{i, k k}\right)$ & $\begin{array}{l}\text { Freac }_{i, k k}: \text { Flow after reaction } \\
\gamma_{i, k k, r r}: \text { Matrix representing reaction stoichiometry }\end{array}$ \\
\hline $\begin{array}{l}\text { Flow } \\
\text { separation }\end{array}$ & $\begin{array}{l}\text { Fout }_{i, k k}=F w_{i, k k} * \text { Split }_{i, k k} \\
\text { Fout }_{i, k k}=F w_{i, k k}-\text { Fout }_{i, k k}-\text { Frec }_{i, k k} \\
\text { Fout }_{i, k k}=\text { Freac }_{i, k k} * S W_{k k} \\
\text { Frec }_{i, k k}=\left(F w_{i, k k}-\text { Fout }_{i, k k}\right) * \operatorname{rec}_{k k} \\
F X_{i, k, k k}=\text { Fout }_{i, k} * S_{k, k k}\end{array}$ & 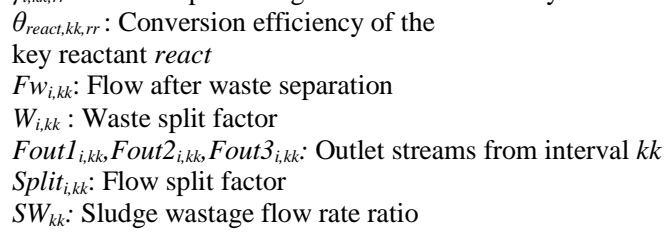 \\
\hline
\end{tabular}




\begin{tabular}{|c|c|c|}
\hline Activation & $y_{k k} * x_{k}^{L O} \leq x_{k} \leq y_{k k} * x_{k}^{U P}$ & $\begin{array}{l}\text { Frec }_{i, k}: \text { External recycle flow } \\
\text { rec }_{k k}: \text { External recycle ratio }\end{array}$ \\
\hline Logical cuts & $\sum_{k k} y_{k k} \leq 1$ & $\begin{array}{l}S_{k, k k}: \text { Parameter containing superstructure information } \\
y_{k k}: \text { Binary variable describing the process interval } \\
x_{k}: \text { Variable bounded by } x_{k}^{L O} \text { and } x_{k}^{U P}\end{array}$ \\
\hline
\end{tabular}

\section{CASE STUDY}

The problem is defined as treatment of domestic wastewater comprising mainly COD, nitrogen and solids as pollutants. The objective is to design the WWTP network with the minimum operational and capital cost possible while satisfying the effluent limitations for organics and nitrogen. The superstructure is defined as shown in Figure 3. It consists of wastewater source (I-1) and sinks for water (VI-1) and sludge (VI-2), primary sedimentation (II-1), pre-denitrification with different SRTs (III-1 and III-2), anaerobic treatment (III-3), different innovative nitrogen removal technologies (IV-1 and IV-2), disinfection options by means of UV, ozone and chloride (V-1, V-2 and V-3) together with by-pass intervals (II-2, III-4, IV-3 and V-4). The database has been developed by following the systematic procedure and the optimization problem was solved.

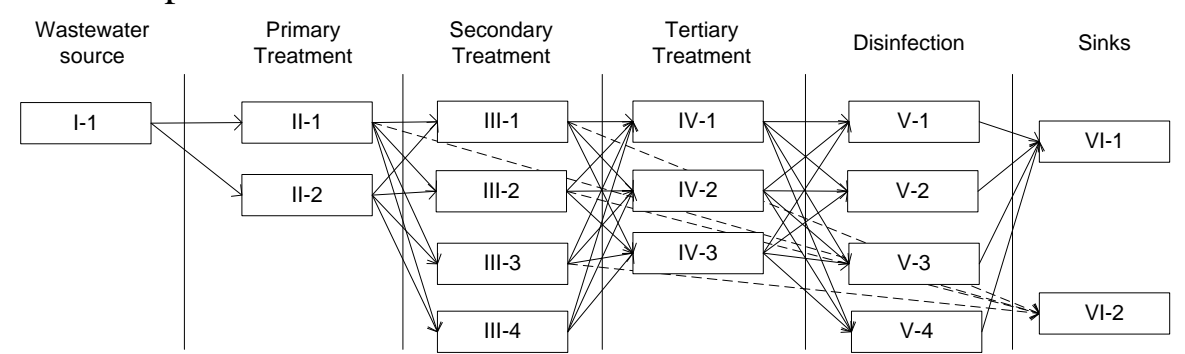

Figure 3. An example superstructure for the case study

The problem was solved under three different scenarios: the first scenario takes only operational cost into account, while the second one considers total annualized cost. The third scenario imposes a stricter effluent limit on the nitrogen concentration. All the scenarios bypassed the primary and tertiary treatment steps together with disinfection. The secondary treatment selection was the high SRT pre-denitrification technology for the first and third scenario and short SRT pre-denitrification for the second scenario. The fact that the short SRT system has a lower capital cost promoted its selection in the second scenario and the high SRT system was favoured due to its ability to remove nitrogen with higher efficiency. Apart from the topology information, the tool also enables the user to track the concentration of the pollutants throughout the treatment line. Moreover, cost breakdown information can also be obtained in the final report.

\section{CONCLUSIONS}

A mathematical programming concept has been introduced in this study to support the early stage decisions on WWTP network selection. By casting the problem as an optimization problem, the decision on which technology to employ is rendered on quantitative metrics which complements the experience based approach used today. Hence the tool is expected to support and facilitate generation and evaluation of ideas for identifying optimal solutions to design new or retrofit existing WWTPs. We also hope that this contribution will open the debate on how we identify novel processes and technologies for WWTPs (be it treatment or resource recovery as the final purpose). This activity is arguably more an art than a science the way it is carried out today.

\section{REFERENCES}


Bozkurt H., Quaglia A., Gernaey K.V. and Sin G. (2013). Early stage design of wastewater treatment plants: A mathematical programming approach. Environmental Modelling \& Software (submitted).

Bozkurt H., Quaglia A., Gernaey K.V. and Sin G. (2014). Superstructure development and optimization under uncertainty for design and retrofit of municipal wastewater treatment plants. ESCAPE24 conference (submitted).

Daigger G.T. (2005). Wastewater treatment plant of the future:Decision analysis approach for increased sustainability. Presented at the Water Security:Policies \& Investments Water Week 2005 Making Decisions on Water Quality. Washington DC.

Hamouda M.A., Anderson W.B. and Huck P.M. (2009). Decision support system in water and wastewater treatment process selection and design: a review. Water Science and Technology, 60 (7), 1757-1770.

Henze M., van Loosdrecht M.C.M., Ekama G. and Brdjanovic D. (2008). Biological Wastewater Treatment Principles, Modelling and Design. IWA Publishing, Glasgow.

Quaglia A., Sarup B., Sin G., Gani R. (2012). Integrated business and engineering framework for synthesis and design of enterprise-wide processing networks. Computers \& Chemical Engineering, 38, 213-223.

Tchobanoglous G., Burton F. L. and Stensel H. D. (2003). Wastewater engineering: Treatment and Reuse. McGraw-Hill Publishing, New York.

WEF and ASCE/EWRI (2010). Design of Municipal Wastewater Treatment Plants. WEF Press, Virginia. 


\title{
Empirical vs. knowledge-based modelling of filtration in submerged anaerobic MBRs (SAnMBRs)
}

\author{
Ángel Robles ${ }^{1}$, Gergö Zajzon ${ }^{2}$, Laura Jurecska ${ }^{3}$, María Victoria Ruano ${ }^{1, *}$, Josep Ribes ${ }^{1}$, Aurora Seco ${ }^{1}$ \\ and José Ferrer ${ }^{4}$

\begin{abstract}
${ }^{1}$ Departament d'Enginyeria Química, Escola Tècnica Superior d'Enginyeria, Universitat de València, josep.ribes; aurora.seco]@uv.es)

${ }^{2}$ Department of Sanitary and Environmental Engineering, Budapest University of Technology and Economics, 1111, Müegyetem rkp. 3, Budapest, Hungary (Email: zajzon.gergo@vkkt.bme.hu)

${ }^{3}$ Department of Microbiology, Eötvös Loránd University, 1117, Pázmány Pèter sètány 1/C, Budapest, Hungary (Email: jurecskalaura@gmail.com)

${ }^{4}$ Institut Universitari d'Investigació d'Enginyeria de l'Aigua i Medi Ambient, IIAMA, Universitat Politècnica de València, Camí de Vera s/n, 46022 Valencia, Spain (Email: jferrer@hma.upv.es).
\end{abstract} \\ Avinguda de la Universitat s/n, 46100 Burjassot, Valencia, Spain (Email: [angel.robles; m.victoria.ruano;
}

\begin{abstract}
The main objective of this study was to compare the capability of empirical and knowledge-based theory to model the filtration process in submerged anaerobic MBRs (SAnMBRs). To this aim, the following three models were developed and validated using data obtained from a SAnMBR system fitted with industrial-scale hollow-fibre membranes: (1) an empirical model; (2) a neural-network based model; and (3) a fuzzy-logic based model. The proposed models represented adequately the filtration process in SAnMBRs, resulting all in a Pearson Product-Moment correlation coefficient $(r)$ above 0.9.
\end{abstract}

\section{Keywords}

Industrial-scale hollow-fibre membranes; knowledge-based modelling; submerged anaerobic MBR

\section{INTRODUCTION}

Further studies are needed in submerged anaerobic MBR (SAnMBR) technology in order to gain more insight into the optimisation of their efficiency, mainly regarding membrane fouling phenomenon. In this respect, mathematical modelling of filtration in SAnMBR technology may help in gaining insights about the aspects that play a key role in membrane fouling (Mannina et al., 2011), and are valuable for the design, prediction, and control of this technology ( $\mathrm{Ng}$ and $\mathrm{Kim}, 2007)$. Models focused on filtration in MBR technology usually rely on parameters that are not measurable, neither on-line, nor with standard laboratory equipment (e.g. soluble microbial products). Moreover, some of them are not able to reproduce the effect of the different stages that form the operating mode of the membrane modules (relaxation, back-flushing...), or cannot be easily coupled to a given biological model. In this respect, some authors (see e.g. Sarioglu et al., 2012) currently tend to develop new simple empirical models that try to reproduce the effect of the most critical variables taking place in the membrane fouling phenomenon: mixed liquor total solids (MLTS) concentration and shear intensity in the membrane tank. However, due to the strongly nonlinear relationships existing between the process inputs and outputs, empirical models could only result in proper results when the process dynamics are bounded by a defined linear zone. In this respect, knowledge-based theory (e.g. neural networks or fuzzy logic) may represent a

Current address: aqualia, gestión integral del agua, S.A., Avenida del Camino de Santiago, 40, 28050 Madrid, Spain (Email: mavictoria.ruano.garcia@fcc.es) 
powerful tool for modelling filtration in SAnMBRs since they allow applying the valuable expert knowledge and allows developing multiple-input-multiple-output process schemes.

The main objective of this study was to compare the capability of empirical and knowledgebased theory to model the filtration process in SAnMBR systems not only in the short term but also in the long term. Since the effect of the main operating conditions on membrane fouling cannot be properly evaluated at the lab scale because they depend heavily on the membrane size (particularly in hollow-fibre (HF) membranes the HF length is a critical parameter), the proposed models were validated in a SAnMBR system fitted with industrialscale HF membrane units. This semi-industrial SAnMBR plant was operated using real wastewater from the pre-treatment of the Carraixet WWTP (Valencia, Spain). Thus, the main disturbances that take place in full-scale plants were reproduced.

\section{MATERIAL AND METHODS}

\section{Semi-industrial SAnMBR plant description}

This study was carried out in a semi-industrial SAnMBR plant fed with the effluent of a fullscale WWTP pre-treatment. It consists of an anaerobic reactor with a total volume of $1.3 \mathrm{~m}^{3}$ connected to two membrane tanks each one with a total volume of $0.8 \mathrm{~m}^{3}$. Each membrane tank includes one ultrafiltration HF membrane commercial system $\left(\right.$ PURON $^{\circledR}$, Koch Membrane Systems, $0.05 \mu \mathrm{m}$ pore size, $30 \mathrm{~m}^{2}$ total filtering area, and outside-in filtration). The membrane system was operated according to a specific schedule involving a combination of different individual stages (back-fluxing, ventilation and degasification) taken from a basic filtration-relaxation cycle. Further details on this SAnMBR system can be found in Robles et al. (2013a).

\section{Filtration process modelling}

\section{Empirical modelling}

The proposed empirical filtration model (resistance-in-series based model, Robles et al., 2013b) considers the following four kinetically governed physical processes: (1) cake layer formation during filtration due to solids deposition; (2) cake layer removal due to membrane scouring by gas sparging; (3) cake layer removal during back-flushing; and (4) irreversible fouling consolidation.

\section{Knowledge-based modelling}

In this study, the following two knowledge-based theories are proposed for modelling filtration in SAnMBRs: (1) neural networks; and (2) fuzzy logic (data not shown). The proposed knowledge-based models calculate the change in the transmembrane pressure (TMP) over time on the basis of the following inputs: $20^{\circ} \mathrm{C}$-standardised transmembrane flux $\left(\mathrm{J}_{20}\right)$, MLTS, specific gas demand per square metre of membrane area $\left(\mathrm{SGD}_{\mathrm{m}}\right)$, and total volume of treated water $\left(V_{T}\right)$.

\section{RESULTS AND DISCUSSION}

Figure 1 provides an example of the results obtained from the short-term validation of both the empirical and neural-network based model proposed in this study. This validation was carried out using experimental data obtained by applying different $\mathrm{J}_{20}$ and $\mathrm{SGD}_{\mathrm{m}}$ values. The results shown in Figure 1a (corresponding to the empirical model) were obtained when operating with a MLTS concentration of $21 \mathrm{~g} \mathrm{~L}^{-1}, \mathrm{SGD}_{\mathrm{m}}$ from approx. 0.13 to $0.4 \mathrm{~m}^{3} \mathrm{~h}^{-1} \mathrm{~m}^{-2}$, and gross $\mathbf{J}_{20}$ from approx. 4 to $12 \mathrm{LMH}$. The results shown in Figure $1 \mathrm{~b}$ (corresponding to the neural-network based model) were obtained when operating with a MLTS concentration 
of $17 \mathrm{~g} \mathrm{~L}^{-1}, \mathrm{SGD}_{\mathrm{m}}$ from approx. 0.17 to $0.3 \mathrm{~m}^{3} \mathrm{~h}^{-1} \mathrm{~m}^{-2}$, and gross $\mathrm{J}_{20}$ from approx. 12 to 26 LMH.

As Figure 1a shows, the results indicate that the empirical model predictions (TMP $\left.{ }_{\text {SIM }}\right)$ accurately reproduce the experimental data $\left(\mathrm{TMP}_{\mathrm{EXP}}\right)$ : an adequate Pearson Product-Moment correlation coefficient $(r)$ of 0.925 was obtained. On the other hand, Figure 1a shows that the empirical model is capable of reproducing the reduction in TMP caused by ventilation (V) or back-flushing (BF) (see, for example, minutes 285 and 615, respectively). Results of the neural-network based model are shown in Figure 1b. It can be seen that the neural network is also capable to reproduce the change in TMP over time when the flux changes. Also in this case, the correlation between experimental data (dTMPexp/dt) and model predictions (dTMPsim/dt) was characterised by a high $r$ value $(r=0.989)$.

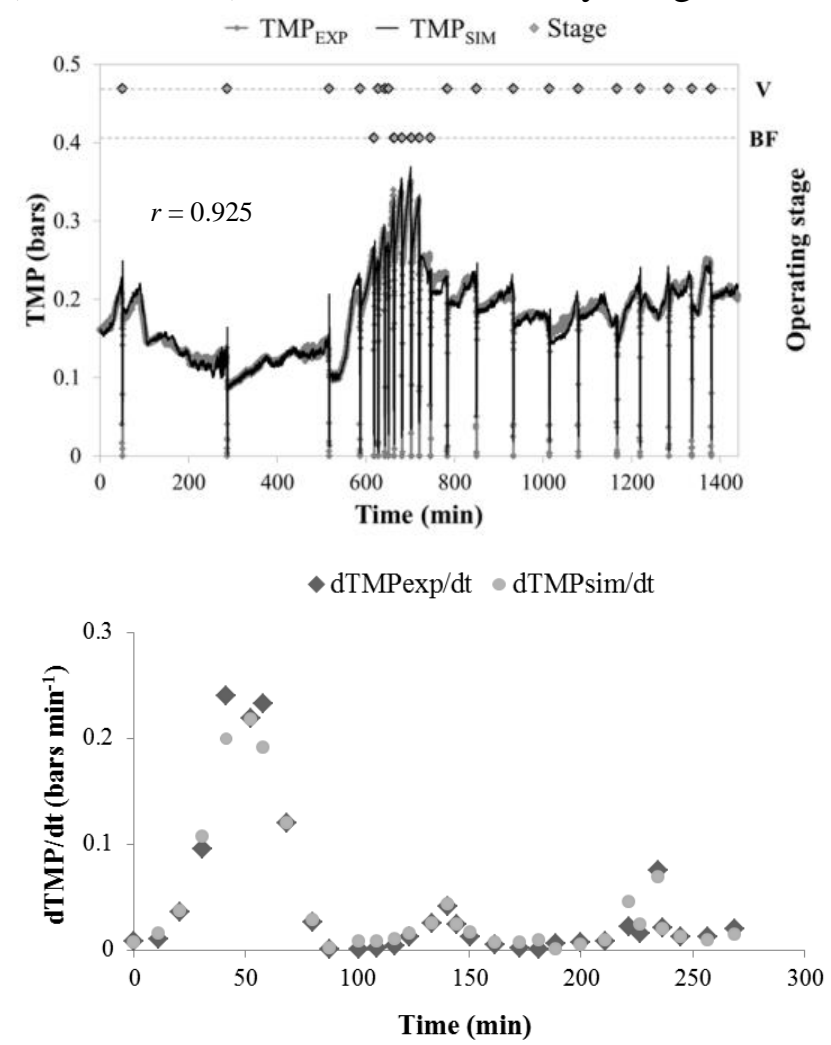

(a)

(b)

Figure 1. Example of the results obtained from the short-term validation of (a) the empirical model and (b) the neural-network based model proposed in this study.

Once the models were calibrated, their generalising capability was evaluated by simulating different operating periods. The performance of the models was evaluated and compared each other.

\section{CONCLUSIONS}

Three models aimed to represent filtration in SAnMBRs were developed and validated: (1) an empirical model; (2) a neural-network based model; and (3) a fuzzy-logic based model. All these models were capable to adequately reproduce the filtration process in SAnMBR technology, resulting in adequate Pearson correlation coefficients (above 0.9).

\section{ACKNOWLEDGEMENT}


This research work was possible thanks to project CTM2011-28595-C02-01/02 (funded by the Spanish Ministry of Economy and Competitiveness jointly with the European Regional Development Fund) and EIT Climate KIC - Pioneers into Practice Programme 2013, which are gratefully acknowledged.

\section{REFERENCES}

Mannina G., Di Bella G. and Viviani G. (2011). An integrated model for biological and physical process simulation in membrane bioreactors (MBRs). J. Membrane Sci. 376, 56-69.

$\mathrm{Ng}$ A.N.L. and Kim A.S. (2007). A mini-review of modeling studies on membrane bioreactor (MBR) treatment for municipal wastewaters. Desalination 212, 261-281.

Robles A., Ruano M.V., Ribes J. and Ferrer J. (2013a). Factors that affect the permeability of commercial hollow-fibre membranes in a submerged anaerobic MBR (HF-SAnMBR) system. Water Res. 47, 1277-1288.

Robles, A., Ruano, M.V., Ribes, J., Seco, A. and Ferrer, J. (2013b) A filtration model applied to submerged anaerobic MBRs (SAnMBRs). J. Membrane Sci. 444, 139-147.

Sarioglu M., Insel G. and Orhon D. (2012). Dynamic in-series resistance modeling and analysis of a submerged membrane bioreactor using a novel filtration mode. Desalination 285, 285-294. 


\title{
Removal of pharmaceuticals in biological wastewater treatment systems: model generalisation and implications for environmental risk predictions
}

\author{
Fabio Polesel ${ }^{1}$, Katherine H. Langford ${ }^{2}$, Stefan Trapp ${ }^{1}$, Kevin V. Thomas ${ }^{2}$, Benedek Gy. Plósz ${ }^{1}$ \\ ${ }^{1}$ Department of Environmental Engineering, Technical University of Denmark, Bygningstorvet, DK-2800 \\ Kgs. Lyngby, Denmark, (Email: fabp@env.dtu.dk; sttr@env.dtu.dk; beep@env.dtu.dk) \\ ${ }^{2}$ Norwegian Institute for Water Research, NIVA, Gaustadalléen 21, NO-0349, Oslo, Norway (Email: \\ katherine.langford@niva.no ; kevin.thomas@niva.no)
}

\begin{abstract}
An Activated Sludge Modelling framework for Xenobiotics (ASM-X) was recently developed to mechanistically predict the fate of pharmaceuticals in a full-scale treatment plant. In this study, we generalized ASM-X to international literature data. Through the generalization, we assessed the influence on the biological removal efficiency of specific factors, namely influent loading dynamics, SRT and retransformation processes (from e.g., human metabolites back to parent chemicals). With regard to the latter, we show that the estimation of removal efficiency based only on parent chemical (a predominant practice in literature) can lead to an underestimation of the environmental risk.
\end{abstract}

Keywords

Pharmaceuticals elimination; ASM-X; model validation; retransformation; hospital WWTP

\section{INTRODUCTION}

Dynamic fate models can represent a cost-saving option to investigate the elimination of xenobiotic trace chemicals in biological wastewater treatment plants (WWTPs). An Activated Sludge Modelling framework for Xenobiotics (ASM-X) was developed and validated in the fate prediction of pharmaceuticals in a full-scale WWTP (Plósz et al., 2010, 2012). These studies highlighted the potential impact of human conjugated metabolites or other commercial chemicals to retransform back to parent forms, leading to a distinction between the concentration of parent $\left(\mathrm{C}_{\mathrm{LI}}\right)$ and retransformable fractions $\left(\mathrm{C}_{\mathrm{CJ}}\right)$ of pharmaceuticals. In this study, we validated ASM-X by comparing predicted removal efficiencies of three pharmaceuticals (sulfamethoxazole, ciprofloxacin and tetracycline) in Bekkelaget WWTP (Oslo, Norway), with published international data whereby sound sampling techniques were used. The validation with literature data, also referred to as generalization (Plósz et al., 2012), aimed at: (i) assessing the underestimation of removal by considering only the parent fraction; (ii) estimating the significance of this underestimation in terms of risk predictions; and (iii) evaluating factors known to affect pharmaceuticals removal. With regard to (iii), we focused on dynamics of influent load of the substances, WWTP operation (e.g., solid retention timeSRT) and retransformation occurring in upstream sewer systems.

\section{MATERIALS AND METHODS}

The full-scale implementation of ASM-X in WEST 2012®, calibrated with the results of batch experiments as presented by Plósz et al. (2010), was used to estimate the elimination of sulfamethoxazole, ciprofloxacin and tetracycline in Bekkelaget WWTP. We distinguished between removal efficiency [-], in the biological treatment, referred to parent fraction (Eq.1) and to both parent and retransformable fraction (Eq. 2)

$$
\begin{aligned}
& \eta_{L I}=\left(C_{L I, \text { in }}-C_{L I, \text { eff }}\right) / C_{L I, \text { in }} \\
& \eta_{T O T}=\left(C_{L I, \text { in }}+C_{C J, \text { in }}-C_{L I, \text { eff }}-C_{C J, \text { eff }}\right) /\left(C_{L I, \text { in }}+C_{C J \text {,in }}\right)
\end{aligned}
$$


where $\mathrm{C}_{\text {in }}$ and $\mathrm{C}_{\text {eff }}\left[\mathrm{ng} \mathrm{\textrm {L } ^ { - 1 }}\right.$ ] were concentrations in secondary influent and effluent, respectively. Estimated removal efficiencies were plotted as a function of the normalized influent load of the chemicals $\left[\mathrm{mg} \mathrm{h}^{-1} 1000 \mathrm{PE}^{-1}\right]$, calculated from the design population equivalent of the WWTP. A literature review was performed for the generalization of ASM-X predictions, with the collection of international data on the full-scale removal of pharmaceuticals. Only data derived from flow-proportional sampling campaigns were included. Additionally, literature studies on the separate treatment of hospital wastewater were selected to characterize a "zero-catchment" scenario, describing the removal of pharmaceuticals in WWTPs with negligible upstream sewer transport.

A preliminary environmental risk assessment of the pharmaceuticals was performed. Predicted environmental concentrations (PECs) were estimated from the effluent concentrations from Bekkelaget WWTP (assuming 10-fold dilution). We distinguished between PECs accounting for only effluent $C_{\mathrm{LI}}$, and for both effluent $\mathrm{C}_{\mathrm{LI}}$ and $\mathrm{C}_{\mathrm{CJ}}$. PECs were then compared to predicted non-effect concentrations (PNECs) reported in literature to assess risk dynamics.

\section{RESULTS AND DISCUSSION (the case of sulfamethoxazole)}

Scenario simulations, considering increased influent loads compared to measurements in Bekkelaget WWTP, were used to generalise ASM-X predictions of sulfamethoxazole removal with literature data. In Fig. 1a, we compared model predictions (5-fold increased influent loading) with removal efficiencies of sulfamethoxazole in municipal WWTPs. Predicted $\eta_{\text {LI }}$ and $\eta_{\text {тот }}$ were consistent with data reported by Göbel et al. (2005, 2007), including a comparable underestimation of the efficiency when the retransformable metabolite (N4-acetyl sulfamethoxazole) was not considered. A similar underestimation error was shown by Yang et al. (2011). Values of $\eta_{\text {LI }}$ reported by Radjenovic et al. (2009) were significantly higher than our estimations and any other literature data at comparable influent loads, suggesting a possible enhancement of biotransformation (due to e.g., operation at high SRT). With regard to the zero-catchment scenario (Fig. 1b), predicted $\eta_{\mathrm{LI}}$ and $\eta_{\text {TOT }}$ at a 25 -fold increased load are in close agreement with values reported by Kovalova et al. (2012).

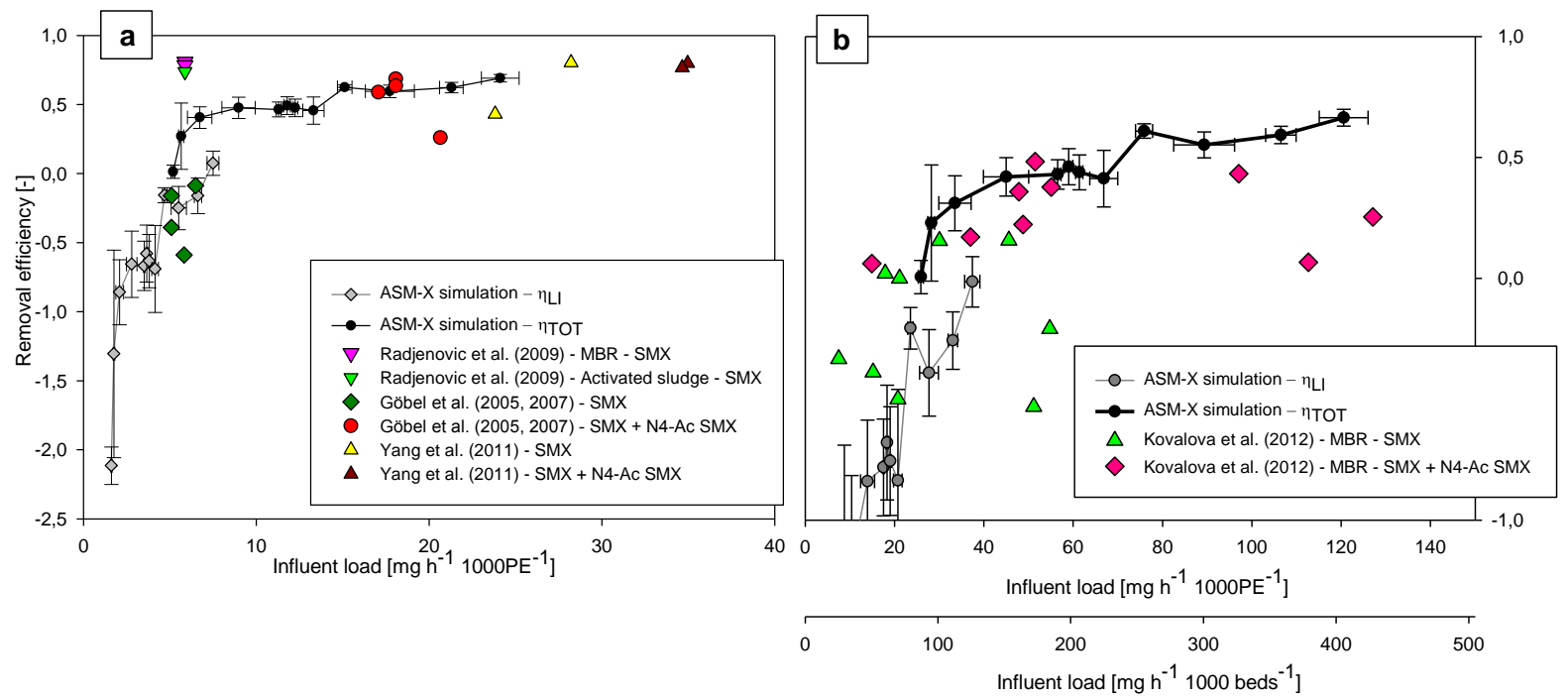

Figure 1. Generalization of ASM-X predicted removal of sulfamethoxazole (SMX) with literature data for municipal WWTPs (a) and for the hospital wastewater treatment-zero-catchment scenario (b). ASM-X predictions of removal efficiency accounted for only parent SMX (grey diamonds) and for parent and retransformable fractions of SMX (black circles). 5-fold and a 25-fold increased influent load, as compared to Plósz et al. (2010), were used for the validation in (a) and (b), respectively. Error bars refer to standard deviations in influent loads and removal efficiencies. 
In Fig. 2, values of the PECs in the recipient water body of the Bekkelaget WWTP were shown. Results obtained in this preliminary assessment suggest that tetracycline (Fig. 2a) and-significantly - ciprofloxacin (Fig. 2b) can represent a considerable chemical risk. The predicted effluent $\mathrm{C}_{\mathrm{CJ}}$ of tetracycline can pose a substantial additional risk (up to $130 \%$ increase as compared to parent-based PEC), whereas the parent-based tetracycline results suggested a marginal violation of the no-effect limit. Estimated as 131-397 times higher than the respective PNEC value, PECs of ciprofloxacin exhibited a marked temporal variability (3fold increase at the peaks).
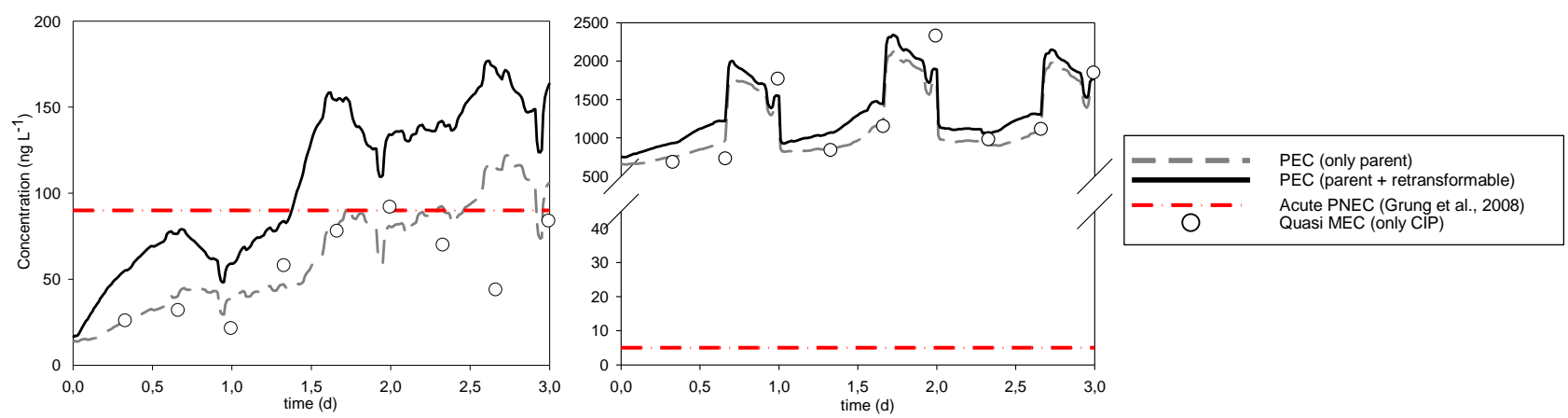

Figure 2. PEC values of tetracycline (a) and ciprofloxacin (b) calculated from ASM-X predictions. For PNEC values used, please refer to Grung et al. (2008). Quasi MECs (measured environmental concentrations) identify effluent measured environmental concentrations divided by a dilution factor.

Overall, these results suggest the importance of using dynamic models for and the necessity of considering retransformable chemical fractions in assessing pharmaceuticals removal in biological WWTPs. Additionally, we show that environmental risk assessments should account for (i) concentrations of retransformable chemicals released in WWTP effluents, potentially representing an additional source of hazard; and (ii) temporal variations in effluent concentrations.

\section{ACKNOWLEDGEMENT}

The authors express special thanks to WWTP operators, in particular Helle Frodahl, and to Xin Yang and Lubomira Kovalova for providing additional details on their study.

\section{REFERENCES}

Göbel, A., Thomsen, A., McArdell, C.S., Joss, A., Giger, W. (2005). Occurrence and sorption behaviour of sulphonamides, macrolides, and trimethoprim in activated sludge treatment. Environ. Sci Technol., 39(11), 3981-3989.

Göbel, A., McArdell, C.S., Joss, A., Siegrist, H., Giger, W. (2007). Fate of sulphonamides, macrolides, and trimethoprim in different wastewater technologies. Sci. Total Environ., 372(2-3), 361-371.

Grung M., Källqvist T., Sakshaug S., Skurtveit S., Thomas K.V. (2008). Environmental assessment of Norwegian priority pharmaceuticals based on the EMEA guideline. Ecotox. Environ. Safe., 71(2), 328340.

Kovalova, L., Siegrist, H., Singer, H., Wittmer, A., McArdell, C.S. (2012). Hospital wastewater treatment by membrane bioreactor: performance and efficiency for organic micropollutant elimination. Environ. Sci. Technol., 46(3), 1536-1545.

Plósz B.G., Leknes, H., Thomas, K.V. (2010). Impacts of competitive inhibition, parent compound formation and partitioning behaviour on antibiotic micro-pollutants removal in activated sludge. Environ. Sci. Technol., 44(2), 734-742.

Plósz, B.G., Langford, K.H., Thomas, K.V. (2012). An activated sludge model for trace xenobiotic chemicals (ASM-X): Assessment of diclofenac and carbamazepine. Biotechnol. Bioeng., 109(11), 2757-2769.

Radjenovic, J., Petrovic, M., Barceló, D. (2009). Fate and distribution of pharmaceuticals in wastewater and sewage sludge of the conventional activated sludge (CAS) and advanced membrane bioreactor (MBR) treatment. Water Res., 43(3), 831-841. 


\section{WWTmod}

Yang, X., Flowers, R., Weinberg., H.S., Singer, P.C. (2011). Occurrence and removal of pharmaceuticals and personal care products (PPCPs) in an advanced wastewater reclamation plant. Water Res., 45(16), 52185228. 


\title{
Modelling aerobic methane oxidation in a municipal WWTP
}

\author{
Matthijs R.J. Daelman ${ }^{1,2, *}$, Tamara Van Eynde ${ }^{2}$, Mark C.M. van Loosdrecht ${ }^{1,3}$ and Eveline I.P. \\ Volcke $^{2}$ \\ ${ }^{1}$ Department of Biotechnology, Delft University of Technology, Julianalaan 67, 2628BC Delft, \\ Netherlands \\ ${ }^{2}$ Department of Biosystems Engineering, Ghent University, Coupure links 653, 9000 Gent, Belgium \\ ${ }^{3}$ KWR Watercycle Research Institute, P.O. Box 1072, 3433PE Nieuwegein, Netherlands \\ *Corresponding author: m.r.j.daelman@tudelft.nl, +31152789175.
}

\begin{abstract}
Methane is an potent greenhouse gas and its emission from municipal wastewater treatment plants should be prevented. One way to do this, would be to promote the biological conversion of methane over stripping in the aeration tanks. In this study we extended Activated Sludge Model $\mathrm{n}^{\circ} 1$ with biological methane oxidation to verify the effect of aeration rate, solids retention time and influent methane concentration on the balance between conversion and stripping. This knowledge helps to stimulate the methane oxidizing capacity of activated sludge to abate methane emissions to the atmosphere.
\end{abstract}

\section{Keywords}

Activated sludge, ASM, climate footprint, greenhouse gases, methane

\section{INTRODUCTION}

Municipal wastewater treatment of gives rise to the emission of the greenhouse gases carbon dioxide, nitrous oxide and methane. Methane is a potent greenhouse gas with a global warming potential of $25 \mathrm{CO}_{2}$-equivalents (IPCC, 2007). In an long-term study on a municipal wastewater treatment plant (WWTP) methane was found to make up $13.5 \%$ of the plants greenhouse gas footprint, exceeding the amount of emitted carbon dioxide related to the plant's electricity and natural gas consumption (Daelman et al., 2013). Globally, sewage treatment accounts for $4 \%$ of the total methane emission (Conrad, 2009).

Basically, the methane that is emitted from a WWTP can be traced back to two sources. Either it is stripped from the incoming wastewater after it has been produced in the sewer (Guisasola et al., 2008), or it is produced during the storage and manipulation of sludge (Daelman et al., 2012). Dissolved methane can be biologically converted, besides being stripped. It was recently discovered that about $80 \%$ of the dissolved methane entering an aerated activated sludge tank was converted with the remainder being stripped (Daelman et al., 2012). Aerobic conversion of methane is performed by methanotrophic bacteria (Ho et al., 2013). Harnessing this methane oxidizing capacity of activated sludge could be a way to avoid the emission of methane. In the end, this could lead to more sustainable wastewater treatment.

The objective of this study was to investigate the effect of a number of operational process conditions on the fate of dissolved methane in an activated sludge plant. To this end, the Activated Sludge Model $n^{\circ} 1$ (ASM1) was extended with aerobic methanotrophic growth. The resulting model, called ASM1m, was implemented in Benchmark Simulation Model $\mathrm{n}^{\circ} 1$ (BSM1) (Copp, 2001) and termed BSM1m Taking into account biological oxidation and stripping of methane, BSM1m is the first model describing dynamic emissions of methane 
during wastewater treatment. As such, it complements existing models for the emission of nitrous oxide in estimating greenhouse gas emissions from WWTPs.

\section{MATERIALS AND METHODS}

ASM1m adds two processes to ASM1: aerobic growth and decay of methanotrophs. The two additional state variables are methane as substrate $\left(\mathrm{S}_{\mathrm{CH} 4}\right)$ and methane oxidizing bacteria $\left(\mathrm{X}_{\mathrm{MOB}}\right)$.

To describe the behaviour of the overall WWTP, ASM1m was implemented in BSM1m, which consists of two anoxic tanks followed by three aerated tanks and a settler and a secondary settling tank (Copp, 2001). BSM1m was used to investigate the effect of the following three operating variables on methane conversion rate and methane stripping: aeration rate (in reactor 3 and 4), influent methane concentration and solids retention time. Table 1 gives an overview of the relevant parameters as well as their default values and the range over which they were varied. Besides the percentage of incoming methane that is stripped, also the conventional BSM1 criteria Effluent Quality (EQ) and Operational Cost Index (OCI) were calculated.

Table 1. Overview of the operating conditions applied in the scenario analysis.

\begin{tabular}{lccc}
\hline Operating condition & Parameter & Default value & Range \\
\hline Aeration rate in reactor 3 and 4 & $k_{L} a_{O_{2}}$ & $240 \mathrm{~d}^{-1}$ & $0-400 \mathrm{~d}^{-1}$ \\
Influent methane concentration & $S_{C H_{4} \text { infl }}$ & $10{\mathrm{~g} \mathrm{COD} \cdot \mathrm{m}^{-3}\left(^{* *}\right)}^{0-50 \mathrm{~g} \mathrm{COD} \cdot \mathrm{m}^{-3}}$ \\
Solids retention time & $\mathrm{SRT}$ & $9.18 \mathrm{~d}^{(* *)}$ & $2-12 \mathrm{~d}$ \\
\hline${ }^{(*)}$ Daelman et al. $(2012)$ & & & \\
${ }^{(*)}$ Calculated from solids balance & & &
\end{tabular}

\section{RESULTS AND DISCUSSION}

Figure shows the influence of the operating conditions on the percentage of incoming methane that is emitted and on the plant's performance in terms of effluent quality and operational costs.

At very low aeration rates $\left(\mathrm{k}_{\mathrm{L}} \mathrm{a}<52 \mathrm{~d}^{-1}\right)$, methanotrophs are outcompeted by ordinary heterotrophs (data not shown), while methane stripping increases with increasing $\mathrm{k}_{\mathrm{L}} \mathrm{a}$, resulting in higher methane emissions. At higher, but still low aeration rates $\left(\mathrm{k}_{\mathrm{L}} \mathrm{a}=52-126 \mathrm{~d}^{-}\right.$ $\left.{ }^{1}\right)$ the increasing methanotrophic growth is reflected in decreasing methane emissions. Beyond the optimum of $126 \mathrm{~d}^{-1}$, stripping takes over from methanotrophic growth and the methane emission increases again. The optimal aeration rate to curb the emission does not coincide with the best effluent quality (lowest EQ) or the lowest operating costs (lowest OCI).

At low influent methane concentrations, most of the methane is converted in the anoxic tank using the oxygen entering with the recycle sludge. As the influent concentration increases, more methane passes through the anoxic tanks to the aeration tanks where it is stripped. Upon a further increase in the influent methane concentration, the conversion in the aeration tanks takes over from the stripping. The impact on the plant's effluent quality and operational cost is negligible. 
The percentage of incoming methane that is emitted decreases with increasing SRT, at least for SRT-values lower than $6 \mathrm{~d}$. When the SRT increases beyond $6 \mathrm{~d}$, the emission first increases and then decreases gradually. This is explained by the growth of methanotrophic biomass (data not shown). For SRT-values increasing up to 6 days, the amount of methanotrophic biomass increases, which is reflected in a decrease of the methane emissions. From an SRT of $6 \mathrm{~d}$ onwards, the biomass increase slows down. The optimal SRT to minimize the emission does not coincide with the best effluent quality (lowest EQ) or the lowest operating costs (lowest OCI).
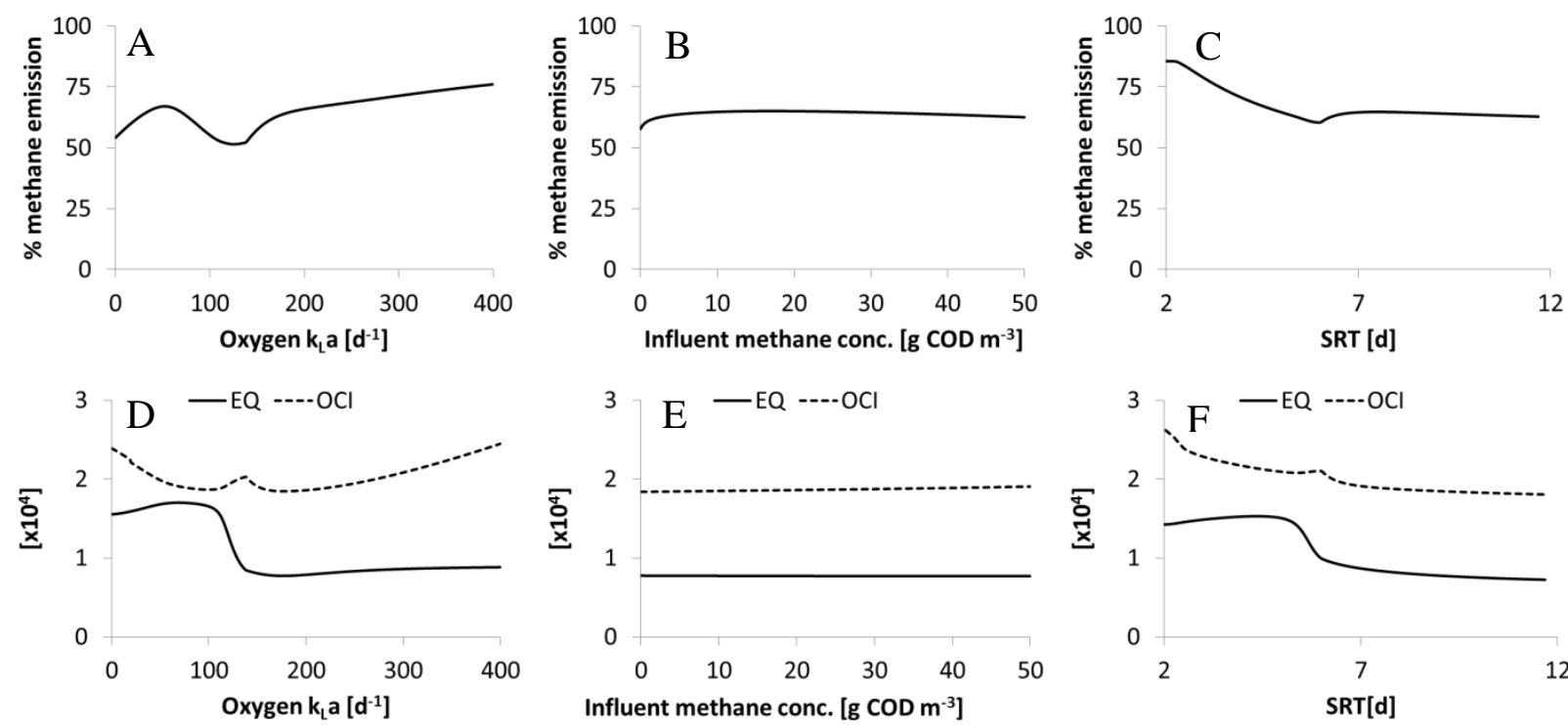

Figure 1. Top: emission of methane as percentage of incoming methane vs. aeration rate (A), influent methane concentration (B) and SRT (C). Bottom: Effluent Quality and Operational Cost Index (for both: the lower the better) vs. aeration rate (D), influent methane concentration (E) and SRT (F).

\section{CONCLUSIONS AND PERSPECTIVES}

- Aerobic methane oxidation during biological wastewater treatment was modelled (ASM1m) and its interaction with methane stripping was assessed in a simulation study for a municipal WWTP.

- Aeration rate, influent methane concentration and solids retention time affect methane emission and could therefore be used for mitigating methane emissions from WWTPs.

- The model will be used to elucidate how the methane emission relates to the plant performance in terms of effluent quality and operational cost, resulting in a multi-criteria analysis.

\section{ACKNOWLEDGEMENTS}

Matthijs Daelman received a joint $\mathrm{PhD}$ grant from Ghent University's Special Research Fund (no. 01SF0510). Ulf Jeppsson is kindly acknowledged for providing the MATLAB code of BSM1.

\section{REFERENCES}

Conrad, R. (2009) The global methane cycle: recent advances in understanding the microbial processes involved. Environmental Microbiology Reports 1(5), 285-292. 
Copp, J.B. (2001) The COST simulation benchmark: description and simulator manual, Office for Official Publications of the European Communities, Luxembourg.

Daelman, M.R.J., van Voorthuizen, E.M., van Dongen, U.G.J.M., Volcke, E.I.P. and van Loosdrecht, M.C.M. (2012) Methane emission during municipal wastewater treatment. Water Research 46(11), 3657-3670.

Daelman, M.R.J., van Voorthuizen, E.M., van Dongen, L.G.J.M., Volcke, E.I.P. and van Loosdrecht, M. (2013) Methane and nitrous oxide emissions from municipal wastewater treatment - results from a long-term study. Water Science and Technology 67(10), 2350-2355.

Guisasola, A., de Haas, D., Keller, J. and Yuan, Z. (2008) Methane formation in sewer systems. Water Research 42(6-7), 1421-1430.

Ho, A., Vlaeminck, S.E., Ettwig, K.F., Schneider, B., Frenzel, P. and Boon, N. (2013) Revisiting Methanotrophic Communities in Sewage Treatment Plants. Applied and Environmental Microbiology 79(8), 2841-2846.

IPCC (2007) Contribution of working group I to the fourth assessment report of the Intergovernmental Panel on Climate Change. Solomon, S., Qin, D., Manning, M., Chen, Z., Marquis, M., Averyt, K.B., Tignor, M. and Miller , H.L. (eds), IPCC, Cambridge, United Kingdom and New York, NY, USA. 


\title{
Innovative Modelling in the Design of the Sacramento Regional Wastewater Treatment Plant for Biological Nutrient Removal
}

\author{
Patrick Dunlap ${ }^{1}$, Andrew Shaw ${ }^{1}$, James Barnard ${ }^{1}$, Heather Phillips ${ }^{2}$, Daniel Wilson ${ }^{3}$ and Ken \\ Abraham $^{3}$ \\ ${ }^{1}$ Black \& Veatch Corporation, 8400 Ward Parkway, Kansas City MO USA (Email: DunlapPJ@bv.com, \\ ShawAR@bv.com,BarnardJL@bv.com) \\ ${ }^{2}$ City of Olathe KS USA (Email: HMPhillips@olatheks.org) \\ ${ }^{3}$ EchoWater Project Management Office, Sacramento CA USA(Email: AbrahamK@sacsewer.com).
}

\section{Keywords}

Modelling, ASM simulators, uncertainty, good modelling practice; ammonia based control, dynamic aeration modeling, dynamic chemical feed control

\section{Background}

The Sacramento Regional County Sanitation District (SRCSD) in California, USA has embarked upon the EchoWater Project to upgrade the Sacramento Regional Wastewater Treatment Plant (SRWTP) to include biological nutrient removal (BNR) in order to meet new standards for discharge and reuse. The permit limits require SRWTP to meet instantaneous daily composite ammonia limit of 3.3/2.0 $\mathrm{mgN} / \mathrm{L}$ (summer/winter), a monthly average ammonia limit of $2.4 / 1.5 \mathrm{mgN} / \mathrm{L}$ (summer/winter), a monthly average nitrate limit of 10 $\mathrm{mgN} / \mathrm{L}$, and to mitigate the effluent total phosphorus to achieve an annual average target of $2.2 \mathrm{mgP} / \mathrm{L}$.

\section{Design Approach}

The process design of the new BNR facility made extensive use of process modelling including several innovative and distinct aspects which are described below. Raw wastewater flows, loads, and characteristics were determined using historical data and special samplings. This data was then used to produce a design case which was a single 6-month influent "itinerary" that included the maximum month, week, and day loadings for flow and COD/N/P loads, and associates the most adverse temperature conditions with these maximum loading periods. Additionally the influent itinerary includes periods which are more representative of typical conditions, and periods which represent minimum loading periods. Diurnal patterns derived from special samplings were applied to these flows and loads to produce an hourly dynamic itinerary. One of the lower loading periods takes place immediately before the maximum month period. The critical period for design is goverened by the ability of the biological process to respond to the extreme swings in loading when transitioning from the lowest load period to the maximum month. Examples of the influent itinerary for COD and TKN can be found in Figure and Figure below.

The inclusion of maximum month, week, and day loadings after a relatively low loading period and along with the high variability of influent flows and loads leads to a design case which is more realistic and stringent than typically used in design. Because of this approach engineering adjustments typically applied to address influent variability were not applied allowing for a more aggressive and economical design. Indeed other failure scenarios which are hard to quantify with design approaches that do not use dynamic models and therefore require the application of additional engineering adjustments can be more accurately estimated using this dynamic approach. 


\section{WWTmod}

Dunlap et al.

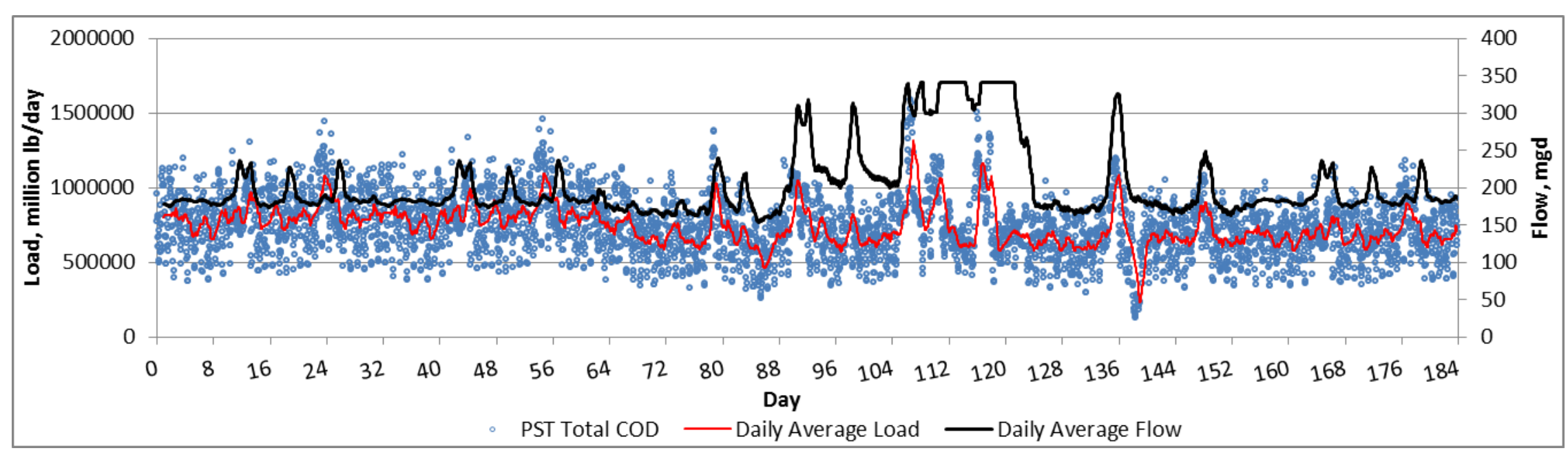

Figure 1 - Influent COD Load itinerary used in modelling $\left(1 \mathrm{lb}=0.45 \mathrm{~kg} \& 1 \mathrm{mgd}=3800 \mathrm{~m}^{3} / \mathrm{day}\right)$

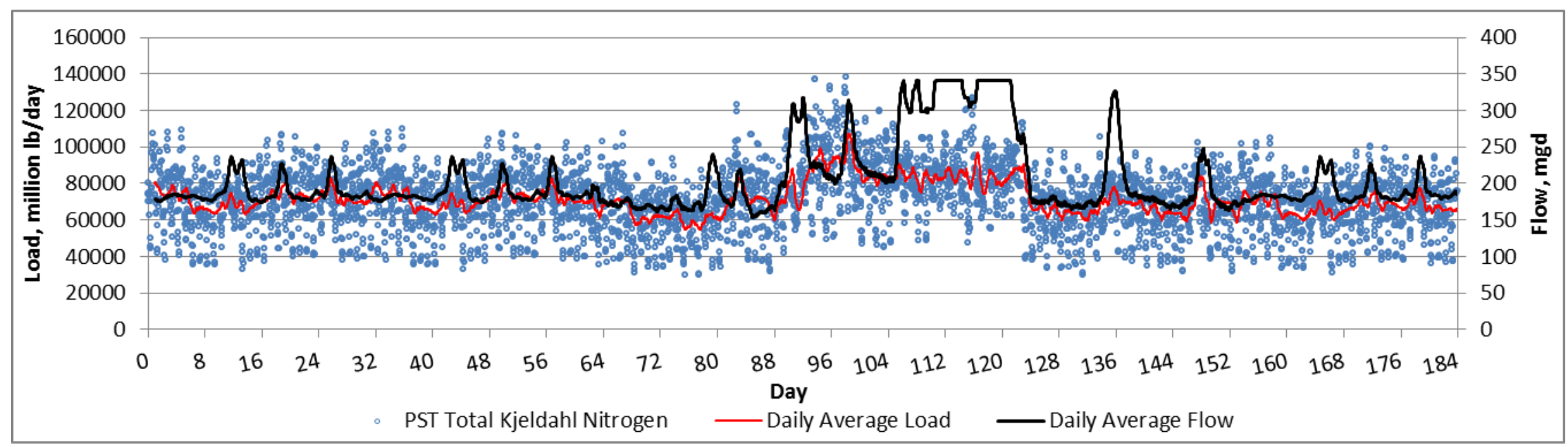

Figure 2 - Influent TKN Load itinerary used in modelling $\left(1 \mathrm{lb}=0.45 \mathrm{~kg} \& 1 \mathrm{mgd}=3800 \mathrm{~m}^{3} / \mathrm{day}\right)$ 


\section{Design SRT Selection}

Selecting an appropriate aerobic solids retention time (SRT) to meet the stringent ammonia requirement of the permit was a primary objective for the dynamic modelling. This was accomplished with a sensitivity analysis on the preliminary design performed only varying SRT in which the impact to peak effluent ammonia and peak solids loading rate (SLR) were monitored, the results of which can be seen in Figure below. Here the peak effluent ammonia crosses the line of the daily composite permit limit when operating below a 4 day SRT aerobic. A 6 day SRT was chosen as it is near the peak SLR for the preliminary design volume and provides a safety factor of about 2 days which allows for some operational problems and plant upsets; one of which will be discussed below. This is significantly below the aerobic SRT which was initially suggested of 7.5 days for a minimum daily average temperature of $16^{\circ} \mathrm{C}$. As the selected SRT and the limiting SLR sets the volume requirement of the basin; the aggressive selection of an aerobic SRT of 6.0 days was able to substantially reduce the required volume from what would be required with a 7.5 day aerobic SRT which a more traditional nitrifying SRT selection criteria would have required.

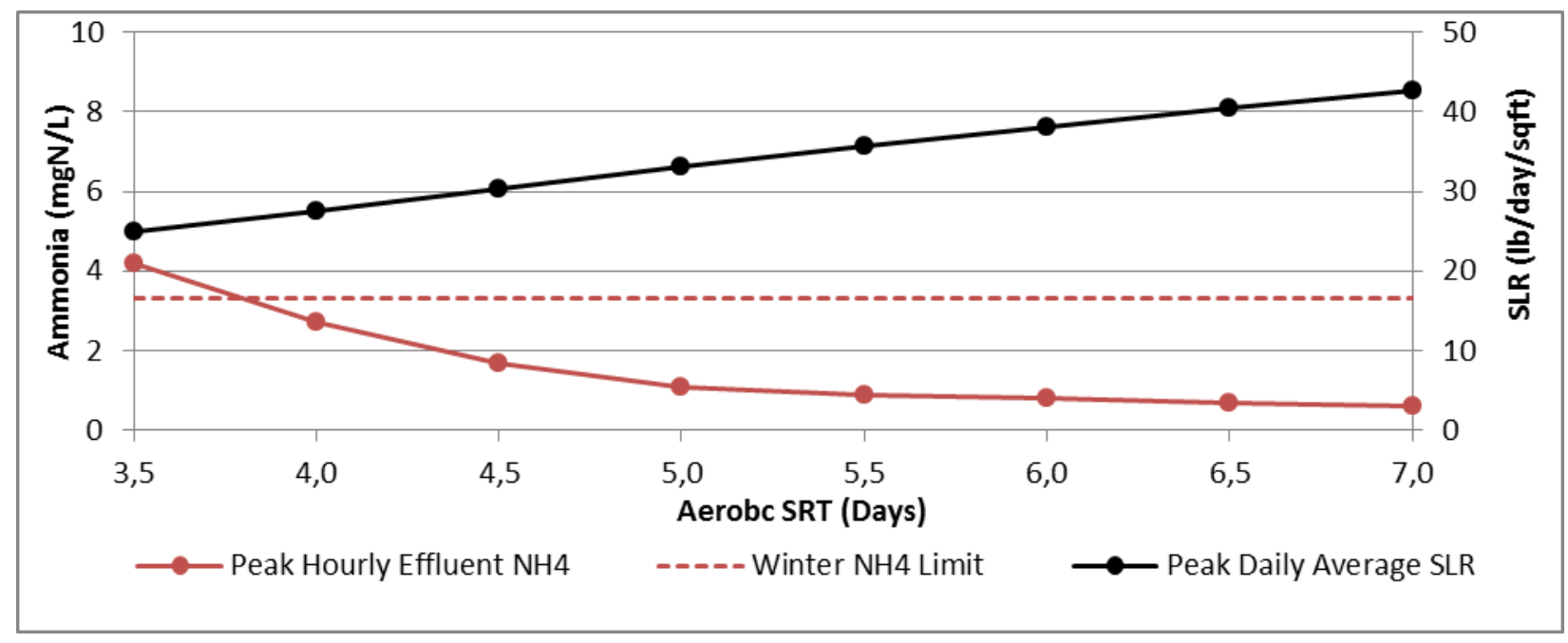

Figure 3 - Sensitivity of Effluent Ammonia and Solids Loading Rate to Aerobic SRT $\left(1 \mathrm{lb} / \mathrm{ft}^{2}=4.88\right.$ $\mathrm{kg} / \mathrm{m}^{2}$ )

\section{Ammonia Control Modelling}

Swing zones are included in the design to minimize the total volume and energy requirements for nitrification while achieving the maximum amount of nitrate removal; a concept which was modelled with the dynamic itinerary and included in the sensitivity analysis discussed previously. Swing zones were controlled in modelling by a feed forward ammonia control system which activated when ammonia in the last dedicated aerobic pass was raised above a set concentration. In modelling the chosen concentrations were $2.0 \mathrm{mg}-\mathrm{N} / \mathrm{L}$ which would cause the first 4 (of 7) swing zones to aerate and $3.5 \mathrm{mg}-\mathrm{N} / \mathrm{L}$ which caused the remaining 3 swing zones to aerate. This dynamic modelling of swing zone control allows for realistic testing of plant performance given an aggressive design and SRT selection. Figure shows the model output ammonia concentration in aeration zone 4, one of the last dedicated aeration zones and the location where ammonia concentration was monitored for feed forward ammonia control in the model. As you can see the model predicts that aeration of the first 4 swing zones is only needed during higher loading months while aeration of all swing zones will only be needed for sporadically for short durations like weeks. 


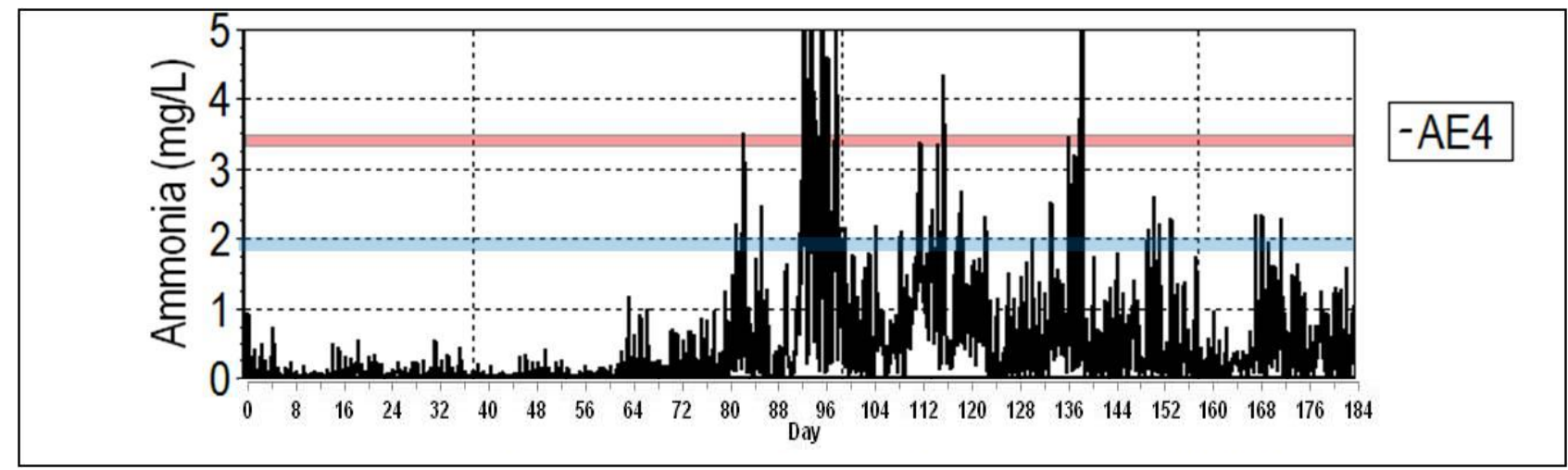

Figure 4 - Ammonia monitoring for feed forward ammonia based control of the swing zones (crossing blue band triggers aeration in first swing zones while red band activates all swing zones)

Given that swing zones were included in the definition of aerobic SRT, reliable operation of the swing zones is important in assuring that the target aerobic SRT is achieved and so failure of the swing zones to aerate when needed was considered a major risk. The risk of noncompliance associated with the chosen SRT due to failure of the swing zones was analyzed by plotting hourly effluent ammonia concentrations as probability distributions under various failure scenarios. In Figure below these probability distributions are given for a scenario in which the ammonia probes which control swing zone aeration have drifted out of calibration by $1 \mathrm{mg}-\mathrm{N} / \mathrm{L}$, and in which the swing zones are not engaged at all due to a complete failure of the control system. The former was modelled by adjusting the set points in the controller from 2.0 and $3.5 \mathrm{mg}-\mathrm{N} / \mathrm{L}$ to 3.0 and $4.5 \mathrm{mg}-\mathrm{N} / \mathrm{L}$ and the latter was modelled by turning off the swing zone aeration controls entirely causing these zones to operate anoxically throughout the entire itinerary. Here we can see that only in the most extreme events when the swing zones were not aerated at all did the effluent ammonia exceed the design limit, and in that event only for a few hours at a time.

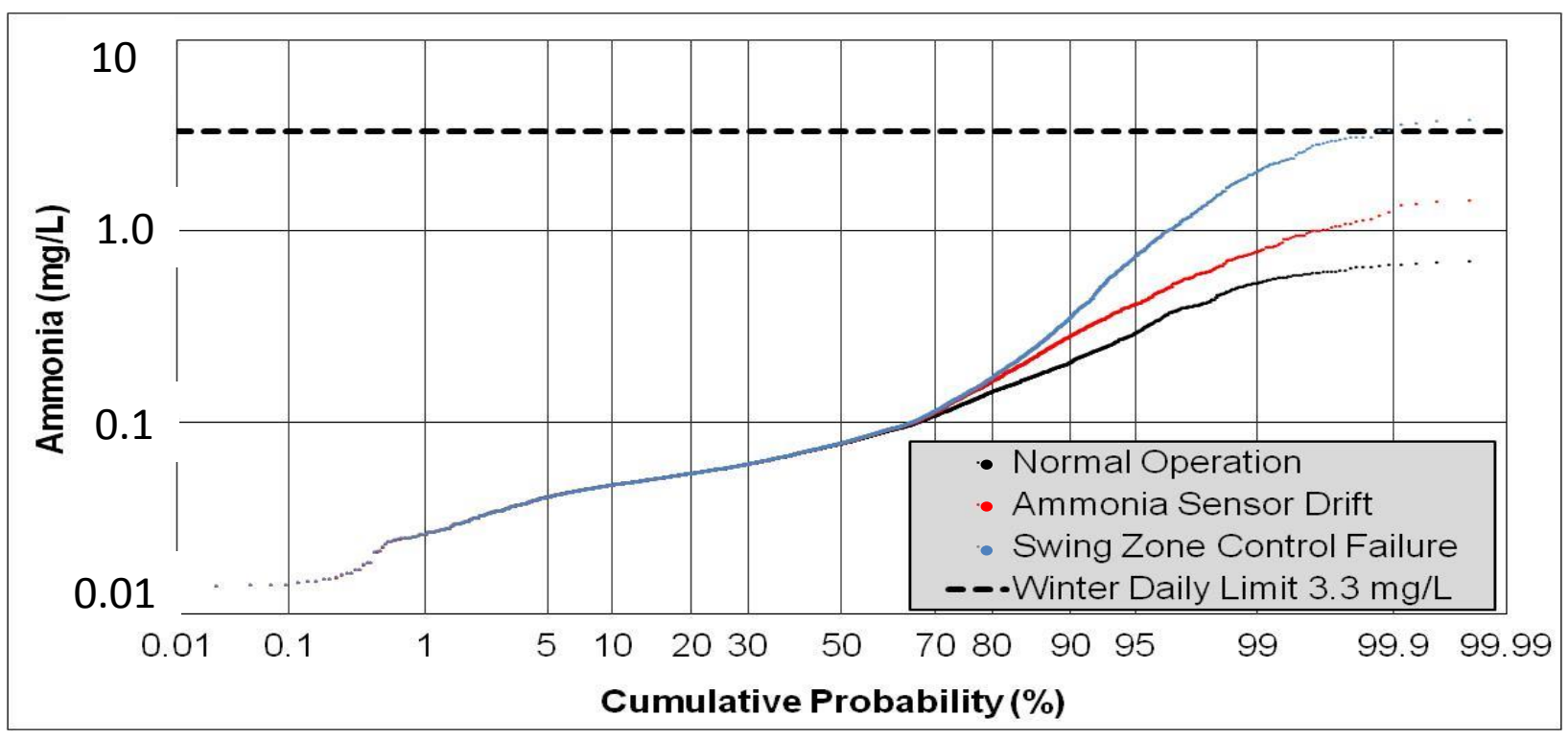

Figure 5 - Probabilty Distribution of effluent ammonia under normal operation and two failure scenarios of ammonia based swing zone control 


\section{Dynamic Aeration Design}

A novel approach to aeration design was used in which the dynamic oxygen uptake rates (OURs) produced by the model for different periods, years, and operating conditions were exported to the aeration model developed by Johnson (1993) to determine the required airflow and number of diffusers at both start up and design conditions. In this method hourly OURs for each zone were exported from the model for the entire itinerary. As can be seen in Figure OURs in individual zones varied significantly over time and maximums/minimums of different zones were not necessarily concurrent, for example the maximum OUR in the first aeration zone is associated with periods of high COD loading while the maximum OUR in the last aeration zone is associated with periods of high TKN loading. Maximum and minimum hour, day, and month values were determined for each zone individually and input to the aeration model to determine the range of airflows to each zone and the appropriate number of diffusers. Additionally the peak oxygen demand or oxygen transfer rate (OTR) across all zones was used to determine the maximum and minimum periods of air demand overall for blower sizing.

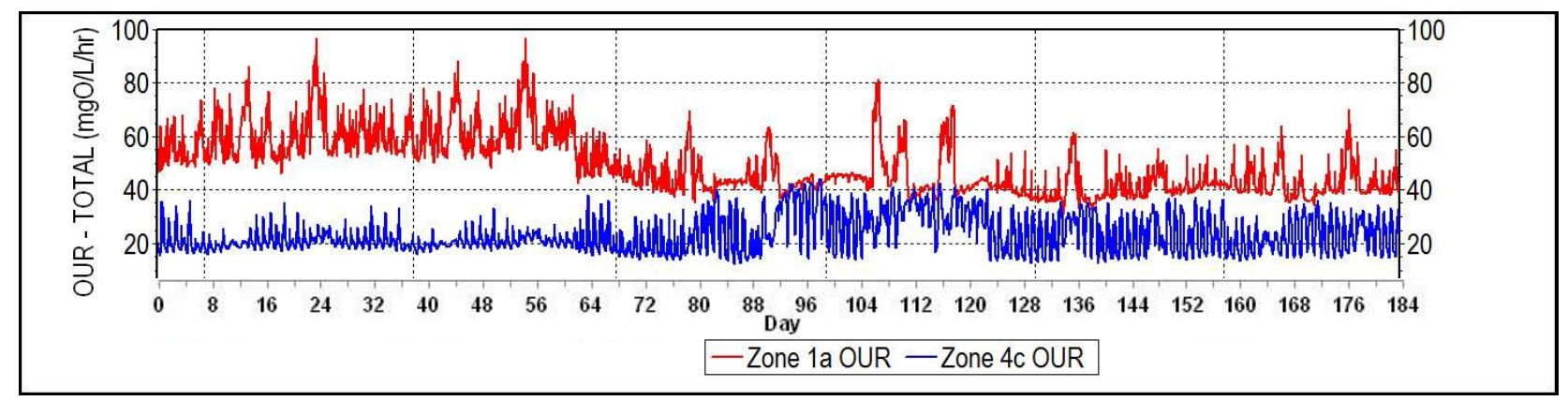

Figure 6 - Example of Dynamic OUR output from two zones.

There were several benefits to dynamic modelling for aeration design. Firstly due to the lack of concurrence of carbonaceous and nitrogenous loads in the provided dynamic itinerary the design airflow was able to be reduced from what would be determined if considering concurrent maximum COD and TKN loads. Secondly modelling the full range of conditions provided data about the variability of airflow demand to each zone which allowed a distribution of airflow demands at each zone to be considered when selecting the number of diffusers per zone.

\section{Chemical Feed Design}

Additionally dynamic control in activated sludge modelling allows carbon feed requirements to be more accurately predicted and has helped in chemical feed system design and chemical feed point selection. Carbon in the form of acetate was fed to the swing zones; controlled by a proportional gain controller which activated in the event of effluent nitrate exceeding a set value with the goal of keeping the monthly average effluent nitrate below $8.0 \mathrm{mg} / \mathrm{L}$. If the swing zone was being aerated at the time another controller was used which override the acetate feed controller. The performance of the acetate feed system can be found in Figure below. Here we see that regular feeding of acetate from day 80 onward is required for the removal of nitrate below $8 \mathrm{mg}-\mathrm{N} / \mathrm{L}$. Here modelling was used to validate the design maximum acetate feed rate determined separately, and the cumulative acetate feed volume 
was tracked to determine the required 7 day storage volume. It is worth noting here that the system is very carbon limited in the provided itinerary due to a high phosphorus concentrtaion. The itinerary influent phosphorus may be revised to a lower concentration upon further investigation.

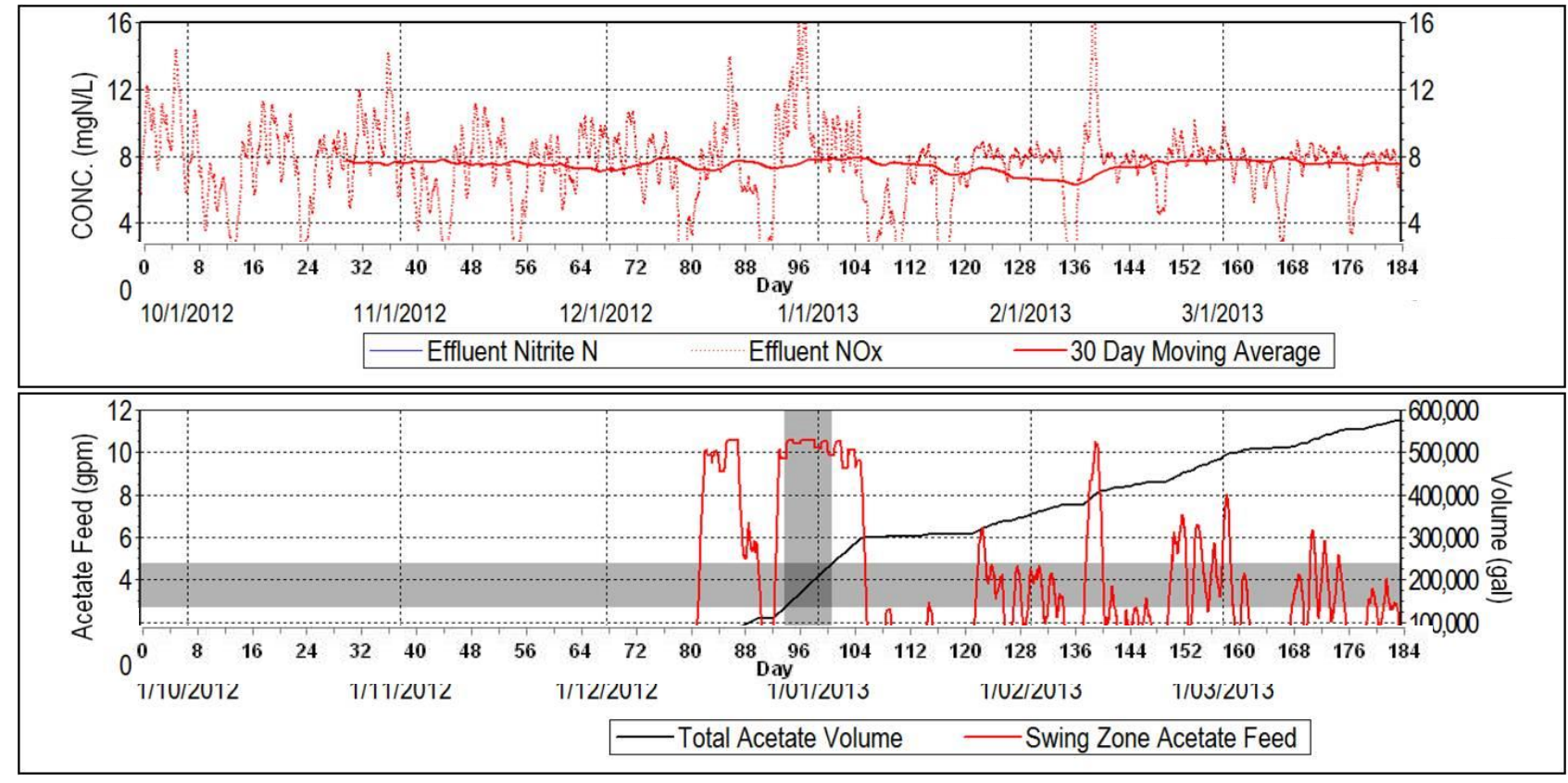

Figure 7 - Effluent Nitrate (top) and Acetate Feed for Nitrate Control (bottom) (1 gallon = 3.78 litres)

\section{Mixed Liquor Fermentation}

Provision of a MLF is a promising option for carbon supplementation for enhanced biological phosphorus removal and is described by Houweling (2010). The first purpose-built MLF in the world was utilized at the Cedar Creek Wastewater Treatment Plant (CCWTP) in Olathe KS (Kobylinski, 2013). Ongoing operational activities, biological process modelling, and bench scale testing at CCWTP are being used to better predict the VFA production capacity of the MLF. The information from this modelling and test work is being used to provide guidance for modelling the MLF proposed for the Sacramento plant.

To calibrate the mixed liquor fermenter; batch tests were performed on Cedar Creek WAS at 4 different Mixed Liquor Suspended Solids (MLSS) concentrations over 6 days with the filtered COD (fCOD) being measured over the duration of the test. A process model was developed using the BioWin simulator platform to generate appropriate WAS characteristics and to simulate the batch fermentation tests of the WAS. The simulation fCOD concentrations were compared to the measured values and the hydrolysis rate was adjusted to match these observations as can be seen in Figure. The adjustment that was made was to increase the hydrolysis factor for anaerobic zones by a factor of 5 (from 0.04 to 0.20 in the simulator defaults). This higher factor matches the hydrolysis factor used as a default in the simulator for anaerobic digesters. This adjustment would seem to take account for the lower ORP which is achieved in the mixed liquor fermenter which is closer to the conditions observed in anaerobic digesters than activated sludge anaerobic zones. Inspection of Figure shows that the current model matches data well for the lower MLSS concentrations but under-predicts fCOD production at higher sludge concentrations. This suggests that the structure of the model for hydrolysis is not correct (second order kinetics with respect to biomass may be more appropriate and will be investigated further). Other research has shown the difficulty of 
accurately predicting hydrolysis rates (Morgenroth, Kommedal, \& Harremoes, 2002). Clearly more investigation of mixed liquor fermentation is needed and currently the authors are partnering with others to do more research on this topic through the Water Environment Research Foundation (WERF).

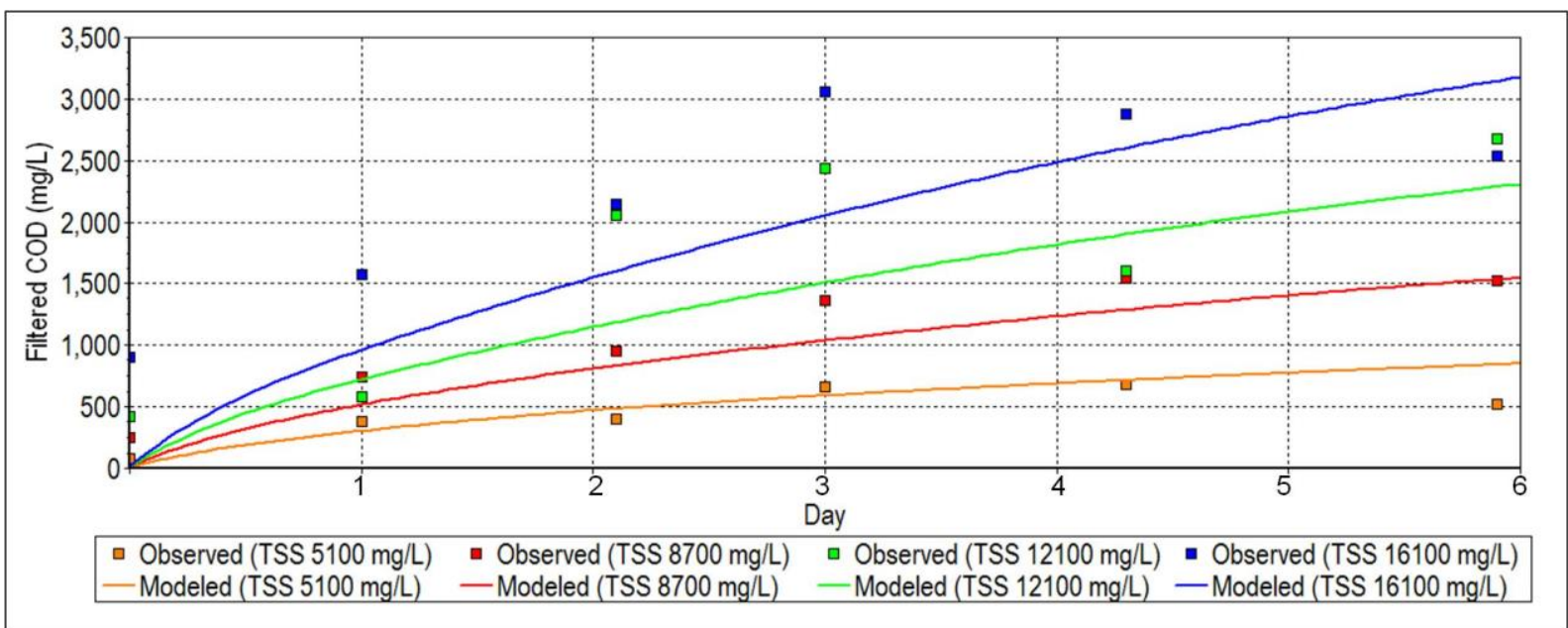

Figure 8 - Production of fCOD through WAS fermentation; experimental and modelling results

To model a continuous MLF, an unaerated activated sludge element and a clarifier were used and the hydrolysis factor for anaerobic zones was adjusted locally in the activated sludge element as described above. SRT in the MLF was set to 1 day while MLF solids were controlled at $9500 \mathrm{mg} / \mathrm{L}$ by adjusting the feed rate of mixed liquor to the MLF. The benefit of the MLF was estimated using the model by tracking the change in VFA and PHA concentration across the fermenter. This showed that with the MLF approximately $30 \%$ more VFA was produced which leads to an improved reduction in $\mathrm{N}$ and $\mathrm{P}$ compared to not using a mixed liquor fermenter. The positive benefit of utilizing a mixed liquor fermenter can be seen in Figure which corresponds to Figure above but without the mixed liquor fermenter. Comparing the two we can see that utilizing a fermenter leads to lower nitrate concentrations when acetate is not supplemented and a reduced total and 7 day storage requirement. Additionally acetate only needs to be supplemented from days 80 to 108 in this case compared to being required from day 80 onward in the scenario without MLF. 

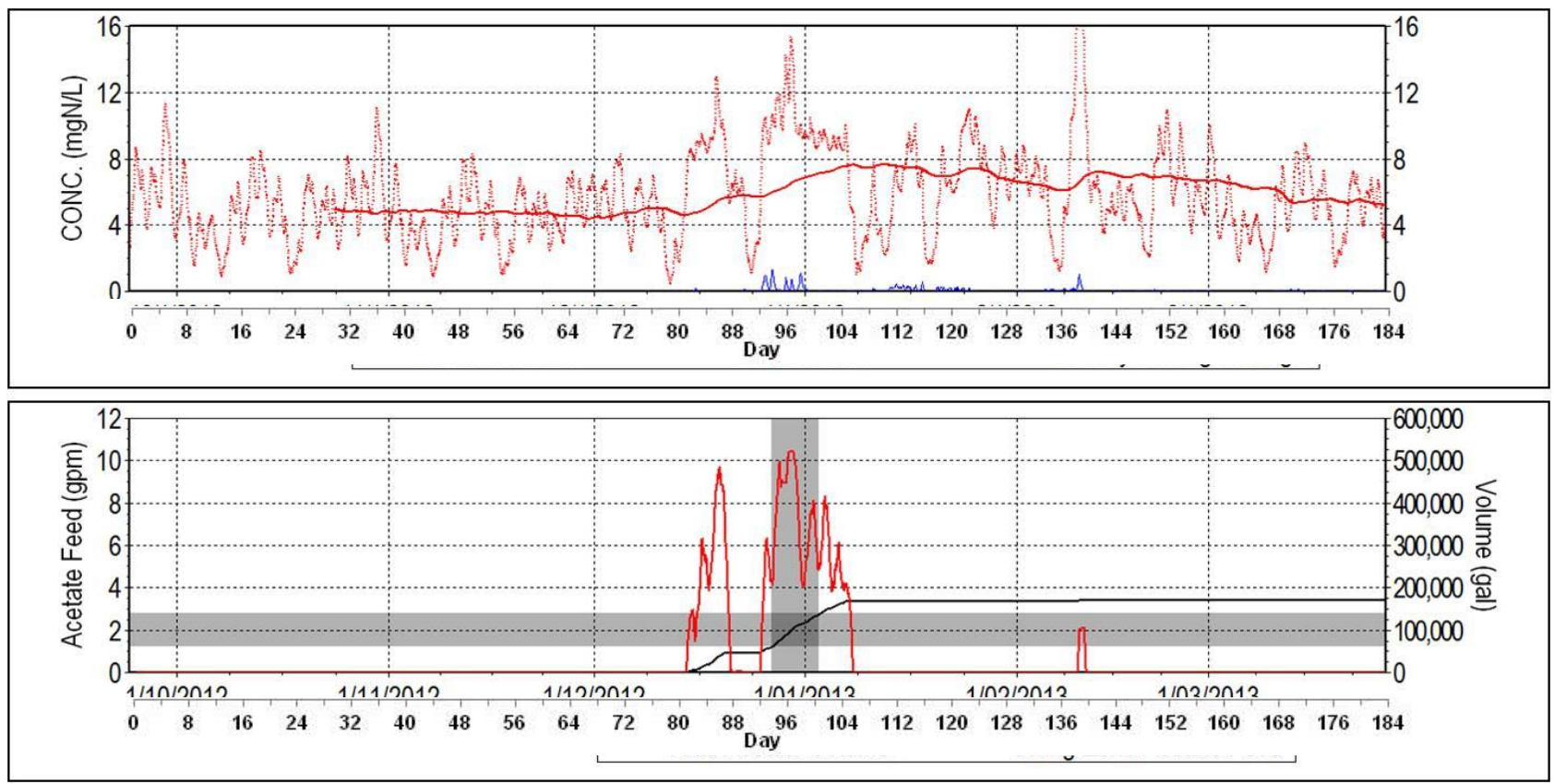

Figure 9 - Effluent Nitrate (top) and Acetate Feed for NOx Control (bottom) w/ MLF (1 gallon = 3.78 litres)

\section{Bibliography}

Houweling, D., Dold, P., \& Barnard, J. (2010). Theoretical Limits to Biological Phosphorus Removal: Rethinking the Influent COD:N:P Ratio. WEFTEC. New Orleans, LA: Water Environment Federation.

Johnson, T. L. (1993). Design Concepts for Activated Sludge Diffused Aeration Systems. (Doctoral Disseration - University of Kansas).

Kobylinski, E., Barnard, J., Massart, N., Kleiwer, A., Crabtree, I., Wirth, M., et al. (2013). Starting up a 5-Stage Bardenhpo Plant with In-Line Fermentation - One Step at a Time. WEFTEC. Chicago: Water Environment Federation.

Morgenroth, E., Kommedal, R., \& Harremoes, P. (2002). Processes and modelling of hydrolysis of particulate organic matter in aerobic wastewater treatment - a review. Water Science and Technology, 45 (6), 25-40. 


\title{
Modeling bioaugmentation with nitrifiers in membrane bioreactors
}

\author{
Alberto Mannucci ${ }^{1}$, Giulio Munz ${ }^{1}$, Gualtiero Mori ${ }^{2}$, Jacek Makinia ${ }^{3}$, Claudio Lubello ${ }^{1}$ and Jan A \\ Oleszkiewicz ${ }^{4}$ \\ ${ }^{1}$ Department of Civil and Environmental Engineering, University of Florence, Via S. Marta n. 3, 50139 \\ Florence, Italy (Email: alberto.mannucci@dicea.unifi.it) \\ ${ }^{2} \mathrm{CER}^{2} \mathrm{CO}$, Consorzio Cuoiodepur Spa, San Romano - San Miniato (PISA - Italy) (Email: \\ gualtiero.mori@cuoiodepur.it) \\ ${ }^{3}$ Faculty of Civil and Environmental Engineering, Gdansk University of Technology, ul. Narutowicza \\ 11/12, 80-233 Gdansk, Poland (Email: jmakinia@pg.gda.pl) \\ ${ }^{4}$ Department of Civil Engineering, University of Manitoba, Winnipeg, Manitoba, Canada R3T 5V6 (Email: \\ oleszkie@cc.umanitoba.ca)
}

\begin{abstract}
The aim of this work was to evaluate bioaugmentation with nitrifiers at pilot scale using two Membrane Bioreactors (MBRs) in order to assess the suitability of state of the art activated sludge models (ASM) in predicting the efficiency of bioaugmentation as a function of operating conditions. It was verified that the difference of temperature between seeding and seeded reactors $(\Delta T)$ is affecting bioaugmentation efficiency and that the experimental data can be accurately simulated when $\Delta \mathrm{T}$ is within a given range (about $10^{\circ} \mathrm{C}$ ). On the contrary, when the temperature is significantly lower in the seeded reactor than in the seeding one, standard ASMs overestimate bioaugmentation efficiency. An ASM able to accurately represent biomass transfer in the presence of high $\Delta \mathrm{T}$ will require the inclusion of mathematical modelling of the effect of temperature time gradients on nitrifiers.
\end{abstract}

Keywords

Activated sludge models, nitrifiers, bioaugmentation, membrane bioreactors.

\section{INTRODUCTION}

Bioaugmentation with nitrifiers is a cost-effective strategy to obtain high nitrification efficiencies at relatively low solids retention time (SRT) (Bartolì et al., 2011; Szoke et al., 2011). It consists of enriching the mixed liquor of the main-stream reactors of an activated sludge system with nitrifying biomass collected from a side stream reactor where the environmental conditions are favourable for nitrifiers growth.

Several phenomena can influence bioaugmentation efficiency (i.e. predation, large temperature differences and different nitrifying biomass in the seeding and the seeded reactor) that are not included in state-of-the-art of activated sludge models and only in few cases bioaugmentation efficiency has been accurately predicted using conventional IWA ASMs (Munz et al, 2012).

Bioaugmentation is not yet easy to predict and control (Van Limbergen et al. 1998) and its success depends on the effective establishment and metabolic adaptation of the added biomass in the treatment system (Satoh et al. 2013).

A Membrane Bioreactor (MBR) offers more accurate control of some important parameter (SRT, total undifferentiated bacteria retention) and this can facilitate the verification of ASMs suitability to predict bioaugmentation.

The use of MBRs both for the seeding and the seeded reactors, ensures similar selective conditions and the possibility of maintaining seeded nitrifiers inside the new environment.

The aim of this work was to model the bioaugmentation process at pilot scale using two MBRs operating in different conditions of ammonia loading rate (ALR), SRT and hydraulic retention time (HRT). 


\section{MATERIAL AND METHODS}

The experimental set-up consisted of two MBRs as shown in Figure 1. The first pilot scale MBR (MBR1- the seeding reactor) consisted of pre-denitrification, nitrification and of a filtration tank equipped with three flat membranes (DF-10 Kubota, Japan). MBR1 was fed with synthetic high strength ammonia influent simulating anaerobic digester supernatant $(650$ $\left.\mathrm{mg} \mathrm{N}-\mathrm{NH}_{4}{ }^{+} \mathrm{L}^{-1} ; 250 \mathrm{mg} \mathrm{COD} \mathrm{L}{ }^{-1}\right)$ and operated for more than 600 days with an SRT of $20 \mathrm{~d}$. The second pilot scale MBR (MBR2 - the seeded reactor) was equipped with a hollow fiber filtration module (Module ZW10 GE-Zenon Environmental) and fed with real domestic wastewater continuously collected from the sewer at the Cuoiodepur WWTP (San Romano -

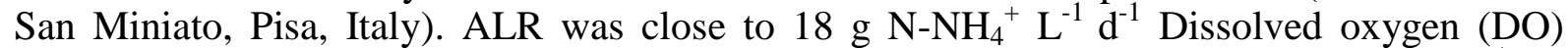
concentration and $\mathrm{pH}$ were the same in both reactors: $\mathrm{pH}=7.5 \pm 0.5, \mathrm{DO}=4 \pm 0.5 \mathrm{mg} \mathrm{L}^{-1}$. In MBR1, temperature was controlled and maintained at $20 \pm 0.5{ }^{\circ} \mathrm{C}$ while in MBR2 temperature depended on environmental temperature without any control and varied from 24 to $6{ }^{\circ} \mathrm{C}$ during the experiment. MBR2 was operated in steady state conditions for more than 400 days without any external seeding before bioaugmentation started with a constant flow of $2.5 \mathrm{~L} \mathrm{~d}^{-1}$ of nitrifying sludge from the filtration tank of MBR1 (Figure 1). Bioaugmentation phase lasted more than $150 \mathrm{~d}$.

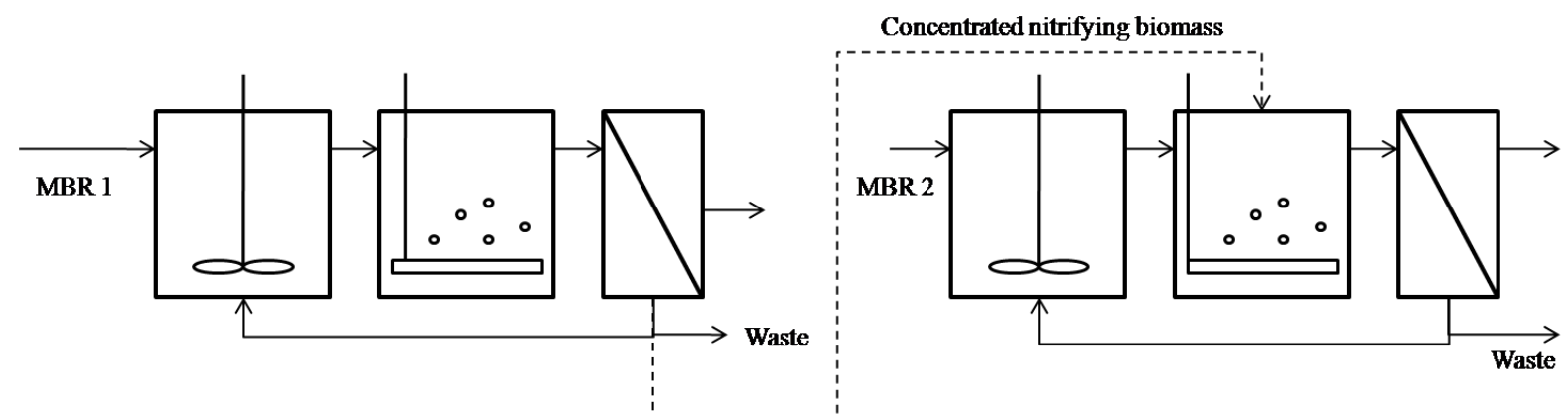

Figure 1 Schematic of the experimental pilot scale set-up

In order to study the kinetic behavior of the MBR pilot plant nitrifying biomass, a series of 7 conventional kinetic batch test were performed. $1 \mathrm{~L}$ of mixed liquor was collected from nitrification tank of the MBR pilot plant and maintained in mixed and aerated conditions until reaching endogenous. A fixed amount of $\mathrm{NH} 4 \mathrm{Cl}$ was dosed in order to obtain an initial concentration that will not be neither limiting nor inhibitory, based on observation and literature data. The $\mathrm{pH}$ was controlled at $8 \pm 0.1$ and the DO higher than $4 \mathrm{mg} \mathrm{L}-1$ through fine bubbles aeration. A sample was collected every 10 minutes and the N-NH4+ was analyzed through colorimetric analysis. Each test was repeated in triplicates.

An activated sludge model with a two-step nitrification-denitrification (ASMN, Hiatt and Grady, 2008) was used to describe the processes (autotrophic and heterotrophic biomass) in both pilot plants and to estimate the amount of active AOB biomass at the time of batch tests according to Munz et al. (2011). The model, that separately represents AOB and NOB populations, was used to calibrate the maximum specific growth rate for $\mathrm{AOB}\left(\mu_{\max , \mathrm{AOB}}\right)$ and the half-saturation constant for ammonia $\left(\mathrm{K}_{\mathrm{NH}}\right)$ with the results of batch tests.

\section{RESULTS AND CONCLUSIONS}

Ammonia Removal Efficiency (ER) estimated in MBR2 under bioaugmented (ON) and non bioaugmented (OFF) period is reported in Figure 2 as a function of the actual temperature. Ammonia RE increase in presence of continuous AOB seeding from MBR1 and 
bioaugmentation efficiency (as RE increasing) depends on MBR2 temperature and presents a peak when temperature was in the range $15-17^{\circ} \mathrm{C}$ (Figure 6).

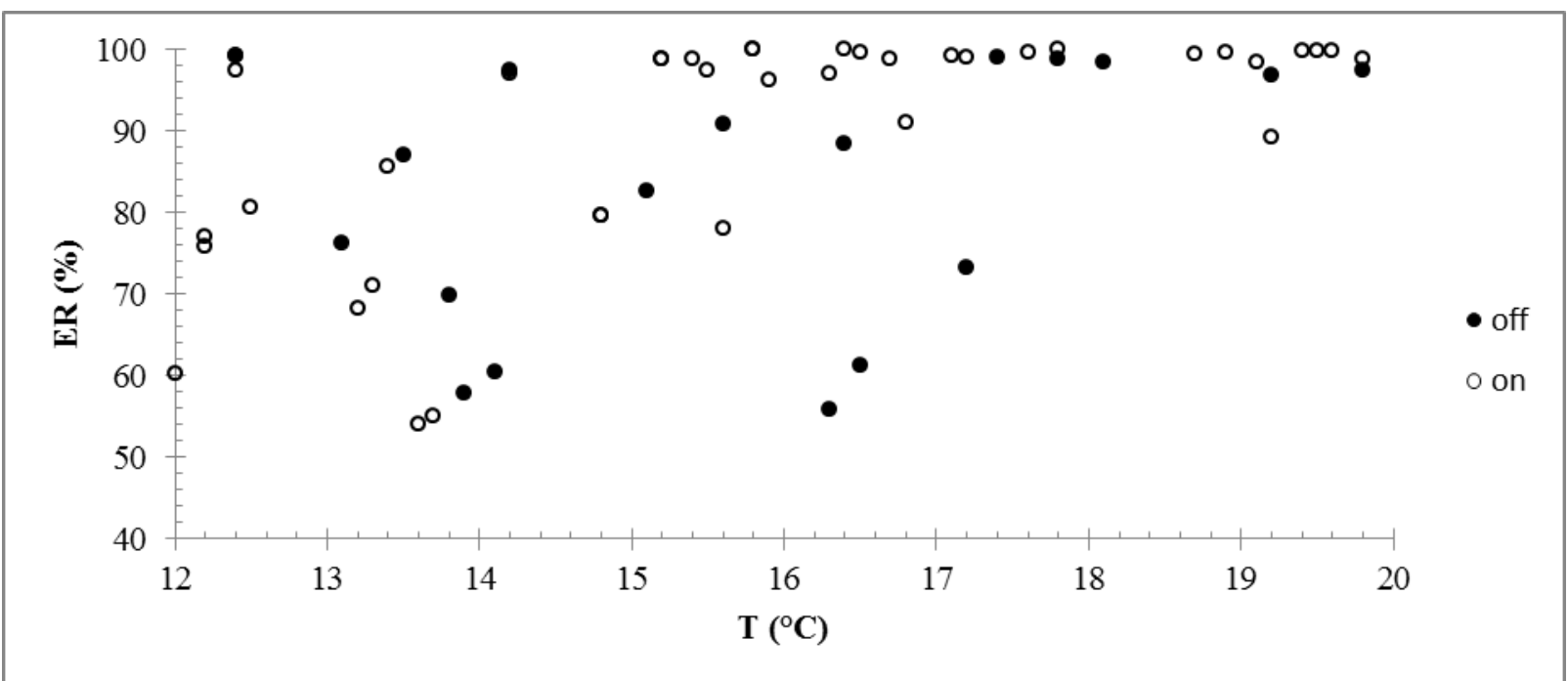

Figure 2 Ammonia Removal Efficiency (ER) in MBR2 under bioaugmented (ON) and non bioaugmented (OFF) period

The AOB concentration of the samples used in kinetic test was determined through the modeling of the nitrification capacity of the MBR1 and MBR2 pilot plants; AOB concentration was then used to calibrate $\mu_{\max , \mathrm{AOB}}$ and $\mathrm{K}_{\mathrm{NH}}$ using ASMN model on the ammonia bulk liquid concentration obtained in conventional batch kinetic tests.

An example of experimental and modeled ammonia concentration for the MBR2 nitrifying biomass is reported in Figure 3.

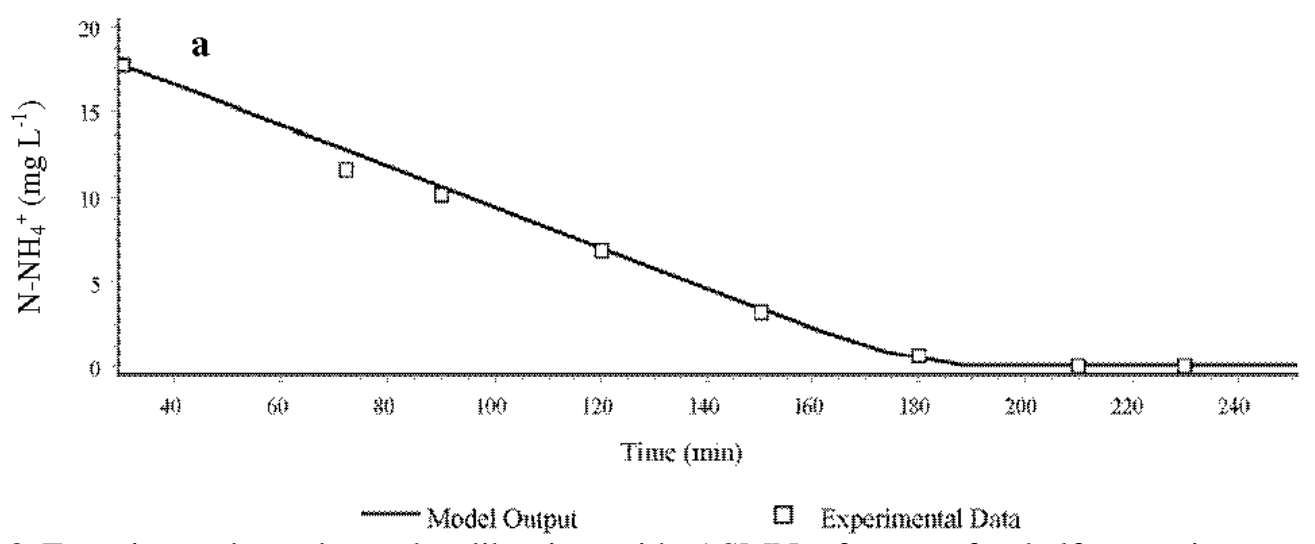

Figure 3 Experimental results and calibration with ASMN of a test for half-saturation constant and maximum specific growth rate estimation

Average values of $\mu_{\max , \mathrm{AOB}}$ and $\mathrm{K}_{\mathrm{NH}}$ obtained from kinetic batch tests and long term monitoring data elaboration are reported in Table 1.

Table 1 Calibrated ASMN parameters in batch tests

\begin{tabular}{|l|c|c|c|c|c|c|c|}
\hline \multirow{2}{*}{ Parameter } & \multirow{2}{*}{ Unit } & \multicolumn{2}{|c|}{ MBR1 } & \multicolumn{2}{c|}{ MBR2 Bioaug OFF. } & \multicolumn{2}{c|}{ MBR2 Bioaug. ON } \\
\cline { 3 - 8 } & Avg. & Std. Dev. & Avg. & Std. Dev. & Avg. & Std. Dev. \\
\hline
\end{tabular}




\begin{tabular}{|c|c|c|c|c|c|c|c|}
\hline $\mathrm{K}_{\mathrm{NH}}$ & $\mathrm{mg} \mathrm{L}^{-1}$ & 0.8 & 0.28 & 0.4 & 0.14 & 0.39 & 0.24 \\
\hline$\mu_{\max , \text { АОВ }}$ & $\mathrm{d}^{-1}$ & 0.95 & 0.025 & 0.85 & 0.015 & 0.84 & 0.019 \\
\hline
\end{tabular}

As reported in Table 1, the main kinetic parameters of AOB in MBR2 were not influenced by continuous seeding of nitrifiers with different kinetics; thus, for further simulations, an unique nitrifying biomass in MBR2 also in presence of bioaugmentation was considered.

ASMN model and calibrated kinetic parameters have been validated on experimental effluent quality data in non-biaougmented period (until day 440) in MBR1 (data not shown) and MBR2 (Figure 4) before modelling the effect of bioaugmentation process (from day 441 to day 525).

Until the difference between temperature in MBR1 and MBR2 $(\Delta \mathrm{T})$ was lower than $10^{\circ} \mathrm{C}(\mathrm{T}$ in MBR1 > $10^{\circ} \mathrm{C}$ ) ASMN model was able to describe MBR2 effluent quality in the presence of bioaugmentation. Differences between experimental data and model output started when $\Delta \mathrm{T}$ is higher than $10^{\circ} \mathrm{C}$ (Figure 5).

The results confirmed the possibility of modeling bioaugmentation effects using a single nitrifying biomass in both seeded and seeding reactors and highlighted the importance of temperature stress.

Adopted model does not consider some important biological adaptation mechanisms reported in literature that can explain bacterial growth reduction due to sudden temperature changes higher than $10^{\circ} \mathrm{C}$.

The observed phenomena can be explained when assuming that the investigated biomass was subject to an adaptation phase and a subsequent partial lag-phase, depending on temperature shock, during which many physiological changes as the induction of the cold-shock proteins occurs (Beales, 2004; Lee et al., 2011).

Due to the presence of a lag phase necessary for the biomass to recover its full activity, the effect of the sudden temperature change should be related to the HRT (in the seeded reactor) and this relationship should be further explored.

As evident from the results of experimental and simulated data reported in Figure 5, where the whole experiment was summarized, $\Delta \mathrm{T}$ between seeding and seeded reactor plays a very important role on the effect of bioaugmentation on nitrification efficiency and has to be taken into consideration in order to improve bioaugmentation modelling.

As reported by Oleszkiewicz and Berquist (1988) and Guo et al. (2010) temperature correction factor depends on the studied temperature range. In this work, temperature in MBR2 reactor varied in the range $7^{\circ} \mathrm{C}-20^{\circ} \mathrm{C}$ in order to study nitrification process in the range of temperature of civil wastewater, but the effect of the same $\Delta \mathrm{T}$ in a different temperature range needs to be evaluated.

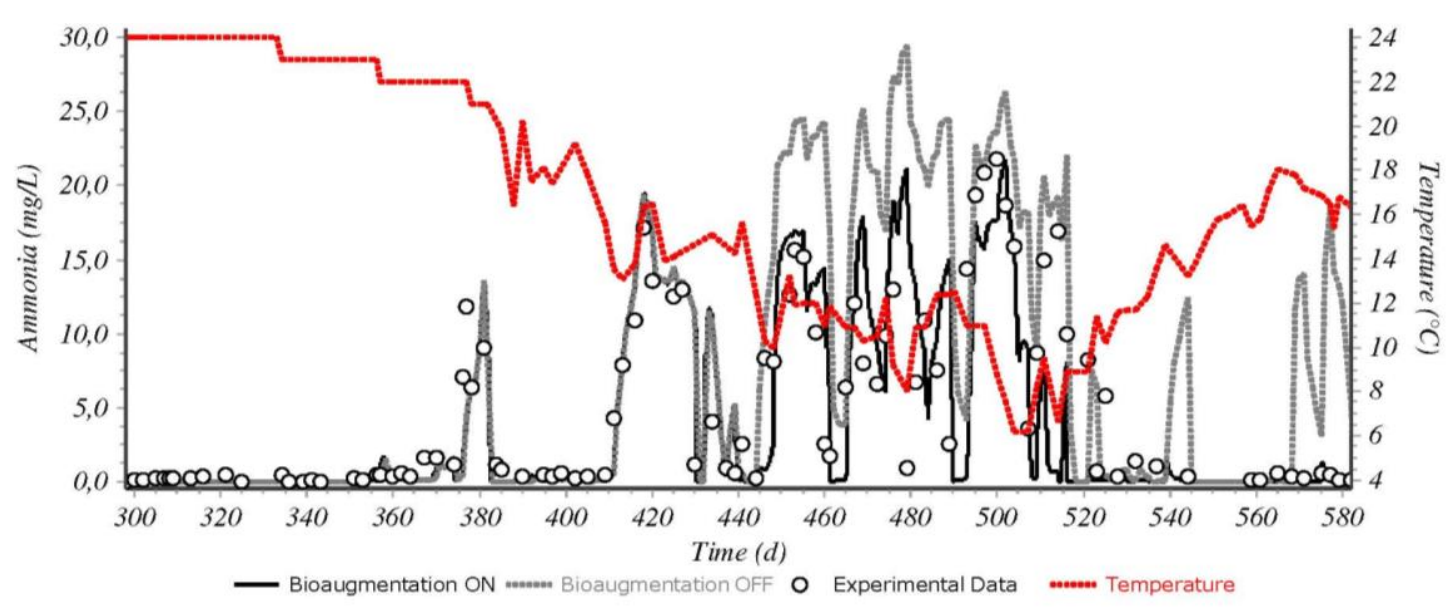


Figure 4 MBR2: Temperature (red line), experimental (white circles) and modeled ammonia effluent concentration using ASMN in the absence (gray dotted line) and in the presence (solid black line) of bioaugmentation.

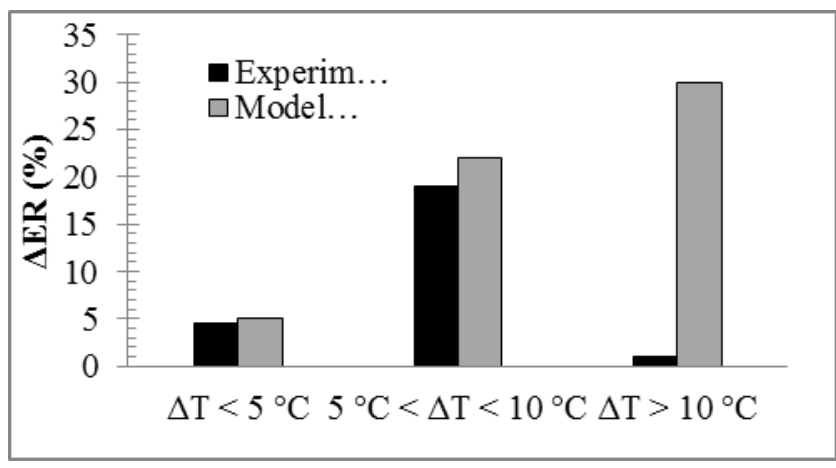

Figure 5 Difference between simulated (gray) and experimental (black) increase of ammonia removal efficiency (ER) due to bioaugmentation as a function of $\Delta \mathrm{T}$.

\section{ACKNOWLEDGEMENTS}

This work was carried out under the project "CARbon BALAncing for nutrient control in wastewater treatment (CARBALA - Marie Curie Irses).

\section{REFERENCES}

Bartrolì A., Carrera J., Perez J. 2011 Bioaugmentation as a tool for improving the start-up and stability of a pilotscale partial nitrification biofilm air lift reactor. Bioresource Technology, 102(6), 4370-4375.

Beales N. 2004 Adaptation of microorganisms to cold temperatures, week acid preservatives, low pH and osmotic stress, a review. Comprehensive Review Food Science Safety, 3, 1-20.

Guo J., Peng Y., Huang H.,Wang S., Ge S., Zhanh J., Wang Z. 2010 Short- and long-term effects of temperature on partial nitrification in a sequencing batch reactor treating domestic wastewater. Journal of Hazardous Materials, 179, 471-479.

Hiatt W. C., Grady C. P. L. Jr. 2008 An updated process model for carbon oxidation, nitrification, and denitrification. Water Environment Research, 80, 2145-2156.

Lee S., Cho K., Kim W., Hwang S. 2011 Acclimatation and activity of ammonia-oxydizing bacteria with respect to variations in zinc concentration, temperature, and microbial population. Bioresource Technology, 102, 4196-4203.

Munz G., Szoke N., Oleszkiewicz J.A. 2012 Effect of ammonia oxidizing bacteria (AOB) kinetics on bioaugmentation. Bioresource Technology, 125, 88-96.

Munz G., Lubello C., Oleszkiewicz J.A. 2011 Modeling the decay of ammonium oxidizing bacteria. Water Research, 45, 2, 557-564.

Oleszkiewicz J.A., Berquist S.A. 1989 Low temperature nitrogen removal in sequencing batch reactors. Water Research, 22, 1163-1171.

Satoh H., Okabe S., Yamaguchi Y., Watanabe Y. 2003 Evaluation of the impact of bioaugmentation and biostimulation by in situ hybridization and microelectrode. Water Research, 37, 2206-2216.

Szoke N., Munz G., Hwang J.H., Oleszkiewicz J. 2011 Two years of full-scale bioaugmentation trials on high purity oxygen activated sludge. Proceedings In: Design, Operation and Economics of Large Waste Water Treatment Plants. Budapest, Hungary, 4-8 September 2011.

Van Limbergen H., Top E.M., Verstraete W. 1998.Bioaugmentation in activated sludge: current features and future perspectives. Applied Microbiology Biotechnology, 50, 16-23. 


\title{
A Green Micro-Algal Growth Model developed in the Activated Sludge Modelling Framework (ASM-A)
}

\author{
Borja Valverde-Pérez ${ }^{1}$, Dorottya Sarolta Wágner ${ }^{2}$, Mariann Sæbø, Jonathan Van Wagenen, Irini \\ Angelidaki, Barth F. Smets, Benedek Gy. Plósz ${ }^{3}$ \\ Department of Environmental Engineering, Technical University of Denmark, Miljøvej, Building 115, DK- \\ 2800, Kgs. Lyngby, Denmark. (Corresponding authors E-mail: ${ }^{1}$ bvape@env.dtu.dk, ${ }^{2}$ dosaw@env.dtu.dk, ${ }^{3}$ \\ beep@env.dtu.dk )
}

\begin{abstract}
Recent research focuses on the use of photobioreactors (PhBRs) as a means to recover wastewater resources and/or as a tertiary treatment process used to polish wastewater treatment plant (WWTP) effluent. Several models have been developed to simulate algae growth. However, there is no accepted process model developed using the systematic approach proposed in the activated sludge modeling (ASM) framework. In this paper, we present a mathematical model developed to simulate green micro-algal growth (ASM-A). It was developed as an extension to the ASM-2d (Henze et al., 2000), hence it can be readily coupled with this commonly used wastewater treatment model. We identified and calibrated a suitable model structure that can describe factors, influencing autotrophic algal growth and nutrient uptake, including macro-nutrient availability and light irradiance in photobioreactors. For model calibration, parameters were estimated through micro-batch (microplate) and a series of batch experiments using a mixed green micro-algal culture isolated in a wastewater pond, growing strictly in suspension. The model was evaluated using independent data obtained in batch experiments with synthetic growth medium.
\end{abstract}

Keywords

Process modeling, nutrient recovery, microalgae, wastewater

\section{INTRODUCTION}

Microalgae photobioreactors offer the potential to recover nutrients contained in wastewater and provide an opportunity for efficient nutrient recycling (Samorí et al., 2013), while serving as tertiary wastewater treatment step. In addition, their biomass can be used for biogas or biodiesel production through anaerobic digestion and the utilization of lipids, respectively. Unlike crop-based biofuels, microalgal biomass does not compete with food production, which qualifies it as a third generation biofuel. However, the production of microalgae has a higher environmental impact (carbon footprint) than the production of crops used for biofuel production. This is due to the comparably higher water demand and energy consumption as well as the greenhouse-gas (GHG) emission associated with the production of nutrients used for cultivation of microalgae (Clarens et al., 2009). With the current technologies, large scale biofuel production from microalgae is neither energetically nor economically favorable, unless wastewater treatment is the primary goal (Lundquist et al., 2010 and Pittman et al., 2011). Moreover, the combination of wastewater treatment with microalgae cultivation eliminates the need to add scarce and potentially costly nutrients like phosphate to the culture medium (Cheng and Ogden, 2011). An accurate model able to simulate algal growth in PhBRs connected with conventional WWTP would be a powerful tool for process design and evaluation of this innovative technology, an area this research addresses.

The main objectives of this study are (i) to develop a micro-algal model in the ASM framework that can combine the bacterial and micro-algal wastewater treatment processes; (ii) to simulate the micro-algal uptake, storage and growth on nitrogen and phosphorous; (iii) to model the co-limitation of various substrates. 


\section{Modelling the growth of green micro-algae}

Several models exist, describing green micro-algal growth. Table 1 summarizes all the models used for model identification in our study. The models range in complexity, from modelling the influence of one single variable on growth e.g. light (Grima et al., 1994; Huesemann et al., 2013) to models incorporating the influence of multiple variables combined e.g. light, nutrient availability, temperature and pH (Ambrose et al., 2006; Wolf et al., 2007; Quinn et al., 2011; Broekhuizen et al., 2012; Guest et al., 2013 or Decostere et al., 2013). Even when the complexity and the number of factors taken into account are high, some of these models are still missing important aspects related to micro-algal growth and their applications. As an example, the PHOBIA model, developed by Wolf et al. (2007), is a biofilm model that includes growth of heterotrophs, nitrifiers and micro-algae, but disregards the algae growth on phosphate, making it inefficient for applications like polishing steps in effluents from WWTP. The modified IWA River Water Quality Model no. 1, described in Broekhuizen et al. (2012), developed to simulate a pilot-scale high rate algae pond, is a model accounting for $\mathrm{pH}$, carbon, oxygen, nitrogen and phosphate. Both models estimate the algal biomass concentration on COD concentration units, which allows closing the mass balances between the different organic components and microbial groups. However, growth on nutrients is described through Monod kinetic formulations and do not account for i) uptake and storage and ii) growth on the stored nutrients - factors crucial to predict nutrient recovery from wastewater. The model by Droop (1973) describes uptake and storage as well as growth on stored nutrients. Models with multiple substrate limitations, in accordance to Droop's approach, are those by Ambrose et al. (2006), Quinn et al. (2011) or Guest et al. (2013). Nevertheless, this approach is still under discussion due to the different approaches to model the nutrient growth limitation (the threshold model or the multiplicative model, described in Bourgaran et al., 2010) and the presence of a nitrogen quota (Richmond 2004). None of these models include the bacterial growth, so coupling them with a WWTP model may fail because they do not take into account the synergies between the different microorganisms (e.g. inorganic carbon or light availabilities due bacterial growth). Although several studies report the growth of algae on different organic substrates (Mata et al., 2010 or Brennan and Owende 2010), none of the models takes into account the mixotrophic or heterotrophic algal growth. Organic carbon sources like glucose or acetate, can enhance algal growth to some extent, but become inhibitors for some algae at high concentrations. Therefore, Moya et al. (1996), proposed a simple model that takes into account the microbial growth rate of algae as a function of light (autotrophic growth) and acetate (heterotrophic growth). While this model would be useful to predict heterotrophic algal growth, the effect of nutrient availability, amongst others, is missing.

The effect of light on algal growth has been modeled by using expressions accounting for different parameters. In certain cases the effect of light on algal growth is modeled by taking into account light inhibition, i.e. Steele, Peeters and Eilers or Haldane (Ambrose et al., 2006, Bouterfas et al., 2002) while in other models photo-inhibition is not included, i.e. Monod, Platt and Jassby, Poisson single-hit models or Smith equations (Ambrose et al., 2006, Bouterfas et al., 2002 and Skjelberd et al., 2012). 


\section{WWTmod}

Valverde-Pérez et al.

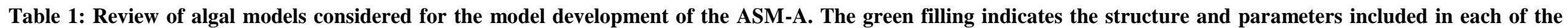
models. P\&E stands for Peeters \& Eilers; P\&J stands for Platt \& Jassby.

\begin{tabular}{|c|c|c|c|c|c|c|c|c|c|c|c|c|c|c|c|c|c|}
\hline \multirow{3}{*}{ Model } & \multicolumn{12}{|c|}{ Autotrophic growth } & \multirow{3}{*}{$\begin{array}{c}\text { Heterotrophic } \\
\text { growth }\end{array}$} & \multicolumn{3}{|c|}{ Bacterial growth } & \multirow{3}{*}{ pH } \\
\hline & \multicolumn{4}{|c|}{ Nutrients } & \multirow{2}{*}{$D I C$} & \multicolumn{7}{|c|}{ Light } & & & & & \\
\hline & Droop & Monod & $N$ & $P$ & & Monod & Haldane & Steele & $P \& E$ & $P \& J$ & Smith & Poisson & & $\boldsymbol{X}_{\boldsymbol{H}}$ & $\boldsymbol{X}_{\boldsymbol{A}}$ & $X_{P A O}$ & \\
\hline $\begin{array}{c}\text { Moya et } \\
\text { al. } 1996\end{array}$ & & & & & & & & & & & & & & & & & \\
\hline $\begin{array}{l}\text { Bouterfas } \\
\text { et al. } 2002\end{array}$ & & & & & & & & & & & & & & & & & \\
\hline $\begin{array}{c}\text { Ambrose } \\
\text { et al. } 2006\end{array}$ & & & & & & & & & & & & & & & & & \\
\hline $\begin{array}{c}\text { Wolf et al. } \\
2007\end{array}$ & & & & & & & & & & & & & & & & & \\
\hline $\begin{array}{l}\text { Bougaran } \\
\text { et al. } 2010\end{array}$ & & & & & & & & & & & & & & & & & \\
\hline $\begin{array}{c}\text { Quinn et } \\
\text { al. } 2011\end{array}$ & & & & & & & & & & & & & & & & & \\
\hline $\begin{array}{c}\text { Broekhuiz } \\
\text { en et al. } \\
2012\end{array}$ & & & & & & & & & & & & & & & & & \\
\hline $\begin{array}{c}\text { Skjelbred } \\
\text { et al., } 2012\end{array}$ & & & & & & & & & & & & & & & & & \\
\hline $\begin{array}{l}\text { Guest et } \\
\text { al. } 2013\end{array}$ & & & & & & & & & & & & & & & & & \\
\hline $\begin{array}{l}\text { Decostere } \\
\text { et al. } 2013\end{array}$ & & & & & & & & & & & & & & & & & \\
\hline $\begin{array}{c}\text { Van } \\
\text { Wagenen } \\
\text { et al. 2014 }\end{array}$ & & & & & & & & & & & & & & & & & \\
\hline $\begin{array}{c}\text { ASM-A } \\
\text { (Wágner } \\
\text { et al. 2014) }\end{array}$ & & & & & & & & & & & & & & & & & \\
\hline
\end{tabular}




\section{ASM-A MODEL DEVELOPMENT}

The model presented in this study was developed in the framework of the well established model for activated sludge ASM-2d (Henze et al., 2000). This ASM model includes all the relevant bacterial groups involved in enhanced biological phorphorus removal systems (EBPR), i.e. heterotrophs, nitrifiers and polyphosphate accumulating organisms (PAOs). However, the expressions included in this section, as well as the model assessment, only refer to the algal processes (Table 2). The units are expressed in accordance with the ASM framework where closed mass balances are used over electron equivalents, expressed in COD, nitrogen and phosphorous and inorganic carbon. ASM nomenclature was followed to make the integration of the algal model into the existing model structures easier.

\section{Uptake and Storage of Nitrogen (R1 and R2)}

Ammonia is the preferred form of nitrogen over nitrate for algal growth. In ASM-A, algal uptake and storage of nitrogen is modelled using ammonia (Eq.1) or nitrate (Eq.2) as nitrogen forms. In ASM-A the uptake and storage of $\mathrm{N}$ is described in relation to the availability of external $\mathrm{N}$ in the wastewater $\left(\mathrm{S}_{\mathrm{NH}} / \mathrm{S}_{\mathrm{NO}}\right)$, as well as to the internal cell quota of $\mathrm{N}\left(\mathrm{X}_{\mathrm{Alg}, \mathrm{N}}\right)$, defined as cell internal storage of $\mathrm{N}$ per total mass of biomass. Nutrient uptake rate decreases as $\mathrm{X}_{\mathrm{Alg}, \mathrm{N}}$ approaches the maximum internal cell quota, $\mathrm{X}_{\mathrm{Alg}, \mathrm{Nmax}}$, in the biomass $\left(\mathrm{X}_{\mathrm{Alg}}\right)$. To take into account the algal preference for ammonia, an inhibition term for nitrate uptake is included when ammonia is available.

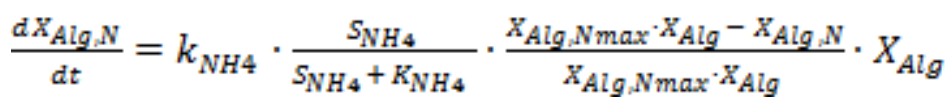

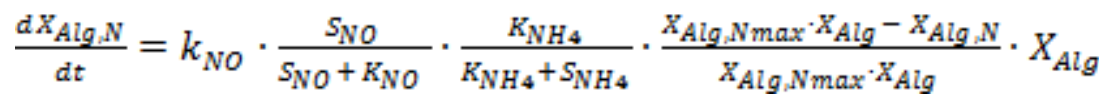

\section{Uptake and Storage of Phosphorous (R3)}

The uptake and storage of $\mathrm{P}$ is defined relative to the availability of external soluble $\mathrm{PO}_{4}{ }^{3-}$ in the wastewater $\left(\mathrm{S}_{\mathrm{PO}}\right)$, as well as to the internal cell quota of $\mathrm{P}\left(\mathrm{X}_{\mathrm{Alg}, \mathrm{PP}}\right)$, defined as cell internal storage of $\mathrm{P}$ per total mass of biomass. Nutrient uptake rate decreases as $\mathrm{X}_{\mathrm{Alg}, \mathrm{PP}}$ approaches the maximum internal cell quota for $\mathrm{P}, \mathrm{X}_{\mathrm{Alg}, \mathrm{PPmax}}$ in the algae biomass $\mathrm{X}_{\mathrm{Alg}}$.

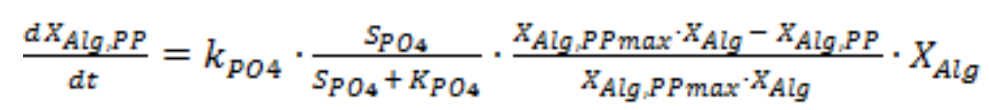

\section{Photoautotrophic Growth (R4)}

Nutrient limitation is described by the Droop model, assuming that growth is dependent on the internal cell quota of the different nutrients. Growth rate decreases as the internal cell quota approaches the minimum subsistence cell quota $\left(\mathrm{X}_{\mathrm{Alg}, \mathrm{Nmin}}\right.$ or $\mathrm{X}_{\mathrm{Alg}, \mathrm{PPmin}}$ ) in the algae biomass. The uptake of dissolved inorganic carbon (DIC) is modeled using Monod kinetics. Light limitation is determined by the photo-synthetically available irradiance passing through the PhBR. We assume that the algae are exposed to a constant light intensity, denoted as $I_{A v}$. To identify a suitable model structure to describe the light influence on micro-algal growth, six different model equations were fitted to the obtained experimental data (not shown) using Sigmaplot $\odot$. Two out of the expressions include the photo-inhibition, i.e. Steele and Peeters and Eilers while in the other models photo-inhibition is not included, i.e. Monod, Platt and 
Jassby, Smith and Poisson single-hit models. Light dependence is modeled using the Steele equation that was chosen based on experimental results (see the results section).

$$
\frac{d x_{A i g}}{d t}=\mu_{A, \max } \cdot\left(1-\frac{x_{A i g N \min } x_{A i g}}{x_{A I g} N}\right) \cdot\left(1-\frac{x_{A i g, P P \min } x_{A i g}}{x_{A i g, P P}}\right) \cdot \frac{s_{D I C}}{s_{D I C+K_{D I C}}} \cdot \frac{I_{A v}}{I_{S}} \cdot e^{1+\frac{I_{A v}}{I_{S}}} \cdot X_{A l g}
$$

\section{Heterotrophic Growth (R5)}

Micro-algae are able to grow on readily biodegradable organic carbon sources. However, high concentrations of organic substrate can result in substrate inhibition of growth (Richmond, 2004). In accordance with the ASM-2d, acetate is assessed as the organic carbon substrate $\left(\mathrm{S}_{\mathrm{A}}\right)$. The Haldane model is employed to describe the effect on growth rate as a function of the substrate concentration. Oxygen serves as a substrate for heterotrophic growth ( $\left.\mathrm{S}_{\mathrm{O} 2}\right)$, and its effect on growth rate is described using the Monod kinetics. The model also takes into account the inhibition due to light intensity of the heterotrophic growth, as well as the nutrient consumption associated with algal growth.

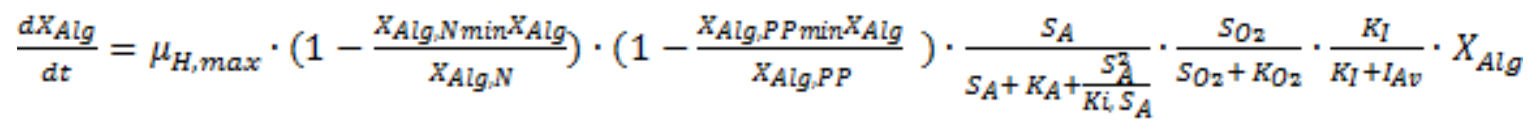

Algal Decay (R6)

The algal decay process includes all forms of algal biomass loss and energy requirements not associated with growth. This includes internal resources used for maintenance, biomass loss during dark respiration, death and lysis that will reduce the amount of active biomass in the culture. In addition, the term includes reduction in biomass due to predators grazing on the algal biomass.

$$
\frac{d x_{A i g}}{d t}=b_{X a l} \times X_{A l g}
$$

\section{MATERIALS AND METHODS}

\section{Process model calibration and implementation}

Table 2 shows the stoichiometry of each of the processes of the ASM-A model. The ASM-A model was implemented as an extension of the simulation model of ASM-2d implemented in Matlab R2012a (The MathWorks, Natick, MA) by Flores-Alsina et al. (2012).

\section{Microorganisms and media}

The mixed green micro-algal consortium used in this study was isolated in a natural pond in contact with wastewater, including mainly Chlorella sp. and Scenedesmus sp. (data not shown). Importantly, the algae grow strictly in suspension, without significant biofilm formation. The mixed culture was cultivated using synthetic cultivation medium, MWC+Se medium (Guillard and Lorenzen, 1972) under standard temperature.

\section{Micro-batch (microplate) experiments}

Micro-batch experiments for assessing exponential growth rate were set up in 24 wells black microplates (VisiPlate, PerkinElmer Inc., Waltham, MA). In this study we refer to the experiments conducted in microplates as micro-batch experiments. Each well can hold a $2 \mathrm{~mL}$ sample. Temperature was regulated at $19.8 \pm 1.0{ }^{\circ} \mathrm{C}$. The inoculated micro-batches were placed on a shaker table (Lab-Saker $L S-X)$, operated at $160 \mathrm{RPM}$. Light was supplied by six $15 \mathrm{~W}$ fluorescent lamps (GroLux, Sylvania ${ }^{\circledR}$, Danvers, MA). In Vivo Fluorescence (IVF) was used 
to measure the algal growth because of its high sensitivity at low biomass concentration. This methodology was successfully applied by Van Wagenen et al. (2014) to determine reliable model parameters describing the light dependence of algal growth for different algae species. In the present work, the methodology is extended to assess the nutrient limitation effect by modifying the MWC+Se medium, progressively decreasing the content of either nitrogen (from ammonia or nitrate) or phosphate in each of the wells. In this way, specific growth rates were obtained as a function of the concentration of the different nutrients.

\section{Batch experiments}

For assessing the effect of nutrient availability on growth, nutrient uptake and storage, batch experiments were set up using $1 \mathrm{~L}$ wide-neck glass bottles (Duran $®$, Germany) with a multiport system allowing for sample extraction and aeration with $\mathrm{CO}_{2}$ enriched air. Light was supplied from the two sides of the batches using $18 \mathrm{~W}$ fluorescent lamps (GroLux, Sylvania®, Danvers, MA). Dilutions were made with the cultivation medium when the optical density reached the value of 0.4 to avoid self-shading in the culture, thus light inhibition. During the batch experiments the limiting substrates were monitored using Hach-Lange test kits and cell density analysis (APHA, 1995). A control batch was run and monitored parallel to the experiments, yielding an independent data set used for model assessment. 


\section{WWTmod}

Valverde-Pérez et al.

Table 2: a) Gujer matrix of the ASM-A model; b) Process rate equations identified in the ASM-A model:

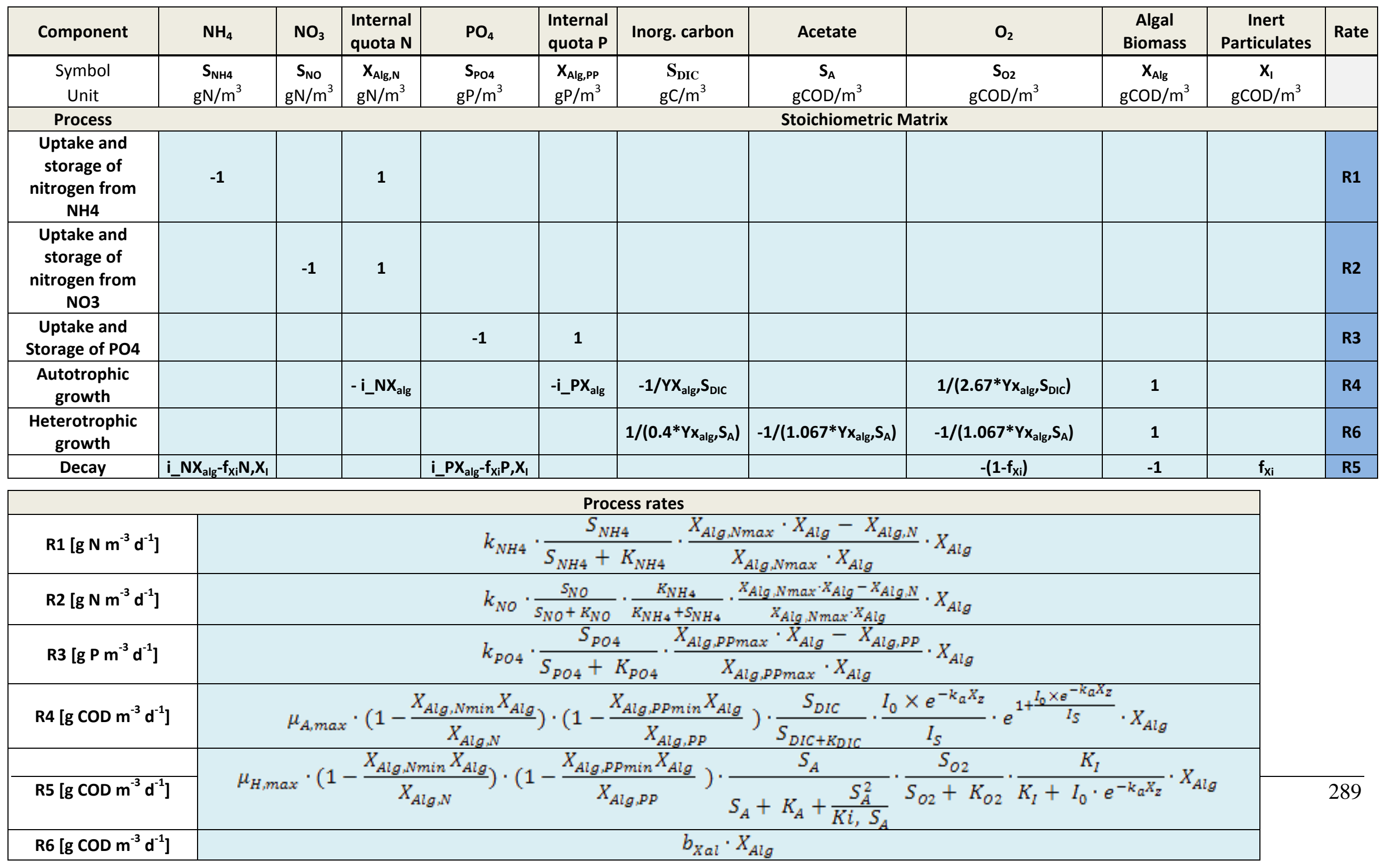




\section{RESULTS AND DISCUSSION}

\section{Model calibration}

Between the six different models of light effect over algal growth, the Steele expression (included in eq. 4) was found to most accurately describe the light dependence of algal growth (Fig. 1). This expression includes the photo-inhibition however, which is not fully-supported by the measured data, and hence further assessment at higher light intensities will be carried out in this project. The maximum microbial growth rate $\left(\mu_{\max }\right)$ is found at an optimal light intensity $\left(\mathrm{I}_{\mathrm{s}}\right)$ of $758.2 \mu \mathrm{mol} \mathrm{m} \mathrm{m}^{-2} \mathrm{~s}^{-1}$, its value being $3.6 \mathrm{~d}^{-1}$.

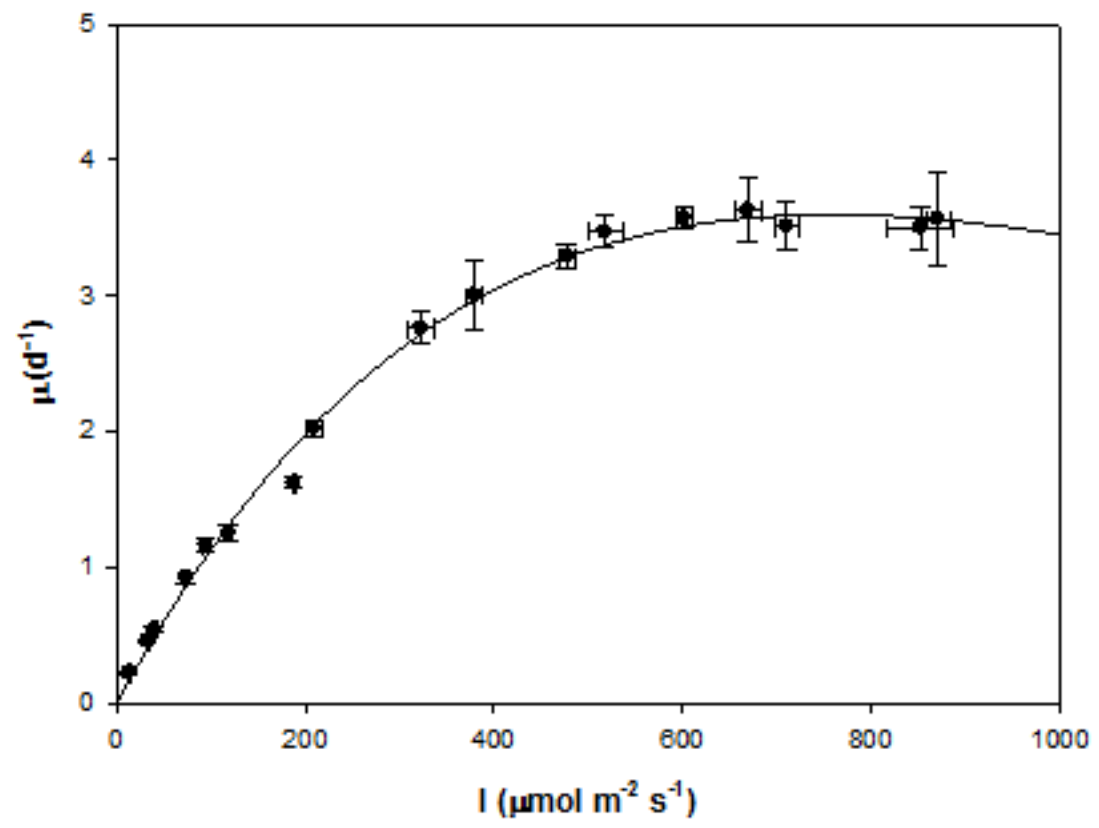

Figure 1: Specific microbial growth rate plotted as function of light intensity according to the Steele equation. Dots represent the value obtained in the micro-batch experiments and the error bars represent the standard deviation on the light intensity and the optical density measured in the micro-batches.

Once the effect of light over algal growth was defined, we conducted micro-batch and batch experiments for model parameter estimation. The nutrient limitation of the microalgae was assessed in both micro-batch and batch experiments. Table 3 shows that results obtained in micro-batch and $1 \mathrm{~L}$ batch experiments are comparable. Van Wagenen et al. (2014) also reports that the parameters describing the light effect over algal growth estimated through micro-batch experiments were comparable to those obtained at larger scale. Therefore, it can be concluded that micro-batch experiments are an efficient tool to estimate the effect of different substrates on microbial growth of micro-algae, saving time and costs.

Table 3: Parameters estimated in micro-batch ( $2 \mathrm{ml})$ and batch (1 1 l) experiments:

\begin{tabular}{|c|c|c|}
\hline Parameter & Microbatch & Batch \\
\hline $\boldsymbol{\mu}_{\text {A,max }}$ & 3.6 & 3.25 \\
\hline $\mathbf{K}_{\text {NH3,Alg }}$ & 0.5 & 0.88 \\
\hline $\mathbf{K}_{\text {NO3,Alg }}$ & - & 3.39 \\
\hline $\mathbf{K}_{\mathbf{P O 4 , A l g}}$ & 0.1 & 0.25 \\
\hline
\end{tabular}


The complete parameter set of the ASM-A, including among others the minimum and maximum internal cell quota for nitrogen and phosphorous, the specific uptake rates and the decay rate, are presented by Wágner et al. (2014).

\section{Model evaluation}

Simulations were carried out using the Matlab implementation of the ASM-A model. A reference parameter set was derived from micro-batch and batch experiments carried out in this study and some parameters were taken from literature (Wágner et al., 2014). The model was calibrated and the prediction was compared with an independent data set from batch experiments. Two cycles were modelled including the point of dilution at the third day, which was done to avoid self-shading effect. Results are shown in Fig. 2:
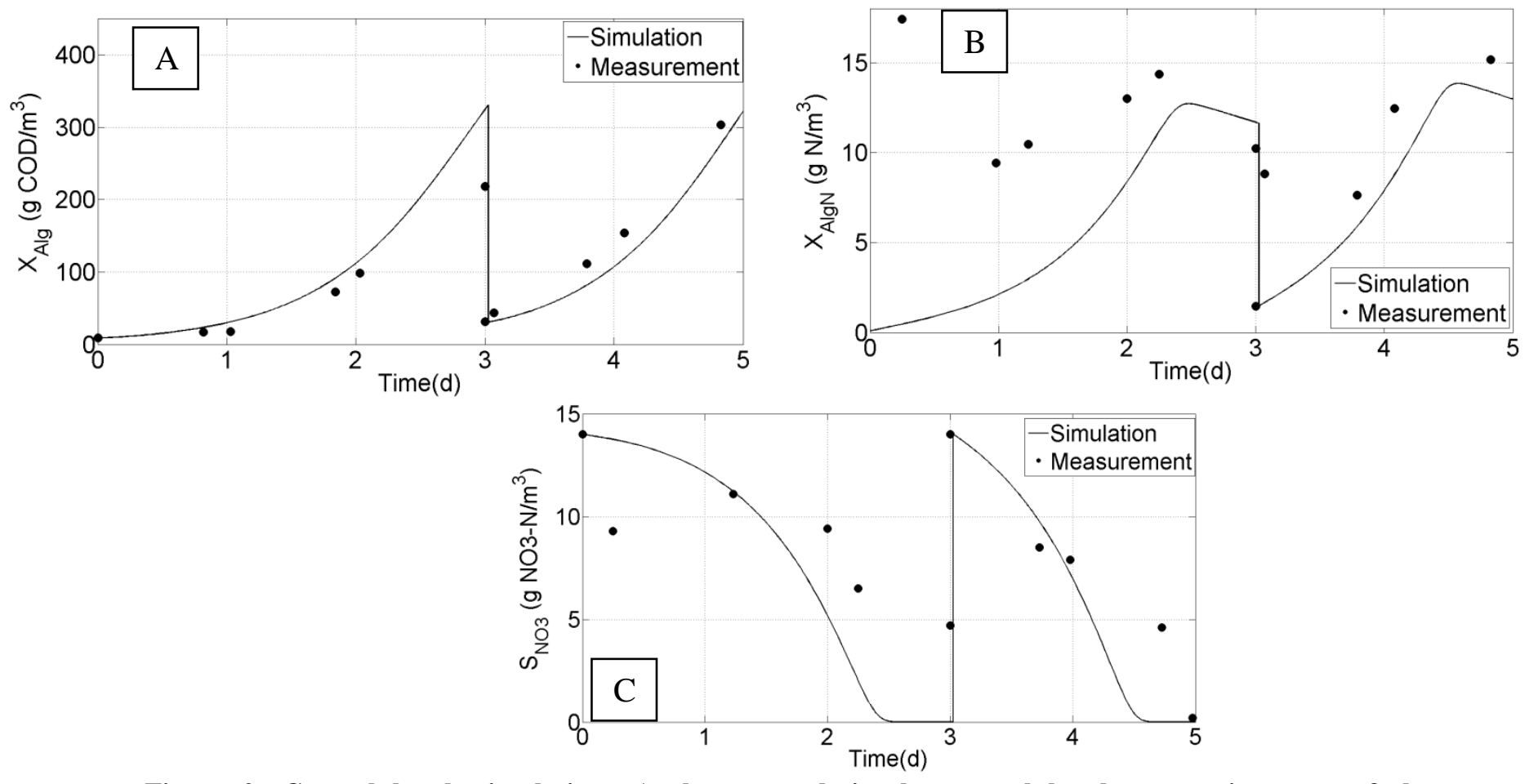

Figure 2: Control batch simulation. a) algae growth in the control batch reactor,in terms of the concentation of algae biomass; b) internal nitrogen storage of algae, in terms of the nitrogen quota; c) bulk nitrate concentration.

According to Fig. 2, ASM-A can predict the algal biomass growth reasonably well during the batch experiment (Fig. 2 a). In these simulations it is assumed that light intensity is constant during the experiments and thus the entire photo-bioreactor is exposed to an average light intensity $\left(\mathrm{I}_{\mathrm{av}}\right)$ measured at the beginning of the experiment. However, since the biomass concentration increases in the batch, the light availability decreases due to self-shading by the algae. The discrepancies between measured and simulated data in Fig. 2, indicate the importance of using a more realistic model describing the light absorption in PhBR. In Fig. 2 $\mathrm{b}$, the prediction of the nitrogen content in biomass shows some limitations at low concentrations. The model predicts well the second cycle of the measurement, however underestimates the measured values in the first cycle. The bulk liquid concentration of nitrate (Fig. 2 c) is reasonably well described by the model. 


\section{CONCLUSION}

In this study, a process model for green micro-algal growth has been identified and developed using the systematic approach of the activated sludge models. The model accurately describes the micro-algal growth under constant light intensity within the culture. In order to improve the estimation of the biomass concentration, the integration of a model for light distribution in $\mathrm{PhBR}$ is needed. The model prediction of nitrogen uptake and storage process needs further improvement. Therefore, our future research activities will focus on the development of a better understanding of the internal nutrient storage in algae in order to reduce the prediction uncertainty derived from the identified model structure.

\section{ACKNOWLEDGEMENT}

D.S. Wágner and B. Valverde-Pérez have contributed equally to the content of this paper. This work was founded by the European Commission, E4WATER Project, FP7-NMP2011.3.4-1 grant agreement 280756 and the InWaTech collaborative agreement between KAIST and DTU.

\section{REFERENCES}

Ambrose R.B. (2006). "Wasp7 benthic algae-model theory and users guide." USEPA, Office of research and development. Athens, Georgia.

APHA (1995) American Public Health Association. Standard Methods for the Examination of Water and Wastewater; APHA: Washington DC, 1995.

Bougaran, G., Bernard, O. and Sciandra, A., (2010). "Modeling continuous cultures of microalgae colimited by nitrogen and phosphorus." Journal of Theoretical Biology, 265, 443-454.

Bouterfas, R., Belkoura, M., Dauta, A. (2002). "Light and temperature effects on the growth rate of three freshwater algae isolated from a eutrophic lake." Hydrobiologia. 489, 207-217.

Brennan, L. and Owende, P. (2010) "Biofuels from microalgae- A review of technologies for production, processing, and extractions of biofuels and co-products." Renewable and Sustainable Energy Reviews, 14, 557-577.

Broekhuizen N., Park J.B.K., McBride G.B. and Craggs, R.J (2012). "Modification, calibration and verification of the IWA River Water Quality Model to simulate a pilot-scale high rate algal pond." Water Research 46, 2911-2926.

Cheng K.C., Ogden K.L. (2011) Algal Biofuels: the research. American Institute of chemical Engineers (AIChE). http/www.aiche.org

Clarens A.F., Ressurreccion E.P., White M.A., Colosi, L.M. (2010). "Environmental life cycle comparison of algae to other bioenergy feedstocks." Environmental Science and Technology 44, 1813-1819.

Decostere, B., Janssens, N., Alvarado, A., Maere, T., Goethals, P., Van Hulle, S.W.H. and Nopens, I. (2013). “A combined respirometer-titrimeter for the determination of microalgae kinetics: experimental data collection and modelling." Chemical Engineering Journal, 222, 85-93.

Droop M.R. (1973). "Some thoughts on nutrient limitation in algae." Journal of Phycology 9 (3), 264-272.

Flores-Alsina X., Gernaey K.V. and Jeppsson U. (2012). "Benchmarking biological nutrient removal in wastewater treatment plants: influence of mathematical model assumptions." Water Science and Technology, 65(8), 1496-1505.

Grima E.M., Camacho F.G., Perez J.A.S., Sevilla J.M.F., Fernandez F.G.A. and Gomez A.C. (1994). “A mathematical model for microalgal growth in light-limited chemostat culture.” Journal of Chemical Technology and Biotechnology 61, 167-173.

Guest, J.S., van Loosdrecht, M.C.M., Skerlos, S.J., and Love, G.N., (2013). "Lumped pathway metabolic model of organic carbon accumulation and mobilization by the alga Chlamydomonas reinhardtii."Environmental Science \& Technology, 47, 3258-3267.

Guillard R.R.L. and Lorenzen C.J. (1972). "Yellow-Green algae with chlorophyllide.” Jounral of Phycology. 8 (1), 10-14

Henze M., Gujer W., Mino T., Matsuo T., Van Loosdrecht M.C.M. (2000). “Activated Sludge Models ASM1, ASM2, ASM2d and ASM3." London: IWA Publishing.

Huesemann, M.H., Van Wagenen, J., Miller, T., Chavis, A., Hobbs, S., and Crowe, B., (2013). “A screening model to predict microalgae biomass growth in photobioreactors and raceway ponds." Biotechnology and 
bioengineering, 110 (6), 1583-1594.

Lundquist, T., Woertz, I., Quinn, N., and Benemann J. (2010). “A realistic technology and engineering assessment of algae biofuel production.” Energy Biosciences Institute 1-178.

Mata, T.M., Martins, A.A. and Caetano, N.S. (2010). "Microalgae for biodiesel production and other applications: a review." Renewable and Sustainable Energy Reviews, 14, 217-232.

Moya, M.J., Sánchez-Guardamino, M.L., Vilavella, A., and Barberá, E. (1997). "Growth of Haematococcus lacustris: A Contribution to Kinetic Modelling." Journal of Chemical Technology and Biotechnology, 68, 303-309.

Pittman, J.K., Dean, A.P., and Olumayowa, O. (2011). "The potential of sustainable algal biofuel production using wastewater resources.” Bioresource Technology 102, 17-25.

Quinn J., de Winter L., Bradley T. (2011). "Microalgae bulk growth model with application to industrial scale systems." Bioresource Technology 102, 5083-5092.

Richmond A. (2004). "Handbook of Microalgal Culture Biotechnology and Applied Phycology." Blackwell Publishing: Oxford.

Samorí, G., Samorí, C., Guerrini, F., and Pistocchi, R. (2013). "Growth and nitrogen removal capacity of Desmodesmus communis and of natural microalgae consortium in a batch culture system in view of urban wastewater treatment: Part 1." Water Research 47,791-801.

Skjelbred B, Edvardsen B, \& Andersen T (2012). "A high-throughput method for measuring growth and loss rates in microalgal cultures." Journal of Applied Phycology, 24(6), 1589-1599.

Van Wagenen, J., Holdt, S.L., De Francisci, D., Valverde-Pérez, B., Plósz, B.Gy. and Angelidaki, I. (2014). "Microplates to increase throughput for screening of microalgae growth potential." Submitted to Applied Phycology.

Verstraete W., Van de Caveye P., Diamantis V. (2009). "Maximum use of resources present in domestic " used water"." Bioresource Technology 100(23), 5537-554.

Wágner, D.S., Valverde-Pérez, B., Sæbø, M., Van Wagenen, J., Angelidaki, I., Smets, B.F., and Plósz, B. Gy. "An activated sludge modeling framework for micro-algal growth in photobioreactors (ASM-A)." In preparation.

Wolf G., Picioreanu, C. and van Loosdrecht, M.C.M., (2007). "Kinetic modeling of phototrophic biofilms: the PHOBIA model.” Biotechnology and bioengineering, 97 (5), 1064-1079. 


\title{
ADM1 implementation with an innovative organic matter characterization methodology: extension to organic micropollutants fate
}

Julie Jimenez, Quentin Aemig, Jean-Philippe Steyer, Dominique Patureau

INRA, UR0050, Laboratoire de Biotechnologie de l'Environnement, Av. des Etangs, Narbonne, F-11100, France (E-mail: julie.jimenez@supagro.inra.fr)

\begin{abstract}
Advanced dynamic anaerobic digestion models, such as ADM1, requires both detailed organic matter characterisation and intimate knowledge of the involved metabolic pathways. In the current study, a methodology for municipal sludge characterization previously developed is used to describe two key parameters: biodegradability and bioaccessibility of organic matter. The methodology is based on coupling sequential chemical extractions with 3D fluorescence spectroscopy. The proposed approach showed a strong application potential for reactor design and advanced control of anaerobic digestion processes. In order to complete the modified model, the organic micropollutants fate modeling is considered, since their degradation is strongly linked with the organic matter biodegradation, and addition of micropollutants kinetics terms in the overall model is proposed.
\end{abstract}

Keywords

Modelling; ADM1, organic matter characterization, bioaccessibility, organic micropollutants

\section{INTRODUCTION}

In the current context, where anaerobic digestion (AD) has become a key process for organic matter treatment and energetic valorization, precise control and prediction of process performance is a must-be. Concomitantly to the organic matter degradation and valorization, some Organic MicroPollutants (OMPs) like Polycyclic Aromatic Hydrocarbons (PAHs) have been shown to be removed during $\mathrm{AD}$ and are strongly correlated with organic matter $(\mathrm{OM})$ removal (Barret et al. 2012). During the biological wastewater treatment, the main pathway of degradation of OMPs is the sorption on sludge (Barret et al., 2012). As a consequence, AD of sludge has to deal with the OMPs degradation, above all from a sanitation and regulations point of view. In terms of OM characterisation and degradation prediction, three concepts are of the most relevance for a particulate substrate such as municipal sludge: bioavailability, bioaccessibility and biodegradability. As OMPs sorbed to the particulate evolves with OM, these concepts would impact them. Recently, a methodology based on chemical sequential extractions and 3D fluorescence spectroscopy has been developed for sewage sludge. A successful correlation with bioaccessibility and biodegradability was found and variables from modified Anaerobic Digestion model $\mathrm{n}^{\circ} 1$ (ADM1) have been characterized (Jimenez et al., 2014). In this study, the methodology is applied before and after AD samples. OMPs measurement was done in each organic matter fractions in order to adapt the ADM1 model to the fractionation developed and to propose a first model of OMPs degradation.

\section{MATERIAL AND METHODS}

\section{Organic matter characterization}

Based on the floc definition, the applied sequential extraction (SE) correlates bioaccessibility of sludge organic matter to its chemical accessibility. The obtained fractions were Dissolved Organic Matter (DOM) obtained by centrifugation and filtration at $0.45 \mu \mathrm{m}$, soluble ExoPolymeric Substances (S-EPS), readily bound EPS (RE-EPS) and Humic Like Substances (HLS) obtained by chemical extractions using salt and/or soda of increasing molarity. The 
non extracted (NE) fraction is the non extractible fraction. Extracts were then measured by fluorescence spectroscopy as explained by (Jimenez et al., 2014).

\section{Batch reactors test}

Two biochemical methane potential (BMP) were operated with a secondary sludge from a wastewater treatment plant in France. The methodology followed was the same than in Angelidaki et al., (2009). Concerning OMPs fate, the first BMP reactor (named BMP1) was operated without PAH addition while the second (BMP2) was operated with PAH addition. OMPs which are sorbed into the particulate fractions SEPS, RE-EPS, HSL and NE were measured after solvent extraction (50:50 v:v of hexane/acetone) and high-performance liquid chromatography analysis.

\section{RESULTS}

\section{New implementation of organic matter variables in ADM1}

Concerning the organic matter degradation, the modified ADM1 model used by Jimenez et al. (2014) has been chosen because (i) the limiting step in sludge AD is the hydrolysis (Contois equation) and (ii) two complex substrates with different hydrolysis rates are considered on the two-shaped curve obtained for methane production rate for sludge. The 3D-SE-LPF methodology is used in order to calculate the non biodegradable fraction, the readily and slowly biodegradable fractions through the Partial Least Square model developed by Jimenez et al. (2014). As shown by the authors, DOM, S-EPS and RE-EPS are the most accessible fractions while HLS and NE are the least one. In order to make more easier the OMP degradation model implementation, ADM1 input variables (readily and slowly biodegradable) are replaced by the extracted fractions : S-EPS and RE-EPS as the particulate COD readily bioacessible and HSL and NE as the particulate COD slowly bioaccessible (cf. Figure 1). Applied on the batch reactor test, methane production curve and output fractions were well predicted as shown by figure 1 by calibrating the maximum growth rate of hydrolytic biomass.
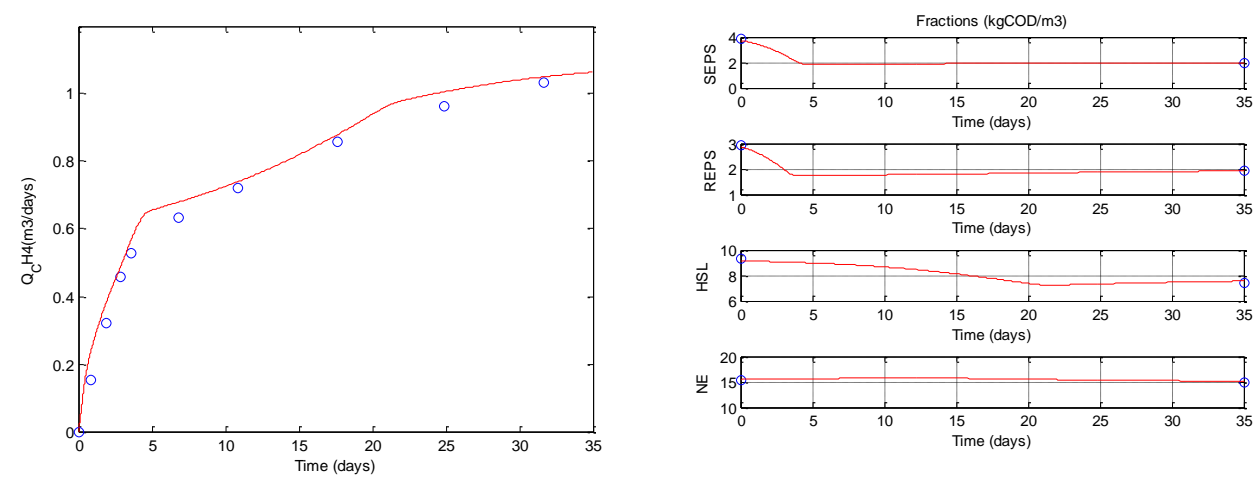

Figure 1. Simulations of methane production and particulate fractions of sludge during BMP tests

\section{OMPs mass balance and model proposition}

Delgadillo-Mirquez (2011) has demonstrated that the OMPs biodegradation are mainly related with the upper biological pathway of anaerobic digestion (hydrolysis and acidogenesis). As a consequence, the bioavailability and bioaccessibility concept of organic matter could be useful for OMPs fate characterization. Consequently, the SE protocol has been applied for OMPs partition assessment into the organic matter compartments in order to estimate their bioavailability. Barret et al. (2012) and Delgadillo (2011) confirmed that the bioavailable fraction is contained in the aqueous phase (free OMPs and sorbed to colloidal matter) and that the bioaccessibility depends on the pollutants sorbed to particles. 
OMPs mass balance obtained during BMP tests are presented in the figure 2 where 3 PAH (Fluorene, Pyrene, Benzo(b)Fluoranthene) from 13 are considered. It appeared that in both BMP1 and BMP2, OMPs degradation was negative in some fractions. One hypothesis would be that during hydrolysis process, OMPs molecules passed from the least accessible fraction to the most one (i.e. DOM). Moreover, in the case of BMP2 where OMPs addition occurred, a bound-residue seemed to be created in the NE from the degradation of OMPs located in HLS.
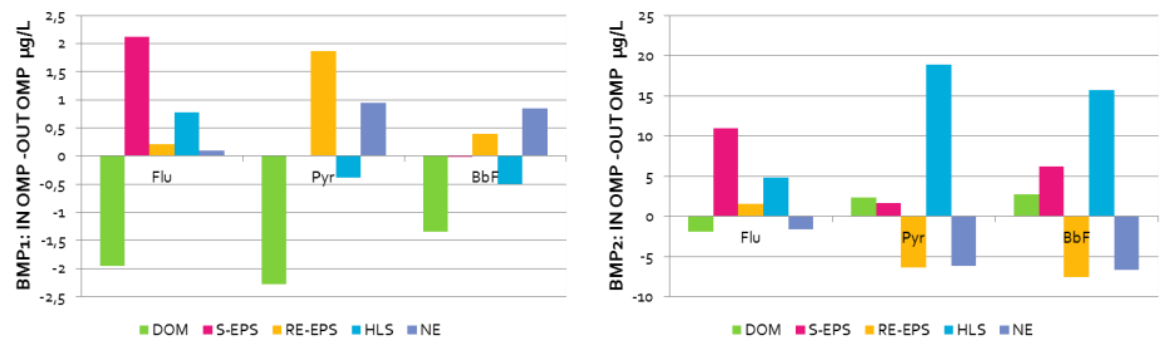

Figure 2. OMPs mass balance during BMP1 and BMP2 tests

Delgadillo et al. (2011) showed that combining bioavailability and cometabolism concepts on a dynamic simplified model allowed the prediction of the OMPs fate in anaerobic digestion. Consequently, OMPs degradation model is proposed (cf. figure 3) based on cometabolism during hydrolysis (with Contois hydrolytic biomass) and during the degradation of the bioavailable fraction DOM into metabolites. Finally, a process of bound residue formation is added.

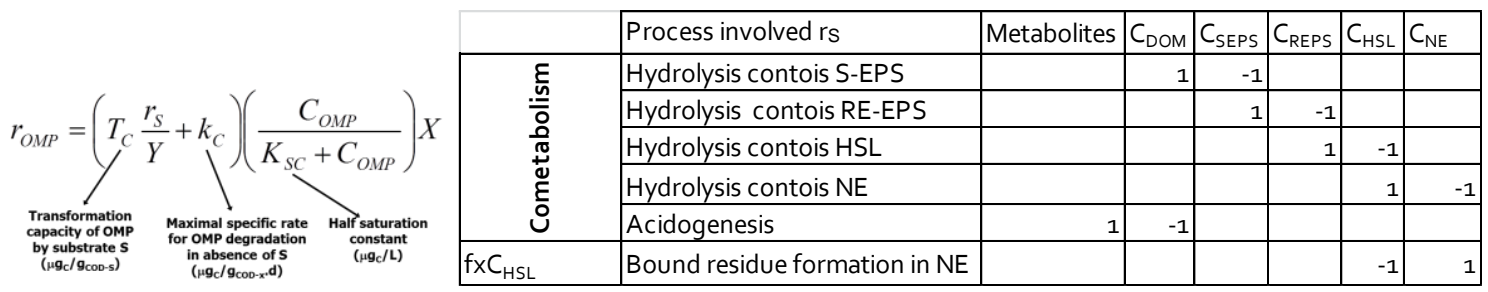

Figure 3. Model proposed for OMPs degradation during anaerobic digestion of sludge

\section{PERSPECTIVES}

Based on previous work, modified ADM1 was able to predict methane production and the different OM particulate fractions simulating bioaccessibility. Concerning OMPs degradation, a new distribution from NE fraction to HLS, RE-EPS, S-EPS and then DOM was observed. Based on these observations, a model was proposed and future data coming from a continuous reactor will provide validation or not of these hypothesis.

\section{REFERENCES}

Angelidaki, I., Alves, M., Bolzonella, D., Borzacconi, L., Campos, J.L., Guwy, A.J., Kalyuzhnyi, S., Jenicek, P. and van Lier, J.B., (2009). Defining the biomethane potential (BMP) of solid organic wastes and energy crops: a proposed protocol for batch assays. Water Science and Technology 59(5), 927-934.

Barret M, Delgadillo L, Trably E, Delgénès N, Braun F, Cea-Barcia G, Steyer J P, and Patureau D, (2012) Anaerobic Removal of Trace Organic Contaminants in Sewage Sludge: 15 Years of Experience. Pedosphere 22, 508-517.

Delgadillo-Mirquez, L., Lardon, L., Steyer, J-P., Patureau, D., (2011) A new dynamic model for bioavailability and cometabolism of micropollutants during anaerobic digestion. Water Research 45 (15), 4511-21.

Jimenez, J., Gonidec, E., Cacho Rivero, J. A., Latrille E., Vedrenne, F., Steyer, J.-P. (2014) Prediction of anaerobic biodegradability and bioaccessibility of municipal sludge by coupling sequential extractions with fluorescence spectroscopy: towards ADM1 implementation. Water Research 50, 359-372. 


\title{
A design-oriented stability analysis of anaerobic co- digestion using ADM1
}

\author{
Sherri Cook $^{1}$, Steven Skerlos ${ }^{1,2}$, and Nancy Love ${ }^{1}$ \\ ${ }^{1}$ Department of Civil and Environmental Engineering, University of Michigan, 1351 Beal Ave, Ann Arbor, \\ MI, 48109, USA (Email: cooksm@umich.edu;nglove@umich.edu) \\ ${ }^{2}$ Department of Mechanical Engineering, University of Michigan, 1301 Beal Ave, Ann Arbor, MI, 48109, \\ USA (Email: skerlos@umich.edu)
}

\begin{abstract}
Co-digestion is the anaerobic digestion of several types of waste and can be used to increase resource recovery. However, co-digestion can exacerbate a digester's tendency to become unstable and stop working. In addition, the quantitative relationship between a diverse set of waste combinations and codigester stability is largely unknown. To address this concern, we created a stability assessment that employs the Anaerobic Digestion Model No. 1 (ADM1) and stability metrics, such as alkalinity concentration, in order to identify influent characteristics that achieve stable operation. The characteristics of particulate-only influent compositions that support stable digester performance have been identified. Also, current design guidelines, which have enabled the effective design and operation of sludge digesters, have been found to be too limited when applied to codigestion systems. Overall, this work contributes to the establishment of case-specific digestion guidelines, which can include re-defining design guidelines for anaerobic co-digestion.
\end{abstract}

\section{Keywords}

anaerobic co-digestion, ADM1, stability, resource recovery

\section{INTRODUCTION \& MODELING APPROACH}

While anaerobically co-digesting several types of wastes can increase the recovery of resources, it can also reduce digester stability and lead to higher costs and lower reliability. Therefore, we developed a digester stability assessment that can be used to establish influent guidelines for codigestion and to improve resource recovery from waste without compromising digester stability.

\section{Stability Index}

Stability indicators are chemical and biological compounds that can indicate if a digester is stable or unstable (Boe et al., 2010; Ferrer et al., 2010; Schoen et al., 2009; Switzenbaum et al., 1990). These stability indicators can be used to compare simulated digester effluent concentrations against values expected for a stable codigestion system. For each indicator, a digester's simulated effluent was compared to the indicator's concentration range in order to rate the digester's stability based on that indicator (e.g., highly stable, borderline stable, or unstable). For example, a stable $\mathrm{pH}$ range was assumed to be $6.1-8.3$, and a digester with a $\mathrm{pH}$ of 6.2 was considered less stable than a digester with a $\mathrm{pH}$ of 7 . Table 1 compiles the stability indicators used in this research along with their target concentration ranges, as found in the literature. Next, each indicator was assigned a weight to represent that indicator's ability to describe a digester's overall stability, which is evaluated by assessing its link to the anaerobic microbial food web, the validity of its concentration range, and its independence from other indicators. Finally, a weighted sum of all the indicators' ratings was calculated to represent the digester's overall stability. 
Table 1. Stability indicators' stable concentraiton ranges and weights used in the stability index.

\begin{tabular}{|c|c|c|c|c|c|}
\hline Indicator & Units & Minimum & Maximum & Weight & Citations \\
\hline $\begin{array}{l}\text { Methane Biogas } \\
\text { Composition }\end{array}$ & $\%$ (by volume) & 55 & $n / a$ & 1 & $\mathrm{AB}$ \\
\hline pH & $(--)$ & 6.1 & 8.3 & 1 & $\mathrm{ABC}$ \\
\hline Alkalinity & $m g \mathrm{CaCO} / \mathrm{L}$ & 2,000 & 20,000 & 1 & BDE \\
\hline Free ammonia & $m g N H 3-N / L$ & $n / a$ & 150 & 1 & BGI \\
\hline $\begin{array}{c}\text { Chemical Oxygen } \\
\text { Demand (COD) } \\
\text { Removal } \\
\end{array}$ & $\%$ (by concentration) & 45 & $n / a$ & 1 & $\mathrm{BL}$ \\
\hline $\begin{array}{c}\text { Volatile Fatty Acids } \\
\text { (VFA) to Alkalinity } \\
\text { Ratio }\end{array}$ & $\begin{array}{c}\text { mg acetate } \\
\text { equivalent/( } m g \\
\left.\mathrm{CaCO}_{3} \text { equivalent }\right)\end{array}$ & $n / a$ & 0.4 & 0.5 & $\mathrm{JK}$ \\
\hline Long Chain Fatty Acids & $m g C O D$ as $L C F A / L$ & $n / a$ & 1,400 & 0.5 & $\mathrm{H}$ \\
\hline $\mathbf{V F A}_{\mathrm{C} 2-\mathrm{C} 5}$ & $\begin{array}{c}m g \text { COD as C2-C5 } \\
V F A s / L\end{array}$ & $n / a$ & 3,700 & 0.5 & A \\
\hline Acetate & mg COD as acetate/L & $n / a$ & 850 & 0.5 & $\mathrm{AF}$ \\
\hline
\end{tabular}

$\mathrm{A}=$ (Ferrer et al., 2010); $\mathrm{B}=($ Tchobanoglous et al., 2003); $\mathrm{C}=$ (Grady et al., 2011); $\mathrm{D}=($ Astals et al., 2012); $\mathrm{E}=($ Alvarez et al., 2010); $\mathrm{F}=$ (Hill et al., 1987); $\mathrm{G}=($ Wang et al., 2012); $\mathrm{H}=($ Neves et al., 2009); $\mathrm{I}=($ Parameswaran and Rittmann, 2012); J=(Schoen et al., 2009); K=(Switzenbaum et al., 1990); L=(Hampton Roads Sanitation District, 2013)

\section{Anaerobic Digestion Simulation \& Uncertainty}

Influent compositions were created to represent a wide range of potential waste combinations, and the digester performance was simulated using the Anaerobic Digestion Model No. 1 (ADM1) (Batstone et al., 2002). In order to represent a large particulate influent space, 10,000 influent compositions were randomly generated using the following criteria: (1) the total organic loading rate (OLR) was within a range of $1-11 \mathrm{~kg} \mathrm{COD} / \mathrm{m}^{3} / \mathrm{d}$, which includes a maximum loading that is about $20 \%$ greater than experimentally determined stable loadings (Björnsson et al., 2000; Ferrer et al., 2010; Westerholm et al., 2012) in order to provide a large range of influent compositions; (2) the loading of carbohydrates, lipids, and proteins were each within a range of $0-11 \mathrm{~kg} \mathrm{COD} / \mathrm{m}^{3} / \mathrm{d}$ in order to represent many different influent composition possibilities; and (3) the loading of inert compounds were within a range of 0 $20 \%$ of the influent's total COD to represent an average inert concentration for a variety of wastes (Zaher et al., 2009).

ADM1 was chosen to simulate the digestion process because it has effectively simulated both experimental and full-scale digesters (e.g., Batstone et al., 2006; Jeppsson, 2007). The differential and algebraic implementation of ADM1 as modified by Rosén and Jeppsson (2006) was used along with ADM1's default biochemical and physicochemical parameters for mesophilic digestion. In addition, alkalinity concentration was estimated from ADM1 outputs using the activated sludge model interface calculations (Nopens et al., 2009). The simulated digester had a volume of $3,400 \mathrm{~m}^{3}$ and perfect mixing was assumed. The temperature was set 


\section{WWTmod}

at 35 Celsius, and the retention time was 20 days. All simulations were run for 1,000 simulated days in order to represent steady-state operation. 


\section{RESULTS AND DISCUSSION}

The value of the stability index was examined by comparing the performance of full-scale and lab-scale digesters with the estimated stability assessment (i.e., stability index rating). Data from 4 full-scale domestic wastewater sludge digesters with stable, long-term operation (Hampton Roads Sanitation District, 2013) were evaluated using the stability index, and the stability index rating was representative of the full-scale digester's stable performance. In addition, data from two lab-scale codigestion reactors, one reactor codigesting domestic wastewater sludge and potato processing industrial waste and the other codigesting manure, slaughterhouse and agricultural waste (Murto et al., 2004), were also evaluated, and the stability index rating was also representative of the experimental codigesters' performance. Testing the stability index calculations with full- scale and lab-scale stable digesters showed the ability of the stability index to estimate stable digester performance. In addition, the stability indicator ranges that are most important to the stability estimation were identified by conducting a Monte Carlo assessment. This assessment found that the minimum percent methane biogas composition, maximum long chain fatty acids (LCFA) concentration, and minimum percent COD removal were all significantly (p-value $<0.5)$ and strongly $(|\rho|>=0.7)$ correlated with overall digester stability for at least one simulated influent composition. More information about the stability bounds for these indicators can improve insight about digester stability.

Wide-ranging influent compositions were simulated using ADM1 and evaluated using this stability index in order to identify a digester's stable influent space, which is comprised of the influent compositions that result in stable operation. Figure 1 shows a digester's stable influent space for particulate influent compositions. In addition, Figure 1 suggests that there are key digester operational considerations. For example, it shows that instability is likely at high OLRs, and this trend is reinforced with experimental data from the literature that shows digester overloading is possible with OLRs greater than $5 \mathrm{kgCOD} / \mathrm{m} 3 / \mathrm{d}$ (Björnsson et al., 2000; Ferrer et al., 2010; Gomez et al., 2006; Grady et al., 2011; Kusowski et al., 2013). Also, the simulations show that there may be a minimum nitrogen requirement for digester stability. The main reason for digester instability in this minimum nitrogen region is due to a lack of biomass growth, as indicated by the total biomass concentration predicted by ADM1. This minimum requirement is around a protein loading of $1 \mathrm{~kg} \mathrm{COD} / \mathrm{m}^{3} / \mathrm{d}$, which equates to a minimum nitrogen loading of $7.5 \mathrm{~mole} / \mathrm{m}^{3} / \mathrm{d}$. Overall, this stable influent space highlights a relationship between influent composition and digester stability that matches current knowledge about stable digester performance under steady-state conditions. Also, it provides the opportunity to inform design guidelines and the selection of wastes in order to achieve reliable anaerobic codigestion.

The applicability of conventional design guidelines to codigestion was investigated by comparing recommended influent guidelines with the modeled stable influent space. The OLR design recommendations is $1-5 \mathrm{~kg} \mathrm{COD} / \mathrm{m}^{3} / \mathrm{d}$ for continuously-stirred tank reactors (Grady et al., 2011). When comparing this recommendation with the stable influent space, the simulations suggests that many stable influents can have an OLR larger than the recommended range. Stable digester operation at OLRs greater than $5 \mathrm{~kg} \mathrm{COD} / \mathrm{m} 3 / \mathrm{d}$ is also seen experimentally (Björnsson et al., 2000; Gomez et al., 2006; Kusowski et al., 2013). Overall, modeling results and experimental data from the literature suggest that the OLR recommendation may be too conservative. 
Another main design guideline is the influent carbon-to-nitrogen $(\mathrm{C} / \mathrm{N})$ ratio. The very general ratio range is 10-90:1 (Wang et al., 2012), with an stated optimum usually around 2030:1 (Stroot et al., 2001; Wang et al., 2012). Data from the experimental literature (Astals et al., 2012; Stroot et al., 2001; Wang et al., 2012; Wu et al., 2010) suggest that the C/N ratio range for stable digestion is based on a specific influent composition, and the stability modeling results also suggest that the $\mathrm{C} / \mathrm{N}$ ratio may have limited applicability. The $\mathrm{C} / \mathrm{N}$ ratio recommendation may be too specific to a type of waste and too focused on an optimal range to provide a good correlation between a large range of influent compositions and general reactor stability.

(a)

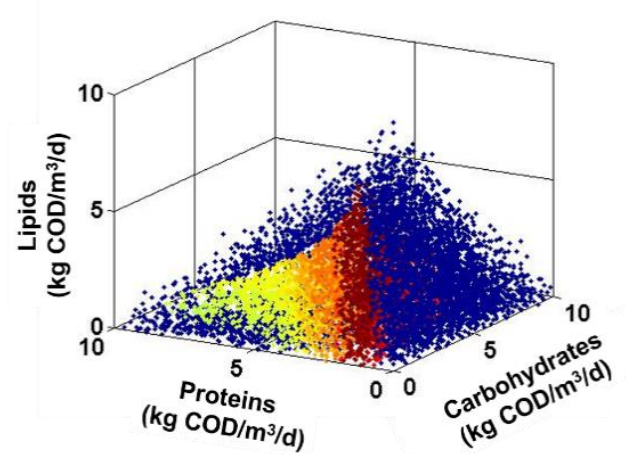

(c)

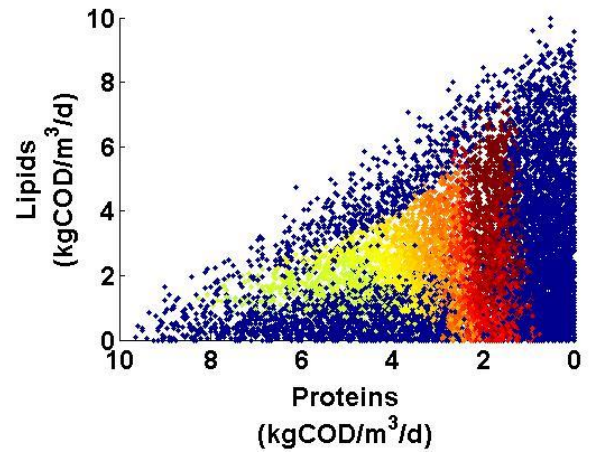

(b)

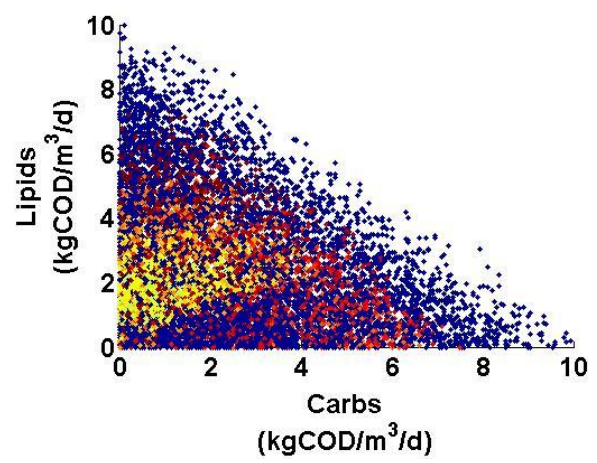

(d)

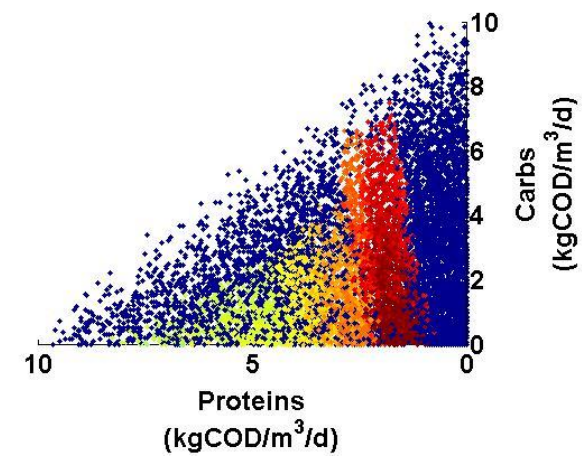

Stability Index Key

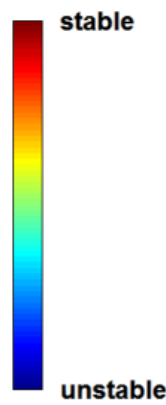

Figure 1. Particulate influent compositions and their relationship to digester stability: (a) 10,000 particulate influent compositions (inert substrate loading not shown); (b-d) influent compositions (each representing a varying concentration of 4 substrates) projected onto 2 substrate loading axes.

\section{CONCLUSIONS}

This work shows that a stable influent space can be estimated using ADM1 and this work's stability index. Also, it shows that a digester's stable influent space for particulate influents may be larger than current design guidelines suggest. Codigestion design guidelines that use substrate composition data (i.e., characterization of carbohydrates, lipids, proteins, etc.), include higher OLRs, and are more inclusive of a diverse range of substrates would be more helpful to improve resource recovery without compromising stability.

\section{ACKNOWLEDGEMENT}


The first author was supported by fellowships from the University of Michigan's Graham Sustainability Institute and Rackham Graduate School.

\section{REFERENCES}

Alvarez, J. a, Otero, L., Lema, J.M., 2010. A methodology for optimising feed composition for anaerobic codigestion of agro-industrial wastes. Bioresour. Technol. 101, 1153-8.

Astals, S., Nolla-Ardèvol, V., Mata-Alvarez, J., 2012. Anaerobic co-digestion of pig manure and crude glycerol at mesophilic conditions: biogas and digestate. Bioresour. Technol. 110, 63-70.

Batstone, D.J., Keller, J., Angelidaki, I., Kalyuzhnyi, S. V., Pavlostathis, S.G., Rozzi, A., Sanders, W.T.M., Siegrist, H., Vavilin, V.A., 2002. IWA Anaerobic Digestion Model No. 1 (ADM1). IWA Publishing, London.

Batstone, D.J., Keller, J., Steyer, J.P., 2006. A review of ADM1 extensions, applications, and analysis: 20022005. Water Sci. Technol. 54, 1.

Björnsson, L., Murto, M., Mattiasson, B., 2000. Evaluation of parameters for monitoring an anaerobic codigestion process. Appl. Microbiol. Biotechnol. 54, 844-849.

Boe, K., Batstone, D.J., Steyer, J.P., Angelidaki, I., 2010. State indicators for monitoring the anaerobic digestion process. Water Res. 44, 5973-5980.

Ferrer, I., Vázquez, F., Font, X., 2010. Long term operation of a thermophilic anaerobic reactor: process stability and efficiency at decreasing sludge retention time. Bioresour. Technol. 101, 2972-2980.

Gomez, X., Cuetos, M., Cara, J., Moran, A., Garcia, A., 2006. Anaerobic co-digestion of primary sludge and the fruit and vegetable fraction of the municipal solid wastes: conditions for mixing and evaluation of the organic loading rate. Renew. Energy 31, 2017-2024.

Grady, C.P.L., Daigger, G.T., Love, N.G., Filipe, C.D.M., 2011. Biological Wastewater Treatment, 3rd ed. IWA Publishing and Alliance House, London.

Hampton Roads Sanitation District, 2013. Yearly Plants Operation Report.

Hill, D., Cobb, S., Bolte, J., 1987. Using volatile fatty acid relationships to predict anaerobic digester failure. Trans. ASABE 30, 496-501.

Jeppsson U. (2007) Investigation of Anaerobic Digestion Alternatives for Henriksdal's WWTP - Supplement. Lund University. Available at: http://ftp.iea.lth.se/publications/Reports/LTH-IEA-7226.pdf.

Kusowski, J., Graziano, S.J., Desing, W.E., Whitlock, D.D., Sigmund, T.W., Zitomer, D.H., 2013. Anaerobic Co-digestion Planning and Research for New Water (Green Bay, WI), in: Proceedings of the Water Environment Federation Technical Exhibition and Conference (WEFTEC). Chicago, IL, pp. 1-20.

Murto, M., Björnsson, L., Mattiasson, B., 2004. Impact of food industrial waste on anaerobic co-digestion of sewage sludge and pig manure. J. Environ. Manage. 70, 101-107.

Neves, L., Oliveira, R., Alves, M.M., 2009. Fate of LCFA in the co-digestion of cow manure, food waste and discontinuous addition of oil. Water Res. 43, 5142-5150.

Nopens, I., Batstone, D.J., Copp, J.B., Jeppsson, U., Volcke, E., Alex, J., Vanrolleghem, P. a, 2009. An ASM/ADM model interface for dynamic plant-wide simulation. Water Res. 43, 1913-1923.

Parameswaran, P., Rittmann, B.E., 2012. Feasibility of anaerobic co-digestion of pig waste and paper sludge. Bioresour. Technol. 124, 163-168.

Rosén C. and Jeppsson U. (2006) Aspects on ADM1 Implementation Within the BSM2 Framework. Lund University. Available at: http://ftp.iea.lth.se/publications/Reports/LTH-IEA-7224.pdf.

Schoen, M. a, Sperl, D., Gadermaier, M., Goberna, M., Franke-Whittle, I., Insam, H., Ablinger, J., Wett, B., 2009. Population dynamics at digester overload conditions. Bioresour. Technol. 100, 5648-5655.

Stroot, P.G., McMahon, K.D., Mackie, R.I., Raskin, L., 2001. Anaerobic codigestion of municipal solid waste and biosolids under various mixing conditions--I. Digester performance. Water Res. 35, 1804-1816.

Switzenbaum, M.S., Giraldo-Gomez, E., Hickey, R.F., 1990. Monitoring of the anaerobic methane fermentation process. Enzyme Microb. Technol. 12, 722-730.

Tchobanoglous, G., Burton, F.L., Stensel, H.D., 2003. Wastewater Engineering: Treatment and Reuse, 4th ed. McGraw Hill, New York, NY.

Wang, X., Yang, G., Feng, Y., Ren, G., Han, X., 2012. Optimizing feeding composition and carbon-nitrogen ratios for improved methane yield during anaerobic co-digestion of dairy, chicken manure and wheat straw. Bioresour. Technol. 120, 78-83.

Westerholm, M., Hansson, M., Schnürer, A., 2012. Improved biogas production from whole stillage by codigestion with cattle manure. Bioresour. Technol. 114, 314-9. 


\section{WWTmod}

Wu, X., Yao, W., Zhu, J., Miller, C., 2010. Biogas and CH(4) productivity by co-digesting swine manure with three crop residues as an external carbon source. Bioresour. Technol. 101, 4042-7.

Zaher, U., Buffiere, P., Steyer, J.-P., Chen, S., 2009. A Procedure to Estimate Proximate Analysis of Mixed Organic Wastes. Water Environ. Res. 81, 407-415. 


\title{
Model-based approach to maximize gas production for high-loaded digestion processes
}

\author{
Ryu Suzuki ${ }^{1}$, Sudhir Murthy ${ }^{1}$, Bernhard Wett $^{2}$ and Imre Takács ${ }^{3}$ \\ ${ }^{1}$ DC Water, 5000 Overlook Ave SW, Washington DC 20010 USA (rsuzuki@ dcwater.com; \\ smurthy@dcwater.com) \\ ${ }^{2}$ ARAconsult, Unterbergerstr.1, A-6020 Innsbruck, Austria \\ ${ }^{3}$ Dynamita, Bordeaux, France
}

\begin{abstract}
A customized Activated Sludge - Anaerobic Digestion Model (ASDM) from BioWin 3.2 by EnviroSim was used to develop a future operational process control strategy for Blue Plains Advanced Wastewater Treatment Plant's (AWTP) anaerobic digesters with

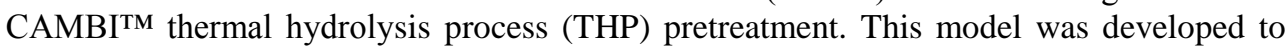
understand the relative importance of free ammonia on gas production and accumulation of volatile fatty acids (VFA). Under any given loading conditions, the operational setpoints of solids retention time (SRT) and free ammonia can be controlled by adjusting the influent solids concentration. This model helps to predict which setpoints would allow for maximum gas production while maintaining digester stability.
\end{abstract}

\section{Keywords}

Modeling; ASDM; anaerobic digestion; ammonia inhibition; thermal hydrolysis

\section{INTRODUCTION}

Anaerobic digesters with CAMBI ${ }^{\mathrm{TM}}$ THP pretreatment are currently being constructed at Blue Plains AWTP located in Washington, DC, United States. Partial solubilization of particulates and disintegration of cells in the THP reduce sludge viscosity, improve bioavailability of organics, and allow the digester to be loaded at higher solids concentrations. However, increased feed concentration and destruction of protein and other complex organic materials also result in free ammonia $\left(\mathrm{NH}_{3}\right)$ levels that are much higher than what is typically seen in conventional mesophilic digesters (Kepp et al., 2001). Free ammonia has inhibitory effects on the methanogens that are responsible for completing the final step in anaerobic digestion systems where acetic acid is converted to methane gas (Eldem, 2005, Wilson, 2009, Wett, 2012). Compared to hydrolysis-limited conventional digestion (Siegrist, 2002, Wilson, 2009), Blue Plains thermal hydrolysis-digestion (THD) is expected to be methanogenesis-limited, and therefore, minimizing the unionized ammonia level becomes a major concern while developing a process control strategy.

One of the key operational controls for Blue Plains is the dilution water feed rate that follows the THP system, which can be used to adjust the digester influent solids concentration, SRT and ammonia concentration. Operating at higher influent concentrations would allow for longer SRT in the digesters but could also result in greater inhibition from higher ammonia concentrations. However, operating at lower solids concentrations could result in lower inhibition but would also reduce digestion time. A calibrated model could assist the plant staff in finding the optimum digester influent concentrations and resulting ammonia concentrations and SRTs that would maximize gas production while maintaining digester stability. 


\section{METHODOLOGY}

\section{Model development}

Various advancements have been made to the original ASDM to simulate the unique impacts of THP and high-loaded digestion process. The effect of THP can be modeled by converting portions of slowly biodegradable $\mathrm{COD}\left(\mathrm{X}_{\mathrm{sp}}\right)$ to readily biodegradable $\mathrm{COD}(\mathrm{rbCOD})$, and active biomass to $\mathrm{X}_{\mathrm{sp}}$, while tracking the release of inert materials and nutrients (Wett et al., 2009). This model was further improved by incorporating ammonia inhibition of aceticlastic methanogens (AM) and alternative degradation pathways through acetic acid oxidizers (ACOX) and hydrogenotrophic methanogens that are more tolerant of higher ammonia levels (Wett et al., 2012). The $\mathrm{NH}_{3}$ inhibition was modeled using the logistic model and the parameters were calibrated using results from bench-scale digesters fed with thermallyhydrolyzed Blue Plains sludge (THD) running in parallel with a conventional mesophilic digester (Wilson et al., 2009). Additionally, the non-ionized form of acetic acid was designated as an inhibitory substrate using the Haldane equation as suggested by Fukuzaki et al. (1990). Detailed descriptions on modeling of THD are provided in previous publications by Wett et al. (2009 and 2012).

The feed sludge characteristics were obtained from a calibrated, full-plant model at Blue Plains. The liquid side treatment at Blue Plains consists of a chemically-enhanced primary treatment (CEPT), a high-rate secondary treatment that is bioaugmented with waste sludge from the nitrification/denitrification (NDN) stage, an NDN system with methanol addition and multimedia filtration. A typical primary sludge (PS) to waste activated sludge (WAS) ratio at Blue Plains is 55/45 but the portion of WAS becomes higher during the winter months when solids decay in the biological reactors slow down.

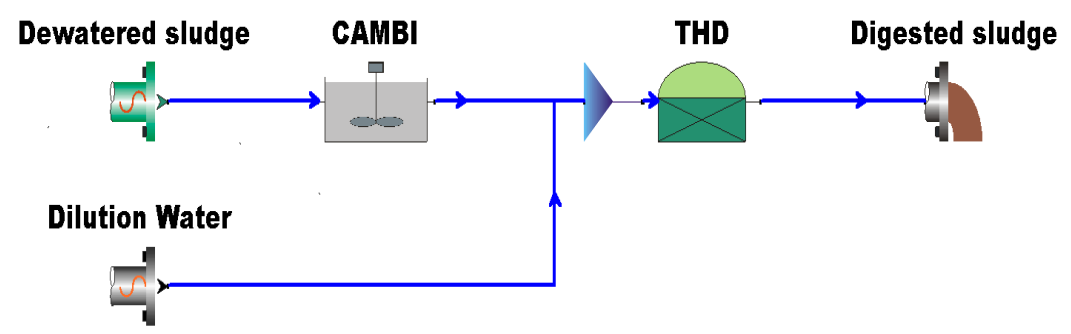

Figure 1. Model configuration in BioWin 3.2.

\section{Experimental Setup}

Three pilot scale digesters were set up to test the effect of varying influent solids concentrations on the digester performance. A blend of PS and WAS dewatered to 25 to $28 \%$ TS at Blue Plains high-solids centrifuge was collected and diluted to approximately 16 to $18 \%$ TS prior to thermal hydrolysis. The pilot thermal hydrolysis system supplied by $\mathrm{CAMBI}^{\mathrm{TM}}$ consisted of a $15 \mathrm{~L}$ reactor, a $50 \mathrm{~L}$ flash tank and a boiler. The reactor was operated at $160^{\circ} \mathrm{C}$ for 30 minutes and flashing pressure of 3.1 bars. TH sludge with concentrations of $10-12.5 \%$ TS was then shipped from Blue Plains to Bucknel University's Anaerobic Digestion Lab to be fed into pilot-scale digesters.

The three digesters were fed at the same solids loading rate of $7 \mathrm{~kg} \mathrm{TS} / \mathrm{m}^{3}$-day but the influent concentrations were adjusted, which in turn determined the target SRT. The operating 
parameters are presented in Table 2. The reactors were operated semi-continuously by batch feeding once a day. The same amount of sludge was withdrawn from the reactor after a 24hour reaction period prior to the next pulse feed. The digester headspace was hooked up to a respirometer to measure the gas production volume and rate. The temperatures inside the reactors were maintained at $38^{\circ} \mathrm{C}$.

Table 1. Operating parameters of the pilot scale digesters

\begin{tabular}{|c|c|c|c|c|}
\hline $\begin{array}{l}\text { Digester } \\
\text { Number }\end{array}$ & $\begin{array}{c}\text { Influent Solids } \\
\text { Concentration } \\
(\% \mathrm{TS})\end{array}$ & $\begin{array}{c}\text { Feed } \\
\text { Rate } \\
\text { (L/day) }\end{array}$ & $\begin{array}{l}\text { Target } \\
\text { SRT } \\
\text { (days) }\end{array}$ & $\begin{array}{c}\text { Solids Loading } \\
\text { Rate } \\
\left(\mathrm{kg} / \mathrm{m}^{3} \text {-day }\right)\end{array}$ \\
\hline 1 & 7.0 & 1 & 10 & 7 \\
\hline 2 & 10.5 & 0.67 & 15 & 7 \\
\hline 3 & 12.5 & 0.56 & 18 & 7 \\
\hline
\end{tabular}

The seed sludge for the digesters came from a previous set of experiments where the impact of $\mathrm{TH}$ reaction temperature was investigated (in preparation). In these experiments, five digesters were fed with sludge from Blue Plains that had been thermally hydrolyzed at reaction temperatures of $130,140,150,160$ and $170^{\circ} \mathrm{C}$. They were operated in the same condition as Digester 2 with influent solids concentration of $10.5 \%$ TS and 15 days SRT. Since these digesters had been operating for close to six months, the anaerobic biomass had been acclimated to high solids conditions and free ammonia concentrations of 130 to 160 $\mathrm{mg} / \mathrm{L}$.
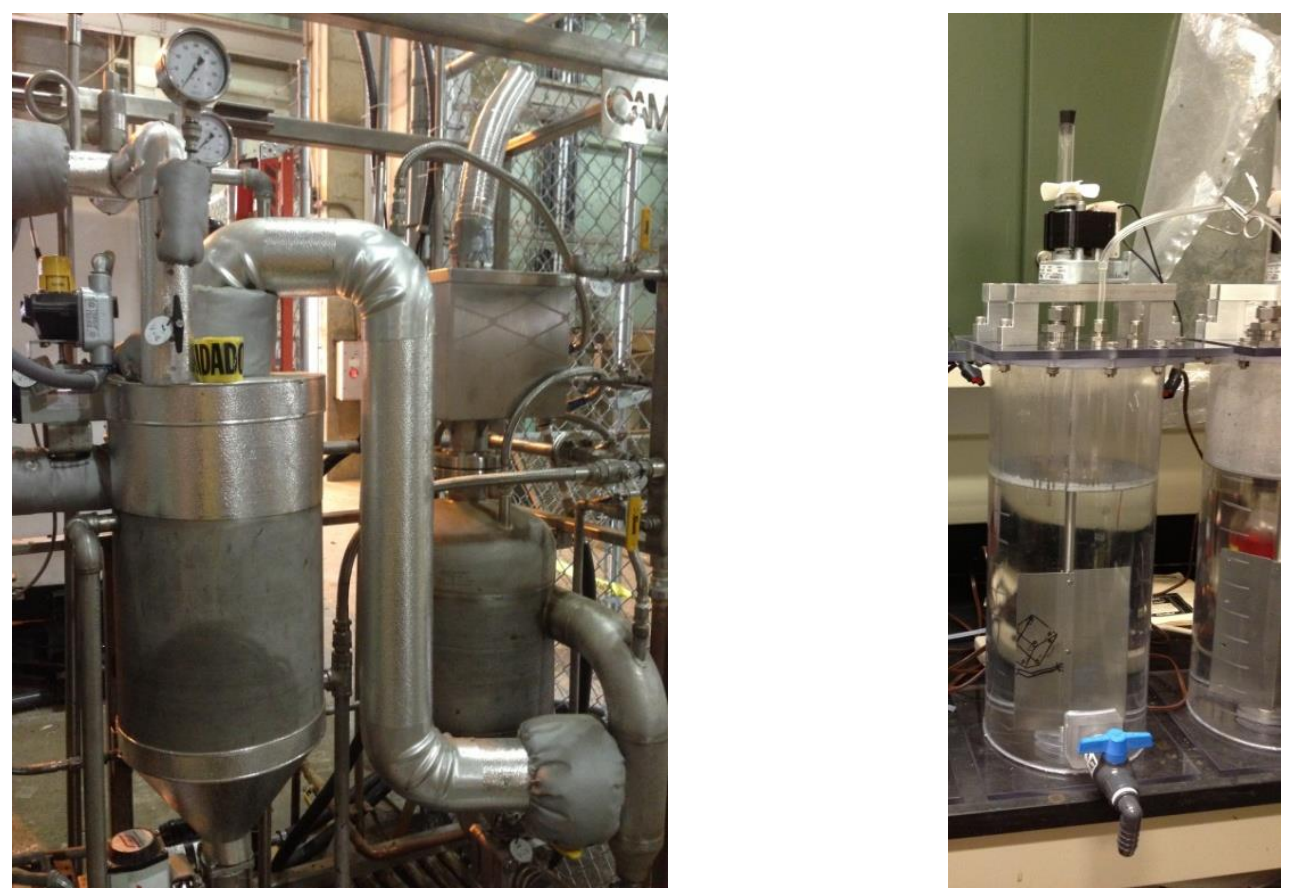

Figure 2. Photos of the pilot TH reactor at Blue Plains [left] and pilot digesters at Bucknell [right].

\section{RESULTS AND DISCUSSION}


Dynamic runs were used to investigate the impact of changing influent concentrations on digester performance. The initial conditions used in the model were assumed to be a steadystate digester that had been operating at 15 days SRT and 10.5\% TS influent. The feed concentrations were adjusted according to Table 1 to simulate the desired SRT.

The measured data and model both show that digester ammonia concentrations can be controlled based on the influent solids concentrations as shown in Figure 3. Even under the same loading rate the total ammonia concentration can be adjusted to from 1,500 to 3,700 $\mathrm{mg} / \mathrm{L}$. The experimental data and model both agreed with a previous study (Kepp et al., 2001) that showed that total ammonia has a stoichiometric relationship with the influent solids concentrations.

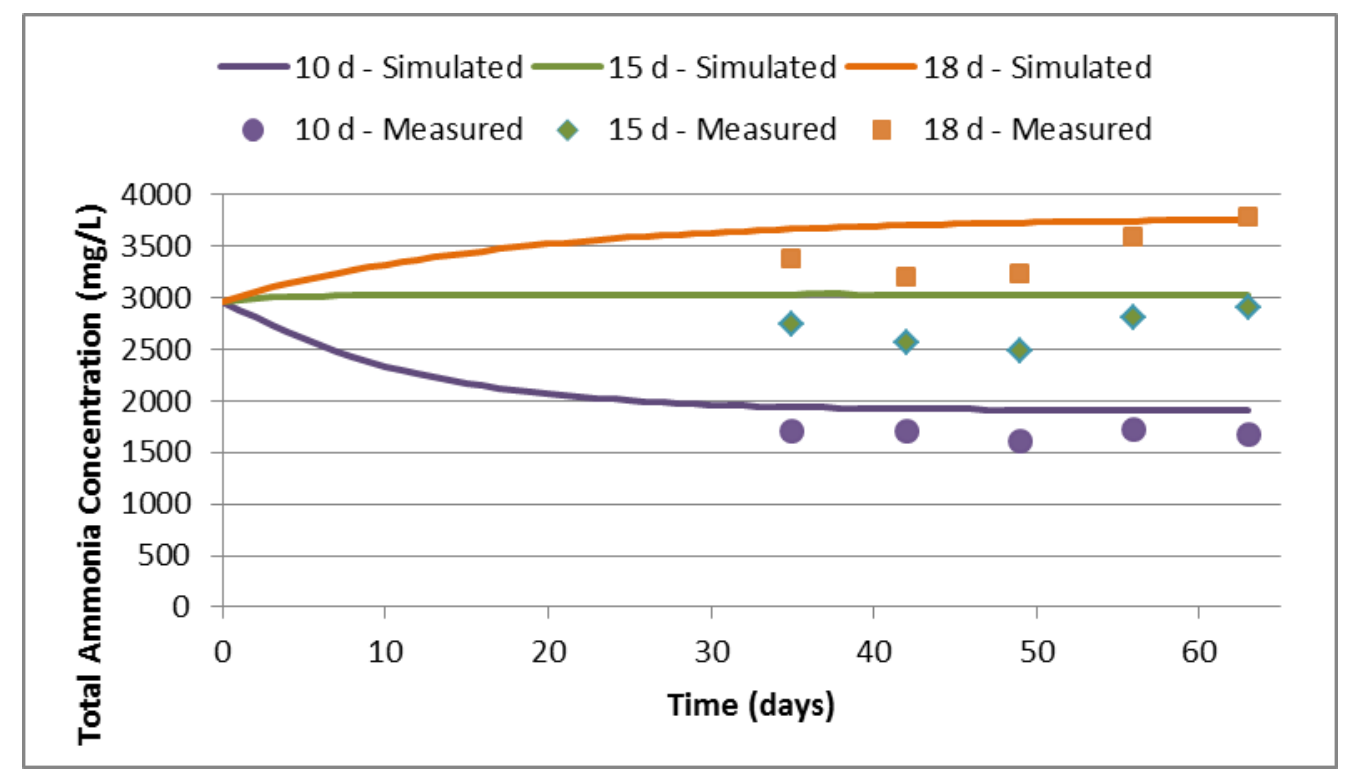

Figure 3. Simulation output and experimental data on the total ammonia concentrations inside the digester.

The model and experimental data also demonstrated that increasing SRT would result in greater specific methane production despite higher ammonia inhibition as shown in Figure 4. The simulation results showed that an increase of even 3 days in the SRT may result in approximately $4 \%$ increase in specific methane production. Our theory is that although THP greatly increases the rbCOD of feed sludge, a large portion of biodegradable COD is still in particulate form and needs to be enzymatically hydrolyzed. That is why the model output showed that total mass of sbCOD was much higher in 10 day SRT compared to 18 day SRT. 


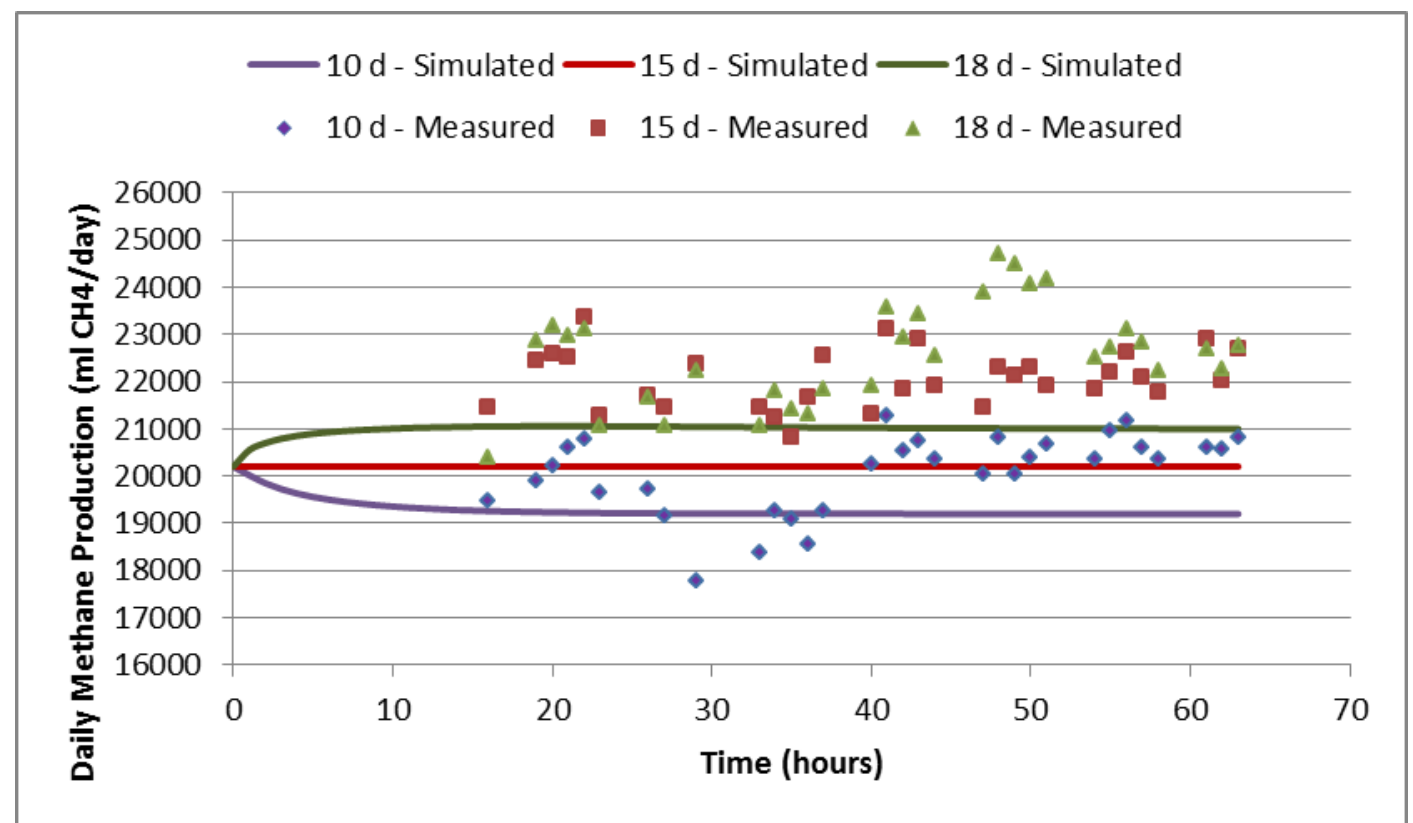

Figure 4. Simulation output compared to experimental results of daily methane production.

However, increasing the ammonia concentration has a negative impact of elevating the VFA levels in the digesters. The current inhibition constants cause the growth rate of methanogens to reduce significantly as the ammonia level reaches what is observed in the digester operated at 18 days SRT. At this point in the experiment, the VFA levels in the 18 days have not reached steady state as the last VFA measurement taken is much higher than the value from the previous week. It is difficult to tell if the VFA levels would increase to the level near what is being predicted by the model.

Another observed model limitation is that the model is predicting that the effect of ammonia inhibition would start to manifest much faster than what the experimental data is indicating. Despite the ammonia level that was increasing quickly in the 18 day SRT reactor, the digester was able to maintain its VFA levels for a longer time than what the model predicted. This may be from the fact that the digester ecology had been exposed to the higher ammonia concentrations for long a time and can withstand a short-term increase in the ammonia level. 


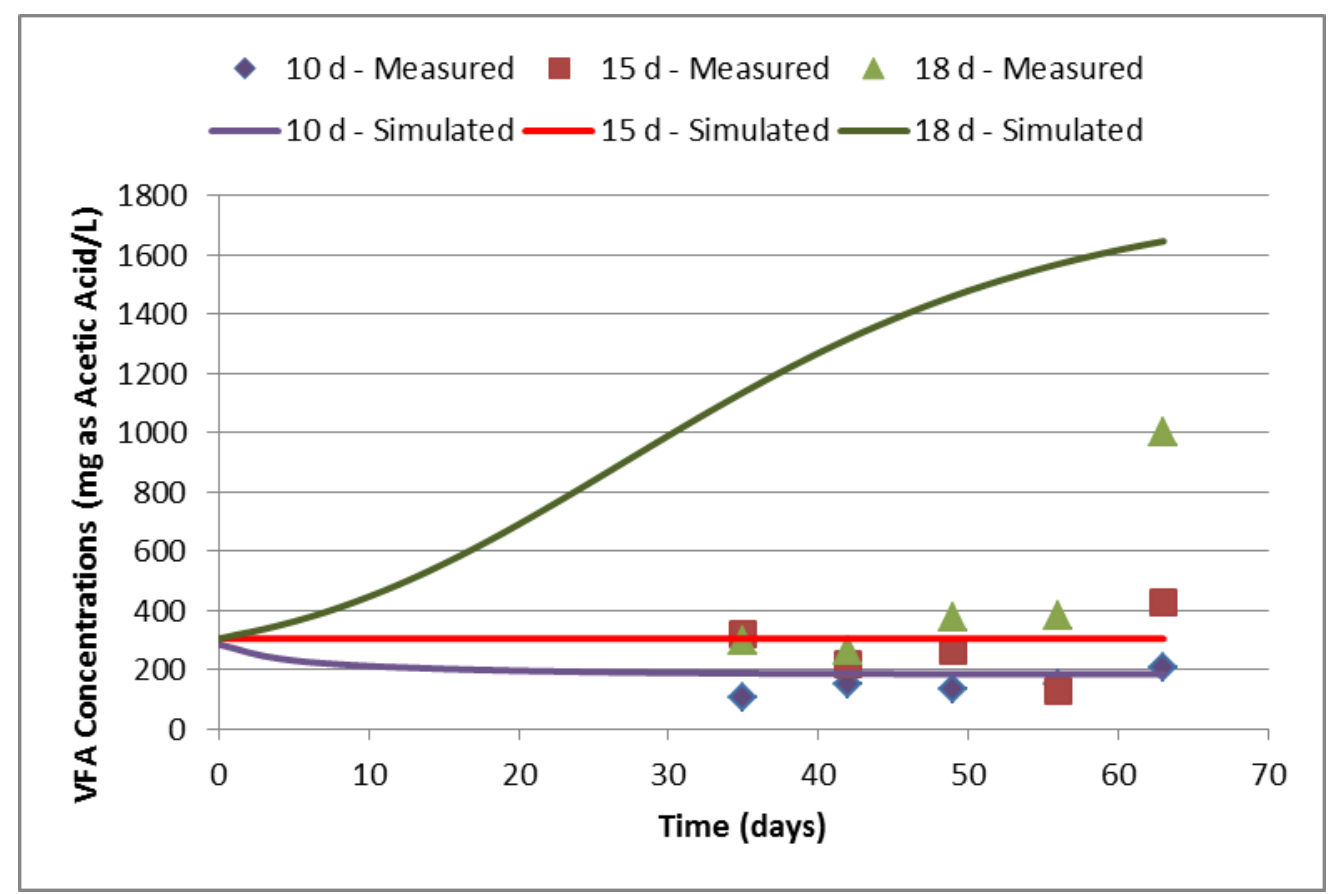

Figure 5. Simulation output compared to experimental results of VFA buildup in digesters.

\section{CONCLUSION}

A model was developed to understand the relative importance of ammonia on gas production and VFA in high-loaded digestion processes. This model helps to predict operational setpoints of SRT and ammonia by controlling the influent solids concentration. Thus, under any given loading conditions, SRT and ammonia can be controlled to maximize the gas production while maintaining digester stability.

\section{REFERENCES}

Eldem, N.O.; Ozturk, I.; Sover, E.; Calli, B.; Akgirav, O., (2005). Ammonia and pH Inhibition in Anaerobic Treatment of Wastewaters, Part I: Experimentation. Toxic/Hazardous Substances and Environmental Engineering; Vol. A39, No. 9, pp. 2405-2420, 2004.

Fukuzaki, S.; Nishio, N.; and Nagai, S. (1990). Kinetics of the Methanogenic Fermentation by Acetate. Applied and Environmental Microbiology. Vol 56, No. 10, 3158-3163.

Gerardi, M.H. (2003). The Microbiology of Anaerobic Digesters. John Wiley \& Sons, Inc., New Jersey

Hattori, S. (2008). Syntrophic Acetate-Oxidizing Microbes in Methanogenic Environments. Microbes Environ. Vol. 23, No.2 , 118-127.

Kepp, U.; Panter, K.; and Solheim, O.E.; (2001). High dry solids digestion. Proc. CIWEM/Aqua Enviro 6th European Biosolids and Organic Residiuals Conference, UK.

Phothilangka, P.; Schoen, M.A.; and Wett, B. (2008). Benefits and drawbacks of thermal pre-hydrolysis for operational performance of wastewater treatment plants. Water Sci Technol. 58(8):1547-53. IWA Publishing, UK

Wett, B.; Murthy, S.; Takács, I.; Wilson, C.A.; Novak, J.T.; Panter, K.; Bailey, W. (2009). Simulation of Thermal Hydrolysis at the Blue Plains AWT: A New Toolkit Developed for Full-Plant Process Design. Proc. WEFTEC, Orlando.

Wett, B; Takács, I.; Batstone, D.; Wilson, C.; Murthy, S. (2012). Anaerobic model for high-loaded or hightemperature digestion - additional pathway of acetate oxidation. Proc. WEFTEC, New Orleans.

Wilson, C.A. (2009). Mechanisms of Methanogenic Inhibition in Advanced Anaerobic Digestion. PhD-thesis Virginia Polytechnic Institute, Blacksburg 


\title{
A Protocol for Optimization of Activated Sludge Mixing
}

\author{
R. W. Samstag ${ }^{1}$ and E. A. Wicklein ${ }^{2}$ \\ ${ }^{1}$ PO Box 10129 Bainbridge Island, WA 98110 (Email: rwsamstag@ stanfordalumni.org) \\ ${ }^{2}$ Carollo Engineers, 1218 Third Avenue, Suite 1600, Seattlle, WA 98110 (Email: ewicklein@ carollo.com)
}

\begin{abstract}
The paper outlines a protocol for comprehensive evaluation of mixing devices for activated sludge tanks using field testing for calibration of computational fluid dynamic (CFD) modelling to compare the impacts of tank and mixing device geometry on mixing and energy efficiency. The protocol includes a CFD model for activated sludge solids settling and transport which captures the influence of solids concentration gradients on fluid motion. This element of the protocol is unique in that almost all analyses completed to date for activated sludge biokinetic modelling or mixing have assumed either 1) complete mixing or 2) neutral density CFD neglecting the true muliphase conditions. To date, the protocol has been applied to several types of mixing devices including jet aeration and mixing, horizontal shaft propeller mixers, and diffused aeration. Field testing of several other types of mixing devices has also been accomplished. The protocol is recommended to optimize design and application of mixing devices for activated sludge service in aerated, anoxic, and anaerobic tanks as part of biological treatment processes. The approach can be extended to incorporate biokinetic models that more accurately predict the impact of tank geometry and mixer configuration on treatment efficiency than can be achieved assuming complete mixing or neutral density CFD.
\end{abstract}

\section{Keywords}

Mixing, activated sludge, modelling, CFD, energy efficiency

\section{INTRODUCTION}

With increasing recognition of the importance of nitrogen and phosphorus removal from wastewater discharges and recognizing the proven economy of biological treatment processes for nutrient removal, the wastewater treatment industry has seen an increase in the use of unaerated tanks for anoxic uptake of nitrate and anaerobic tanks to facilitate phosphorus removal from wastewater effluents. Anaerobic tanks are also increasingly used to improve activated sludge settleability. In order to facilitate these treatment goals, biological treatment tanks with significant concentrations of suspended solids must be mechanically mixed. With this increasing importance of mixing in standard biological treatment processes, optimization of tank geometry and mixer configuration becomes more important. We want the most efficient mixing in two senses: 1) we want near uniform distribution of suspended solids across our treatment tanks and 2) we want to use tank geometries and mixer configurations that minimize consumption of energy. It is towards this optimization of mixing and energy efficiency that the proposed protocol aims.

\section{PROTOCOL APPROACH}

The elements of the proposed protocol include the following:

- Field testing for comparison with CFD results

- Development of CFD models for the conditions of the field test

- Calibration of the CFD models

- Development of CFD models for alternate basin geometry and mixer type and configuration and comparison of CFD results from alternative geometries and mixing devices in terms of mixing and energy efficiency 
Samstag and Wicklein.

\section{Field Testing}

Field tests used by the authors to date have included: 1) solids profiles, 2) velocity profiles, 3) flow pattern distribution tests, and 4) residence time distribution dye tests. In the current paper only solids profiles will be discussed.

Solids profile tests are arguably the most important data for evaluation of mixers for activated sludge service. In suspended growth wastewater applications a uniform distribution of solids concentrations at the lowest power level is the primary goal. Solids profile tests can be implemented in the field in a number of ways. Solids samples can be withdrawn by Kemmerer samplers or by a series of sample pumps using the techniques developed by Robert Crosby (Bender and Crosby, 1980) or by solids probes. In the Crosby technique a grid of approximately 25 samples are withdrawn across the tank width and depth at locations chosen to illustrate mixer influence. The samples are then analyzed for suspended solids content (Standard Methods 2540D.) For measurements discussed below we used an Insite Instrumentation Group Model 3150 probe.

Detailed TSS measurements for four vertical shaft mixers have been conducted by Carollo Engineers for the Orange County Utilities' (OCU) South Water Reclamation Facility (SWRF) in Orlando, Florida. A cross sectional grid of 25 data points were measured for each mixer. Three hydrofoil mixers from different manufacturers were compared to a hyperboloid mixer. Hydrofoil I had three flat impellers each with a downward bending trailing edge. Hydrofoil II had three curving impeller blades of relatively large diameter. Hydrofoil III was constructed of a single flat plate with three downward folding projections. These impellers all produce a downward pumping action through the impeller. The Hyperboloid I mixer also produced a downward pumping action using a series of upwardly projecting shallow ridges of hyperboloid shape. Measurements were taken over the course of a one-hour period on different days for each mixer. The deviation of the measured concentration from the average concentration across the entire section was then calculated. This deviation may be considered the coefficient of variation $(\mathrm{CoV})$ of the sample data for each mixer.

Contour plots of the measured concentration data for two of the hydrofoil mixers are presented in Figure 1. The contour plots indicate an asymmetry across the tank. This asymmetry is thought to have been caused by upstream conditions in the racetrack tank. The three hydrofoils were all arranged on their vertical shafts at approximately one third depths in the tanks. The one hyperboloid mixer was nearer to the bottom of the reactor tank. The contour data for this mixer (not shown) indicated relatively uniform concentrations across the tank, but significantly higher concentrations in the tank bottom.

Carollo Engineers also conducted limited field tests of jet mixing and aeration of an operating sequencing batch reactor (SBR) at the Blacks Ford Regional Water Reclamation Facility (BFRWRF) of the JEA Utility in Jacksonville, Florida. The tests (Samstag et al., 2012) were conducted to establish solids concentration profiles under normal operating conditions for use in calibrating CFD modelling. Solids concentration measurements were taken at multiple depths at two locations at the edge of the operating SBR using a calibrated optical solids measurement probe. Mixed liquor suspended solids (MLSS) measurements were made during mixed cycles with both air and pumping operational and during pumping-only mix cycles. 


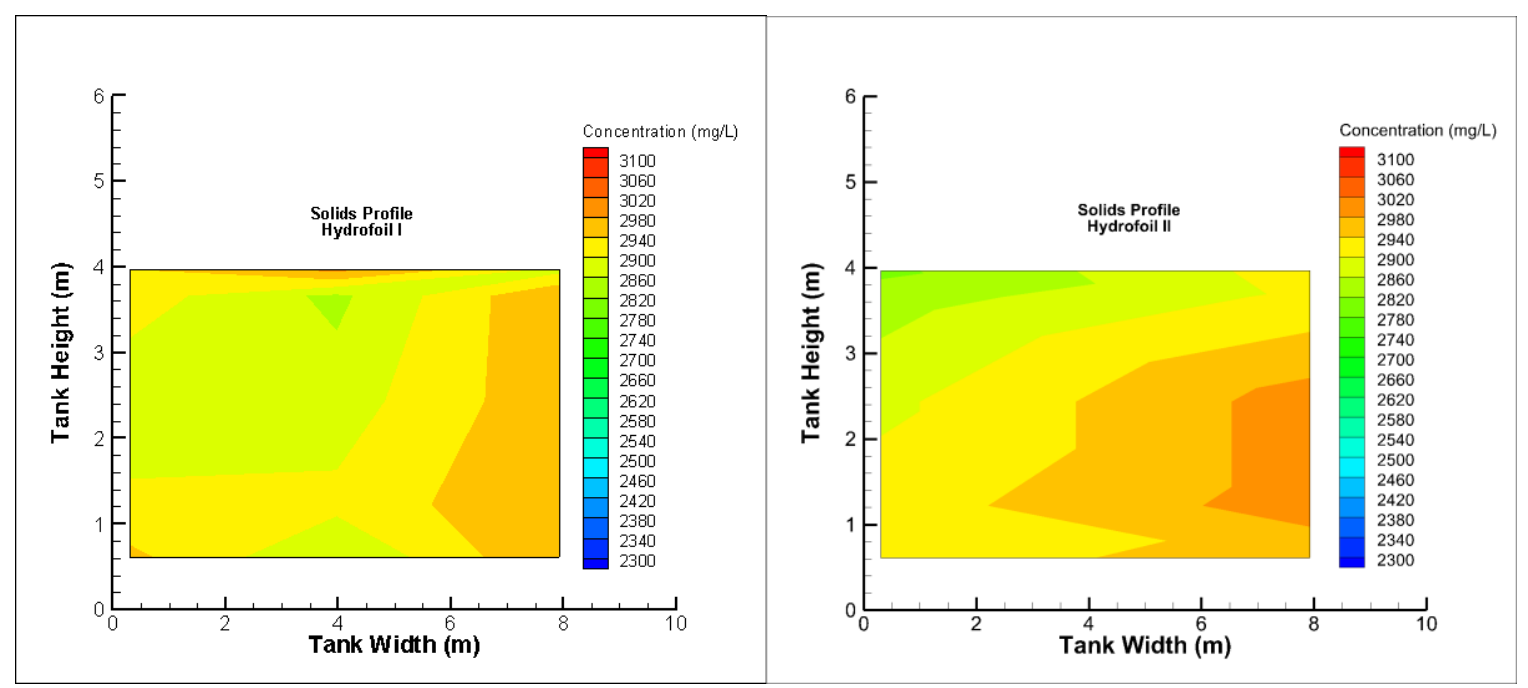

Figure 1. Solids profiles for two vertical shaft mixers.

\section{Development of CFD models for the conditions of the field test}

The first step in development of a calibrated CFD model is to produce a geometric and computational mesh of the field-tested reactor. Modern tools for CFD allow construction of extremely detailed models of complex geometries and optimized computational meshes. The authors have ANSYS GAMBIT, Version 2.4.6 for both modeling and meshing for the case studies referenced here.

Figure 2 presents an illustration of the three-dimensional geometric model and a projection of the computational mesh prepared for the BFRWRF SBR tanks for CFD analysis. The model shows the three jet headers, the main header pump intake, the auxiliary header pumps and intakes, and the effluent decanters. The effluent decanters were not required for the flow simulation, but were included to simulate the fluid environment. The polyhedral computational mesh of approximately one million cells is shown projected onto model surfaces.
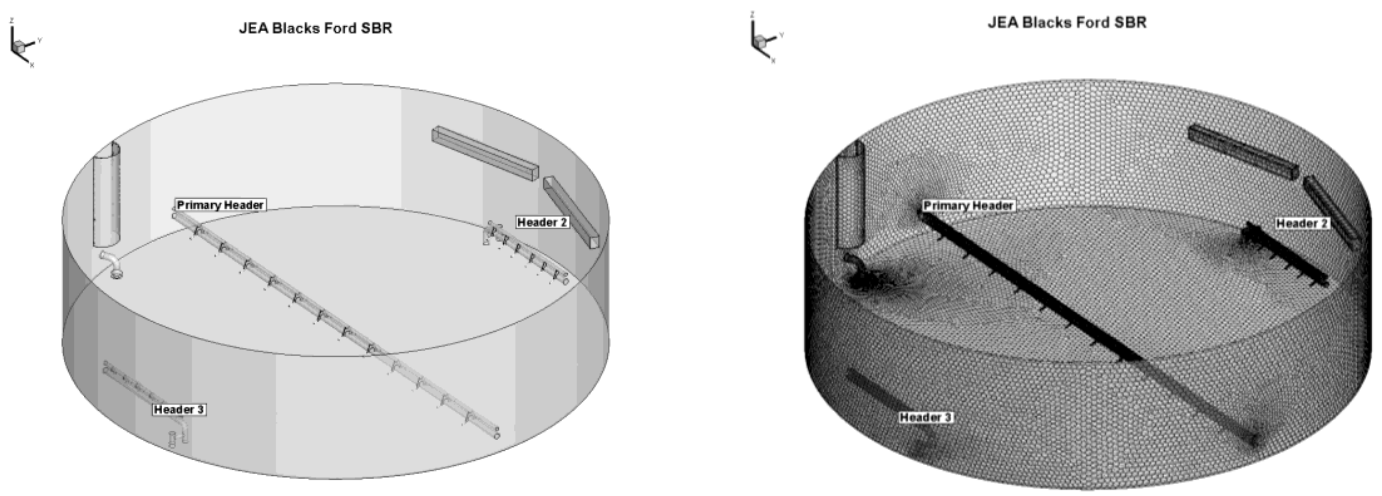

Figure 2. SBR Tank Geometric Model and Computational Mesh.

\section{Calibration of the CFD models}

Modern CFD models can be used without calibration. The physics of CFD have been verified within the tolerance of most field measurements many times before. A possible exception is in the area of turbulence estimation. It is widely believed that the k-epsilon turbulence model is appropriate for the types of recirculating flows commonly seen in activated sludge 
sedimentation tanks, but calibration is more important where the CFD is used as a base for solids transport or biokinetic models, since many of the empirical parameters used in these models are much less well established than the physical parameters applicable to fluid flow. Calibration techniques have included solids settling rate testing, solids profile matching, and clarifier sludge blanket matching during dynamic flows. See Griborio et al. (2008), Wicklein and Samstag (2009), Samstag and Griborio (2010), and Samstag et al. (2010). For this protocol we demonstrate the use of one of the most useful techniques, solids profile matching. The authors used Fluent Version 13 on a computing platform of 64-bit workstations with multiple CPU cores running a 64-bit Windows XP operating system.

Figure 3 presents contour plots of CFD simulations of dynamic solids profiles for the BFRWRF under conditions of normal operation with aeration on and with only the recirculation pumps on. The simulation of the aerated condition produced a good match to the fully mixed condition seen in the field tests. The solids profile match for simulation of pumped mixing after aeration had been turned off for 25 minutes confirmed the development of relatively clear water in the top of the tank that was seen in the field tests. See Samstag et al., 2012.

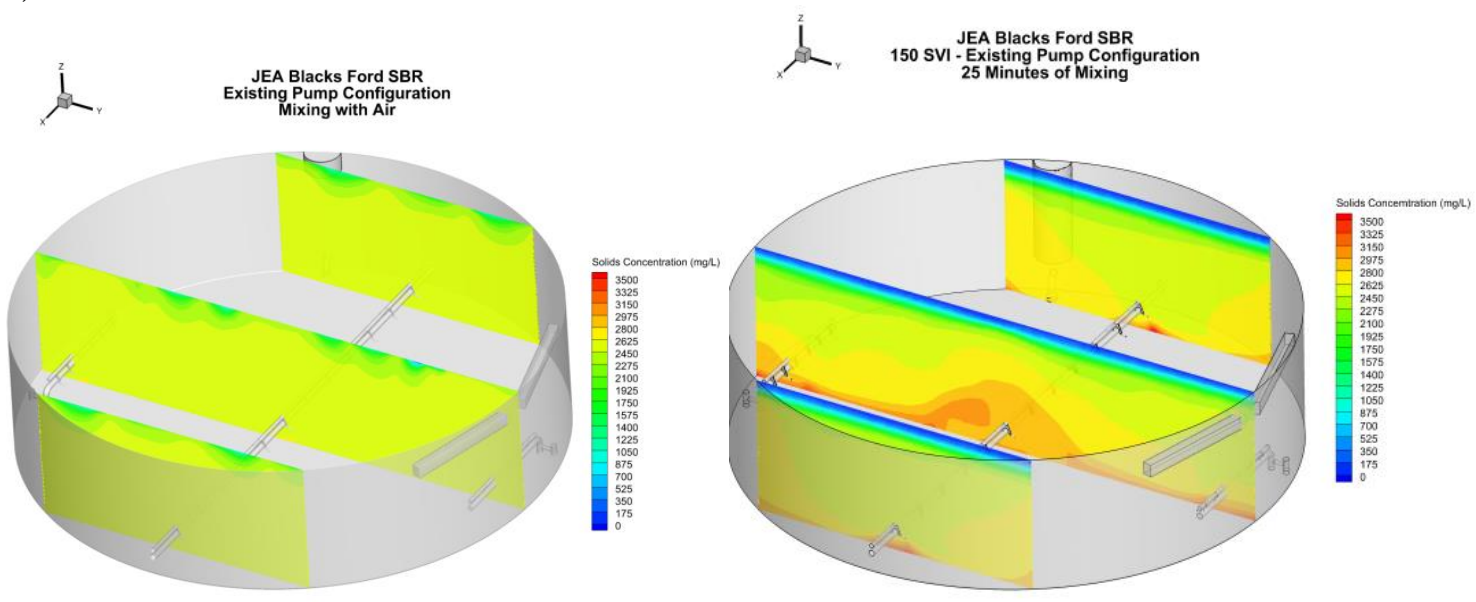

Figure 3. Solids profiles of CFD results with (left) and without (right) aeration.

These simulations were conducted using a user defined function (UDF) for solids settling and transport with coupling of the influence of solids gradients on the density profile and fluid flow. Hindered settling velocities were calculated based on a sludge volume index (SVI) of 150 $\mathrm{mL} / \mathrm{g}$, using the revised Daigger equation (Daigger, 1995). Further details are presented in Samstag et al. (2012).

The work did not include velocity profiling of the SBR tanks. This provides another opportunity for calibration. Calibration of velocity fields has been accomplished in sedimentation tanks. The early work of Larsen (1977) is compared to CFD in Wicklein and Samstag (2009) Samstag et al (2010) compares drogue velocity measurements to CFD model results.

Figure 4 presents the predicted velocity profile for the SBR tanks at the BFRWRF from the CFD model. With the aeration on, higher velocities penetrate to the upper reaches of the tank resulting in complete solids mixing. With aeration turned off very high velocities at the exit from the mixing jets dissipate rapidly in the tank leaving velocities less than $0.1 \mathrm{~m} / \mathrm{s}$ in most of the tank. 


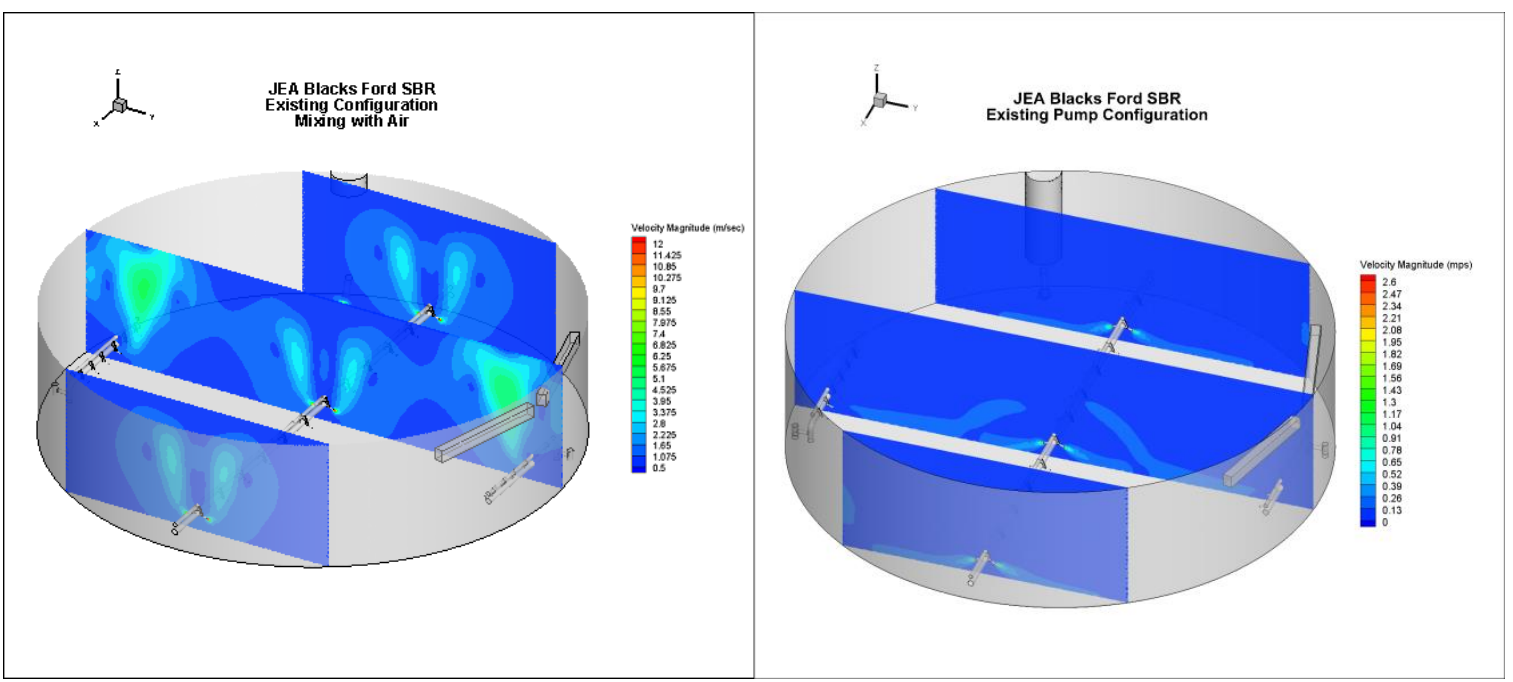

Figure 4. Velocity profiles for air mixing (left) and pumped mixing (right).

The importance of including density coupling in the CFD simulation is illustrated in Figure 5. The figure compares the results from simulation of 25 minutes of pumped mixing in the SBR tanks after turning off aeration with the density couple active (left side) to a neutral density simulation where the effect of concentration gradients on the density field was turned off (right side). Without including the density couple, the CFD simulation predicts relatively complete mixing for the pumped mix condition. This result is unrealistic based on the field tests. Since neutral density CFD simulation of mixing is common in the industry, this is a significant finding.
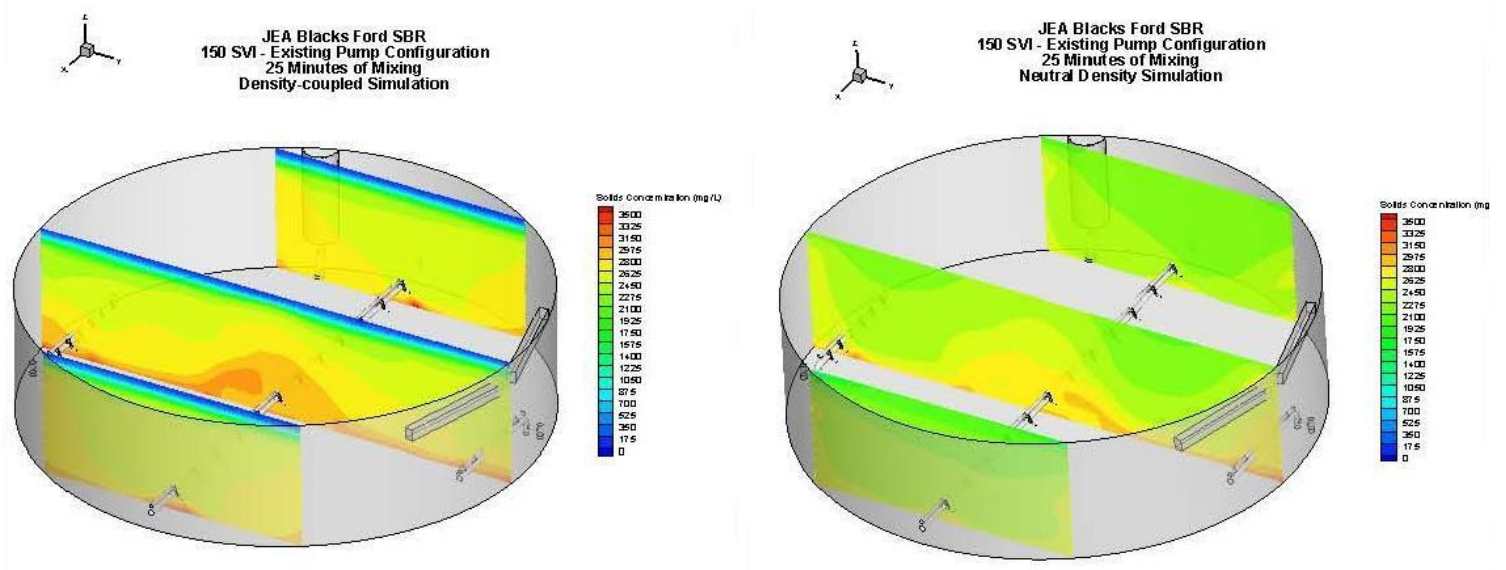

Figure 5. Comparison of solids profiles from density-coupled and neutral density models

\section{CFD Model Alternatives}

After calibration of the CFD model to conditions of operation in the field, the CFD model can be used to consider alternative configurations to improve operation. For the BFRWRF project, for example, a series of alternatives were considered in which the jet velocity was increased in an effort to improve solids mixing during the pumped mix cycle. CFD simulations were developed for four different jet velocities from $2.5 \mathrm{~m} / \mathrm{sec}$ to $4.0 \mathrm{~m} / \mathrm{sec}$. The simulations indicated that increasing the velocity to $3.0 \mathrm{~m} / \mathrm{sec}$ would reduce solids deposition in the tank after 25 minutes of pumped mixing after aeration is turned off, but that increasing the jet 
velocity to $4.0 \mathrm{~m} / \mathrm{sec}$ would be required to achieve a $\mathrm{CoV}$ for solids concentration less than 10 percent. Power requirements for this increase in mixing intensity would triple the installed power for this facility. These results showed that the original design was significantly inadequate to meet a specification of $\mathrm{CoV}$ less than 10 percent.

In another project Carollo investigated the efficiency of solids mixing in the plug flow racetrack tank. Two different mixers were investigated using an un-calibrated CFD model incorporating density-coupled solids settling and transport. This project also included a twofluid model of the aerated zone in a side-sloped reactor.

Table 1 presents summary date for mixing efficiency derived from the field tests and calibrated and un-calibrated CFD simulations discussed above for:

- Pumped jet mixing

- Vertical hydrofoil mixers

- Vertical hyperboloid mixers

- Horizontal propeller mixers

The data indicate that the hydrofoil, hyperboloid, and horizontal propeller mixers have the potential for much greater power efficiency than pumped mixing. The equivalent power required for these mixers for a $\mathrm{CoV}$ of 10 percent varied in the range of 1.4 to $8.5 \mathrm{~W} / \mathrm{m} 3$. These values are almost an order of magnitude greater than for pumped jet mixing.

\section{CONCLUSIONS}

A protocol has been developed for comparisons of the efficiency of activated sludge mixing systems using field-calibrated CFD models. A crucial difference in this protocol from previous work is to incorporate the effects of solids settling and transport on fluid motion. Elements of the protocol have been applied to jet aeration and mixing, vertical shaft hyperboloid mixers, horizontal propeller mixers, and diffused aeration. We propose this protocol as a comprehensive approach to optimizing activated sludge mixing and urge its adoption in future studies.

\section{ACKNOWLEDGEMENTS}

This work has been accomplished as part of Carollo Engineers' CFD modelling practice for the named clients. Support for this work by these clients and by Carollo Engineers is gratefully acknowledged. Field testing was assisted by staff from Carollo Engineers Orlando office, including Roderick Reardon, Sudhan Paranjape, and Erica Stone, whose help was greatly appreciated. 


\section{WWTmod}

Samstag and Wicklein.

Table 1. Comparison of required volumetric power input for different mixer types.

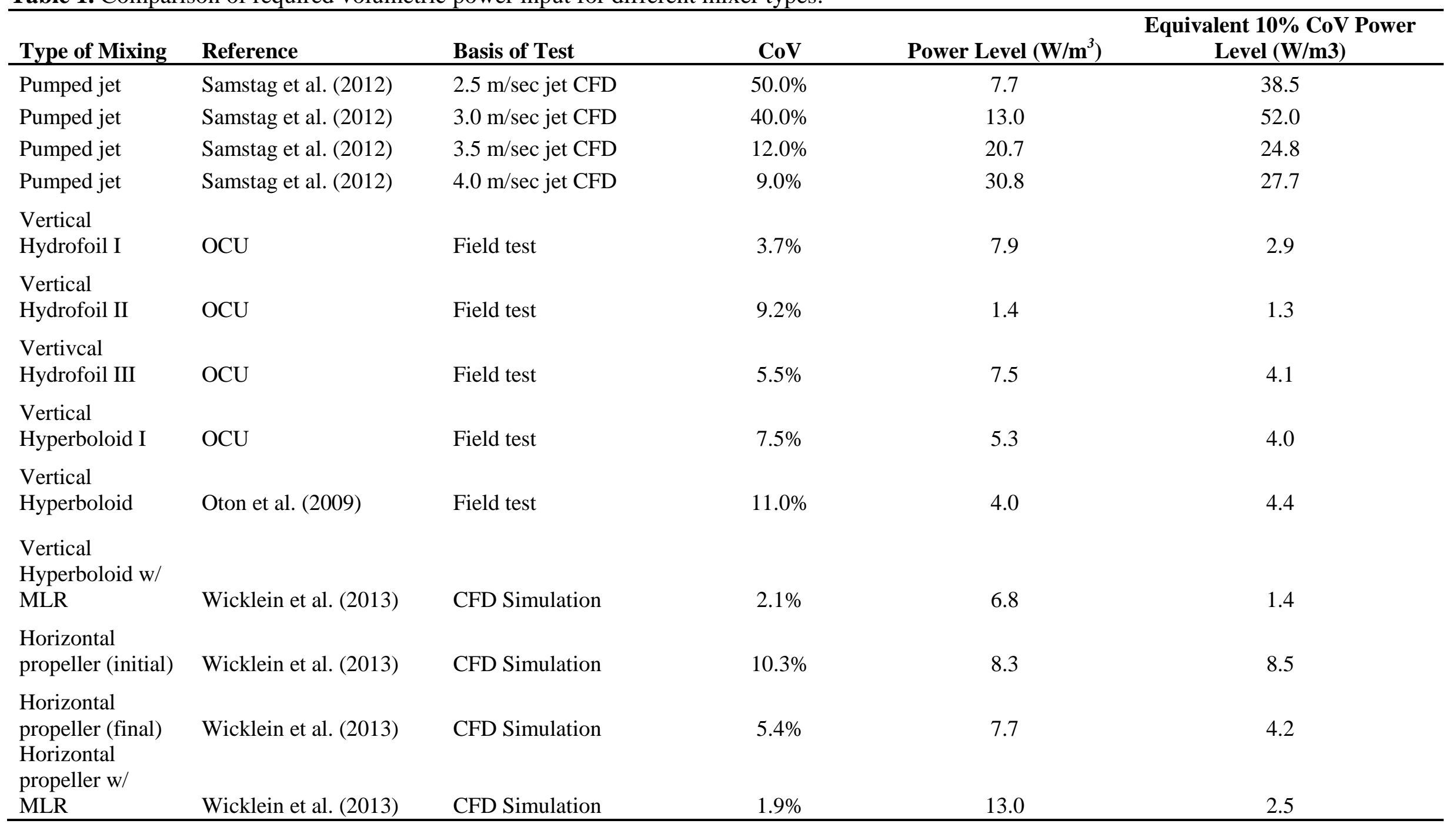




\section{REFERENCES}

American Public Health Association (APHA), the American Water Works Association (AWWA), and the Water Environment Federation (WEF) (2013) Standard Methods for the Examination of Water and Wastewater. Online Edition (http://www.standardmethods.org/store/ProductView.cfm?ProductID=63).

Bosma, A.J. and Reitsma, B.A. (2007) Hydraulic Design of Activated sludge Tanks with CFD. Proceedings of the COMSOL Users Conference, Grenoble.

Crosby. R. M. and Bender, J.H. (1980) Hydraulic Considerations That Affect Secondary Clarifier Performance. EPA Technology Transfer, United States Environmental Protection Agency, 905N80001.

Daigger, G.T. (1995) Development of Refined Clarifier Operating Diagrams Using Updated Settling Characteristics Database. Water Environment Research, 67, 95.

Griborio, A.; Rohrbacher, J., Taylor, R., Pitt, P., and Latimer, R. (2008) Evaluation of Wet Weather Strategies and Clarifier Optimization Using State-of-the-Art Tool. Proceedings of the Water Environment Federation, WEFTEC Conference.

Larsen, P. (1977) On the Hydraulics of Rectangular Settling Basins, Experimental and Theoretical Studies. Department of Water Resources Engineering. Lund Institute of Technology / University of Lund, Report No. 1001, Lund, Sweden.

Oton, S. et. al. (2009) The Fine Line Between Thorough Mixing and Energy Consumption. WEF Nutrient Removal Conference Proceedings, 2009.

Samstag R. and Griborio, A. (2010) Calibration and validation of CFD Models - Case Study: CFD Modelling of Secondary Clarifiers. Second International Water Association/Water Environment Federation Wastewater Treatment Modeling Seminar, Mont-Sainte-Anne, Quebec, Canada.

Samstag, R.W., Wicklein, E.A., Reardon, R. D., Leetch, R. J., Parks, R. M., and Groff, C. D. (2012) Field and CFD Analysis of Jet Aeration and Mixing. Proceedings of the Water Environment Federation 84th Annual Technical Conference and Exposition; Orlando, Florida

Samstag, R., Zhou, S., Chan, R., Royer, C., and Brown, K. (2010) Comprehensive Evaluation of Secondary Sedimentation Performance. Proceedings of the Water Environment Federation 82nd Annual Technical Conference and Exposition, New Orleans, LA.

Wicklein E. A. and Samstag R.W. (2009) Comparing Commercial and Transport CFD Models for Secondary Sedimentation. Proceedings of the Water Environment Federation 81st Annual Technical Conference and Exposition; Orlando, Florida.

Wicklein, E. A., K. Rogers, R. Hunt, A. Gharagozian, M. Cocke, and P. Roy. (2013) CFD Modeling of Activated Sludge Mixing in an Oxidation Ditch Conversion. Proceedings of the $85^{\text {th }}$ Annual WEFTEC Conference, Chicago, Illinois. 


\title{
A general three-dimensional extension to ADM1: the importance of an integrated fluid flow model
}

\author{
David L. F. Gaden ${ }^{1}$ and Eric L. Bibeau ${ }^{2}$ \\ ${ }^{1}$ Department of Mechanical \& Manufacturing Engineering, University of Manitoba, R3T 2N2, Winnipeg, \\ Canada (Email: david_gaden@umanitoba.ca) \\ ${ }^{2}$ Department of Mechanical \& Manufacturing Engineering, University of Manitoba, R3T 2N2, Winnipeg, \\ Canada (Email: Eric.Bibeau@ad.umanitoba.ca)
}

\begin{abstract}
The current state-of-the-art model for anaerobic digesters is Anaerobic Digestion Model No. 1 (ADM1). It is a bulk model with a framework that ignores spatial variations, leading to several inherent limitations. Anaerobic Digestion Model with Multi-Dimensional Architecture (ADM-MDA) is an extension to ADM1 that incorporates spatial discretization and computational fluid dynamics (CFD). A comparison between ADM1 and ADM-MDA shows that under some conditions, spatial variation alone can make the difference between a healthy digester and digester failure. These findings underscore the importance of CFD in digester simulations. This paper presents the results of this four-year numerical model development project.
\end{abstract}

Keywords

3D; ADM1; CFD; modelling; OpenFOAM; spatial resolution

\section{INTRODUCTION}

Anaerobic digestion modelling has traditionally been more focused on the biochemistry of digestion, and less focused on the fluid flow and reactor geometry. Anaerobic Digestion Model No. 1 (ADM1) (Batstone, Keller et al., 2002) is such a model, with a solid biochemical foundation, but little, if any, consideration of fluid flow. Intuitively this makes sense, as the biochemistry of anaerobic digesters is considerably more complicated than the fluid flow, particularly considering the fluid velocities can be near zero. However, fluid flow and thermal gradients may impact the model more significantly than this line of thinking suggests. There have been numerous experimental studies into fluid, such as Karim, Hoffmann et al. (2005a, 2005b), who studied the efficacy of various mixing strategies at different waste concentrations. Numerical studies into digester fluid flow also have been reported, although these generally ignore biochemistry, including $\mathrm{Wu}$ and Chen (2008), who performed a computational fluid dynamics (CFD) study into flow in lab-scale and pilot-scale digesters, focusing on the effect of using a non-Newtonian fluid model. Although sparse, a few studies exist that combine biochemistry and fluid flow, including Fleming (2002) who created a CFD model with biochemistry based on Hill's monod reaction model (1983a, 1983b). Other studies have accomplished some aspects of fluid flow and biochemistry, such as Batstone, Hernandez, et al. (2005), which used compartmental ADM1 simulations to establish spatial variation in a plug-flow reactor. To further address fluid flow and biochemistry, this project implements Anaerobic Digestion Model with Multi-Dimensional Architecture (ADM-MDA) (Gaden, 2013), a three-dimensional full implementation of ADM1 with an integrated CFD flow model. 


\section{MODEL DEVELOPMENT}

Extending the governing equations of ADM1 from a bulk model to three dimensions is achieved by including spatial variables in the derivation. For instance, the mass balance for a species changes from:

$$
\frac{d S_{v a r}}{d t}-\frac{V_{l}}{Q_{l}}\left(S_{v a r, i n}-S_{v a r}\right)=r_{v a r}
$$

to:

$$
\frac{\partial S_{v a r}}{\partial t}+\nabla \bullet\left(\mathbf{U} S_{v a r}\right)-\nabla \bullet \Gamma_{v a r} \boldsymbol{\nabla} S_{v a r}=r_{v a r}
$$

However, solving the new equation set is non-trivial as the introduction of spatial resolution changes a differential-algebraic equation set (DAE) to a partial differential algebraic equation set (PDAE), and few suitable solvers exist.

This project develops Coupled-Reaction-Advection-Flow Transient Solver (CRAFTS) to handle the new numerical framework. CRAFTS is a general reaction solver for single-phase, incompressible fluid flows. It allows users to define their own variables, reactions, inhibitions, coefficients and control logic without requiring any programming. CRAFTS also has a framework for User-Defined Functions (UDFs) that allows for custom algebraic algorithms, such as ADM1's Newton-Raphson ion model. CRAFTS is a novel PDAE solver that also employs a novel programmable logic controller (PLC) emulator. CRAFTS is built using OpenFOAM®, a free and open source CFD suite.

Gaden (2013) presents the full details of the model and its development.

\section{CASE SETUP}

To evaluate model performance, this study compares the performance of ADM1 against ADM-MDA on a case involving a $100 \mathrm{~m}^{3}$ rectangular digester. In this case, fluid injection events of occur daily for two minutes at a velocity of $0.05 \mathrm{~ms}^{-1}$, giving a hydraulic retention time of only 8.33 days. Fluid mixing events occur for ten minutes every hour.

\section{RESULTS}

Most variables show little differences between the two models, however, the continuum assumption of ADM1 leads to subtle differences in mass exchanged during each fluid injection event. These differences can amplify quickly with highly sensitive dynamic systems, such as ADM1. Figure 1a shows the two models have a significant disagreement with the total acetate concentration, $S_{a c}$. ADM1 suggests a stable digester, whereas ADMMDA indicates an unhealthy digester. This disagreement can be explained by the fact that acetate degraders can be seen washing out only in ADM-MDA, Figure $1 \mathrm{~b}$. 


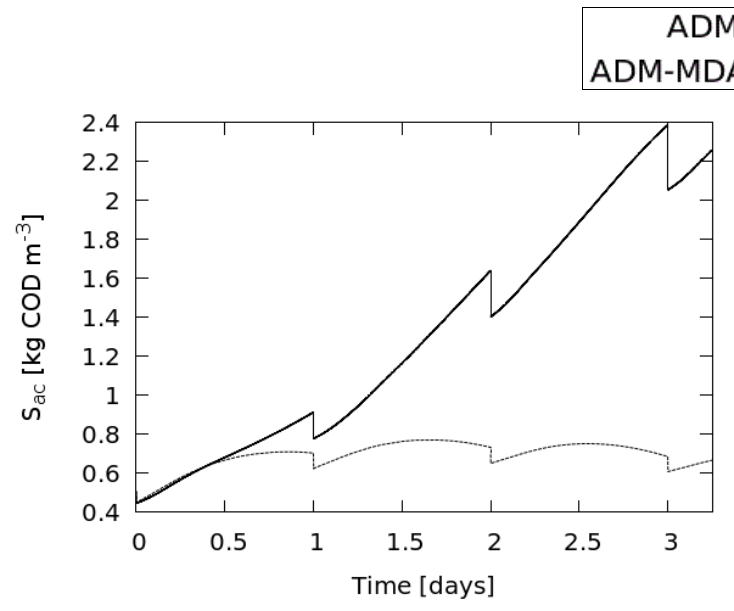

Figure 1a. Total acetate concentration, $S_{a c}$

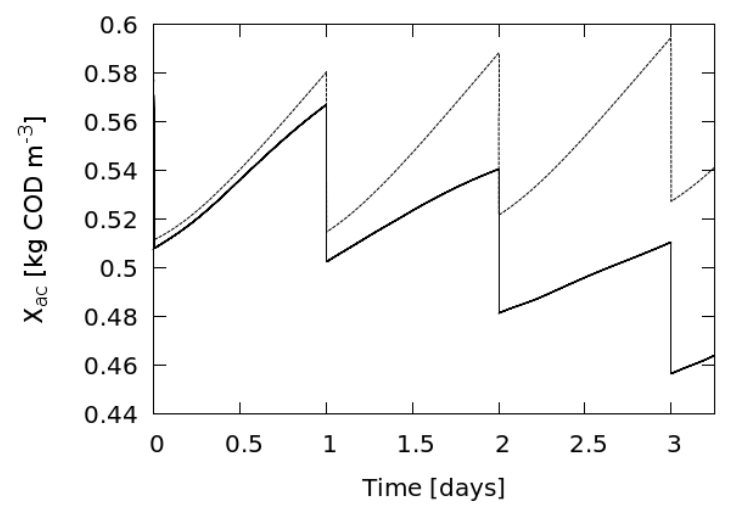

Figure 1b. Acetogenic biomass, $X_{a c}$

The spatial data that ADM-MDA provides can also be useful for design purposes. For instance, the model showed dissolved methane building up at the centre of the reactor, Figure 2. This suggests a change in mixing strategy might improve gas transfer rate. By way of contrast, ADM1 does not include spatial data.

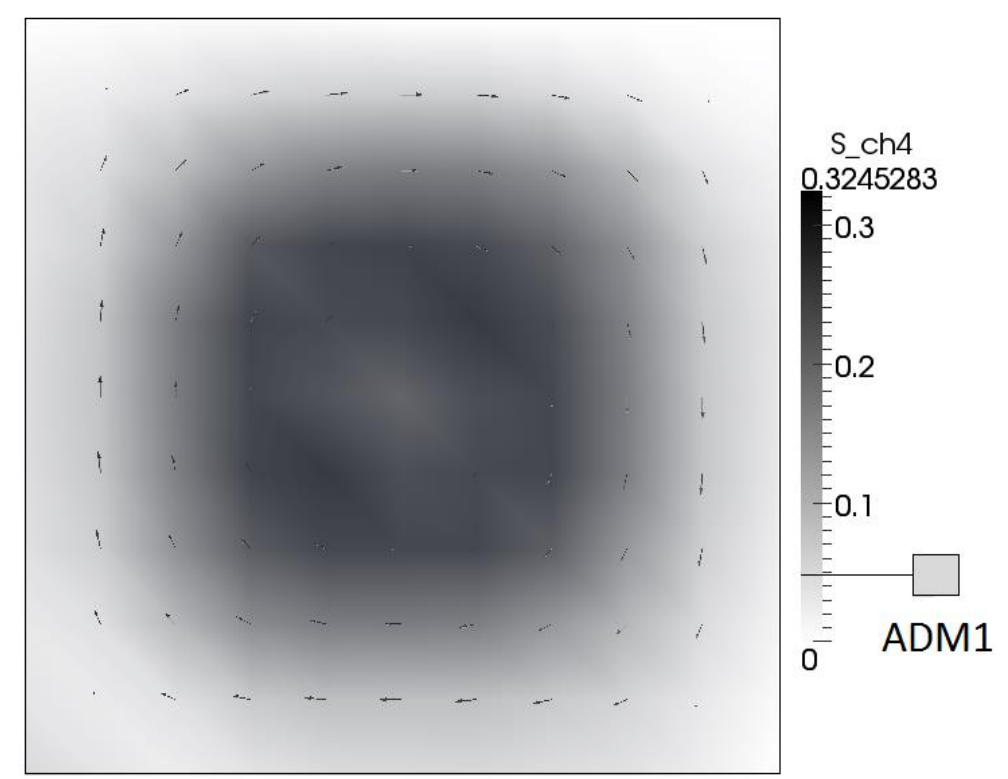

Figure 2. ADM-MDA

Dissolved methane concentration, $\mathrm{S}_{\mathrm{ch} 4}$, spatial distribution at $\mathrm{t}=56,651 \mathrm{~s}(15 \mathrm{~h} 44 \mathrm{~m} 11 \mathrm{~s})$.

\section{FUTURE DEVELOPMENT}

This research project has produced a strong foundation for a spatially-resolved implementation of ADM1; however, the second-order accuracy inherent in CRAFTS' finite volume method can conflict with the numerical stiffness of ADM1. This may limit the practicality of the model, such as reduce the maximum mesh size. There are several promising areas for model improvement, including the gas model and the transport model. Source code from this research endeavour is being released as free and open source software to the ADM1 modelling community to facilitate further model developments, (Gaden, Bibeau, 2013). 


\section{ACKNOWLEDGEMENT}

This research was jointly funded by Manitoba Hydro and by the Natural Sciences and Engineering Research Council of Canada (NSERC). The authors would like to thank the many people that assisted with this project, including significant contributions from Dr. Scott J. Ormiston, Dr. Hrvoje Jasak, and Dr. Ivor Clifford.

\section{REFERENCES}

Batstone D. J., Hernandez J. L. A., Schmidt J. E. 2005. "Hydraulics of laboratory and full-scale upflow anaerobic sludge blanket (UASB) reactors," Biotechnology and Bioengineering, v. 91, n. 3, pp. 387-391.

Batstone D. J., Keller J., Angelidaki I., Kalyuzhni S. V., Pavlostathis S. G., Rozzi A., Sanders W. T. M., Siegrist H. and Vavlin V. A. 2002. Anaerobic Digester Model No.1 (ADM1). Scientific and Technical Report No.13, IWA Task Group for Mathematical Modelling of Anaerobic Digestion Processes. IWA Publishing, London, United Kingdom.

Fleming J. G. 2002. "Novel simulation of anaerobic digestion using computational fluid dynamics," Ph.D. Thesis, Faculty of Mechanical Engineering, North Carolina State University, North Carolina, United States.

Gaden D. L. F. 2013. Modelling Anaerobic Digesters in Three Dimensions: Integration of Biochemistry with Computational Fluid Dynamics. Ph.D. thesis. University of Manitoba, Canada. Available at http://hdl.handle.net/1993/22096 (2013-09-23)

Gaden D. L. F., Bibeau E. L. 2013. Toolset for OpenFOAM. 26 September. doi:10.5203/sc_gad_1

Hill D. T. 1983a. "Simplified monod kinetics of methane fermentation of animal wastes," Agricultural Wastes, v. 5, pp. 1-16.

Hill D. T. 1983b. "Energy consumption relationships for mesophilic and thermophilic digestion of animal manures," Transactions of the American Society of Agricultural Engineering, v. 26, n. 3, pp. 841-848.

Karim K., Hoffmann R., Klasson K. T., Al-Dahhan M. H. 2005a. "Anaerobic digestion of animal waste: effect of mode of mixing," Water Research, v. 39, pp. 3597,3606.

Karim K., Hoffmann R., Klasson T., Al-Dahhan M. H. 2005b. "Anaerobic digestion of animal waste: waste strength versus impact of mixing," Bioresource Technology, v. 96, pp. 1771-1781.

Wu B., Chen S. 2008. "CFD simulation of non-Newtonian fluid flow in anaerobic digesters," Biotechnology and Bioengineering, v. 99, n. 3, pp. 700-711. 


\title{
Modelling bacterial selection during the plug-flow feeding phase of aerobic granular sludge biofilm reactors
}

\author{
David G. Weissbrodt ${ }^{1,2,3}$, Christof Holliger ${ }^{1}$, Eberhard Morgenroth ${ }^{2,3}$ \\ ${ }^{1}$ Laboratory for Environmental Biotechnology, Ecole Polytechnique Fédérale de Lausanne, Station 6, 1015 \\ Lausanne, Switzerland (Email: christof.holliger@epfl.ch) \\ ${ }^{2}$ Institute of Environmental Engineering, ETH Zürich, Wolfgang-Pauli-Strasse 15, 8093 Zürich, \\ Switzerland \\ (Email: david.weissbrodt@ifu.baug.ethz.ch; eberhard.morgenroth@ifu.baug.ethz.ch) \\ ${ }^{3}$ Department of Process Engineering, Eawag, Ueberlandstrasse 133, 8600 Duebendorf, Switzerland \\ (Email: david.weissbrodt@eawag.ch; eberhard.morgenroth@eawag.ch)
}

\begin{abstract}
A mathematical model was developed to investigate hydraulic transport and bacterial selection during slow up-flow anaerobic feeding $\left(0.9 \mathrm{~m} \mathrm{~h}^{-1}\right)$ of wastewater across the settled bed of granular sludge biofilms used in column-type sequencing-batch reactors to remove nutrients. A plug-flow regime with dispersion was identified from residence time distribution data (RMSE $\left.<0.010, \mathrm{R}^{2}=0.999\right)$. Metabolic formulations allowed assessing the effect of environmental conditions on the competition of polyphosphate- (PAO, Accumulibacter) and glycogen-accumulating organisms (GAO, Competibacter) for the uptake of acetate during anaerobic feeding. Since PAO and GAO metabolisms rely on distinct dynamics of intracellular storage polymers, the feeding phase length at nominal flowrate was shown to impact bacterial selection. In addition, acetate was preferentially consumed by PAO under alkaline conditions ( $\mathrm{pH} 7.5-8.0)$ independently of temperature $\left(10-30^{\circ} \mathrm{C}\right)$. GAO were only able to outcompete PAO under combined acidic ( $\mathrm{pH}$ 6.0-6.5) and higher mesophilic $\left(25-30^{\circ} \mathrm{C}\right)$ conditions. However, the difference in uptake rates was only half of the one obtained under conditions selecting for PAO. The model can support the assessment of spatial stratification of conversion processes across the bed and the design of operation and bed geometries towards optimal bacterial resource management in granular sludge.
\end{abstract}

\section{Keywords}

Biological nutrient removal; granular sludge biofilms; feeding phase; reactor regime; $\mathrm{PAO} / \mathrm{GAO}$

\section{INTRODUCTION}

Efficient biological nutrient removal (BNR) in intensified sequencing-batch reactors (SBR) using aerobic granular sludge (AGS) biofilms requires optimal management of the bacterial resource. Preferential selection of polyphosphate- (PAO, e.g. Accumulibacter) over glycogenaccumulating organisms (GAO, e.g. Competibacter) is required for enhanced biological phosphorus removal. PAO and GAO are selected under slow up-flow anaerobic feeding regime across the settled bed of AGS (de Kreuk and van Loosdrecht, 2004). Operation with selective purge of upper bed fractions can favor PAO over GAO (Winkler et al., 2011). Similarly to activated sludge systems (Oehmen et al., 2010), $\mathrm{pH}$ and temperature trigger PAO and GAO selection in AGS (Weissbrodt et al., 2013). Since the PAO/GAO competition relies on their ability to take up volatile fatty acids (VFA) under anaerobic conditions, a hydraulicmetabolic mathematical model was developed here to investigate the effect of combined feeding and environmental conditions on bacterial selection in AGS.

\section{MATERIAL AND METHODS}


Investigations were conducted in a lab-scale column-type reactor with a volume of $1.6 \cdot 10^{-3}$ $\mathrm{m}^{3}$ and a height-to-diameter ratio of 9.66 . The AGS bed of $0.33 \mathrm{~m}$ comprised $543 \mathrm{~g}$ of wet granular biomass with a biofilm density of $1120 \mathrm{~kg} \mathrm{~m}^{-3}$ and a homogeneous size distribution over the height $(1.7 \pm 1.2 \mathrm{~mm})$. The influent was fed at a slow nominal flowrate of $1.2 \cdot 10^{-3} \mathrm{~m}^{3}$ $\mathrm{h}^{-1}$ corresponding to superficial and interstitial velocities of 0.44 and $0.90 \mathrm{~m} \mathrm{~h}^{-1}$, respectively.

A one-dimensional plug-flow hydraulic transport model with dispersion was implemented in Berkeley Madonna and calibrated based on residence time distributions (RTD) recorded at the bed and reactor outlets with on-line electrical conductivity after step-change of the inlet concentration of an inert $\mathrm{NaBr}$ tracer, according to Gujer (2008). Axial and radial dispersion coefficients were identified from the dimensionless variance of the normalized probability function of residence time. The calibrated hydraulic transport model was coupled to structured formulations of anaerobic metabolisms of Accumulibacter and Competibacter in function of $\mathrm{pH}(6.0-8.0)$ and temperature $\left(10-30^{\circ} \mathrm{C}\right)$ (Lopez-Vazquez et al., 2009). Since VFA uptake by PAO and GAO rely on dynamics of intracellular storage polymers that are not transported with the flow, and cannot reach a steady-state, the feeding phase length $(0.25-2.5 \mathrm{~h})$ was studied as additional factor of bacterial selection.

\section{RESULTS AND DISCUSSION}

The structure of the calibrated hydraulic transport model comprised 50 mixed compartments in series and dispersion of $36 \%$ in volume and $7 \%$ in flowrate (RMSE $<0.010, \mathrm{R}^{2}=0.999$ ). The wastewater flow was related to laminar regime $\left(\operatorname{Re}_{\mathrm{bed}}=0.25<1\right)$. The dimensionless coefficient of axial dispersion in the z-direction of flow $\left(\mathrm{N}_{\mathrm{Dz}}=0.047\right)$, that corresponded to an absolute value $\left(\mathrm{D}_{\mathrm{z}}\right)$ of $1.5 \cdot 10^{-2} \mathrm{~m}^{2} \mathrm{~h}^{-1}$, was in the domain of large amount of dispersion (0.025-0.200) for packed bed bioreactors (Albuquerque and Santana, 2004). The radial dispersion transverse to the direction of flow $\left(\mathrm{N}_{\mathrm{Dt}}=0.299, \mathrm{D}_{\mathrm{t}}=9.6 \cdot 10^{-2} \mathrm{~m}^{2} \mathrm{~h}^{-1}\right)$ was even more higher in this AGS system with low Peclet numbers $\left(\mathrm{Pe}_{\mathrm{z}}=21.3\right.$ and $\left.\mathrm{Pe}_{\mathrm{t}}=3.4<100\right)$. According to RTDs measured at bed and reactor outlets, no mixing occurs between the influent and the treated wastewater volume above the bed.

Under non-selective reference simulation conditions in a bed of $50 \mathrm{~cm}$ with 60 min feeding, $20^{\circ} \mathrm{C}$, pH 7.0, $64 \mathrm{~kg}_{\text {TSS }} \mathrm{m}_{\text {bed }}{ }^{-3}$ with $67 \%$ vss and $50 \%$ of active cells related to $25 \%$ PAO and $25 \% \mathrm{GAO}$, both populations removed acetate at equal volumetric rate $\left(1.25 \mathrm{~kg}_{\mathrm{COD} \mathrm{h}} \mathrm{m}^{-3}\right)$. Prolonged feeding of 120 and $150 \mathrm{~min}$ at nominal flowrate switched off GAO and PAO activities by full depletion of glycogen and polyphosphate, respectively. Simulations displayed preferential uptake of acetate by PAO under alkaline conditions (e.g. pH 8.0) independently from temperature $\left(10-30^{\circ} \mathrm{C}\right)$ (Figure 1$)$. GAO were only able to outcompete PAO under combined acidic and higher mesophilic conditions (e.g. $\mathrm{pH} 6.0$ and $30^{\circ} \mathrm{C}$ ). However, the difference in acetate uptake rates in the bed was only half of the one obtained under conditions selecting for PAO. These results met with multifactorial experiments conducted in AGS-SBRs (Weissbrodt et al., 2013). Contrary to $\mathrm{pH}$, temperature significantly affected bed height requirements for full acetate uptake under anaerobic feeding.

\section{CONCLUSIONS}

Modelling hydraulic transport of wastewater during slow anaerobic feeding across AGS beds should integrate axial and radial dispersion components. For the application, the present approach highlighted that fill-and-draw phases can efficiently be implemented in column-type AGS-SBRs. The feeding phase length and indirectly the working volume impact bacterial 
selection. Slightly alkaline conditions, e.g. by addition of lime in the influent, efficiently select for PAO. The amount of AGS should be adapted in function of temperature for full anaerobic VFA uptake during feeding.

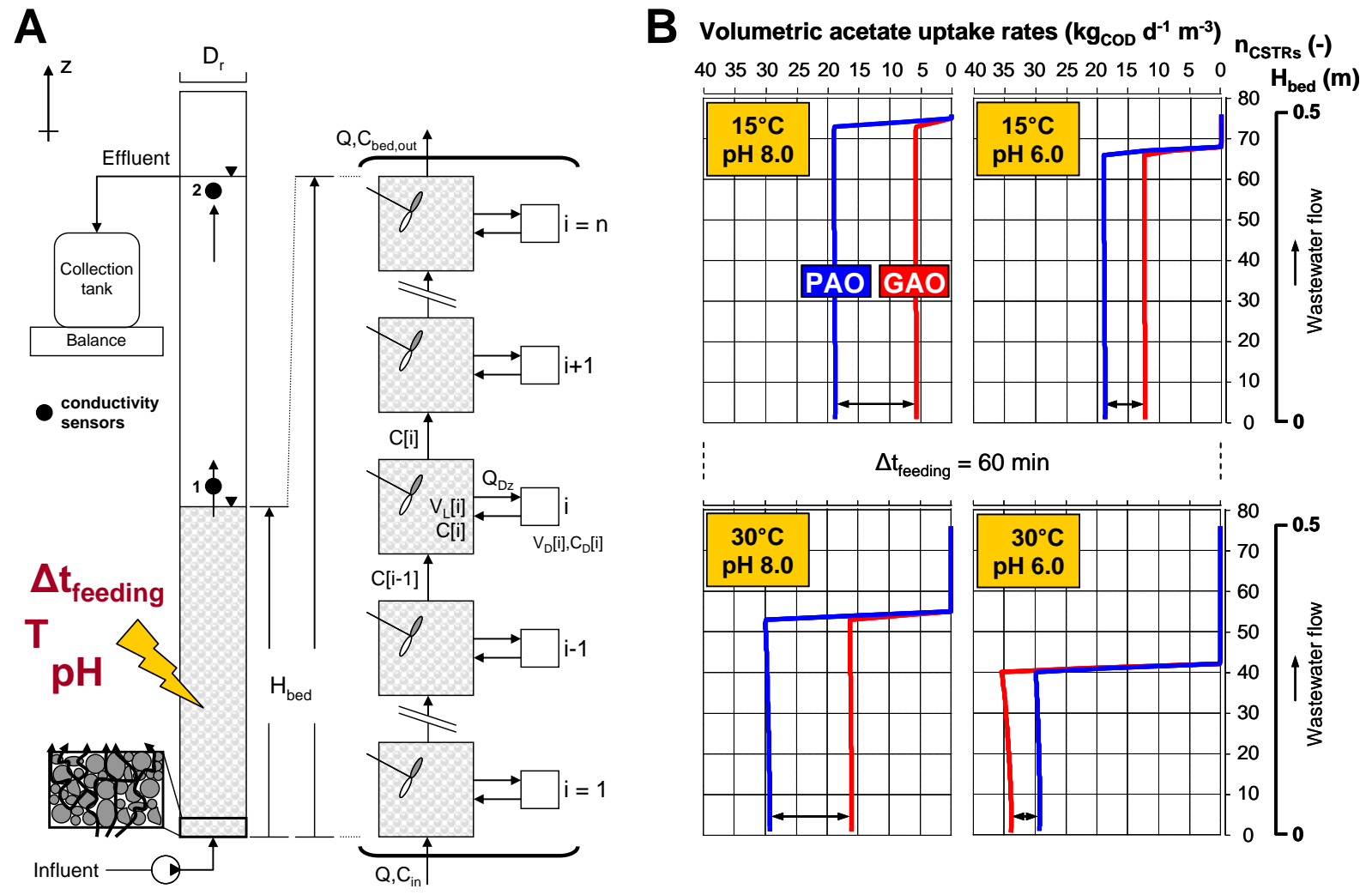

Figure 1. Structure of the plug-flow hydraulic transport model with dispersion (A) and impact of $\mathrm{pH}$ and temperature on acetate uptake by PAO and GAO (B) under slow up-flow anaerobic feeding of wastewater across the bed of AGS.

\section{ACKNOWLEDGEMENT}

This study was financed by the Swiss National Science Foundation at EPFL, Grants no. 205321-120536 and 200020-138148, and by internal funding at ETH Zürich and Eawag.

\section{REFERENCES}

Albuquerque, A. and Santana, F. (2004). Hydrodynamic behaviour of a biological packed bed under different hydraulic and organic loading. In: Reis, A.H. and Miguel, A.F. (eds), International Conference on Applications of Porous Media, Evora, Portugal, pp. 319-327.

de Kreuk, M.K. and van Loosdrecht, M.C.M. (2004). Selection of slow growing organisms as a means for improving aerobic granular sludge stability. Water Sci. Technol. 49(11-12), 9-17.

Gujer, W. (2008). Systems Analysis for Water Technology, Springer Verlag, Berlin, Heidelberg.

Lopez-Vazquez, C.M., Oehmen, A., Hooijmans, C.M., Brdjanovic, D., Gijzen, H.J., Yuan, Z. and van Loosdrecht, M.C.M. (2009). Modelling the PAO-GAO competition: Effects of carbon source, pH and temperature. Water Res. 43(2), 450-462.

Oehmen, A., Lopez-Vazquez, C.M., Carvalho, G., Reis, M.A.M. and van Loosdrecht, M.C.M. (2010). Modelling the population dynamics and metabolic diversity of organisms relevant in anaerobic/anoxic/aerobic enhanced biological phosphorus removal processes. Water Res. 44(15), 4473-4486.

Weissbrodt, D.G., Schneiter, G.S., Furbringer, J.M. and Holliger, C. (2013). Identification of trigger factors selecting for polyphosphate- and glycogen-accumulating organisms in aerobic granular sludge sequencing batch reactors. Water Res., in press. 


\section{WWTmod}

Winkler, M.K.H., Bassin, J.P., Kleerebezem, R., de Bruin, L.M.M., van den Brand, T.P.H. and van Loosdrecht, M.C.M. (2011). Selective sludge removal in a segregated aerobic granular biomass system as a strategy to control PAO-GAO competition at high temperatures. Water Res. 45(11), 3291-3299. 


\title{
Should activated sludge models consider influent seeding of nitrifiers? Field characterization of nitrifying bacteria
}

\author{
Shameem Jauffur, Siavash Isazadeh and Dominic Frigon
}

Department of Civil Engineering and Applied Mechanics, McGill University, 817 Sherbrooke St. West, Montreal, Quebec, H3A 0C3, Canada (Email of corresponding author: dominic.frigon@mcgill.ca).

\begin{abstract}
This study reveals the presence of nitrifying bacteria in influent municipal wastewaters reaching fullscale biological wastewater treatment plants (WWTPs). Respirometric assays showed that the influent nitrifiers could be activated following metabolic induction. We also show that there is a potential of the nitrifiers in the influent stream to actually seed activated sludge bioreactors. Influent-incurred nitrifier seeding affects model performance and influences ammonium $\left(\mathrm{NH}_{4}{ }^{+}\right)$removal. Simulation studies showed that the impact of nitrifier seeding by influent is more prominent at low temperatures. At $4{ }^{\circ} \mathrm{C}$, a nitrifier seed of $5 \mathrm{mg}-\mathrm{COD}_{\text {biomass }} / \mathrm{L}$ induces a $30 \%$ reduction in residual $\mathrm{NH}_{4}{ }^{+}$level and a $17 \%$ gain in solids retention time (SRT) as compared to unseeded conditions. These findings support the need to fine-tune process modelling pertaining to $\mathrm{NH}_{4}{ }^{+}$removal in wastewaters and provide a novel potential means of sustaining nitrification in cold temperature through seeding of influent with nitrifiers.
\end{abstract}

Keywords

Ammonia; influent; modelling, nitrification, nitrifying bacteria

\section{INTRODUCTION}

Ammonia $\left(\mathrm{NH}_{3}\right)$ represents a serious environmental hazard and a deadly threat to fish and aquatic life due to its toxicity (Campos et al. 2008). The most widely applied process worldwide for ammonia removal from municipal wastewater is nitrification: the aerobic biological conversion of $\mathrm{NH}_{3}$ to nitrate $\left(\mathrm{NO}_{3}{ }^{-}\right)$via nitrite $\left(\mathrm{NO}_{2}{ }^{-}\right)$as a secreted intermediate (Mahvi et al. 2008). This microbial-induced catalyzed oxidation is a key process in wastewater treatment and effectively reduces the toxicity associated with $\mathrm{NH}_{3}$ and the biochemical oxygen demand (BOD) of $\mathrm{NH}_{3} / \mathrm{NH}_{4}{ }^{+}$. However, nitrification is a fragile temperature sensitive process (Van Dyke et al. 2003). It is, therefore, not surprising why many biological wastewater treatment systems carrying out nitrogen removal have encountered failures during winter season (Ilies and Mavinic 2001; Kim et al. 2006). Yet, since $\mathrm{NH}_{3}$ is toxic to fish and other aquatic life during all seasons, stricter effluent standards are being implemented and enforced to protect aquatic environments like in the case of North American jurisdictions which have adopted year round regulations for the discharge of total ammonia nitrogen (Canada Fisheries Act 2012; U.S.A Federal Register 2013). Consequently, the conversion of $\mathrm{NH}_{3}$ into $\mathrm{NO}_{3}{ }^{-}$is a requirement even during the winter season, which drives the increase of the design solids retention time (SRT) to prevent nitrifier washout (Rittmann and McCarty 2001). As a consequence, the footprints of these wastewater treatment plants (WWTPs) and the necessary capital investment are higher than for warmer climates.

Nitrifying microorganisms are clustered in few evolutionary lineages within the prokaryotic and archeal domains. They are functionally classified as (1) Ammonia Oxidizing Bacteria (AOB) and Ammonia Oxidizing Archea (AOA), which oxidize $\mathrm{NH}_{3}$ to $\mathrm{NO}_{2}{ }^{-}$, and (2) Nitrite Oxidizing Bacteria (NOB), which convert $\mathrm{NO}_{2^{-}}$to $\mathrm{NO}_{3^{-}}$(Schramm 2003). AOB and NOB share a close symbiotic relationship with each other forming densely packed microcolonies 
and cell clusters in wastewater treatment systems, since the product of $\mathrm{NH}_{3}$ oxidation by $\mathrm{AOB}$ is the substrate for NOB, and whose accumulation is inhibitory to AOB (Daims et al. 2006).

So far the possible seeding of nitrifiers in terms of AOB and NOB by influent wastewaters to activated sludge bioreactors has been overlooked. Even current best practices for biological wastewater treatment modelling, such as the International Water Association (IWA) consensus Activated Sludge Models (ASMs), assume no active biomass in municipal wastewater at the entrance of treatment facilities. If nitrifier seeding subsists in wastewater treatment systems, this may cause models to underestimate nitrification in extreme situations like cold temperature, and lead to over-sizing of aerated bioreactors. Evidencing the existence of significant influent nitrifier seeding may trigger important reviews of wastewater treatment system design practices for cold climate.

To our knowledge, no studies have been conducted so far to unravel the presence of nitrifiers in influent municipal wastewaters reaching full-scale biological wastewater treatment facilities. In this study, we provide answers to the following questions which we believe will promote our understanding of the ecophysiological implications and dynamics of nitrifiers across activated sludge wastewater treatment systems: Are autotrophic nitrifiers present and active in influents of full-scale municipal WWTPs? Are the influent nitrifying populations the same as those present in mixed liquors or in other words, is seeding possible and observed? And given the level of observed potential seeding, what type of gains in design SRT could be made if seeding is considered during modelling?

\section{MATERIALS AND METHODS}

\section{Site description and sample collection}

Influent and Mixed Liquor Suspended Solids (MLSS) samples were collected from 8 fullscale biological WWTPs located in the region of Montreal in Quebec, Canada. The treatment plants use the activated sludge type process and have different layouts and configurations (Table 1). 24-hour composite influent samples and grab MLSS samples were collected at a depth of $1 \mathrm{~m}$ during the Winter 2013. The biomass was spun by micro-centrifugation in 1.5 $\mathrm{ml}$ eppendorf tubes and periodically frozen at $-20{ }^{\circ} \mathrm{C}$ until time of analysis.

Table 1. Description of activated sludge wastewater treatment plants

\begin{tabular}{|c|c|c|c|c|c|c|c|}
\hline \multirow{2}{*}{ WWTPs } & \multicolumn{2}{|c|}{ Geographic location } & \multirow{2}{*}{$\begin{array}{c}\text { Plant } \\
\text { process }^{\mathrm{a}}\end{array}$} & \multirow{2}{*}{$\begin{array}{l}\text { Flow rate } \\
\left(\mathrm{m}^{3} / \text { day }\right)\end{array}$} & \multirow{2}{*}{$\begin{array}{l}\text { SRT } \\
\text { (day) }\end{array}$} & \multirow{2}{*}{$\begin{array}{l}\text { HRT } \\
(\mathrm{hr})\end{array}$} & \multirow{2}{*}{$\begin{array}{c}\text { Influent } \\
\text { composition }(\%)\end{array}$} \\
\hline & Latitude N & Longitude W & & & & & \\
\hline Cowansville & $45^{\circ} 13^{\prime} 16.55^{\prime \prime}$ & $72^{\circ} 46^{\prime} 30.41^{\prime \prime}$ & $\mathrm{CA}$ & 14,000 & 10 & 18 & $90: 10$ \\
\hline Farnham & $45^{\circ} 17^{\prime} 21.90^{\prime \prime}$ & $72^{\circ} 59^{\prime} 35.05^{\prime \prime}$ & EA & 6,000 & 80 & 48 & $80: 20$ \\
\hline Granby & $45^{\circ} 22^{\prime} 17.45^{\prime \prime}$ & $72^{\circ} 46 \prime 23.98^{\prime \prime}$ & $\mathrm{CA}$ & 55,000 & 7 & 20 & $50: 50$ \\
\hline LaPrairie & $45^{\circ} 24^{\prime} 16.48^{\prime \prime}$ & $73^{\circ} 33^{\prime} 22.06^{\prime \prime}$ & EA & 65,000 & 7 & 15 & $45: 55$ \\
\hline Marieville & $45^{\circ} 26^{\prime} 20.28^{\prime \prime}$ & $73^{\circ} 9^{\prime} 51.40^{\prime \prime}$ & EA & 5,000 & 25 & 12 & $80: 20$ \\
\hline Pincourt & $45^{\circ} 23^{\prime} 25.30^{\prime \prime}$ & $74^{\circ} 1^{\prime} 37.34^{\prime \prime}$ & EBPR & 6,000 & 15 & 8 & $90: 10$ \\
\hline Salaberry & $45^{\circ} 13^{\prime} 34.61^{\prime \prime}$ & $74^{\circ} 4^{\prime} 20.44^{\prime \prime}$ & EBPR & 57,000 & 25 & 12 & $27: 6: 57$ \\
\hline Vaudreuil & $45^{\circ} 23^{\prime} 25.30^{\prime \prime}$ & $74^{\circ} 1^{\prime} 37.34^{\prime \prime}$ & $\mathrm{SBR}+\mathrm{EBPR}$ & 18,000 & 5 & 3 & $50: 50$ \\
\hline
\end{tabular}




\section{DNA extraction and PCR amplification of $\operatorname{amo} A$ and $n x r B$ genes}

Genomic DNA was extracted from $0.25 \mathrm{~g}$ of decanted samples (influent and mixed liquor) using the $M O B I O$ UltraClean ${ }^{\mathrm{TM}}$ Fecal DNA Kit (Carlsbad, CA). The extracted DNA samples were diluted to $12 \mathrm{ng}$ and used to determine the presence of nitrifiers by PCR amplifying specific genes using barcoded primers. Barcode multiplexing allows simultaneous sequencing and retrieving of samples based on the identification of sample specific Multiplex Identifier (MID) tag (Liu and Jansson 2010). Ammonia oxidizing bacteria (AOB) were analyzed by targeting the $a m o A$ functional gene using the forward primer amoA- $1 F$ GGG GTT TCT ACT GGT GGT and reverse primer $a m o A-2 R$ CCC CTC TGC AAA GCC TTC TTC (Rotthauwe et al. 1997) while the nitrite oxidizing bacteria (NOB) population was studied by targeting the $n x r B$ gene using the forward primer $n x r B-F 169$ TAC ATG TGG TGG AAC A and reverse primer 616R CGG TTC TGG TCR ATC A (Maixner 2009). Each 50 $\mu$ l of PCR reaction mixture contained $2.5 \mu \mathrm{l}$ of $0.5 \mathrm{M}$ forward primer, $2.5 \mu 1$ of $0.5 \mathrm{M}$ reverse primer, $10 \mu \mathrm{l}$ of $1 \mathrm{x}$ PCR colorless buffer (Bioline), $2.75 \mu 1$ of $2.75 \mathrm{mM} \mathrm{MgCl}_{2}, 0.5 \mu 1$ of $250 \mu \mathrm{M}$ dNTP mixture, $2 \mu \mathrm{l}$ of 12ng DNA template, $0.5 \mu \mathrm{l}$ of 2.5 units Taq DNA Polymerase (Bioline) and 29.25 $\mu \mathrm{l}$ of UltraPure $^{\mathrm{TM}}$ DNase/RNase-Free Distilled Water (Invitrogen). The PCR thermocycling conditions for amoA gene fragment amplification were as follows: $95{ }^{\circ} \mathrm{C}$ for $4 \mathrm{~min}, 35$ cycles of $95{ }^{\circ} \mathrm{C}$ for $40 \mathrm{~s}, 56{ }^{\circ} \mathrm{C}$ for $30 \mathrm{~s}, 72{ }^{\circ} \mathrm{C}$ for $1 \mathrm{~min}$ followed by a final extended elongation at $72{ }^{\circ} \mathrm{C}$ for $10 \mathrm{~min}$. The thermal profiles used for the amplification of $n x r B$ gene target sequence were as follows: $95{ }^{\circ} \mathrm{C}$ for $5 \mathrm{~min}, 35$ cycles of $95{ }^{\circ} \mathrm{C}$ for $40 \mathrm{~s}, 62{ }^{\circ} \mathrm{C}$ for $40 \mathrm{~s}, 72{ }^{\circ} \mathrm{C}$ for $1 \mathrm{~min}$ followed by a final extended elongation at $72{ }^{\circ} \mathrm{C}$ for $10 \mathrm{~min}$. The PCR amplicons were purified using the $M O B I O$ UltraClean ${ }^{\mathrm{TM}}$ PCR Clean-UP Kit.

\section{GX FLS Titanium 454-pyrosequencing}

The amplicon concentration of each sample was determined using the Quant-iT ${ }^{\mathrm{TM}}$ PicoGreen kit and normalized to a concentration of $30 \mathrm{ng} / \mu \mathrm{l}$. The PCR products were pooled and their quality assessed by the Bioanalyzer 2100 (Agilent Technologies). Purified amplicons were subjected to emulsion PCR (emPCR) based on Roche-454 Life Science Protocol and then pyrosequenced by the GS FLX Titanium Sequencing machine. The sequencing run was performed at the McGill University and Genome Quebec Innovation Centre (Montreal, QC) on $1 / 4^{\text {th }}$ PicoTiter plate.

\section{Sequence data analysis}

The amoA and $n x r B$ gene sequences were trimmed and filtered using the QIIME Pipeline (Caporaso et al. 2010) to retain only good quality sequences devoid of primers and barcodes. Quality filtered sequences (minimum read length of $200 \mathrm{bp}$, quality score higher than 25 and without ambiguous bases and mismatches) were clustered at $97 \%$ sequence similarity and assigned to taxonomic operational taxonomic units (OTUs) using the RDP classifier (FunGene Pipeline and repository) (Wang et al. 2007). Bacterial diversity analyses (alpha and beta) were performed using the BiodiversityR package of the R-software, version 3.0.1, based on standardized OTU abundance data.

\section{Respirometric-response assessment of influent nitrifying biomass}

In order to assess the metabolic status of nitrifying biomass in the influent wastewater, approximately 8-10 L of influent were collected from the LaPrairie WWTP and the biosolids were concentrated to about $2500 \mathrm{mg} / \mathrm{l}$. The concentrated biosolids were used to perform respirometric assays by batch respirometry using the Challenge Technology ${ }^{\mathrm{TM}}$ AER-208 Respirometer System to stimulate oxygen uptake rate (OUR) of AOB and NOB populations 
through the addition of $\mathrm{NH}_{4}{ }^{+}$and $\mathrm{NO}_{2}^{-}$respectively as electron donors and measuring the resulting OUR profiles (Chandran and Smets 2005).

\section{Steady-state modelling of $\mathrm{NH}_{4}{ }^{+}$removal}

Residual $\mathrm{NH}_{4}{ }^{+}\left(S_{N H}\right)$ concentration from nitrification was simulated to compare the impacts of temperature and SRT under nitrifier seeding and non-seeding conditions. The steady-state equations were derived using mass balances on the control volume (reactor and settling tank) (Rittmann and McCarty 2001). $S_{N H}$ concentration resulting from treatment of a given bioreactor under non-seeding condition was computed using equation (1). The impact of nitrifier seeding on the treatment system was expressed by reformulating Eq. (1) to Eq. (2) to

$$
S_{N H}=\frac{K_{N H, A}}{\frac{\theta_{x} \hat{q} Y_{A}}{1+b \theta_{x}}-1}
$$
incorporate a term defining nitrifying biomass in the influent stream $\left(X_{A}^{a}\right)$ and mixed liquor $\left(X_{A}\right)$. Default stoichiometric, kinetic and composition model parameters were adopted

$$
S_{N H}=\frac{K_{N H, A}}{\frac{\theta_{x} \hat{q} Y_{A}}{1+b \theta_{x}-\frac{\theta_{x}}{\theta} \cdot \frac{X_{A}^{o}}{X_{A}}}}-1
$$

Where,

$S_{N H} \quad$ Residual effluent $\mathrm{NH}_{4}{ }^{+}$

$K_{N H, A}$ Saturation constant for $\mathrm{NH}_{4}{ }^{+}$

$\theta_{x} \quad$ Solids retention time

$\theta \quad$ Hydraulic retention time

$b \quad$ Endogenous respiration rate

$\hat{q} \quad$ Max. substrate uptake rate

$Y_{A} \quad$ Nitrifier yield coefficient Nitrifying biomass in influent Nitrifying biomass in MLSS

from ASM3 to perform the calculations (Henze et al. 2000).

\section{RESULTS AND DISCUSSION}

\section{Presence of nitrifiers in influent}

Nitrifiers (both AOB and NOB) were detected in all municipal influents collected from the 8 WWTPs. Scrutinizing the sequences at $97 \%$ sequence similarity resulted in an average of 360 distinct OTUs in the influent and 236 OTUs in the mixed liquor samples for the AOB sequences. An inferior number of OTUs was detected for the NOB sequences with an average of 96 OTUs in the influent and 76 OTUs in the mixed liquor samples. The significant $(P<0.05)$ lower diversity in the mixed liquor samples suggests that less OTUs dominate the mixed liquor samples than the number of OTUs entering the plants. Explicit comparison of the nitrifying AOB OTUs in the influent and mixed liquor samples by cluster analysis showed no clear distinction between these 2 types of sample, i.e., all influent and all mixed liquor samples did not cluster together (Figure 1a).

a)

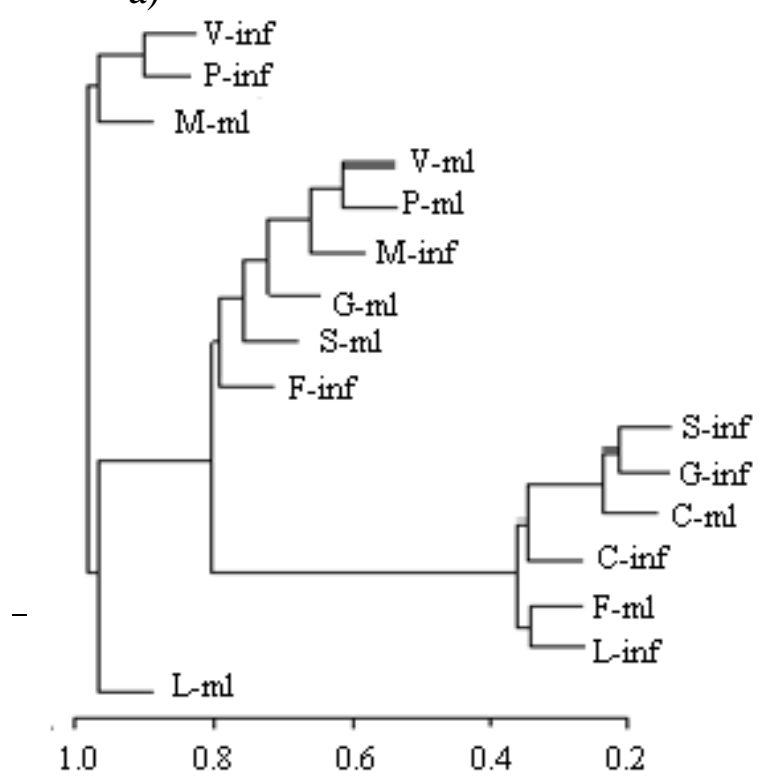

b)

Yet, in general, influent and mixed liquor samples from the same plant also did not cluster together. However, since

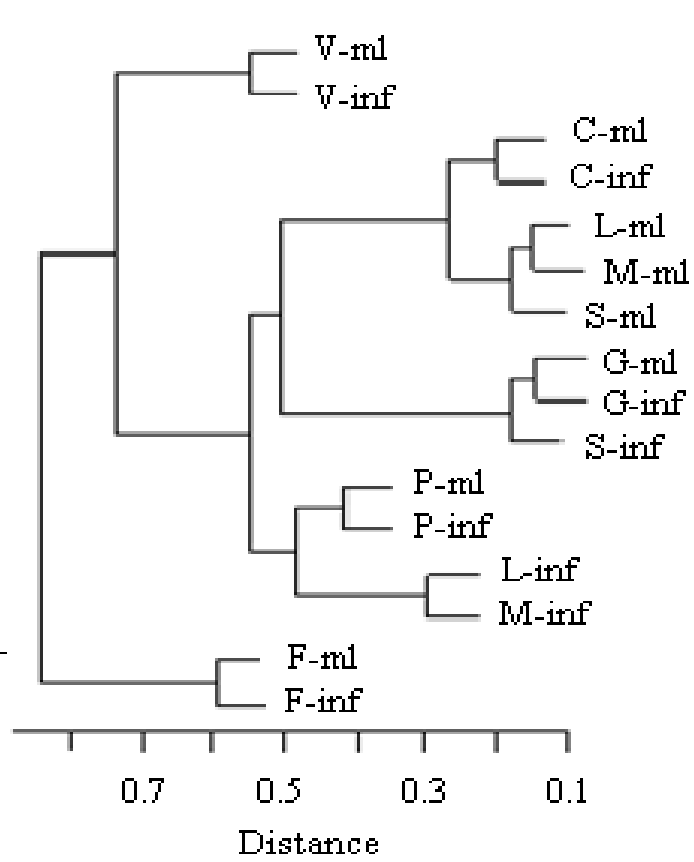


the OTUs detected in the influents were also observed in the mixed liquors, seeding from the influent is possible. Comparison of the biotic similarity between the influent and mixed liquor AOB community assemblages of the same WWTP, using the Bray-Curtis and Kulczinski similarity indices based on standardized abundance datasets, showed that an average of $24 \%$ of AOB OTUs were shared between the two types of sample matrices with the shared OTU reaching as high as $68 \%$ in the case of Cowansville wastewater treatment facility. The most abundant AOB OTU in the influent also occurred as the most abundant AOB OTU in the mixed liquor for all the WWTPs.

Seeding was more apparent for the NOB populations with influent and mixed liquor NOB sequences clustering together for Vaudreuil, Cowansville, Granby, Pincourt and Farnham WWTPs (Figure 1b). Abundance-based similarity indices (Bray-Curtis and Kulczinski) showed an even higher sharing of NOB OTUs as compared to the AOB taxonomic units, averaging $61 \%$ with the highest degree of OTU sharing attaining as high as $86 \%$ in the case of Granby WWTP. Similar to the AOB abundance pattern, the most abundant NOB OTUs in the influent were also found to be most abundant ones in the mixed liquor.

Stochastic models describing bacterial community assemblies predict that random immigration of bacteria plays a crucial role in shaping bacterial communities (Ofiţeru et al. 2010). The present study supports this prediction and allude the potential seeding of autotrophic nitrifying bacteria from influent streams to the activated sludge. The scale of bacterial immigration from the source community is likely to be dependent on the size of the source bacterial reservoir with the immigration rate being higher when the source community size is small (Curtis and Sloan 2006). Hence, seeding of nitrifiers in wastewater treatment systems may be more significant as compared to seeding of heterotrophic bacterial populations since nitrifiers are much less diverse than heterotrophs. According to Curtis et al. (2006), AOB have a low diversity in WWTPs with only 100-200 species growing in WWTPs in a global bacterial community of $10^{27}$. This is line with our findings where we detected AOB populations comprising of 100-395 species and NOB constituting of even smaller populations with only 50-100 species, in the activated sludge samples.

\section{Effects of nitrifier seeding on modelling approaches}

The potential of biological activated sludge systems to perform nitrification is significantly limited at low temperatures, thereby requiring larger aeration tank sizes and longer aerobic SRTs. This is intimately linked to the slow growth rate of nitrifying bacterial biomass at low temperatures (Grady et al. 1999). Based on mass balance considerations, it had been estimated that supplementing a system with $0.1 \mathrm{~g}$ of nitrifiers per day per $\mathrm{g}$ of nitrifiers already present can effectively reduce the SRT up to $45 \%$ at $8{ }^{\circ} \mathrm{C}$ (Salem et al. 2003). The respirometric assays we performed showed that the influent $\mathrm{AOB}$ and $\mathrm{NOB}$ populations responded immediately to the addition of electron donors, and that they could attain full metabolic activity within $6.5 \mathrm{~h}$ and $4.5 \mathrm{~h}$

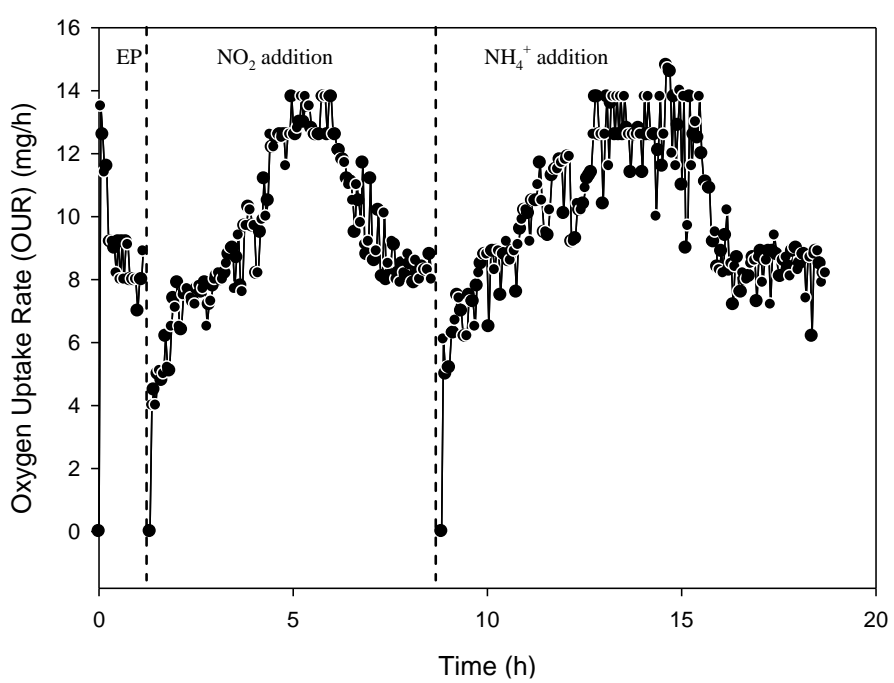

Figure 2. OUR profile obtained from respirometric analysis. $\mathrm{NO}_{2}^{-}$and $\mathrm{NH} 4+$ were added as substrate at $1.25 \mathrm{~h}$ and $8.50 \mathrm{~h}$ respectively. EP-Endogenous Phase. 
respectively (Figure 2). This is indicative of active nitrifiers continuously entering WWTPs via the influent and hints towards potential seeding of activated sludge systems. Based on the activity test data, the level of nitrifiers in the influent of LaPrairie WWTP was estimated at 5 mg-COD biomass $/ \mathrm{L}$, which corresponded to a seeding level at this site of $0.2-0.3 \mathrm{~g}$ of nitrifiers per day per gram of nitrifiers already present. The level of biomass observed in the influent of LaPrairie WWTP justifies further considering potential seeding for modelling and design purposes.

Modelling the $\mathrm{NH}_{4}{ }^{+}$removal from wastewater under the level of nitrifier seeding observed at LaPrairie WWTP and non-seeding scenarios shows that influent nitrifier seeding does not seem to make a significant difference on $S_{N H}$ concentrations at temperatures above $10{ }^{\circ} \mathrm{C}$ (Figure 3). However, as temperature decreases an accrued effect is observed on the $S_{N H}$ concentrations when nitrifying biomass seeding is considered. At 4 ${ }^{\circ} \mathrm{C}$, a seeding of $5 \mathrm{mg}-\mathrm{COD}_{\text {biomass }} / \mathrm{L}$ of nitrifying biomass reduced the $S_{N H}$ concentration by $30 \%$ as compared to unseeded condition. The same simulations showed that nitrifier seeding could allow a reduction of the SRT by approximately $17 \%$ to reach the same $S_{N H}$ level as for the unseeded conditions. These findings demonstrate the kind of gains that can be made in design if seeding of nitrifiers is considered. This may be of interest to modellers and designers, especially at the design stage of wastewater treatment systems since determining the size of activated sludge reactors during design is done by considering the minimum SRT capable of

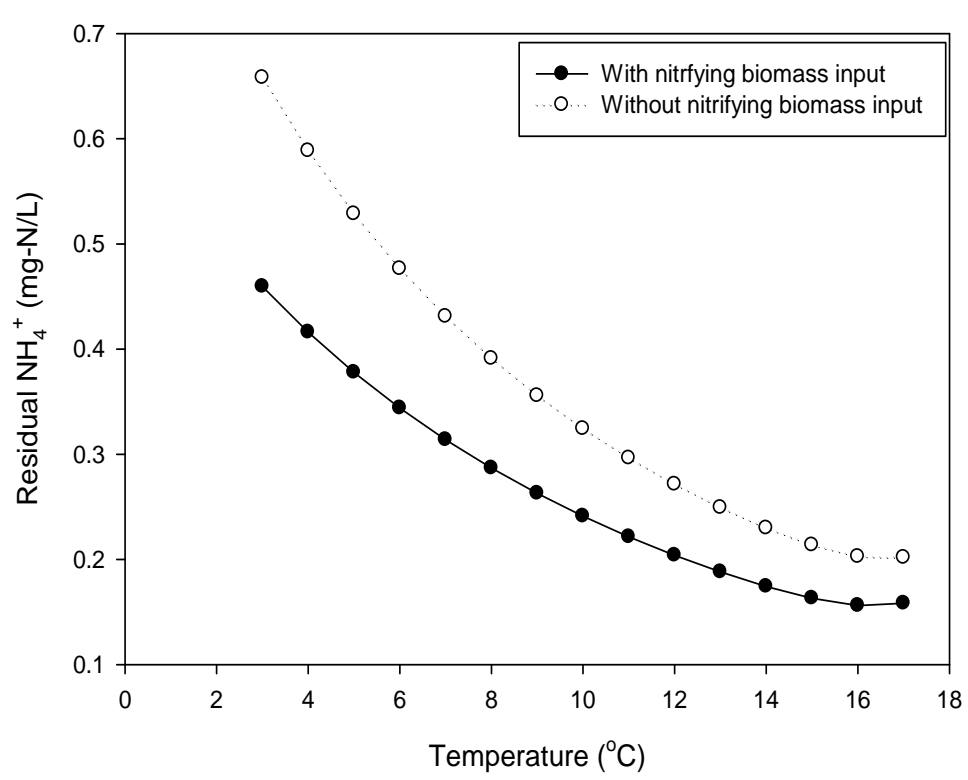

Figure 3. Simulation of $\mathrm{NH}_{4}^{+}$residual concentrations from nitrification in an activated sludge system under influentnitrifier seeding $(5 \mathrm{mg} / \mathrm{L})$ and non-seeding conditions at different temperatures. sustaining high enough activity to provide satisfactory $\mathrm{NH}_{4}{ }^{+}$removal. Considering nitrifier seeding from the influent stream may help reduce the size of aeration tanks at the design stage. In turn, alleviating over-sizing of reactors would translate in reductions in capital expenditure for the construction of wastewater treatment infrastructures. Potentially, this would also reduce the costs of operation because lower oxygen demands would follow as the oxygen demand by the plant is proportional to the SRT (Rittmann and McCarty 2001). However, although our study shows the existence of potential seeding from influent municipal wastewaters to activated sludge bioreactors, the extent of such seeding still needs to be assessed. Reproducing seeding scenarios in lab or pilotscale bioreactors using actual influent nitrifiers from full-scale WWTPs may help to elucidate this aspect which will represent yet another step in understanding this phenomenon.

\section{CONCLUSIONS}

It is the first time that the presence of nitrifying bacteria (both $\mathrm{AOB}$ and NOB) has been revealed in influent municipal wastewaters reaching wastewater treatment facilities. Our findings advocate the existence of potential seeding of nitrifiers from influent streams to full- 
scale activated sludge reactors that may be contributing to the nitrification process in these systems. Simulation of $\mathrm{NH}_{4}{ }^{+}$removal showed that nitrification is enhanced at low temperature under seeding conditions as compared to unseeded scenarios. This may require fine-tuning of process modelling by incorporating seeding especially at low temperatures where the impact may be significant. Such aspect may prove useful in reactor design and improving operation optimality with the aim of sustaining nitrification all year-round including extreme winter seasons. It also paves the way for the implementation of a novel potential means to cope with nitrification in cold temperature by actually seeding influent wastewaters to increase the nitrifier fractions in sewer systems.

\section{ACKNOWLEDGEMENT}

The Funding for this work was provided by the Natural Sciences and Engineering Research Council (NSERC) of Canada and Air Liquide Canada. We extend our gratitude to operators of the WWTPs for granting us permission to collect samples and providing us with operational data.

\section{REFERENCES}

Campos J.L., Carvalho S., Portela R., Mosquera-Corral A. and Méndez R. 2008 Kinetics of denitrification using sulphur compounds: Effects of $\mathrm{S} / \mathrm{N}$ ratio, endogenous and exogenous compounds. Bioresource Technology, 99(5), 1293-1299.

Canada Fisheries Act. 2012 http://laws-lois.justice.gc.ca/eng/regulations/sor-2012-139/page-1.html (accessed 04 February 2014).

Caporaso J.G., Kuczynski J., Stombaugh J., Bittinger K., Bushman F.D., Costello E.K., Fierer N., Peña A.G., Goodrich J.K., Gordon J.I., Huttley G.A., Kelley S.T., Knights D., Koenig J.E., Ley R.E., Lozupone C.A., McDonald D., Muegge B.D., Pirrung M., Reeder J., Sevinsky J.R., Turnbaugh P.J., Walters W.A., Widmann J., Yatsunenko T., Zaneveld J. and Knight R. 2010 QIIME allows analysis of highthroughput community sequencing data. Nature Methods, 7(5), 335-336.

Chandran K. and Smets B.F. 2005 Optimizing experimental design to estimate ammonia and nitrite oxidation biokinetic parameters from batch respirograms. Water Research, 39(20), 4969-4978.

Curtis T.P. and Sloan W.T. 2006 Towards the design of diversity: stochastic models for community assembly in wastewater treatment plants. Water Science and Technology, 54(1), 227-236.

Daims H., Maixner F., Lücker S., Stoecker K., Hace K. and Wagner M. 2006 Ecophysiology and niche differentiation of Nitrospira-like bacteria, the key nitrite oxidizers in wastewater treatment plants. Water Science and Technology, 54(1), 21-27.

Grady L., Daigger G. and Lim H. 1999 Biological wastewater treatment, Marcel Dekker, New York.

Henze M., Gujer W., Mino T. and Loosdrecht M.v. 2000 Activated sludge models ASM1, ASM2, ASM2d and ASM3. International Water Association Task Group on Mathematical Modelling for Design and Operation of Biological Wastewater, Treatment, IWA Pub., London.

Ilies P. and Mavinic D.S. 2001 Biological nitrification and denitrification of a simulated high ammonia landfill leachate using 4-stage Bardenpho systems: system startup and acclimation. Canadian Journal of Civil Engineering, 28(1), 85-97.

Kim D.-J., Lee D.-I. and Keller J. 2006 Effect of temperature and free ammonia on nitrification and nitrite accumulation in landfill leachate and analysis of its nitrifying bacterial community by FISH. Bioresource Technology, 97(3), 459-468.

Liu W.T. and Jansson J.K. 2010 Environmental molecular microbiology, Caister Academic Press, Norfolk, UK.

Mahvi A.H., Nabizadh R., Pishrafti M.H. and Zarei T. 2008 Evaluation of Single Stage USBF in Removal of Nitrogen and Phosphorus from Wastewater. European Journal of Scientific Research, 23(2), 204-211.

Maixner F. 2009 The ecophysiology of nitrite-oxidizing bacteria in the genus Nitrospira: Novel aspects and unique features. PhD thesis, University of Wien, Austria.

Ofiţeru I.D., Lunn M., Curtis T.P., Wells G.F., Criddle C.S., Francis C.A., Sloan W.T. and Tiedje J.M. 2010 Combined niche and neutral effects in a microbial wastewater treatment community. Proceedings of the National Academy of Sciences of the United States of America, 107(35), 15345-15350. 


\section{WWTmod}

Rittmann B. and McCarty P. 2001 Environmental biotechnology : principles and applications, McGraw-Hill, Boston.

Rothauwe J.H., Witzel K.P. and Liesack W. 1997 The ammonia monooxygenase structural gene amoA as a functional marker: molecular fine-scale analysis of natural ammonia-oxidizing populations. Applied and Environmental Microbiology, 63(12), 4704-4712.

Salem S., Berends D.H.J.G., Heijnen J.J. and Van Loosdrecht M.C.M. 2003 Bio-augmentation by nitrification with return sludge. Water Research, 37(8), 1794-1804.

Schramm A. 2003 In Situ Analysis of Structure and Activity of the Nitrifying Community in Biofilms, Aggregates, and Sediments. Geomicrobiology Journal, 20(4), 313-333.

U.S.A Federal Register 2013 http://www.gpo.gov/fdsys/pkg/FR-2013-08-22/pdf/2013-20307.pdf (accessed 04 February 2014).

Van Dyke S., Jones S. and Ong S.K. 2003 Cold weather nitrogen removal deficiencies of aerated lagoons. Environmental Technology, 24(6), 767-777. 


\title{
Generation of (synthetic) influent data for performing wastewater treatment modelling studies
}

\author{
Xavier Flores-Alsina ${ }^{1}$, Christoph Ort $^{2}$, Cristina Martin ${ }^{3,4}$, Lorenzo Benedetti ${ }^{5}$, Evangelina Belia ${ }^{6}$, Laura \\ Snip $^{1}$, Ramesh Saagi ${ }^{7}$, Mansour Talebizadeh ${ }^{4}$, Peter A. Vanrolleghem ${ }^{4}$, Ulf Jeppsson ${ }^{7}$, Krist V. Gernaey ${ }^{1}$ \\ ${ }^{1}$ Center for Process Engineering and Technology (PROCESS), Department of Chemical and Biochemical \\ Engineering, Technical University of Denmark (DTU), Building 229, DK-2800 Kgs. Lyngby, Denmark \\ ${ }^{2}$ Eawag, Swiss Federal Institute of Aquatic Science and Technology, CH 8600 Dübendorf, Switzerland \\ ${ }^{3}$ Deusto Institute of Technology - University of Deusto, Avda. Universidades 24, 48007 Bilbao, Spain \\ ${ }^{4}$ modelEAU, Département de génie civil et de génie des eaux, Université Laval, 1065 Avenue de la Médecine, \\ Québec G1V 0A6, QC, Canada \\ ${ }^{5}$ Waterways, Via del Ferrone 88, 50023 Impruneta (Florence), Italy \\ ${ }^{6}$ Primodal Inc., 145 Aberdeen, Québec QC, G1R 2C9, Canada \\ ${ }^{7}$ Division of Industrial Electrical Engineering and Automation (IEA), Lund University, Box 118, SE-221 00 \\ Lund, Sweden
}

\begin{abstract}
The success of many modelling studies strongly depends on the availability of sufficiently long influent time series - the main disturbance of a typical wastewater treatment plant (WWTP) - representing the inherent natural variability at the plant inlet as accurately as possible. This is an important point since most modelling projects suffer from a lack of realistic data representing the influent wastewater dynamics. The objective of this paper is to show the advantages of creating synthetic data when performing modelling studies for WWTPs. This study reviews the different principles that influent generators can be based on, in order to create realistic influent time series. In addition, the paper summarizes the variables that those models can describe: influent flow rate, temperature and traditional/emerging pollution compounds, weather conditions (dry/wet) as well as their temporal resolution (from minutes to years). The importance of calibration/validation is addressed and the authors critically analyse the pros and cons of manual versus automatic and frequentistic vs Bayesian methods. The presentation will focus on potential engineering applications of influent generators, illustrating the different model concepts with case studies. The authors have significant experience using these types of tools and have worked on interesting case studies that they will share with the audience. Discussion with experts at the WWTmod seminar shall facilitate identifying critical knowledge gaps in current WWTP influent disturbance models. Finally, the outcome of these discussions will be used to define specific tasks that should be tackled in the near future to achieve more general acceptance and use of WWTP influent generators.
\end{abstract}

Keywords: Disturbance generators, dynamics, flow, influents, pollution loads, uncertainty

\section{INTRODUCTION}

The use of activated sludge models (ASM) (Henze et al., 2000) is constantly growing and both industry and academia are increasingly applying these tools when performing wastewater treatment plant (WWTP) engineering studies. The level of detail and the specific data required for a modelling exercise strongly depend on the project objectives. In general, the more specific the results of the simulation study, the more detailed the required set of data (Cierkens et al., 2012). However, due to the high cost of measuring campaigns, many simulation studies of full-scale WWTPs suffer from a lack of sufficiently long and detailed time series for flow rates, temperature and nutrient/pollutant concentrations representing realistic wastewater influent dynamics. For this reason, model-based influent generators are an alternative that has recently gained considerable interest (Gernaey et al., 2011).

\section{METHODS}

Literature offers a wide range of tools generating influent characteristics by means of mathematical models. The paper will analyse in detail:

- The shift in methods to generate influent dynamics from the simpler (black box) to the more complex (grey/white box) models including a more detailed description of the 
phenomena taking place in the urban drainage system with more/less equations/model parameters (Fig 1). The type of approach will determine: 1) model parsimony (limiting the number of parameters); 2) model transparency (by using model parameters that have a physical meaning); and 3) model flexibility (easily extended to other applications) (Gernaey et al., 2011).

- The data availability to create/re-create the different influents. Here, different relevant questions are to be answered: "Do I have measurements and can I therefore apply statistical analysis to obtain longer time series? "Are measurements entirely missing and do I need a model that can provide realistic patterns without measurements?"

A more in depth analysis about methods will be complemented with the critical review carried out by Martin and Vanrolleghem (2013).
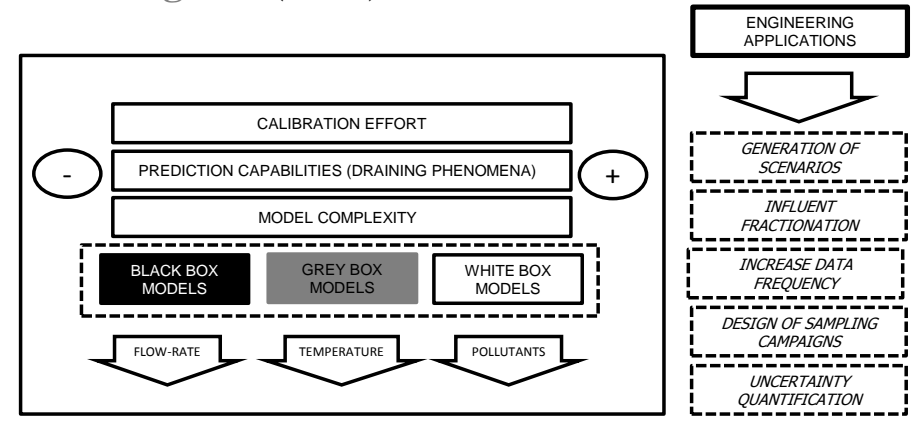

Figure 1. Methods, characteristics, modelled compounds and engineering applications of influent generators.

\section{COMPOUNDS AND TEMPORAL RESOLUTION}

Another point of discussion will focus on what kind of compounds (and their temporal resolution) can be described with the current models. For example:

- Generation/frequency of (dry weather) flow rate, temperature, traditional components (COD, TSS, TN and TP) and emerging components (pharmaceuticals, illicit drugs);

- Generation/frequency of wet weather flow, temperature and traditional/emerging components.

Most of the models used to describe traditional compounds are based on intensive measuring campaigns carried out during the 90s (Butler et al., 1995). In addition to the description of traditional pollutants some of these models can also describe emerging compounds. For example, De Keyser et al. (2010) developed a database summarizing different emission patterns for 26 priority pollutants (daily/weekly/seasonal /annual). Lindblom et al. (2006) and Snip et al. (2013) upgraded the phenomenological influent model presented by Gernaey et al. (2011) including the behaviour of bisphenol A, pyrene and some pharmaceuticals (antibiotics, painkillers, mood stabilizers). Ort et al. (2005) developed a conceptual stochastic model to characterise short-term variations of benzotriazole concentrations (a chemical contained in dishwasher detergents), which can be easily adapted to any down-the-drain household chemical. Additional model complexity is necessary to describe the behaviour of all these elements in wet-weather conditions (Gernaey et al., 2011). Nevertheless some of the wet-weather generators are simplified and may not correctly represent the rainfall properties, the build-up/wash-off (pollution) and rainfall/run-off (water). Specifically, associated soil models currently do not include physico-chemical descriptions of moisture properties and some transport models are not capable to correctly describe the first-flush effect after a (heavy) storm event (Martin and Vanrolleghem, 2013). For these reasons the effect on flow rate, substances and temperature might be systematically under- or overestimated. 


\section{CALIBRATION AND VALIDATION}

Another important point that will be discussed is related to several aspects that should be considered during calibration/validation of such influent generation models:

- In most black box models, parameter values are identified after processing long time series. However, these parameters are adjusted to fit the inputs and outputs and do not have any physical/biological/chemical meaning. On the other hand, grey and white box models are based on parameters that correspond to measurements or physical characteristics of the catchment.

- The traditional calibration procedure uses a trial and error process of parameter adjustments. Often, the goodness-of-fit of the calibrated model is basically a visual judgement comparing simulated and observed data. This process is subjective and can be quite long and tedious unless the process engineer has a good knowledge about the model behaviour (Flores-Alsina et al., 2013). Automatic calibration has the advantage that it can (in some cases) accelerate the process and be objective as it is based on quantitative goodness-of-fit criteria.

- Frequentist analysis has demonstrated to work quite well in identifiable systems (Omlin and Reichert, 1999). Nevertheless, when the models present: 1) some apparent identifiability problems (Omlin and Reichert, 1999); or, 2) some structural uncertainty in the model formulation (Neumann and Gujer, 2008), this approach is no longer valid and other approaches based on Bayesian statistics are recommended. However, the calibration effort increases substantially when using more elaborate methods (Lindblom et al., 2011; Rieckermann et al., 2011; Talebizadeh et al., 2013) (Fig 1).

\section{ENGINEERING APPLICATIONS}

The engineering applications of influent generators are various (Fig 1):

1. Increase data frequency: Sub-hour frequency of influent data is required when the model is used to test control strategies and wet-weather operation. Characterization of the influent implies a large effort and high costs when analysing samples for a series of pollutants. Recent developments in measurement technology have made sensors more reliable and cheap. Still, several standard lab analyses, such as COD, cannot be performed reliably in on-line mode in the influent of a WWTP (Olsson et al., 2012). In these situations, influent generators can certainly increase the frequency of influent data and provide additional dynamics not revealed by measurements (Devisscher et al., 2006; Gernaey et al., 2011; Flores-Alsina et al., 2013).

2. Design of sampling strategies: Grey / Black box influent generators can account for, amongst other factors, different types of dynamics, levels of occurrence and the effect of pumping strategies in the sewer when (mathematically) describing the occurrence of traditional/emerging pollutants. This feature can be extremely useful when designing sampling campaigns. Ort et al. (2010) demonstrated that errors of 50\% or more are possible for 24-h composite samples when the compound is not sampled at a sufficiently high frequency.

3. Fractionation: Influent fractionators can easily be plugged in to the time series created by influent generators. The main idea is to correlate the model state variables used in the ASM models (Henze et al., 2000) with their analytical measurements. For example, Grau et al. (2007) and Germaey et al. (2011) proposed two alternatives based on different principles. The first approach is based on an optimizer that finds suitable fractionation parameters according to the available data. The second approach uses (fixed) parameter values in order to convert for example $\mathrm{COD}_{\text {sol }}$ into non-biodegradable $\left(\mathrm{S}_{\mathrm{I}}\right)$ and biodegradable $\left(\mathrm{S}_{\mathrm{S}}\right)$ soluble substrates using the ASM1 (Henze et al., 2000). 
4. Uncertainty/Sensitivity analysis of influent profiles: The use of probability distribution functions in some of the influent generator models combined with Monte Carlo simulations might help to quantify the range and/or uncertainty of simulated data (wastewater properties). These simulation outputs can be used to better design WWTPs using probabilistic concepts rather than safety factors (Rousseau et al., 2001; Belia et al., 2009; Flores-Alsina et al., 2012; Talebizadeh et al., 2013) or to test the robustness of control strategies (Benedetti et al., 2006; Flores-Alsina et al., 2008; ).

5. Generation of scenarios: Dynamics and complexity of factors influencing wastewater systems make reliable predictions very difficult, i.e. the characteristics of the catchment area can change substantially over the years. For this reason, it is necessary to improve the planning and design of wastewater treatment infrastructures through methodologies that systematically account for uncertain futures (Dominguez and Gujer, 2006). The use of the presented tools can be very beneficial to answer "what-if" questions (Gernaey et al., 2011; Flores-Alsina et al., 2013; Martin and Vanrolleghem, 2013).

\section{PURPOSE OF THE PAPER}

The main objective of this presentation/paper is to demonstrate the advantages of influent generators (reduce the cost of measuring campaigns, fill data gaps, create additional scenarios) with several illustrative case studies. The second purpose is to identify critical knowledge gaps related to model development, calibration procedures and increasing the number of (wastewater) engineering applications. Comments received at the conference will be included in subsequent influent generator model upgrades (the authors are actively working on model development), thus addressing modellers' needs. This will finally achieve a more general acceptance and - equally important - common standards on model building and calibration of influent generators.

\section{ACKNOWLEDGEMENTS}

Dr. Flores-Alsina, Mr. Saagi and Miss Snip acknowledge the Marie Curie Program of the European Union's $7^{\text {th }}$ Framework Programme FP7 2007-2013 under the REA agreements 329349 (PROTEUS) and 289193 (SANITAS). Peter Vanrolleghem holds the Canada Research Chair in Water Quality Modelling

\section{REFERENCES}

Belia E., Amerlinck Y., Benedetti L., Johnson B., Sin G., Vanrolleghem P.A., Gernaey K.V., Gillot S., Neumann M.B., Rieger L., Shaw A., Villez K. (2009). Wastewater treatment modelling: Dealing with uncertainties. Wat. Sci. Tech., 60(8), 1929-1941.

Benedetti L., Bixio D., Vanrolleghem P.A. (2006). Assessment of WWTP design and upgrade options: Balancing costs and risks of standards' exceedance. Wat. Sci. Tech., 54(6-7), 371-378.

Butler D., Friedler E., Gatt K. (1995). Characterising the quantity and quality of domestic wastewater inflows. Wat. Sci. Tech., 31(7), 13-24.

Cierkens K., Plano S., Benedetti L., Weijers S., de Jonge J., Nopens, I. (2012). Impact of influent data frequency and model structure on the quality of WWTP model calibration and uncertainty. Wat. Sci. Tech., 65(2), 233-242. Devisscher M., Ciacci G., Fé L., Benedetti L., Bixio, D., Thoeye C., De Gueldre G., Marsili-Libelli S., Vanrolleghem, P.A. (2006). Estimating costs and benefits of advanced control for wastewater treatment plants the MAgIC methodology. Wat. Sci. Tech., 53(4-5): 215-223.

De Keyser W., Gevaert V., Verdonck F., De Baets B., Benedetti L. (2010). An emission time series generator for pollutant release modelling in urban areas. Environ. Modell. Softw., 25(4), 554-561.

Dominguez D., Gujer W. (2006). Evolution of a wastewater treatment plant challenges traditional design concepts. Water Res., 40(7), 1389-1396.

Flores-Alsina X., Rodríguez-Roda I., Sin G., Gernaey K. (2008). Multi-criteria evaluation of wastewater treatment plant control strategies under uncertainty. Water Res., 42, 4485-4497. 
Flores-Alsina X., Corominas L., Neumann M.B., Vanrolleghem P.A (2012). Assessing the use of activated sludge processs design guidelines in wastewater treatment plant projects. A methodology based on global sensitivity analysis. Environ. Modell. Softw., 38(12), 50-58.

Flores-Alsina X., Saagi R., Lindblom E., Thirsing C., Thornberg., Gernaey K.V., Jeppsson U. (2013). Calibration and validation of a phenomenological influent pollutant disturbance scenario generator using fullscale data. Water Res. Submitted.

Gernaey K.V., Flores-Alsina X., Rosen C., Benedetti L., Jeppsson U. (2011). Dynamic influent pollutant disturbance scenario generation using a phenomenological modelling approach. Environ. Modell. Softw., 26(11), 1255-1267.

Grau P., Beltrán S., de Gracia M., Ayesa E. (2007). New mathematical procedure for the automatic estimation of influent characteristics in WWTPs. Wat. Sci. Tech., 56(8), 95-106.

Henze M., Gujer W., Mino T., van Loosdrecht M.C.M. (2000). Activated Sludge Models ASM1, ASM2, ASM2d and ASM3. IWA Scientific and Technical Report No. 9, IWA Publishing, London, UK.

Lindblom E., Gernaey K.V., Henze M., Mikkelsen P.S. (2006). Integrated modelling of two xenobiotic organic compounds. Wat. Sci. Tech., 54(6-7), 213-221.

Lindblom E., Ahlman S., Mikkelsen P.S. (2011). Uncertainty-based calibration and prediction with a stormwater surface accumulation-washoff model based on coverage of sampled $\mathrm{Zn}, \mathrm{Cu}, \mathrm{Pb}$ and $\mathrm{Cd}$ field data. Water Res., 45(13), 3823-3835.

Martin C., Vanrolleghem P.A. (2013). Analyzing, completing and generating influent data for WWTP modelling: a critical review. Environ. Modell. Softw. Submitted

Neumann M., Gujer W. (2008). Underestimation of uncertainty in statistical regression of environmental models: Influence of model structure uncertainty. Env. Sci. Tech., 42(11), 4037-4043.

Olsson G. (2012). ICA and me: A subjective review. Water Res., 46(6), 1586-1624.

Omlin M., Reichert P. (1999). A comparison of techniques for the estimation of model prediction uncertainty. Ecol. Model., 115(1), 45-59.

Ort C., Lawrence M.G., Reungoat J., Mueller J.F. (2010). Sampling for PPCPs in sewers: Comparison of different sampling modes and optimization strategies. Env. Sci. Tech., 44(16), 6289-6296.

Ort C., Schaffner C., Giger W., Gujer W. (2005). Modeling stochastic load variations in sewer systems. Wat. Sci Tech., 52(5), 113-22.

Rieckermann J., Anta J., Scheidegger A., Ort C. (2011). Assessing wastewater micropollutant loads with approximate Bayesian computations. Env. Sci. Tech., 45(10), 4399-4406.

Rousseau D., Verdonck F., Moerman O., Carrette R., Thoeye C., Meirlaen J., Vanrolleghem P.A. (2001). Development of a risk assessment based technique for design/retrofitting of WWTPs. Wat. Sci. Tech., 43(7), 287-294.

Snip L., Flores-Alsina X., Plosz B., Jeppsson U., Gernaey K.V. (2013). Extending the BSM platform with occurrence, transport and fate of micropollutants. Environ. Modell. Softw. Submitted

Talebizadeh M., Belia L., Vanrolleghem P.A. (2014). Influent generator for probabilistic design of nutrient removal wastewater treatment plants. Submitted to 4th IWA/WEF Wastewater Treatment Modelling Seminar (WWTmod2014), Spa, Belgium, March 30 - April 2, 2014. 


\title{
A simple yet efficient colour-based system analysis tool for ASM
}

\author{
Amerlinck, Y. ${ }^{1}$, Cierkens, K. ${ }^{1}$, Nopens, I. ${ }^{1}$ \\ ${ }^{1}$ BIOMATH, Department of Mathematical Modeling, Statistics and Bioinformatics, Ghent University, \\ Coupure Links 653, 9000 Gent, Belgium (Email: youri.amerlinck@ugent.be; katrijn.cierkens@ugent.be; \\ ingmar.nopens@ugent.be).
}

\begin{abstract}
Model-based analysis and optimization of wastewater treatment plants usually consists of numerous simulations and large data sets generated. Finding specific information in these data sets is not always a trivial task especially when this information is hidden in temporary algebraic variables that are not easily accessible or not defined as such. An extremely simple yet effective colour-based evaluation method is proposed for system analysis, e.g. for bottleneck identification. The tool proved very useful in evaluating large amounts of data and in taking certain decisions. Including the tool in simulation platforms could facilitate ASM model analysis and provide transparency for both experienced and inexperienced modellers.
\end{abstract}

Keywords

Post-processing; bottleneck identification; data evaluation; ASM analysis

\section{INTRODUCTION}

Present-day wastewater treatment plant (WWTP) optimization is largely based on modelbased analysis. However this analysis usually results in the execution of a large number of simulations and the generation of large data sets. Trying to find explanations why one scenario (unexpectedly) performs better than the other or even trying to determine which scenario is the better one requires "number crunching". Visual inspection of time series contains the risk of overlooking things, whereas summarizing time series into single numbers (average, minimum, maximum, etc.) results in significant loss of information. Simple colourbased evaluation methods might be a third approach to facilitate the data evaluation. In addition, the description of process rates or kinetics has become increasingly complex and typically multiple switching functions are joined to yield the overall process rate. Although of profound importance, these switching functions are somewhat "hidden" and often not explicitly available as output variable, meaning that intermediate calculation results are not directly accessible for the modeller. Detailed analysis of these switching functions can provide useful insight. The goal of this paper is to demonstrate the proposed system analysis tool based on an illustrative example.

\section{MATERIALS AND METHODS}

A method is proposed for the analysis of kinetic expressions that consist of a product of switching functions. These switching functions, e.g. Monod functions (Monod, 1942), are used to describe the effect of a limiting factor on a particular biological process or to (de)activate a process when a factor is exceeding a threshold. Typically, several switching functions are joined together and finally result in having a process run in the range between its maximal rate and zero. However, when a rate drops, it is not always obvious from the simulation which switching function is actually the limiting factor due to their joined nature and the fact that they are not separately calculated as algebraic states or output variables. Hence, these switching functions contain valuable "hidden" information on the activity of the process. Using colour coding for all distinctive switching functions allows for a fast inspection of the impact of all switching functions on the overall process rate and to detect 
which factor is limiting. The method was applied using the WWTP model of Eindhoven (Amerlinck et al., 2013) to evaluate the evolution of enhanced biological phosphorus removal (EBPR), nitrification and denitrification activity in scenarios with reduced phosphate concentration in the inlet (induced by the application of dissolved air flotation (DAF)). As an illustrative example, the process rate limitation of autotrophic growth is shown here. In the ASM2d model (Henze et al., 2000) the growth rate of autotrophs (Equation 1) contains switching functions for oxygen $\left(\mathrm{S}_{\mathrm{O}}\right)$, ammonium $\left(\mathrm{S}_{\mathrm{NH}}\right)$, phosphate $\left(\mathrm{S}_{\mathrm{PO}}\right)$ and alkalinity $\left(\mathrm{S}_{\mathrm{ALK}}\right)$.

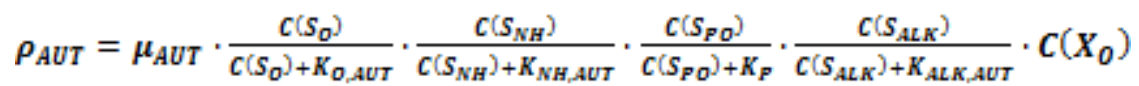

Equation 13

\section{RESULTS AND DISCUSSION}

Dynamic simulation of a scenario with reduced phosphate concentration in the inlet of the biological treatment (Figure ), resulting from the application of a DAF, showed an unexpected but significant increase in ammonium concentration while oxygen levels and the amount of nitrifiers seemed sufficiently high.

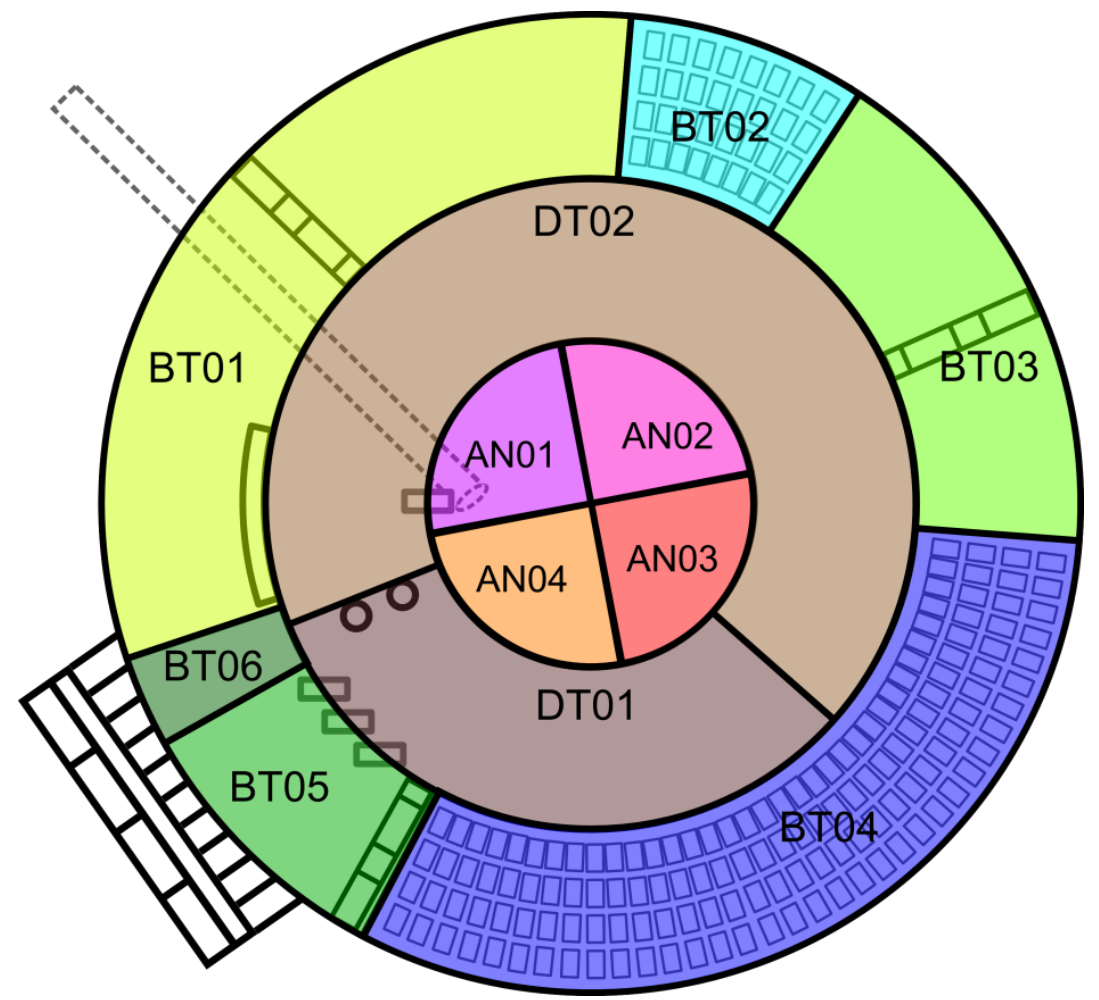

Figure 1. The circular modified UCT configuration of the activated sludge tanks at the WWTP of Eindhoven and the mapping of tanks in series used in the model.

At some points in time phosphate was rather low but it could not be deduced directly from the dynamic simulation results that this was causing the decrease in nitrification activity. In contrast, the proposed method gave a clear view on this aspect. Table shows the individual effect of the distinct Monod switching functions (row 1-4) and the overall autotrophic growth rate as a fraction of the maximum growth rate (row 5, being the product of rows 1-4), for the different reaction zones, averaged over the entire simulation period. Colours evolve from 
green, indicating a high value (no limitation) over orange to red, indicating a low value (severe limitation).

From Table it can be clearly seen that phosphate is not limiting in the anaerobic (AN01 through AN04) and anoxic zones (DT01 and DT02). The limiting factor in these zones is obviously the lack of oxygen. However, in the aerobic zones (BT01 through BT06) a relation can be seen between the limitation in the autotrophic growth rate and the Monod switching function for phosphate. I.e. while the Monod switching functions for alkalinity, ammonium and oxygen are high (and thus not limiting), the Monod switching function for phosphate and as a consequence also the overall autotrophic growth rate is low.

Table 1. Oxygen limitation (in the anaerobic and anoxic zones) and phosphate limitation (in the aerobic zones) of the overall autotrophic growth visualized through the impact of the different Monod terms for the different sections of the activated sludge tank, averaged over the entire simulation period, using a colour-based system analysis tool.

\begin{tabular}{|lccccccccccc|c|c|}
\hline AVERAGE & AN01 & AN02 & AN03 & AN04 & DT01 & DT02 & BT01 & BT02 & BT03 & BT04 & BT05 & BT06 \\
\hline Monod S_ALK & 0.93 & 0.93 & 0.92 & 0.92 & 0.93 & 0.93 & 0.93 & 0.93 & 0.93 & 0.93 & 0.93 & 0.93 \\
Monod S_NH & 1.00 & 1.00 & 1.00 & 1.00 & 1.00 & 1.00 & 0.99 & 0.99 & 0.99 & 0.99 & 0.98 & 0.98 \\
Monod S_O & 0.06 & 0.00 & 0.00 & 0.00 & 0.02 & 0.06 & 0.77 & 0.75 & 0.70 & 0.88 & 0.88 & 0.85 \\
Monod S_PO & 0.98 & 0.99 & 0.99 & 0.99 & 0.80 & 0.76 & 0.36 & 0.29 & 0.28 & 0.29 & 0.29 & 0.29 \\
\hline Overall & 0.05 & 0.00 & 0.00 & 0.00 & 0.01 & 0.01 & 0.21 & 0.13 & 0.10 & 0.21 & 0.20 & 0.19 \\
\hline
\end{tabular}

Table 10 shows the dynamic behaviour of the same autotrophic growth rate (as a fraction of the maximum growth rate; similar as row 5 of Table but dynamic instead of averaged). Table on the other hand shows only the Monod term for phosphate as nutrient for growth (similar as row 4 of Table but dynamic). Also from Table 10 and Table this relation, between the autotrophic growth rate and the Monod switching function for phosphate, can be deduced. I.e. when the Monod term for phosphate reaches higher percentages (green colour) the growth of autotrophs increases (yellow to green colours). The switching functions for oxygen $\left(\mathrm{S}_{\mathrm{O}}\right)$, ammonium $\left(\mathrm{S}_{\mathrm{NH}}\right)$ and alkalinity $\left(\mathrm{S}_{\mathrm{ALK}}\right)$ remain high and do not show the same trend (results not shown), although they lower the activity of the nitrification process slightly.

Table 10. Recovery over time of the growth rate of autotrophs for the different sections of the activated sludge tank shown using a colour-based evaluation method.

\begin{tabular}{|c|c|c|c|c|c|c|c|c|c|c|c|c|}
\hline Time & AN01 & AN02 & AN03 & AN04 & DT01 & DT02 & BT01 & ВT02 & ВТ03 & ВT04 & ВT05 & ВT06 \\
\hline 11.962 & 0.05 & 0.00 & 0.00 & 0.00 & 0.00 & 0.01 & 0.28 & 0.16 & 0.11 & 0.12 & 0.12 & 0.13 \\
\hline 11.965 & 0.05 & 0.00 & 0.00 & 0.00 & 0.00 & 0.01 & 0.29 & 0.17 & 0.12 & 0.14 & 0.14 & 0.14 \\
\hline 11.969 & 0.05 & 0.00 & 0.00 & 0.00 & 0.00 & 0.01 & 0.31 & 0.19 & 0.13 & 0.15 & 0.15 & 0.16 \\
\hline 11.972 & 0.05 & 0.00 & 0.00 & 0.00 & 0.00 & 0.01 & 0.32 & 0.21 & 0.15 & 0.17 & 0.17 & 0.18 \\
\hline 11.976 & 0.05 & 0.00 & 0.00 & 0.00 & 0.00 & 0.01 & 0.34 & 0.23 & 0.17 & 0.20 & 0.20 & 0.21 \\
\hline 11.979 & 0.05 & 0.00 & 0.00 & 0.00 & 0.00 & 0.01 & 0.36 & 0.26 & 0.20 & 0.24 & 0.24 & 0.25 \\
\hline 11.983 & 0.05 & 0.00 & 0.00 & 0.00 & 0.00 & 0.01 & 0.38 & 0.29 & 0.23 & 0.29 & 0.30 & 0.30 \\
\hline 11.986 & 0.05 & 0.00 & 0.00 & 0.00 & 0.00 & 0.01 & 0.41 & 0.33 & 0.27 & 0.36 & 0.36 & 0.37 \\
\hline 11.990 & 0.05 & 0.00 & 0.00 & 0.00 & 0.00 & 0.01 & 0.44 & 0.37 & 0.30 & 0.43 & 0.43 & 0.43 \\
\hline 11.993 & 0.05 & 0.00 & 0.00 & 0.00 & 0.00 & 0.01 & 0.47 & 0.40 & 0.32 & 0.49 & 0.49 & 0.49 \\
\hline 11.997 & 0.05 & 0.00 & 0.00 & 0.00 & 0.00 & 0.01 & 0.49 & 0.42 & 0.32 & 0.53 & 0.53 & 0.53 \\
\hline 12.000 & 0.05 & 0.00 & 0.00 & 0.00 & 0.00 & 0.01 & 0.50 & 0.43 & 0.31 & 0.57 & 0.57 & 0.56 \\
\hline 12.004 & 0.05 & 0.00 & 0.00 & 0.00 & 0.00 & 0.00 & 0.51 & 0.43 & 0.29 & 0.60 & 0.60 & 0.59 \\
\hline 12.007 & 0.05 & 0.00 & 0.00 & 0.00 & 0.00 & 0.00 & 0.52 & 0.44 & 0.28 & 0.62 & 0.62 & 0.61 \\
\hline 12.011 & 0.05 & 0.00 & 0.00 & 0.00 & 0.00 & 0.00 & 0.53 & 0.44 & 0.27 & 0.64 & 0.64 & 0.62 \\
\hline 12.014 & 0.05 & 0.00 & 0.00 & 0.00 & 0.00 & 0.00 & 0.54 & 0.45 & 0.27 & 0.66 & 0.65 & 0.64 \\
\hline 12.018 & 0.05 & 0.00 & 0.00 & 0.00 & 0.00 & 0.00 & 0.55 & 0.46 & 0.27 & 0.67 & 0.67 & 0.65 \\
\hline 12.021 & 0.05 & 0.00 & 0.00 & 0.00 & 0.00 & 0.00 & 0.56 & 0.46 & 0.28 & 0.68 & 0.68 & 0.66 \\
\hline 12.024 & 0.05 & 0.00 & 0.00 & 0.00 & 0.00 & 0.00 & 0.57 & 0.47 & 0.28 & 0.69 & 0.69 & 0.67 \\
\hline 12.028 & 0.05 & 0.00 & 0.00 & 0.00 & 0.00 & 0.00 & 0.58 & 0.48 & 0.29 & 0.70 & 0.70 & 0.68 \\
\hline
\end{tabular}


Table 3. Recovery of the phosphate limitation over time shown through the impact of the Monod term of phosphate for the different sections of the activated sludge tank using a colour-based evaluation method.

\begin{tabular}{|c|c|c|c|c|c|c|c|c|c|c|c|c|}
\hline Time & AN01 & AN02 & AN03 & AN04 & DT01 & DT02 & ВT01 & ВT02 & BT03 & BT04 & BT05 & ВT06 \\
\hline 11.962 & 0.99 & 0.99 & 0.99 & 0.99 & 0.96 & 0.95 & 0.36 & 0.21 & 0.15 & 0.15 & 0.15 & 0.15 \\
\hline 11.965 & 0.99 & 0.99 & 0.99 & 0.99 & 0.96 & 0.95 & 0.37 & 0.23 & 0.17 & 0.16 & 0.16 & 0.17 \\
\hline 11.969 & 0.99 & 0.99 & 0.99 & 0.99 & 0.96 & 0.95 & 0.39 & 0.25 & 0.19 & 0.18 & 0.18 & 0.19 \\
\hline 11.972 & 0.99 & 0.99 & 0.99 & 0.99 & 0.96 & 0.95 & 0.41 & 0.28 & 0.21 & 0.21 & 0.21 & 0.21 \\
\hline 11.976 & 0.99 & 0.99 & 0.99 & 0.99 & 0.96 & 0.95 & 0.44 & 0.31 & 0.24 & 0.24 & 0.24 & 0.25 \\
\hline 11.979 & 0.99 & 0.99 & 0.99 & 0.99 & 0.95 & 0.95 & 0.47 & 0.35 & 0.29 & 0.29 & 0.29 & 0.30 \\
\hline 11.983 & 0.99 & 0.99 & 0.99 & 0.99 & 0.95 & 0.95 & 0.50 & 0.40 & 0.35 & 0.35 & 0.35 & 0.37 \\
\hline 11.986 & 0.99 & 0.99 & 0.99 & 0.99 & 0.95 & 0.95 & 0.55 & 0.47 & 0.42 & 0.43 & 0.44 & 0.45 \\
\hline 11.990 & 0.99 & 0.99 & 0.99 & 0.99 & 0.95 & 0.95 & 0.59 & 0.53 & 0.49 & 0.51 & 0.52 & 0.53 \\
\hline 11.993 & 0.99 & 0.99 & 0.99 & 0.99 & 0.95 & 0.95 & 0.64 & 0.59 & 0.57 & 0.59 & 0.59 & 0.60 \\
\hline 11.997 & 0.99 & 0.99 & 0.99 & 0.99 & 0.95 & 0.95 & 0.68 & 0.65 & 0.63 & 0.65 & 0.65 & 0.66 \\
\hline 12.000 & 0.99 & 0.99 & 0.99 & 0.99 & 0.95 & 0.95 & 0.72 & 0.69 & 0.68 & 0.70 & 0.70 & 0.71 \\
\hline 12.004 & 0.99 & 0.99 & 0.99 & 0.99 & 0.95 & 0.95 & 0.75 & 0.73 & 0.72 & 0.73 & 0.73 & 0.74 \\
\hline 12.007 & 0.99 & 0.99 & 0.99 & 0.99 & 0.95 & 0.94 & 0.77 & 0.75 & 0.75 & 0.76 & 0.76 & 0.77 \\
\hline 12.011 & 0.99 & 0.99 & 0.99 & 0.99 & 0.95 & 0.94 & 0.79 & 0.78 & 0.77 & 0.78 & 0.78 & 0.79 \\
\hline 12.014 & 0.99 & 0.99 & 0.99 & 0.99 & 0.95 & 0.94 & 0.81 & 0.80 & 0.79 & 0.80 & 0.80 & 0.81 \\
\hline 12.018 & 0.98 & 0.99 & 0.99 & 0.99 & 0.95 & 0.94 & 0.82 & 0.81 & 0.81 & 0.82 & 0.82 & 0.82 \\
\hline 12.021 & 0.98 & 0.99 & 0.99 & 0.99 & 0.95 & 0.94 & 0.83 & 0.83 & 0.83 & 0.83 & 0.83 & 0.84 \\
\hline 12.024 & 0.98 & 0.99 & 0.99 & 0.99 & 0.94 & 0.94 & 0.84 & 0.84 & 0.84 & 0.84 & 0.84 & 0.85 \\
\hline 12.028 & 0.98 & 0.99 & 0.99 & 0.99 & 0.94 & 0.94 & 0.85 & 0.85 & 0.85 & 0.85 & 0.85 & 0.86 \\
\hline
\end{tabular}

\section{CONCLUSION}

A simple yet effective colour-based system analysis tool for ASM was illustrated for supporting model analysis. The tool allowed expert modellers to make a fast system analysis given the large amount of simulation outputs. The tool also facilitated discussion with and reporting for the non-expert modellers and proved to be a valuable tool in the decisionmaking process.

\section{ACKNOWLEDGEMENT}

The authors would like to thank Waterboard the Dommel for the financial support and the many in-depth discussions.

\section{REFERENCES}

Amerlinck, Y., T. Flameling, T. Maere, S. Weijers, and I. Nopens, 2013, Practical application of dynamic process models for wastewater treatment plant optimization: Work in progress, Proceedings of the Water Environment Federation, WEFTEC 2013, Chicago, IL, USA.

Henze, M., W. Gujer, T. Mino, and M. van Loosdrecht, 2000, Activated Sludge Models: ASM1, ASM2, ASM2d and ASM3: IWA Scientific and Technical Report: London, IWA Publishing.

Monod, J., 1942, Recherches sur la Croissance des Cultures Bactériennes (2nd edn.) Hermann, Paris. 


\title{
Modelling $\mathrm{N}_{2} \mathrm{O}$ dynamics in the engineered $\mathrm{N}$ cycle: Evaluation of alternate model structures
}

\author{
Barth F. Smets ${ }^{1}$, Carles Pellicer-Nàcher ${ }^{1}$, Carlos Domingo-Félez ${ }^{1}$, Marlene Mark Jensen ${ }^{1}$, Elham \\ Ramin $^{1}$, Benedek Gy. Plósz ${ }^{1}$, Gürkan Sin ${ }^{2}$, Krist V. Gernaey². \\ ${ }^{1}$ Department of Environmental Engineering, Technical University of Denmark, Miljøvej 113, $2800 \mathrm{Kgs.}$ \\ Lyngby, Denmark (Email: bfsm@env.dtu.dk; capn@env.dtu.dk; mmaj@env.dtu.dk; elhr@env.dtu.dk; \\ beep@env.dtu.dk; cadf@env.dtu.dk; agzm@env.dtu.dk) \\ ${ }^{2}$ Department of Chemical and Biochemical Engineering, Technical University of Denmark, Søltofts Plads \\ 227, 2800 Kgs. Lyngby, Denmark (Email: gsi@kt.dtu.dk; kvg@kt.dtu.dk)
}

\begin{abstract}
Research on nitrous oxide $\left(\mathrm{N}_{2} \mathrm{O}\right)$ formation in engineered wastewater systems has experienced an exponential development in the recent years due to the important environmental impact of this greenhouse gas. These efforts have crystalized in a large number of publications that aim to identify the importance of the main microbial processes responsible for its production and consumption. The conceptualization of these pathways in mathematical models has the potential to become a key tool to increase our understanding on the complex interrelationships within these ecosystems and develop strategies to minimize the carbon footprint of wastewater treatment plants. The present contribution aims to summarize the recent developments in this field and makes use of standard indentifiability measures to show how the choice of experimental protocols and model structures can potentially impact their calibration.
\end{abstract}

\section{Keywords}

Modelling; nitrous oxide; wastewater, calibration, uncertainty

\section{INTRODUCTION}

Process modelling can be a valuable tool to predict and minimize the environmental footprint of nitrogen removal processes in wastewater treatment plants. Consequently, several models have been proposed to date in order to describe the production and dynamics of nitrous oxide $\left(\mathrm{N}_{2} \mathrm{O}\right)$ both from aerobic ammonium oxidizing bacteria (AOB, e.g. Ni et al., 2011) and heterotrophic bacteria (HB, e.g. Hiatt and Grady Jr., 2008). Recent studies have identified $\mathrm{AOB}$ as the main driver for $\mathrm{N}_{2} \mathrm{O}$ emissions during biological nitrogen removal operations (Wunderlin et al., 2013).

While some of these models make use of parametric correlations obtained from the fitting of experimental data (Houweling et al., 2011), most of the documented modelling approaches are pseudo-mechanistic, meaning that the proposed biochemical processes producing or consuming $\mathrm{N}_{2} \mathrm{O}$ are mathematically outlined with traditional biokinetic formulations ( $\mathrm{Ni}$ et al., 2011; Mampaey et al., 2013; Ni et al., 2013a). A recent study has attempted to examine the ability of different model structures to predict the $\mathrm{N}_{2} \mathrm{O}$ production mechanisms by AOB (Ni et al., 2013b). However, the methodology used was biased by an ambiguous selection of calibrated model parameters. Furthermore, the wide range of model structures used result in comparably low number of parameter values published in this field, which in turn does not allow for a consensus-based set.

The present contribution makes an in-depth and robust assessment of the predictive capabilities of existing model structures by using standard metrics for parameter identifiability under well-defined experimental conditions. 


\section{MATERIALS AND METHODS}

Three models describing $\mathrm{N}_{2} \mathrm{O}$ production by AOB (Ni et al., 2011; Mampaey et al., 2013; Ni et al., 2013a) were implemented in Aquasim 2.1 (Reichert, 1994). The parameter sets used in each model were the ones suggested in each study. The following reactor was modelled in all three cases: it had a volume of 3L, it was considered completely mixed, and it contained a population of AOB which corresponded to about $10 \%$ of the active VSS fraction in the assayed sludge (2500 mg-VSS/L, values derived from Ekama and Wentzel, 2008, and Saunders et al., 2013). Four experimental scenarios were further considered for each model, all of them had a total duration of 12.5 hours:

- Case A: A batch test where three $\mathrm{NH}_{4}{ }^{+}$pulses of $6 \mathrm{mg} \mathrm{N} / \mathrm{L}$ were added at times $0,3.3$, and 7.2 hours in the presence of a controlled dissolved oxygen (DO) concentration of $2 \mathrm{mg} / \mathrm{L}$.

- Case B: A batch test where three $\mathrm{NH}_{4}{ }^{+}$pulses of $6 \mathrm{mg} \mathrm{N} / \mathrm{L}$ were added under uncontrolled DO conditions at the same times as previously indicated.

- Case C: A chemostat test at an $\mathrm{NH}_{4}{ }^{+}$load of $0.1 \mathrm{~g} \mathrm{~N} /$ day where the aeration is turned off 3.3 hours after the beginning of the experiment and turned back on 3.3 hours after the disturbance.

- Case D: A chemostat test at an $\mathrm{NH}_{4}{ }^{+}$load of $0.1 \mathrm{~g} \mathrm{~N} /$ day and constant aeration rate where the $\mathrm{NH}_{4}{ }^{+}$load is increased 20 fold after 3.3 hours of operation and is brought to the initial value 3.3 hours after the disturbance.

The metrics described by Brun et al (2001), i.e. the mean square sensitivity measure $\left(\delta^{\mathrm{msqr}}\right)$ and the collinearity index $(\gamma)$, were adapted for the assessment of the structural identifiability of the considered model. Both identifiability parameters were coded as scripts in MATLAB (Sin and Vanrolleghem, 2007). The numerical data required to drive those functions was generated with a batch version of Aquasim run in parallel by using the implemented model files.

\section{RESULTS AND DISCUSSION}

The study of the local sensitivity functions for the considered models is summarized in Figure 1 (only for Case B). Ideally, higher values for the mean sensitivity measurement $\left(\delta^{\mathrm{msqr}}\right)$ are preferred, as they reflect a higher impact of the model parameters on the model output and hence a higher probability of describing experimental data. This measurement should agree with a low collinearity index (below 5), which forms a measure of the higher identifiability of the feasible parameter subsets that can describe of $\mathrm{N}_{2} \mathrm{O}$ dynamics. Here, even though there is a higher percentage of identifiable parameter subsets for the model proposed by Mampaey et al. (i.e. \% of all subsets with an identifiability index below 5), they have on average a lower sensitivity (reflected by an average mean squared sensitivity measure computed for all the considered model parameters), which may complicate model calibration. 
$\square$ Average sensitivity factor

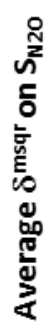
$\left.\begin{array}{c}1,5 \\ 1 \\ 0,5 \\ 0\end{array}\right]$
$\Delta \%$ of identifiable subsets

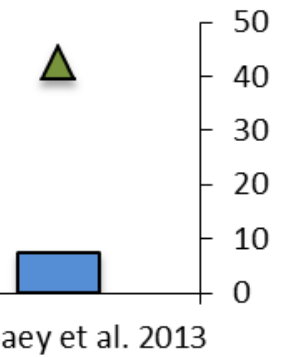

Figure 1. Average model sensitivities and percentage of identifiable parameter subsets wrt $\mathrm{N}_{2} \mathrm{O}$ dynamics in selected model structures.

In contrast, there is a smaller fraction of identifiable parameter subsets in both models by $\mathrm{Ni}$ et al. However, their sensitivity is clearly larger, suggesting that a careful selection of parameter subsets (so that they are identifiable) can make the calibration of these models easier in comparison to the model suggested by Mampaey et al. This higher degree of sensitivity could derive from a higher degree of simplification in the processes involved in $\mathrm{N}_{2} \mathrm{O}$ production and consumption in both models by $\mathrm{Ni}$ et al. (4 processes are involved versus 5 in Mampaey et al.).

Using the model by $\mathrm{Ni}$ et al. (2011) as an example (Figure 2), it can be seen how the experimental conditions can impact the sensitivity of the parameters involved in $\mathrm{N}_{2} \mathrm{O}$ production. Those experimental protocols with higher associated oxygen dynamics (i.e., cases $\mathrm{B}$ and $\mathrm{C}$ ) result in modelling scenarios that yield parameters with higher average sensitivity on the $\mathrm{N}_{2} \mathrm{O}$ concentration, which should ease the calibration of the considered model. Similar trends were obtained for the other two models studied.

$\Delta \%$ of identifiable subsets

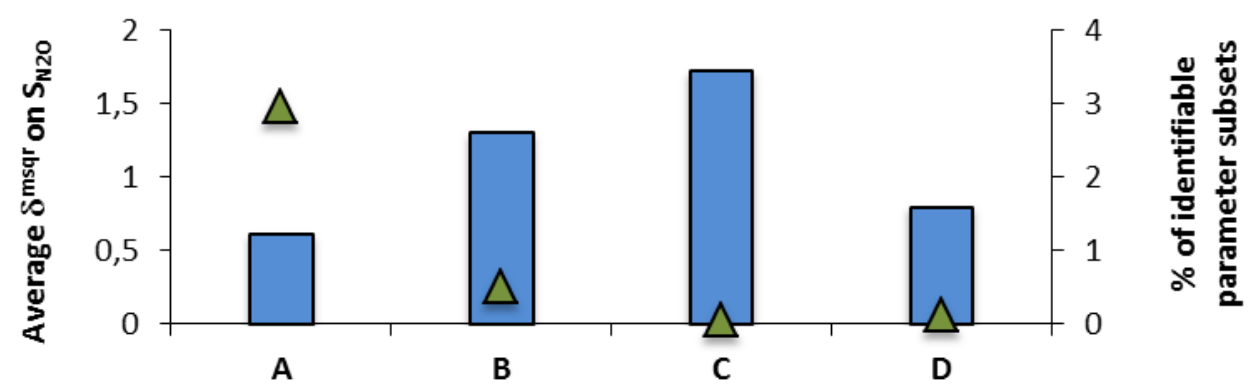

Figure 2. Average model sensitivities and percentage of identifiable parameter subsets wrt $\mathrm{N}_{2} \mathrm{O}$ dynamics in $\mathrm{Ni}$ et al. 2011 under the modelled experimental cases. Case A: Batch, cnt DO; Case B: Batch, uncontrolled DO; Case C: Chemostat, negative pulse in aeration; Case $D$ : Chemostat, $\mathrm{NH}_{4}{ }^{+}$load pulse.

\section{CONCLUSIONS}

It is possible to conclude that the selection of a model structure and an experimental protocol both have a direct impact on the calibration procedure of process models describing $\mathrm{N}_{2} \mathrm{O}$ production by AOB. Further work should facilitate the preparation of experimental protocols for calibration of existing process models for $\mathrm{N}_{2} \mathrm{O}$ dynamics.

\section{ACKNOWLEDGEMENT}


The present research work was supported by a grant from the Danish Research Council for Strategic research to BFSM.

\section{REFERENCES}

Ekama, G.A. \& Wentzel, M.C. (2008). Organic material removal. In: M. Henze, M. C. M. van Loosdrecht, G. A. Ekama, \& D. Brdjanovic (eds.). Biological wastewater treatment: Principles, modelling and design. London: IWA Publishing, p. 511.

Hiatt, W.C. \& Grady Jr., C.P.L. (2008). An updated process model for carbon oxidation, nitrification, and denitrification. Water Environ. Res. 80 (11). p.pp. 2145-2156.

Houweling, D., Wunderlin, P., Dold, P., Bye, C., Joss, A. \& Siegrist, H. (2011). $\mathrm{N}_{2} \mathrm{O}$ Emissions: Modeling the effect of process configuration and diurnal loading patterns. Water Environ. Res. 83 (12). p.pp. 21312139.

Mampaey, K.E., Beuckels, B., Kampschreur, M.J., Kleerebezem, R., van Loosdrecht, M.C.M. \& Volcke, E.I.P. (2013). Modelling nitrous and nitric oxide emissions by autotrophic ammonia-oxidizing bacteria. Environ. Technol. 34 (12). p.pp. 1555-1566.

Ni, B.-J., Ruscalleda, M.M., Pellicer-Nàcher, C. \& Smets, B.F. (2011). Modeling nitrous oxide production during biological nitrogen removal via nitrification and denitrification: extensions to the general ASM models. Environ. Sci. Technol. 45 (18). p.pp. 7768-7776.

Ni, B.-J., Ye, L., Law, Y., Byers, C. \& Yuan, Z. (2013a). Mathematical modeling of nitrous oxide $\left(\mathrm{N}_{2} \mathrm{O}\right)$ emissions from full-scale wastewater treatment plants. Environ. Sci. Technol. 47 (14). p.pp. $7795-7803$.

Ni, B.-J., Yuan, Z., Chandran, K., Vanrolleghem, P. a \& Murthy, S. (2013b). Evaluating four mathematical models for nitrous oxide production by autotrophic ammonia-oxidizing bacteria. Biotechnol. Bioeng. 110 (1). p.pp. 153-63.

Reichert, P. (1994). Aquasim - a tool for simulation and data analysis of aquatic systems. Water Sci. Technol. 30 (2). p.pp. 21-30.

Saunders, A.M., Larsen, P. \& Nielsen, P.H. (2013). Comparison of nutrient-removing microbial communities in activated sludge from full-scale MBRs and conventional plants. Water Sci. Technol. 68 (2). p.pp. 366371.

Sin, G. \& Vanrolleghem, P.A. (2007). Extensions to modeling aerobic carbon degradation using combined respirometric-titrimetric measurements in view of activated sludge model calibration. Water Research. 41 (15) p.pp. 3345-3358.

Wunderlin, P., Lehmann, M.F., Siegrist, H., Tuzson, B., Joss, A., Emmenegger, L. \& Mohn, J. (2013). Isotope signatures of $\mathrm{N}_{2} \mathrm{O}$ in a mixed microbial population system: constraints on $\mathrm{N}_{2} \mathrm{O}$ producing pathways in wastewater treatment. Environ. Sci. Technol. 47 (3). p.pp. 1339-1348. 


\title{
Modelling simultaneous anaerobic methane and ammonium removal in a granular sludge reactor
}

\author{
M.-K. H. Winkler ${ }^{1}$, T.P.W. Vannecke ${ }^{1}$, A. Bogdan ${ }^{1}$ and E. I. P. Volcke ${ }^{1}$ \\ ${ }^{1}$ Department of Biosystems Engineering, Ghent University, Coupure links 653, 9000 Gent, Belgium \\ (E-mail: Mari.Winkler@UGent.be, Eveline.Volcke@UGent.be, Thomas.Vannecke@UGent.be)
}

Keywords Anammox, anaerobic methane oxidation, granular sludge, modelling, simulation

\begin{abstract}
A mathematical model was applied to evaluate the influence of microbial kinetics of the process operation on the coexistence of nitrite dependent anaerobic methane oxidizing bacteria (N-DAMO) and anaerobic ammonium oxidizing bacteria (Anammox) in a single granule. Only if the influent contained the exact stoichiometric ratios at which both bacteria got enough nitrite, complete ammonium and methane removal could be realized. At high nitrogen biomass loadings N-DAMO lost the competition against Anammox bacteria. In addition, was the influence of granular size evaluated showing that a granules size of up to $0.75 \mathrm{~mm}$ enabled a simultaneous ammonium and methane removal efficiency above $85 \%$.
\end{abstract}

\section{INTRODUCTION}

During anaerobic digestion methane is formed which can be used for energy generation through cogeneration. However, some of the methane remains in a dissolved state in the effluent of the anaerobic digestion (reject water) and may escape into the atmosphere during downstream processing. Given the large global warming potential of methane, being about $25 \mathrm{CO}_{2}$ equivalents over a 100 year time horizon, even small quantities of methane emissions can largely affect the carbon footprint of a wastewater treatment plant (WWTP). Since reject water typically contains high ammonium concentrations and little or no organic carbon, Anammox-based system are often applied to remove nitrogen in an autotrophic way. To prevent greenhouse gas emissions, it would clearly be beneficial if dissolved methane could be biologically removed from the reject water at the same time as nitrogen. Recently a new bacterium (Candidatus Methylomirabilis oxyfera) was discovered capable of oxidizing methane with nitrite as electron and converting it to nitrogen gas and carbon dioxide (Ettwig et al, 2010). A combination of biological anaerobic methane oxidation (N-DAMO) and anaerobic ammonium oxidation (Anammox) could hence solve the methane emissions caused from reject water. Some early experiments have already been done, demonstrating the possible coexistence of both bacteria performing simultaneous removal of methane and ammonium (Leusken et al., 2011). Since both bacteria have a doubling time of more than ten days proper biomass retention is needed to handle large volumetric flows and loading capacities such as encountered in a WWTP (Ettwig et al., 2010; Strous et al., 1998). Granular sludge reactors are a type of biofilm reactors in which biomass is grown in the form of dense, fast-settling granules, resulting in compact systems which allows a high loading rate due to a large biofilm surface area in the reactor. Therefore, granules offer a good option for simultaneous growth of both N-DAMO and Anammox bacteria combine both slow growing bacteria in one granule. Due to the slow growth rates of the involved bacteria, experimental work aiming at process optimization can be very time consuming. Mathematical models have been shown earlier to be useful to optimize the performance of granular sludge reactors (Batstone et al., 2004; De Kreuk et al., 2007). In this study, a mathematical model is applied to evaluate the influence of process operation on the coexistence of anaerobic methane and ammonium oxidizing bacteria in a single granule.

\section{GRANULAR SLUDGE REACTOR MODEL}

A one-dimensional biofilm model was set up to describe growth and decay of anaerobic methane oxidizers (N-DAMO) and anaerobic ammonium oxidizers (Anammox) in a granular sludge reactor. The model was implemented in the Aquasim software. The reactor volume was considered to be fixed at $400 \mathrm{~m}^{3}$. Spherical biomass particles (granules) are grown from an initial radius of $0.01 \mathrm{~mm}$ to a 
predefined steady state granule radius such that the reactor eventually contains $100 \mathrm{~m}^{3}$ of particulate material, comprising both active biomass as well as inerts generated during endogenous respiration. The reactor behaviour has been simulated at a flow rate of $2500 \mathrm{~m}^{3} \cdot \mathrm{d}^{-1}$ at a total nitrogen concentration of $1000 \mathrm{~g} \mathrm{~N} . \mathrm{m}^{-3}$ and $200 \mathrm{~g}$ COD. $\mathrm{m}^{-3}$ of methane, respectively. These values are based on typical values as measured in reject water (Bandara et al., 2011). Furthermore, it was assumed that the total nitrogen concentration fed into the system consisted of $430 \mathrm{~g} \mathrm{~N} . \mathrm{m}^{-3}$ of ammonium and $570 \mathrm{~g} \mathrm{~N} . \mathrm{m}^{-3}$ of nitrite, corresponding to stoichiometric-optimal nitrite:methane and nitrite:ammonium ratios for conversion with methane and ammonium, by N-DAMO and Anammox, respectively.

\section{RESULTS AND DISCUSSION- Competition for nitrite and ammonium}

N-DAMO bacteria mainly grew in inner part of the granules, which can be explained by their slower growth rate, whereas Anammox mainly grew in the outer part of the granule. The inner part of the granules was consisting of inerts (Figure 1). In the model different $\mathrm{NO}_{2}{ }^{-} /$totalN ratios (total $\mathrm{N}$ $\left.1000 \mathrm{mgN} / \mathrm{m}^{3}\right)$ were tested at a fixed COD concentration $\left(200 \mathrm{gCOD} / \mathrm{m}^{3}\right)$ to study at which influent composition both bacteria could coexist. At lower ratios (ammonium in access) N-DAMO bacteria were out competed by Anammox bacteria. However, at ratios close to the stoichiometric equilibrium $\left(\mathrm{NO}_{2}, \mathrm{NH}_{4}, \mathrm{CH}_{4}\right)$ needed to completely remove methane and ammonium, N-DAMO bacteria could retain in the system. At high ratios Anammox bacteria got restricted by ammonium leading to an accumulation of nitrite in the bulk (Figure 2).

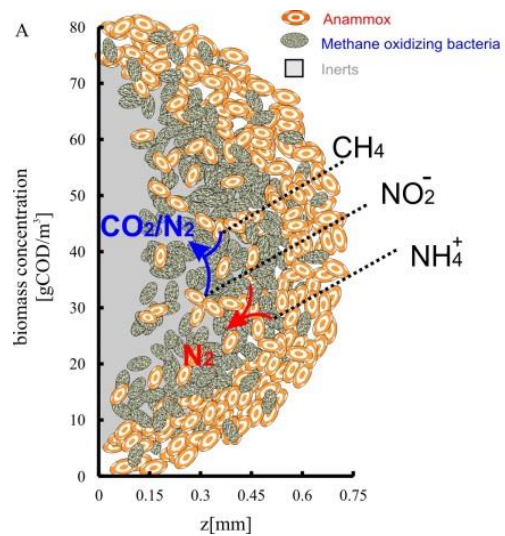

Figure 1. A) schematic view as well as B) modelling image of a distribution of $(\diamond)$ Anammox and ( $\mathbf{\square}) \mathrm{N}-$ DAMO bacteria as well as $(\bullet)$ inerts as a function of granular diameter.

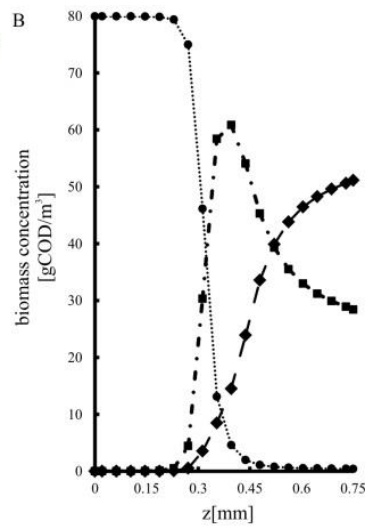

Figure 2. Reactor performance in terms of $(\bullet)$ ammonium, $(\mathrm{x})$ methane, $(\boldsymbol{\Delta})$ nitrate, $(\diamond)$ nitrite, and $(-) \mathrm{N}_{2}$ at different nitrite over nitrogen ratios.

\section{Influence of volumetric biomass loading rate and granular size}

For granule radius in a range of 0.1-0.75 mm both types of bacteria coexisted in approximately equal amounts leading to a simultaneous methane and ammonium removal above $85 \%$. At higher granule sizes inerts increase due to substrate depletion in the inner core of the granule hence restricting microbial growth. In addition, N-DAMO bacteria decrease at higher granules radius due the lower diffusion coefficient of methane compared to ammonium. Bigger granules favoured Anammox bacteria whereas smaller granules enabled the coexistence of both bacteria in one granule (Figure 3). The effect of changing the total biomass volume on the bulk liquid concentrations as well as on the biomass fractions of both bacteria was evaluated based on media containing the stoichiometric ratios needed for a complete ammonium and methane removal (Figure 3). When only little biomass was in the reactor (biomass volume lower than $50 \mathrm{~m}^{3}$ ) Anammox dominated the system (Figure 4 phase I). At a biomass volume from $50 \mathrm{~m}^{3}$ until $100 \mathrm{~m}^{3}$ both bacteria started to compete for substrate and space (no inerts). During this transition period $\left(50 \mathrm{~m}^{3}\right.$ until $\left.100 \mathrm{~m}^{3}\right)$ the removal efficiencies, which could be explained by ammonium and nitrite affinity constants (data not shown). From $100 \mathrm{~m}^{3}$ on N-DAMO bacteria dominated the granule and the oscillation in bulk liquid concentrations stopped. When N-DAMO dominated the system $\left(>100 \mathrm{~m}^{3}\right)$ simultaneous ammonium and methane removal could be realized (Figure 4 phase II). The results showed that the volumetric biomass loading rate needs to be sufficiently low to allow simultaneous methane and ammonium removal. 

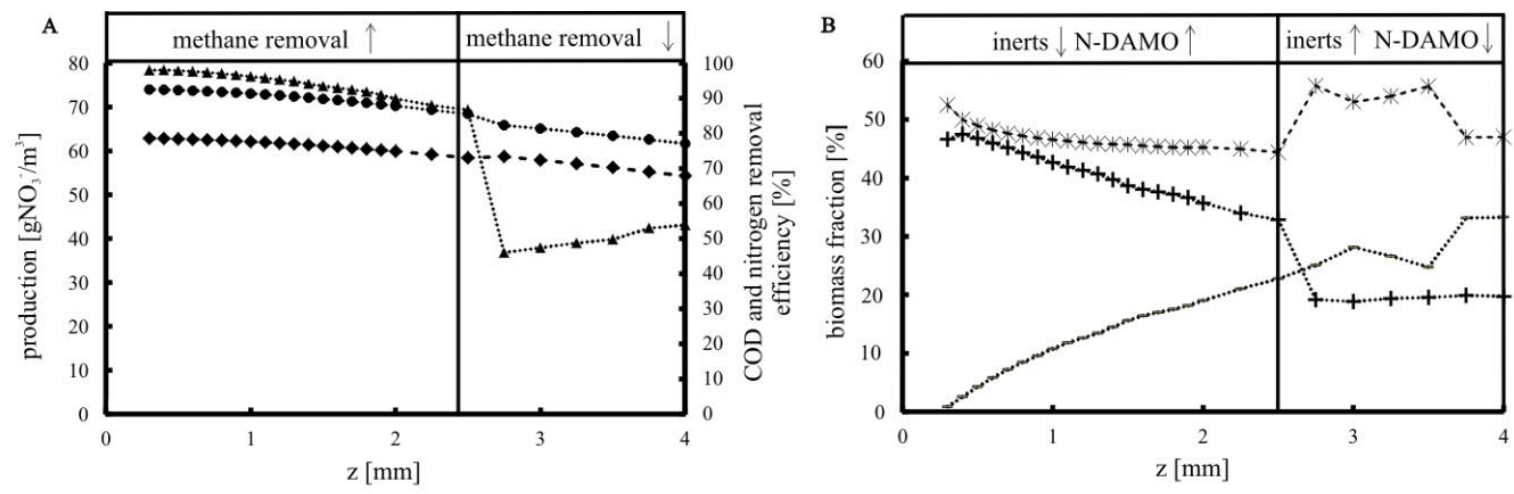

Figure 3. Influence of granule diameter on (A) reactor performance in terms of methane $(\mathbf{A})$ ammonium $(\bullet)$ removal efficiency and nitrate production $(\bullet)$ as well as (B) biomass concentrations of Anammox (Ж), $\mathrm{N}-\mathrm{DAMO}(+)$, and interts

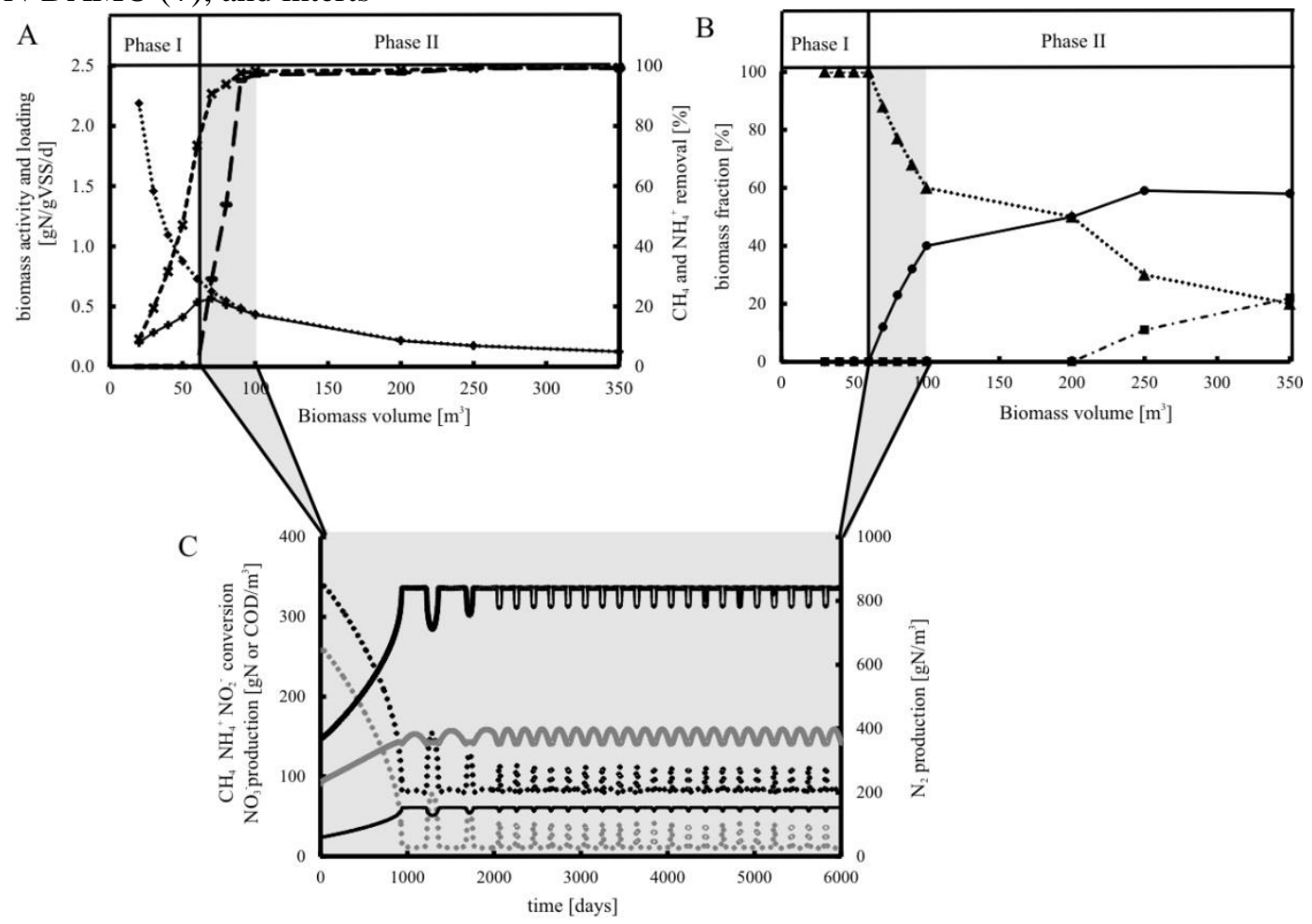

Figure 4. (A) Reactor performance of ( $\bullet$ biomass loading, (+) biomass activity, (x) nitrogen removal efficiency, and (-) methane removal efficiency as well as (B) biomass profiles of ( $\boldsymbol{\Delta})$ Anammox bacteria, $(\bullet)$ N-DAMO bacteria, and (-) inerts at different biomass volume fractions in the reactor. The total volume of the reactor was considered to be $400 \mathrm{~m}^{3}$. In phase I (A) no methane was removed and (B) no N-DAMO were present. In phase II (A) methane was removed (B) and N-DAMO grew in the system. (C) only in this period oscillations occurred.

\section{CONCLUSIONS}

- Simultaneous anaerobic methane and ammonium removal, through N-DAMO and Anammox bacteria respectively, was described for the first time through a mathematical model, which was subsequently applied to simulate these reactions in a granule sludge reactor.

- Bigger granules favoured Anammox bacteria whereas smaller granules enabled the coexistence of both bacteria in one granule

- The volumetric biomass loading rate needs to be sufficiently low to allow simultaneous methane and ammonium removal. At high loading rates, N-DAMO are outcompeted by Anammox bacteria.

- The stability of simultaneous anaerobic methane and ammonium removal is severely affected by the ammonium and nitrite affinity constants of N-DAMO and Anammox bacteria. 


\section{REFERENCES}

Bandara W.M., Satoh H., Sasakawa M., Nakahara Y., Takahashi, M., Okabe S., (2011), Removal of residual dissolved methane gas in an upflow anaerobic blanket reactor treating low-strength wastewater at a low temperature with degassing membrane, Water Research, 45(11), 3533-3540.

Batstone D.J., Keller J.,Blackall L.L. (2004), The influence of substrate kinetics on the microbial community structure in granular anaerobic biomass, Water Research, 38(6) 1390-1404.

Ettwig K.F., Butler M.K., Le Paslier D., Pelletier E., Mangenot S., Kuypers M., et al., (2010), Nitritedriven anaerobic methane oxidation by oxygenic bacteria, Nature, 464, 543-548.

de Kreuk M.K., Picioreanu C., Hosseini M., Xavier J.B., van Loosdrecht M.C.M. (2007),Kinetic Model of a Granular Sludge SBR:Influences on Nutrient Removal, Biotechnology and Bioengineering,97(4)801-815.

Leusken F.A., Sanchez J., van Alen T.A., Sanabria J., Op den Camp H.J.M., Jetten M.S.M., Kartal B., (2011), Simultaneous Nitrite-Dependent Anaerobic Methane and Ammonium Oxidation Processes. Applied Environmental Microbiology, 77(9), 6802-6807.

Strous M., Heijnen J.J., Kuenen, J.G., Jetten M.S.M., (1998), The sequencing batch reactor as a powerful tool for the study of slowly growing anaerobic ammonium-oxidizing microorganisms, Applied Microbiology and Biotechnology, 50(5), 589-596. 


\title{
Risk assessment modelling of $\mathrm{N}_{2} \mathrm{O}$ production in activated sludge systems: Quality not Quantity
}

\author{
Jose Porro $^{1,2}$, Costanza Milleri ${ }^{3}$, Joaquim Comas ${ }^{1}$, Ignasi Rodriguez-Roda ${ }^{1,4}$, Maite Pijuan ${ }^{4}$, Lluís \\ Corominas ${ }^{4}$, Lisha Guo ${ }^{5}$, Matthijs Daelman ${ }^{6,7}$, Eveline Volcke ${ }^{6}$, Mark van Loosdrecht ${ }^{7}$, Peter A. \\ Vanrolleghem ${ }^{5}$, and Ingmar Nopens ${ }^{2}$ \\ ${ }^{1}$ Laboratory of Chemical and Environmental Engineering (LEQUiA), University of Girona, Faculty of Sciences, \\ Campus Montilivi s/n, 17071, Girona, Spain \\ ${ }^{2}$ BIOMATH, Department of Mathematical Modelling, Statistics and Bioinformatics, Ghent University, Coupure \\ Links 653, 9000 Gent, Belgium \\ ${ }^{3}$ University of Florence, Civil \& Environmental Engineering Dept., Via S. Marta 3, 50139, Firenze, Italy \\ ${ }^{4}$ ICRA, Catalan Institute for Water Research, Scientific and Technological Park of the University of Girona, H2O \\ Building, Emili Grahit 101, 17003 Girona, Spain \\ ${ }^{5}$ modelEAU, Département de génie civil et de génie des eaux, Université Laval, Québec G1V 0A6, QC, Canada \\ ${ }^{6}$ Department of Biosystems Engineering, Ghent University, Coupure Links 653, 9000 Gent, Belgium \\ ${ }^{7}$ Department of Biotechnology, Delft University of Technology, Julianalaan 67, 2628 BC Delft, Netherlands
}

\begin{abstract}
A knowledge-based risk assessment modelling approach is proposed to provide a qualitative means of benchmarking WWTP design and control strategies in terms of risk of $\mathrm{N}_{2} \mathrm{O}$ production. The approach makes use of ASM model output variables corresponding to conditions that have been specifically linked to the risk of WWTP $\mathrm{N}_{2} \mathrm{O}$ production in the literature, and applies a fuzzy logic rule-based system to qualitatively assign risk of $\mathrm{N}_{2} \mathrm{O}$ production, as opposed to predicitng actual emission. To demonstrate the proof of concept, the qualitative $\mathrm{N}_{2} \mathrm{O}$ risk model was used to interpret mathematical simulation data and distinguish risk of $\mathrm{N}_{2} \mathrm{O}$ production resulting from two different aeration control strategies (DO set points of $2 \mathrm{mg} \cdot \mathrm{L}^{-1}$ and $1.3 \mathrm{mg} \cdot \mathrm{L}^{-1}$ ). The approach demonstrated its potential in assessing risk of $\mathrm{N}_{2} \mathrm{O}$ production on a plant-wide level, as well as the reactor level, which allowed diagnosing specific risks and identifying opportunities for mitigation. Results also demonstrated how the $\mathrm{N}_{2} \mathrm{O}$ risk model tool can be helpful in selecting appropriate mechanistic $\mathrm{N}_{2} \mathrm{O}$ production models through its risk diagnosis. The $\mathrm{N}_{2} \mathrm{O}$ risk assessment model can also serve as a practical decision support tool for qualitatively assessing multicriteria control strategies as seen in the $\mathrm{N}_{2} \mathrm{O}$ risk, effluent quality, and operational cost benchmarking results. The tool is flexible and can be used not only with mathematical model output data, but also online, or SCADA data for examining risk of $\mathrm{N}_{2} \mathrm{O}$ production for current and historical plant operations.
\end{abstract}

\section{Keywords}

Activated Sludge Model (ASM), nitrous oxide, qualitative modelling, risk assesment modelling

\section{INTRODUCTION}

A considerable amount of focus has been placed on modelling full-scale wastewater treatment plant (WWTP) nitrous oxide $\left(\mathrm{N}_{2} \mathrm{O}\right)$ emissions in recent years given their high global warming potential. As a result, several promising mechanistic models have been developed (Yu et al., 2010; Ni et al., 2011; Houweling et al., 2011; Law et al., 2012; Ni et al., 2012; Guo and Vanrolleghem, 2013; Mampaey et al., 2013; Ni et al., 2013). However, there is not yet a rigorously validated and consensus-based model. This is largely due to the complex and interactive nature of the processes leading to $\mathrm{N}_{2} \mathrm{O}$ emissions from activated sludge systems, including ammonia-oxidizing bacteria (AOB) cell metabolism and gene expressions ( $\mathrm{Yu}$ et al., 2010, Chandran et al., 2011), AOB and nitrite-oxidizing bacteria (NOB) kinetic rates (Foley et al., 2010), mass transfer processes, and the dynamic operational and environmental conditions that impact the propensity of full-scale microbial populations for producing $\mathrm{N}_{2} \mathrm{O}$ during both nitrification and denitrification (Kampschreur et al., 2009; Foley et al., 2010, 
Chandran et al., 2011). As researchers continue to make strides in reaching a consensus on $\mathrm{N}_{2} \mathrm{O}$ dominant pathways, model validation, and implementing and calibrating multiple $\mathrm{N}_{2} \mathrm{O}$ pathway models, a knowledge-based risk assessment modelling approach is proposed to complement the progression of the mechanistic description of $\mathrm{N}_{2} \mathrm{O}$ production, and provide a qualitative means of benchmarking WWTP design and control strategies. A similar knowledge-based risk assessment modelling approach (AS risk model) has been successfully developed and implemented by Comas et al. (2008) for diagnosing the risk of microbiology related solids separation problems, such as filamentous bulking, foaming, and rising sludge, resulting from various activated sludge control strategies. Parallels between modelling activated sludge solids separation problems and $\mathrm{N}_{2} \mathrm{O}$ production, such as the lack of validated mechanistic models and interest in more holistic benchmarking of control strategies, thus motivated the extension of this risk assessment modelling concept for heuristically diagnosing WWTP $\mathrm{N}_{2} \mathrm{O}$ production.

\section{METHODS}

The methodology for developing the $\mathrm{N}_{2} \mathrm{O}$ risk model is generally consistent with that of the AS risk model development (Comas et al., 2008). The proposed integrated mathematical / knowledge-based risk assessment modelling approach makes use of ASM state variables corresponding to conditions that have been specifically linked to the risk of WWTP $\mathrm{N}_{2} \mathrm{O}$ production in the literature (Kampschreur et al., 2009; Foley et al., 2010; Ahn et al., 2010; GWRC, 2011), but not yet formalized in a modelling platform through which $\mathrm{N}_{2} \mathrm{O}$ risk can be assessed with other criteria in various WWTP simulation scenarios. Therefore, a knowledge base of the operational conditions/parameters associated with risk of $\mathrm{N}_{2} \mathrm{O}$ production via heterotrophic denitrification and AOB nitrification/denitrification pathways was compiled and then classified in terms of low, medium, and high risk according to values found in the literature correlating to low, medium, and high $\mathrm{N}_{2} \mathrm{O}$ production in either full-scale or lab-scale studies. This knowledge was then represented in a fuzzy logic, IF / THEN rule-based system implemented in both Matlab and Excel, through which a qualitative risk score can be dynamically assigned for each variable representing the operational risk condition. The risk score is based on scale from 0 to 1 , with 1 representing the highest risk.

To demonstrate the proof of concept of $\mathrm{N}_{2} \mathrm{O}$ risk assessment modelling, the risk model was implemented for only three of the several risk parameters defined in the knowledge base: high nitrite $\left(\mathrm{NO}_{2}{ }^{-}\right)$for nitrification and denitrification reactors, and low dissolved oxygen (DO) and ammonia oxidation rate (AOR) via DO for nitrification reactors (Table 1). This portion of the $\mathrm{N}_{2} \mathrm{O}$ risk model was applied to the Benchmark Simulation Model No. 2 (BSM2), a five reactor (two anoxic and three aerobic) MLE configuration. Two different control scenarios were compared: Scenario 1 - DO set point of Activated Sludge Unit (ASU) No. 4 (ASU4) is 2 $\mathbf{m g} \cdot \mathbf{L}^{\mathbf{- 1}}$, and Scenario 2 - DO setpoint of ASU4 is $\mathbf{1 . 3} \mathbf{~ m g} \cdot \mathbf{L}^{\mathbf{- 1}}$, with $\mathrm{k}_{\mathrm{L}}$ a set proportionally as $1.5 \mathrm{k}_{\mathrm{L}} \mathrm{a}, \mathrm{k}_{\mathrm{L}} \mathrm{a}$, and $0.5 \mathrm{k}_{\mathrm{L}}$ a for the aerobic reactors ASU3, ASU4, and ASU5, respectively. The model implemented in Porro et al. (2011), which includes two-step nitrification and four-step denitrification, was used since the $\mathrm{NO}_{2}{ }^{-}$state variable could be used for implementing the $\mathrm{N}_{2} \mathrm{O}$ risk model for the high $\mathrm{NO}_{2}^{-}$condition, whereas the original BSM2 platform implementing ASM1 only includes single-step nitrification and, hence, no $\mathrm{NO}_{2}^{-}$variable. Although the model used in Porro et al. (2011) also includes the implementation of mechanistic models for $\mathrm{N}_{2} \mathrm{O}$ production, the $\mathrm{N}_{2} \mathrm{O}$ variables are ignored since the purpose of the paper is demonstrating a qualitative approach to assessing control strategies for $\mathrm{N}_{2} \mathrm{O}$ production risk as opposed to a quantitative approach (i.e. mechanistically predicting $\mathrm{N}_{2} \mathrm{O}$ concentrations). The ASM model output data was then input into the Excel version of the risk 
model to plot dynamic $\mathrm{N}_{2} \mathrm{O}$ risk based upon the corresponding BSM2 model output state variables. Similarly to Corominas et al. (2012) and Guo et al. (2012), Operational Cost Index (OCI), which includes energy costs, and Effluent Quality Index (EQI) per Nopens et al. (2010) were also compared for the two scenarios along with overall $\mathrm{N}_{2} \mathrm{O}$ risk to demonstrate the $\mathrm{N}_{2} \mathrm{O}$ risk assessment model's potential in multi-criteria decision support.

\section{RESULTS AND DISCUSSION}

Table 2 summarizes $\mathrm{N}_{2} \mathrm{O}$ risk model results in each of the reactors by average overall risk, from taking the maximum risk of the three individual risk parameters results for each time step, and by percent of time under high risk, with high risk being a risk score of greater than or equal to 0.8, as defined by Comas et al. (2008). Also summarized in Table 2 is the average overall risk score for all of the reactors, as well as the percent of time under high risk accounting for all reactors, or the percent of the total simulation time in which at least one reactor was under high risk. As anticipated, the two different DO control set points resulted in different conditions in each of the reactors, and hence, noticeable differences in average overall risk scores and time under high risk between the two scenarios. Obviously the largest differences are seen in the aerobic reactors, since the only change between the scenarios was the DO set point. These differences in risk results in the aerobic reactors are due to the DO concentration itself, as low DO implicates the potential for $\mathrm{N}_{2} \mathrm{O}$ production via AOB denitrification (Kampschreur et al., 2009; Tallec et al., 2008), and higher DO implicates $\mathrm{N}_{2} \mathrm{O}$ production via the hydroxylamine oxidation pathway (Law et al., 2012), as well as the $\mathrm{NO}_{2}{ }^{-}$ concentrations, which implicate AOB denitrification (Kampschreur et al., 2009). Inspecting the $\mathrm{N}_{2} \mathrm{O}$ production risk results in each of the reactors, ASU5 reactor stands out for Scenario 2 , with an average overall risk score of 0.95 and 96 percent of the time under high risk.

To give a sense of the $\mathrm{N}_{2} \mathrm{O}$ risk model tool's capabilities, Figure $\mathbf{1}$ is provided to illustrate further inspection of the risk results, comparing plots of both the individual and overall risk in ASU5 for both scenarios. As the DO set point is lower in Scenario 2, and ASU5 has a $\mathrm{k}_{\mathrm{L}} \mathrm{a}$ that is half that of ASU4 where the DO set point is controlled, it can be understood why the DO levels are significantly lower and hence the $\mathrm{N}_{2} \mathrm{O}$ production risk, due to low DO, significantly higher. To add to the risk of ASU5, the lower DO concentrations also lead to higher $\mathrm{NO}_{2}^{-}$concentrations compared to Scenario 1 due to the difference in oxygen halfsaturation constants between AOB and NOB (Hanaki et al.,1990; Mota et al., 2005), and therefore, higher risk due to high $\mathrm{NO}_{2}^{-}$concentrations. As the ASU5 DO concentrations in both scenarios are less than the low risk threshold $\left(<1.8 \mathrm{mg} \cdot \mathrm{L}^{-1}\right)$ for AOR risk, $\mathrm{N}_{2} \mathrm{O}$ production risk due to AOR (hydroxylamine oxidation pathway) is always zero in both scenarios for ASU5. As the two remaining risk parameters with high risk values account for AOB denitrification, one could surmise that the particular conditions for ASU5 lend to $\mathrm{N}_{2} \mathrm{O}$ production via the AOB denitrification pathway. This highlights the capability of the tool in helping to hypothesize pathways, and therefore, also to select mechanistic models of $\mathrm{N}_{2} \mathrm{O}$ production. As the specific risks can be diagnosed, the $\mathrm{N}_{2} \mathrm{O}$ risk model tool also demonstrates its potential in identifying opportunities for mitigating $\mathrm{N}_{2} \mathrm{O}$ production risk. In this case, it is clear that better control of the DO in ASU5, or better distribution of the air between ASU3 and ASU5 could help to minimize the risk in ASU5, and hence the overall risk for Scenario 2 since ASU5 was under high risk 96 percent of the simulation time. The opportunity to better distribute air among reactors was also noted by Guo et al. (2012) for minimizing greenhouse gas mass transfer and emissions into the air as it is related to the $\mathrm{k}_{\mathrm{L}} \mathrm{a}$. However, in this case, the $\mathrm{N}_{2} \mathrm{O}$ risk model assesses only the effect of DO on risk of production and not mass transfer and emissions. It is also clear from these results that looking at only average overall risk 
alone, may not fully diagnose the potential $\mathrm{N}_{2} \mathrm{O}$ production risk as the average overall risk score for each scenario only differed by 0.07 . Since different reactors can be under high risk at different times, it is helpful to also consider the amount of time in which at least one reactor is under high risk.

Table 3 summarizes the $\mathrm{N}_{2} \mathrm{O}$ risk, EQI, and OCI results for both scenarios. As anticipated the lower DO control resulted in some cost savings based upon the OCI; however, the EQI decreased slightly, and the average overall $\mathrm{N}_{2} \mathrm{O}$ risk score increased slightly. However, the time under high risk for all five reactors increased significantly, by 1.5 times to almost 100 percent of the simulation time. Depending upon objectives, this information could be helpful in determining whether the five percent savings in the OCI is worth increasing the time under high $\mathrm{N}_{2} \mathrm{O}$ production risk to almost 100 percent of the time. This information could also help in decision making by prompting further investigation into the conditions in ASU5. For example, if better control or distribution of the air among ASU3 and ASU5 is feasible, as suggested previously, then risk could potentially be mitigated, while still realizing the same cost savings since essentially the same amount of air would be added, just distributed differently.

\section{CONCLUSIONS AND PERPESCTIVES}

The integrated mathematical / knowledge-based risk assessment modelling concept by Comas et al. (2008) has been adapted for assessing the risk of $\mathrm{N}_{2} \mathrm{O}$ production in WWTPs. The qualitative $\mathrm{N}_{2} \mathrm{O}$ risk model approach was used to interpret mathematical simulation data and distinguish risk of $\mathrm{N}_{2} \mathrm{O}$ production resulting from two different aeration control strategies. The approach demonstrated potential for assessing risk of $\mathrm{N}_{2} \mathrm{O}$ production on a plant-wide level, as well as the reactor level, which allowed diagnosing specific risks and identifying opportunities for mitigation. Results also demonstrated how the $\mathrm{N}_{2} \mathrm{O}$ risk model tool can complement the application of mechanistic models of $\mathrm{N}_{2} \mathrm{O}$ production through the implication of specific $\mathrm{N}_{2} \mathrm{O}$ production pathways in the risk diagnosis, which can then be used in hypothesizing underlying mechanisms and selecting appropriate mechanistic $\mathrm{N}_{2} \mathrm{O}$ production models. The $\mathrm{N}_{2} \mathrm{O}$ risk assessment model can also serve as a practical decision support tool for qualitatively assessing multi-criteria control strategies as seen in the results. As the Excel version of the risk assessment model was used in this study, the results not only demonstrate the potential application of the tool with mathematical model output data, but also with online, or SCADA data for operators interested in making use of the available knowledge and examining risk of $\mathrm{N}_{2} \mathrm{O}$ production for current and historical plant operations. Work is ongoing confirming AOR values/risk and to test the entire knowledge base with full-scale data from various measurement campaigns.

\section{ACKNOWLEDGEMENT}

The research work of Jose Porro is financed by People Program (Marie Curie Actions) of the European Union's Seventh Framework Programme FP7/2007-2013, 579 under REA agreement 289193 (SANITAS). Maite Pijuan also acknowledges the Ramon y Cajal research fellowship (RYC2009-04959) and project CTM 2011-27163 provided by the Spanish Government. Peter Vanrolleghem holds the Canada Research Chair in Water Quality Modelling. 
Table 1. Portion of $\mathrm{N}_{2} \mathrm{O}$ production risk knoweldge base included in $\mathrm{N}_{2} \mathrm{O}$ risk assessment model

\begin{tabular}{|c|c|c|c|c|c|c|c|c|c|}
\hline \multirow{2}{*}{$\begin{array}{l}\text { Process/ } \\
\text { Condition }\end{array}$} & \multirow{2}{*}{$\begin{array}{c}\text { Operational } \\
\text { Parameter / Condition }\end{array}$} & \multirow{2}{*}{ ASM Variable } & & \multicolumn{3}{|c|}{ Risk Classification } & \multirow{2}{*}{ Mechanism } & \multirow{2}{*}{$\begin{array}{c}\text { References for } \\
\text { Operational Risk } \\
\text { Parameter } \\
\text { Identification } \\
\end{array}$} & \multirow{2}{*}{$\begin{array}{l}\text { References for } \\
\text { Parameter Values }\end{array}$} \\
\hline & & & & Low & Medium & High & & & \\
\hline \multirow[b]{2}{*}{ Denitrification } & \multirow[b]{2}{*}{ high $\mathrm{NO}_{2}$} & \multirow[b]{2}{*}{$\mathrm{NO}_{2}$} & range & $<0.2$ & $0.2-0.5$ & $>0.5$ & \multirow{2}{*}{$\begin{array}{c}\text { - Heterotrophic } \\
\text { denitrification } \\
\text { - AOB denitrification }\end{array}$} & \multirow{2}{*}{$\begin{array}{l}\text { Kampschreur et al. } \\
\text { 2009; Foley et al., } \\
\text { 2010; Ahn et al., 2010; } \\
\text { GWRC, } 2011\end{array}$} & \multirow[b]{2}{*}{ GWRC, 2011} \\
\hline & & & units & & $\mathrm{mg} / \mathrm{L}$ & & & & \\
\hline \multirow{6}{*}{ Nitrification } & \multirow[b]{2}{*}{ high $\mathrm{NO}_{2}$} & \multirow[b]{2}{*}{ NO2 } & range & $<0.2$ & $0.2-0.5$ & $>0.5$ & \multirow{2}{*}{$\mathrm{AOB}$ denitrification } & Kampschreur et al. & \multirow[b]{2}{*}{ GWRC, 2011} \\
\hline & & & units & & $\mathrm{mg} / \mathrm{L}$ & & & $\begin{array}{c}\text { 2009; Foley et al., } \\
\text { 2010; Ahn et al., 2010; } \\
\text { GWRC, } 2011\end{array}$ & \\
\hline & \multirow{2}{*}{ low DO } & \multirow{2}{*}{ DO } & range & $>1.5$ & $0.4-1.5$ & $<0.4$ & \multirow{2}{*}{$A O B$ denitrification } & \multirow{2}{*}{ Kampschreur et al. 2010} & \multirow{2}{*}{ Tallec et al., 2008} \\
\hline & & & units & & $\mathrm{mg} / \mathrm{L}$ & & & & \\
\hline & \multirow{2}{*}{$\begin{array}{l}\text { Non-limiting DO, NH4, } \\
\text { AOR }\end{array}$} & \multirow{2}{*}{ DO } & range & $<1.8$ & 2.15 & $>2.5$ & \multirow[t]{2}{*}{ AOB nitrification } & \multirow{2}{*}{$\begin{array}{c}\text { Ahn et al., 2010, } \\
\text { Chandran et al., 2011, } \\
\text { Law et al., } 2012\end{array}$} & \multirow{2}{*}{ Law et al., 2012} \\
\hline & & & units & & $\mathrm{O} 2 \mathrm{mg} / \mathrm{L}$ & & & & \\
\hline
\end{tabular}

Table 2. Summary of overall $\mathrm{N}_{2} \mathrm{O}$ risk results

\begin{tabular}{|c|c|c|c|c|c|c|c|c|c|c|c|c|}
\hline & \multicolumn{2}{|c|}{ ASU1 } & \multicolumn{2}{|c|}{ ASU2 } & \multicolumn{2}{|c|}{ ASU3 } & \multicolumn{2}{|c|}{ ASU4 } & \multicolumn{2}{|c|}{ ASU5 } & \multicolumn{2}{|c|}{ Overall } \\
\hline & $\begin{array}{c}\text { Average } \\
\text { Overall } \\
\text { Risk }\end{array}$ & $\begin{array}{c}\text { \% of Time } \\
\text { Under High } \\
\text { Risk }\end{array}$ & $\begin{array}{c}\text { Average } \\
\text { Overall } \\
\text { Risk }\end{array}$ & $\begin{array}{c}\text { \% of Time } \\
\text { Under High } \\
\text { Risk }\end{array}$ & $\begin{array}{c}\text { Average } \\
\text { Overall } \\
\text { Risk }\end{array}$ & $\begin{array}{c}\% \text { of Time } \\
\text { Under High } \\
\text { Risk }\end{array}$ & $\begin{array}{c}\text { Average } \\
\text { Overall } \\
\text { Risk }\end{array}$ & $\begin{array}{c}\% \text { of Time } \\
\text { Under High } \\
\text { Risk }\end{array}$ & $\begin{array}{c}\text { Average } \\
\text { Overall } \\
\text { Risk }\end{array}$ & $\begin{array}{c}\% \text { of Time } \\
\text { Under High } \\
\text { Risk }\end{array}$ & $\begin{array}{c}\text { Average } \\
\text { Overall } \\
\text { Risk }\end{array}$ & $\begin{array}{c}\text { \% of Time } \\
\text { Under High } \\
\text { Risk }\end{array}$ \\
\hline Scenario 1_DO2 & 0.58 & 21 & 0.31 & 21 & 0.56 & 33 & 0.41 & 10 & 0.51 & 19 & 0.47 & 64 \\
\hline Scenario 2_D01.3 & 0.44 & 13 & 0.11 & 2.4 & 0.74 & 50 & 0.46 & 30 & 0.95 & 96 & 0.54 & 98 \\
\hline
\end{tabular}

Table 3. Summary of Scenario Benchmarking Results

\begin{tabular}{lcc}
\hline & Scenario1 DO_2 & Scenario2 DO_1.3 \\
\hline Time Under High $\mathbf{N}_{\mathbf{2}} \mathbf{O}$ Risk (\%) & 64 & 98 \\
Average Overall $\mathbf{N}_{\mathbf{2}} \mathbf{O}$ Risk Score & 0.47 & 0.54 \\
EQI $\left(\mathrm{kg} \mathrm{poll} \cdot \mathrm{d}^{-1}\right)$ & 5612 & 5694 \\
OCI $(-)$ & 10537 & 10023 \\
\hline
\end{tabular}


Porro et al.

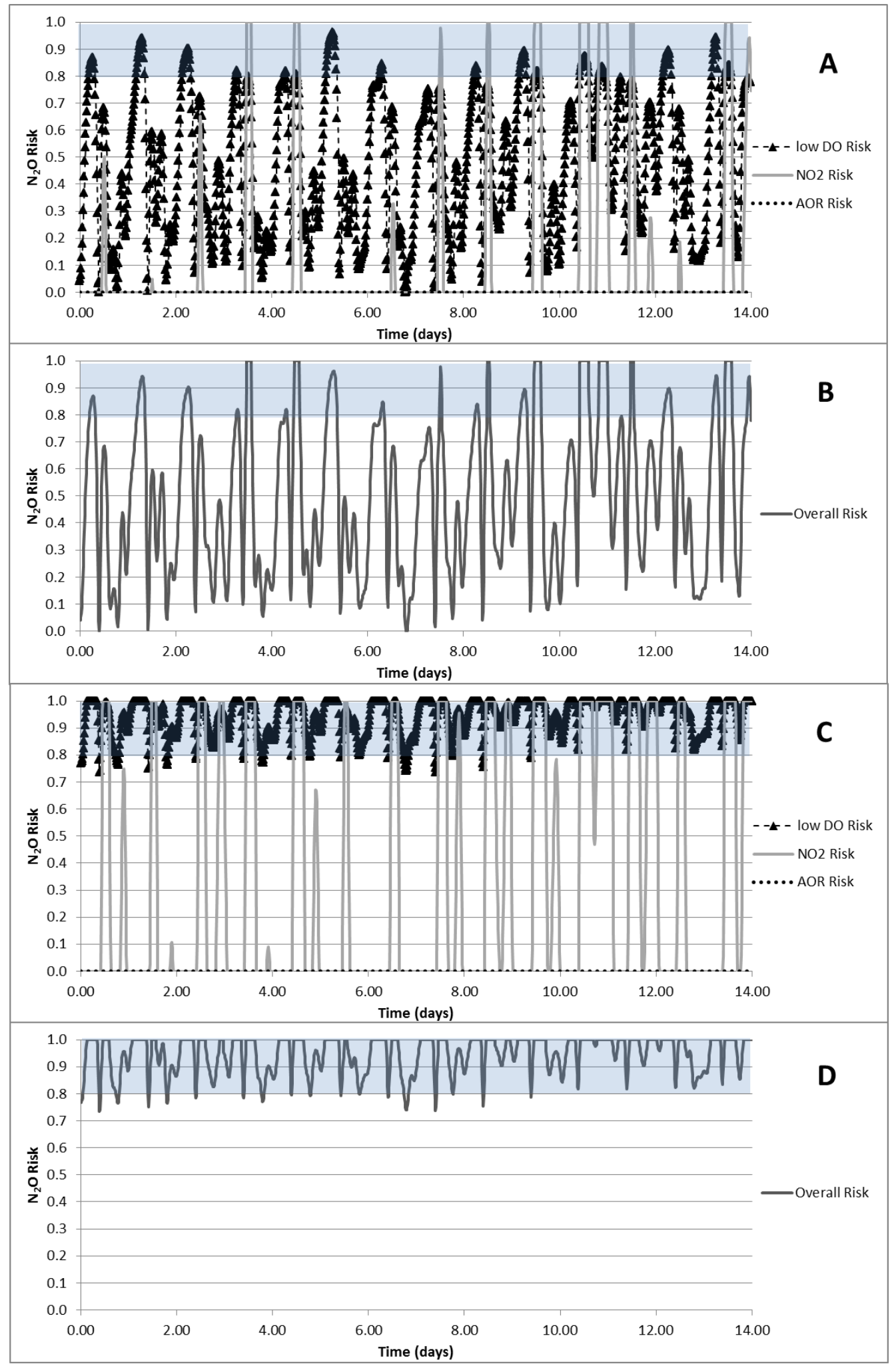

Figure 1 Dynamic $\mathrm{N}_{2} \mathrm{O}$ risk results for ASU5: Scenario 1_DO2 individual (A) and overall (B) risks, and Scenario 2_DO1.3 individual (C) and overall (D) risks. High risk $(\geq 0.8)$ is shaded in grey. 


\section{REFERENCES}

Ahn, J.H., Kim, S., Park, H., Rahm, B., Pagilla, K., Chandran, K. (2010). N $\mathrm{N}_{2} \mathrm{O}$ Emissions from Activated Sludge Processes, 2008a-2009: Results of a National Monitoring Survey in the United States. Environ. Sci. Technol., 44 (12), 4505-11.

Chandran, K., Stein, L., Klotz, M., van Loosdrecht, M. (2011) Nitrous Oxide Production by Lithotrophic Ammonia-Oxidizing Bacteria and Implications for Engineered Nitrogen-Removal Systems. Biochemical Society Transactions, 39, 1832-1837.

Comas, J., Rodriguez-Roda, I., Gernaey, K.V., Rosen, C., Jeppsson, U., Poch, M. (2008). Risk Assessment Modeling of MicrobiologyRelated Solids Separation Problems in Activated Sludge Systems. Environmental Modeling \& Software., 23, $1250-1261$.

Corominas L., Flores-Alsina X., Snip L. and Vanrolleghem P.A. (2012 ). Comparison of Different Modelling Approaches to Better Understand Greenhouse Gas Emissions from Wastewater Treatment Plants. Biotechnol. Bioeng., 109 (11), $2855-2863$.

Foley, J., de Haas, D., Yuan, Z., Lant, P. (2010). Nitrous Oxide Generation in Full-Scale Biological Nutrient Removal Wastewatertreatment Plants. Water Res., 44, 831-844.

Global Water Research Coalition (GWRC) (2011). $\mathrm{N}_{2} \mathrm{O}$ and $\mathrm{CH}_{4}$ Emission from Wastewater Collection and Treatment Systems State of the Science Report: Report of the GWRC Research Strategy Workshop, Montreal, Canada, September 2010, Report 29.

Guo L., Porro, J., Sharma, K., Amerlinck, A., Benedetti, L., Nopens, I., Shaw, A., Van Hulle, S.W.H., Yuan, Z., and Vanrolleghem, P.A. (2012). Towards a benchmarking tool for minimizing wastewater utility greenhouse gas footprints. Water Sci. Technol., 66(11), $2483-2495$.

Guo L. and Vanrolleghem, P.A. (2013). Calibration and Validation of an Activated Sludge Model for Greenhouse Gases No. 1 (ASMG1): Prediction of Temperature-Dependent $\mathrm{N}_{2} \mathrm{O}$ Emission Dynamics. Bioprocess Biosyst. Eng., DOI 10.1007/s00449-013-0978-3.

Hanaki, K., Chalermraj, W., and Shinichiro, O. (1990). Nitrification at low levels of dissolved oxygen with and without organic loading in a suspended-growth reactor. Water Res., 24, 297-302.

Houweling, D., Dold, P., Wunderlin, P., Joss, A., Siegrist, H. (2011). N ${ }_{2}$ O Emissions: Impact of Process Configuration and Diurnal Loading Patterns. In: Proceedings of IWA/WEF Nutrient Recovery and Management 2011 Conference, Miami, FL (USA), 9-12 January 2011.

Kampschreur, M.J.; Temmink, H.; Kleerebezem, R.; Jetten, M.S.M.; van Loosdrecht, M.C.M. (2009). Nitrous Oxide Emission During Wastewater Treatment. Water Res., 43(17), 4093-4103.

Law, Y., Ni, B. J., Lant, P., Yuan, Z. (2012). Nitrous oxide (N2O) production by an enriched culture of ammonia oxidizing bacteria depends on its ammonia oxidation rate. Water Res., 46 (10) 3409-3419.

Mampaey K.E., Beuckels B, Kampschreur M.J., Kleerebezem R, van Loosdrecht M.C.M. and Volcke E.I.P. (2013). Modelling nitrous and nitric oxide emissions by autotrophic ammonia-oxidizing bacteria. Environ. Technol., 34(12), 1555-1566.

Mota, C., Head, M., Ridenoure, J., Cheng, J., de los Reyes, F. (2005). Effects of Aeration Cycles on Nitrifying Bacterial Populations and Nitrogen Removal in Intermittently Aerated Reactors. Appl. Environ. Microbiol., 71 (12), 8565-8572.

Ni, B.J.,Ruscalleda, M., Pellicer-Nacher, C., Smets, B.F. (2011). Modeling Nitrous Oxide Production During Biological Nitrogen Removal via Nitrification and Denitrification: Extensions to the Genreal ASM Models. Environ. Sci. Technol., 45, 7768-7776.

Ni, B.J., Yuan, Z., Chandran, K., Vanrolleghem, P.A., Murthy, S. (2012). Evaluating Mathematical Models for $\mathrm{N}_{2} \mathrm{O}$ Production by Ammonia-Oxidising Bacteria: Towards a Unified Model. In: Proceedings: 3rd IWA/WEF Wastewater Treatment Modelling Seminar, WWTmod 2012, Mont-Sainte-Anne, Quebec, Canada, 26-28 February 2012.

Ni, B.J.,Ye, L., Law, Y., Byers, C., Yuan, Z. (2013). Mathematical Modeling of Nitrous Oxide ( $\left.\mathrm{N}_{2} \mathrm{O}\right)$ Emissions from Full-Scale Wastewater Treatment Plants. Environ. Sci. Technol., 47 (14), 7795-7803.

Nopens I., Benedetti L., Jeppsson U., Pons M.-N., Alex J., Copp J., Gernaey K., Rosen C., Steyer J.-P. and Vanrolleghem P.A. (2010). Benchmark Simulation Model No 2 - Finalisation of plant layout and default control strategy. Water Sci. Technol., 62(9), 1967 1974.

Porro, J., Guo, L., Sharma, K., Benedetti, L., Van Hulle, S.W.H., Vanrolleghem, P.A., Amerlinck, Y., Yuan, Z., Shaw, A., and Nopens, I. (2011). Towards a benchmarking tool for minimizing wastewater utility greenhouse gas footprints. In: Proceedings: 8th IWA Symposium on Systems Analysis and Integrated Assessment, Watermatex 2011, San Sebastian, Spain, 20 - 22 June 2011.

Tallec, G., Garnier, J., Billen, G., Gousailles, M., 2008. Nitrous oxide emissions from denitrifying activated sludge of urban wastewater treatment plants, under anoxia and low oxygenation. Bioresource Technol. 99 (7), 2200-2209.

Yu, R., Kampschreur, M. J., Loosdrecht, M. C. M. V., Chandran, K. (2010). Mechanisms and Specific Directionality of Autotrophic Nitrous Oxide and Nitric Oxide Generation during Transient Anoxia. Environ. Sci. Technol., 44, 1313-1319. 


\title{
Estimation of apparent nitrification kinetics as the key for reliable greenhouse gas emission prediction
}

\author{
Haydee De Clippeleir ${ }^{1}$, Imre Takacs ${ }^{2}$, Bernhard Wett ${ }^{3}$, Kartik Chandran ${ }^{1}$ and Sudhir Murthy ${ }^{4}$ \\ ${ }^{1}$ Columbia University, New York City, NY, USA (Email: $\underline{h d 2296 @ \text { columbia.edu) }}$ \\ 2Dynamita (Email: imre@dynamita.com) \\ ${ }^{3}$ ARAconsult (email:wett@araconsult.at \\ ${ }^{4}$ DC Water, Washington DC, USA (Email: Sudhir.Murthy@dcwater.com )
}

\begin{abstract}
This paper offers a modeling approach for estimation of greenhouse gas emissions based on apparent differential AOB-NOB rates estimation and NirK kinetics as the main key parameters. This simplified greenhouse gas model was calibrated with data obtained from a full-scale experiment on the mainstream deammonification stage at the Strass WWTP showing increased $\mathrm{N}_{2} \mathrm{O}$ emissions at higher DO set-points. The latter was directly correlated with the increased AOB-NOB differential at higher DO set-points resulting in increased nitrite accumulation.
\end{abstract}

\section{INTRODUCTION}

The formation of $\mathrm{N}_{2} \mathrm{O}$ and $\mathrm{NO}$ is considered complex and difficult to predict due to the interplay of many possible factors, contributors and mechanisms within the contributors. As aerobic ammonium oxidizing bacteria (AOB) are believed to be the main contributors to the $\mathrm{N}_{2} \mathrm{O}$ and $\mathrm{NO}$ emissions in wastewater treatment plants (Chandran et al. 2011, Ni et al. 2013), the main focus in research, measuring campaigns and modeling studies goes to understanding the $\mathrm{AOB}$ mechanisms of $\mathrm{NO} / \mathrm{N}_{2} \mathrm{O}$ production in detail. Two main routes are distinguished in those studies and referred to as the forward (through $\mathrm{NH}_{2} \mathrm{OH}$ ) and reverse (through nitrite) route. The forward route is mainly linked to peaks in ammonium loading, while the reverse route is mainly dependent on nitrite accumulation as shown in pure or enriched AOB cultures (Chandran et al. 2011).

Simulation of $\mathrm{N}_{2} \mathrm{O}$ emissions based on the different routes studied in these detailed AOB studies becomes quite complex especially as the relative contributions of both routes and its kinetics are not known and calibration could be difficult as measurements of all possible intermediates and gene expressions in real systems is scarce and difficult to accomplish. In this study we propose a simplified approach based on apparent rate differential estimation between $\mathrm{AOB}$ and nitrite oxidizers (NOB) considering nitrite as the main precursor for $\mathrm{N}_{2} \mathrm{O}$ emission. Model calibration was done based on greenhouse emission data obtained from the full-scale plant in Strass. In this system mainstream deammonification was applied and greenhouse gas emissions were deliberately increased by increasing the dissolved oxygen (DO) set-point and nitrogen loading (addition of filtrate) resulting in higher nitrite accumulation.

\section{EXPERIMENT}

Greenhouse gas emissions were measured on the B-stage of the Strass WWTP (Austria) in which a full-scale mainstream deammonification trial was taking place at that moment (Wett et al. 2012). During normal operations the B-stage was operated with a fully anoxic predenitrification stage followed by a nitrification stage (carrousel) at a DO set-point at the end of the aeration zones of $2 \mathrm{mg} \mathrm{O} / \mathrm{L}$ (reactors Naer3b and Naer4b in schematic Figure 4). The plant was fed with $6000 \mathrm{~kg} \mathrm{COD} / \mathrm{d}$ and $560 \mathrm{~kg} \mathrm{~N} / \mathrm{d}$ (raw sewage). To test the relation between the operational conditions and the greenhouse gas emissions, the DO set-point in the 
nitrification zone was changed for $2.5 \mathrm{~h}$ to 1,3 and $2 \mathrm{mg} \mathrm{O}_{2} / \mathrm{L}$ subsequently. At DO set-points of 3 and $2 \mathrm{mg} \mathrm{O}_{2} / \mathrm{L}$ the nitrogen load was increased with $10 \%$ by addition of filtrate to the mainstream reactor. The latter allowed for continuous aeration during the testing (NH4-based control). Oxygen profiles within the nitrification carrousel are given in Table 1.

Table 1: Observed DO profiles in the nitrification carrousel at different DO set-points

\begin{tabular}{lcccccccc}
\hline $\begin{array}{l}\text { DO } \\
\text { set-point }\end{array}$ & \multicolumn{7}{c}{ Observed DO concentration $\left(\mathbf{m g} \mathbf{O}_{2} / \mathbf{L}\right)$} \\
& Nanox3a & Nanox3b & Naer3a & Naer3b & Nanox4a & Nanox4b & Naer4a & Naer4b \\
\hline 1 & 0.7 & 0.1 & 0.4 & $\mathbf{1}$ & 0.7 & 0.1 & 0.4 & $\mathbf{1}$ \\
2 & 0.8 & 0.15 & 0.7 & $\mathbf{2}$ & 0.8 & 0.15 & 0.7 & $\mathbf{2}$ \\
3 & 2 & 1.2 & 2 & $\mathbf{3}$ & 2 & 1.2 & 2 & $\mathbf{3}$ \\
\hline
\end{tabular}

\section{RESULTS AND DISCUSSION}

\section{Why is the apparent differential $A O B-N O B$ rate the key parameter?}

\section{Forward $\mathrm{N}_{2} \mathrm{O}$ route}

High ammonium peak loadings are described as triggers for $\mathrm{N}_{2} \mathrm{O}$ production and believed to happen through the forward pathway. However, ammonium peak loadings will also results in a dynamic nitrite accumulation due to a larger differential between AOB and NOB rates at high ammonium residual levels (Figure 1). Therefore, the reverse $\mathrm{N}_{2} \mathrm{O}$ pathway starting from this accumulated nitrite could play a more important role than described right now, especially as hydroxylamine accumulation remains very minor. No hydroxylamine could be detected in the B-stage of the WWTP in Strass (Austria) under different loading conditions and ammonium residual concentrations (data not shown).

\section{Reverse $\mathrm{N}_{2} \mathrm{O}$ route}

The reverse pathway is described to be mainly dependent on oxygen conditions (Chandran et al 2011). Measurements of $\mathrm{NO}$ and $\mathrm{N}_{2} \mathrm{O}$ emissions in the mainstream B-stage of the wastewater treatment plant in Strass (Austria) showed increased emissions when operating at higher dissolved oxygen (DO) concentrations, while you would expect the opposite based on Chandran et al. (2011) (Figure 2). This effect could be explained by a higher nitrite accumulation at higher DO compared to lower DO operation ( 0.5 compared to $4 \mathrm{mg} \mathrm{NO} \mathrm{N}_{2} \mathrm{~N} / \mathrm{L}$ at 1 and $3 \mathrm{mg} \mathrm{O} / \mathrm{L}$, respectively) as the apparent oxygen affinity coefficient for AOB was higher than for NOB and the differential in rates happens mainly at high DO (Figure 2).
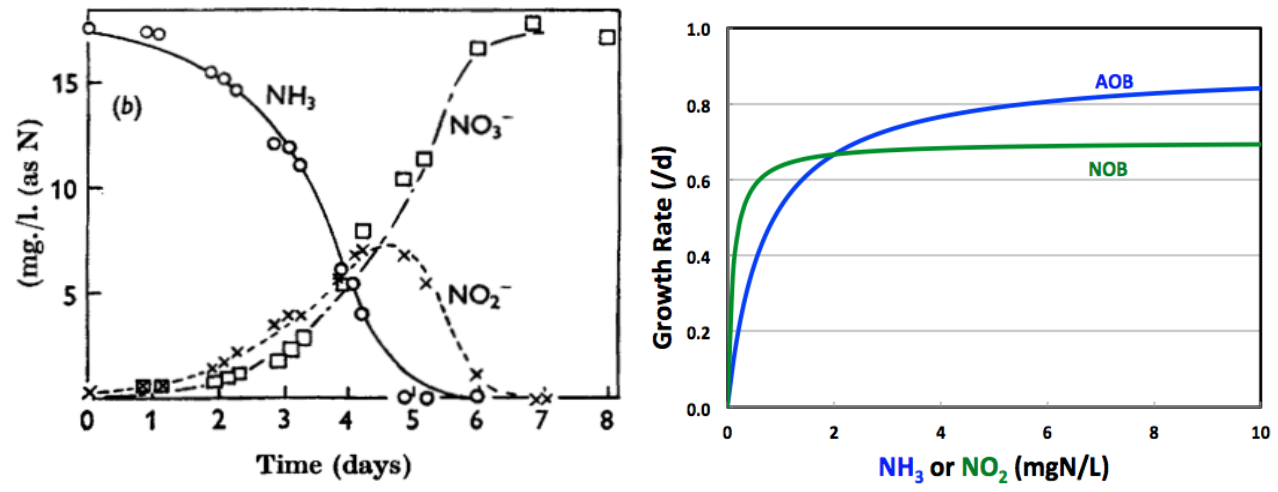

Figure 1: Simulation of Monod kinetics of $\mathrm{AOB}$ and NOB explains the nitrite accumulation at higher ammonium residual concentration (Downing et al 1964) which represents ammonium peak loading conditions (based on WEFTEC presentation of Dold P., 2012) 

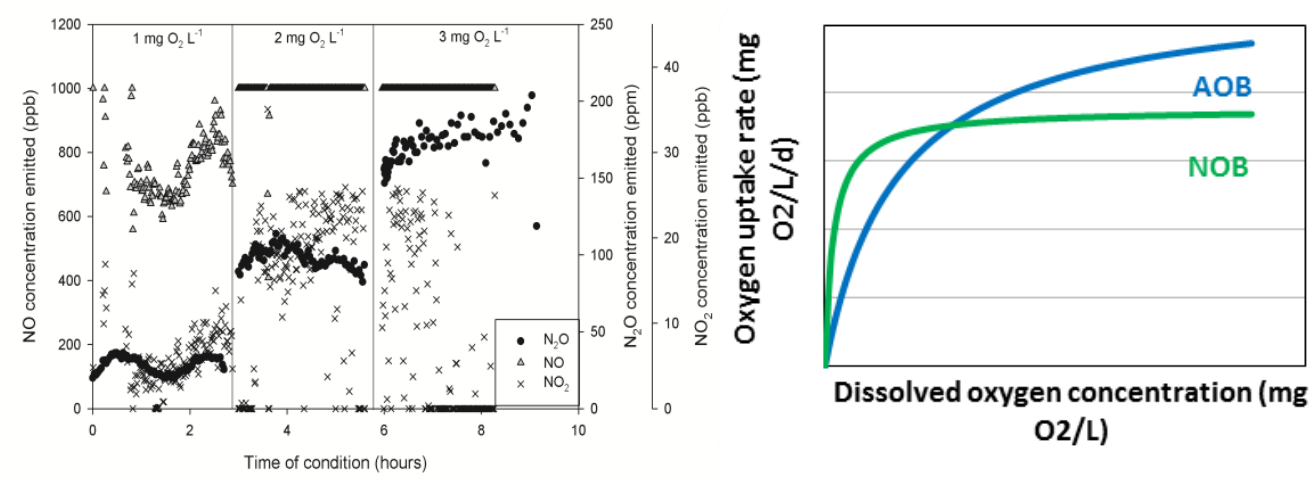

Figure 2: left: Increased levels of $\mathrm{N}_{2} \mathrm{O}$ emission going from low $\left(1 \mathrm{mg} \mathrm{O}_{2} / \mathrm{L}\right)$ to high $\mathrm{DO}(3 \mathrm{mg} \mathrm{O} / \mathrm{L})$ operation in the B-stage of the WWTP in Strass (Austria), right: Simulation of AOB and NOB kinetics measured at that time in the full-scale reactor showing increased differential rate between AOB and NOB at high DO.

\section{Nitrite/NirK kinetics as the basis of $\mathrm{N}_{2} \mathrm{O} / \mathrm{NO}$ formation}

The key genes in the $\mathrm{N}_{2} \mathrm{O}$ pathway starting from nitrite (reverse route) are nitrite reductase (NirK) and nitric oxide reductase (Nor). Expression of NirK is dependent on nitrite levels, ammonium presence and oxygen levels ( $\mathrm{Yu}$ et al. 2010). The nitrite concentration dependency, which is the results of the differential AOB-NOB rates, was determined in a batch experiment. The nitrite affinity coefficient for $\mathrm{NO}$ and $\mathrm{N}_{2} \mathrm{O}$ production was observed to be $1 \mathrm{mg} \mathrm{NO} \mathrm{N}_{2} \mathrm{~N} / \mathrm{L}$ in the presence of ammonium and transient anoxia operation (Figure 3). Besides nitrite levels, implementation of an oxygen switch for NirK expression simulating the first order decay of nirK during aerobic conditions is often needed to explain the decrease in $\mathrm{N}_{2} \mathrm{O} / \mathrm{NO}$ emission after the transition from anoxic to aerobic (Yu et al 2010, Wett 2012) and was therefore incorporated in the model.

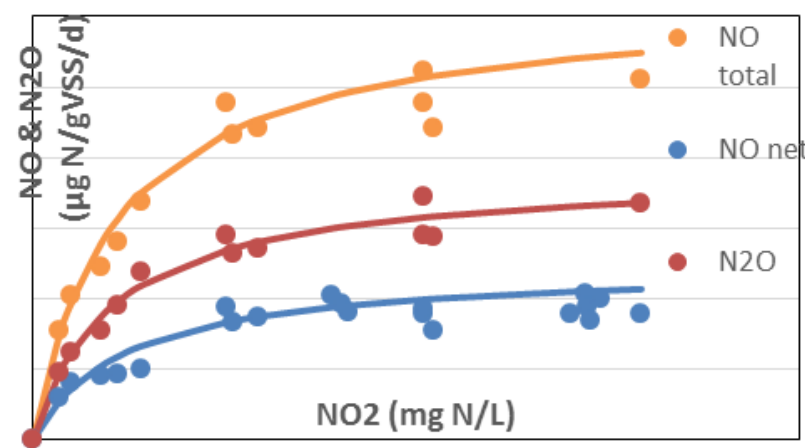

Figure 3: Left: Monod model fit for $\mathrm{NO}$ and $\mathrm{N}_{2} \mathrm{O}$ specific production rate of B-stage sludge from Blue Plains advanced WWTP (Washington DC) depending on the nitrite concentration in the system during transient anoxia (10/10 minutes) and in the presence of excess ammonium (10 $\mathrm{mg} \mathrm{N} / \mathrm{L})$.

\section{GHG Model}

The Strass plant (Austria) was modelled using the same 4-step nitrification/denitrification and GHG model (Sumo-N in the Sumo simulator) as described in the GHG workshop at WWTMod 2012. The model contains the Hyatt and Grady (2008) heterotrophic denitrification model and the autotrophic denitrification concept (Mampaey et al. 2013), extended by the nirK enzyme indicator (Table 2). The latter concept was originally based on the observation that during transition periods from anoxic to aerobic conditions GHGemissions first tend to increase and then at continued aeration concentrations decrease. Enzyme measurements ( $\mathrm{Yu}$ et al. 2010) indicated during periods of nitrite availability a corresponding accumulation specifically of NirK known to catalyse NO-formation and and a 
more pronounced depletion of this enzym at increasing DO concentrations. The enzymactivity growth- and decay-approach is considered as a method to describe the dynamics of NO-formation depending on preceding process conditions. The calibration consisted of setting up the plant configuration with the proper loading and DO values, and adjusting half saturation values in order to obtain the right $\mathrm{N}_{2} \mathrm{O}$ release proportions (Table 3 ).

Table 2: Rate expressions used in the model to describe the kinetics for $\mathrm{NO}$ and $\mathrm{N}_{2} \mathrm{O}$ production by $\mathrm{AOB}$

\begin{tabular}{|c|c|}
\hline $\begin{array}{l}\text { Processes } \\
\text { by AOB }\end{array}$ & Rate expression \\
\hline $\mathrm{NO}_{2} \rightarrow \mathrm{NO}$ & $\mu_{\mathrm{AOB}} * \mathrm{X}_{\mathrm{AOB}} * \operatorname{Msat}\left(\mathrm{S}_{\mathrm{O} 2} ; \mathrm{k}_{\mathrm{O} 2, \mathrm{AOB}}\right) * \operatorname{Msat}\left(\mathrm{S}_{\mathrm{NH}} ; \mathrm{k}_{\mathrm{NHx}, \mathrm{nut}}\right) * \eta_{\mathrm{NO} 2, \mathrm{AOB}} * \mathrm{X}_{\mathrm{ENZ}, \mathrm{AOB}} * \mathrm{Msat}\left(\mathrm{S}_{\mathrm{NO} 2} ; \mathrm{k}_{\mathrm{NO} 2, \mathrm{AOB}}\right)$ \\
\hline $\mathrm{NO} \rightarrow \mathrm{N}_{2} \mathrm{O}$ & $\mu_{\mathrm{AOB}} * \mathrm{X}_{\mathrm{AOB}} * \operatorname{Msat}\left(\mathrm{S}_{\mathrm{O} 2} ; \mathrm{k}_{\mathrm{O} 2, \mathrm{AOB}}\right) * \operatorname{Msat}\left(\mathrm{S}_{\mathrm{NH}} ; \mathrm{k}_{\mathrm{NHx}, \mathrm{nut}}\right) * \eta_{\mathrm{NO}, \mathrm{AOB}} * \operatorname{Msat}\left(\mathrm{S}_{\mathrm{NO}} ; \mathrm{k}_{\mathrm{NO}, \mathrm{AOB}}\right)$ \\
\hline Enzyme activation & $\begin{array}{l}\mu_{\mathrm{ENZ}, \mathrm{AOB}} * \mathrm{X}_{\mathrm{AOB}} * \operatorname{Msat}\left(\mathrm{S}_{\mathrm{NO} 2} ; \mathrm{k}_{\mathrm{NO} 2, \mathrm{AOBENZ}}\right) * \operatorname{Max}\left(0 ;\left(1-\mathrm{X}_{\mathrm{ENZ}, \mathrm{AOB}}\right) /\left(\left(1-\mathrm{X}_{\mathrm{ENZ}, \mathrm{AOB}}\right)+\mathrm{k}_{\mathrm{scaling}}\right)\right) * \\
\left(\mathrm{k}_{\mathrm{scaling}}+1\right)\end{array}$ \\
\hline Enzyme decay & $\mathrm{b}_{\mathrm{ENZ}, \mathrm{AOB}} * \mathrm{Msat}\left(\mathrm{X}_{\mathrm{ENZ}, \mathrm{AOB}} ; \mathrm{k}_{\mathrm{ENZ}, \mathrm{AOB}}\right) * \mathrm{Msat}\left(\mathrm{S}_{\mathrm{O} 2} ; \mathrm{k}_{\mathrm{O} 2, \mathrm{AOBENZ}}\right)$ \\
\hline
\end{tabular}

Table 3: Parameters used in the GHG model for the Strass case study.

\begin{tabular}{|c|c|c|c|}
\hline Parameter & Symbol & Value & Unit \\
\hline $\mathrm{O}_{2}$ half saturation coefficient for AOB & $\mathrm{k}_{\mathrm{O} 2, \mathrm{AOB}}$ & 0.4 & $\mathrm{mg} \mathrm{O}_{2} / \mathrm{L}$ \\
\hline $\mathrm{O}_{2}$ half saturation coefficient for NOB & $\mathrm{k}_{\mathrm{O} 2 \mathrm{NOB}}$ & 0.4 & $\mathrm{mg} \mathrm{O}_{2} / \mathrm{L}$ \\
\hline $\mathrm{O}_{2}$ inhibition coefficient $\mathrm{OHO}$ & $\mathrm{k}_{\mathrm{O} 2 \mathrm{OHO}}$ & 0.05 & $\mathrm{mg} \mathrm{O}_{2} / \mathrm{L}$ \\
\hline $\mathrm{NO}_{2}$ half saturation coefficient for $\mathrm{AOB}$ & $\mathrm{k}_{\mathrm{NO} 2, \mathrm{AOB}}$ & 0.2 & $\mathrm{mg} \mathrm{NO} 2-\mathrm{N} / \mathrm{L}$ \\
\hline $\mathrm{NO}_{2}$ half saturation coefficient for $\mathrm{NOB}$ & $\mathrm{k}_{\mathrm{NO} 2, \mathrm{NOB}}$ & 0.2 & $\mathrm{mg} \mathrm{NO}{ }_{2}-\mathrm{N} / \mathrm{L}$ \\
\hline $\mathrm{NO}_{2}$ half saturation coefficient for $\mathrm{OHO}$ & $\mathrm{k}_{\mathrm{NO} 2, \mathrm{OHO}}$ & 1 & $\mathrm{mg} \mathrm{NO}{ }_{2}-\mathrm{N} / \mathrm{L}$ \\
\hline NO half saturation coefficient for AOB & $\mathrm{k}_{\mathrm{NO}, \mathrm{AOB}}$ & 0.001 & mg NO-N/L \\
\hline $\mathrm{NO}$ half saturation coefficient for $\mathrm{OHO}$ & $\mathrm{k}_{\mathrm{NO}, \mathrm{OHO}}$ & 0.001 & mg NO-N/L \\
\hline $\mathrm{N}_{2} \mathrm{O}$ half saturation coefficient for $\mathrm{OHO}$ & $\mathrm{k}_{\mathrm{N} 2 \mathrm{O}, \mathrm{OHO}}$ & 0.1 & $\mathrm{mg} \mathrm{N}_{2} \mathrm{O}-\mathrm{N} / \mathrm{L}$ \\
\hline $\mathrm{NO}_{2}$ nirK enzyme half saturation for $\mathrm{AOB}$ & $\mathrm{k}_{\mathrm{NO} 2, \mathrm{AOBENZ}}$ & 0.1 & $\mathrm{mg} \mathrm{NO}-\mathrm{N} / \mathrm{L}$ \\
\hline $\mathrm{O}_{2}$ half saturation for enzyme decay in AOB & $\mathrm{k}_{\mathrm{O} 2, \mathrm{AOBENZ}}$ & 0.1 & $\mathrm{mg} \mathrm{O}_{2} / \mathrm{L}$ \\
\hline Maximum enzyme activation rate & $\mu_{\mathrm{ENZ,AOB}}$ & 1 & $\mathrm{~d}^{-1}$ \\
\hline Correction factor for $\mathrm{NO}$ production by $\mathrm{AOB}$ & $\eta_{\mathrm{NO} 2, \mathrm{AOB}}$ & 1 & - \\
\hline Correction factor for $\mathrm{N}_{2} \mathrm{O}$ production by $\mathrm{AOB}$ & $\eta_{\mathrm{NO}, \mathrm{AOB}}$ & 1 & - \\
\hline Maximum growth rates for $\mathrm{AOB}$ & $\mu_{\mathrm{AOB}}$ & 0.9 & $d^{-1}$ \\
\hline Maximum growth rates for NOB & $\mu_{\mathrm{NOB}}$ & 0.7 & $d^{-1}$ \\
\hline Maximum growth rates for $\mathrm{OHO}$ & $\mu_{\mathrm{OHO}}$ & 6 & $\mathrm{~d}^{-1}$ \\
\hline
\end{tabular}

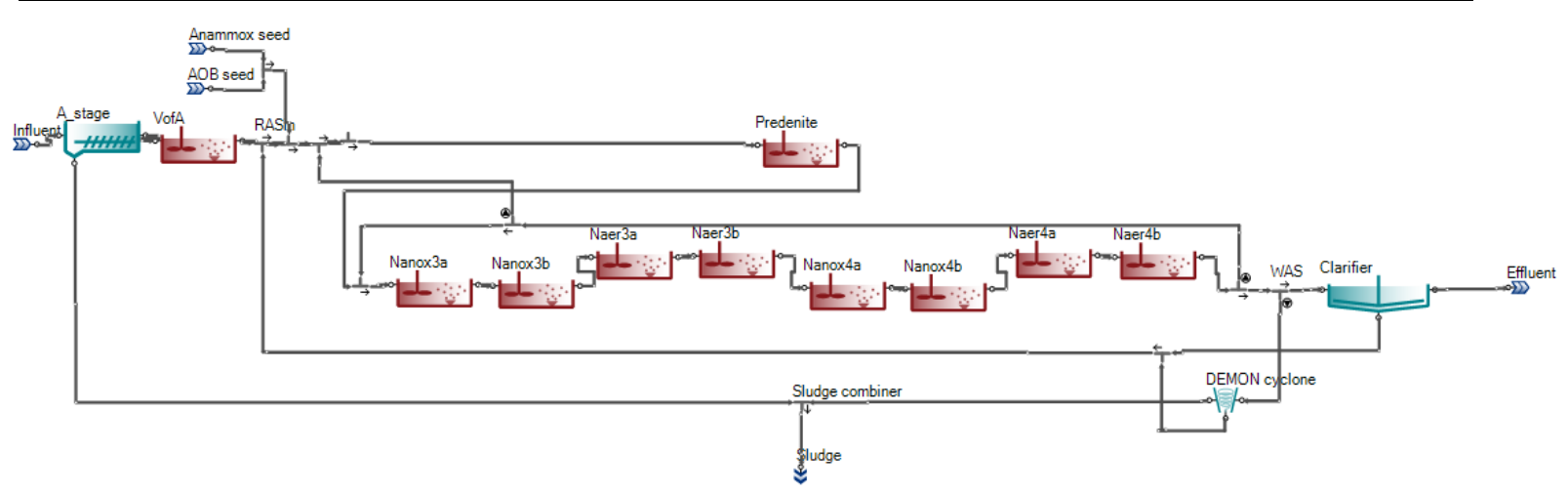

Figure 4: Sumo configuration of the Strass Mainstream Deammonification process

Oxygen profiles were calibrated by adjusting the $\mathrm{kLa}_{\mathrm{O} 2}$ to 240,280 and $350 \mathrm{~d}^{-1}$ for DO setpoints 1.2 and $3 \mathrm{mg} \mathrm{O} / \mathrm{L}$. Due to the fact that this experiment was done at full scale (less defined boundaries) and due to the complexity of NOB out-selection during mainstream 
deammonification (Al-Omari et al. 2014) modeled nitrite accumulation values were slightly lower than the observed values. Nitrite concentrations of $0.3,1.3$ and $1.7 \mathrm{mg} \mathrm{NO} \mathrm{N}_{2} \mathrm{~N} / \mathrm{L}$ were obtained by the model at 1,2 and $3 \mathrm{mg} \mathrm{O} / 2$, respectively, while an increase of 0.5 to $4 \mathrm{mg}$ $\mathrm{NO}_{2}-\mathrm{N} / \mathrm{L}$ was observed during the experiment. Although absolute nitrite levels were lower, the model showed the stepwise increase in $\mathrm{N}_{2} \mathrm{O}$ emission with increasing DO set-point and thus increasing AOB-NOB differential (Figure 5).

It should be noted that the $\mathrm{N}_{2} \mathrm{O}$ emissions obtained in this experiment were very high $(3,9$ and $15 \%$ of $\mathrm{N}$ load at 1,2 and $3 \mathrm{mg} \mathrm{O}_{2} / \mathrm{L}$ ) and were enhanced on purpose during this experiment. It was not the attempt of this experiment to minimize emission but rather test potential conditions which would increase the emissions. Moreover, no adaptation of the system towards the transient condition applied in this experiment (oxygen and loading) was allowed which further maximized the potential effect. The emission observed in this experiment are therefore not expected during long term mainstream deammonification operation as optimal balances between NOB out-selection and anoxic nitrite removal by anammox or denitrification will decrease nitrite accumulation especially at the transition between anoxic to aerobic conditions. Moreover, adaptation can occur which will further decrease the emissions ( $\mathrm{Yu}$ et al 2010).

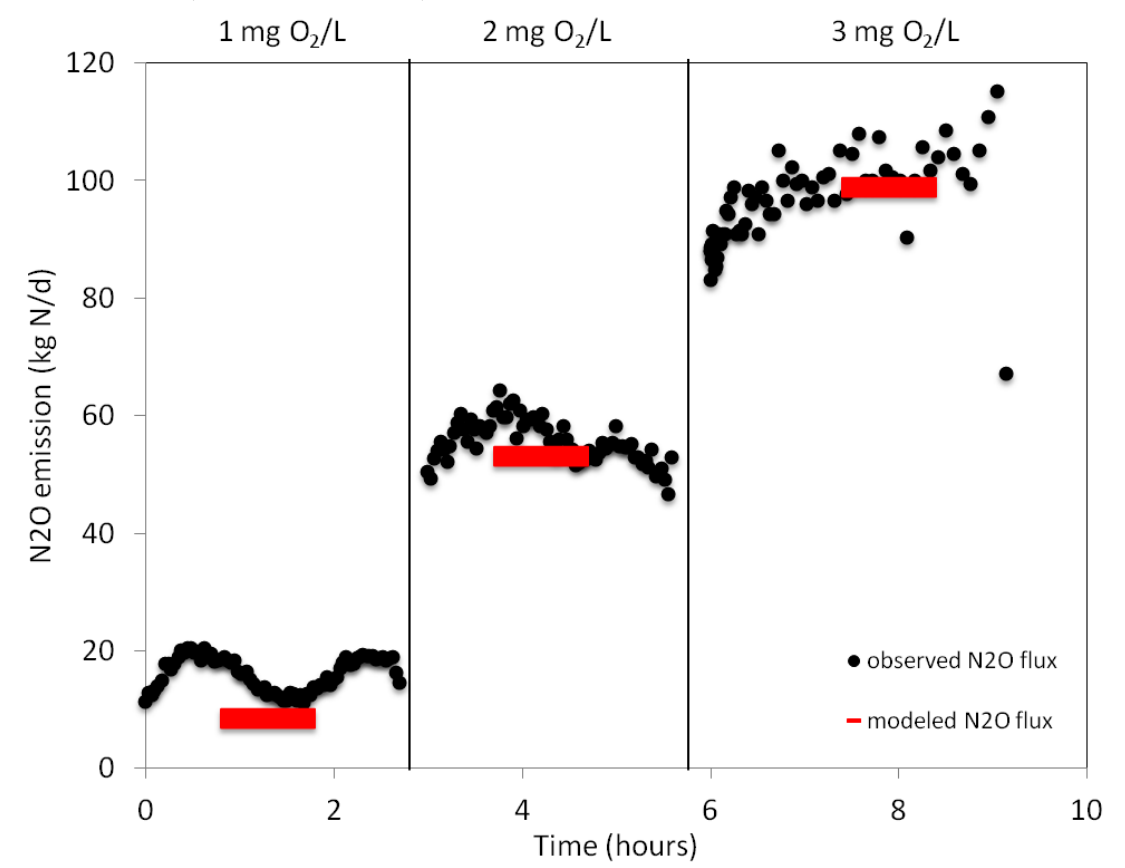

Figure 5: Modeled vs observed $\mathrm{N}_{2} \mathrm{O}$ emission at increasing DO set-points and subsequently higher nitrite accumulation

\section{CONCLUSION}

This study that with a simplified GHG model only focused on the reverse $\mathrm{N}_{2} \mathrm{O}$ pathways, increased $\mathrm{N}_{2} \mathrm{O}$ at higher DO concentrations and thus higher AOB-NOB differential could be predicted.

\section{REFERENCES}

Al-Omari, A. Wett, B., Murthy, S. De Clippeleir, H., Han, M., Regmi, P., Bott, C. and Nopens, I. (2014) Model based evaluation of mechanisms and benefits of nitrogen short-cut processes. WWTmod 2014.

Chandran, K., Stein, L.Y., Klotz, M.G. and van Loosdrecht, M.C.M. (2011) Nitrous oxide production by lithotrophic ammmonia-oxidizing bacteria and implications for engineered nitrogen removal systems. Biochamical Society Transactions 39(6), 1832-1837. 


\section{WWTmod}

Hiatt, W.C. and Grady, C.P. (2008) An updated process model for carbon oxidation, nitrification, and denitrification. Water Research 80(11), 2145-56

Mampaey, K., Beuckels, B., Kampschreur,M., Kleerebezem, R. Van Loosdrecht, M., Volcke, E. (2013)Modelling nitrous and nitric oxide emissions by autotrophic ammonia-oxidizing bacteria. Environmental Technology.

Ni, B-J, Ye, L., Law, Y., Byers, C., Yuan, Z. (2013). Mathematical Modeling of Nitrous Oxide (N2O) Emissions from Full-Scale Wastewater Treatment Plants. Environ. Sci. Technol. dx.doi.org/10.1021/es4005398

Shaw, A.R., Takács, I., Pagilla, K.R. and Murthy, S. (2013) "A new approach to assess the dependency of extant half-saturation coefficients on maximum process rates and estimate intrinsic coefficients" Water Research (In Press) 2013

B. Wett, A. Omari, S.M. Podmirseg, M. Han, S. Murthy, C. Bott, M. Hell, I. Takács, G. Nyhuis, M. Gómez Brandón, M. O’Shaughnessy (2012). Going for mainstream deammonification from bench- to full-scale for maximized resource efficiency. Water Science and Technology, 68/2, 283-289

Wett B., Chandran, K., Murthy, S., Takacs, I. (2012) Workshop presentation WWTmod 2012.

Yu, R., Kampschreur, M.J., van Loosdrecht, M.C.M and Chandran, K. (2010) Mechanisms and specific directionality of autotrophic nitrous oxide and nitric oxide generation during transient anoxia. Envir, Sci. Technol. 44(4), 1313-1319. 